Cochrane Database of Systematic Reviews

\title{
Interventions to prevent hypothermia at birth in preterm and/or low birth weight infants (Review)
}

McCall EM, Alderdice F, Halliday HL, Vohra S, Johnston L

McCall EM, Alderdice F, Halliday HL, Vohra S, Johnston L.

Interventions to prevent hypothermia at birth in preterm and/or low birth weight infants.

Cochrane Database of Systematic Reviews 2018, Issue 2. Art. No.: CD004210.

DOI: 10.1002/14651858.CD004210.pub5.

www.cochranelibrary.com 
TABLE OF CONTENTS

PLAIN LANGUAGE SUMMARY

SUMMARY OF FINDINGS

BACKGROUND

OBJECTIVES

METHODS

Figure 1.

RESULTS

Figure 2.

Figure 3.

Figure 4.

Figure 5.

DISCUSSION

\section{AUTHORS' CONCLUSIONS}

ACKNOWLEDGEMENTS

REFERENCES

CHARACTERISTICS OF STUDIES

DATA AND ANALYSES

Analysis 1.1. Comparison 1 Plastic wrap or bag versus routine care, Outcome 1 Core body temperature $\left({ }^{\circ} \mathrm{C}\right)$ on admission to NICU or up to 2 hours after birth.

Analysis 1.2. Comparison 1 Plastic wrap or bag versus routine care, Outcome 2 Hypothermia on admission to NICU: core body temperature $<36.5^{\circ} \mathrm{C}$ or skin temperature $<36^{\circ} \mathrm{C}$.

Analysis 1.3. Comparison 1 Plastic wrap or bag versus routine care, Outcome 3 Outside normothermic range on admission to NICU or up to 2 hours after birth.

Analysis 1.4. Comparison 1 Plastic wrap or bag versus routine care, Outcome 4 Core body temperature $\left({ }^{\circ} \mathrm{C}\right)$ at 1 hour after birth.

Analysis 1.5. Comparison 1 Plastic wrap or bag versus routine care, Outcome 5 Core body temperature $\left({ }^{\circ} \mathrm{C}\right)$ at 90 minutes after birth.

Analysis 1.6. Comparison 1 Plastic wrap or bag versus routine care, Outcome 6 Core body temperature $\left({ }^{\circ} \mathrm{C}\right)$ at 2 hours after birth.

Analysis 1.7. Comparison 1 Plastic wrap or bag versus routine care, Outcome 7 Core body temperature $\left({ }^{\circ} \mathrm{C}\right)$ post stabilisation. . Analysis 1.8. Comparison 1 Plastic wrap or bag versus routine care, Outcome 8 Hypothermia post stabilisation: core body temperature $<36.5^{\circ} \mathrm{C}$ or skin temperature $<36^{\circ} \mathrm{C}$.

Analysis 1.9. Comparison 1 Plastic wrap or bag versus routine care, Outcome 9 Outside normothermic range post stabilisation.

Analysis 1.10 . Comparison 1 Plastic wrap or bag versus routine care, Outcome 10 Core body temperature $\left({ }^{\circ} \mathrm{C}\right) 30$ minutes after initial NICU admission temperature was taken.

Analysis 1.11. Comparison 1 Plastic wrap or bag versus routine care, Outcome 11 Core body temperature $\left({ }^{\circ} \mathrm{C}\right) 1$ hour after initial NICU admission temperature was taken.

Analysis 1.12. Comparison 1 Plastic wrap or bag versus routine care, Outcome 12 Core body temperature $\left({ }^{\circ} \mathrm{C}\right) 90$ minutes after initial NICU admission temperature was taken.

Analysis 1.13. Comparison 1 Plastic wrap or bag versus routine care, Outcome 13 Core body temperature $\left({ }^{\circ} \mathrm{C}\right) 2 \mathrm{hours}$ after initial NICU admission temperature was taken.

Analysis 1.14. Comparison 1 Plastic wrap or bag versus routine care, Outcome 14 Hyperthermia on admission to NICU: core body temperature $>37.5^{\circ} \mathrm{C}$.

Analysis 1.15. Comparison 1 Plastic wrap or bag versus routine care, Outcome 15 Hyperthermia post stabilisation: core body temperature $\geq 37.5^{\circ} \mathrm{C}$

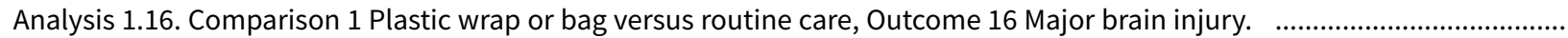
Analysis 1.17. Comparison 1 Plastic wrap or bag versus routine care, Outcome 17 Intraventicular haemorrhage (all grades). ... Analysis 1.18. Comparison 1 Plastic wrap or bag versus routine care, Outcome 18 Intraventricular haemorrhage (grades III and IV).

Analysis 1.19. Comparison 1 Plastic wrap or bag versus routine care, Outcome 19 Mortality (death within hospital stay or at 6 months' corrected gestation).

Analysis 1.20. Comparison 1 Plastic wrap or bag versus routine care, Outcome 20 Arterial oxygen saturation (percentage). ..... Analysis 1.21. Comparison 1 Plastic wrap or bag versus routine care, Outcome 21 Bicarbonate (mmol/L). 
Analysis 1.22. Comparison 1 Plastic wrap or bag versus routine care, Outcome $22 \mathrm{Blood}$ gas $\mathrm{pH}$ (first).

Analysis 1.23. Comparison 1 Plastic wrap or bag versus routine care, Outcome 23 Blood gas $\mathrm{pH}<7.25$.

Analysis 1.24. Comparison 1 Plastic wrap or bag versus routine care, Outcome 24 Blood glucose concentration (mmol/L) (first).

Analysis 1.25. Comparison 1 Plastic wrap or bag versus routine care, Outcome 25 Blood glucose $<2.6 \mathrm{mmol} / \mathrm{L}$.

Analysis 1.26. Comparison 1 Plastic wrap or bag versus routine care, Outcome $26 \mathrm{Blood}$ glucose $>6 \mathrm{mmol} / \mathrm{L}$.

Analysis 1.27. Comparison 1 Plastic wrap or bag versus routine care, Outcome 27 Blood glucose concentration mmol/L at 120 minutes after birth.

Analysis 1.28. Comparison 1 Plastic wrap or bag versus routine care, Outcome 28 Bronchopulmonary dysplasia (BPD).

Analysis 1.29. Comparison 1 Plastic wrap or bag versus routine care, Outcome 29 BPD steroids.

Analysis 1.30. Comparison 1 Plastic wrap or bag versus routine care, Outcome 30 Duration of hospitalisation (days).

Analysis 1.31. Comparison 1 Plastic wrap or bag versus routine care, Outcome 31 Duration of hospitalisation (days).

Analysis 1.32. Comparison 1 Plastic wrap or bag versus routine care, Outcome 32 Duration of continuous positive airway pressure (CPAP) (days).

Analysis 1.33. Comparison 1 Plastic wrap or bag versus routine care, Outcome 33 Duration of oxygen therapy (days). ............

Analysis 1.34. Comparison 1 Plastic wrap or bag versus routine care, Outcome 34 Duration of ventilation (days).

Analysis 1.35. Comparison 1 Plastic wrap or bag versus routine care, Outcome 35 Gastrointestinal perforation.

Analysis 1.36. Comparison 1 Plastic wrap or bag versus routine care, Outcome 36 Intubation in delivery room.

.....

Analysis 1.37. Comparison 1 Plastic wrap or bag versus routine care, Outcome 37 Necrotising enterocolitis (NEC).

Analysis 1.38. Comparison 1 Plastic wrap or bag versus routine care, Outcome 38 Patent ductus arteriosus (PDA).

Analysis 1.39. Comparison 1 Plastic wrap or bag versus routine care, Outcome 39 Pneumothorax.

Analysis 1.40. Comparison 1 Plastic wrap or bag versus routine care, Outcome 40 Pulmonary haemorrhage.

Analysis 1.41. Comparison 1 Plastic wrap or bag versus routine care, Outcome 41 Requirement for bubble continuous positive airway pressure (BCPAP).

Analysis 1.42. Comparison 1 Plastic wrap or bag versus routine care, Outcome 42 Requirement for ventilation. .......................

Analysis 1.43. Comparison 1 Plastic wrap or bag versus routine care, Outcome 43 Respiratory distress syndrome (RDS). .........

Analysis 1.44. Comparison 1 Plastic wrap or bag versus routine care, Outcome 44 Retinopathy of prematurity (ROP). .............

Analysis 1.45. Comparison 1 Plastic wrap or bag versus routine care, Outcome 45 Sepsis (late).

Analysis 1.46. Comparison 1 Plastic wrap or bag versus routine care, Outcome 46 Sepsis (early).

Analysis 2.1. Comparison 2 Plastic wrap versus routine care (interhospital neonatal transport), Outcome 1 Core body temperature $\left({ }^{\circ} \mathrm{C}\right)$ on admission to NICU or up to 2 hours after birth.

Analysis 2.2. Comparison 2 Plastic wrap versus routine care (interhospital neonatal transport), Outcome 2 Hypothermia on admission to NICU: core body temperature $<36.5^{\circ} \mathrm{C}$ or skin temperature $<36^{\circ} \mathrm{C}$.

Analysis 2.3. Comparison 2 Plastic wrap versus routine care (interhospital neonatal transport), Outcome 3 Decrease in temperature (axillary ${ }^{\circ} \mathrm{C}$ ) from baseline before transport to NICU admission.

Analysis 2.4. Comparison 2 Plastic wrap versus routine care (interhospital neonatal transport), Outcome 4 Hyperthermia on admission to NICU: core body temperature $>37.5^{\circ} \mathrm{C}$.

Analysis 2.5. Comparison 2 Plastic wrap versus routine care (interhospital neonatal transport), Outcome 5 Base excess. ........ Analysis 2.6. Comparison 2 Plastic wrap versus routine care (interhospital neonatal transport), Outcome 6 Blood gas pH. ...... Analysis 2.7. Comparison 2 Plastic wrap versus routine care (interhospital neonatal transport), Outcome 7 Duration of oxygen therapy (days).

Analysis 2.8. Comparison 2 Plastic wrap versus routine care (interhospital neonatal transport), Outcome 8 Hemo glucose test. .

Analysis 2.9. Comparison 2 Plastic wrap versus routine care (interhospital neonatal transport), Outcome 9 Hypoglycaemia (blood glucose level $<40 \mathrm{mg} / \mathrm{dL}$ within 2 hours of birth).

Analysis 2.10. Comparison 2 Plastic wrap versus routine care (interhospital neonatal transport), Outcome 10 Severe metabolic acidosis.

Analysis 3.1. Comparison 3 Plastic bag with previous drying versus routine care, Outcome 1 Core body temperature $\left({ }^{\circ} \mathrm{C}\right) 30$ minutes after birth.

Analysis 3.2. Comparison 3 Plastic bag with previous drying versus routine care, Outcome 2 Core body temperature $\left({ }^{\circ} \mathrm{C}\right) 1$ hour after birth.

Analysis 3.3. Comparison 3 Plastic bag with previous drying versus routine care, Outcome 3 Core body temperature $\left({ }^{\circ} \mathrm{C}\right) 90$ minutes after birth.

Analysis 3.4. Comparison 3 Plastic bag with previous drying versus routine care, Outcome 4 Core body temperature $\left({ }^{\circ} \mathrm{C}\right) 2$ hours after birth.

Analysis 3.5. Comparison 3 Plastic bag with previous drying versus routine care, Outcome 5 Hypothermia. 
Analysis 3.6. Comparison 3 Plastic bag with previous drying versus routine care, Outcome 6 Glucose concentration (mmol/L) at 120 minutes after birth.

Analysis 4.1. Comparison 4 Plastic cap versus routine care (no cap), Outcome 1 Core body temperature $\left({ }^{\circ} \mathrm{C}\right.$ ) on admission to NICU or up to 2 hours after birth.

Analysis 4.2. Comparison 4 Plastic cap versus routine care (no cap), Outcome 2 Hypothermia on admission to NICU: core body temperature $<36.4^{\circ} \mathrm{C}$.

Analysis 4.3. Comparison 4 Plastic cap versus routine care (no cap), Outcome 3 Outside normothermic range on admission to NICU or up to 2 hours after birth.

Analysis 4.4. Comparison 4 Plastic cap versus routine care (no cap), Outcome 4 Core body temperature $\left({ }^{\circ} \mathrm{C}\right) 1$ hour after initial NICU admission temperature was taken.

Analysis 4.5. Comparison 4 Plastic cap versus routine care (no cap), Outcome 5 Major brain injury.

Analysis 4.6. Comparison 4 Plastic cap versus routine care (no cap), Outcome 6 Mortality (death within hospital stay). ...........

Analysis 4.7. Comparison 4 Plastic cap versus routine care (no cap), Outcome 7 Bicarbonate (mmol/L).

Analysis 4.8. Comparison 4 Plastic cap versus routine care (no cap), Outcome 8 Blood gas pH (first).

Analysis 4.9. Comparison 4 Plastic cap versus routine care (no cap), Outcome 9 First serum glucose concentration (mmol/L) on admission to NICU.

Analysis 4.10. Comparison 4 Plastic cap versus routine care (no cap), Outcome 10 Intubation at birth.

Analysis 5.1. Comparison 5 Plastic bag and plastic cap versus routine care, Outcome 1 Core body temperature $\left({ }^{\circ} \mathrm{C}\right)$ on admission to NICU or up to 2 hours after birth.

Analysis 5.2. Comparison 5 Plastic bag and plastic cap versus routine care, Outcome 2 Core body temperature $\left({ }^{\circ} \mathrm{C}\right)$ at 10 th minute of life.

Analysis 5.3. Comparison 5 Plastic bag and plastic cap versus routine care, Outcome 3 Core body temperature $\left({ }^{\circ} \mathrm{C}\right)$ at 15 th minute of life.

Analysis 5.4. Comparison 5 Plastic bag and plastic cap versus routine care, Outcome 4 Core body temperature $\left({ }^{\circ} \mathrm{C}\right)$ at 30 th minute of life.

Analysis 5.5. Comparison 5 Plastic bag and plastic cap versus routine care, Outcome 5 Core body temperature $\left({ }^{\circ} \mathrm{C}\right)$ at 1 hour of life.

Analysis 5.6. Comparison 5 Plastic bag and plastic cap versus routine care, Outcome 6 Core body temperature $\left({ }^{\circ} \mathrm{C}\right) 1$ hour after initial NICU admission temperature was taken.

Analysis 5.7. Comparison 5 Plastic bag and plastic cap versus routine care, Outcome 7 Core body temperature $\left({ }^{\circ} \mathrm{C}\right) 2$ hours after initial NICU admission temperature was taken.

Analysis 5.8. Comparison 5 Plastic bag and plastic cap versus routine care, Outcome 8 Hyperthermia: core body temperature $>37.0^{\circ} \mathrm{C}$.

Analysis 5.9. Comparison 5 Plastic bag and plastic cap versus routine care, Outcome 9 Hyponatraemia (serum sodium concentration < $130 \mathrm{mmol} / \mathrm{L})$.

Analysis 5.10. Comparison 5 Plastic bag and plastic cap versus routine care, Outcome 10 Weight (grams) at fifth day of life. .... Analysis 6.1. Comparison 6 Plastic bag with previous drying versus plastic bag without previous drying, Outcome 1 Core body temperature $\left({ }^{\circ} \mathrm{C}\right) 30$ minutes after birth.

Analysis 6.2. Comparison 6 Plastic bag with previous drying versus plastic bag without previous drying, Outcome 2 Core body temperature $\left({ }^{\circ} \mathrm{C}\right) 1$ hour after birth.

Analysis 6.3. Comparison 6 Plastic bag with previous drying versus plastic bag without previous drying, Outcome 3 Core body temperature $\left({ }^{\circ} \mathrm{C}\right) 90$ minutes after birth.

Analysis 6.4. Comparison 6 Plastic bag with previous drying versus plastic bag without previous drying, Outcome 4 Core body temperature $\left({ }^{\circ} \mathrm{C}\right) 2$ hours after birth.

Analysis 6.5. Comparison 6 Plastic bag with previous drying versus plastic bag without previous drying, Outcome 5 Hyperthermia.

Analysis 6.6. Comparison 6 Plastic bag with previous drying versus plastic bag without previous drying, Outcome 6 Glucose concentration $(\mathrm{mmol} / \mathrm{L})$ at 2 hours after birth.

Analysis 7.1. Comparison 7 Plastic cap versus plastic bag, Outcome 1 Core body temperature $\left({ }^{\circ} \mathrm{C}\right)$ on admission to NICU or up to 2 hours after birth.

Analysis 7.2. Comparison 7 Plastic cap versus plastic bag, Outcome 2 Hypothermia on admission to NICU: core body temperature $<36.4^{\circ} \mathrm{C}$.

Analysis 7.3. Comparison 7 Plastic cap versus plastic bag, Outcome 3 Outside normothermic range on admission to NICU or up to 2 hours after birth.

Analysis 7.4. Comparison 7 Plastic cap versus plastic bag, Outcome 4 Core body temperature $\left({ }^{\circ} \mathrm{C}\right) 1$ hour after initial temperature on admission to NICU taken. 
Analysis 7.5. Comparison 7 Plastic cap versus plastic bag, Outcome 5 Hyperthermia on admission to NICU: core body temperature $>37.5^{\circ} \mathrm{C}$.

Analysis 7.6. Comparison 7 Plastic cap versus plastic bag, Outcome 6 Major brain injury.

Analysis 7.7. Comparison 7 Plastic cap versus plastic bag, Outcome 7 Mortality (death at discharge).

Analysis 7.8. Comparison 7 Plastic cap versus plastic bag, Outcome 8 Bicarbonate concentration (mmol/L).

Analysis 7.9. Comparison 7 Plastic cap versus plastic bag, Outcome 9 Blood gas $\mathrm{pH}$.

Analysis 7.10. Comparison 7 Plastic cap versus plastic bag, Outcome 10 First serum glucose concentration (mmol/L) on admission to NICU.

Analysis 7.11. Comparison 7 Plastic cap versus plastic bag, Outcome 11 Intubation at birth.

Analysis 8.1. Comparison 8 Plastic bag versus plastic wrap, Outcome 1 Core body temperature $\left({ }^{\circ} \mathrm{C}\right) 20$ minutes after birth. ..... Analysis 8.2. Comparison 8 Plastic bag versus plastic wrap, Outcome 2 Core body temperature $\left({ }^{\circ} \mathrm{C}\right) 40$ minutes after birth. ..... Analysis 8.3. Comparison 8 Plastic bag versus plastic wrap, Outcome 3 Core body temperature $\left({ }^{\circ} \mathrm{C}\right) 1$ hour after birth. ............ Analysis 8.4. Comparison 8 Plastic bag versus plastic wrap, Outcome 4 Decrease in core body temperature $\left({ }^{\circ} \mathrm{C}\right)$ during 1 hour after birth.

Analysis 8.5. Comparison 8 Plastic bag versus plastic wrap, Outcome 5 Hypothermia within 1 hour after birth: core body temperature $<36.5^{\circ} \mathrm{C}$ or skin temperature $<36^{\circ} \mathrm{C}$.

Analysis 8.6. Comparison 8 Plastic bag versus plastic wrap, Outcome 6 Moderate hypothermia within 1 hour after birth: core body temperature $32^{\circ} \mathrm{C}$ to $35.9^{\circ} \mathrm{C}$.

Analysis 8.7. Comparison 8 Plastic bag versus plastic wrap, Outcome 7 Outside normothermic range on admission to NICU or up to 2 hours after birth.

Analysis 9.1. Comparison 9 Plastic total body wrap (body + head) versus plastic body wrap (head uncovered), Outcome 1 Core body temperature $\left({ }^{\circ} \mathrm{C}\right)$ on admission to NICU.

Analysis 9.2. Comparison 9 Plastic total body wrap (body + head) versus plastic body wrap (head uncovered), Outcome 2 Hypothermia on admission to NICU: core body temperature $<36.5^{\circ} \mathrm{C}$.

Analysis 9.3. Comparison 9 Plastic total body wrap (body + head) versus plastic body wrap (head uncovered), Outcome 3 Mild hypothermia on admission to NICU: core body temperature $36^{\circ} \mathrm{C}$ to $36.4^{\circ} \mathrm{C}$.

Analysis 9.4. Comparison 9 Plastic total body wrap (body + head) versus plastic body wrap (head uncovered), Outcome 4 Moderate hypothermia on admission to NICU: core body temperature $<32.0^{\circ} \mathrm{C}$ to $35.9^{\circ} \mathrm{C}$.

Analysis 9.5. Comparison 9 Plastic total body wrap (body + head) versus plastic body wrap (head uncovered), Outcome 5 Outside normothermic range on admission to NICU or within 2 hours after birth.

Analysis 9.6. Comparison 9 Plastic total body wrap (body + head) versus plastic body wrap (head uncovered), Outcome 6 Core body temperature $\left({ }^{\circ} \mathrm{C}\right) 1$ hour after admission to NICU.

Analysis 9.7. Comparison 9 Plastic total body wrap (body + head) versus plastic body wrap (head uncovered), Outcome 7 Hyperthermia on admission to NICU: core body temperature $>37.5^{\circ} \mathrm{C}$.

Analysis 9.8. Comparison 9 Plastic total body wrap (body + head) versus plastic body wrap (head uncovered), Outcome 8 Intraventricular haemorrhage.

Analysis 9.9. Comparison 9 Plastic total body wrap (body + head) versus plastic body wrap (head uncovered), Outcome 9 Periventricular leukomalacia.

Analysis 9.10. Comparison 9 Plastic total body wrap (body + head) versus plastic body wrap (head uncovered), Outcome 10 Mortality.

Analysis 9.11. Comparison 9 Plastic total body wrap (body + head) versus plastic body wrap (head uncovered), Outcome 11 Bronchopulmonary dysplasia (BPD).

Analysis 9.12. Comparison 9 Plastic total body wrap (body + head) versus plastic body wrap (head uncovered), Outcome 12 Necrotising enterocolitis.

Analysis 10.1. Comparison 10 Plastic bag and plastic hat versus plastic bag and cotton hat, Outcome 1 Core body temperature $\left({ }^{\circ} \mathrm{C}\right)$ on admission to NICU or up to 2 hours after birth.

Analysis 10.2. Comparison 10 Plastic bag and plastic hat versus plastic bag and cotton hat, Outcome 2 Core body temperature $\left({ }^{\circ} \mathrm{C}\right) 1$ hour after admission to NICU.

Analysis 10.3. Comparison 10 Plastic bag and plastic hat versus plastic bag and cotton hat, Outcome 3 Core body temperature $\left({ }^{\circ} \mathrm{C}\right) 2$ hours after admission to NICU.

Analysis 11.1. Comparison 11 Stockinette cap versus routine care (no cap), Outcome 1 Core body temperature $\left({ }^{\circ} \mathrm{C}\right.$ ) on admission to NICU or up to 2 hours after birth.

Analysis 11.2. Comparison 11 Stockinette cap versus routine care (no cap), Outcome 2 Hypothermia on admission to NICU: core body temperature $<36.5^{\circ} \mathrm{C}$ or skin temperature $<36^{\circ} \mathrm{C}$.

Analysis 12.1. Comparison 12 Skin-to-skin care versus routine care, Outcome 1 Hypothermia: skin temperature $<35.5^{\circ} \mathrm{C}$ for 2 consecutive recordings. 
Analysis 12.2. Comparison 12 Skin-to-skin care versus routine care, Outcome 2 Hypoglycaemia: blood glucose level $<2.6 \mathrm{mmol} /$ L.

Analysis 13.1. Comparison 13 Thermal mattress versus routine care, Outcome 1 Core body temperature $\left({ }^{\circ} \mathrm{C}\right)$ on admission to NICU or up to 2 hours after birth.

Analysis 13.2. Comparison 13 Thermal mattress versus routine care, Outcome 2 Hypothermia on admission to NICU: core body temperature $<36.5^{\circ} \mathrm{C}$ or skin temperature $<36^{\circ} \mathrm{C}$.

Analysis 13.3. Comparison 13 Thermal mattress versus routine care, Outcome 3 Moderate hypothermia on admission to NICU: core body temperature $<36^{\circ} \mathrm{C}$.

Analysis 13.4. Comparison 13 Thermal mattress versus routine care, Outcome 4 Hypothermia on admission to NICU: core body temperature $<35^{\circ} \mathrm{C}$.

Analysis 13.5. Comparison 13 Thermal mattress versus routine care, Outcome 5 Outside normothermic range on admission to NICU or up to 2 hours after birth.

Analysis 13.6. Comparison 13 Thermal mattress versus routine care, Outcome 6 Hyperthermia on admission to NICU: core body temperature $>37.5^{\circ} \mathrm{C}$.

Analysis 13.7. Comparison 13 Thermal mattress versus routine care, Outcome 7 Mortality.

Analysis 13.8. Comparison 13 Thermal mattress versus routine care, Outcome 8 Major brain injury - IVH (grade $\mathbb{I I}$ or $\mathbb{N}$ ) . ........ Analysis 13.9. Comparison 13 Thermal mattress versus routine care, Outcome 9 Major brain injury - cystic periventricular leukomalacia.

Analysis 13.10. Comparison 13 Thermal mattress versus routine care, Outcome 10 Bronchopulmonary dysplasia (BPD). ........ Analysis 13.11. Comparison 13 Thermal mattress versus routine care, Outcome 11 Duration of hospital stay (days). ............... Analysis 13.12. Comparison 13 Thermal mattress versus routine care, Outcome 12 Duration of ventilation (days). .................. Analysis 13.13. Comparison 13 Thermal mattress versus routine care, Outcome 13 Duration of oxygen requirements (days). ... Analysis 13.14. Comparison 13 Thermal mattress versus routine care, Outcome 14 Hypoglycaemia.

Analysis 13.15. Comparison 13 Thermal mattress versus routine care, Outcome 15 Metabolic acidosis.

Analysis 13.16. Comparison 13 Thermal mattress versus routine care, Outcome 16 Necrotising enterocolitis (NEC).

Analysis 13.17. Comparison 13 Thermal mattress versus routine care, Outcome 17 Sepsis.

Analysis 14.1. Comparison 14 Thermal mattress versus plastic wrap or bag, Outcome 1 Core body temperature $\left({ }^{\circ} \mathrm{C}\right)$ on admission to NICU or up to 2 hours after birth.

Analysis 14.2. Comparison 14 Thermal mattress versus plastic wrap or bag, Outcome 2 Hypothermia on admission to NICU (core body temperature $<36.5^{\circ} \mathrm{C}$ or skin temperature $<36^{\circ} \mathrm{C}$ ).

Analysis 14.3. Comparison 14 Thermal mattress versus plastic wrap or bag, Outcome 3 Outside normothermic range on admission to NICU or up to 2 hours after birth.

Analysis 14.4. Comparison 14 Thermal mattress versus plastic wrap or bag, Outcome 4 Hyperthermia on admission to NICU: core body temperature $>37.5^{\circ} \mathrm{C}$.

Analysis 14.5. Comparison 14 Thermal mattress versus plastic wrap or bag, Outcome 5 Brain injury (IVH grade I or $\mathbb{I}$ ) . ........ Analysis 14.6. Comparison 14 Thermal mattress versus plastic wrap or bag, Outcome 6 Major brain injury (IVH grade $\mathbb{I}$ or $\mathbb{V}$ ) . . Analysis 14.7. Comparison 14 Thermal mattress versus plastic wrap or bag, Outcome 7 Mortality (death before discharge). .... Analysis 14.8. Comparison 14 Thermal mattress versus plastic wrap or bag, Outcome 8 Bronchopulmonary dysplasia (BPD). .. Analysis 14.9. Comparison 14 Thermal mattress versus plastic wrap or bag, Outcome 9 Hypotension during first 24 hours of life.

Analysis 14.10. Comparison 14 Thermal mattress versus plastic wrap or bag, Outcome 10 Necrotising enterocolitis (NEC). ..... Analysis 14.11. Comparison 14 Thermal mattress versus plastic wrap or bag, Outcome 11 Patent ductus arteriosus (PDA). ..... Analysis 14.12. Comparison 14 Thermal mattress versus plastic wrap or bag, Outcome 12 PDA - medication only. .................. Analysis 14.13. Comparison 14 Thermal mattress versus plastic wrap or bag, Outcome 13 PDA - ligation. ............................... Analysis 14.14. Comparison 14 Thermal mattress versus plastic wrap or bag, Outcome 14 Retinopathy of prematurity (ROP all grades).

Analysis 14.15. Comparison 14 Thermal mattress versus plastic wrap or bag, Outcome 15 Retinopathy of prematurity (ROP laser therapy).

Analysis 14.16. Comparison 14 Thermal mattress versus plastic wrap or bag, Outcome 16 Spontaneous intestinal perforation (SIP).

Analysis 14.17. Comparison 14 Thermal mattress versus plastic wrap or bag, Outcome 17 Worst base deficit in first 24 hours of life.

Analysis 14.18. Comparison 14 Thermal mattress versus plastic wrap or bag, Outcome 18 Worst pH in first 24 hours of life. ..... Analysis 15.1. Comparison 15 Plastic bag and thermal mattress versus plastic bag only, Outcome 1 Core body temperature $\left({ }^{\circ} \mathrm{C}\right)$ on admission to NICU or up to 2 hours of after birth. 
Analysis 15.2. Comparison 15 Plastic bag and thermal mattress versus plastic bag only, Outcome 2 Hypothermia: core body temperature $<36.5^{\circ} \mathrm{C}$ on admission to NICU.

Analysis 15.3. Comparison 15 Plastic bag and thermal mattress versus plastic bag only, Outcome 3 Outside normothermic range on admission to NICU or up to 2 hours after birth.

Analysis 15.4. Comparison 15 Plastic bag and thermal mattress versus plastic bag only, Outcome 4 Hyperthermia on admission to NICU: core body temperature $>37.5^{\circ} \mathrm{C}$.

Analysis 15.5. Comparison 15 Plastic bag and thermal mattress versus plastic bag only, Outcome 5 Major brain injury. ........... Analysis 15.6. Comparison 15 Plastic bag and thermal mattress versus plastic bag only, Outcome 6 Mortality (death before hospital discharge).

Analysis 15.7. Comparison 15 Plastic bag and thermal mattress versus plastic bag only, Outcome 7 Chronic lung disease. ...... Analysis 15.8. Comparison 15 Plastic bag and thermal mattress versus plastic bag only, Outcome 8 Coagulation support. ....... Analysis 15.9. Comparison 15 Plastic bag and thermal mattress versus plastic bag only, Outcome 9 Inotrope use.

Analysis 15.10. Comparison 15 Plastic bag and thermal mattress versus plastic bag only, Outcome 10 Intubated during admission.

Analysis 15.11. Comparison 15 Plastic bag and thermal mattress versus plastic bag only, Outcome $11 \geq 1$ dose surfactant during admission.

APPENDICES

WHAT'S NEW

HISTORY

CONTRIBUTIONS OF AUTHORS 
[Intervention Review]

\section{Interventions to prevent hypothermia at birth in preterm and/or low birth weight infants}

Emma M McCall1 ${ }^{\text {, Fiona Alderdice }}{ }^{2}$, Henry L Halliday ${ }^{3}$, Sunita Vohra ${ }^{4}$, Linda Johnston $5,6,7$

1School of Nursing and Midwifery, Queen's University Belfast, Belfast, UK. 2National Perinatal Epidemiology Unit, Nuffield Department of Population Health, University of Oxford, Oxford, UK. ${ }^{3}$ Retired Honorary Professor of Child Health, Queen's University Belfast, Belfast, UK. ${ }^{4}$ Department of Pediatrics, University of Alberta, Edmonton, Canada. ${ }^{5}$ Lawrence S Bloomberg Faculty of Nursing, University of Toronto, Toronto, Canada. ${ }^{6}$ Soochow University, Taipei, Taiwan. ${ }^{7}$ The University of Melbourne, Melbourne, Australia

Contact: Emma M McCall, School of Nursing and Midwifery, Queen's University Belfast, Medical Biology Centre, 97 Lisburn Road, Belfast, Northern Ireland, UK. e.mccall@qub.ac.uk.

Editorial group: Cochrane Neonatal Group.

Publication status and date: New search for studies and content updated (conclusions changed), published in Issue 2, 2018.

Citation: McCall EM, Alderdice F, Halliday HL, Vohra S, Johnston L. Interventions to prevent hypothermia at birth in preterm and/or low birth weight infants. Cochrane Database of Systematic Reviews 2018, Issue 2. Art. No.: CD004210. DOI: 10.1002/14651858.CD004210.pub5.

Copyright () 2018 The Cochrane Collaboration. Published by John Wiley \& Sons, Ltd.

\section{A B S T R A C T}

\section{Background}

Newborn admission temperature is a strong predictor of outcomes across all gestations. Hypothermia immediately after birth remains a worldwide issue and, if prolonged, is associated with harm. Keeping preterm infants warm is difficult even when recommended routine thermal care guidelines are followed in the delivery room.

\section{Objectives}

To assess the efficacy and safety of interventions designed for prevention of hypothermia in preterm and/or low birth weight infants applied within 10 minutes after birth in the delivery room, compared with routine thermal care or any other single/combination of intervention(s) also designed for prevention of hypothermia in preterm and/or low birth weight infants applied within 10 minutes after birth in the delivery room.

\section{Search methods}

We used the standard search strategy of Cochrane Neonatal to search the Cochrane Central Register of Controlled Trials (CENTRAL; 2016, Issue 5), MEDLINE via PubMed (1966 to 30 June 2016), Embase (1980 to 30 June 2016), and CINAHL (1982 to 30 June 2016). We also searched clinical trials databases, conference proceedings, and reference lists of retrieved articles for randomised controlled trials and quasi-randomised trials.

\section{Selection criteria}

Trials using randomised or quasi-randomised allocations to test interventions designed to prevent hypothermia (apart from 'routine' thermal care) applied within 10 minutes after birth in the delivery room for infants at $<37$ weeks' gestation and/or birth weight $\leq 2500$ grams.

\section{Data collection and analysis}

We used Cochrane Neonatal methods when performing data collection and analysis.

\section{Main results}

Twenty-five studies across 15 comparison groups met the inclusion criteria, categorised as: barriers to heat loss (18 studies); external heat sources (three studies); and combinations of interventions (four studies). 


\section{Barriers to heat loss}

\section{Plastic wrap or bag versus routine care}

Plastic wraps improved core body temperature on admission to the neonatal intensive care unit (NICU) or up to two hours after birth (mean difference (MD) $0.58^{\circ} \mathrm{C}, 95 \%$ confidence interval (CI) 0.50 to $0.66 ; 13$ studies; 1633 infants), and fewer infants had hypothermia on admission to the NICU or up to two hours after birth (typical risk ratio (RR) $0.67,95 \% \mathrm{Cl} 0.62$ to 0.72 ; typical risk reduction (RD) $-0.25,95 \% \mathrm{Cl}-0.29$ to -0.20; number needed to treat for an additional beneficial outcome (NNTB) 4, 95\% Cl 4 to $5 ; 10$ studies; 1417 infants). Risk of hyperthermia on admission to the NICU or up to two hours after birth was increased in infants in the wrapped group (typical RR $3.91,95 \% \mathrm{Cl} 2.05$ to 7.44 ; typical RD $0.04,95 \% \mathrm{Cl} 0.02$ to 0.06 ; number needed to treat for an additional harmful outcome (NNTH) $25,95 \% \mathrm{Cl} 17$ to $50 ; 12$ studies; 1523 infants), but overall, fewer infants receiving plastic wrap were outside the normothermic range (typical $\mathrm{RR} 0.75,95 \% \mathrm{Cl} 0.69$ to 0.81 ; typical RD $-0.20,95 \% \mathrm{Cl}-0.26$ to -0.15 ; NNTH 5, $95 \% \mathrm{Cl} 4$ to 7 ; five studies; 1048 infants).

Evidence was insufficient to suggest that plastic wraps or bags significantly reduce risk of death during hospital stay or other major morbidities, with the exception of reducing risk of pulmonary haemorrhage.

Evidence of practices regarding permutations on this general approach is still emerging and has been based on the findings of only one or two small studies.

\section{External heat sources}

Evidence is emerging on the efficacy of external heat sources, including skin-to-skin care (SSC) versus routine care (one study; 31 infants) and thermal mattress versus routine care (two studies; 126 infants).

SSC was shown to be effective in reducing risk of hypothermia when compared with conventional incubator care for infants with birth weight $\geq 1200$ and $\leq 2199$ grams (RR $0.09,95 \% \mathrm{Cl} 0.01$ to 0.64 ; RD $-0.56,95 \% \mathrm{Cl}-0.84$ to -0.27 ; NNTB 2, 95\% Cl 1 to 4). Thermal (transwarmer) mattress significantly kept infants $\leq 1500$ grams warmer $\left(\mathrm{MD} 0.65^{\circ} \mathrm{C}, 95 \% \mathrm{Cl} 0.36\right.$ to 0.94$)$ and reduced the incidence of hypothermia on admission to the NICU, with no significant difference in hyperthermia risk.

\section{Combinations of interventions}

Two studies (77 infants) compared thermal mattresses versus plastic wraps or bags for infants at $\leq 28$ weeks' gestation. Investigators reported no significant differences in core body temperature nor in the incidence of hypothermia, hyperthermia, or core body temperature outside the normothermic range on admission to the NICU.

Two additional studies (119 infants) compared plastic bags and thermal mattresses versus plastic bags alone for infants at < 31 weeks' gestation. Meta-analysis of these two studies showed improvement in core body temperature on admission to the NICU or up to two hours after birth, but an increase in hyperthermia. Data show no significant difference in the risk of having a core body temperature outside the normothermic range on admission to the NICU nor in the risk of other reported morbidities.

\section{Authors' conclusions}

Evidence of moderate quality shows that use of plastic wraps or bags compared with routine care led to higher temperatures on admission to NICUs with less hypothermia, particularly for extremely preterm infants. Thermal mattresses and SSC also reduced hypothermia risk when compared with routine care, but findings are based on two or fewer small studies. Caution must be taken to avoid iatrogenic hyperthermia, particularly when multiple interventions are used simultaneously. Limited evidence suggests benefit and no evidence of harm for most short-term morbidity outcomes known to be associated with hypothermia, including major brain injury, bronchopulmonary dysplasia, retinopathy of prematurity, necrotising enterocolitis, and nosocomial infection. Many observational studies have shown increased mortality among preterm hypothermic infants compared with those who maintain normothermia, yet evidence is insufficient to suggest that these interventions reduce risk of in-hospital mortality across all comparison groups. Hypothermia may be a marker for illness and poorer outcomes by association rather than by causality. Limitations of this review include small numbers of identified studies; small sample sizes; and variations in methods and definitions used for hypothermia, hyperthermia, normothermia, routine care, and morbidity, along with lack of power to detect effects on morbidity and mortality across most comparison groups. Future studies should: be adequately powered to detect rarer outcomes; apply standardised morbidity definitions; focus on longer-term outcomes, particularly neurodevelopmental outcomes.

\section{PLAIN LANGUAGE SUMMARY}

\section{Interventions to prevent hypothermia at birth in preterm and/or low birth weight infants}

Review question: What is known about the efficacy and safety of interventions designed to prevent hypothermia in preterm and/or low birth weight babies applied within 10 minutes after birth in the delivery room, compared with routine thermal care or any other single/ combination of intervention(s)? 
Background: Preventing low body temperature at birth in preterm and low birth weight babies may be important for survival and longterm outcomes. Babies rely on external help to maintain temperature, particularly in the first 12 hours of life. For vulnerable babies born preterm or at low birth weight, abnormally low body temperature (hypothermia) is a worldwide issue across all climates and has been linked to a variety of complications including death. Preventive action is taken by reducing heat loss and/or providing warmth through external heat sources. Precautionary steps routinely include ensuring a warm delivery room; drying immediately after birth, especially the head; wrapping in prewarmed dry blankets (including the head); prewarming surfaces; and eliminating draughts.

Search date: We used the standard search strategy of the Cochrane Neonatal Review Group to search CENTRAL (2016, ISsue 5), MEDLINE (1966 to 30 June 2016), Embase (1980 to 30 June 2016), and the Cumulative Index to Nursing and Allied Health Literature (CINAHL; 1982 to 30 June 2016). We also searched clinical trials databases, conference proceedings, and reference lists of retrieved articles for randomised controlled trials and quasi-randomised trials.

Key results: This review identified 25 studies involving 2433 babies; researchers used additional preventive actions in the first 10 minutes of life to prevent problems with hypothermia. Use of plastic coverings, heated mattresses, and skin-to-skin contact kept infants warmer (and within normal body temperature ranges) than routine preventive action. However, care must be taken, particularly when these methods are combined, to avoid the unintended effect of making babies too warm, which may be harmful. Limitations consist of small numbers of babies and studies included in some comparison groups; variations in methods and definitions used for normal body temperature and routine care; and differences in materials used.

Although this review confirmed that some of these measures are effective in preventing hypothermia, results across all studies show no reduction in deaths and only limited improvement in short-term complications or illnesses normally associated with being too cold. Findings suggest that perhaps hypothermia is a marker for poorer outcomes, particularly in the most immature and smallest babies, rather than a direct cause. Review authors recommend that future studies should be large enough to detect changes for rarer illnesses, should define these illnesses in the same way so they can be combined across studies, and should focus on longer-term consequences.

Quality of the evidence: Overall for the main comparison group (plastic wraps or bags vs routine care), we are moderately confident that trial results and our conclusions are reliable. Across the remaining comparison groups, evidence is insufficient to allow firm judgements mainly because numbers of studies and sample sizes are small.

In comparisons of plastic wraps or bags versus routine care to keep preterm or low birth weight babies warm, we rated the quality of evidence as moderate for key outcomes. Across outcomes reporting on babies' regulation of their body temperature, we suspect that some small trials showing that the intervention did not keep these babies warmer may not have been published, findings of studies were not in agreement, or evidence was based on small numbers of studies or events. For major complications of brain injury and bleeding into the lung (pulmonary haemorrhage), the number of events was too small or findings were based on only one study. We suspect that some small trials reporting deaths may not have been published; however this was unlikely to have affected review findings. 


\section{SUMMARY OF FINDINGS}

\section{Summary of findings for the main comparison. Plastic wrap or bag compared with routine care in preterm and/or low birth weight infants}

\section{Plastic wrap or bag compared with routine care in preterm and/or low birth weight infants}

Patient or population: preterm and/or low birth weight infants

Setting: maternity hospitals and neonatal units

Intervention: plastic wrap or bag

Comparison: routine care

\begin{tabular}{|c|c|c|c|c|c|c|}
\hline \multirow[t]{2}{*}{ Outcomes } & \multicolumn{2}{|c|}{ Anticipated absolute effects* $(95 \% \mathrm{Cl})$} & \multirow{2}{*}{$\begin{array}{l}\text { Relative effect } \\
(95 \% \mathrm{Cl})\end{array}$} & \multirow{2}{*}{$\begin{array}{l}\text { No. of partici- } \\
\text { pants } \\
\text { (studies) }\end{array}$} & \multirow{2}{*}{$\begin{array}{l}\text { Quality of the } \\
\text { evidence } \\
\text { (GRADE) }\end{array}$} & \multirow[t]{2}{*}{ Comments } \\
\hline & $\begin{array}{l}\text { Risk with routine } \\
\text { care }\end{array}$ & $\begin{array}{l}\text { Risk with plastic } \\
\text { wrap or bag }\end{array}$ & & & & \\
\hline $\begin{array}{l}\text { Core body temperature }\left({ }^{\circ} \mathrm{C}\right) \\
\text { on admission to NICU or up } \\
\text { to } 2 \text { hours after birth }\end{array}$ & $\begin{array}{l}\text { Mean core body tem- } \\
\text { perature }\left({ }^{\circ} \mathrm{C}\right) \text { on ad- } \\
\text { mission to NICU or } \\
\text { up to } 2 \text { hours after } \\
\text { birth ranged from } \\
34.80 \text { to } 36.2 \text {. }\end{array}$ & $\begin{array}{l}\text { MD } 0.58 \text { higher } \\
\text { ( } 0.50 \text { higher to } \\
0.66 \text { higher) }\end{array}$ & - & $\begin{array}{l}1633 \\
(13 \mathrm{RCTs})\end{array}$ & $\begin{array}{l}\oplus \oplus \oplus \ominus \\
\text { MODERATEa,b,c }\end{array}$ & $\begin{array}{l}\text { Publication bias was attributed to } \\
\text { non-significant smaller trials. We re- } \\
\text { moved smaller trials with statistical } \\
\text { significance, leading to a balanced } \\
\text { funnel plot. Conclusions were simi- } \\
\text { lar. }\end{array}$ \\
\hline \multirow{2}{*}{$\begin{array}{l}\text { Hypothermia on admission } \\
\text { to NICU: core body tempera- } \\
\text { ture }<36.5^{\circ} \mathrm{C} \text { or skin temper- } \\
\text { ature }<36^{\circ} \mathrm{C}\end{array}$} & Study population & & \multirow{2}{*}{$\begin{array}{l}\text { RR } 0.67 \\
\text { (0.62 to } 0.72)\end{array}$} & \multirow{2}{*}{$\begin{array}{l}1417 \\
\text { (10 RCTs) }\end{array}$} & \multirow{2}{*}{$\begin{array}{l}\oplus \oplus \oplus \odot \\
\text { MODERATEb,c }\end{array}$} & \multirow{2}{*}{$\begin{array}{l}\text { Publication bias was attributed to } \\
\text { non-significant smaller trials. We re- } \\
\text { moved smaller trials with statistical } \\
\text { significance, leading to a balanced } \\
\text { funnel plot. Conclusions were simi- } \\
\text { lar. }\end{array}$} \\
\hline & 738 per 1000 & $\begin{array}{l}495 \text { per } 1000 \\
(458 \text { to } 531 \text { ) }\end{array}$ & & & & \\
\hline \multirow{2}{*}{$\begin{array}{l}\text { Hyperthermia on admission } \\
\text { to NICU: core body tempera- } \\
\text { ture }>37.5^{\circ} \mathrm{C}\end{array}$} & Study population & & \multirow{2}{*}{$\begin{array}{l}\text { RR } 3.91 \\
\text { (2.05 to } 7.44)\end{array}$} & \multirow{2}{*}{$\begin{array}{l}1523 \\
\text { (12 RCTs) }\end{array}$} & \multirow{2}{*}{$\begin{array}{l}\oplus \oplus \oplus \ominus \\
\text { MODERATEe }\end{array}$} & \\
\hline & 12 per 1000 & $\begin{array}{l}46 \text { per } 1000 \\
\text { ( } 24 \text { to } 87 \text { ) }\end{array}$ & & & & \\
\hline $\begin{array}{l}\text { Major brain injury (within } \\
\text { hospital stay) }\end{array}$ & Study population & & $\begin{array}{l}\text { RR } 0.78 \\
\text { (0.47 to } 1.27 \text { ) }\end{array}$ & $\begin{array}{l}1100 \\
(5 \mathrm{RCTs})\end{array}$ & $\begin{array}{l}\oplus \oplus \oplus \ominus \\
\text { MODERATE }\end{array}$ & \\
\hline
\end{tabular}

Major brain injury (within 
(29 to 79$)$

\begin{tabular}{|c|c|c|c|c|c|c|}
\hline \multirow{2}{*}{$\begin{array}{l}\text { Pulmonary haemorrhage } \\
\text { (within hospital stay) }\end{array}$} & \multicolumn{2}{|c|}{ Study population } & \multirow{2}{*}{$\begin{array}{l}\text { RR } 0.60 \\
(0.38 \text { to } 0.95)\end{array}$} & \multirow{2}{*}{$\begin{array}{l}796 \\
(1 \mathrm{RCT})\end{array}$} & \multirow{2}{*}{$\begin{array}{l}\oplus \oplus \oplus \odot \\
\text { MODERATE } g\end{array}$} & \\
\hline & 112 per 1000 & $\begin{array}{l}67 \text { per } 1000 \\
(43 \text { to } 106)\end{array}$ & & & & \\
\hline \multirow{2}{*}{$\begin{array}{l}\text { Mortality (death within hos- } \\
\text { pital stay or at } 6 \text { months' } \\
\text { corrected gestation) }\end{array}$} & \multicolumn{2}{|c|}{ Study population } & \multirow{2}{*}{$\begin{array}{l}\text { RR } 0.91 \\
\text { (0.73 to } 1.15 \text { ) }\end{array}$} & \multirow{2}{*}{$\begin{array}{l}1447 \\
\text { (10 RCTs) }\end{array}$} & \multirow{2}{*}{$\begin{array}{l}\oplus \oplus \oplus \odot \\
\text { MODERATEc,h }\end{array}$} & \multirow{2}{*}{$\begin{array}{l}\text { Publication bias was unlikely to have } \\
\text { affected findings of the meta-analy- } \\
\text { sis. }\end{array}$} \\
\hline & 168 per 1000 & $\begin{array}{l}153 \text { per } 1000 \\
\text { (123 to } 194)\end{array}$ & & & & \\
\hline
\end{tabular}

${ }^{*}$ The risk in the intervention group (and its 95\% confidence interval) is based on the assumed risk in the comparison group and the relative effect of the intervention (and its $95 \% \mathrm{Cl})$.

Cl: confidence interval; MD: mean difference; NICU: neonatal intensive care unit; OIS: Office of Information Services; RCT: randomised controlled trial; RR: risk ratio.

\section{GRADE Working Group grades of evidence.}

High quality: We are very confident that the true effect lies close to that of the estimate of the effect.

Moderate quality: We are moderately confident in the effect estimate: The true effect is likely to be close to the estimate of the effect, but there is a possibility that it is substantially different.

Low quality: Our confidence in the effect estimate is limited: The true effect may be substantially different from the estimate of the effect.

Very low quality: We have very little confidence in the effect estimate: The true effect is likely to be substantially different from the estimate of effect.

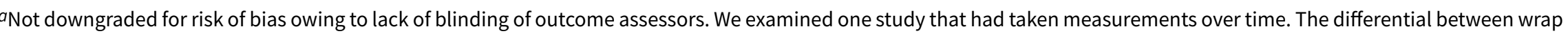
and routine care groups appeared to increase with time, which is not what would be expected if the difference was due to knowledge of assignment groups.

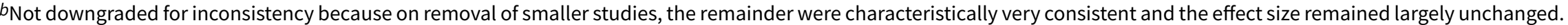

'Downgraded one level for publication bias.

$d$ Downgraded one level for inconsistency (considerable differences in effect size across studies).

eDowngraded one level for imprecision (conclusions were based on 50 incidences of hyperthermia in total - these were sufficient to show significance but with wide $95 \%$ Cls).

fDowngraded one level for imprecision (numbers of incidences were below OIS guidance).

gDowngraded one level for imprecision (evidence came from only one study).

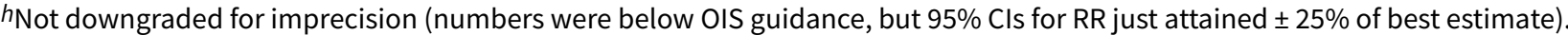




\section{B A C K G R O U N D}

Keeping preterm infants sufficiently warm immediately after birth, especially during resuscitation, is difficult even when routine thermal care guidelines are followed. Hypothermia is defined by the World Health Organization (WHO) as a core body temperature $<36.5^{\circ} \mathrm{C}$, or a skin temperature $<36^{\circ} \mathrm{C}$ (WHO 1997). However, temperature ranges for preterm infants vary, depending on the site of measurement and the device used (Knobel-Dail 2015). Globally, newborn hypothermia remains a challenge in both resource-poor and resource-rich settings and across all climates. Lunze 2013 reported hypothermia prevalence rates in a systematic review of literature pertaining to low- and middle-income countries. Hospital rates varied widely from $8 \%\left(<34.5^{\circ} \mathrm{C}\right)$ within 12 hours of birth (Guinea-Bissau, Africa) to $85 \%\left(<36^{\circ} \mathrm{C}\right)$ on admission (Harare, Zimbabwe, Africa), and community rates ranged from $11 \%$ (< $35.6^{\circ} \mathrm{C}$ ) on the first day of life (Haryana, India) to $92 \%\left(<36.5^{\circ} \mathrm{C}\right)$ during the first month (Sarlahi, Nepal) (Lunze 2013). The Vermont Oxford Network (VON) 2016 database summary, including infants of birth weight from 501 to 1500 grams, shows an unadjusted network rate of $14.6 \%$ ( 8067 of 55,246 infants) for core body temperature $<36^{\circ} \mathrm{C}$ within the first hour after admission to neonatal intensive care units (NICUs) (VON 2017). The highest rates occurred amongst the smallest $(22.1 \%$ of 501 to 750 grams) and most immature infants $(26.2 \%$ at $<24$ weeks' gestation) (VON 2017). These data were collated from 988 participating centres across 28 countries of high- or upper-middle-income status (The World Bank 2016). The VON also analysed admission temperature data for 454,617 very low birth weight (VLBW) infants surviving for 12 hours or longer in 1112 NICUs during the period from 2009 to 2016 (VON 2017a). Although these data show improvement in admission hypothermia rates (temperatures $<36.5^{\circ} \mathrm{C}$ ) ranging from $52.6 \%$ (2009) to $38.2 \%$ (2016), nearly 4 in 10 infants (excluding early deaths) were cold on admission to the NICU. Similarly, in the UK, for infants born in 2015 and 2016, the National Neonatal Audit Programme (NNAP) highlighted hypothermia as an ongoing area of concern, with $28 \%(2054$ of $7351 ; 2015)$ and $25 \%$ (1930 of $7758 ; 2016)$ of infants at $<32$ weeks' gestation having a recorded temperature below the recommended temperature range $\left(36.5^{\circ} \mathrm{C}\right.$ to $\left.37.5^{\circ} \mathrm{C}\right)$ within one hour of birth (NNAP 2016; NNAP 2017). Consequently, implementation, or sustained use, of evidence-based thermal care strategies is needed to address this issue.

\section{Description of the condition}

The newborn cannot shiver (Scopes 1963) and relies on interventions for protection against exposure to cold. The ability to maintain an equilibrium between heat loss and heat gain despite variation in environmental temperatures is restricted during the first 12 hours of life (Buczkowski-Bickmann 1992; Smales 1978). After birth, deep body and skin temperature of the term newborn can drop at a rate of approximately $0.1^{\circ} \mathrm{C}$ and $0.3^{\circ} \mathrm{C}$ per minute, respectively, unless immediate action is taken (Adamson 1965a). Although cold stress may be important for initiating breathing, and induced cooling may help protect the brain of asphyxiated term or near term newborns (Harned 1970), prolonged exposure to cold should be avoided, especially in the preterm infant. Factors that increase the risk of hypothermia include prematurity and decreasing birth weight (Chang 2015; Laptook 2007; Miller 2011; Mullany 2010); intrauterine growth restriction (Borse 1997; Hey 1975); and asphyxia, congenital anomalies such as gastroschisis, and damage to the central nervous system
(Buczkowski-Bickmann 1992). Extended periods of cold stress have been associated with harmful side effects including hypoglycaemia (Elliott 1957), respiratory distress and chronic lung disease (Boo 2013; Costeloe 2000; Pomerance 1974), necrotising enterocolitis (Yu 1984), hypoxia and metabolic acidosis (Adamson 1965; Gandy 1964), coagulation defects (Chadd 1972), delayed readjustment from foetal to newborn circulation (Stephenson 1970), acute renal failure and intraventricular haemorrhage (Boo 2013; Miller 2011), poor weight gain (Glass 1968), late-onset sepsis (Laptook 2007), and death (de Almeida 2014; Elliott 1957; Miller 2011).

The association between admission hypothermia and mortality is well documented in the literature. The 2015 International Liaison Committee on Resuscitation critically appraised 36 observational studies (published between 1964 and 2014) demonstrating increased risk of mortality associated with hypothermia at admission in non-asphyxiated infants at birth (Perlman 2015; Wyllie 2015). Review authors initially judged this evidence to be of low quality but subsequently upgraded the quality to moderate on the basis of effect size, dose effect, and single direction of evidence. Across all gestations, admission temperature was shown to be a strong predictor of mortality and morbidity. Laptook 2007 indicated that for every $1^{\circ} \mathrm{C}$ decrease in admission temperature, the odds of in-hospital mortality increased by $28 \%$ and the odds of late-onset sepsis increased by $11 \%$ in a cohort of 5277 low birth weight infants. This study also highlighted a birth weight/intubation interaction, with admission temperature showing an average difference of $1.4^{\circ} \mathrm{C}$ between an infant of 401 and 1400 grams compared with $0.4^{\circ} \mathrm{C}$ for birth weight alone. More recently, Wilson 2016 and Lyu 2015 assessed the association between admission temperature and neonatal mortality and morbidity in population cohort studies of 5697 very preterm infants from 19 regions in 11 European countries (Wilson 2016), as well as 9833 infants at < 33 weeks' gestation in Canadian NICUs (Lyu 2015). Use of mixed-effects generalised adjusted linear models revealed that an admission temperature $<35^{\circ} \mathrm{C}$ was associated with increased early (one to six days) neonatal death (risk ratio (RR) 2.41, 95\% confidence interval (CI) 1.45 to 4.00 ) and late (7 to 28 days) neonatal death (RR $1.79,95 \%$ $\mathrm{Cl} 1.15$ to 2.78 ) but showed no association after 28 days of life (Wilson 2016). Overall, for every $1^{\circ} \mathrm{C}$ rise in admission temperature, mortality fell by 15\% (Wilson 2016). Lyu 2015 identified an admission temperature range (axillary or rectal) of $36.5^{\circ} \mathrm{C}$ to $37.2^{\circ} \mathrm{C}$ associated with the lowest rates of a composite mortality/ morbidity outcome and with individual major morbidities (major brain injury, severe retinopathy of prematurity, bronchopulmonary dysplasia, necrotising enterocolitis, nosocomial infection/sepsis, and duration of ventilation). However, Wilson 2016 found no association between admission temperature and neonatal morbidity when adjusting analyses for obstetrical characteristics. These retrospective observational studies had several limiting factors, including lack of standardised practice for temperature measurement (site, timing, and instrument) and lack of data for potential confounding factors such as maternal temperature or use of humidified gases. Further investigation is needed to explore the pathways from admission hypothermia to mortality, and to ascertain whether hypothermia is a step in the causal pathway possibly via late sepsis (Laptook 2007), or, alternatively, whether hypothermia is a marker for illness and poorer outcomes by association rather than by causality.

Rapid postnatal fall in body temperature is attributable to a combination of physical characteristics (e.g. large surface area in 
relation to body weight and a thin layer of insulating fat) and environmental factors in the delivery room. Extent of total heat loss and the four modes of heat exchange (conduction, convection, radiation, and evaporation) are influenced by ambient air temperature, pressure and relative humidity, and temperature of surrounding surfaces (Capobianco 1980; Thomas 1994). Increased rate of heat loss is mainly caused by evaporation of amniotic fluid from the skin surface when the wet newborn moves from the warm environment of the uterus into a cool, dry delivery room (Adamson 1965a; Hammarlund 1980). To maintain core body temperature within the normal range of $36.5^{\circ} \mathrm{C}$ to $37.5^{\circ} \mathrm{C}$ (skin temperature of $0.5^{\circ} \mathrm{C}$ to $1.0^{\circ} \mathrm{C}$ lower) (Hey 1970; Oliver 1965), the term infant produces heat from breakdown of brown fat (nonshivering thermogenesis) and peripheral vasoconstriction (Davis 1980; Stern 1970). When skin temperature falls to $35^{\circ} \mathrm{C}$ to $36^{\circ} \mathrm{C}$, non-shivering thermogenesis is initiated (Bruck 1961). The WHO classifies a core body temperature for newborns from $36^{\circ} \mathrm{C}$ to $36.4^{\circ} \mathrm{C}$ as mild hypothermia, from $32^{\circ} \mathrm{C}$ to $35.9^{\circ} \mathrm{C}$ as moderate hypothermia, and $<32^{\circ} \mathrm{C}$ as severe (WHO 1997). Preterm infants have the combined disadvantages of a large surface area in relation to body weight, decreased fat for heat production and insulation, decreased glycogen stores, immature skin, which increases water loss, and poor vascular control. They experience even higher evaporative heat losses than term infants on the first day, especially at low ambient relative humidity (Hammarlund 1979). For each millilitre of water that evaporates from the skin, 560 calories of heat is lost (Rutter 2000). Currently, no formal definition of 'normal' temperatures for preterm infants is accepted, and methods and accuracy of temperature measurement continue to be debated (Bailey 2000; Smith 2004; Smith 2013a).

The external (skin environment) temperature gradient is pivotal in influencing the infant's response to cold (Adamson 1965); here the healthcare professional can intervene in the delivery room to minimise the risk of hypothermia. Standard care includes ensuring a warm delivery room at a minimum of $25^{\circ} \mathrm{C}$ (WHO 1997), drying the infant thoroughly immediately after birth (especially the head) (Bloom 1994), removing any wet blankets, wrapping in a prewarmed blanket, prewarming any contact surfaces, eliminating draughts, and maintaining close proximity to outside walls (Capobianco 1980). If available, radiant warmers for resuscitation and stabilisation allow easy access and are effective in preventing heat loss, provided the infant is immediately dried and placed under the prewarmed heater (Dahm 1972; Du 1969). Although the infant gains heat via radiation, potential losses through convection and evaporation are increased, and these losses are exacerbated if drying is inadequate. Servo-control is advantageous for avoidance of overheating or underheating if absorption of heat is obstructed by coverings. Since this review was first published (McCall 2005), standard thermal care has evolved, and emerging research evidence encompasses several other preventive measures that were originally considered to exceed routine management.

In practice, achieving optimal ambient delivery room temperatures for preterm infants may prove difficult (Chitty 2013; Knobel 2005a). Reilly 2015 reported that $86 \%$ of infants enrolled in the multicentre Heat Loss Prvention (HeLP) trial were initially resuscitated in delivery room ambient temperatures $\leq 25^{\circ} \mathrm{C}$. In a small randomised controlled trial $(\mathrm{RCT})(\mathrm{N}=91)$ conducted in Eastern China (Jia 2012), researchers found that mean core body temperature was significantly higher in infants at $\leq 32$ weeks' gestation who were born in a warm room (ambient temperature range $24^{\circ} \mathrm{C}$ to $26^{\circ} \mathrm{C}$; mean (standard deviation - SD) 25.1 (0.6)) compared with those born in regular rooms (ambient temperature set at $20^{\circ} \mathrm{C}$ to $23^{\circ} \mathrm{C}$; mean (SD) 22.5 (0.6)). This increase in ambient temperature was associated with a $0.5^{\circ} \mathrm{C}$ higher mean NICU admission temperature and a $31.9 \%$ reduction in the rate of hypothermia. However, this study excluded use of additional thermal care interventions, resulting in a high rate of hypothermia even in warm rooms. Another larger RCT ( $N=825$ infants) determined that an increase in ambient operating room temperature to $23^{\circ} \mathrm{C}$ (in addition to gestation-specific thermal care practices) at the time of caesarean delivery reduces the rate of neonatal hypothermia (core body temperature $<36.5^{\circ} \mathrm{C}$ ) on arrival to the admitting nursery compared with the standard temperature of $20^{\circ} \mathrm{C}(35 \%$ vs $50 \% ; \mathrm{P}<$ 0.001) (Duryea 2016). Data also show a trend towards increased hyperthermia (core body temperature $\geq 38.0^{\circ} \mathrm{C}$ ) in the intervention group. Hypothermia was less frequent at lower gestations because these infants received additional preventive measures such as plastic poncho and cap (<32 weeks' gestation) and warmed gel mattresses ( $<28$ weeks' gestation). For preterm infants $(n=147)$, data show no differences in rates of hypothermia and hyperthermia between groups. Ninety-three per cent of 62 surgeons stated that ambient temperatures of $23^{\circ} \mathrm{C}$ would be considered acceptable if improvement in neonatal outcomes could be demonstrated. Bhatt 2007 recommended that all delivery rooms should be fitted with individual thermostat and humidity controls to allow adjustment for preterm delivery by gestational age and birth weight. This supports recent American Heart Association (AHA), European Resuscitation Council (ERC), and International Liaison Committee on Resuscitation (ILCOR) guidance on temperature maintenance in the delivery room (Perlman 2015). For preterm infants at < 32 weeks' gestation under radiant warmers, a combination of interventions is recommended, which may include ambient temperature of $23^{\circ} \mathrm{C}$ to $25^{\circ} \mathrm{C}$ as recommended by Perlman 2015 and delivery room temperature above $25^{\circ} \mathrm{C}$ for preterm infants at $<28$ weeks' gestation as recommended by Wyllie 2015. However, in practice, even with a thermostat, it may not be possible to effectively change the delivery room temperature in time for a delivery.

\section{Description of the intervention}

For the purposes of this review, studies to investigate the effectiveness of additional measures to reduce heat loss in the immediate postnatal period fall into two main groups.

\section{- Barriers to heat loss.}

\section{- External heat sources.}

Interventions in the first group focus mainly on reducing evaporative heat losses (LeBlanc 1991); they include wraps and/ or head coverings made from a variety of materials (Chaput 1979; Coles 1979; Holzman 1985; Lang 2004). Baum 1968 tested a polyester suit lined with aluminium, known as the 'silver swaddler', which was designed to prevent hypothermia by reducing all modes of heat transfer to the environment. This was effective for infants with birth weight $>3000$ grams, but because the material is opaque, it is not practical for use during resuscitation. Transparent plastic coverings such as bubble wrap (Besch 1971), as well as single-layer gowns (Hobbs 1975), are effective in the delivery room for fullterm healthy newborns and those with birth weight $>2000$ grams, respectively. Use of an occlusive polyethylene transparent wrap or bag immediately at birth improved admission temperatures in non-randomised studies of infants at $<27$ weeks' gestation 
(Bredemeyer 2005), between 28 and 30 weeks (marginal below 28 weeks) (Ibrahim 2009), and at < 33 weeks' gestation (Lenclen 2002), as well as in very low birth weight infants and extremely low birth weight infants when compared with routine care (Abd-El Hamid 2012; Carroll 2010). Investigators have also used hoods or heat shields that are not in contact with the infant's body, in conjunction with a radiant warmer or incubator (Baumgart 1981; Bell 1980). Researchers have used barrier creams, waxes, and protective films such as Aquaphor to reduce heat loss in immature infants, but these substances normally are not applied within 10 minutes of birth (Nopper 1996).

Interventions in the second group include heated mattresses and the more inherent approach of skin-to-skin care (SSC) or kangaroo mother care (KMC). Low-cost heated-water-filled cot mattresses have been shown to be as effective as air-heated incubators in healthy preterm and/or low birth weight infants (Gray 2004; Green-Abate 1994; Sarman 1989; Sarman 1992), and as effective as space-heated rooms in low-resource settings (Green-Abate 1994); they are also effective in decreasing hypothermia during transport of very low birth weight infants (L'Herault 2001). More recently, exothermic chemical gel (sodium acetate) mattresses have been utilised during newborn resuscitation and stabilisation of preterm infants (Almeida 2009; Ibrahim 2010; Pinheiro 2011). These mattresses emit latent heat of crystallisation when activated (Carmichael 2007). The Transwarmer mattress was tested in vitro, and investigators stressed the importance of activation before use to avoid unnecessary heat losses that may occur as a consequence of placing an infant on a non-activated mattress (McCarthy 2012). During 'immediate birth or very early skin-to-skin' contact, the infant is placed on the mother's chest or abdomen during the first minute after birth and suctioned, and, if well enough, the infant is thoroughly dried and placed in a light blanket. A hat may be placed on the infant's head to minimise heat loss (Moore 2016). The benefits of uninterrupted skin-to-skin contact immediately after birth are well documented and include improvements in thermal control and physiological stability; however, SSC has been focussed primarily on healthy term infants or poststabilisation preterm and/or low birth weight infants (Boundy 2016; Britton 1980; Christensson 1992; Phillips 2013). Of 86 studies included in a recent systematic review (RCTs and observational studies) that documented initiation time of SSC, only seven (8\%) reported initiation immediately after birth, and $41(48 \%)$ presented stability criteria for initiation (Boundy 2016). Fourteen studies that initiated SSC only after stabilisation, or when other criteria were met, reported temperature as a continuous outcome; two studies did not provide information on timing of initiation. Mean body temperature was significantly higher in the intervention group compared with the control group (14 studies; mean difference (MD) $0.24^{\circ} \mathrm{C}$; $95 \%$ $\mathrm{Cl} 0.15$ to 0.33 ), although heterogeneity was high. This effect was similar for preterm ( $<37$ weeks' gestation) and low birth weight (<2500 grams) infant subgroups. KMC significantly reduced the risk of hypothermia compared with conventional care (typical RR $0.22,95 \% \mathrm{Cl} 0.12$ to 0.41 ; nine studies). In a small study comprising 26 infants at $\leq 26$ weeks' gestation (postnatal age two to nine days), Karlsson 2012 demonstrated that SSC can be safely initiated and facilitates temperature control during the first week of life, even among those receiving intensive care. A recent cohort of 90 moderately preterm infants at 32 to 34 weeks' gestation in Norway prospectively assessed the feasibility and safety of early SSC in the delivery room after vaginal birth compared with immediate transfer to the NICU in a conventional incubator (Kristoffersen 2016). Data show no statistically significant temperature differences between the two groups at 30 minutes after birth. However, this study was at risk of bias owing to differences in measurement sites for temperature (SSC (rectal) and incubator (axillary)) and underpowering of the study. Nevertheless, investigators implemented SSC immediately after birth with no additional resources and found SSC to be both feasible and safe for this group of infants.

In recent years, several studies have focussed on new strategies devised to maintain preterm infants' warmth at birth, such as use of humidified air during respiratory support to improve admission temperatures. In 2010, te Pas and colleagues, in a small, prospective observational cohort study, demonstrated a reduction in moderate hypothermia (core body temperature $<36^{\circ} \mathrm{C}$ ) in the heated group (53\% vs $19 \%$; $P<0.001)$ among infants at $\leq 32$ weeks' gestation. Trial authors recommended further research to assess short- and long-term outcomes and cost versus benefit (te Pas 2010). The bench test study Shearman 2012, which also emphasised the need for more research into safety issues pertaining to heating and humidifying gas at resuscitation, supported this decision before any other clinical trials were initiated. Subsequently, a more recent RCT ( $N=203)$ assessed the effect on admission temperature of adding heated humidified gases (HHGs) during respiratory support at birth and during transport of infants at $<32$ weeks' gestation (Meyer 2015). Standard thermal care employed at the time of the study included warm delivery rooms $\left(25^{\circ} \mathrm{C}\right.$ to $\left.26^{\circ} \mathrm{C}\right)$, preheated radiant warmer surfaces, occlusive body wrap (without drying), and placement of hats. This study showed that use of HHG appeared to be safe and possibly beneficial for infants at $<28$ weeks' gestation. Fewer infants $(31 \%)$ in the HHG group had temperatures outside the normothermic range $\left(36.5^{\circ} \mathrm{C}\right.$ to $\left.37.5^{\circ} \mathrm{C}\right)$ compared with infants in the cold, dry gas group (58\%) $(P=0.03)$. Similarly, McGrory 2017 conducted an RCT ( $N=273)$ at two Australian centres to assess whether use of HHG during stabilisation of infants at $<30$ weeks' gestation decreases hypothermia rates at NICU admission (core body temperature $<36.5^{\circ} \mathrm{C}$ ). A significant reduction in admission hypothermia ( $27 \%$ in the HHG group vs $43 \%$ in the control group) was reported without an overall increased risk of hyperthermia (core body temperature $>37.5^{\circ} \mathrm{C}$ ), although the trend towards an increased incidence of hyperthermia for lower gestations in the HHG group suggests the need for caution and careful temperature monitoring. McGrory 2017 also noted the need to balance benefits, costs, and risks of introducing an additional piece of delivery room equipment and indicated that consideration should be given to other low-cost interventions such as increasing ambient temperature or using exothermic mattresses.

All these interventions have potential disadvantages, for example, Newton 2003 reported that significantly more infants (at < 30 weeks' gestation) wrapped in polyethylene bags were hyperthermic $\left(>37^{\circ} \mathrm{C}\right)$ when compared with unwrapped historical control infants. Brun 1997 noted that use of a chemical hot pack during resuscitation of a newborn infant resulted in third-degree burns, and recommended that these should not be used unless the peak temperature of the pack is $<44^{\circ} \mathrm{C}$. McCarthy 2011 and McCarthy 2013 reported that in infants at $<31$ weeks' gestation, using an exothermic mattress as well as a polyethylene bag caused increased hyperthermia on admission to the NICU when compared with only placing infants in polyethylene bags. Ibrahim 2010 reported a higher incidence of admission hyperthermia (usually transient) associated with use of acetate gel mattresses 
in combination with polyethylene bags at resuscitation in preterm infants at $<28$ weeks' gestation. However, Pinheiro 2011 concluded that chemical warming packs added to plastic wrapping are useful for reducing hypothermia during stabilisation of very low birth weight infants without producing significant hyperthermia, and that their usefulness outweighs any potential rare adverse effects such as focal skin injury. Increasingly, these interventions are used in combination, and it is imperative that clinicians carefully monitor infant temperatures to avert harm, because hyperthermia also increases risks of neonatal mortality and morbidity (Perlman 2015).

\section{How the intervention might work}

Interventions to reduce hypothermia at birth should decrease total heat losses or should provide external heat without compromising accessibility during resuscitation; these interventions should have minimal side effects (such as hyperthermia, burns, maceration, or infection).

\section{Why it is important to do this review}

Neonatal hypothermia after birth remains a world wide issue across all climates (Costeloe 2000; Lunze 2013; NNAP 2016; VON 2017), as well as all levels of income (Ali 2012; Christensson 1988; Jaleel 2011; Johanson 1992; Kumar 2009; Laptook 2007; Tafari 1973), and, if prolonged, can lead to harm. Over 50 years ago, Silverman 1958 and Day 1964 showed that reducing heat losses in preterm infants in the first few days after birth increased survival rates. The association of hypothermia with neonatal mortality and adverse clinical outcomes is well known. Early intervention in the delivery room, particularly for preterm infants undergoing resuscitation (Laptook 2008), is therefore a matter of high priority if hypothermia is to be prevented. Soll 2008 re-emphasised the need to address and understand the consequences of poor thermal care for the newborn to improve clinical outcomes. Cordaro 2012 suggested that admission hypothermia in low birth weight preterm infants is not a "complication of prematurity; it is a consequence of healthcare provider inattentiveness." Current concerted and collaborative efforts to reduce the incidence of hypothermia on admission to neonatal units have included rapid cycle quality improvement initiatives and implementation of 'intervention bundles', such as staff education, checklists, consistent room air temperature, use of polyethylene bags or caps and thermal mattresses, and transfer in a warmed incubator (Arrindell 2012; Caldas 2017; DeMauro 2013; Harer 2017; Ho 2007; Kaplan 2009; Kumar 2012; Lee 2008; Lewis 2011; Silvestri 2012; Yip 2017). Manai 2013 reported that the rate of admission hypothermia in preterm low birth weight infants was reduced from $44 \%$ to $0 \%$ within a two-year period at a regional NICU in San Jose. This study applied a multi-disciplinary, standardised, evidence-based thermal management approach before delivery and through NICU admission, implemented via the rapid cycle PlanDo-Study-Act quality improvement method. Additional studies in high-income settings adopting this approach have reported comparable successes in prevention of hypothermia without incurring hyperthermia or other adverse complications (Pearlman 2012; Pinheiro 2011; Pinheiro 2014; Russo 2014). The primary objective of adopting evidence-based thermal care practices during stabilisation and transfer to the NICU is to achieve and maintain normothermia while avoiding both iatrogenic hypothermia and hyperthermia (McCall 2014). Therefore, this review provides healthcare professionals with a critical appraisal of available evidence, with focus on individual or combinations of interventions applied within 10 minutes after birth in the delivery room, and is limited to preterm and/or low birth weight infants because these infants are most susceptible to adverse effects of hypothermia. Longer-term thermal management and spatial or environmental strategies for increasing warming are beyond the scope of this review, which provides an update of a Cochrane Review first published as McCall 2005, and later updated in McCall 2008 and McCall 2010.

\section{O B J E C T IVES}

\section{Primary}

To assess the efficacy and safety of interventions designed for prevention of hypothermia in preterm and/or low birth weight infants applied within 10 minutes after birth in the delivery room, compared with routine thermal care or any other single/ combination of intervention(s) also designed for prevention of hypothermia in preterm and/or low birth weight infants applied within 10 minutes after birth in the delivery room.

\section{Secondary}

To assess effects of these interventions on complications associated with preterm birth, hypothermia, and adverse outcomes.

\section{METHODS}

\section{Criteria for considering studies for this review}

\section{Types of studies}

All trials using randomised or quasi-randomised allocation to test a specific intervention(s) designed to prevent hypothermia immediately after birth.

\section{Types of participants}

Preterm infants at $<37$ weeks' gestation (according to best obstetrical estimate at time of delivery) or low birth weight infants weighing $\leq 2500$ grams, for whom intervention(s) to prevent hypothermia is/are applied within 10 minutes after birth in the delivery room. These infants and small-for-gestation infants were eligible for inclusion.

Excluded were Infants with major congenital malformations, especially abdominal wall defects.

\section{Types of interventions}

Any intervention(s) applied within 10 minutes after birth in the delivery room apart from ROUTINE THERMAL CARE, which was defined as any of the following routine practices: providing a warm delivery room (at a minimum temperature of $25^{\circ} \mathrm{C}$ - rarely achieved in practice), drying the infant immediately after birth, removing wet blankets and wrapping in a prewarmed blanket, prewarming any contact surfaces, avoiding draughts, and, in developed countries (The World Bank 2016), using radiant warmers or incubators.

We studied the following interventions.

- Barriers to heat loss applied to any part of the body of the preterm and/or low birth weight infant within 10 minutes after birth in the delivery room. 
- Coverings such as transparent plastic wraps and bags made of low-density polyethylene (LDPE) or linear low-density polyethylene (LLDPE) or polyvinylidene chloride (PVDC).

- Semipermeable membranes such as Opsite or Tegaderm.

- Other additional swaddling materials or wraps (excluding delivery room blankets) such as the 'silver swaddler'.

- External heat sources (non-routine) initiated within 10 minutes after birth in the delivery room.

- Skin-to-skin care.

- Heated/gel/chemical mattresses.

\section{Types of outcome measures}

\section{Primary outcomes}

- Temperature of the infant taken on admission to the neonatal intensive care unit (NICU) or up to two hours after birth. We assessed temperature as both continuous and dichotomous variables.

- We accepted rectal, axillary, oral, or tympanic temperature measurements as equivalent core body temperature, and we accepted abdominal skin temperature for skin temperature. When both core temperature and skin temperature were recorded, core temperature took priority. When multiple temperatures were recorded (i.e. within different time frames up to two hours after birth), the lowest temperature recorded took priority.

- A core body temperature $<36.5^{\circ} \mathrm{C}$ or a skin temperature $<$ $36^{\circ} \mathrm{C}$ indicated the presence of hypothermia within control and intervention groups.

- For hypothermia, we used core body temperature and skin temperature subgroupings as defined by WHO 1997 to determine three levels of severity.

- Mild hypothermia or cold stress: core body temperature $36^{\circ} \mathrm{C}$ to $36.4^{\circ} \mathrm{C}$, or skin temperature $35.5^{\circ} \mathrm{C}$ to $35.9^{\circ} \mathrm{C}$.

- Moderate hypothermia: core body temperature $32^{\circ} \mathrm{C}$ to $35.9^{\circ} \mathrm{C}$, or skin temperature $31.5^{\circ} \mathrm{C}$ to $35.4^{\circ} \mathrm{C}$.

- Severe hypothermia: core body temperature $<32^{\circ} \mathrm{C}$, or skin temperature $<31.5^{\circ} \mathrm{C}$.

\section{Secondary outcomes}

We categorised these outcomes as morbidity and as adverse outcomes due to the intervention.

\section{Morbidity}

- Hypoglycaemia (defined by a blood glucose level $<2.0 \mathrm{mmol} / \mathrm{L}$ within two hours of birth)

- Respiratory distress syndrome (RDS) (defined by clinical signs of grunting, flaring, retractions, cyanosis in room air, tachypnoea and a radiological picture of reticulogranular mottling, and air bronchogram)

- Surfactant given at any time

- Intubation in the delivery room

- Requirement for ventilation and duration of ventilation (days)

- Length of stay (days)

- Mortality: death within seven days, death within 28 days, and/or death during hospital stay

- Severe metabolic acidosis (defined by a $\mathrm{pH}<7.20$ and/or a base deficit $>10 \mathrm{mmol} / \mathrm{L}$ within the first three days of life)
- Intraventricular haemorrhage (defined according to the criteria of head ultrasonography performed before 14 days of life) (Lee 2000; Papile 1978)

- Patent ductus arteriosus (defined by clinical diagnosis + treatment with indomethacin or ibuprofen or surgical ligation, or both) (Lee 2000)

- Chronic lung disease (defined by oxygen dependency at 36 weeks' postmenstrual age for an infant born at $\leq 32$ weeks' gestation) (Lee 2000; Shennan 1988)

- Necrotising enterocolitis (defined according to the criteria of Bell 1978 as stage 2 or higher and classified as medical (clinical symptoms and signs + evidence of pneumatosis on abdominal radiographs), or according to the criteria of Lee 2000 and classified as surgical (histological evidence of NEC on surgical specimen of intestine))

- Acute renal failure (defined by Stapleton 1987 as a serum creatinine level $>1.5 \mathrm{mg} / \mathrm{dL}(>130 \mu \mathrm{mol} / \mathrm{L}$ ) and oliguria (urine output $<1 \mathrm{~mL} / \mathrm{kg} / \mathrm{h})$ )

\section{Adverse outcomes due to the intervention}

- Hyperthermia (defined as temperature on admission to NICU or within two hours of birth $\geq 38^{\circ} \mathrm{C}$ )

- Burns within three days of birth

- Maceration within three days of birth

- Skin or systemic infection secondary to intervention within the first week of birth (defined by a culture of pathogenic bacteria from normally sterile body tissue or fluid)

- Antibiotic course of five days or longer started within the first seven days of birth

- Interference with resuscitation and other practices (e.g. umbilical vein catheter placement for fluid replacement, chest tube insertion)

- Fluid problems such as dehydration or fluid overload, electrolyte imbalance such as hypernatraemia (serum sodium > $150 \mathrm{mmol} / \mathrm{L}$ ) or hyponatraemia (<130 mmol/L)

- Any other unexplained adverse outcome attributed to the intervention within seven days of birth

- Negative psychological outcomes (perception of care by parents)

\section{Search methods for identification of studies}

We used the standard Cochrane search strategy (Higgins 2011).

\section{Electronic searches}

We used the criteria and standard methods of Cochrane and the Cochrane Neonatal Review Group (see the Cochrane Neonatal Group search strategy for specialized register). We conducted a comprehensive search that included the following.

- Cochrane Central Register of Controlled Trials (CENTRAL; 2016, Issue 5) in the Cochrane Library.

- MEDLINE via PubMed (1966 to 30 June 2016).

- Embase (1980 to 30 June 2016).

- Cumulative Index to Nursing and Allied Health Literature (CINAHL; 1982 to 30 June 2016).

We have detailed the MEDLINE search strategy in Appendix 1. We devised similar search strategies using appropriate terminology for 
each electronic database plus database-specific limiters for RCTs and neonates (see Appendix 2 for full search strategies for each database). We applied no language restrictions.

We searched clinical trials registries for ongoing and recently completed trials.

- US National Institutes of Health Database (clinicaltrials.gov).

- World Health Organization International Trials Registry and Platform (www.whoint/ictrp/search/en/).

- International Standard Randomised Controlled Trial Number Registry (ISRCTN Registry).

In addition, we searched the following databases.

- Online Computer Library Center (OCLC) WorldCat (January 2013).

- Database of Abstracts of Reviews of Effects (DARE) (1994 to January 2013).

- Conference/symposia proceedings via the British Library's Electronic Table of Contents (ZETOC) (1993 to January 2013).

- International Statistical Institute (ISI) proceedings (1990 to January 2013).

We conducted the current update of this review in two phases.

- Phase one: search end date January 2013.

- Phase two: search end date June 2016.
After consultation with the Cochrane Neonatal Review Group, we restricted phase two to full manuscripts of RCTs published in the English language; therefore publication and language bias cannot be ruled out.

\section{Searching other resources}

We handsearched the reference lists of any articles selected for inclusion in this review to identify additional relevant articles.

\section{Data collection and analysis}

We followed the standard Cochrane method for conducting a systematic review, as described in the Cochrane Handbook for Systematic Reviews of Interventions (Higgins 2011).

\section{Selection of studies}

We designed the search strategy and searched electronic databases in association with Faculty Librarians at Queen's University Belfast. Two independent review authors (EC, FA) separately assessed the full list of titles and abstracts for eligibility and retrieved the full text of those considered relevant. Each review author clearly stated reasons for exclusion of studies. One included study required formal translation into English language (Farhadi 2012). We documented the selection process using a PRISMA flow chart (see Figure 1). We produced a Characteristics of included studies table for each study considered eligible for inclusion, as well as a Characteristics of excluded studies table for each study excluded after examination (we included the reason for exclusion). 
Figure 1. Study flow diagram: review update.

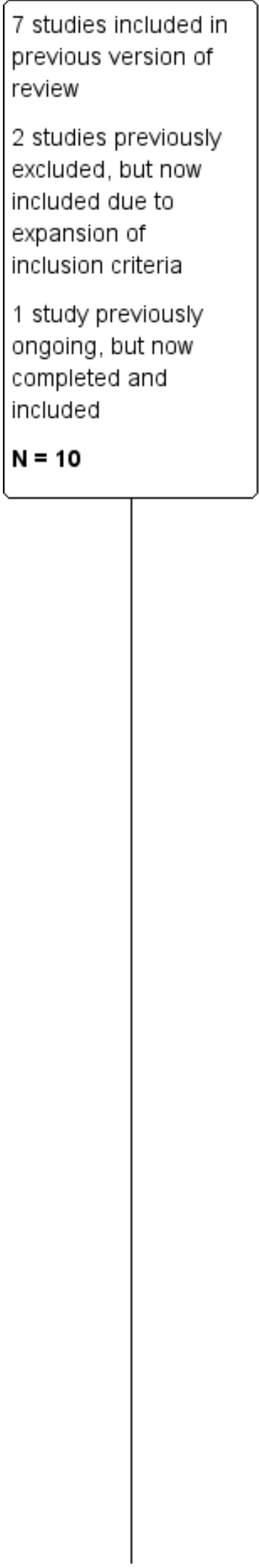

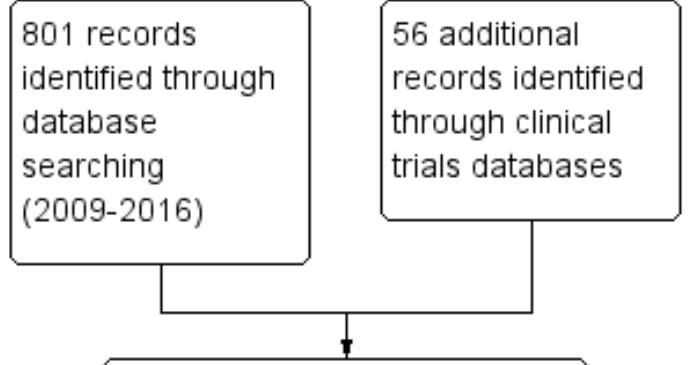

797 records after duplicates removed

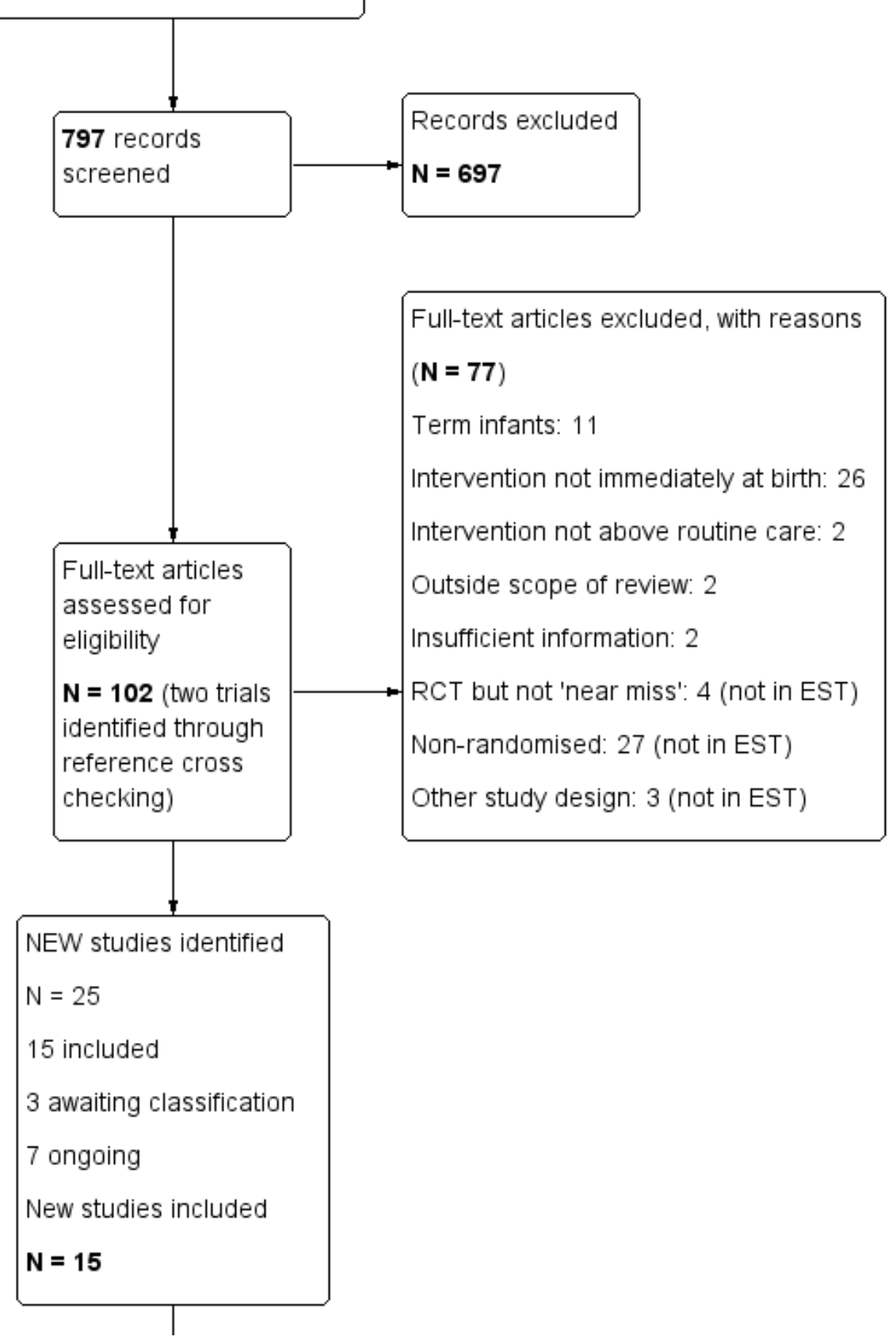


Figure 1. (Continued)

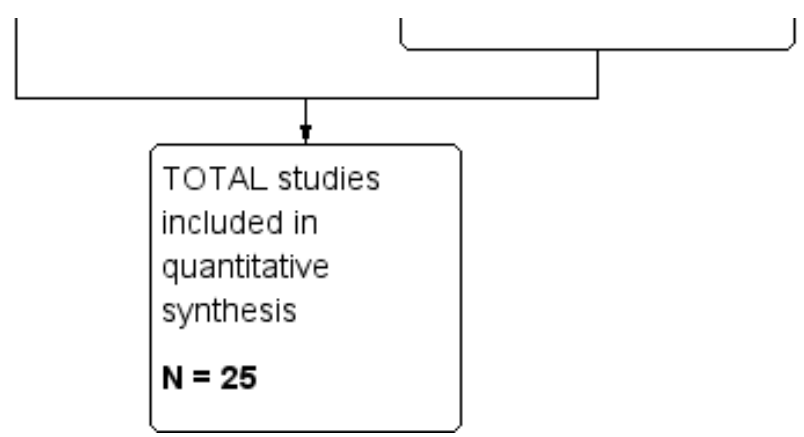

\section{Data extraction and management}

Two independent review authors (EC, FA) separately extracted, assessed, and coded all data for each study using a form that was designed specifically for this review. We replaced any standard error of the mean with the corresponding standard deviation. We resolved disagreements by discussion and corresponded with study investigators regarding further methodological data and results, as required. We extracted important information with respect to trial characteristics, participant characteristics, intervention characteristics, and outcome measures.

\section{Assessment of risk of bias in included studies}

Two independent review authors (EC, FA) separately assessed studies that fulfilled the criteria for inclusion for risk of bias and extracted data using prepared proformas. We judged risk of bias according to the six domains outlined in the risk of bias tool provided in the Cochrane Handbook for Systematic Reviews of Interventions (Higgins 2011).

- Random sequence generation.

- Allocation concealment.

- Blinding of participants and healthcare providers/personnel.

- Blinding of outcome assessors.

- Incomplete outcome data.

- Selective reporting.

- Other potential threats to validity.

Team members reached complete agreement and sought additional information from investigators for 18 included trials (Bhavsar 2015; Caglar 2014; Cardona Torres 2012; Chantaroj 2011; Farhadi 2012; Gathwala 2010; Knobel 2005; Leadford 2013; Leslie 2007; Mathew 2012; McCarthy 2013; Reilly 2015; Rohana 2011; Smith 2013; Tescon-delos Santos 2012; Trevisanuto 2010; Vohra 1999; Vohra 2004). We did not blind review authors to authors nor to institutions. In three instances, a team member was also an author of a selected trial, so we excluded that team member from the study appraisal process. We detailed the methodological information retrieved in the Characteristics of included studies tables.

In addition, we assessed the following issues and entered our findings into the 'Risk of bias' table.

\section{Selection bias (random sequence generation and allocation concealment)}

For each included trial, we planned to categorise the risk of selection bias as follows.

\section{Random sequence generation}

- Low risk: Investigators describe a random component in the sequence generation process, such as referring to a random number table, using a computer random number generator, tossing a coin, shuffling cards or envelopes, throwing dice, drawing lots, or minimising.

- High risk: Investigators describe a non-random component in the sequence generation process such as sequence generated by odd or even date of birth, sequence generated by some rule based on date or day of admission, sequence generated by some rule based on hospital or clinic record number, allocation by judgement of the clinician, allocation by preference of the participant, allocation based on the results of a laboratory test or series of tests, or allocation by availability of the intervention.

- Unclear risk: No or unclear information is provided.

\section{Allocation concealment}

For each included trial, we planned to categorise risk of bias regarding allocation concealment as follows.

- Low risk: Participants and investigators enrolling participants could not foresee assignment because one of the following, or an equivalent method, was used to conceal allocation: central allocation (including telephone, web-based, and pharmacy-controlled randomisation), sequentially numbered drug containers or identical appearance, or sequentially numbered sealed opaque envelopes.

- High risk: Participants and investigators enrolling participants could possibly foresee assignments and thus introduce selection bias, such as allocation based on open random allocation schedule (e.g. a list of random numbers), unsealed or non-opaque envelopes, alternation or rotation, date of birth, or case record number.

- Unclear risk: No or unclear information is provided.

\section{Blinding (performance bias)}

For each included trial, we planned to categorise the methods used to blind study personnel from knowledge of which intervention a participant received.

- Low risk: Investigators describe no blinding or incomplete blinding, but review authors judge that the outcome is not likely to be influenced by lack of blinding; blinding of participants and of key study personnel is ensured, and it is unlikely that blinding could have been broken. 
- High risk: Investigators describe no blinding or incomplete blinding, and the outcome is likely to be influenced by lack of blinding; blinding of key trial participants and personnel is attempted, but it is likely that blinding could have been broken, and the outcome is likely to be influenced by lack of blinding.

- Unclear risk: No or unclear information is provided.

\section{Blinding (detection bias)}

For each included trial, we planned to categorise the methods used to blind outcome assessors from knowledge of which intervention a participant received.

- Low risk: Investigators describe no blinding or incomplete blinding, but review authors judge that the outcome is not likely to be influenced by lack of blinding; blinding of participants and of key study personnel is ensured, and it is unlikely that blinding could have been broken.

- High risk: Investigators describe no blinding of outcome assessment, but review authors judge that the outcome measurement is not likely to be influenced by lack of blinding; blinding of outcome assessment is ensured, but it is likely that blinding could have been broken, and the outcome measurement is likely to be influenced by lack of blinding.

- Unclear risk: No or unclear information is provided.

\section{Incomplete outcome data (attrition bias)}

For each included trial and for each outcome, we planned to describe the completeness of data including attrition and exclusions from the analysis.

- Low risk.

- No missing outcome data.

- Reasons for missing outcome data unlikely to be related to true outcome (for survival data, censoring unlikely to introduce bias).

- Missing outcome data balanced in numbers across intervention groups, with similar reasons for missing data across groups.

- For dichotomous outcome data, the proportion of missing outcomes compared with observed event risk is not enough to have a clinically relevant impact on the intervention effect estimate.

- For continuous outcome data, plausible effect size (difference in means or standardised difference in means) among missing outcomes is not enough to have a clinically relevant impact on observed effect size.

- Missing data have been imputed by appropriate methods.

- High risk.

- Reason for missing outcome data likely to be related to true outcome, with imbalance in numbers or reasons for missing data across intervention groups.

- For dichotomous outcome data, proportion of missing outcomes compared with observed event risk enough to induce clinically relevant bias in intervention effect estimate.

- For continuous outcome data, plausible effect size (difference in means or standardised difference in means) among missing outcomes enough to induce clinically relevant bias in observed effect size.
- "As-treated" analysis done with substantial departure of the intervention received from that assigned at randomisation.

- Potentially inappropriate application of simple imputation.

- Unclear risk: No or unclear information is provided.

\section{Selective reporting (reporting bias)}

For each included trial, we planned to describe how we investigated the risk of selective outcome reporting bias and what we found. We planned to access all protocols of included trials through clinical trials registries (clinicaltrials.gov; controlled-trials.com; who.int/ ictrp) and through direct contact with trial authors.

We planned to assess methods as follows.

- Low risk: Study protocol is available and all of the trial's prespecified (primary and secondary) outcomes of interest in the review have been reported in the prespecified way; or study protocol is not available but it is clear that published reports include all expected outcomes, including those that were prespecified (convincing text of this nature may be uncommon).

- High risk: Not all of the trial's prespecified primary outcomes have been reported; one or more primary outcomes is reported via measurements, analysis methods, or subsets of data (e.g. subscales) that were not prespecified; one or more reported primary outcomes were not prespecified (unless clear justification for their reporting is provided, such as an unexpected adverse effect); one or more outcomes of interest in the review are reported incompletely so they cannot be entered into a meta-analysis; or the study report fails to include results for a key outcome that would be expected to have been reported for such a trial.

- Unclear risk: No or unclear information is provided (study protocol was not available).

\section{Other potential sources of bias (other bias)}

For each included trial, we planned to describe any important concerns that we had about other possible sources of bias (e.g. whether a potential source of bias is related to the specific study design used).

We planned to assess whether each trial was free of other problems that could put it at risk of bias.

- Low risk: Trial appears to be free of other sources of bias.

- High risk: Trial has at least one important risk of bias (e.g. trial had a potential source of bias related to the specific study design used or has been claimed to have been fraudulent or had some other problem).

- Unclear risk: Risk of bias may be present, but information is insufficient to assess whether an important risk of bias exists, or rationale or evidence that an identified problem will introduce bias is insufficient.

\section{Measures of treatment effect}

We calculated risk ratios (RRs) and 95\% confidence intervals (Cls) for dichotomous outcomes. From the risk difference (RD), we calculated the number needed to treat for an additional beneficial outcome (NNTB) or the number needed to treat for an additional harmful outcome (NNTH) along with $95 \%$ confidence limits. We 
calculated mean differences (MDs) and 95\% confidence limits for continuous outcomes.

\section{Unit of analysis issues}

The unit of analysis was the participating infant in individually randomised trials. When a trial had multiple arms, we assigned each comparison, if independent, to the appropriate separate comparison group for meta-analysis. We found no clusterrandomised trials, but they are eligible for inclusion. Planned analyses would include adjusting sample sizes using the methods described in the Cochrane Handbook for Systematic Reviews of Interventions (Section 16.3.4 or 16.3.6) (Higgins 2011) along with an estimate of the intracluster correlation coefficient (ICC) derived from the trial, from a similar trial, or from a study of a similar population. We would perform a subgroup analysis to investigate effects of the randomisation unit if feasible.

\section{Dealing with missing data}

We contacted trial authors regarding missing data (methodological data, outcome data for subgroups, and missing data, such as standard deviations) via a number of strategies, namely, the contact email address provided on the published manuscript, "Researchgate" (ResearchGate 2016), and institutional email addresses. We excluded studies to the Characteristics of excluded studies table or assigned them to the Characteristics of studies awaiting classification table when we had insufficient information to determine the study design and efforts to contact trial authors were unsuccessful.

\section{Assessment of heterogeneity}

We estimated treatment effects of individual trials and examined heterogeneity between trials by inspecting forest plots and quantifying the impact of heterogeneity using the $\mathrm{I}^{2}$ statistic: low $(>25 \%$ and $<50 \%)$, moderate $(\geq 50 \%$ and $<75 \%)$, and high $(\geq 75 \%)$ (Higgins 2003). If we detected substantial statistical heterogeneity, we explored possible causes (e.g. differences in study quality, participants, intervention regimens, or outcome assessments) by performing post hoc subgroup analyses.

\section{Assessment of reporting biases}

We assessed the symmetry of funnel plots for publication bias when we included 10 or more trials in a meta-analysis. When possible, we investigated the possibility of reporting bias for included studies by comparing the primary and secondary outcomes reported in full manuscripts with prespecified outcomes as published in study protocols or in online clinical trials registers (US National Institutes of Health Database, available at http://ClinicalTrials.gov; International Standard Randomised Controlled Trial Number Registry, available at http://controlled-trials.com; World Health Organization International Trials Registry and Platform, available at http://apps.who.int/trialsearch/).

\section{Data synthesis}

We performed meta-analysis using Review Manager software version 5.3 (RevMan 2014) as supplied by Cochrane. For estimates of typical risk ratio and risk difference, we used the Mantel-Haenszel method. For measured quantities, we used the inverse variance method. We performed all meta-analyses using the fixed-effect model.

\section{Quality of evidence}

We used the Grading of Recommendations Assessment, Development and Evaluation (GRADE) approach, as outlined in the GRADE Handbook (Schünemann 2013), to assess the quality of evidence for the following (clinically relevant) outcomes for the main comparison group.

- Core body temperature $\left({ }^{\circ} \mathrm{C}\right)$ on admission to NICU or up to two hours after birth.

- Hypothermia on admission to NICU: core body temperature < $36.5^{\circ} \mathrm{C}$ or skin temperature $<36^{\circ} \mathrm{C}$.

- Core body temperature $\left({ }^{\circ} \mathrm{C}\right)$ one hour after initial NICU admission temperature was taken.

- Hyperthermia on admission to NICU: core body temperature > $37.5^{\circ} \mathrm{C}$.

- Major brain injury within hospital stay.

- Pulmonary haemorrhage within hospital stay.

- Mortality (death within hospital stay or at six months' corrected gestation).

Two independent review authors (EM, FA) and a statistician (MS) assessed the quality of evidence for each of the outcomes listed above. We considered evidence from RCTs as high quality but downgraded the evidence one level for serious (or two levels for very serious) limitations on the basis of the following: design (risk of bias), consistency across studies, directness of evidence, precision of estimates, and presence of publication bias. We used the GRADEpro Guideline Development Tool (GRADEpro GDT) to create a 'Summary of findings' table to report the quality of the evidence.

The GRADE approach results in an assessment of the quality of a body of evidence according to one of four grades.

- High: We are very confident that the true effect lies close to that of the estimate of the effect.

- Moderate: We are moderately confident in the effect estimate: The true effect is likely to be close to the estimate of the effect, but there is a possibility that it is substantially different.

- Low: Our confidence in the effect estimate is limited: The true effect may be substantially different from the estimate of the effect.

- Very low: We have very little confidence in the effect estimate: The true effect is likely to be substantially different from the estimate of effect.

\section{Subgroup analysis and investigation of heterogeneity}

We planned to carry out subgroup analyses by intervention, by birth weight/gestational age, and by income grouping of the country of study, to determine whether effectiveness varies according to:

- Interventions applied.

- Birth weight and gestation within the following categories. - Birth weight (< 1500 grams; 1500 to 2500 grams).

- Gestation (<28 weeks; 28 to 32 weeks; 33 to 37 weeks).

We determined the income grouping for the country in which each included trial was conducted using the World Bank list of economies: low-income, lower middle-income, upper middle- 
income, and high-income (The World Bank 2016). "Low-income and middle-income economies are sometimes referred to as developing economies. The use of the term is convenient; it is not intended to imply that all economies in the group are experiencing similar development or that other economies have reached a preferred or final stage of development. Classification by income does not necessarily reflect development status" (The World Bank 2013).

However, the subgroups reported in the included studies were not compatible with those prespecified. Therefore, we carried out post facto subgroup analyses based on reported gestation and birth weight subcategories when appropriate within each comparison group.

\section{Sensitivity analysis}

We planned to explore the impact of the level of bias by undertaking sensitivity analyses. If needed, we planned to incorporate summary assessments of risk of bias into explicit measures of the quality of evidence using the GRADE system (GRADEpro GDT) for the primary outcome measure (core body temperature on admission to NICU or up to two hours after birth) and for key secondary outcome measures.

\section{RE S U L T S}

\section{Description of studies}

See the Characteristics of included studies table, the 'Risk of bias' table (Figure 2), and Characteristics of excluded studies, Characteristics of studies awaiting classification, and Characteristics of ongoing studies tables. 
Figure 2. Risk of bias summary: review authors' judgements about each risk of bias item for each included study.

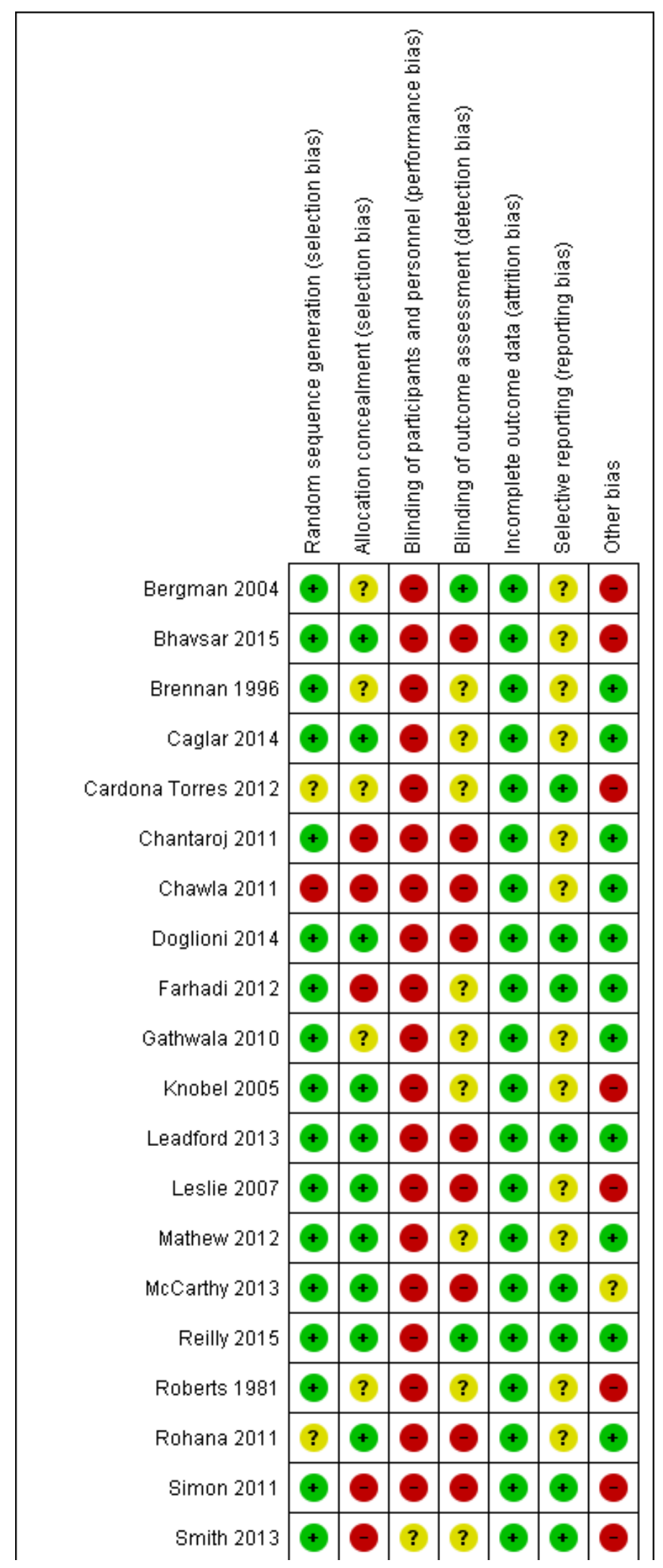


Figure 2. (Continued)

\begin{tabular}{|c|c|c|c|c|c|c|c|}
\hline Smith 2013 & + & - & ? & ? & + & + & \\
\hline Talakoub 2015 & + & $?$ & $?$ & $?$ & + & $?$ & + \\
\hline Tescon-delos Santos 2012 & & & & + & + & $?$ & \\
\hline Trevisanuto 2010 & + & + & & $?$ & + & + & + \\
\hline Vohra 1999 & $\oplus$ & + & & - & + & $?$ & \\
\hline Vohra 2004 & + & + & & ) & + & $?$ & + \\
\hline
\end{tabular}

\section{Results of the search}

Figure 1 shows the flow diagram for screening, selection, and assessment of studies generated by the 2009 to 2016 updated literature searches.

In the previous version of this review (McCall 2010), we included seven studies (Bergman 2004; Brennan 1996; Knobel 2005; Roberts 1981; Trevisanuto 2010; Vohra 1999; Vohra 2004), and we excluded 90 studies. One study was awaiting assessment (Punnahitananda 2008), and one study that was ongoing at that time - Vohra 2004 [pers comm]) - has since been completed and published (Reilly 2015).

For this update, we excluded Punnahitananda 2008 (see Characteristics of excluded studies table) and included Reilly 2015 (see Characteristics of included studies table). In addition, in the light of expansion of our inclusion criteria (i.e. to include comparisons with any other single/combination of intervention(s) also designed for prevention of hypothermia in preterm and/or low birth weight infants applied within 10 minutes after birth in the delivery room), we reassessed previously excluded studies and included two - Mathew 2008 (subset) and NCT00603837 - that have since been published in full (Mathew 2012; Simon 2011). We identified 25 new studies through our updated searches and after screening and assessment included 15 of them (Bhavsar 2015; Caglar 2014; Cardona Torres 2012; Chantaroj 2011; Chawla 2011; Doglioni 2014; Farhadi 2012; Gathwala 2010; Leadford 2013; Leslie 2007; McCarthy 2013; Rohana 2011; Smith 2013; Talakoub 2015; Tescon-delos Santos 2012). We are awaiting further details from investigators on two additional studies (Ahmed 2013; Castro 2007), and on one study currently under review for publication (Nimbalkar 2015). We included these in the Characteristics of studies awaiting classification table. In addition, we classified seven studies as ongoing (CRTI/2014/11/005200; CTRI/2016/02/006673; ISRCTN13184012; NCT01604317; NCT02189746; NCT02250079; NCT02311972), details of which we provided in the Characteristics of ongoing studies table. We also excluded 43 new randomised or quasi-randomised controlled trials and provided reasons for their exclusion in the Characteristics of excluded studies table. We excluded 27 non-randomised studies but, as in previous versions of this review, did not include them in the Characteristics of excluded studies table.

Overall, building upon previous versions of this review (McCall 2005; McCall 2008; McCall 2010), we identified 35 studies, of which we included 25 (Bergman 2004; Bhavsar 2015; Brennan 1996; Caglar 2014; Cardona Torres 2012; Chantaroj 2011; Chawla 2011;
Doglioni 2014; Farhadi 2012; Gathwala 2010; Knobel 2005; Leadford 2013; Leslie 2007; Mathew 2012; McCarthy 2013; Reilly 2015; Roberts 1981; Rohana 2011; Simon 2011; Smith 2013; Talakoub 2015; Tescon-delos Santos 2012; Trevisanuto 2010; Vohra 1999; Vohra 2004); three studies are awaiting classification (Ahmed 2013; Castro 2007; Nimbalkar 2015), and seven studies are ongoing (CRTI/2014/11/005200; CTRI/2016/02/006673; ISRCTN13184012; NCT01604317; NCT02189746; NCT02250079; NCT02311972). One RCT recently published outside the current search dates became known to the review authors and is therefore also awaiting classification in the next update (Shafie 2017).

\section{Included studies}

In all, we included in this review 25 studies involving 2481 randomised infants (2433 completing the studies): two theses (Brennan 1996; Roberts 1981), 22 published manuscripts (Bhavsar 2015; Bergman 2004; Caglar 2014; Cardona Torres 2012; Chantaroj 2011; Chawla 2011; Doglioni 2014; Farhadi 2012; Gathwala 2010; Knobel 2005; Leadford 2013; Mathew 2012; McCarthy 2013; Reilly 2015; Rohana 2011; Simon 2011; Smith 2013; Talakoub 2015; Tescon-delos Santos 2012; Trevisanuto 2010; Vohra 1999; Vohra 2004), and one conference abstract coupled with additional unpublished information received from trial authors (Leslie 2007).

\section{Participants and settings}

Investigators categorised participants by gestation (all preterm) in 17 studies (Caglar 2014; Chawla 2011; Doglioni 2014; Farhadi 2012; Knobel 2005; Leslie 2007; Mathew 2012; McCarthy 2013; Reilly 2015; Roberts 1981; Rohana 2011; Smith 2013; Talakoub 2015; Tescondelos Santos 2012; Trevisanuto 2010; Vohra 1999; Vohra 2004), by birth weight (all low birth weight) in two studies (Bergman 2004; Brennan 1996), and by both gestation and birth weight inclusion criteria in six studies (Bhavsar 2015; Cardona Torres 2012; Chantaroj 2011; Gathwala 2010; Leadford 2013; Simon 2011).

The upper gestational age eligibility criterion across studies was within the extremely preterm category ( $<28$ weeks' gestation) in two studies (Reilly 2015; Vohra 2004); within the very preterm category ( 28 to 31 weeks' gestation) in 10 studies (Chawla 2011; Doglioni 2014; Knobel 2005; Leslie 2007; Mathew 2012; McCarthy 2013; Simon 2011; Smith 2013; Trevisanuto 2010; Vohra 1999); within the moderately preterm category (32 to 33 weeks' gestation) in six studies (Caglar 2014; Chantaroj 2011; Gathwala 2010; Farhadi 2012; Rohana 2011; Talakoub 2015), and within the late preterm category (34 to 36 weeks' gestation) in five studies (Bhavsar 2015; Cardona Torres 2012; Leadford 2013; Roberts 
1981; Tescon-delos Santos 2012). Two additional studies with no gestational age criteria included infants weighing $\geq 1200$ to $\leq 2199$ grams and weighing $\leq 1500$ grams, respectively (Bergman 2004; Brennan 1996). The most frequently reported exclusion criterion was congenital malformations, particularly neural tube defects, omphalocoele, gastroschisis, or other open lesions that would cause greater than normal heat loss at delivery.

Most included studies were single-centred. Four studies were conducted at two or more participating centres, namely, Bergman 2004 and Talakoub 2015 (two centres), Doglioni 2014 (three centres), and Reilly 2015 (39 centres). Sample size ranged from 24 randomised (24 completing the study) in a small single-centred study (Brennan 1996), to 813 randomised (801 completing the study) in a large multi-centred study (Reilly 2015); median sample size across all studies was 62 randomised. Four studies were conducted in lower middle-income countries: India (Bhavsar 2015; Gathwala 2010), Zambia (Leadford 2013), and the Phillippines (Tescon-delos Santos 2012); and seven in upper middle-income countries: South Africa (Bergman 2004), Mexico (Cardona Torres 2012), Thailand (Chantaroj 2011), Turkey (Caglar 2014), Iran (Farhadi 2012; Talakoub 2015) and Malaysia (Rohana 2011). All other studies took place in high-income countries: USA (Brennan 1996; Chawla 2011; Knobel 2005; Mathew 2012; Roberts 1981; Simon 2011), Canada (Vohra 1999; Vohra 2004), Ireland (McCarthy 2013), Australia (Smith 2013), Italy (Doglioni 2014; Trevisanuto 2010), UK (Leslie 2007), and at multiple centres in high-income countries (Reilly 2015).

\section{Interventions}

Interventions included in this review fall into three major groups: barriers to heat loss, external heat sources, and combinations of interventions.

- Eleven comparisons (18 studies: 2121 infants randomised, 2080 completing the studies) fell within the barriers to heat loss category: plastic wrap or bag versus routine care (Cardona Torres 2012; Chantaroj 2011; Farhadi 2012; Gathwala 2010; Knobel 2005; Leadford 2013; Reilly 2015; Rohana 2011; Smith 2013; Talakoub 2015; Trevisanuto 2010; Vohra 1999; Vohra 2004), plastic wrap versus routine care during interhospital neonatal transport (Bhavsar 2015), plastic bag with previous drying versus routine care (Cardona Torres 2012), plastic cap versus routine care (no cap) (Trevisanuto 2010), plastic bag and plastic cap versus routine care (Talakoub 2015; Tescon-delos Santos 2012), plastic bag with previous drying versus plastic bag without previous drying (Cardona Torres 2012), plastic cap versus plastic bag (Trevisanuto 2010), plastic bag versus plastic wrap (Caglar 2014), plastic total body wrap (body + head) versus plastic body wrap (head uncovered) (Doglioni 2014), plastic bag and plastic hat versus plastic bag and cotton hat (Talakoub 2015), and stockinet cap versus routine care (no cap) (Roberts 1981).

- Two comparisons (three studies: 161 infants randomised,157 completing the studies) fell within the external heat sources category: skin-to-skin care versus routine care (Bergman 2004), and thermal mattress versus routine care (Brennan 1996; Chawla 2011).

- Two comparisons (four studies: 199 infants randomised, 196 completing the studies) fell within the combinations of interventions category: thermal mattress versus plastic wrap or bag (Mathew 2012; Simon 2011), and plastic bag and thermal mattress versus plastic bag only (Leslie 2007; McCarthy 2013).

All interventions were applied immediately after birth in the delivery room. Available information indicates that all studies used a combination of external heat sources (radiant warmer/ drop light, warmer table, transport incubator) and caps as part of routine thermal care (control group and/or intervention group). Three studies also employed plastic barriers or shields as part of routine care (Bergman 2004; Chantaroj 2011; Chawla 2011). Bergman 2004 compared skin-to-skin contact versus routine care. Infants in the routine care group were immediately transferred to a prewarmed servo-controlled closed incubator, which remained with the mother in the delivery ward for the first hour. If the skin temperature became $<36^{\circ} \mathrm{C}$, a cap and booties were applied and a heat shield was placed over the infant. If this was insufficient, a sheet of plastic was framed over the foot end of the heat shield and the outlet of warm air funnelled over the infant. Chawla 2011 compared thermal mattress versus routine care for infants at $<32$ weeks' gestation. All infants received routine thermal care including hats and radiant warmers and were transported to the NICU in prewarmed incubators set at $37^{\circ} \mathrm{C}$. In addition, infants at $<28$ weeks' gestation were also placed without drying in a reclosable plastic bag below the neck. Chantaroj 2011 compared plastic bag versus routine care, and after initial stabilisation, infants in the routine care group were covered with a polyvinyl wrap as part of standard care before transfer to the NICU. With evolving routine thermal care throughout the lifetime of this review, more recent studies have considered the use of plastic wraps or bags applied immediately at birth as part of routine thermal care (e.g. Caglar 2014; Leslie 2007; McCarthy 2013). However, for the purpose of assigning included studies to a comparison group in this review, 'plastic wrap or bag' has remained an intervention/ active comparator rather than being referred to as 'routine care', in keeping with the prespecified definition at the review protocol stage.

During two studies, researchers attempted to keep the ambient delivery room temperature at $26^{\circ} \mathrm{C}$ for preterm births (Chantaroj 2011; Knobel 2005). Investigators reported a range of routine delivery room ambient temperatures from a cool $20^{\circ} \mathrm{C}$ to $21^{\circ} \mathrm{C}$ (Farhadi 2012), through $24^{\circ} \mathrm{C}$ (Trevisanuto 2010), through $25^{\circ} \mathrm{C}$ (Leadford 2013), to a warm $26^{\circ} \mathrm{C}$ to $28^{\circ} \mathrm{C}$ (Tescon-delos Santos 2012). Despite attempts to keep delivery room temperatures static, researchers reported ranges of $18^{\circ} \mathrm{C}$ to $31^{\circ} \mathrm{C}$ (Knobel 2005). We have provided full details of additional thermal care measures in the Characteristics of included studies table.

\section{Outcomes}

Four studies (Gathwala 2010; Mathew 2012; Talakoub 2015; Tescondelos Santos 2012) reported the primary outcome measure (temperature of the infant on admission to NICU or up to two hours after birth) as a continuous variable, one study as a dichotomous variable (derived from skin temperature in ${ }^{\circ} \mathrm{C}$ ) (Bergman 2004), and the remaining 20 studies as both a continuous and a dichotomous variable (Bhavsar 2015; Brennan 1996; Caglar 2014; Cardona Torres 2012; Chantaroj 2011; Chawla 2011; Doglioni 2014; Farhadi 2012; Knobel 2005; Leadford 2013; Leslie 2007; McCarthy 2013; Reilly 2015; Roberts 1981; Rohana 2011; Simon 2011; Smith 2013; Trevisanuto 2010; Vohra 1999; Vohra 2004). Overall, across studies, 18 studies reported core body temperature in ${ }^{\circ} \mathrm{C}$ axillary (Bhavsar 2015; Brennan 1996; Caglar 2014; Cardona Torres 2012; 
Chawla 2011; Doglioni 2014; Farhadi 2012; Leadford 2013; Leslie 2007; Mathew 2012; Reilly 2015; Roberts 1981; Rohana 2011; Simon 2011; Smith 2013; Talakoub 2015; Tescon-delos Santos 2012; Trevisanuto 2010), four studies ${ }^{\circ} \mathrm{C}$ rectal (Chantaroj 2011; Knobel 2005; Vohra 1999; Vohra 2004), and two studies both axillary and rectal (Gathwala 2010; McCarthy 2013); one study reported skin temperature in ${ }^{\circ} \mathrm{C}$ (Bergman 2004). Definitions of hypothermia were not consistent across studies. One large multi-centred study was powered for all-cause mortality at discharge (or at six months' corrected gestation, if the infant remained in hospital), reporting baseline and poststabilisation core body temperature $\left({ }^{\circ} \mathrm{C}\right.$ axillary) as secondary outcomes (Reilly 2015).

Earlier studies provided limited reporting of prespecified secondary outcomes, but more recent studies have reported important secondary outcome measures, including mortality, major brain injury, bronchopulmonary dysplasia (BPD), necrotising enterocolitis (NEC), retinopathy of prematurity (ROP), sepsis, and adverse events, particularly hyperthermia on admission to the NICU.

\section{Summary descriptions of individual studies}

We have presented the main characteristics of included studies in the Characteristics of included studies table.

\section{Excluded studies}

In all, for this update, 43 new randomised or quasi-randomised controlled trials did not meet our inclusion criteria or were considered to be 'near misses' and are included in the Characteristics of excluded studies table. Primary reasons for exclusion include the following: Participants were term (i.e. at $\geq 37$ weeks' gestation) ( $n=11$ studies); the intervention was not applied immediately at birth (within 10 minutes) in the delivery room (n $=26$ studies); the intervention was not considered to be over and above routine thermal care ( $\mathrm{n}=2$ studies); the intervention was considered to be beyond the remit of this review ( $n=2$ studies); and methodological information was insufficient for an informed decision regarding eligibility ( $\mathrm{n}=2$ studies).

We have provided reasons for exclusion for all individual studies ( $=132$ studies) in the Characteristics of excluded studies table.

\section{Risk of bias in included studies}

Figure 2 presents the risk of bias summary detailing review authors' judgements about each risk of bias item for each included study. Additional details and the review authors' supporting statements for risk of bias are available in the Characteristics of included studies table.

\section{Allocation}

Generation of the allocation sequence was adequate (computer generated, lot drawing, random number tables) in most studies (84\%), inadequate in two studies (Chawla 2011; Tescon-delos Santos 2012), and unclear in two studies (Cardona Torres 2012; Rohana 2011). Allocation concealment was adequate in 52\% of studies, inadequate in six studies (Chantaroj 2011; Chawla 2011; Farhadi 2012; Simon 2011; Smith 2013; Tescon-delos Santos 2012), and unclear in six studies (Bergman 2004; Brennan 1996; Cardona Torres 2012; Gathwala 2010; Roberts 1981; Talakoub 2015). Four studies reported using sealed opaque envelopes but did not explicitly state that these were sequentially numbered; we therefore assessed these studies as having high risk for selection bias (Chantaroj 2011; Farhadi 2012; Simon 2011; Smith 2013). Nine studies also employed random sequences balanced in blocks of two, four, or six participants (Bhavsar 2015; Chantaroj 2011; Farhadi 2012; Leslie 2007; Mathew 2012; McCarthy 2013; Roberts 1981; Trevisanuto 2010; Vohra 2004). We noted some potential for inadequate allocation concealment and therefore cannot rule out selection bias. However, on assessment, we did not penalise studies for this. Overall, across both domains, we considered 12 studies (48\%) to be at low risk of potential selection bias (Bhavsar 2015; Caglar 2014; Doglioni 2014; Knobel 2005; Leadford 2013; Leslie 2007; Mathew 2012; McCarthy 2013; Reilly 2015; Trevisanuto 2010; Vohra 1999; Vohra 2004).

\section{Blinding}

Across $92 \%$ of included studies, we noted no attempt to blind healthcare participants and personnel to the intervention (unclear but unlikely in two studies - Smith 2013; Talakoub 2015). However, lack of blinding is often not feasible for non-pharmacological interventions (Boutron 2008). Outcome measures were objective and so were less likely to be biased than subjective outcome measures. For our primary outcome measure - core body or skin temperature - we conducted a simple linear regression analysis using the temperature time series (birth to 120 minutes) for one intervention and control reported in Cardona Torres 2012 . We found a significant linear trend $(P<0.029)$ and widening of the mean temperature difference between control and intervention groups (polyethylene bag without previous drying). This pattern of results suggests that detection bias was unlikely. In addition, we assessed secondary outcome data for key morbidities throughout the neonatal stay when personnel were less likely to be aware of the original allocation group. Only three studies (Bergman 2004; Reilly 2015; Tescon-delos Santos 2012) reported any attempt to blind outcome assessors or the data analysis team to the intervention; therefore, we cannot rule out potential biases for the other studies.

\section{Incomplete outcome data}

No studies reported incomplete outcome data $\geq 20 \%$. All randomised infants completed the trial in most (64\%) studies. Of the remaining 10 studies (Bergman 2004; Bhavsar 2015; Cardona Torres 2012; Reilly 2015; Rohana 2011; Simon 2011; Smith 2013; Talakoub 2015; Vohra 1999; Vohra 2004), nine studies clearly stated reasons for missing data. Data show losses to follow-up as follows: Bergman 2004 (4 of 35, or 3\%), Bhavsar 2015 (5 of 101, or $5 \%$ ), Cardona Torres 2012 (9 of 99, or 9\%), Reilly 2015 (12 of 813 , or $1.5 \%$ ), Rohana 2011 (5 of 115, or 4\%), Simon 2011 (3 of 39, or 8\%), Smith 2013 (3 of 95, or 3\%), Talakoub 2015 ( 2 of 98, or 2\%), Vohra 1999 (3 of 62 , or $5 \%$ ), and Vohra 2004 ( 2 of 55, or 4\%). Two studies carried out an intention-to-treat analysis including all infants initially enrolled (Reilly 2015; Simon 2011). We categorised all studies as having low risk of attrition bias.

\section{Selective reporting}

We did not have access to study protocols for 16 included studies; therefore we assessed potential risk of reporting bias as limited. We accessed online clinical trial registries or protocols published in journals for nine studies and found that all or most study outcomes detailed at the time of registration were also reported in the published manuscript; therefore we categorised these as having low risk of reporting bias (Cardona Torres 2012; Doglioni 2014; Farhadi 2012; Leadford 2013; McCarthy 2013; Reilly 2015; Simon 
2011; Smith 2013; Trevisanuto 2010). Information was insufficient for assessment of risk of bias for the remaining 16 studies.

\section{Other potential sources of bias}

Overall, 14 studies (56\%) appeared to be free from any additional sources of bias. We categorised one study as having unclear risk of other sources of potential bias owing to the significantly longer time from birth to admission for infants in the plastic bag + thermal mattress group when compared with the plastic bag group (24 vs 19 minutes; $P=0.008$ ) (McCarthy 2013). Trial authors presented findings derived from a regression analysis that explored the relationship between time to admission and admission temperature, showing some evidence of a positive correlation between time to admission and rectal temperature in both groups, although the association was weak. Further multiple regression analyses suggest that maternal temperature and transport incubator temperature had an effect on infant temperature and the presence of hyperthermia, although these associations were not significant enough to explain differences between the two groups. Trial authors suggested that increased time from birth to admission in the plastic bag and thermal mattress group may have been a consequence of non-masking of treatment allocation. This may have resulted in greater vigilance among carers regarding infants who were not in the plastic bag and mattress group; we therefore moved these infants to the NICU faster group (shorter time from birth to admission) to avoid hypothermia.

We categorised 10 studies as having high risk of other sources of potential bias based on the following details.

- Bergman 2004 showed potential for selection bias in that the assigned research nurse was unavailable for 99 potentially eligible mother-infant dyads. The aetiology of hypothermia in these infants may have differed from the causes studied. In addition, recruitment for this study was terminated on the basis of significant results after an interim analysis was conducted.

- Data from Bhavsar 2015 show that infants in the intervention group were significantly $(P<0.05)$ heavier than those in the control group. This fact could have potentially influenced the effect size because smaller infants are more susceptible to heat loss.

- For Cardona Torres 2012, we found potential for selection bias in that infants in the intervention group were significantly smaller than those in the control group. This could have been a consequence of poor randomisation.

- Knobel 2005 attempted to maintain delivery room temperature at $26^{\circ} \mathrm{C}$ for all preterm deliveries, but actual temperatures ranged from $18.9^{\circ} \mathrm{C}$ to $31.1^{\circ} \mathrm{C}$. On post hoc analysis, we found that warmer delivery room temperatures were associated with higher admission temperatures, but only the subgroup of infants who were both delivered in warm rooms and placed in plastic bags had a mean temperature $>36.4^{\circ} \mathrm{C}$. Data show no significant differences between intervention and control groups for mean delivery room temperature. After controlling for delivery room temperature, we found that the mean temperature on admission to the NICU in the intervention group was still $0.6^{\circ} \mathrm{C}$ higher than in the control group.

- Leslie 2007 was underpowered (powered to detect a difference in mean temperature (intervention and control) of $0.8^{\circ} \mathrm{C}$ ).

- Roberts 1981 reported some imbalance between study groups in mean delivery room axillary temperatures for infants <
2000 grams. Analysis of covariance shows that delivery room axillary temperature had a significant effect on infant axillary temperature on admission to the NICU. When we statistically equalised the two groups with respect to delivery room axillary temperature, we noted no significant differences between the two interventions.

- Simon 2011 showed potential for selection bias in that the assigned researcher was unavailable for 141 of 148 excluded cases. The aetiology of hypothermia in these infants may have differed from the causes studied. Trial authors noted greater variability of use between practitioners when the intervention was polyethylene wrap as opposed to the thermal mattress. In some cases, displacement of the wrap could have resulted in heat loss; therefore we cannot rule out performance bias.

- Smith 2013 showed potential for performance bias when the infant underwent prolonged resuscitation, or when placement of a nappy or umbilical lines tended to dislodge the NeoWrap, potentially resulting in heat loss and therefore an underestimated effect size. Times to arrival to the NICU from the delivery room varied.

- Tescon-delos Santos 2012 reported that trial authors provided additional swaddling with prewarmed blankets for infants with a recorded axillary temperature $<36.5^{\circ} \mathrm{C}$, which prevented further occurrence of hypothermia and potentially masked any true effect of the intervention (polyethylene body wrap and cap). A higher incidence of further swaddling in the control group resulted in higher mean temperatures during the first 15 minutes of life. This additional swaddling may also have been influenced by greater attention of healthcare providers (not blinded to the intervention) to the control group. Therefore we cannot rule out performance bias.

- Vohra 1999 showed some imbalance in birth weight between study groups. However the birth weight adjusted difference in mean rectal temperature of $1.54^{\circ} \mathrm{C}$ for the smaller group remained significant. For infants at $<28$ weeks' gestation, mean birth weight was 914 grams (SD 163) for the plastic wrap group and 742 grams (SD 206) for the non-wrap group; therefore, results could be potentially biased towards the wrap group because the non-wrap group was lighter and more vulnerable to heat loss.

In addition, Reilly 2015 reported several protocol violations among treatment groups, including delayed application of the wrap, wrap opening during the study period, and early removal of the wrap. However, data show significantly higher mean baseline and poststabilisation temperatures for the wrap group. Other violations included use of adjunct heat sources in small numbers of infants in both wrap and non-wrap groups, which was unlikely to affect findings. We therefore assigned this study low risk of other bias.

\section{Effects of interventions}

See: Summary of findings for the main comparison Plastic wrap or bag compared with routine care in preterm and/or low birth weight infants

\section{Results of meta-analyses}

In all, we included in this review 25 studies involving 2481 randomised infants (2433 completing the studies). We presented 15 comparison groups across three main categories: barriers to heat loss, external (additional) heat sources, and combinations 
of interventions. The barriers to heat loss category consisted of 11 comparisons: plastic wrap or bag versus routine care (Cardona Torres 2012; Chantaroj 2011; Farhadi 2012; Gathwala 2010; Knobel 2005; Leadford 2013; Reilly 2015; Rohana 2011; Smith 2013; Talakoub 2015; Trevisanuto 2010; Vohra 1999; Vohra 2004), plastic wrap versus routine care during interhospital neonatal transport (Bhavsar 2015), plastic bag with previous drying versus routine care (Cardona Torres 2012), plastic cap versus routine care (no cap) (Trevisanuto 2010), plastic bag and plastic cap versus routine care (Talakoub 2015; Tescon-delos Santos 2012), plastic bag with previous drying versus plastic bag without previous drying (Cardona Torres 2012), plastic cap versus plastic bag (Trevisanuto 2010), plastic bag versus plastic wrap (Caglar 2014), plastic total body wrap (body + head) versus plastic body wrap (head uncovered) (Doglioni 2014), plastic bag and plastic hat versus plastic bag and cotton hat (Talakoub 2015), and stockinet cap versus routine care (no cap) (Roberts 1981). The external heat source category consisted of two comparison groups: skin-to-skin care versus routine care (Bergman 2004), and thermal mattress versus routine care (Brennan 1996; Chawla 2011). Two additional comparison groups compared combinations of interventions: plastic wrap or bag versus thermal mattress (Mathew 2012; Simon 2011), and plastic bag + thermal mattress versus plastic bag only (Leslie 2007; McCarthy 2013).

\section{Barriers to heat loss category}

\section{Plastic wrap or bag versus routine care (Comparison 1)}

\section{Primary outcomes}

Core body temperature $\left({ }^{\circ} \mathrm{C}\right)$ of the infant taken on admission to the NICU or up to two hours after birth (Outcome 1.1)

Thirteen studies comprising 1633 infants reported core body temperature $\left({ }^{\circ} \mathrm{C}\right.$ rectal or axillary) on admission to the NICU (Chantaroj 2011; Farhadi 2012; Gathwala 2010; Knobel 2005; Reilly 2015; Rohana 2011; Smith 2013; Talakoub 2015; Trevisanuto 2010; Vohra 1999; Vohra 2004), at 30 minutes after birth (Cardona Torres 2012), and at one hour after birth (Leadford 2013). Reilly 2015 defined baseline temperature as temperature taken after cardiorespiratory stabilisation upon direct admission to the NICU or after arrival to the NICU if immediate resuscitation took place in the delivery room. Most studies reported that the time from birth to admission to the NICU ranged from around 20 minutes to 45 minutes; therefore, we included the 30-minute temperature measurement from the Cardona Torres 2012 study, rather than the primary endpoint of 120 minutes. Each individual study showed a significant effect in favour of the intervention group (plastic wrap or bag) for infants across gestations, with the exception of Vohra 1999, which showed no significant differences in effect for the subgroup of infants at 28 to 31 weeks' gestation. Four studies did not provide subgroup data (Cardona Torres 2012; Chantaroj 2011; Gathwala 2010; Leadford 2013); we therefore grouped them together in the 'all infant' category with a range of gestations from 26 to 37 weeks. Although infants in the Knobel 2005 and Trevisanuto 2010 studies had gestation $<29$ completed weeks and Smith $2013 \leq 29$ completed weeks, we included these infants in the $<28$ completed weeks subgroup for meta-analysis. Four studies provided data for the subgroup $\geq 28$ completed weeks, with a range of gestation from 28 to 33 weeks (Farhadi 2012; Rohana 2011; Talakoub 2015; Vohra 1999). The intervention consisted of plastic bags in 10 studies (Cardona Torres 2012; Chantaroj 2011; Farhadi 2012; Gathwala 2010; Knobel 2005; Leadford 2013; Talakoub 2015; Trevisanuto 2010; Vohra 1999; Vohra 2004), and three studies provided plastic wrap (or a bag cut similar to wrap) as the intervention (Reilly 2015; Rohana 2011; Smith 2013).

Outcome 1.1.1: Overall for infants at $<37$ weeks' gestation, data show a statistically significant difference in core body temperature on admission to the NICU favouring the intervention group (plastic wrap or bag) compared with those who received routine care immediately after birth in the delivery room (mean difference (MD) $0.58^{\circ} \mathrm{C}, 95 \%$ confidence interval $(\mathrm{Cl}) 0.50$ to $0.66 ; 13$ studies; $\mathrm{N}=$ 1633) (Analysis 1.1). However, the overall test for homogeneity and for each subgroup of infants failed, with an $\mathrm{I}^{2}$ value ranging from $59 \%$ to $61 \%$ (moderate heterogeneity).

Outcome 1.1.2: For infants at $<28$ weeks' gestation, data show a statistically significant difference in core body temperature on admission to the NICU favouring the intervention group (plastic wrap or bag) when compared with those who received routine care immediately after birth in the delivery room $\left(\mathrm{MD} 0.65^{\circ} \mathrm{C}, 95 \% \mathrm{Cl}\right.$ 0.52 to 0.79 ; eight studies; $N=1171$ ) (Analysis 1.1 ). However, the test for homogeneity failed, with an $\mathrm{I}^{2}$ value of $61 \%$ (moderate heterogeneity).

Outcome 1.1.3: For infants at $\geq 28$ weeks' gestation, data show a statistically significant difference in core body temperature on admission to the NICU favouring the intervention group (plastic wrap or bag) when compared with those who received routine care immediately after birth in the delivery room (MD $0.56^{\circ} \mathrm{C}, 95 \%$ $\mathrm{Cl} 0.34$ to 0.78 ; four studies, $\mathrm{n}=200$ ) (Analysis 1.1 ). However, the test for homogeneity failed, with an $\mathrm{I}^{2}$ value of $60 \%$ (moderate heterogeneity).

\section{Publication bias and investigation of heterogeneity}

Owing to the substantial heterogeneity observed, we inspected a funnel plot comparison for plastic wrap or bag versus routine care for core body temperature $\left({ }^{\circ} \mathrm{C}\right)$ on admission to the NICU or up to two hours after birth for possible publication bias (Figure 3). When we removed Vohra 2004 from the meta-analysis of all studies ( $<37$ weeks' gestation), data show no change in the effect measure (MD $0.58^{\circ} \mathrm{C}, 95 \% \mathrm{Cl} 0.50$ to $0.66 ; 12$ studies; $\mathrm{N}=1580$ ). Heterogeneity remained moderate at $61 \%$. Publication bias was potentially problematic for the subgroup of studies presenting data for infants at $<28$ weeks' gestation, suggesting that non-effect, smaller studies have not been published. To overcome this, we removed from the meta-analysis the following studies with $95 \%$ confidence limits > 1, with an $I^{2}$ value of $0 \%$ : Rohana 2011; Vohra 1999; and Vohra 2004 (MD $0.57^{\circ} \mathrm{C}, 95 \% \mathrm{Cl} 0.42$ to 0.71 ; five studies; $\mathrm{N}=1063$ ). For the study subgroup presenting data for infants at $\geq 28$ weeks' gestation, upon removal of Farhadi $2012\left(\mathrm{MD} 0.49^{\circ} \mathrm{C}\right.$, $95 \% \mathrm{Cl} 0.26$ to 0.72 ; three studies; $\mathrm{N}=178$ ), heterogeneity remained moderate, with an $\mathrm{I}^{2}$ value of $55 \%$. 
Figure 3. Funnel plot of comparison: 1 Plastic wrap or bag versus routine care, outcome: 1.1 Core body temperature $\left({ }^{\circ} \mathrm{C}\right)$ on admission to NICU or up to 2 hours after birth.

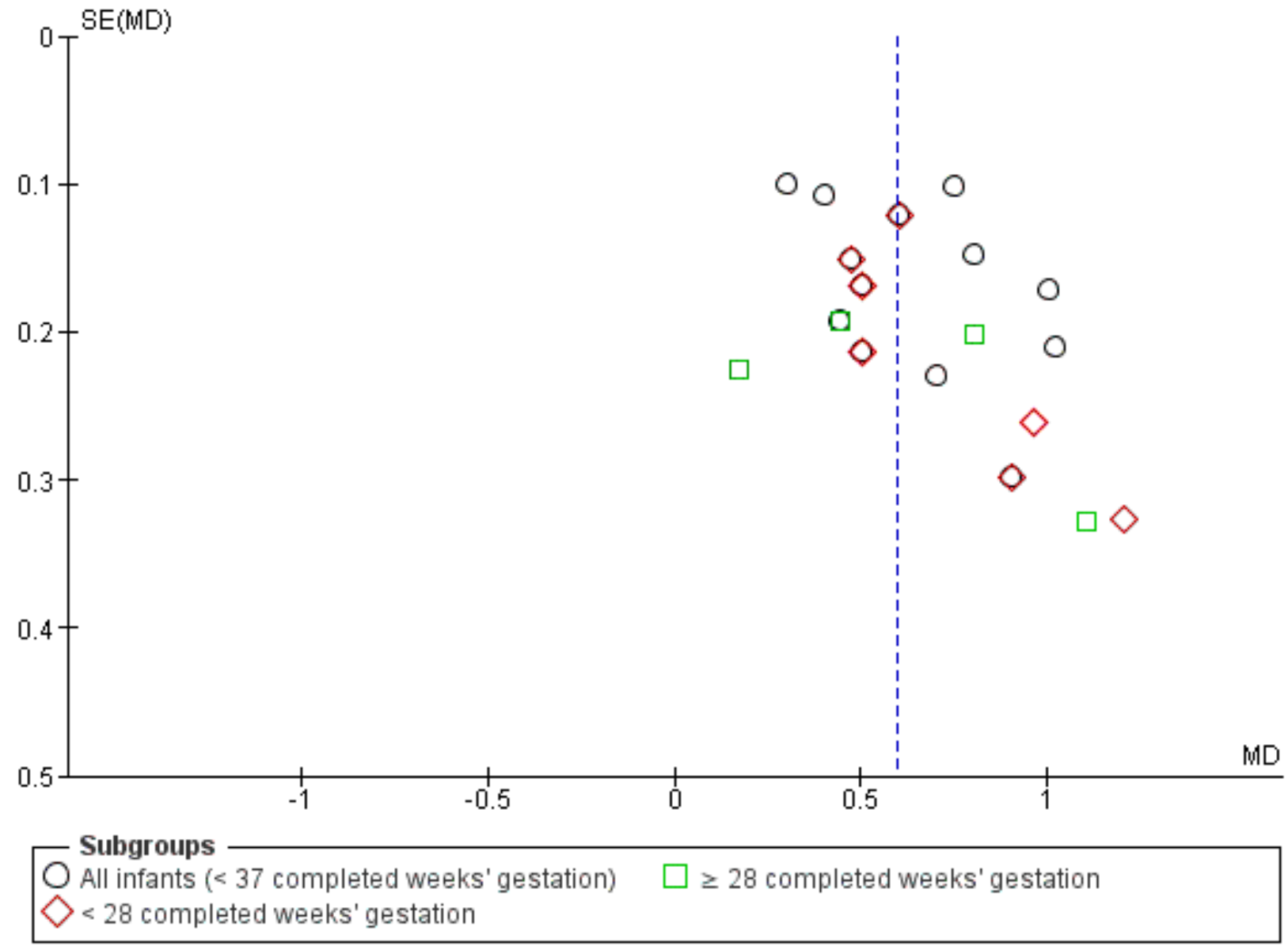

Hypothermia on admission to the NICU (core body temperature < $36.5^{\circ} \mathrm{C}$, or skin temperature $<36^{\circ} \mathrm{C}$ ) (Outcome 1.2 )

Ten studies comprising 1417 infants, in addition to reporting core body temperature on admission to the NICU as a continuous variable, provided data in a dichotomous format in terms of the incidence of hypothermia in intervention and control groups (plastic wrap or bag and routine care) (Cardona Torres 2012; Chantaroj 2011; Farhadi 2012; Knobel 2005; Leadford 2013; Reilly 2015; Rohana 2011; Trevisanuto 2010; Vohra 1999; Vohra 2004). Cardona Torres 2012 reported hypothermia throughout the study period of 120 minutes, and Leadford 2013 reported hypothermia one hour after birth. Most studies defined hypothermia as a core body temperature (rectal or axillary) $<36.5^{\circ} \mathrm{C}$ on admission to the NICU, and two studies defined hypothermia as a core body temperature (rectal or axillary) $<36.4^{\circ} \mathrm{C}$ on admission to the NICU (Knobel 2005; Trevisanuto 2010). Reilly 2015 reported hypothermia defined as core body temperature (axillary) $<36.5^{\circ} \mathrm{C}$ at baseline (after cardiorespiratory stabilisation upon direct admission to the NICU, or after arrival to the NICU if immediate resuscitation took place in the delivery room). Cardona Torres 2012 provided no clear definition. For Vohra 1999 and Vohra 2004, we derived data from a graph of core body temperatures on admission to the NICU versus birth weight for control and intervention groups; core body temperature (rectal) was defined as $<35.0^{\circ} \mathrm{C}$, and no subgroup data were available.

Most individual studies showed a significant effect in favour of the intervention (plastic wrap or bag group), with the exception of Chantaroj 2011, which showed no differences in effect for infants at $<28$ weeks' gestation; however the number of infants in this subgroup was very small. The small trials of Vohra 1999 and Cardona Torres 2012 showed borderline significant effects favouring the plastic wrap or bag group.

Outcome 1.2.1: Overall, for infants at $<37$ weeks' gestation, plastic wrap or bag significantly reduced risk of hypothermia on admission to the NICU (typical RR $0.67,95 \% \mathrm{Cl} 0.62$ to 0.72 ; typical risk difference (RD) $-0.25,95 \% \mathrm{Cl}-0.29$ to $-0.20 ; 10$ studies; $\mathrm{N}=1417$ ) (Analysis 1.2). Four infants would have to be wrapped in plastic to prevent one infant from becoming hypothermic (NNTB 4, 95\% $\mathrm{Cl} 4$ to 5). The overall test for homogeneity failed, with an $\mathrm{I}^{2}$ value of $65 \%$ (moderate heterogeneity) for RR and $70 \%$ (moderate heterogeneity) for RD, as well as for subgroup differences, with an $I^{2}$ value of $78.5 \%$ (high heterogeneity) for RR and $91.6 \%$ (high heterogeneity) for RD.

Outcome 1.2.2: For infants at $<28$ weeks' gestation, plastic wrap or bag significantly reduced risk of hypothermia on admission to NICU 
(typical RR 0.70, 95\% Cl 0.65 to 0.77 ; typical RD $-0.23,95 \% \mathrm{Cl}-0.29$ to -0.18 ; six studies; $\mathrm{N}=1029$ ) (Analysis 1.2).

This finding is consistent with those for outcome measure 1.1.2. Four infants would have to be wrapped in plastic to prevent one infant from becoming hypothermic (NNTB 4, 95\% $\mathrm{Cl} 4$ to 6). The test for homogeneity passed with an $I^{2}$ value of $44 \%$ (low heterogeneity) for RR but with $\mathrm{I}^{2}$ of $67 \%$ (moderate heterogeneity) for RD.

Outcome 1.2.3: For infants at $\geq 28$ weeks' gestation, plastic wrap or bag significantly reduced risk of hypothermia on admission to the NICU (typical RR $0.17,95 \% \mathrm{Cl} 0.07$ to 0.43 ; typical RD $-0.71,95 \%$ $\mathrm{Cl}-0.89$ to -0.52 ; two studies; $\mathrm{N}=55$ ) (Analysis 1.2). This finding is consistent with those reported for outcome measure 1.1.3. One infant would have to be wrapped in plastic to prevent one infant from becoming hypothermic (NNTB $195 \% \mathrm{Cl} 1$ to 2). The test for homogeneity passed with an $\mathrm{I}^{2}$ value of $0 \%$ for both RR and RD.

Computational errors can occur when a zero count is seen in one or both arms of a study, as in Cardona Torres 2012 and Vohra 1999. RevMan software compensates by adding 0.5 to each cell.
Publication bias and investigation of heterogeneity

Owing to the substantial heterogeneity noted above, we inspected a funnel plot comparison (SE(log (RR)) vs RR) for plastic wrap or bag versus routine care for hypothermia on admission to the NICU for possible publication bias. Figure 4 shows that publication bias may be present, suggesting that non-effect, smaller studies have not been published. Upon removal from the meta-analysis of small studies showing a significant effect with $\mathrm{SE}(\log (\mathrm{RR}))$ above 0.3 (Cardona Torres 2012; Chantaroj 2011; Farhadi 2012; Vohra 1999; Vohra 2004), the overall test for heterogeneity passed with an $I^{2}$ value of $0 \%$, and the effect size remained highly significant (typical RR $0.72,95 \% \mathrm{Cl} 0.67$ to 0.78 ; five studies; $N=1167$ ). For the subgroup of studies presenting data for infants at $<28$ weeks' gestation, upon removal of two studies from the meta-analysis (Farhadi 2012; Vohra 2004), the overall test for heterogeneity passed with $I^{2}$ of $0 \%$, and the effect size remained highly significant (typical RR $0.73,95 \% \mathrm{Cl}$ 0.67 to 0.79 ; four studies; $N=958$ ).

Figure 4. Funnel plot of comparison: 1 Plastic wrap or bag versus routine care, outcome: 1.2 Hypothermia on admission to NICU: core body temperature $<36.5^{\circ} \mathrm{C}$ or skin temperature $<36^{\circ} \mathrm{C}$.

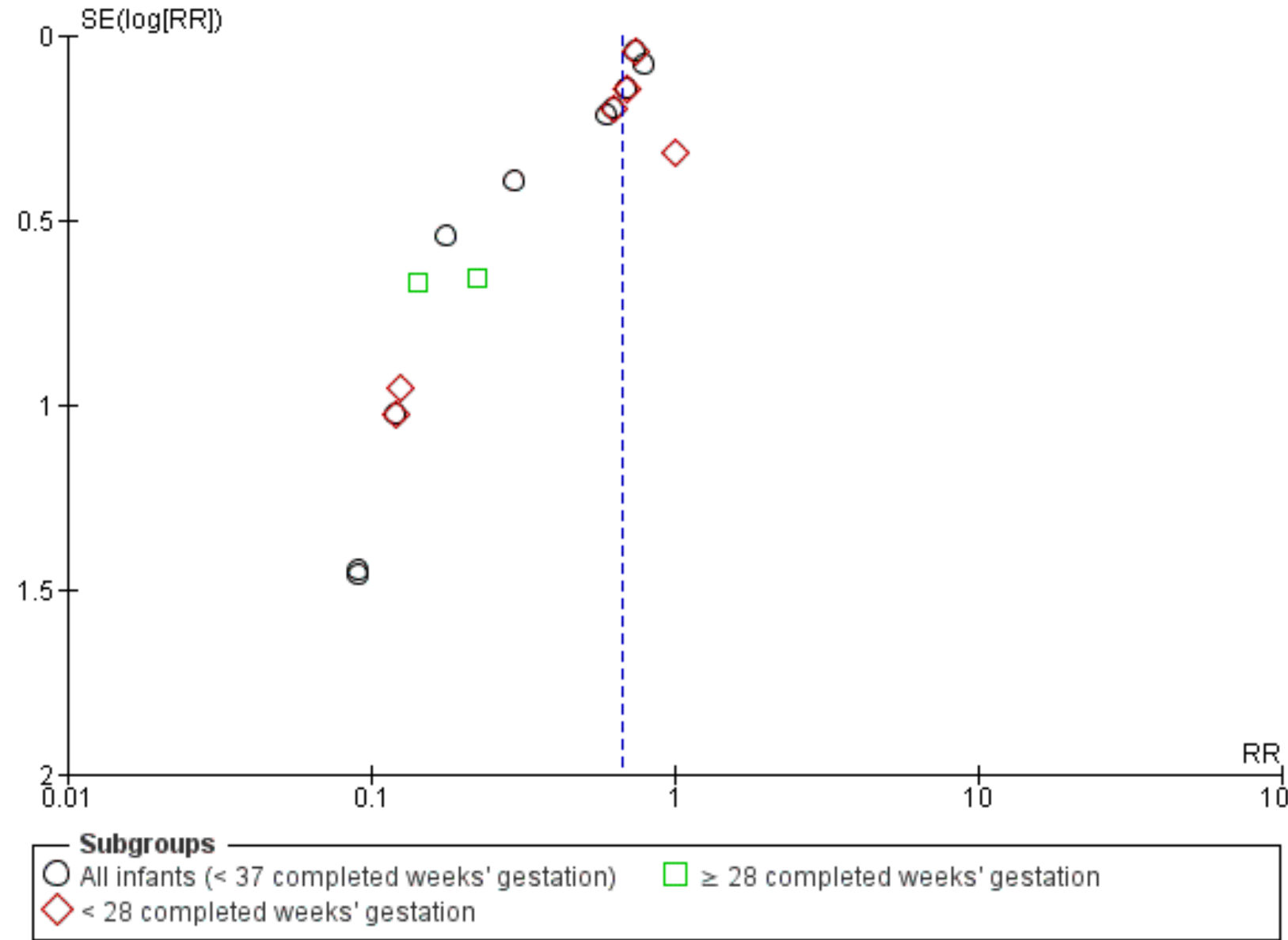


Outside normothermic range on admission to the NICU or up to two hours after birth (Outcome 1.3)

Five studies comprising 1048 infants reported this outcome, or it was derived from definitions of hypothermia and hyperthermia as the incidence of a core body temperature outside the normothermic range on admission to the NICU (Chantaroj 2011; Farhadi 2012; Leadford 2013; Reilly 2015; Trevisanuto 2010). Chantaroj 2011 and Farhadi 2012 defined normothermia as core body temperature (rectal or axillary) of $36.5^{\circ} \mathrm{C}$ to $37.5^{\circ} \mathrm{C}$, Reilly 2015 as core body temperature (axillary) of $36.5^{\circ} \mathrm{C}$ to $37.4^{\circ} \mathrm{C}$ (baseline), Trevisanuto 2010 as core body temperature (axillary) of $36.4^{\circ} \mathrm{C}$ to $37.5^{\circ} \mathrm{C}$, and Leadford 2013 as core body temperature (axillary) of $36.5^{\circ} \mathrm{C}$ to $37.5^{\circ} \mathrm{C}$ at one hour after birth. Most individual studies showed a significant effect favouring the intervention (plastic wrap or bag group), with the exception of Chantaroj 2011, which showed no difference in effect for infants at $<28$ weeks' gestation; however, the number of infants in this subgroup was very small. Trevisanuto 2010 showed a borderline significant effect favouring the plastic wrap or bag group. This was not a prespecified outcome at the review protocol stage.

Outcome 1.3.1: Overall, for infants at < 37 weeks' gestation, plastic wrap or bag significantly reduced the risk of a core body temperature outside the normothermic range on admission to the NICU (typical RR $0.75,95 \% \mathrm{Cl} 0.69$ to 0.81 ; typical RD -0.20 , $95 \% \mathrm{Cl}-0.26$ to -0.15 ; five studies; $\mathrm{N}=1048$ ) (Analysis 1.3 ). Five infants would have to be wrapped in plastic to prevent one infant from having a core body temperature outside the normothermic range (NNTB 5, 95\% Cl 4 to 7 ). The overall test for homogeneity failed, with an $1^{2}$ value of $77 \%$ (high heterogeneity) for RR and $87 \%$ (high heterogeneity) for RD, and also for subgroup differences with an $\mathrm{I}^{2}$ value of $77 \%$ (high heterogeneity) for RR and $92.5 \%$ (high heterogeneity) for RD.

Outcome 1.3.2: For infants at $<28$ weeks' gestation, plastic wrap or bag significantly reduced the risk of being outside the normothermic range on admission to the NICU (typical RR 0.81, 95\% $\mathrm{Cl} 0.75$ to 0.88 ; typical RD $-0.15,95 \% \mathrm{Cl}-0.21$ to -0.10 ; three studies; $\mathrm{N}=871$ ) (Analysis 1.3). Seven infants would have to be wrapped in plastic to prevent one infant from having a core body temperature outside the normothermic range on admission to the NICU (NNTB $7,95 \% \mathrm{Cl} 5$ to 10$)$. The test for homogeneity passed, with an $\mathrm{I}^{2}$ value of $0 \%$ for RR and $\mathrm{I}^{2}$ of $38 \%$ (low heterogeneity) for RD.

Outcome 1.3.3: For infants at $\geq 28$ weeks' gestation, plastic wrap or bag significantly reduced the risk of being outside the normothermic range on admission to the NICU (RR $0.14,95 \% \mathrm{Cl}$ 0.04 to 0.52 ; RD $-0.76,95 \% \mathrm{Cl}-0.98$ to -0.53 ; one study; $\mathrm{N}=33$ ) (Analysis 1.3). One infant would have to be wrapped in plastic to prevent one infant from having a core body temperature outside the normothermic range on admission to the NICU (NNTB 1, 95\% Cl 1 to 2$)$.

\section{Core body temperature one hour after birth (Outcome 1.4)}

One study reported this outcome in terms of core body temperature in ${ }^{\circ} \mathrm{C}$ (axillary) every 15 minutes until two hours after birth (Cardona Torres 2012). This was not a prespecified outcome at the review protocol stage.

Outcome 1.4.1: For infants at $\geq 28$ and $<37$ weeks' gestation and birth weight $\geq 1000$ and $\leq 2499$ grams, a statistically significant difference in core body temperature one hour after birth favoured the intervention (plastic wrap or bag) group when compared with the group that received routine care immediately after birth in the delivery room ( $\mathrm{MD} 0.40^{\circ} \mathrm{C}, 95 \% \mathrm{Cl} 0.19$ to 0.61 ; one study; $\mathrm{N}=60$ ) (Analysis 1.4).

\section{Core body temperature 90 minutes after birth (Outcome 1.5)}

One study reported this outcome in terms of core body temperature in ${ }^{\circ} \mathrm{C}$ (axillary) every 15 minutes until two hours after birth (Cardona Torres 2012). This was not a prespecified outcome at the review protocol stage.

Outcome 1.5.1: For infants at $\geq 28$ and $<37$ weeks' gestation and birth weight $\geq 1000$ and $\leq 2499$ grams, a statistically significant difference in core body temperature 90 minutes after birth favoured the intervention (plastic wrap or bag) group when compared with the group that received routine care immediately after birth in the delivery room $\left(\mathrm{MD} 0.40^{\circ} \mathrm{C}, 95 \% \mathrm{Cl} 0.18\right.$ to 0.62 ; one study; $\mathrm{N}=60$ ) (Analysis 1.5).

\section{Core body temperature two hours after birth (Outcome 1.6)}

One study reported this outcome in terms of core body temperature in ${ }^{\circ} \mathrm{C}$ (axillary) every 15 minutes until two hours after birth (Cardona Torres 2012). This was not a prespecified outcome at the review protocol stage.

Outcome 1.6.1: For infants at $\geq 28$ and $<37$ weeks' gestation and birth weight $\geq 1000$ and $\leq 2499$ grams, a statistically significant difference in core body temperature two hours after birth favoured the intervention (plastic wrap or bag) group when compared with the group that received routine care immediately after birth in the delivery room $\left(\mathrm{MD} 0.30^{\circ} \mathrm{C}, 95 \% \mathrm{Cl} 0.13\right.$ to 0.47 ; one study; $\mathrm{N}=60$ ) (Analysis 1.6).

\section{Core body temperature post stabilisation (Outcome 1.7)}

Two studies comprising 911 infants reported this outcome in terms of core body temperature in ${ }^{\circ} \mathrm{C}$ (axillary) post stabilisation in the NICU (Reilly 2015; Rohana 2011). Reilly 2015 defined post stabilisation as temperature taken after admission procedures were completed and the infant was left to rest in a stable thermoneutral environment. Rohana 2011 defined post stabilisation as temperature taken once respiratory support, peripheral lines, and cardiorespiratory monitor probes had been secured. This was not a prespecified outcome at the review protocol stage. Each individual study showed a significant effect in favour of the intervention (plastic wrap or bag) group for infants at $<28$ weeks' gestation; however Rohana 2011 showed no (borderline) significant difference in effect for the subgroup of infants at $\geq 28$ weeks' gestation.

Outcome 1.7.1: Overall, for infants at $\geq 24$ and $\leq 33$ weeks' gestation, a statistically significant difference in core body temperature post stabilisation favoured the intervention (plastic wrap or bag) group when compared with the group that received routine care immediately after birth in the delivery room (MD $0.43^{\circ} \mathrm{C}, 95 \% \mathrm{Cl} 0.22$ to 0.64 ; two studies; $\mathrm{N}=911$ ) (Analysis 1.7 ). The overall test for homogeneity passed with an $\mathrm{I}^{2}$ value of $0 \%$.

Outcome 1.7.2: For infants at $<28$ weeks' gestation, a statistically significant difference in core body temperature post stabilisation favoured the intervention (plastic wrap or bag) group when compared with the group that received routine care immediately after birth in the delivery room $\left(\mathrm{MD} 0.44^{\circ} \mathrm{C}, 95 \% \mathrm{Cl} 0.20\right.$ to 0.67 ; two 
studies; $\mathrm{N}=838$ ) (Analysis 1.7 ). The test for homogeneity passed with an $\mathrm{I}^{2}$ value of $0 \%$.

Outcome 1.7.3: For infants at $\geq 28$ weeks' gestation, a borderline statistically significant difference in core body temperature post stabilisation favoured the intervention (plastic wrap or bag) group when compared with the group that received routine care immediately after birth in the delivery room $\left(\mathrm{MD} 0.40^{\circ} \mathrm{C}, 95 \% \mathrm{Cl}\right.$ -0.00 to 0.80 ; one study; $N=73$ ) (Analysis 1.7 ).

\section{Hypothermia post stabilisation: core body temperature $<36.5^{\circ} \mathrm{C}$, or} skin temperature $<36^{\circ} \mathrm{C}$ (Outcome 1.8)

Two studies comprising 841 infants reported this outcome in terms of incidence of hypothermia in intervention and control groups (plastic wrap or bag and routine care) (Farhadi 2012; Reilly 2015). Hypothermia post stabilisation was defined as core body temperature (axillary) $<36.5^{\circ} \mathrm{C}$. Reilly 2015 defined 'post stabilisation' as temperature taken after admission procedures were completed and the infant was left to rest in a stable thermoneutral environment; we utilised incidence of hypothermia one hour after admission to the NICU for Farhadi 2012. This was not a prespecified outcome at the review protocol stage. Each individual study showed a significant effect in favour of the intervention (plastic wrap or bag) group across gestations.

\section{Overall}

For infants at $\leq 32$ weeks' gestation, plastic wrap or bag significantly reduced the risk of hypothermia post stabilisation (typical RR 0.76 , $95 \% \mathrm{Cl} 0.65$ to 0.88 ; typical RD $-0.13,95 \% \mathrm{Cl}-0.19$ to -0.06 ; two studies; $N=841$ ) (Analysis 1.8). Eight infants would have to be wrapped in plastic to prevent one infant from being hypothermic post stabilisation (NNTB 8, 95\% Cl 5 to 17). The overall test for homogeneity failed with an $\mathrm{I}^{2}$ value of $76 \%$ (high heterogeneity) for RR and $I^{2}$ of $91 \%$ (high heterogeneity) for RD, and for subgroup differences with $\mathrm{I}^{2}$ values of $74.7 \%$ (moderate heterogeneity) for RR and $90.7 \%$ (high heterogeneity) for RD.

Outcome 1.8.1: For infants at $\leq 32$ weeks' gestation (when no subgroup data were available), plastic wrap or bag significantly reduced the risk of hypothermia post stabilisation (RR $0.05,95 \%$ $\mathrm{Cl} 0.00$ to 0.76 ; RD $-0.50,95 \% \mathrm{Cl}-0.72$ to -0.28 ; one study; $\mathrm{N}=40$ ) (Analysis 1.8). Two infants would have to be wrapped in plastic to prevent one infant from being hypothermic post stabilisation (NNTB 2, 95\% $\mathrm{Cl} 1$ to 4).

Outcome 1.8.2: For infants at $\geq 24$ and $<28$ weeks' gestation, plastic wrap or bag significantly reduced the risk of hypothermia post stabilisation (RR $0.79,95 \% \mathrm{Cl} 0.69$ to $0.92 ; \mathrm{RD}-0.11,95 \% \mathrm{Cl}-0.18$ to -0.04 ; one study; $\mathrm{N}=801$ ) (Analysis 1.8 ). Nine infants would have to be wrapped in plastic to prevent one infant from being hypothermic post stabilisation (NNTB 9, $95 \% \mathrm{Cl} 6$ to 25 ).

\section{Outside the normothermic range post stabilisation (Outcome 1.9)}

Two studies comprising 841 infants reported this outcome, or it was derived from definitions of hypothermia and hyperthermia in terms of incidence outside the normothermic range post stabilisation in intervention and control groups (plastic wrap or bag and routine care) (Reilly 2015), as well as one hour after admission to the NICU (Farhadi 2012). Reilly 2015 defined normothermia as core body temperature (rectal or axillary) of $36.5^{\circ} \mathrm{C}$ to $37.4^{\circ} \mathrm{C}$, and Farhadi 2012 as core body temperature (axillary) of $36.5^{\circ} \mathrm{C}$ to $37.5^{\circ} \mathrm{C}$. This was not a prespecified outcome at the review protocol stage. Farhadi 2012 showed a significant effect favouring the intervention (plastic wrap or bag) group for infants at $\leq 32$ weeks' gestation (when no subgroup data were available), and Reilly 2015 showed no significant difference in effect for the subgroup of infants at $<28$ weeks' gestation.

\section{Overall}

For infants at $\leq 32$ weeks' gestation, data show no significant difference (borderline) in the risk of having a core body temperature outside the normothermic range post stabilisation (typical RR 0.88 , $95 \% \mathrm{Cl} 0.78$ to 1.00 ; typical $\mathrm{RD}-0.07,95 \% \mathrm{Cl}-0.13$ to 0.00 ; two studies; $N=841$ ) (Analysis 1.9). The overall test for homogeneity failed with an $I^{2}$ value of $80 \%$ (high heterogeneity) for RR and $I^{2}$ of $90 \%$ (high heterogeneity) for RD, and with $\mathrm{I}^{2}$ values of $79.6 \%$ (high heterogeneity) for RR and $90.1 \%$ (high heterogeneity) for RD for subgroup differences.

Outcome 1.9.1: For infants at $\leq 32$ weeks' gestation (when no subgroup data were available), plastic wrap or bag significantly reduced the risk of having a core body temperature outside the normothermic range one hour after admission to the NICU (RR 0.10, $95 \% \mathrm{Cl} 0.01$ to 0.71 ; RD $-0.45,95 \% \mathrm{Cl}-0.69$ to -0.21 ; one study; $\mathrm{N}=$ 40) (Analysis 1.9). Two infants would have to be wrapped in plastic to prevent one infant from being outside the normothermic range (NNTB 2, 95\% $\mathrm{Cl} 2$ to 5).

Outcome 1.9.2: For infants at $\geq 24$ and $<28$ weeks' gestation, data show no significant difference in the risk of having a core body temperature outside the normothermic range post stabilisation (RR $0.92,95 \% \mathrm{Cl} 0.81$ to 1.04 ; RD $-0.05,95 \% \mathrm{Cl}-0.12$ to 0.02 ; one study; $\mathrm{N}=801$ ) (Analysis 1.9).

\section{Core body temperature $\mathbf{3 0}$ minutes after the initial NICU admission temperature was taken (Outcome 1.10)}

One study reported this outcome in terms of core body temperature in ${ }^{\circ} \mathrm{C}$ (axillary) 30 minutes after the initial NICU admission temperature was taken (Smith 2013). This was not a prespecified outcome at the review protocol stage.

Outcome 1.10.1: For infants at $\leq 29$ weeks' gestation, data show a statistically significant difference in core body temperature 30 minutes after the initial NICU temperature was taken favouring the intervention (plastic wrap or bag) group over the group that received routine care immediately after birth in the delivery room (MD $0.57^{\circ} \mathrm{C}, 95 \% \mathrm{Cl} 0.27$ to 0.87 ; one study; $\mathrm{N}=92$ ) (Analysis 1.10 ).

\section{Core body temperature one hour after the initial NICU admission temperature was taken (Outcome 1.11)}

Six studies comprising 373 infants reported this outcome in terms of core body temperature in ${ }^{\circ} \mathrm{C}$ (rectal or axillary) one hour after the initial NICU admission temperature was taken (Farhadi 2012; Gathwala 2010; Smith 2013; Talakoub 2015; Trevisanuto 2010; Vohra 2004). This was not a prespecified outcome at the review protocol stage. The purpose for collection of this outcome measure was to ascertain whether the intervention (plastic wrap or bag) prevented rather than delayed the postnatal fall in body temperature immediately after birth. Most individual studies showed an effect favouring the plastic wrap or bag, with the exception of Gathwala 2010 for infants at $\leq 32$ weeks' gestation and birth weight $\leq 1500$ grams, Talakoub 2015 for infants at 28 to 32 weeks' gestation, and Vohra 2004 for infants at $<28$ weeks' 
gestation, which tended towards favouring the plastic wrap or bag group.

Outcome 1.11.1: Overall, for infants at $<37$ weeks' gestation, a statistically significant difference shown for core body temperature one hour after the initial NICU admission temperature was taken favours the intervention (plastic wrap or bag) group when compared with the group that received routine care immediately after birth in the delivery room (MD $0.36^{\circ} \mathrm{C}, 95 \% \mathrm{Cl} 0.25$ to 0.47 ; six studies; $\mathrm{N}=373$ ) (Analysis 1.11). The overall test for homogeneity failed with an $\mathrm{I}^{2}$ value of $80 \%$ (high heterogeneity) and $\mathrm{I}^{2}$ of $74.6 \%$ (moderate heterogeneity) for subgroup differences.

Outcome 1.11.2: For infants at $<28$ weeks' gestation, a statistically significant difference shown for core body temperature one hour after the initial NICU admission temperature was taken favoured the intervention (plastic wrap or bag) group when compared with the group that received routine care immediately after birth in the delivery room ( $\mathrm{MD} 0.49^{\circ} \mathrm{C}, 95 \% \mathrm{Cl} 0.33$ to 0.66 ; four studies; $\mathrm{N}=227$ ) (Analysis 1.11). The test for homogeneity passed with an $\mathrm{I}^{2}$ value of $24 \%$.

Outcome 1.11.3: For infants at $\geq 28$ weeks' gestation, a statistically significant difference shown for core body temperature one hour after the initial NICU admission temperature was taken favoured the intervention (plastic wrap or bag) group when compared with the group that received routine care immediately after birth in the delivery room ( $\mathrm{MD} 0.72^{\circ} \mathrm{C}, 95 \% \mathrm{Cl} 0.48$ to 0.96 ; two studies; $\mathrm{N}=86$ ) (Analysis 1.11). The test for homogeneity passed with an $\mathrm{I}^{2}$ value of $36 \%$ (low heterogeneity).

\section{Core body temperature 90 minutes after the initial NICU admission} temperature was taken (Outcome 1.12)

One study reported this outcome in terms of core body temperature in ${ }^{\circ} \mathrm{C}$ (axillary) 90 minutes after the initial NICU admission temperature was taken (Smith 2013). This was not a prespecified outcome at the review protocol stage.

Outcome 1.12.1: For infants at $\leq 29$ weeks' gestation, a statistically significant difference shown for core body temperature 90 minutes after the initial NICU admission temperature was taken favoured the intervention (plastic wrap or bag) group when compared with the group that received routine care immediately after birth in the delivery room ( $\mathrm{MD} 0.56^{\circ} \mathrm{C}, 95 \% \mathrm{Cl} 0.27$ to 0.85 ; one study; $\mathrm{N}=92$ ) (Analysis 1.12).

\section{Core body temperature two hours after the initial NICU admission temperature was taken (Outcome 1.13)}

Two studies comprising 156 infants reported this outcome in terms of core body temperature in ${ }^{\circ} \mathrm{C}$ (axillary) two hours after the initial NICU admission temperature was taken (Smith 2013; Talakoub 2015). This was not a prespecified outcome at the review protocol stage. Each individual study showed a significant effect in favour of the intervention (plastic wrap or bag) group for infants across gestations.

\section{Overall}

Overall, for infants at $\leq 32$ weeks' gestation, a statistically significant difference shown for core body temperature two hours after the initial NICU admission temperature was taken favoured the intervention (plastic wrap or bag) group when compared with the group that received routine care immediately after birth in the delivery room ( $\mathrm{MD} 0.37^{\circ} \mathrm{C}, 95 \% \mathrm{Cl} 0.16$ to 0.59 ; two studies; $\mathrm{N}=156$ ) (Analysis 1.13). The test for homogeneity failed with an $\mathrm{I}^{2}$ value of $64 \%$ (moderate heterogeneity).

Outcome 1.13.1: For infants at $\leq 29$ weeks' gestation, a statistically significant difference was shown for core body temperature two hours after the initial NICU admission temperature was taken favouring the intervention (plastic wrap or bag) group when compared with the group that received routine care immediately after birth in the delivery room $\left(\mathrm{MD} 0.28^{\circ} \mathrm{C}, 95 \% \mathrm{Cl} 0.04\right.$ to 0.52 ; one study; $\mathrm{N}=92$ ) (Analysis 1.13).

Outcome 1.13.2: For infants at 28 to 32 weeks' gestation, a statistically significant difference was shown for core body temperature two hours after the initial NICU admission temperature was taken favouring the intervention (plastic wrap or bag) group when compared with the group that received routine care immediately after birth in the delivery room (MD $0.74^{\circ} \mathrm{C}, 95 \%$ $\mathrm{Cl} 0.26$ to 1.22 ; one study; $\mathrm{N}=64$ ) (Analysis 1.13).

\section{Secondary outcomes}

Hyperthermia on admission to the NICU: core body temperature > $37.5^{\circ} \mathrm{C}$ (Outcome 1.14)

Twelve studies comprising 1523 infants reported this outcome in terms of incidence of hyperthermia in the intervention and control groups (plastic wrap or bag and routine care). Some investigators defined hyperthermia as core body temperature (rectal or axillary) $>37.5^{\circ} \mathrm{C}$ (Chantaroj 2011; Farhadi 2012; Smith 2013; Trevisanuto 2010; Vohra 2004), Reilly 2015 as core body temperature (axillary) $\geq 37.5^{\circ} \mathrm{C}$, and Leadford 2013 as core body temperature (axillary) > $38^{\circ} \mathrm{C}$; five studies did not provide a clear definition (Cardona Torres 2012; Gathwala 2010; Knobel 2005; Talakoub 2015; Vohra 1999). This was not the prespecified definition at the review protocol stage. For the purposes of this meta-analysis, review authors interpreted 'no risk of hyperthermia' as reported in Talakoub 2015 as no hyperthermia in intervention or control groups (trial authors have been contacted).

Each individual study showed no significant difference in risk of hyperthermia across gestations, with the exception of a large multi-centre study, which showed that plastic wrap significantly increased the risk of being hyperthermic on admission to the NICU for infants at $<28$ weeks' gestation (Reilly 2015).

Outcome 1.14.1: Overall, for infants at $<37$ weeks' gestation, plastic wrap or bag significantly increased the risk of being hyperthermic on admission to the NICU (typical RR 3.91, 95\% CI 2.05 to 7.44 ; typical RD $0.04,95 \% \mathrm{Cl} 0.02$ to 0.06 ; twelve studies; $\mathrm{N}=$ 1523) (Analysis 1.14). Twenty-five infants would have to be wrapped in plastic to have one infant hyperthermic on admission to the NICU (NNTH 25, 95\% Cl 17 to 50). The overall test for homogeneity passed with an $\mathrm{I}^{2}$ value of $0 \%$ for RR and $19 \%$ for RD. For subgroup differences, homogeneity passed with an $\mathrm{I}^{2}$ value of $0 \%$ for RR and $47.7 \%$ (low heterogeneity) for RD.

Note: RR was not estimable for five studies owing to zero events in both arms.

Outcome 1.14.2: For infants at $<28$ weeks' gestation, plastic wrap or bag significantly increased the risk of being hyperthermic on admission to the NICU (typical RR 4.00, 95\% CI 2.04 to 7.83; typical $\mathrm{RD} 0.05,95 \% \mathrm{Cl} 0.03$ to 0.08 ; seven studies; $\mathrm{N}=1121$ ) (Analysis 1.14). 
Twenty infants would have to be wrapped in plastic to have one infant hyperthermic on admission to the NICU (NNTH 20, 95\% Cl 13 to 33 ). The overall test for homogeneity passed with an $\mathrm{I}^{2}$ value of $14 \%$ for RR and $0 \%$ for RD.

Outcome 1.14.3: For infants at $\geq 28$ weeks' gestation, data show no statistically significant differences between the two interventions (plastic bag and routine care) for being hyperthermic on admission to the NICU (typical RR (not estimable); typical RD 0.00, 95\% CI -0.05 to 0.05 ; three studies; $N=138$ ) (Analysis 1.14). The overall test for homogeneity passed with an $I^{2}$ value of $0 \%$ for RD.

In addition, Rohana 2011 reported that two infants in the wrap group were hyperthermic $\left(>37.5^{\circ} \mathrm{C}\right)$ within the first 12 hours of life (for infants at $\geq 24$ and $\leq 33$ weeks' gestation).

\section{Publication bias and investigation of heterogeneity}

Data show no evidence of publication bias.

\section{Hyperthermia post stabilisation: core body temperature $>37.5^{\circ} \mathrm{C}$ (Outcome 1.15)}

One study reported this outcome in terms of incidence of hyperthermia in intervention and control groups (plastic wrap or bag and routine care) post stabilisation (Reilly 2015). Investigators defined hyperthermia as core body temperature (axillary) $\geq 37.5^{\circ} \mathrm{C}$. Reilly 2015 defined post stabilisation as temperature taken after admission procedures were completed and the infant was left to rest in a stable thermoneutral environment. This was not our prespecified definition at the review protocol stage.

Outcome 1.15.1: For infants at $\geq 24$ and $<28$ weeks' gestation, plastic wrap or bag significantly increased risk of being hyperthermic post stabilisation (RR $2.35,95 \% \mathrm{Cl} 1.38$ to 4.00 ; RD $0.06,95 \% \mathrm{Cl} 0.02$ to 0.10 ; one study; $\mathrm{N}=801$ ) (Analysis 1.15 ). Seventeen infants would have to be wrapped in plastic to have one infant hyperthermic post stabilisation (NNTH 17, 95\% Cl 10 to 50).

\section{Major brain injury}

\section{Major brain injury (defined as sonographic evidence of} intraventricular haemorrhage (IVH) with ventricular dilatation, parenchymal haemorrhagic infarction, or periventricular leukomalacia) (Outcome 1.16)

One review author $(\mathrm{HH})$ reviewed data definitions for brain injury (all grades) across all studies and allocated individual outcomes to an appropriate composite outcome for the purposes of meta-analysis. This composite outcome was not prespecified at the review protocol stage. Five studies comprising 1100 infants reported this outcome (Knobel 2005; Reilly 2015; Rohana 2011; Smith 2013; Trevisanuto 2010). Knobel 2005 and Trevisanuto 2010 defined major brain injury as sonographic evidence of IVH with ventricular dilatation, parenchymal haemorrhagic infarction, or periventricular leukomalacia. Rohana 2011 provided data for severe IVH grade III and N . Reilly 2015 provided data separately for severe IVH grade $\mathbb{I I}$ and $\mathbb{V}$ and for cystic periventricular leukomalacia. These were not mutually exclusive; therefore we used cystic periventricular leukomalacia in this meta-analysis. Smith 2013 provided data for cystic periventricular leukomalacia. Each individual study showed no significant differences in effect across gestations.

Overall
For infants at $\leq 33$ weeks' gestation, data show no significant difference in risk of major brain injury between infants who received plastic wrap or bag and those given routine care (typical RR 0.78 , $95 \% \mathrm{Cl} 0.47$ to 1.27 ; typical RD $-0.01,95 \% \mathrm{Cl}-0.04$ to 0.01 ; five studies; $\mathrm{N}=1100$ ) (Analysis 1.16 ). The overall test for homogeneity passed with an $\mathrm{I}^{2}$ value of $0 \%$ for both RR and RD and for subgroup differences.

Outcome 1.16.1: For infants at $\leq 33$ weeks' gestation (when no subgroup data were available), data show no significant difference in risk of major brain injury between infants who received plastic wrap or bag and those given routine care (RR $0.30,95 \% \mathrm{Cl} 0.03$ to 2.60; RD $-0.05,95 \% \mathrm{Cl}-0.12$ to 0.03 ; one study; $\mathrm{N}=110$ ) (Analysis 1.16).

Outcome 1.16.2: For infants at $<28$ weeks' gestation, data show no significant difference in risk of major brain injury between infants who received plastic wrap or bag and those given routine care (typical RR $0.83,95 \% \mathrm{Cl} 0.50$ to 1.39; typical RD $-0.01,95 \% \mathrm{Cl}-0.04$ to 0.02 ; four studies; $\mathrm{n}=990$ ) (Analysis 1.16). The test for homogeneity passed with an $\mathrm{I}^{2}$ value of $0 \%$ for both RR and RD.

\section{Brain injury (Outcome 1.17)}

Three studies comprising 880 infants reported this outcome in terms of incidence of all grades of IVH ( $I$ to $\mathbb{V}$ ) in intervention and control groups (plastic wrap or bag and routine care) (Farhadi 2012; Reilly 2015; Smith 2013). Each individual study showed no significant difference in effect for infants at $<28$ weeks' gestation.

Outcome 1.17.1: For infants at $<28$ weeks' gestation, data show no significant difference in risk of any grade IVH between infants who received plastic wrap or bag and those given routine care (typical RR $0.92,95 \% \mathrm{Cl} 0.77$ to 1.09 ; typical RD $-0.03,95 \% \mathrm{Cl}-0.09$ to 0.03 ; three studies; $\mathrm{N}=880$ ) (Analysis 1.16). The test for homogeneity passed with an $\mathrm{I}^{2}$ value of $0 \%$ for both RR and RD.

\section{Intraventricular haemorrhage grades III and IV (Outcome 1.18)}

One study (for which data have not been meta-analysed in outcome 1.16) reported this outcome in terms of incidence of severe IVH (grades II and $\mathbb{N}$ ) in intervention and control groups (plastic wrap and routine care) (Reilly 2015).

Outcome 1.18.1: For infants at $\geq 24$ and $<28$ weeks' gestation, data show no significant difference in risk of major brain injury between infants who received plastic wrap and those given routine care (RR $0.99,95 \% \mathrm{Cl} 0.67$ to 1.46 ; RD $-0.00,95 \% \mathrm{Cl}-0.05$ to 0.05 ; one study; $\mathrm{N}=753$ ) (Analysis 1.18).

\section{Mortality (death before hospital discharge or at six months' corrected age) (Outcome 1.19)}

Ten studies comprising 1447 infants reported this outcome in terms of incidence of death within hospital stay or at six months' corrected age if infants in hospital in intervention and control groups (plastic wrap or bag and routine care) remain in hospital (Chantaroj 2011; Farhadi 2012; Knobel 2005; Leadford 2013; Reilly 2015; Rohana 2011; Smith 2013; Trevisanuto 2010; Vohra 1999; Vohra 2004). Mortality figures for Vohra 2004 included two infants who died in the delivery room. The study with greatest weighting $(64.6 \%)$ in the meta-analysis was powered for all-cause mortality (at discharge or at six months' corrected gestation, if the infant remains in hospital) as the primary outcome measure (Reilly 2015). Mortality was a secondary outcome measure in the remaining nine 
studies. Each individual study showed no significant difference in effect across gestations.

Outcome 1.19.1: Overall, for infants at $<37$ weeks' gestation, results of the meta-analysis generally favour the intervention group (plastic wrap or bag), with the exception of Leadford 2013. However, overall data show no significant differences in risk of death within hospital stay (or at six months' corrected age) between infants who received plastic wrap or bag and those given routine care (typical RR $0.91,95 \% \mathrm{Cl} 0.73$ to 1.15 ; typical RD $-0.01,95 \% \mathrm{Cl}-0.05$ to $0.02 ; 10$ studies; $\mathrm{N}=1447$ ) (Analysis 1.19). The overall test for homogeneity passed with an $\mathrm{I}^{2}$ value of $0 \%$ for RR and $22 \%$ for RD and for subgroup differences an $\mathrm{I}^{2}$ value of $0 \%$.

Outcome 1.19.2: For infants at $<28$ weeks' gestation, data show no significant difference in risk of death within hospital stay (or at six months' corrected age) between infants who received plastic wrap or bag and those given routine care (typical RR 0.92, 95\% Cl 0.72 to 1.18 ; typical RD $-0.02,95 \% \mathrm{Cl}-0.06$ to 0.03 ; six studies; $\mathrm{N}=1114$ ) (Analysis 1.19). The test for homogeneity passed with an $\mathrm{I}^{2}$ value of $0 \%$ for RR but an $I^{2}$ value of $56 \%$ (moderate heterogeneity) for RD.
Outcome 1.19.3: For infants at 28 to 31 weeks' gestation, data show no significant difference in risk of death within hospital stay (or at six months' corrected age) between infants who received plastic wrap or bag and those given routine care (RR not estimable; RD 0.00, $95 \% \mathrm{Cl}-0.09$ to 0.09 ; one study; $\mathrm{N}=41$ ) (Analysis 1.19 ).

\section{Publication bias and investigation of heterogeneity}

We inspected a funnel plot comparison (SE(log (RR)) vs RR) of mortality for plastic wrap or bag versus routine care for core body temperature in ${ }^{\circ} \mathrm{C}$ for possible publication bias (Figure 5). Findings suggest possible publication bias, but it is unlikely that this would affect findings of the meta-analysis. On removal of two studies (Smith 2013; Vohra 1999), the test for homogeneity passed with an

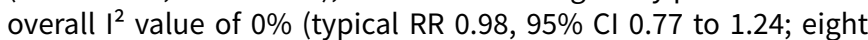
studies; $\mathrm{N}=1298$ ), as did the test for the subgroup differences at < 28 weeks' gestation (typical RR 1.0, $95 \% \mathrm{Cl} 0.77$ to 1.28 ; four studies; $\mathrm{N}=1006)$.

Figure 5. Funnel plot of comparison: 1 Plastic wrap or bag versus routine care, outcome: 1.19 Mortality (death within hospital stay or at 6 months' corrected gestation).

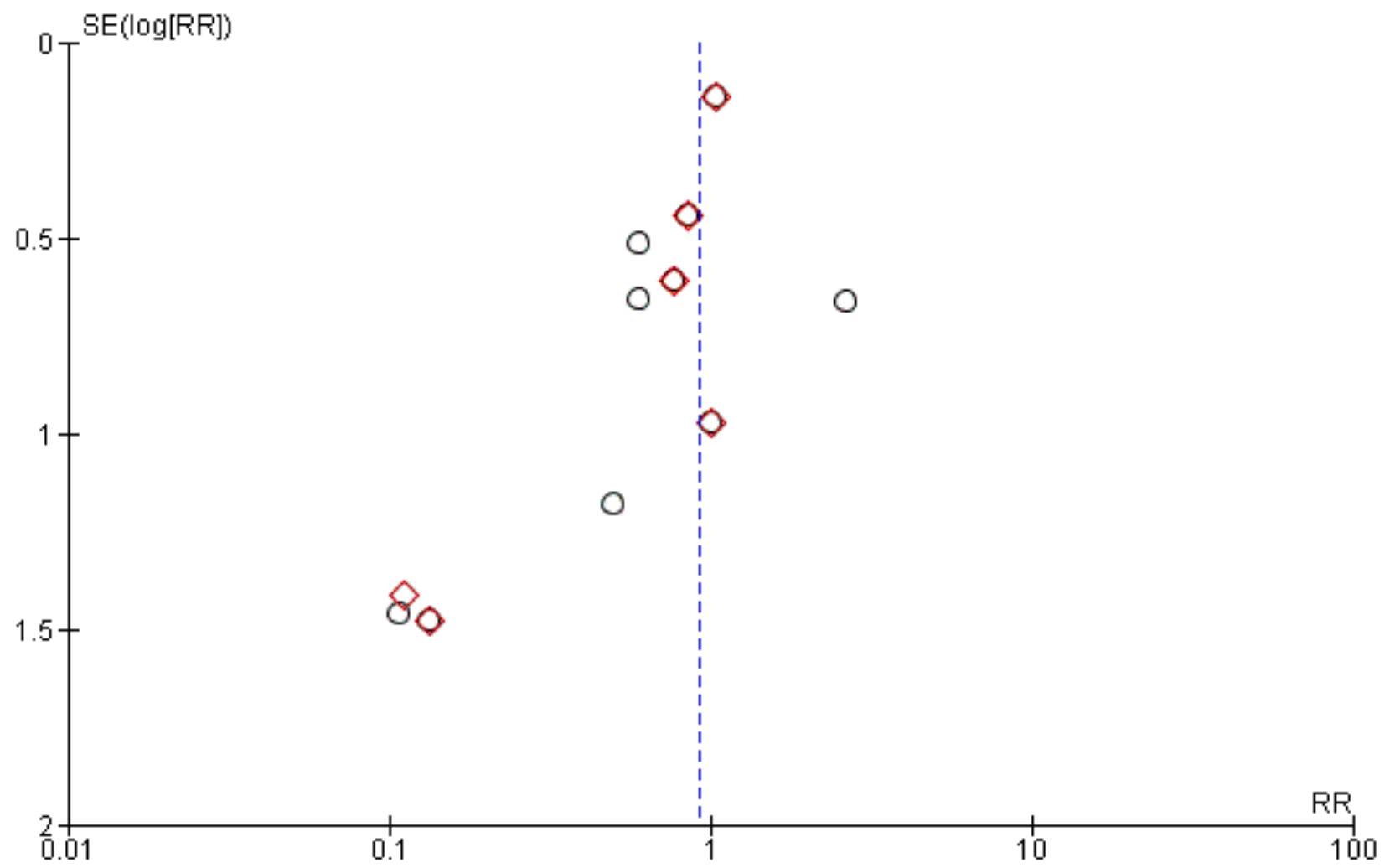

Subgroups

All infants (<37 weeks' gestation)

$<28$ completed weeks' gestation

\section{$\geq 28$ completed weeks' gestation}




\section{Other secondary outcomes}

\section{Arterial oxygen saturation (percentage) (Outcome 1.20)}

One study reported this outcome in terms of percentage oxygen saturation on admission to the NICU (Farhadi 2012). This was not a prespecified outcome at the review protocol stage.

Outcome 1.20.1: For infants at $\leq 32$ weeks' gestation, data show no statistically significant differences between the two interventions (plastic bag and routine care) for arterial oxygen saturation (MD $-4.10 \%, 95 \% \mathrm{Cl}-16.00$ to 7.80 ; one study; $\mathrm{N}=40$ ) (Analysis 1.20 ).

\section{Bicarbonate (mmol/L) (Outcome 1.21)}

Two studies comprising 117 infants reported this outcome in terms of bicarbonate (mmol/L) (Trevisanuto 2010; Vohra 2004). This was not a prespecified outcome at the review protocol stage. Each individual study showed no significant differences in effect for infants at $<28$ weeks' gestation.

Outcome 1.21.1: For infants at < 28 weeks' gestation, data show no statistically significant differences between the two interventions (plastic bag and routine care) for bicarbonate (MD $0.31 \mathrm{mmol} / \mathrm{L}$, $95 \% \mathrm{Cl}-0.72$ to 1.35 ; two studies; $\mathrm{N}=117$ ) (Analysis 1.21 ). The test for homogeneity passed with an $\mathrm{I}^{2}$ value of $0 \%$.

\section{First blood gas pH (Outcome 1.22)}

Two studies comprising 117 infants reported this outcome in terms of first blood gas pH (Trevisanuto 2010; Vohra 2004). This was not a prespecified outcome at the review protocol stage. Each individual study showed no significant differences in effect for infants at $<28$ weeks' gestation. However, Vohra 2004 is tending towards favouring routine care, and Trevisanuto 2010 is tending towards favouring plastic bag.

Outcome 1.22.1: For infants at $<28$ weeks' gestation, data show no statistically significant differences between the two interventions (plastic bag and routine care) for first blood gas $\mathrm{pH}$ (MD 0.01, $95 \% \mathrm{Cl}-0.02$ to 0.04 ; two studies; $\mathrm{N}=117$ ) (Analysis 1.22). The test for homogeneity failed with an $\mathrm{I}^{2}$ value of $70 \%$ (moderate heterogeneity). Because $\mathrm{pH}$ is on a logarithmic scale, it would be more appropriate to measure this outcome as a median value and range or interquartile range (IQR) to avoid its use as a continuous variable such as that prespecified at the review protocol stage: severe metabolic acidosis defined as $\mathrm{pH}<7.20$ and/or base deficit $>10 \mathrm{mmol} / \mathrm{L}$ within the first three days of life.

\section{First blood gas $\mathrm{pH}<7.25$ (Outcome 1.23)}

One study reported this outcome in terms of incidence of a first blood gas $\mathrm{pH}<7.25$ in intervention and control groups (plastic wrap and routine care) (Smith 2013).

Outcome 1.23.1: For infants at $\leq 29$ weeks' gestation, data show no significant differences in risk of first blood gas $\mathrm{pH}<7.25$ between infants who received plastic wrap or bag and those given routine care (RR $0.81,95 \% \mathrm{Cl} 0.55$ to $1.20 ; \mathrm{RD}-0.11,95 \% \mathrm{Cl}-0.33$ to 0.10 ; one study; $\mathrm{N}=84$ ) (Analysis 1.23).

\section{First blood glucose concentration (mmol/L) (Outcome 1.24)}

Four studies comprising 195 infants reported this outcome in terms of first blood glucose concentration (mmol/L) (Chantaroj 2011; Farhadi 2012; Trevisanuto 2010; Vohra 2004). When data were presented as $\mathrm{mg} / \mathrm{dL}$ (USA Standard), we converted these to $\mathrm{mmol}$ /
L (UK Standard). This was not a prespecified outcome at the review protocol stage, although hypoglycaemia defined by a blood glucose level $<2 \mathrm{mmol} / \mathrm{L}$ within two hours of birth was prespecified at the review protocol stage. Each individual study showed no significant differences in effect across gestations. Trevisanuto 2010 tended towards favouring plastic bag for infants at $<28$ weeks' gestation.

Overall

For infants at $\leq 32$ weeks' gestation, data show no statistically significant differences between the two interventions (plastic bag and routine care) for first blood glucose concentration (MD -0.14 $\mathrm{mmol} / \mathrm{L}, 95 \% \mathrm{Cl}-0.50$ to 0.21 ; four studies; $\mathrm{N}=195$ ). Overall, the test for homogeneity passed with an $\mathrm{I}^{2}$ value of $11 \%$ but failed for subgroup differences with an $1^{2}$ value of $51.4 \%$ (moderate heterogeneity).

Outcome 1.24.1: For infants at $\leq 32$ weeks' gestation (for whom no subgroup data were available), data show no statistically significant differences between the two interventions (plastic bag and routine care) for first blood glucose concentration (MD $-0.62 \mathrm{mmol} / \mathrm{L}, 95 \%$ $\mathrm{Cl}-1.37$ to 0.12 ; two studies; $N=78$ ) (Analysis 1.24). The test for homogeneity passed with an $I^{2}$ value of $0 \%$.

Outcome 1.24.2: For infants at $<28$ weeks' gestation, data show no statistically significant differences between the two interventions (plastic bag and routine care) for first blood glucose concentration (MD $-0.00 \mathrm{mmol} / \mathrm{L}, 95 \% \mathrm{Cl}-0.41$ to 0.41 ; two studies; $\mathrm{N}=117$ ) (Analysis 1.24). The test for homogeneity passed with an $\mathrm{I}^{2}$ value of $0 \%$.

\section{First blood glucose concentration $<2.6 \mathrm{mmol} / \mathrm{L}$ (Outcome 1.25)}

One study reported this outcome in terms of incidence of a first blood glucose concentration $<2.6 \mathrm{mmol} / \mathrm{L}$ in intervention and control groups (plastic wrap and routine care) (Smith 2013). This was not a prespecified outcome at the review protocol stage.

Outcome 1.25.1: For infants at $\leq 29$ weeks' gestation, data show no significant difference in risk of first blood glucose concentration < $2.6 \mathrm{mmol} / \mathrm{L}$ between infants who received plastic wrap and those given routine care (RR $0.66,95 \% \mathrm{Cl} 0.31$ to 1.43 ; RD $-0.10,95 \% \mathrm{Cl}$ -0.28 to 0.08 ; one study; $N=85$ ) (Analysis 1.25).

\section{First blood glucose concentration $>6.0 \mathrm{mmol} / \mathrm{L}$ (Outcome 1.26)}

One study reported this outcome of blood glucose concentration $>6.0 \mathrm{mmol} / \mathrm{L}$ in intervention and control groups (plastic wrap and routine care) (Smith 2013). This was not a prespecified outcome at the review protocol stage.

Outcome 1.26.1: For infants at $\leq 29$ weeks' gestation, data show no significant difference in risk of first blood glucose concentration > $6.0 \mathrm{mmol} / \mathrm{L}$ between infants who received plastic wrap and those given routine care ( $\mathrm{RR} 1.07,95 \% \mathrm{Cl} 0.23$ to $5.02 ; \mathrm{RD} 0.00,95 \% \mathrm{Cl}-0.10$ to 0.11 ; one study; $\mathrm{N}=85$ ) (Analysis 1.26).

\section{Blood glucose concentration (mmol/L) 120 minutes after birth (Outcome 1.27)}

One study reported this outcome as a median value and IQR (Cardona Torres 2012). This was not a prespecified outcome at the review protocol stage.

Outcome 1.27.1: For infants at $<37$ weeks' gestation, median blood glucose concentration (mmol/L) at 120 minutes after birth 
was comparable in intervention (plastic wrap or bag) and control (routine care) groups (one study; $\mathrm{N}=60 ; \mathrm{P}>0.05$ ) (Analysis 1.27).

\section{Bronchopulmonary dysplasia (BPD) (Outcome 1.28)}

One study reported this outcome in terms of a collapsed category analysis (Reilly 2015). Investigators provided no clear definition. This was not a prespecified outcome at the review protocol stage.

Outcome 1.28.1: For infants at $\geq 24$ and $<28$ weeks' gestation, data show no significant difference in risk of BPD between infants who received plastic wrap or bag and those given routine care (RR 0.99, $95 \% \mathrm{Cl} 0.86$ to 1.15 ; RD $-0.00,95 \% \mathrm{Cl}-0.08$ to 0.07 ; one study; $\mathrm{N}=$ 702) (Analysis 1.28).

\section{Bronchopulmonary dysplasia (BPD) steroids (outcome 1.29)}

One study reported this outcome in terms of incidence of BPD steroids in intervention and control groups (plastic wrap or bag and routine care) (Reilly 2015). This was not a prespecified outcome at the review protocol stage.

Outcome 1.29.1: For infants at $\geq 24$ and $<28$ weeks' gestation, data show no significant difference in risk of BPD steroids between infants who received plastic wrap or bag and those given routine care (RR $0.94,95 \% \mathrm{Cl} 0.71$ to $1.25 ; \mathrm{RD}-0.01,95 \% \mathrm{Cl}-0.07$ to 0.04 ; one study; $\mathrm{N}=796$ ) (Analysis 1.29).

\section{Duration of hospitalisation (days) (Outcome 1.30)}

Two studies comprising 88 infants reported this outcome in terms of duration of hospital stay in days (Chantaroj 2011; Knobel 2005). Each individual study showed no significant difference in effect across gestations.

\section{Overall}

For infants at $\leq 32$ weeks' gestation, data show no statistically significant differences between the two interventions (plastic bag and routine care) for duration of hospitalisation (MD -6.35 days, $95 \% \mathrm{Cl}-17.27$ to 4.56; two studies; $\mathrm{N}=126$ ) (Analysis 1.30). Overall, the test for homogeneity passed with an $I^{2}$ value of $0 \%$ overall and for subgroup differences.

Outcome 1.30.1: For infants at $\leq 32$ weeks' gestation or birth weight $<1500$ grams (when no subgroup data were available), data show no statistically significant differences between the two interventions (plastic bag and routine care) for duration of hospitalisation (MD -7.50 days, $95 \% \mathrm{Cl}-24.17$ to 9.17 ; one study; $\mathrm{N}$ = 38) (Analysis 1.30).

Outcome 1.30.2: For infants at $<28$ weeks' gestation, data show no statistically significant differences between the two interventions (plastic bag and routine care) for duration of hospitalisation (MD -5.49 days, $95 \% \mathrm{Cl}-19.93$ to 8.95 ; one study; $\mathrm{N}=88$ ) (Analysis 1.30).

\section{Duration of hospitalisation (days) (Outcome 1.31)}

One study reported this outcome as a median value with IQR (Rohana 2011).

Outcome 1.31.1: For infants at 24 to 33 weeks' gestation, median duration of hospitalisation was comparable in the intervention (plastic wrap) and control groups (routine care) (one study; $\mathrm{N}=110$; $\mathrm{P}=0.29$ ) (Analysis 1.31).
Duration of continuous positive airway pressure (CPAP) (days) (Outcome 1.32)

One study reported this outcome as a median value with IQR (Rohana 2011). This was not a prespecified outcome at the review protocol stage.

Outcome 1.32.1: For infants at 24 to 33 weeks' gestation, median duration (days) of CPAP was comparable in the intervention (plastic wrap) and control (routine care) groups (one study; $\mathrm{N}=110 ; \mathrm{P}=0.29$ ) (Analysis 1.32).

\section{Duration of oxygen therapy (days) (Outcome 1.33)}

One study reported this outcome in terms of duration of oxygen therapy (days) (Knobel 2005). This was not a prespecified outcome at the review protocol stage.

Outcome 1.33.1: For infants at $<29$ weeks' gestation, data show no statistically significant differences between the two interventions (plastic bag and routine care) for duration of oxygen therapy (MD -6.51 days, $95 \% \mathrm{Cl}-23.30$ to 10.28 ; one study; $\mathrm{N}=88$ ) (Analysis 1.33).

\section{Duration of ventilation (days) (Outcome 1.34)}

One study reported this outcome as a median value with IQR (Rohana 2011).

Outcome 1.34.1: For infants at 24 to 33 weeks' gestation, median duration of ventilation (days) was comparable in the intervention (plastic wrap) and control (routine care) groups (one study; $\mathrm{N}=110$; $\mathrm{P}=0.30$ ) (Analysis 1.34).

\section{Gastrointestinal perforation (Outcome 1.35)}

One study reported this outcome in terms of incidence of gastrointestinal perforation in the intervention and control groups (plastic wrap and routine care) (Reilly 2015). This was not a prespecified outcome at the review protocol stage.

Outcome 1.35.1: For infants at $\geq 24$ and $<28$ weeks' gestation, data show no significant differences in risk of gastrointestinal perforation between infants who received plastic wrap and those given routine care ( $\mathrm{RR} 1.25,95 \% \mathrm{Cl} 0.68$ to $2.28 ; \mathrm{RD} 0.01,95 \% \mathrm{Cl}-0.02$ to 0.04 ; one study; $\mathrm{N}=795$ ) (Analysis 1.35 ).

\section{Intubation in delivery room (Outcome 1.36)}

One study reported this outcome in terms of incidence of intubation in the delivery room in intervention and control groups (plastic bag and routine care) (Trevisanuto 2010).

Outcome 1.36.1: For infants at $<29$ weeks' gestation, data show no significant differences in risk of intubation in the delivery room between infants who received plastic bag and those given routine care (RR $1.00,95 \% \mathrm{Cl} 0.63$ to 1.58 ; $\mathrm{RD} 0.00,95 \% \mathrm{Cl}-0.24$ to 0.24 ; one study; $\mathrm{N}=64$ ) (Analysis 1.36).

\section{Necrotising enterocolitis (NEC) (Outcome 1.37)}

Two studies comprising 907 infants reported this outcome in terms of incidence of NEC In intervention and control groups (plastic wrap and routine care) (Reilly 2015; Rohana 2011). Each individual study showed no significant difference in effect across gestations.

Overall for infants at $\geq 24$ and $\leq 33$ weeks' gestation, data show no significant differences in risk of NEC between infants who received plastic wrap and those given routine care (typical RR 1.01, 95\% 
$\mathrm{Cl} 0.65$ to 1.58 ; typical RD $0.00,95 \% \mathrm{Cl}-0.03$ to 0.04 ; two studies; $\mathrm{N}=907$ ) (Analysis 1.37). Overall, the test for homogeneity passed with an $I^{2}$ value of $29 \%$ (low heterogeneity) for RR and 35\% (low heterogeneity) for RD, and for subgroup differences an $\mathrm{I}^{2}$ value of $28.6 \%$ (low heterogeneity) for RR and 26.9\% (low heterogeneity) for $\mathrm{RD}$.

Outcome 1.37.1: For infants at $\geq 24$ and $\leq 33$ weeks' gestation (when no subgroup data were available), data show no significant differences in risk of NEC between infants who received plastic wrap and those given routine care (RR 5.98, 95\% Cl 0.29 to 121.75 ; RD $0.04,95 \% \mathrm{Cl}-0.02$ to 0.10 ; one study; $\mathrm{N}=110$ ) (Analysis 1.37 ).

Outcome 1.37.2: For infants at $\geq 24$ and $<28$ weeks' gestation, data show no significant differences in risk of NEC between infants who received plastic wrap and those given routine care (RR 0.95, 95\% $\mathrm{Cl} 0.60$ to 1.49 ; RD $0.00,95 \% \mathrm{Cl}-0.04$ to 0.03 ; one study; $\mathrm{N}=797$ ) (Analysis 1.37).

\section{Patent ductus arteriosus (PDA) (Outcome 1.38)}

Two studies comprising 905 infants reported this outcome in terms of incidence of PDA in intervention and control groups (plastic wrap and routine care) (Reilly 2015; Rohana 2011). Each individual study showed no significant differences in effect across gestations.

Overall for infants at $\geq 24$ and $\leq 33$ weeks' gestation, data show no significant differences in risk for PDA between infants who received plastic wrap and those given routine care (typical RR $0.90,95 \% \mathrm{Cl}$ 0.78 to 1.03 ; typical RD $-0.05,95 \% \mathrm{Cl}-0.12$ to 0.01 ; two studies; $\mathrm{N}=$ 905) (Analysis 1.38). Overall, the test for homogeneity passed with an $\mathrm{I}^{2}$ value of $0 \%$ for RR and for RD, and for subgroup differences.

Outcome 1.38.1: For infants at $\geq 24$ and $\leq 33$ weeks' gestation (when no subgroup data were available), data show no significant differences in risk of PDA between infants who received plastic wrap and those given routine care (RR $0.80,95 \% \mathrm{Cl} 0.39$ to 1.62 ; RD -0.05 , $95 \% \mathrm{Cl}-0.21$ to 0.11 ; one study; $\mathrm{N}=110$ ) (Analysis 1.38 ).

Outcome 1.38.2: For infants at $\geq 24$ and $<28$ weeks' gestation, data show no significant differences in risk of PDA between infants who received plastic wrap and those given routine care (RR 0.90, 95\% $\mathrm{Cl} 0.79$ to 1.04 ; RD $-0.05,95 \% \mathrm{Cl}-0.12$ to 0.02 ; one study; $\mathrm{N}=795$ ) (Analysis 1.38).

\section{Pneumothorax (Outcome 1.39)}

One study reported this outcome in terms of incidence of pneumothorax in the intervention and control groups (plastic wrap and routine care) (Reilly 2015). This was not a prespecified outcome at the review protocol stage.

Outcome 1.39.1: For infants at $\geq 24$ and $<28$ weeks' gestation, data show no significant differences in risk of pneumothorax between infants who received plastic wrap and those given routine care (RR $0.98,95 \% \mathrm{Cl} 0.56$ to 1.69 ; RD $-0.00,95 \% \mathrm{Cl}-0.03$ to 0.03 ; one study; $\mathrm{N}=796$ ) (Analysis 1.39).

\section{Pulmonary haemorrhage (Outcome 1.40)}

One study reported this outcome in terms of incidence of pulmonary haemorrhage during hospital stay in intervention and control groups (plastic wrap and routine care) (Reilly 2015). This was not a prespecified outcome at the review protocol stage.
Outcome 1.40.1: For infants at $\geq 24$ and $<28$ weeks' gestation, plastic wrap significantly reduced risk of pulmonary haemorrhage during hospital stay (RR $0.60,95 \% \mathrm{Cl} 0.38$ to $0.95 ; \mathrm{RD}-0.04,95 \% \mathrm{Cl}$ -0.08 to -0.01 ; one study; $\mathrm{N}=796$ ) (Analysis 1.40 ). Twenty-five infants would have to be wrapped in plastic to prevent one infant from having a pulmonary haemorrhage (NNTB $25,95 \% \mathrm{Cl} 13$ to 100 ).

Requirement for bubble continuous positive airway pressure (BCPAP) (Outcome 1.41)

One study reported this outcome in terms of incidence of requirement for BCPAP in the intervention and control groups (plastic wrap and routine care) (Smith 2013). This was not a prespecified outcome at the review protocol stage.

Outcome 1.41.1: For infants at $\leq 29$ weeks' gestation, data show no significant difference in risk of requirement for BCPAP between infants who received plastic wrap and those given routine care (RR $0.97,95 \% \mathrm{Cl} 0.81$ to 1.18 ; RD $-0.02,95 \% \mathrm{Cl}-0.18$ to 0.13 ; one study; $\mathrm{N}=92$ ) (Analysis 1.41).

\section{Requirement for ventilation (Outcome 1.42)}

One study reported this outcome in terms of incidence of requirement for ventilation in intervention and control groups (plastic wrap and routine care) (Smith 2013).

Outcome 1.42.1: For infants at $\leq 29$ weeks' gestation, data show no significant differences in risk of requirement for ventilation between infants who received plastic wrap and those given routine care (RR $0.92,95 \% \mathrm{Cl} 0.70$ to 1.20 ; $\mathrm{RD}-0.06,95 \% \mathrm{Cl}-0.25$ to 0.13 ; one study; $\mathrm{N}=92$ ) (Analysis 1.42).

\section{Respiratory distress syndrome (RDS) (Outcome 1.43)}

Two studies comprising 910 infants reported this outcome in terms of incidence of RDS in intervention and control groups (plastic wrap and routine care) (Reilly 2015; Rohana 2011). Each individual study showed no significant differences in effect across gestations.

Overall for infants at $\geq 24$ and $\leq 33$ weeks' gestation, data show no significant differences in risk of RDS between infants who received plastic wrap and those given routine care (typical RR 1.01, 95\% $\mathrm{Cl} 0.97$ to 1.06 ; typical RD $0.01,95 \% \mathrm{Cl}-0.03$ to 0.05 ; two studies; $\mathrm{N}=910$ ) (Analysis 1.43). Overall, the test for homogeneity passed with an $I^{2}$ value of $0 \%$ overall for RR and RD, and for subgroup differences.

Outcome 1.43.1: For infants at $\geq 24$ and $\leq 33$ weeks' gestation (when no subgroup data were available), data show no significant differences in risk of RDS between infants who received plastic wrap and those given routine care (RR $1.04,95 \% \mathrm{Cl} 0.78$ to 1.38 ; RD 0.02 , $95 \% \mathrm{Cl}-0.16$ to 0.20 ; one study; $\mathrm{N}=110$ ) (Analysis 1.43 ).

Outcome 1.43.2: For infants at $\geq 24$ and $<28$ weeks' gestation, data show no significant differences in risk of RDS between infants who received plastic wrap and those given routine care (RR 1.01, 95\% $\mathrm{Cl} 0.97$ to 1.05 ; RD $0.01,95 \% \mathrm{Cl}-0.03$ to 0.04 ; one study; $\mathrm{N}=800$ ) (Analysis 1.43).

\section{Retinopathy of prematurity (ROP) (Outcome 1.44)}

One study reported this outcome in terms of incidence of ROP in intervention and control groups (plastic wrap and routine care) (Reilly 2015). This was not a prespecified outcome at the review protocol stage. 
Outcome 1.44.1: For infants at $\geq 24$ and $<28$ weeks' gestation, data show no significant differences in risk of gastrointestinal perforation between infants who received plastic wrap and those given routine care ( $\mathrm{RR} 1.00,95 \% \mathrm{Cl} 0.72$ to $1.40 ; \mathrm{RD} 0.00,95 \% \mathrm{Cl}-0.06$ to 0.06 ; one study; $\mathrm{N}=606$ ) (Analysis 1.44).

\section{Sepsis (late) (Outcome 1.45)}

Two studies comprising 830 infants reported this outcome in terms of incidence of late sepsis in intervention and control groups (plastic wrap and routine care) (Reilly 2015; Smith 2013). This was not a prespecified outcome at the review protocol stage. Each individual study showed no significant differences in effect for infants at $\leq 29$ weeks' gestation.

Outcome 1.45.1: For infants at $\leq 29$ weeks' gestation, data show no significant differences in risk of late sepsis between infants who received plastic wrap and those given routine care (typical RR 0.88 , $95 \% \mathrm{Cl} 0.70$ to 1.10 ; typical RD $-0.03,95 \% \mathrm{Cl}-0.09$ to 0.02 ; two studies; $\mathrm{N}=830$ ) (Analysis 1.45 ). The test for homogeneity passed with an $\mathrm{I}^{2}$ value of $9 \%$ for RR and $0 \%$ for RD.

\section{Sepsis (early) (Outcome 1.46)}

Two studies comprising 883 infants reported this outcome in terms of incidence of early sepsis in intervention and control groups (plastic wrap and routine care) (Reilly 2015; Smith 2013). This was not a prespecified outcome at the review protocol stage. Each individual study showed no significant differences in effect for infants at $\leq 29$ weeks' gestation.

Outcome 1.46.1: For infants at $\leq 29$ weeks' gestation, data show no significant differences in risk of early sepsis between infants who received plastic wrap and those given routine care (typical RR $0.69,95 \% \mathrm{Cl} 0.38$ to 1.29 ; typical RD $-0.02,95 \% \mathrm{Cl}-0.04$ to 0.01 ; two studies; $\mathrm{N}=883$ ) (Analysis 1.46). The test for homogeneity passed with an $I^{2}$ value of $0 \%$ for RR and RD.

\section{Other secondary outcomes}

Leadford 2013 reported no significant differences in mean temperature after 24 hours of admission or in length of stay among infants admitted to the NICU (14 in the intervention group; nine in the control group). In addition, investigators did not document hypotension, hypoglycaemia, seizures in first 24 hours after birth, BPD, pneumothorax, major brain injury, bowel perforation, or pulmonary haemorrhage in any of these infants.

Researchers did not report the following secondary outcome measures (as predefined at the review protocol stage) for any of the included studies in this comparison group: surfactant given at any time, severe metabolic acidosis, chronic lung disease, or acute renal failure.

\section{Adverse occurrences due to the intervention}

Cardona Torres 2012 reported that the wrap procedure was well accepted by neonatal staff and did not interfere with resuscitation, and that none of the infants for whom polyethylene bags were used developed skin lesions or infection. Chantaroj 2011 stated that researchers identified no adverse events including hyperthermia, skin maceration, and infection in the control or polyethylene bag groups. Similarly, Gathwala 2010 reported no hyperthermia in the vinyl bag or control group. Farhadi 2012 made no reference to any adverse occurrences due to the intervention, other than one reported case of hyperthermia in the Zipkif plastic bag group. Knobel 2005 reported that one participant in the intervention group was hyperthermic with a rectal admission temperature of $38.3^{\circ} \mathrm{C}$; the delivery room environmental temperature was $26.7^{\circ} \mathrm{C}$. These investigators also reported that the clear, pliable polyurethane bag did not interfere with assessment (visualisation, auscultation, palpation) nor with resuscitative interventions. Leadford 2013 reported that none of the infants in either group were hyperthermic, and no infants developed skin side effects attributable to plastic bags. Rohana 2011 reported no other complications associated with the polyethylene wrap apart from two cases of hyperthermia in the wrap group. Trevisanuto 2010 reported that two infants in the plastic wrap group had an axillary admission temperature $>37.5^{\circ} \mathrm{C}\left(37.6^{\circ} \mathrm{C}\right.$ and $38^{\circ} \mathrm{C}$, respectively). Staff in the Smith 2013 study experienced some problems with the plastic wrap with regard to placement of the saturation probe and umbilical lines and the fact that prolonged resuscitation tended to displace the wrap. Investigators in Vohra 1999 described no occurrences of adverse events attributable to the intervention (i.e. hyperthermia, infection, skin maceration, or interference with resuscitation) among infants in the intervention and control groups. Vohra 2004 reported that the wrap procedure was accepted by neonatal staff and did not interfere with resuscitation in the delivery room. These investigators indicated that two infants in the intervention group had a rectal temperature on admission above $37.5^{\circ} \mathrm{C}$.

None of the included studies reported the following secondary outcome measures (adverse occurrences) (as predefined at the review protocol stage) for this comparison group: burns, antibiotics, or negative psychological outcomes.

\section{Plastic wrap versus routine care interhospital neonatal transport (Comparison 2)}

One study comprising 96 infants compared polyethylene wrap versus routine care for infants at $<37$ weeks' gestation and birth weight $\leq 2500$ grams who were born in maternity homes or hospitals and subsequently underwent interhospital transport to the NICU (Bhavsar 2015).

\section{Primary outcomes}

Core body temperature $\left({ }^{\circ} \mathrm{C}\right)$ of the infant taken on admission to NICU or up to two hours after birth (Outcome 2.1)

One study reported this outcome in terms of core body temperature in ${ }^{\circ} \mathrm{C}$ (axillary) on admission to NICU before the infant was unwrapped (Bhavsar 2015).

Outcome 2.1.1: For infants at $<37$ weeks' gestation with birth weight $\leq 2500$ grams, data show a statistically significant difference for core body temperature on admission to the NICU favouring the intervention (plastic wrap) group when compared with the group that received routine care immediately after birth in the delivery room ( $\mathrm{MD} 0.70^{\circ} \mathrm{C}, 95 \% \mathrm{Cl} 0.32$ to 1.08 ; one study; $\mathrm{N}=96$ ) (Analysis 2.1).

\section{Hypothermia on admission to NICU (core body temperature $<36.5^{\circ} \mathrm{C}$ or skin temperature $<36^{\circ} \mathrm{C}$ ) (Outcome 2.2)}

Bhavsar 2015, in addition to reporting core body temperature on admission to the NICU as a continuous variable, provided data in a dichotomous format in terms of the incidence of hypothermia among intervention and control groups (plastic wrap and routine 
care). Researchers defined hypothermia as core body temperature (axillary) $<35^{\circ} \mathrm{C}$ on admission to the NICU.

Outcome 2.2.1: For infants at $<37$ weeks' gestation with birth weight $\leq 2500$ grams, plastic wrap significantly reduced risk of hypothermia on admission to the NICU (RR $0.48,95 \% \mathrm{Cl} 0.27$ to $0.84 ; \mathrm{RD}-0.27,95 \% \mathrm{Cl}-0.45$ to -0.08 ; one study; $\mathrm{N}=96$ ). Four infants would have to be wrapped in plastic to prevent one from becoming hypothermic (NNTB 4, 95\% Cl 2 to 13) (Analysis 2.2).

Decrease in core body temperature $\left({ }^{\circ} \mathrm{C}\right)$ from baseline before transport to NICU admission (Outcome 2.3)

One study reported this outcome in terms of a decrease in core body temperature from baseline before transport to NICU admission (Bhavsar 2015). This was not a prespecified outcome at the review protocol stage.

Outcome 2.3.1: For infants at $<37$ weeks' gestation with birth weight $\leq 2500$ grams, data show a statistically significant difference in decreased temperature from baseline before transport favouring the intervention (plastic wrap) group when compared with the group that received routine care immediately after birth in the delivery room ( $\mathrm{MD}-0.40^{\circ} \mathrm{C}, 95 \% \mathrm{Cl}-0.61$ to -0.19 ; one study; $\mathrm{N}=96$ ) (Analysis 2.3).

\section{Secondary outcomes}

Hyperthermia on admission to NICU: core body temperature $>37.5^{\circ} \mathrm{C}$ (Outcome 2.4)

Bhavsar 2015, in addition to reporting core body temperature on admission to the NICU as a continuous variable, provided data in a dichotomous format in terms of incidence of hyperthermia in the intervention and control groups (plastic wrap and routine care). Study authors defined hyperthermia as a core body temperature (axillary) $>37.5^{\circ} \mathrm{C}$ on admission to the NICU.

Outcome 2.4.1: For infants at $<37$ weeks' gestation with birth weight $\leq 2500$ grams, data show no significant differences in risk of hyperthermia on admission to the NICU between infants who received plastic wrap and those given routine care (RR 2.88, 95\% $\mathrm{Cl} 0.12$ to $68.98 ; \mathrm{RD} 0.02,95 \% \mathrm{Cl}-0.03$ to 0.08 ; one study; $\mathrm{N}=96$ ) (Analysis 2.4).

\section{Base excess (Outcome 2.5)}

One study reported this outcome in terms of base excess (Bhavsar 2015). Trial authors did not clearly state the measurement time point. This was not a prespecified outcome at the review protocol stage.

Outcome 2.5.1: For infants at $<37$ weeks' gestation with birth weight $\leq 2500$ grams, data show no statistically significant differences between the two intervention groups (plastic wrap and routine care) for base excess (MD $0.60,95 \% \mathrm{Cl}-0.49$ to 1.69 ; one study; $\mathrm{N}=96$ ) (Analysis 2.5).

\section{Blood gas pH (Outcome 2.6)}

One study reported this outcome in terms of blood gas $\mathrm{pH}$ (Bhavsar 2015). Researchers did not clearly state the measurement time point. This was not a prespecified outcome at the review protocol stage.

Outcome 2.6.1: For infants at $<37$ weeks' gestation with birth weight $\leq 2500$ grams, data show no statistically significant differences between the two intervention groups (plastic wrap and routine care) in blood gas $\mathrm{pH}(\mathrm{MD} 0.00,95 \% \mathrm{Cl}-0.02$ to 0.02 ; one study; $\mathrm{N}=96$ ) (Analysis 2.6).

\section{Duration of oxygen therapy (days) (Outcome 2.7)}

One study reported this outcome in terms of duration of oxygen therapy (days) (Bhavsar 2015). This was not a prespecified outcome at the review protocol stage.

Outcome 2.7.1: For infants at $<37$ weeks' gestation with birth weight $\leq 2500$ grams, data show no statistically significant differences between the two interventions (plastic wrap and routine care) for duration of oxygen therapy (days) (MD -2.39, 95\% $\mathrm{Cl}-8.27$ to 3.49 ; one study; $\mathrm{N}=96$ ) (Analysis 2.7).

\section{Blood glucose level ( $\mathrm{mg} / \mathrm{dL}$ ) within two hours of birth (Outcome 2.8)}

One study reported this outcome in terms of results of hemo glucose tests $(\mathrm{mg} / \mathrm{dL}$ ) (Bhavsar 2015). This was not a prespecified outcome at the review protocol stage.

Outcome 2.8.1: For infants at $<37$ weeks' gestation with birth weight $\leq 2500$ grams, data show no statistically significant differences between the two interventions (plastic wrap and routine care) for blood glucose level within two hours of birth (MD $7.20,95 \% \mathrm{Cl}-2.03$ to 16.43 ; one study; $\mathrm{N}=96$ ) (Analysis 2.8).

\section{Hypoglycaemia (blood glucose $<40 \mathrm{mg} / \mathrm{dL}$ ) within two hours after} birth (Outcome 2.9)

One study reported this outcome in terms of incidence of blood glucose $<40 \mathrm{mg} / \mathrm{dL}(2.3 \mathrm{mmol} / \mathrm{L})$ within two hours after birth in intervention and control groups (plastic wrap and routine care) (Bhavsar 2015). This was not the prespecified definition of hypoglycaemia at the review protocol stage.

Outcome 2.9.1: For infants at $<37$ weeks' gestation and birth weight $\leq 2500$ grams, plastic wrap significantly reduced risk of hypoglycaemia within two hours after birth (RR $0.55,95 \% \mathrm{Cl} 0.31$ to 0.98 ; RD $-0.20,95 \% \mathrm{Cl}-0.39$ to -0.02 ; one study; $\mathrm{N}=96$ ). Five infants would have to be wrapped in plastic to prevent one from becoming hypoglycaemic (NNTB 5, 95\% Cl 3 to 50) (Analysis 2.9).

\section{Severe metabolic acidosis (Outcome 2.10)}

One study reported this outcome in terms of incidence of $\mathrm{pH}<7.20$ and/or base deficit $>10 \mathrm{mmol} / \mathrm{L}$ within the first three days of life among intervention and control groups (plastic wrap and routine care) (Bhavsar 2015).

Outcome 2.10.1: For infants at $<37$ weeks' gestation with birth weight $\leq 2500$ grams, data show no significant differences in risk of severe metabolic acidosis within the first three days after birth between infants who received plastic wrap and those given routine care (RR $1.15,95 \% \mathrm{Cl} 0.38$ to 3.52 ; $\mathrm{RD} 0.02,95 \% \mathrm{Cl}-0.11$ to 0.14 ; one study; $\mathrm{N}=96$ ) (Analysis 2.10).

\section{Other secondary outcomes}

None of the included studies reported the following secondary outcome measures (as predefined at the review protocol stage) for this comparison group: respiratory distress syndrome, surfactant given at any time, intubation in the delivery room, requirement for ventilation, duration of ventilation, length of stay, mortality, IVH, PDA, chronic lung disease, NEC, or acute renal failure for infants in intervention and control groups. 


\section{Adverse occurrences due to the intervention}

None of the included studies in this comparison group reported the following secondary outcome measures (adverse occurrences) (as predefined at the review protocol stage): burns, maceration, infection, antibiotics, skin maceration, interference with resuscitation, fluid problems, or negative psychological outcomes for infants in intervention and control groups.

\section{Plastic bag with previous drying versus routine care (Comparison 3)}

One study comprising 60 infants compared polyethylene bag with previous drying versus routine care for infants at $\geq 28$ and < 37 weeks' gestation and birth weight $\geq 1000$ and $\leq 2499$ grams (Cardona Torres 2012).

\section{Primary outcomes}

Core body temperature $\left({ }^{\circ} \mathrm{C}\right)$ of the infant taken on admission to NICU or up to two hours after birth

One study reported this outcome in terms of time to achieve a mean core body temperature (axillary) of $36.5^{\circ} \mathrm{C}$ and core body temperature in ${ }^{\circ} \mathrm{C}$ (axillary) of the infant every 15 minutes until two hours after birth (Cardona Torres 2012). These were not prespecified outcomes at the review protocol stage. For the purposes of this review, we have reported core body temperature in ${ }^{\circ} \mathrm{C}$ (axillary) at 30 minutes, 1 hour, 90 minutes, and 2 hours after birth.

\section{Core body temperature $\left({ }^{\circ} \mathrm{C}\right)$ of the infant taken at 30 minutes after birth (Outcome 3.1)}

One study reported this outcome in terms of core body temperature in ${ }^{\circ} \mathrm{C}$ (axillary) at 30 minutes after birth (Cardona Torres 2012). This was not a prespecified outcome at the review protocol stage.

Outcome 3.1.1: For infants at $\geq 28$ and $<37$ weeks' gestation and birth weight $\geq 1000$ and $\leq 2499$ grams, data show a statistically significant difference for core body temperature 30 minutes after birth favouring the intervention (plastic bag with previous drying) group when compared with the group that received routine care immediately after birth in the delivery room $\left(\mathrm{MD} 0.30^{\circ} \mathrm{C}, 95 \% \mathrm{Cl} 0.12\right.$ to 0.48 ; one study; $\mathrm{N}=60$ ) (Analysis 3.1).

\section{Core body temperature $\left({ }^{\circ} \mathrm{C}\right)$ of the infant taken at one hour after birth} (Outcome 3.2)

One study reported this outcome in terms of core body temperature in ${ }^{\circ} \mathrm{C}$ (axillary) 60 minutes after birth (Cardona Torres 2012). This was not a prespecified outcome at the review protocol stage.

Outcome 3.2.1 For infants at $\geq 28$ and $<37$ weeks' gestation with birth weight $\geq 1000$ and $\leq 2499$ grams, data show a statistically significant difference for core body temperature 60 minutes after birth favouring the intervention (plastic bag with previous drying) group when compared with the group that received routine care immediately after birth in the delivery room $\left(\mathrm{MD} 0.30^{\circ} \mathrm{C}, 95 \% \mathrm{Cl} 0.11\right.$ to 0.49; one study; $\mathrm{N}=60$ ) (Analysis 3.2) (Cardona Torres 2012).

\section{Core body temperature $\left({ }^{\circ} \mathrm{C}\right)$ of the infant taken at 90 minutes after} birth (Outcome 3.3)

One study reported this outcome in terms of core body temperature in ${ }^{\circ} \mathrm{C}$ (axillary) 90 minutes after birth (Cardona Torres 2012). This was not a prespecified outcome at the review protocol stage.
Outcome 3.3.1: For infants at $\geq 28$ and $<37$ weeks' gestation with birth weight $\geq 1000$ and $\leq 2499$ grams, data show a statistically significant difference for core body temperature 90 minutes after birth favouring the intervention (plastic bag with previous drying) group when compared with the group that received routine care immediately after birth in the delivery room $\left(\mathrm{MD} 0.30^{\circ} \mathrm{C}, 95 \% \mathrm{Cl} 0.12\right.$ to 0.48 ; one study' $\mathrm{N}=60$ ) (Analysis 3.3).

Core body temperature $\left({ }^{\circ} \mathrm{C}\right)$ of the infant taken at two hours after birth (Outcome 3.4)

One study reported this outcome in terms of core body temperature in ${ }^{\circ} \mathrm{C}$ (axillary) 120 minutes after birth (Cardona Torres 2012). This was not a prespecified outcome at the review protocol stage.

Outcome 3.4.1: For infants at $\geq 28$ and $<37$ weeks' gestation with birth weight $\geq 1000$ and $\leq 2499$ grams, data show a statistically significant difference for core body temperature 120 minutes after birth favouring the intervention (plastic bag with previous drying) group when compared with the group that received routine care immediately after birth in the delivery room $\left(\mathrm{MD} 0.40^{\circ} \mathrm{C}, 95 \% \mathrm{Cl} 0.24\right.$ to 0.56 ; one study; $\mathrm{N}=60$ ) (Analysis 3.4).

\section{Hypothermia on admission to the NICU (core body temperature < $36.5^{\circ} \mathrm{C}$ or skin temperature $<36^{\circ} \mathrm{C}$ ) (Outcome 3.5)}

One study reported this outcome in terms of incidence of hypothermia in the intervention and control groups (plastic bag with previous drying and routine care) (Cardona Torres 2012). However, investigators provided no clear definition of hypothermia.

Outcome 3.5.1: For infants at $\geq 28$ and $<37$ weeks' gestation with birth weight $\geq 1000$ and $\leq 2499$ grams, data show no significant difference in risk of hypothermia on admission to the NICU between infants who received plastic bag with previous drying and those given routine care (RR $0.09,95 \% \mathrm{Cl} 0.01$ to 1.57 ; RD $-0.17,95 \% \mathrm{Cl}$ -0.31 to -0.03 ; one study; $\mathrm{N}=60$ ) (Analysis 3.5).

Computational errors can occur one or both arms of a study have a zero count, as is the case in Cardona Torres 2012. RevMan software compensates by adding 0.5 to each cell.

\section{Secondary outcomes}

Blood glucose concentration (mmol/L) 120 minutes after birth (Outcome 3.6)

One study reported this outcome (Cardona Torres 2012). When data were presented as $\mathrm{mg} / \mathrm{dL}$ (USA Standard), we converted these to $\mathrm{mmol} / \mathrm{L}$ (UK Standard). This was not a prespecified outcome at the review protocol stage.

Outcome 3.6.1: One study reported this outcome as a median value with interquartile range (IQR) (Cardona Torres 2012). For infants at $\geq 28$ and $<37$ weeks' gestation with birth weight $\geq 1000$ and $\leq 2499$ grams, median glucose concentrations at 120 minutes after birth were comparable in the intervention (plastic bag with previous drying) and control (routine care) groups (one study; $\mathrm{N}=$ 60: $P>0.05$ ) (Analysis 3.6).

\section{Other secondary outcomes}

Cardona Torres 2012 reported no differences between groups for heart rate, respiratory rate, or blood pressure levels (measured by the flushing technique with a sphygmomanometer at 15 minutes, 1 hour, and 2 hours after birth). 
None of the included studies reported the following secondary outcome measures (as predefined at the review protocol stage) for this comparison group: hypoglycaemia, respiratory distress syndrome, surfactant given at any time, intubation in the delivery room, requirement for ventilation, duration of ventilation, length of stay, mortality, severe metabolic acidosis, IVH, PDA, chronic lung disease, NEC, or acute renal failure.

\section{Adverse occurrences due to the intervention}

None of the included studies reported the following secondary outcome measures (adverse occurrences) (as predefined at the review protocol stage) for this comparison group: hyperthermia, burns, maceration, antibiotics, fluid problems, or negative psychological outcomes.

Cardona Torres 2012 reported that none of the neonates in the plastic bag with previous drying group developed any skin lesion, infection, or hyperthermia (definition not reported), and that the wrap procedure was well accepted by neonatal staff and did not interfere with resuscitation.

\section{Plastic cap versus routine care (no cap) (Comparison 4)}

One study comprising 64 infants compared polyethylene caps versus routine care for infants at $<29$ weeks' gestation (Trevisanuto 2010).

\section{Primary outcomes}

Core body temperature $\left({ }^{\circ} \mathrm{C}\right)$ of the infant taken on admission to the NICU or up to two hours after birth (Outcome 4.1)

One study reported this outcome in terms of core body temperature in ${ }^{\circ} \mathrm{C}$ (axillary) on admission to the NICU (Trevisanuto 2010).

Outcome 4.1.1: For infants at $<29$ weeks' gestation, data show a statistically significant difference for core body temperature on admission to the NICU favouring the intervention (plastic cap) group when compared with the group that received routine care immediately after birth in the delivery room $\left(\mathrm{MD} 0.80^{\circ} \mathrm{C}, 95 \% \mathrm{Cl} 0.41\right.$ to 1.19; one study; $\mathrm{N}=64$ ) (Analysis 4.1).

\section{Hypothermia on admission to the NICU (core body temperature < $36.4^{\circ} \mathrm{C}$ ) (Outcome 4.2 )}

Trevisanuto 2010 in addition to reporting core body temperature on admission to the NICU as a continuous variable, provided data in a dichotomous format in terms of incidence of hypothermia in the intervention and control groups (plastic cap and routine care). Investigators defined hypothermia as a core body temperature (axillary) $<36.4^{\circ} \mathrm{C}$ on admission to the NICU.

Outcome 4.2.1: For infants at $<29$ weeks' gestation, plastic cap significantly reduced risk of hypothermia on admission to the NICU (RR $0.48,95 \% \mathrm{Cl} 0.32$ to 0.73 ; RD $-0.47,95 \% \mathrm{Cl}-0.67$ to -0.27 ; one study; $\mathrm{N}=64)$. This finding is consistent with those for Outcome measure 4.1.1. Two infants would have to wear a plastic cap to prevent one infant from becoming hypothermic (NNTB 2, 95\% Cl 2 to 4) (Analysis 4.2).

\section{Outside the normothermic range on admission to the NICU or up to two hours after birth (Outcome 4.3)}

We derived this outcome for one study in terms of incidence of core body temperature (axillary) outside the normothermic range in the intervention and control groups (plastic cap and routine care)
(Trevisanuto 2010). Investigators defined normothermic range as core body temperature (axillary) within the range $36.4^{\circ} \mathrm{C}$ to $37.5^{\circ} \mathrm{C}$ on admission to the NICU. This was not a prespecified outcome at the review protocol stage.

Outcome 4.3.1: For infants at $<29$ weeks' gestation, plastic cap significantly reduced the risk of having a core body temperature outside the normothermic range on admission to the NICU (RR $0.48,95 \% \mathrm{Cl} 0.32$ to 0.73 ; RD $-0.47,95 \% \mathrm{Cl}-0.67$ to -0.27 ; one study; $\mathrm{N}=64$ ). Two infants would have to receive a plastic cap to prevent one infant from having a core body temperature outside the normothermic range on admission to the NICU (NNTB 2, 95\% CI 2 to 4) (Analysis 4.3).

Core body temperature taken one hour after initial admission temperature to the NICU was taken (Outcome 4.4)

One study reported this outcome in terms of core body temperature in ${ }^{\circ} \mathrm{C}$ (axillary) taken one hour after the initial NICU admission was taken (Trevisanuto 2010). This was not a prespecified outcome at the review protocol stage.

Outcome 4.4.1: For infants at $<29$ weeks' gestation, data show a statistically significant difference for core body temperature taken one hour after initial NICU admission temperature was taken favouring the intervention (plastic cap) group when compared with the group that received routine care immediately after birth in the delivery room $\left(\mathrm{MD} 0.80^{\circ} \mathrm{C}, 95 \% \mathrm{Cl} 0.46\right.$ to 1.14 ; one study; $\mathrm{N}=64$ ) (Analysis 4.4).

\section{Secondary outcomes}

Major brain injury (defined as sonographic evidence of IVH with ventricular dilatation, parenchymal haemorrhagic infarction, or periventricular leukomalacia) (Outcome 4.5)

One study reported this outcome in terms of incidence of major brain injury (defined as sonographic evidence of IVH with ventricular dilatation, parenchymal haemorrhagic infarction, or periventricular leukomalacia) in intervention and control groups (plastic cap and routine care) (Trevisanuto 2010). This was not a prespecified outcome at the review protocol stage.

Outcome 4.5.1: For infants at $<29$ weeks' gestation, data show no significant difference in risk of major brain injury between infants who received plastic cap and those given routine care (RR 1.50, 95\% $\mathrm{Cl} 0.27$ to 8.38 ; RD $0.03,95 \% \mathrm{Cl}-0.10$ to 0.16 ; one study; $\mathrm{N}=64$ ) (Analysis 4.5).

\section{Mortality (death within hospital stay) (Outcome 4.6)}

One study reported this outcome in terms of incidence of death within hospital stay in intervention and control groups (plastic cap and routine care) (Trevisanuto 2010).

Outcome 4.6.1: For infants at $<29$ weeks' gestation, data show no significant differences in risk of death within hospital stay between infants who received plastic cap and those given routine care (RR $1.50,95 \% \mathrm{Cl} 0.27$ to 8.38 ; RD $0.03,95 \% \mathrm{Cl}-0.10$ to 0.16 ; one study; $\mathrm{N}=64$ ) (Analysis 4.6).

\section{Bicarbonate (mmol/L) (Outcome 4.7)}

One study reported this outcome (Trevisanuto 2010). This was not a prespecified outcome at the review protocol stage. 
Outcome 4.7.1: For infants at $<29$ weeks' gestation, data show no statistically significant differences between the two interventions (plastic cap and routine care) for bicarbonate (MD $1.00 \mathrm{mmol} / \mathrm{L}$, $95 \% \mathrm{Cl}-0.25$ to 2.25 ; one study; $\mathrm{N}=64$ ) (Analysis 4.7 ).

\section{Blood gas pH (first) (Outcome 4.8)}

One study reported this outcome in terms of first blood $\mathrm{pH}$ (Trevisanuto 2010). This was not a prespecified outcome at the review protocol stage.

Outcome 4.8.1: For infants at $<29$ weeks' gestation, data show no statistically significant differences between the two interventions (plastic cap and routine care) for first blood gas $\mathrm{pH}$ (MD 0.01, 95\% $\mathrm{Cl}-0.03$ to 0.05 ; one study; $\mathrm{N}=64$ ) (Analysis 4.8). Because $\mathrm{pH}$ is on a logarithmic scale, it would be more appropriate to measure this outcome as a median value and range or IQR, or to avoid its use as a continuous variable such as that prespecified at the review protocol stage: severe metabolic acidosis defined as $\mathrm{pH}<7.20$ and/or base deficit $>10 \mathrm{mmol} / \mathrm{L}$ within the first three days of life.

\section{First serum glucose concentration ( $\mathrm{mmol} / \mathrm{L}$ ) on admission to the NICU} (Outcome 4.9)

One study reported this outcome in terms of first serum glucose concentration $(\mathrm{mmol} / \mathrm{L}$ ) on admission to the NICU (Trevisanuto 2010). This was not a prespecified outcome at the review protocol stage.

Outcome 4.9.1: For infants at $<29$ weeks' gestation, data show no statistically significant differences between the two interventions (plastic cap and routine care) for first serum glucose concentration (MD $0.10 \mathrm{mmol} / \mathrm{L}, 95 \% \mathrm{Cl}-0.42$ to 0.62 ; one study; $\mathrm{N}=64$ (Analysis 4.9).

\section{Intubation at birth (Outcome 4.10)}

One study reported this outcome in terms of incidence of intubation at birth in the intervention and control groups (plastic cap and routine care) (Trevisanuto 2010).

Outcome 4.10.1: For infants at $<29$ weeks' gestation, data show no significant differences in risk of intubation at birth between infants who received plastic cap and those given routine care (RR 0.82, 95\% $\mathrm{Cl} 0.49$ to 1.37 ; RD $-0.09,95 \% \mathrm{Cl}-0.34$ to 0.15 ; one study; $\mathrm{N}=64$ ) (Analysis 4.10).

\section{Other secondary outcomes}

None of the included studies reported the following secondary outcome measures (as predefined at the review protocol stage) for this comparison group: hypoglycaemia, respiratory distress syndrome, surfactant given at any time, requirement for ventilation, duration of ventilation, severe metabolic acidosis, IVH, PDA, chronic lung disease, NEC, or acute renal failure.

\section{Adverse occurrences due to the intervention}

Trevisanuto 2010 reported that no infants had hyperthermia (axillary temperature on admission $>37.5^{\circ} \mathrm{C}$ ) in the plastic cap group or among those receiving routine care immediately at birth. None of the included studies reported the following secondary outcome measures (adverse occurrences) (as predefined at the review protocol stage) for this comparison group: burns, infection, antibiotics, skin maceration, interference with resuscitation, or negative psychological outcomes for infants in either group.

\section{Plastic bag and plastic cap versus routine care (Comparison 5)}

Two studies comprising 122 infants compared plastic bag + plastic cap versus routine care for infants at $\geq 28$ and $\leq 32$ weeks' gestation (Talakoub 2015), nor for infants at $\geq 28$ and $\leq 36$ weeks' gestation (Tescon-delos Santos 2012).

\section{Primary outcomes}

Core body temperature $\left({ }^{\circ} \mathrm{C}\right)$ of the infant taken on admission to the NICU or up to two hours after birth (Outcome 5.1)

Two studies comprising 122 infants reported this outcome in terms of core body temperature in ${ }^{\circ} \mathrm{C}$ (axillary) at the 10th, 15th, 30th, and 60th minutes of life (Tescon-delos Santos 2012), as well as upon admission to the NICU (Talakoub 2015; Tescon-delos Santos 2012), and at one and two hours after the initial NICU admission temperature was taken (Tescon-delos Santos 2012). Each individual study showed a significant effect - as in Talakoub 2015 - or provided data tending towards favouring the intervention (plastic wrap or bag) - as in Tescon-delos Santos 2012.

Outcome 5.1.1: For infants at $\geq 28$ and $\leq 36$ weeks' gestation, data show a statistically significant difference for core body temperature on admission to the NICU favouring the intervention (plastic bag + plastic cap) group when compared with the group that received routine care immediately after birth in the delivery room (MD $0.32^{\circ} \mathrm{C}, 95 \% \mathrm{Cl} 0.15$ to 0.50 ; two studies; $\mathrm{N}=122$ ) (Analysis 5.1 ).

The test for homogeneity failed with an $\mathrm{I}^{2}$ value of $90 \%$ (high heterogeneity). This heterogeneity could possibly be attributed to differences in included gestational ages at $\geq 28$ and $\leq 32$ weeks - as in Talakoub 2015 - and at $\geq 28$ and $\leq 36$ weeks - as in Tescon-delos Santos 2012.

Core body temperature $\left({ }^{\circ} \mathrm{C}\right)$ of the infant taken at the 10th minute of life (Outcome 5.2)

One study reported this outcome in terms of core body temperature in ${ }^{\circ} \mathrm{C}$ (axillary) at the 10th minute of life (Tescon-delos Santos 2012).

Outcome 5.2.1: For infants at $\geq 28$ and $\leq 36$ weeks' gestation, data show no statistically significant differences between the two interventions (plastic bag + plastic cap and routine care) for core body temperature at the 10 th minute of life $\left(M D-0.27^{\circ} \mathrm{C}, 95 \% \mathrm{Cl}\right.$ -0.55 to 0.01 ; one study; $\mathrm{N}=58$ ) (Analysis 5.2).

Core body temperature $\left({ }^{\circ} \mathrm{C}\right)$ of the infant taken at the 15 th minute of life (Outcome 5.3)

One study reported this outcome in terms of core body temperature in ${ }^{\circ} \mathrm{C}$ (axillary) at the 15th minute of life (Tescon-delos Santos 2012).

Outcome 5.3.1: For infants at $\geq 28$ and $\leq 36$ weeks' gestation, data show no statistically significant differences between the two interventions (plastic bag + plastic cap and routine care) for core body temperature at the 15 th minute of life $\left(\mathrm{MD}-0.26^{\circ} \mathrm{C}, 95 \% \mathrm{Cl}\right.$ -0.56 to 0.05 ; one study; $N=58$ ) (Analysis 5.3).

Core body temperature $\left({ }^{\circ} \mathrm{C}\right)$ of the infant taken at the 30th minute of life (Outcome 5.4)

One study reported this outcome in terms of core body temperature in ${ }^{\circ} \mathrm{C}$ (axillary) at the 30th minute of life (Tescon-delos Santos 2012).

Outcome 5.4.1: For infants at $\geq 28$ and $\leq 36$ weeks' gestation, data show no statistically significant differences between the two 
interventions (plastic bag + plastic cap and routine care) for core body temperature (axillary ${ }^{\circ} \mathrm{C}$ ) at the 30 th minute of life (MD $0.06^{\circ} \mathrm{C}$, $95 \% \mathrm{Cl}-0.23$ to 0.35 ; one study; $\mathrm{N}=58$ ) (Analysis 5.4).

\section{Core body temperature $\left({ }^{\circ} \mathrm{C}\right)$ of the infant taken at one hour of life (Outcome 5.5)}

One study reported this outcome in terms of core body temperature in ${ }^{\circ} \mathrm{C}$ (axillary) at one hour of life (Tescon-delos Santos 2012).

Outcome 5.5.1: For infants at $\geq 28$ and $\leq 36$ weeks' gestation, data show no statistically significant differences between the two interventions (plastic bag + plastic cap and routine care) for core body temperature (axillary ${ }^{\circ} \mathrm{C}$ ) at one hour of life (MD $-0.02^{\circ} \mathrm{C}, 95 \%$ $\mathrm{Cl}-0.32$ to 0.27 ; one study; $\mathrm{N}=58$ ) (Analysis 5.5).

Core body temperature $\left({ }^{\circ} \mathrm{C}\right)$ of the infant taken at one hour after the initial NICU admission temperature was taken (Outcome 5.6)

One study reported this outcome in terms of core body temperature in ${ }^{\circ} \mathrm{C}$ (axillary) one hour after the initial NICU temperature was taken (Talakoub 2015).

Outcome 5.6.1: For infants at $\geq 28$ and $\leq 32$ weeks' gestation, data show a statistically significant difference for core body temperature one hour after the initial NICU admission temperature was taken favouring the intervention (plastic bag + plastic cap) group when compared with the group that received routine care immediately after birth in the delivery room $\left(\mathrm{MD} 0.83^{\circ} \mathrm{C}, 95 \% \mathrm{Cl} 0.37\right.$ to 1.29 ; one study; $\mathrm{N}=64$ ) (Analysis 5.6).

Core body temperature $\left({ }^{\circ} \mathrm{C}\right)$ of the infant taken at two hours after the initial NICU admission temperature was taken (Outcome 5.7)

One study reported this outcome in terms of core body temperature in ${ }^{\circ} \mathrm{C}$ (axillary) two hours after the initial NICU admission temperature was taken (Talakoub 2015).

Outcome 5.7.1: For infants at $\geq 28$ and $\leq 32$ weeks' gestation, data show a statistically significant difference for core body temperature two hours after the initial NICU admission temperature was taken favouring the intervention (plastic bag + plastic cap) group when compared with the group that received routine care immediately after birth in the delivery room $\left(\mathrm{MD} 1.11^{\circ} \mathrm{C}, 95 \% \mathrm{Cl} 0.65\right.$ to 1.57 ; one study; $\mathrm{N}=64$ ) (Analysis 5.7).

\section{Secondary outcomes}

Hyperthermia (core body temperature $>37.0^{\circ} \mathrm{C}$ on admission to the NICU (Outcome 5.8)

Two studies comprising 122 infants reported this outcome (Talakoub 2015; Tescon-delos Santos 2012). Tescon-delos Santos 2012, in addition to reporting core body temperature on admission to the NICU as a continuous variable, provided data in a dichotomous format in terms of incidence of hyperthermia in the intervention and control groups (plastic bag + plastic cap and routine care). Investigators defined hyperthermia as a core body temperature (axillary) $>37.0^{\circ} \mathrm{C}$ on admission to the NICU. Talakoub 2015 did not provide a clear definition for hyperthermia but reported no hyperthermia in either group.

Outcome 5.8.1: For infants at $\geq 28$ and $\leq 36$ weeks' gestation, data show no significant difference in risk of hyperthermia between infants who received plastic bag + plastic cap and those given routine care (typical RR $0.18,95 \% \mathrm{Cl} 0.02$ to 1.39 ; typical RD -0.08 , $95 \% \mathrm{Cl}-0.16$ to 0.00 ; one study; $\mathrm{N}=122$ ) (Analysis 5.8 ).
The test for homogeneity failed with an $\mathrm{I}^{2}$ value of $87 \%$ (high heterogeneity) for RD. This heterogeneity could possibly be attributed to differences in included gestational ages at $\geq 28$ and $\leq$ 32 weeks - in Talakoub 2015 - and at $\geq 28$ and $\leq 36$ weeks - in Tescondelos Santos 2012.

\section{Hyponatraemia (Outcome 5.9)}

One study reported this outcome in terms of incidence of serum sodium concentration $<130 \mathrm{mmol} / \mathrm{L}$ in intervention and control groups (plastic bag + plastic cap and routine care) (Tescon-delos Santos 2012).

Outcome 5.9.1: For infants at $\geq 28$ and $\leq 36$ weeks' gestation, data show no significant differences in risk of hyponatraemia between infants who received plastic bag + plastic cap and those given routine care (RR $1.43,95 \% \mathrm{Cl} 0.35$ to 5.83 ; $\mathrm{RD} 0.04,95 \% \mathrm{Cl}-0.13$ to 0.21 ; one study; $\mathrm{N}=58$ ) (Analysis 5.9).

\section{Weight (grams) at fifth day of life (Outcome 5.10)}

One study reported this outcome in terms of weight (grams) on the fifth day of life or the last day of life if death occurred before the fifth day (Tescon-delos Santos 2012). This outcome measure was designed to assess whether an infant had greater than $20 \%$ body weight loss by the fifth day of life. This was not a prespecified outcome at the review protocol stage.

Outcome 5.10.1: For infants at $\geq 28$ and $\leq 36$ weeks' gestation, data show no statistically significant differences between the two interventions (plastic bag + plastic cap and routine care) for weight (grams) on the fifth day of life (MD - $74.20 \mathrm{~g}, 95 \% \mathrm{Cl}-301.63$ to 153.23; one study; $\mathrm{N}=58$ ) (Analysis 5.10).

Outcome 5.10.2: For infants at $\geq 28$ and $\leq 36$ weeks' gestation, data show no reported cases when the infant lost more than $20 \%$ body weight by the fifth day of life.

\section{Other secondary outcomes}

Tescon-delos Santos 2012 reported no cases of skin infection in terms of inflammation of the skin caused by a pathogenic organism (bacteria, virus, or fungi) and no cases of systemic infection (culture-positive sepsis) in both intervention (plastic bag + plastic cap) and control (routine care) groups.

Investigators did not report the following secondary outcome measures (as predefined at the review protocol stage) for any of the included studies in this comparison group: hypoglycaemia, respiratory distress syndrome, surfactant given at any time, intubation in the delivery room, requirement for ventilation, duration of ventilation, length of stay, severe metabolic acidosis, IVH, PDA, chronic lung disease, NEC, or acute renal failure.

\section{Adverse occurrences due to the intervention}

Tescon-delos Santos 2012 reported no cases of skin burns or maceration in both intervention (plastic bag + plastic cap) and control (routine care) groups. None of the included studies reported the following secondary outcome measures (adverse occurrences) (as predefined at the review protocol stage) for this comparison group: antibiotics or negative psychological outcomes. 
Plastic bag with previous drying versus plastic bag without previous drying (Comparison 6)

One study comprising 60 infants compared polyethylene bag with previous drying versus polyethylene bag without previous drying for infants at $\geq 28$ and $<37$ weeks' gestation with birth weight $\geq 1000$ and $\leq 2499$ grams (Cardona Torres 2012).

\section{Primary outcomes}

Core body temperature $\left({ }^{\circ} \mathrm{C}\right)$ of the infant taken on admission to the NICU or up to two hours after birth

One study reported this outcome in terms of time to achieve a mean core body temperature (axillary) of $36.5^{\circ} \mathrm{C}$ and core body temperature in ${ }^{\circ} \mathrm{C}$ (axillary) of the infant every 15 minutes until two hours after birth (Cardona Torres 2012). These were not prespecified outcomes at the review protocol stage. For the purposes of this review, we have reported core body temperature in ${ }^{\circ} \mathrm{C}$ (axillary) at 30 minutes, 1 hour, 90 minutes, and 2 hours after birth

\section{Core body temperature $\left({ }^{\circ} \mathrm{C}\right) 30$ minutes after birth (Outcome 6.1)}

One study reported this outcome in terms of core body temperature in ${ }^{\circ} \mathrm{C}$ (axillary) 30 minutes after birth (Cardona Torres 2012). This was not a prespecified outcome at the review protocol stage.

Outcome 6.1.1: For infants at $\geq 28$ and $<37$ weeks' gestation with birth weight $\geq 1000$ and $\leq 2499$ grams, data show no statistically significant differences between the two interventions (plastic bag with previous drying vs plastic bag without previous drying) for core body temperature 30 minutes after birth $\left(\mathrm{MD} 0.0^{\circ} \mathrm{C}, 95 \% \mathrm{Cl}-0.17\right.$ to 0.17 ; one study; $\mathrm{N}=60$ ) (Analysis 6.1).

\section{Core body temperature $\left({ }^{\circ} \mathrm{C}\right)$ of the infant taken at one hour after birth} (Outcome 6.2)

One study reported this outcome in terms of core body temperature in ${ }^{\circ} \mathrm{C}$ (axillary) 60 minutes after birth (Cardona Torres 2012). This was not a prespecified outcome at the review protocol stage.

Outcome 6.2.1: For infants at $\geq 28$ and $<37$ weeks' gestation with birth weight $\geq 1000$ and $\leq 2499$ grams, data show no statistically significant differences between the two interventions (plastic bag with previous drying vs plastic bag without previous drying) for core body temperature 60 minutes after birth $\left(\mathrm{MD}-0.10^{\circ} \mathrm{C}, 95 \% \mathrm{Cl}-0.28\right.$ to 0.08 ; one study; $N=60$ ) (Analysis 6.2).

\section{Core body temperature $\left({ }^{\circ} \mathrm{C}\right)$ of the infant taken at 90 minutes after birth (Outcome 6.3)}

One study reported this outcome in terms of core body temperature in ${ }^{\circ} \mathrm{C}$ (axillary) 90 minutes after birth (Cardona Torres 2012). This was not a prespecified outcome at the review protocol stage.

Outcome 6.3.1: For infants at $\geq 28$ and $<37$ weeks' gestation with birth weight $\geq 1000$ and $\leq 2499$ grams, data show no statistically significant differences between the two interventions (plastic bag with previous drying vs plastic bag without previous drying) for core body temperature 90 minutes after birth $\left(\mathrm{MD}-0.10^{\circ} \mathrm{C}, 95 \% \mathrm{Cl}-0.29\right.$ to 0.09 ; one study; $\mathrm{N}=60$ ) (Analysis 6.3).
Core body temperature $\left({ }^{\circ} \mathrm{C}\right)$ of the infant taken at two hours after birth (Outcome 6.4)

One study reported this outcome in terms of core body temperature in ${ }^{\circ} \mathrm{C}$ (axillary) 120 minutes after birth (Cardona Torres 2012). This was not a prespecified outcome at the review protocol stage.

Outcome 6.4.1: For infants at $\geq 28$ and $<37$ weeks' gestation with birth weight $\geq 1000$ and $\leq 2499$ grams, data show no statistically significant differences between the two interventions (plastic bag with previous drying vs plastic bag without previous drying) for core body temperature 120 minutes after birth $\left(\mathrm{MD} 0.10^{\circ} \mathrm{C}, 95 \% \mathrm{Cl}-0.04\right.$ to 0.24 ; one study; $\mathrm{N}=60$ ) (Analysis 6.4).

\section{Secondary outcomes}

Hyperthermia on admission to the NICU: core body temperature > $37.5^{\circ} \mathrm{C}$ (Outcome 6.5)

Cardona Torres 2012, in addition to reporting core body temperature on admission to the NICU as a continuous variable, provided data in a dichotomous format in terms of incidence of hyperthermia in the intervention and comparison groups (plastic bag with previous drying vs plastic bag without previous drying). Investigators did not provide a clear definition of hyperthermia. Data show one case of hyperthermia (definition not reported) in the plastic bag without previous drying group $\left(37.7^{\circ} \mathrm{C}\right)$.

Outcome 6.5.1: For infants at $\geq 28$ and $<37$ weeks' gestation with birth weight $\geq 1000$ and $\leq 2499$ grams, data show no significant differences in risk of hyperthermia between infants who received plastic bag with previous drying and those given plastic bag without previous drying (RR $0.33,95 \% \mathrm{Cl} 0.01$ to 7.87 ; RD $-0.03,95 \% \mathrm{Cl}-0.12$ to 0.05 ; one study; $\mathrm{N}=60$ ) (Analysis 6.5).

Blood glucose concentration ( $\mathrm{mmol} / \mathrm{L}$ ) at 120 minutes after birth (Outcome 6.6)

One study reported this outcome as a median value with IQR (Cardona Torres 2012). Data were converted to the International System of Units (SI) $\mathrm{mmol} / \mathrm{L}$ from $\mathrm{mg} / \mathrm{dL}$. This was not a prespecified outcome at the review protocol stage.

Outcome 6.6.1: For infants at $\geq 28$ and $<37$ weeks' gestation with birth weight $\geq 1000$ and $\leq 2499$ grams, median blood glucose concentrations at 120 minutes after birth were comparable in the intervention (plastic bag with drying) and active comparator groups (plastic bag without drying) (one study; $\mathrm{N}=60 ; \mathrm{P}>0.05$ ) (Analysis 6.6).

\section{Other secondary outcomes}

Cardona Torres 2012 reported no hypothermia (clear definition not provided) in the plastic bag with pr without previous drying groups.

None of the included studies reported the following secondary outcome measures (as predefined at the review protocol stage) for this comparison group: hypoglycaemia, respiratory distress syndrome, surfactant given at any time, intubation in the delivery room, requirement for ventilation, duration of ventilation, length of stay, mortality, severe metabolic acidosis, IVH, PDA, chronic lung disease, NEC, and acute renal failure.

\section{Adverse occurrences due to the intervention}

Cardona Torres 2012 reported that none of the neonates in the plastic bag with or without previous drying group developed 
lesions of the skin or infection. Cardona Torres 2012 also reported that the wrap procedure was well accepted by neonatal staff and did not interfere with resuscitation.

None of the included studies reported the following secondary outcome measures (adverse occurrences) (as predefined at the review protocol stage) for this comparison group: burns, maceration, antibiotics, fluid problems, or negative psychological outcomes.

\section{Plastic cap versus plastic bag (no cap) (Comparison 7)}

One study comprising 64 infants compared polyethylene caps versus polyethylene bags for infants at $<29$ weeks' gestation (Trevisanuto 2010).

\section{Primary outcomes}

Core body temperature $\left({ }^{\circ} \mathrm{C}\right)$ of the infant taken on admission to the NICU or up to two hours after birth (Outcome 7.1)

One study reported this outcome in terms of core body temperature in ${ }^{\circ} \mathrm{C}$ (axillary) on admission to the NICU (Trevisanuto 2010).

Outcome 7.1.1: For infants at $<29$ weeks' gestation, data show no statistically significant differences between the two interventions (plastic cap and plastic bag) for core body temperature on admission to the NICU (MD $0.30^{\circ} \mathrm{C}, 95 \% \mathrm{Cl}-0.12$ to 0.72 ; one study; $\mathrm{N}=64$ ) (Analysis 7.1).

\section{Hypothermia on admission to the NICU (core body temperature $<$ $36.4^{\circ} \mathrm{C}$ ) (Outcome 7.2 )}

Trevisanuto 2010, in addition to reporting core body temperature on admission to the NICU as a continuous variable, provided data in a dichotomous format in terms of incidence of hypothermia in the intervention and comparison groups (plastic cap and plastic bag). Investigators defined hypothermia as a core body temperature (axillary) $<36.4^{\circ} \mathrm{C}$ on admission to the NICU.

Outcome 7.2.1: For infants at $<29$ weeks' gestation, data show no significant differences in risk of hypothermia on admission to the NICU between infants who received plastic cap and those given plastic bag (RR $0.70,95 \% \mathrm{Cl} 0.43$ to 1.13 ; RD $-0.19,95 \% \mathrm{Cl}-0.43$ to 0.05; one study; $\mathrm{N}=64$ ) (Analysis 7.2).

\section{Outside normothermic range on admission to the NICU or up to two hours after birth (Outcome 7.3)}

We derived this outcome for one study in terms of incidence of core body temperature (axillary) outside the normothermic range of $36.4^{\circ} \mathrm{C}$ to $37.5^{\circ} \mathrm{C}$ on admission to the NICU among intervention and comparison groups (plastic cap and plastic bag) (Trevisanuto 2010). This was not a prespecified outcome at the review protocol stage.

Outcome 7.3.1: For infants at $<29$ weeks' gestation, data show no significant difference (borderline) in risk of having a core body temperature outside the normothermic range on admission to the NICU between infants who received plastic cap and those given plastic bag (RR $0.64,95 \% \mathrm{Cl} 0.40$ to $1.01 ; \mathrm{RD}-0.25,95 \% \mathrm{Cl}-0.49$ to -0.01; one study; $\mathrm{N}=64$ ) (Analysis 7.3).
Core body temperature taken one hour after initial NICU admission temperature was taken (Outcome 7.4)

One study reported this outcome in terms of core body temperature in ${ }^{\circ} \mathrm{C}$ (axillary) (Trevisanuto 2010). This was not a prespecified outcome at the review protocol stage.

Outcome 7.4.1: For infants at $<29$ weeks' gestation, data show a borderline statistically significant difference for core body temperature taken one hour after the initial NICU admission temperature was taken favouring the intervention (plastic cap) group when compared with the group that received plastic bag immediately after birth in the delivery room $\left(\mathrm{MD} 0.30^{\circ} \mathrm{C}, 95 \% \mathrm{Cl} 0.00\right.$ to 0.60 ; one study; $\mathrm{N}=64$ ) (Analysis 7.4 ).

\section{Secondary outcomes}

Hyperthermia on admission to the NICU: core body temperature > $37.5^{\circ} \mathrm{C}$ (Outcome 7.5 )

Trevisanuto 2010, in addition to reporting core body temperature on admission to the NICU as a continuous variable, provided data in a dichotomous format in terms of incidence of hyperthermia among intervention and comparison groups (plastic cap and plastic bag). Investigators defined hyperthermia as a core body temperature (axillary) $>37.5^{\circ} \mathrm{C}$ on admission to the NICU.

Outcome 7.5.1: For infants at $<29$ weeks' gestation, data show no significant difference in risk of hyperthermia on admission to the NICU between infants who received plastic cap and those given plastic bag (RR $0.20,95 \% \mathrm{Cl} 0.01$ to 4.01 ; RD $-0.06,95 \% \mathrm{Cl}-0.16$ to 0.04 ; one study; $\mathrm{N}=60$ ) (Analysis 7.5).

\section{Major brain injury (defined as sonographic evidence of IVH with ventricular dilatation, parenchymal haemorrhagic infarction, or periventricular leukomalacia) (Outcome 7.6)}

One study reported this outcome in terms of incidence of major brain injury defined as sonographic evidence of IVH with ventricular dilatation, parenchymal haemorrhagic infarction, or periventricular leukomalacia in the intervention and comparison groups (plastic cap and plastic bag) (Trevisanuto 2010). This was not a prespecified outcome at the review protocol stage.

Outcome 7.6.1: For infants at $<29$ weeks' gestation, data show no significant difference in risk of major brain injury between infants who received plastic cap and those given plastic bag (RR 1.50, 95\% $\mathrm{Cl} 0.27$ to 8.38 ; RD $0.03,95 \% \mathrm{Cl}-0.10$ to 0.16 ; one study; $\mathrm{N}=64$ ) (Analysis 7.6).

\section{Mortality (death within hospital stay) (Outcome 7.7)}

One study reported this outcome in terms of incidence of death within hospital stay in the intervention and comparison groups (plastic cap and plastic bag) (Trevisanuto 2010).

Outcome 7.7.1: For infants at $<29$ weeks' gestation, data show no significant difference in risk of death within hospital stay between infants who received plastic cap and those given plastic bag (RR $1.50,95 \% \mathrm{Cl} 0.27$ to 8.38 ; RD $0.03,95 \% \mathrm{Cl}-0.10$ to 0.16 ; one study; $\mathrm{N}=64$ ) (Analysis 7.7).

\section{Bicarbonate concentration (mmol/L) (Outcome 7.8)}

One study reported this outcome in terms of bicarbonate concentration (mmol/L) (Trevisanuto 2010). This was not a prespecified outcome at the review protocol stage. 
Outcome 7.8.1: For infants at $<29$ weeks' gestation, data show a borderline statistically significant difference for bicarbonate concentration favouring the intervention (plastic cap) group when compared with the group that received plastic bag immediately after birth in the delivery room (MD $1.00 \mathrm{mmol} / \mathrm{L}, 95 \% \mathrm{Cl} 0.02$ to 1.98; one study; $\mathrm{N}=64$ ) (Analysis 7.8).

\section{Blood gas pH (first) (Outcome 7.9)}

One study reported this outcome in terms of first blood gas $\mathrm{pH}$ (Trevisanuto 2010). This was not a prespecified outcome at the review protocol stage. Because $\mathrm{pH}$ is on a logarithmic scale, it would be more appropriate to measure this outcome as a median value with range or IQR, or to avoid its use as a continuous variable such as those prespecified at the review protocol stage: severe metabolic acidosis as defined by $\mathrm{pH}<7.20$ and/or base deficit $>10 \mathrm{mmol} / \mathrm{L}$ within the first three days of life.

Outcome 7.9.1: For infants at $<29$ weeks' gestation, data show no statistically significant differences between the two interventions (plastic cap and plastic bag) for first blood gas pH (MD -0.02, 95\% Cl -0.06 0.02; one study, $n=64$ ) (Analysis 7.9).

\section{First serum glucose concentration (mmol/L) (Outcome 7.10)}

One study reported this outcome in terms of first serum glucose concentration $(\mathrm{mmol} / \mathrm{L})$ on admission to the NICU (Trevisanuto 2010).

Outcome 7.10.1: For infants at $<29$ weeks' gestation, data show no statistically significant differences between the two interventions (plastic cap and plastic bag) for first serum glucose concentration on admission to the NICU (MD $0.00 \mathrm{mmol} / \mathrm{L}, 95 \% \mathrm{Cl},-0.50$ to 0.50 ; one study; $\mathrm{N}=64$ ) (Analysis 7.10).

\section{Intubation at birth (Outcome 7.11)}

One study reported this outcome in terms of incidence of intubation at birth in the intervention and comparison groups (plastic cap and plastic bag) (Trevisanuto 2010).

Outcome 7.11.1: For infants at $<29$ weeks' gestation, data show no significant difference in risk of intubation at birth between infants who received plastic cap and those given plastic bag (RR 0.82, 95\% $\mathrm{Cl} 0.49$ to 1.37 ; RD $-0.09,95 \% \mathrm{Cl}-0.34$ to 0.15 ; one study; $\mathrm{N}=64$ ) (Analysis 7.11).

\section{Other secondary outcomes}

None of the included studies reported the following secondary outcome measures (as predefined at the review protocol stage) for this comparison group: hypoglycaemia, respiratory distress syndrome, surfactant given at any time, requirement for ventilation, duration of ventilation, severe metabolic acidosis, IVH, PDA, chronic lung disease, NEC, or acute renal failure.

\section{Adverse occurrences due to the intervention}

Trevisanuto 2010 reported that use of an oximetry indicates opening of the bag during placement of the sensor, which reduces the temperature protective effect.

None of the included studies reported the following secondary outcome measures (adverse occurrences) (as predefined at the review protocol stage) for this comparison group: burns, infection, antibiotics, skin maceration, interference with resuscitation, or negative psychological outcomes for infants in the intervention and control groups.

\section{Plastic bag versus plastic wrap (Comparison 8)}

One study comprising 59 infants compared vinyl bag versus polyethylene wrap for infants at $\leq 32$ weeks' gestation (Caglar 2014).

\section{Primary outcomes}

Core body temperature $\left({ }^{\circ} \mathrm{C}\right)$ of the infant taken on admission to the NICU or up to two hours after birth

One study reported this outcome in terms of core body temperature in ${ }^{\circ} \mathrm{C}$ (axillary) at 20,40, and 60 minutes after birth and decrease in core body temperature during the first hour after birth (Caglar 2014).

\section{Core body temperature $\left({ }^{\circ} \mathrm{C}\right)$ of the infant taken at 20 minutes after birth (Outcome 8.1)}

Outcome 8.1.1: For infants at $\leq 32$ weeks' gestation, data show a statistically significant difference for core body temperature taken at 20 minutes after birth favouring the intervention (plastic bag) group when compared with the group that received plastic wrap immediately after birth in the delivery room $\left(\mathrm{MD} 0.14^{\circ} \mathrm{C}, 95 \% \mathrm{Cl} 0.08\right.$ to 0.20 ; one study; $\mathrm{N}=59$ ) (Analysis 8.1).

\section{Core body temperature $\left({ }^{\circ} \mathrm{C}\right)$ of the infant taken at 40 minutes after birth (Outcome 8.2)}

Outcome 8.2.1: For infants at $\leq 32$ weeks' gestation, data show a statistically significant difference for core body temperature taken at 40 minutes after birth favouring the intervention (plastic bag) group when compared with the group that received plastic wrap immediately after birth in the delivery room $\left(\mathrm{MD} 0.30^{\circ} \mathrm{C}, 95 \% \mathrm{Cl} 0.24\right.$ to 0.36 ; one study; $\mathrm{N}=59$ ) (Analysis 8.2).

Core body temperature $\left({ }^{\circ} \mathrm{C}\right)$ of the infant taken at one hour after birth (Outcome 8.3)

Outcome 8.3.1: For infants at $\leq 32$ weeks' gestation, data show a statistically significant difference for core body temperature taken at one hour after birth favouring the intervention (plastic bag) group when compared with the group that received plastic wrap immediately after birth in the delivery room $\left(\mathrm{MD} 0.35^{\circ} \mathrm{C}, 95 \% \mathrm{Cl} 0.29\right.$ to 0.41 ; one study; $\mathrm{N}=59$ ) (Analysis 8.3).

Decrease in core body temperature $\left({ }^{\circ} \mathrm{C}\right)$ of the infant during one hour after birth (Outcome 8.4)

Outcome 8.4.1: For infants at $\leq 32$ weeks' gestation, data show a statistically significant difference for decrease in core body temperature during the first hour after birth favouring the intervention (plastic bag) group when compared with the group that received plastic wrap immediately after birth in the delivery room ( $\mathrm{MD}-1.34^{\circ} \mathrm{C}, 95 \% \mathrm{Cl}-2.22$ to -0.46 ; one study; $\mathrm{N}=59$ ) (Analysis 8.4).

Hypothermia within one hour after birth (core body temperature < $36.5^{\circ} \mathrm{C}$ or skin temperature $<36^{\circ} \mathrm{C}$ ) (Outcome 8.5)

Caglar 2014, in addition to reporting core body temperature on admission to NICU as a continuous variable, provided data in a dichotomous format in terms of incidence of hypothermia in the intervention and comparison groups (plastic bag and plastic wrap). Investigators defined hypothermia as core body temperature (axillary) $<36.4^{\circ} \mathrm{C}$; cold stress as $36^{\circ} \mathrm{C}$ to $36.4^{\circ} \mathrm{C}$; 
moderate hypothermia as $32^{\circ} \mathrm{C}$ to $35.9^{\circ} \mathrm{C}$; and severe hypothermia as $<32^{\circ} \mathrm{C}$.

Outcome 8.5.1: For infants at $\leq 32$ weeks' gestation, data show no significant difference in risk of hypothermia within one hour after birth between infants who received plastic bags and those given plastic wrap (RR $0.84,95 \% \mathrm{Cl} 0.63$ to 1.12 ; RD $-0.14,95 \% \mathrm{Cl}-0.35$ to 0.08; one study; $\mathrm{N}=59$ ) (Analysis 8.5).

\section{Moderate hypothermia within one hour after birth (core body temperature $32^{\circ} \mathrm{C}$ to $35.9^{\circ} \mathrm{C}$, or skin temperature $31.5^{\circ} \mathrm{C}$ to $35.4^{\circ} \mathrm{C}$ ) (Outcome 8.6)}

One study reported this outcome in terms of incidence of hypothermia in the intervention and comparison groups (plastic bag and plastic wrap) (Caglar 2014). Investigators defined moderate hypothermia as core body temperature within the range $32^{\circ} \mathrm{C}$ to $35.9^{\circ} \mathrm{C}$.

Outcome 8.6.1: For infants at $\leq 32$ weeks' gestation, data show no significant difference (borderline) in risk of hypothermia within one hour after birth between infants who received plastic bag and those given plastic wrap (RR $0.56,95 \% \mathrm{Cl} 0.31$ to 1.02 ; RD $-0.29,95 \% \mathrm{Cl}$ -0.54 to -0.03 ; one study; $\mathrm{N}=59$ ) (Analysis 8.6).

\section{Outside normothermic range on admission to the NICU or up to two} hours after birth (Outcome 8.7)

One study reported this outcome in terms of incidence of core body temperature (axillary) outside the normothermic range in intervention and comparison groups (plastic bag and plastic wrap) (Caglar 2014). Investigators defined the range of normothermia as core body temperature (axillary) within the range $36.5^{\circ} \mathrm{C}$ to $37.5^{\circ} \mathrm{C}$ within one hour after birth. This was not a prespecified outcome at the review protocol stage.

Outcome 8.7.1: For infants at $\leq 32$ weeks' gestation, data show no significant difference in risk of having a core body temperature outside the normothermic range within one hour after birth between infants who received plastic bag and those given plastic wrap (RR 0.84, 95\% Cl 0.63 to 1.12; RD $-0.14,95 \% \mathrm{Cl}-0.35$ to 0.08 ; one study; $\mathrm{N}=59$ ) (Analysis 8.7).

\section{Other secondary outcomes}

Data show no reported severe hypothermia in the intervention (plastic bag) and control (plastic wrap) groups. Studies provided mortality figures as baseline characteristics. with three reported deaths in the plastic bag group and one reported death in the plastic wrap group. The time frame for mortality is unclear. Included studies did not report the following secondary outcome measures (as predefined at the review protocol stage) for this comparison group: hypoglycaemia, respiratory distress syndrome, surfactant given at any time, requirement for ventilation, duration of ventilation, length of stay, mortality, severe metabolic acidosis, IVH, PDA, chronic lung disease, NEC, or acute renal failure for infants in the intervention and control groups. However, trial authors reported type of ventilatory support, surfactant intake, Apgar score @ 5 minutes and 11 minutes, cord gas $\mathrm{pH}$, blood sugar at delivery, and mortality as baseline characteristics of infants.

\section{Adverse occurrences due to the intervention}

Data show no reported hyperthermia in the intervention (plastic bag) and control (plastic wrap) groups (deduced from hypothermia and normothermia data). Researchers did not report the following secondary outcome measures (adverse occurrences) (as predefined at the review protocol stage) for any of the included studies in this comparison group: burns, maceration, infection, antibiotics, skin maceration, interference with resuscitation, fluid problems, or negative psychological outcomes for infants in the intervention and control groups.

\section{Plastic total body wrap (body + head) versus plastic body wrap (head uncovered) (Comparison 9)}

One study comprising 100 infants compared total body wrap (polyethylene bags covering body and head, face exposed) versus body wrap (polyethylene bags up to shoulders, head uncovered) for infants at <29 weeks' gestation (Doglioni 2014).

\section{Primary outcomes}

Core body temperature $\left({ }^{\circ} \mathrm{C}\right)$ of the infant taken on admission to the NICU or up to two hours after birth (Outcome 9.1)

One study reported this outcome in terms of core body temperature in ${ }^{\circ} \mathrm{C}$ (axillary) on admission to the NICU (Doglioni 2014).

Outcome 9.1.1: For infants at $<29$ weeks' gestation, data show no statistically significant differences between the two interventions (plastic total body wrap and plastic wrap, head uncovered) for core body temperature on admission to the NICU $\left(\mathrm{MD} 0.10^{\circ} \mathrm{C}, 95 \% \mathrm{CI}\right.$ -0.18 to 0.38 ; one study; $N=100$ ) (Analysis 9.1).

Hypothermia on admission to the NICU (core body temperature < $36.5^{\circ} \mathrm{C}$ or skin temperature $<36^{\circ} \mathrm{C}$ ) (Outcome 9.2)

Doglioni 2014, in addition to reporting core body temperature on admission to the NICU as a continuous variable, provided data in a dichotomous format in terms of incidence of hypothermia in the intervention and comparison groups (plastic total body wrap and plastic wrap, head uncovered). Investigators defined hypothermia as a core body temperature (axillary) $<36.5^{\circ} \mathrm{C}$; mild hypothermia as $36^{\circ} \mathrm{C}$ to $36.4^{\circ} \mathrm{C}$; and moderate hypothermia as $32^{\circ} \mathrm{C}$ to $35.9^{\circ} \mathrm{C}$ on admission to the NICU.

Outcome 9.2.1: For infants at $<29$ weeks' gestation, data show no significant difference in risk of hypothermia on admission to the NICU between infants who received plastic total body wrap and those given plastic wrap (head uncovered) (RR $0.87,95 \% \mathrm{Cl} 0.55$ to $1.37 ; \mathrm{RD}-0.06,95 \% \mathrm{Cl}-0.25$ to 0.13 ; one study; $\mathrm{N}=100$ ) (Analysis 9.2).

Mild hypothermia on admission to the NICU (core body temperature $32^{\circ} \mathrm{C}$ to $35.9^{\circ} \mathrm{C}$ ) (Outcome 9.3 )

Outcome 9.3.1: For infants at $<29$ weeks' gestation, data show no significant difference in risk of mild hypothermia on admission to the NICU between infants who received plastic total body wrap and those given plastic wrap (head uncovered) (RR $1.08,95 \% \mathrm{Cl} 0.56$ to 2.05; RD 0.02, 95\% Cl-0.15 to 0.19; one study; $\mathrm{N}=100$ ) (Analysis 9.3).

Moderate hypothermia on admission to the NICU (core body temperature $32^{\circ} \mathrm{C}$ to $35.9^{\circ} \mathrm{C}$ ) (Outcome 9.4)

Outcome 9.4.1: For infants at $<29$ weeks' gestation, data show no significant difference in risk of moderate hypothermia on admission to the NICU between infants who received plastic total body wrap and those given plastic wrap (head uncovered) (RR 0.60, $95 \% \mathrm{Cl} 0.24$ to 1.53 ; RD $-0.08,95 \% \mathrm{Cl}-0.22$ to 0.06 ; one study; $\mathrm{N}=$ 100) (Analysis 9.4). 
Outside the normothermic range on admission to the NICU or within two hours after birth (Outcome 9.5)

We derived this outcome from one study in terms of incidence of core body temperature (axillary) outside the normothermic range of $36.5^{\circ} \mathrm{C}$ to $37.5^{\circ} \mathrm{C}$ on admission to the NICU in the intervention and comparison groups (plastic total body wrap and plastic wrap, head uncovered) (Doglioni 2014 ). This was not a prespecified outcome at the review protocol stage.

Outcome 9.5.1: For infants at $<29$ weeks' gestation, data show no significant difference in risk of having a core body temperature outside the normothermic range on admission to the NICU between infants who received plastic total body wrap and those given plastic wrap (head uncovered) (RR $0.88,95 \% \mathrm{Cl} 0.59$ to 1.32 ; RD $-0.06,95 \%$ $\mathrm{Cl}-0.26$ to 0.14 ; one study; $\mathrm{N}=100$ ) (Analysis 9.5).

\section{Core body temperature $\left({ }^{\circ} \mathrm{C}\right)$ of the infant taken one hour after} admission to the NICU (Outcome 9.6)

One study reported this outcome in terms of core body temperature in ${ }^{\circ} \mathrm{C}$ (axillary) one hour after admission to the NICU (Doglioni 2014).

Outcome 9.6.1: For infants at $<29$ weeks' gestation, data show no statistically significant difference between the two interventions (plastic total body wrap and plastic wrap, head uncovered) for core body temperature one hour after admission to the NICU (MD $-0.10^{\circ} \mathrm{C}, 95 \% \mathrm{Cl}-0.47$ to 0.27 ; one study; $\mathrm{N}=100$ ) (Analysis 9.6).

\section{Secondary outcomes}

\section{Hyperthermia on admission to the NICU (core body temperature >} $37.5^{\circ} \mathrm{C}$ ) (Outcome 9.7)

Doglioni 2014, in addition to reporting core body temperature on admission to the NICU as a continuous variable, provided data in a dichotomous format in terms of incidence of hyperthermia in the intervention and comparison groups (plastic total body wrap and plastic wrap, head uncovered). Investigators defined hyperthermia as core body temperature (axillary) $>37.5^{\circ} \mathrm{C}$.

Outcome 9.7.1: For infants at $<29$ weeks' gestation, data show no significant difference in risk of hyperthermia on admission to the NICU after birth between infants who received plastic total body wrap and those given plastic wrap (head uncovered) (RR 1.00, $95 \% \mathrm{Cl} 0.21$ to 4.72 ; RD $0.00,95 \% \mathrm{Cl}-0.09$ to 0.09 ; one study; $\mathrm{N}=$ 100) (Analysis 9.7). One infant in the wrap group had an axillary temperature $>38^{\circ} \mathrm{C}$.

\section{Major brain injury}

One study reported this outcome in terms of incidence of major brain injury in the intervention and comparison groups (plastic total body wrap and plastic wrap, head uncovered), as defined by sonographic evidence of IVH with ventricular dilatation, parenchymal haemorrhagic infarction, or periventricular leukomalacia (Doglioni 2014).

\section{Major brain injury: intraventricular haemorrhage (IVH) (Outcome 9.8)}

One study reported this outcome in terms of incidence of severe IVH in the intervention and comparison groups (plastic total body wrap and plastic wrap, head uncovered) (Doglioni 2014).

Outcome 9.8.1: For infants at $<29$ weeks' gestation, data show no significant difference in risk of severe IVH between infants who received plastic total body wrap and those given plastic wrap (head uncovered) (RR $0.80,95 \% \mathrm{Cl} 0.34$ to 1.86 ; RD $-0.04,95 \% \mathrm{Cl}-0.19$ to 0.11 ; one study; $\mathrm{N}=100$ ) (Analysis 9.8).

Major brain injury: periventricular leukomalacia (PVL) (Outcome 9.9)

One study reported this outcome in terms of incidence of severe PVL in intervention and comparison groups (plastic total body wrap and plastic wrap, head uncovered) (Doglioni 2014).

Outcome 9.9.1: For infants at $<29$ weeks' gestation, data show no significant difference in risk of PVL between infants who received plastic total body wrap and those given plastic wrap (head uncovered) (RR 1.00, 95\% Cl 0.06 to $15.55 ; \mathrm{RD} 0.00,95 \% \mathrm{Cl}-0.05$ to 0.05 ; one study; $\mathrm{N}=100$ ) (Analysis 9.9).

\section{Mortality (death within hospital stay or at six months' corrected gestation) (Outcome 9.10)}

One study reported this outcome in terms of incidence of death before discharge from hospital or at six months' corrected gestation in the intervention and comparison groups (plastic total body wrap and plastic wrap, head uncovered) (Doglioni 2014).

Outcome 9.10.1: For infants at $<29$ weeks' gestation, data show no significant difference in risk of death between infants who received plastic total body wrap and those given plastic wrap (head uncovered) (RR $0.29,95 \% \mathrm{Cl} 0.06$ to $1.31 ; \mathrm{RD}-0.10,95 \% \mathrm{Cl}-0.21$ to 0.01 ; one study; $\mathrm{N}=100$ ) (Analysis 9.10).

\section{Bronchopulmonary dysplasia (BPD) (Outcome 9.11)}

One study reported this outcome in terms of incidence of oxygen dependency at 36 postconceptional weeks in the intervention and comparison groups (plastic total body wrap and plastic wrap, head uncovered) (Doglioni 2014 ). Investigators defined BPD as chronic lung disease at the review protocol stage.

Outcome 9.11.1: For infants at $<29$ weeks' gestation, data show no significant difference in risk of BPD between infants who received plastic total body wrap and those given plastic wrap (head uncovered) (RR $1.00,95 \% \mathrm{Cl} 0.59$ to $1.69 ; \mathrm{RD} 0.00,95 \% \mathrm{Cl}-0.19$ to 0.19 ; one study; $\mathrm{N}=100$ ) (Analysis 9.11).

\section{Necrotising enterocolitis (NEC) (Outcome 9.12)}

One study reported this outcome in terms of incidence of NEC in the intervention and comparison groups (plastic total body wrap and plastic wrap, head uncovered) (Doglioni 2014).

Outcome 9.12.1: For infants at $<29$ weeks' gestation, data show no significant difference in the risk of NEC between infants who received plastic total body wrap and those given plastic wrap (head uncovered) (RR $1.00,95 \% \mathrm{Cl} 0.41$ to 2.46 ; RD $0.00,95 \% \mathrm{Cl}-0.14$ to 0.14 ; one study; $\mathrm{N}=100$ ) (Analysis 9.12).

\section{Other secondary outcomes}

None of the included studies reported the following secondary outcome measures (as predefined at the review protocol stage) for this comparison group: hypoglycaemia, respiratory distress syndrome, surfactant given at any time, requirement for ventilation, duration of ventilation, length of stay, severe metabolic acidosis, PDA, or acute renal failure for infants in the intervention and control groups.

Adverse occurrences due to the intervention 
Doglioni 2014 reported that the wrapping procedure was similar in both groups and was well accepted by neonatal teams.

None of the included studies reported the following secondary outcome measures (adverse occurrences) (as predefined at the review protocol stage) for this comparison group: burns, maceration, infection, antibiotics, skin maceration, interference with resuscitation, fluid problems, or negative psychological outcomes for infants in the intervention and control groups.

\section{Plastic bag and plastic hat versus plastic bag (body only) and cotton hat (Comparison 10)}

One study comprising 64 infants compared polyethylene bag + polyethylene hat versus polyethylene bag + cotton hat for infants at $\geq 28$ and $\leq 32$ weeks' gestation (Talakoub 2015).

\section{Primary outcomes}

Core body temperature $\left({ }^{\circ} \mathrm{C}\right)$ of the infant taken on admission to the NICU or up to two hours after birth (Outcome 10.1)

One study reported this outcome in terms of core body temperature in ${ }^{\circ} \mathrm{C}$ (axillary) on admission to the NICU (Talakoub 2015).

Outcome 10.1.1: For infants at $\geq 28$ and $\leq 32$ weeks' gestation, data show a statistically significant difference in core body temperature on admission to the NICU favouring the intervention (plastic bag + plastic hat) group when compared with the group given plastic bag + cotton hat immediately after birth in the delivery room (MD $0.32^{\circ} \mathrm{C}$, $95 \% \mathrm{Cl} 0.02$ to 0.62 ; one study; $\mathrm{N}=64$ ) (Analysis 10.1 ).

Core body temperature $\left({ }^{\circ} \mathrm{C}\right)$ of the infant taken one hour after admission (Outcome 10.2)

One study reported this outcome in terms of core body temperature in ${ }^{\circ} \mathrm{C}$ (axillary) one hour after admission to the NICU (Talakoub 2015).

Outcome 10.2.1: For infants at $\geq 28$ and $\leq 32$ weeks' gestation, data show a statistically significant difference in core body temperature one hour after admission to the NICU favouring the intervention (plastic bag + plastic hat) group when compared with the group that received plastic bag + cotton hat immediately after birth in the delivery room ( $\mathrm{MD} 0.37^{\circ} \mathrm{C}, 95 \% \mathrm{Cl} 0.03$ to 0.71 ; one study; $\mathrm{N}=64$ ) (Analysis 10.2).

\section{Core body temperature $\left({ }^{\circ} \mathrm{C}\right)$ of the infant taken two hours after} admission (Outcome 10.3)

One study reported this outcome in terms of core body temperature in ${ }^{\circ} \mathrm{C}$ (axillary) two hours after admission to the NICU (Talakoub 2015).

Outcome 10.3.1: For infants at $\geq 28$ and $\leq 32$ weeks' gestation, data show a statistically significant difference in core body temperature two hours after admission to the NICU favouring the intervention (plastic bag + plastic hat) group when compared with the group that received plastic bag + cotton hat immediately after birth in the delivery room $\left(\mathrm{MD} 0.37^{\circ} \mathrm{C}, 95 \% \mathrm{Cl} 0.11\right.$ to 0.63 ; one study; $\mathrm{N}=64$ ) (Analysis 10.3).

\section{Other secondary outcomes}

None of the included studies reported the following secondary outcome measures (as predefined at the review protocol stage) for this comparison group: hypothermia, hypoglycaemia, respiratory distress syndrome, surfactant given at any time, intubation in the delivery room, requirement for ventilation, duration of ventilation, length of stay, mortality, severe metabolic acidosis, IVH, PDA, chronic lung disease, NEC, or acute renal failure for infants in the intervention and control groups.

\section{Adverse occurrences due to the intervention}

Talakoub 2015 reported no hyperthermic infants in the 'plastic bag and plastic hat' and 'plastic bag + cotton hat' groups. Investigators provided no definition of hyperthermia. None of the included studies reported the following secondary outcome measures (adverse occurrences) (as predefined at the review protocol stage) for this comparison group: burns, maceration, infection, antibiotics, skin maceration, interference with resuscitation, fluid problems, or negative psychological outcomes for infants in the intervention and control groups.

\section{Stockinet cap versus routine care (no cap) (Comparison 11)}

One study comprising 40 infants compared stockinette cap versus routine care (Roberts 1981). This study reported figures for all infants at $\geq 32$ and $\leq 36$ weeks' gestation, and also for the subgroup of infants $<2000$ grams birth weight. We have tried to disaggregate the data using the information available to obtain data for the subgroup of infants of birth weight $\geq 2000$ grams. These subgroup analyses by birth weight were not prespecified in the protocol for this review.

\section{Primary outcomes}

Core body temperature $\left({ }^{\circ} \mathrm{C}\right)$ on admission to the NICU or up to two hours after birth (Outcome 11.1)

One study reported this outcome in terms of core body temperature in ${ }^{\circ} \mathrm{C}$ (axillary) on admission to the NICU (Roberts 1981).

Outcome 11.1.1: Overall, for infants at $\geq 32$ and $\leq 36$ weeks' gestation (birth weight range 1360 to 2965 grams), data show no statistically significant difference between the two interventions (stockinette cap and routine care) for core body temperature on admission to the NICU (MD $0.10^{\circ} \mathrm{C}, 95 \% \mathrm{Cl}-0.21$ to 0.41 ; one study; $\mathrm{N}=40$ ) (Analysis 11.1)

Outcome 11.1.2: For infants of birth weight $<2000$ grams, data show a borderline statistically significant difference in core body temperature on admission to the NICU favouring the intervention (stockinette cap) group when compared with the group that received routine care immediately after birth in the delivery room ( $\mathrm{MD} 0.70^{\circ} \mathrm{C}, 95 \% \mathrm{Cl}-0.01$ to 1.41 ; one study; $\mathrm{N}=10$ ) (Analysis 11.1 ).

Outcome 11.1.3: For infants of birth weight $\geq 2000$ grams, data show no statistically significant difference between the two interventions (stockinette cap and routine care) for core body temperature on admission to the $\mathrm{NICU}\left(\mathrm{MD} 0.00^{\circ} \mathrm{C}, 95 \% \mathrm{Cl}-0.37\right.$ to 0.37 ; one study; $\mathrm{N}=30$ ) (Analysis 11.1).

The test for homogeneity passed with an $\mathrm{I}^{2}$ value of $32.7 \%$ (low heterogeneity) for subgroup differences.

Hypothermia on admission to the NICU (core body temperature < $36.5^{\circ} \mathrm{C}$, or skin temperature $<36^{\circ} \mathrm{C}$ ) (Outcome 11.2)

Roberts 1981, in addition to reporting core body temperature on admission to the NICU as a continuous variable, provided data in a dichotomous format in terms of incidence of hypothermia in the 
intervention and control groups (stockinette cap and routine care). Investigators defined hypothermia as a core body temperature (axillary) $<36^{\circ} \mathrm{C}$ on admission to the NICU.

Outcome 11.2.1: For infants at $\geq 32$ and $\leq 36$ weeks' gestation (birth weight range 1360 to 2965 grams), data show no significant difference in risk of hypothermia between infants who received stockinette cap and those given routine care (RR 0.90, 95\% Cl 0.48 to $1.71 ; \mathrm{RD}-0.05,95 \% \mathrm{Cl}-0.36$ to 0.26 ; one study; $\mathrm{N}=40$ ) (Analysis 11.2).

\section{Secondary outcomes}

No included studies reported the following secondary outcome measures (as predefined at the review protocol stage) for this comparison group: hypoglycaemia, respiratory distress syndrome, surfactant given at any time, intubation in the delivery room, requirement for ventilation, duration of ventilation, length of stay, mortality, severe metabolic acidosis, IVH, PDA, chronic lung disease, NEC, and acute renal failure.

\section{Adverse occurrences due to the intervention}

No included studies reported the following secondary outcome measures (adverse occurrences) (as predefined at the review protocol stage) for this comparison group: hyperthermia, burns, maceration, skin or systemic infection, antibiotics, interference with resuscitation and other practices, fluid problems, or negative psychological outcomes.

\section{External heat sources category}

\section{Skin-to-skin care versus routine care (Comparison 12)}

One study comprising 31 infants compared skin-to-skin care versus routine (conventional incubator) care for infants of birth weight $\geq$ 1200 and $\leq 2199$ grams (Bergman 2004).

\section{Primary outcomes}

\section{Hypothermia (skin temperature $<35.5^{\circ} \mathrm{C}$ for two consecutive} recordings) (Outcome 12.1)

One study reported this outcome in terms of incidence of skin temperature remaining below $35.5^{\circ} \mathrm{C}$ for two consecutive readings (five-minute intervals for the first hour, thereafter 15minute intervals during the six-hour observation period) in the intervention and control groups (skin-to-skin care and routine care) (Bergman 2004). This was not a prespecified outcome at the review protocol stage.

Outcome 12.1.1: For infants of birth weight $\geq 1200$ and $\leq$ 2199 grams, skin-to-skin contact significantly reduced risk of hypothermia within six hours of birth (RR $0.09,95 \% \mathrm{Cl} 0.01$ to 0.64 ; $\mathrm{RD}-0.56,95 \% \mathrm{Cl}-0.84$ to -0.27 ; one study; $\mathrm{N}=31$ ). Two infants would have to receive skin-to-skin contact to prevent one infant from becoming hypothermic (NNTB 2, 95\% Cl 1 to 4) (Analysis 12.1).

\section{Secondary outcomes}

\section{Hypoglycaemia (blood glucose $<2.6 \mathrm{mmol} / \mathrm{L}$ ) (Outcome 12.2)}

One study reported this outcome in terms of incidence of confirmed laboratory estimation of blood glucose $<2.6 \mathrm{mmol} / \mathrm{L}$ within the six-hour observation period (when blood glucose was measured by heel prick at one, three, and six hours) in the intervention and control groups (skin-to-skin care and routine care) (Bergman 2004).
This was not the prespecified definition of hypoglycaemia at the review protocol stage.

Outcome 12.2.1: For infants of birth weight $\geq 1200$ and $\leq 2199$ grams, data show no significant difference in risk of hypoglycaemia between infants who received skin-to-skin contact and those given conventional incubator care (RR $0.24,95 \% \mathrm{Cl} 0.03$ to 2.06 ; RD -0.18 , $95 \% \mathrm{Cl}-0.43$ to 0.08 ; one study; $\mathrm{N}=31$ ) (Analysis 12.2 ).

\section{Other secondary outcomes}

This study reported the following additional outcome measures: heart rate $<100$ or $>180$ per minute for two consecutive recordings, apnoea longer than 20 seconds, oxygen saturation below $87 \%$ for two consecutive recordings despite supplementation with nasal prong oxygen, fraction of inspired oxygen $\left(\mathrm{FiO}_{2}\right)$ up to 0.60 , and CPAP up to $5 \mathrm{~cm}$ water, and overall data (transfers to NICU, exceeded parameters, mean SCRIP score within first six hours and number of perfect scores, mean SCRIP score in the sixth hour, and number of perfect scores).

None of the included studies reported the following secondary outcome measures (as predefined at the review protocol stage) for this comparison group: respiratory distress syndrome, surfactant given at any time, intubation in the delivery room, requirement for ventilation, duration of ventilation, length of stay, mortality, severe metabolic acidosis, IVH, PDA, chronic lung disease, NEC, or acute renal failure.

\section{Adverse occurrences due to the intervention}

Bergman 2004 reported that "there were no adverse events related to the intervention".

\section{Thermal mattress versus routine care (Comparison 13)}

Two studies comprising 126 infants compared thermal mattress versus routine care (Brennan 1996; Chawla 2011). Brennan 1996 compared the transwarmer infant transport mattress versus routine care for infants $\leq 1500$ grams, and Chawla 2011 compared the transwarmer mattress versus routine care for infants at $<32$ weeks' gestation. Although infants in the Chawla 2011 study were at $<32$ weeks' gestation, these infants were included in the birth weight $\leq 1500$ grams subgroup for meta-analysis and are referred to as such in the text. Chawla 2011 used additional thermal care measures (plastic bag without drying below the neck) for infants at $<28$ weeks' gestation, which was considered to be part of routine thermal care practices. Brennan 1996, the earlier study, did not employ such additional thermal care measures. Chawla 2011 reported that 40 neonates received both plastic bag and the transwarmer mattress, and 35 received plastic bag without the transwarmer mattress. In total, 43 infants were included in the < 28 weeks' gestation subgroup. Because all infants did not receive a plastic bag, we chose to include the data from Chawla 2011 in this comparison group rather than in comparison group 15 (plastic bag and thermal mattress vs plastic bag only).

\section{Primary outcomes}

Core body temperature $\left({ }^{\circ} \mathrm{C}\right)$ of the infant taken on admission to the NICU or up to two hours after birth (Outcome 13.1)

Two studies comprising 126 infants reported core body temperature in ${ }^{\circ} \mathrm{C}$ (axillary) on admission to the NICU (Brennan 1996; Chawla 2011). Each individual study showed a significant 
effect in favour of the intervention (thermal mattress) group for infants with birth weight $\leq 1500$ grams.

Outcome 13.1.1: For infants of birth weight $\leq 1500$ grams, data show a statistically significant difference in core body temperature on admission to the NICU favouring the intervention (thermal mattress) group when compared with the group that received routine care immediately after birth in the delivery room (MD $0.65^{\circ} \mathrm{C}, 95 \% \mathrm{Cl} 0.36$ to 0.94 ; two studies; $\mathrm{N}=126$ ) (Analysis 13.1 ).

However, the test for homogeneity failed with an $\mathrm{I}^{2}$ value of $85 \%$ (high heterogeneity). We note that this failure most likely is due to differences in methods between the two studies.

\section{Hypothermia on admission to the NICU (core body temperature < $36.5^{\circ} \mathrm{C}$, or skin temperature $<36^{\circ} \mathrm{C}$ ) (Outcome 13.2)}

Brennan 1996 and Chawla 2011, in addition to reporting core body temperature on admission to the NICU as a continuous variable, provided data in a dichotomous format in terms of incidence of hypothermia in the intervention and control groups (thermal mattress and routine care). Brennan 1996 defined hypothermia as core body temperature (axillary) $<36.5^{\circ} \mathrm{C}$ on admission to the NICU, and Chawla 2011 as core body temperature (axillary) $<36^{\circ} \mathrm{C}$ on admission to the NICU. Data for these outcome measures are presented separately.

Outcome 13.2.1: For infants of birth weight $\leq 1500$ grams, the thermal mattress significantly reduced risk of hypothermia on admission to the NICU (RR $0.30,95 \% \mathrm{Cl} 0.11$ to 0.83 ; RD -0.58 , 95\% $\mathrm{Cl}-0.91$ to -0.26 ; one study; $\mathrm{N}=24$ ) (Analysis 13.2). Two infants would have to receive a thermal mattress to prevent one infant from becoming hypothermic (NNTB 2, 95\% $\mathrm{Cl} 1$ to 4).

\section{Hypothermia on admission to the NICU (core body temperature < $36.0^{\circ} \mathrm{C}$ ) (Outcome 13.3)}

Chawla 2011 also reported hypothermia as a core body temperature (axillary) $<36^{\circ} \mathrm{C}$ on admission to the NICU. Brennan 1996 presented individual core body temperatures as continuous outcomes so data were derived for moderate hypothermia as defined in the Chawla 2011 study. Therefore, two studies comprising 126 infants reported hypothermia as core body temperature (axillary) $<36^{\circ} \mathrm{C}$ on admission to the NICU (Brennan 1996; Chawla 2011). Each individual study showed a significant effect in favour of the intervention (thermal mattress) group for infants with birth weight $\leq 1500$ grams.

Outcome 13.3.1: For infants of birth weight $\leq 1500$ grams, a thermal mattress significantly reduced risk of hypothermia (core body temperature (axillary) $<36^{\circ} \mathrm{C}$ ) on admission to the NICU (typical RR $0.49,95 \% \mathrm{Cl} 0.32$ to 0.76 ; typical RD $-0.30,95 \% \mathrm{Cl}-0.46$ to -0.14 ; two studies; $\mathrm{N}=126$ ) (Analysis 13.3). Three infants would have to receive a thermal mattress to prevent one infant from becoming hypothermic (NNTB 3, 95\% $\mathrm{Cl} 2$ to 7 ).

However, the test for homogeneity failed with an $1^{2}$ value of $62 \%$ (moderate heterogeneity) for RR, and $73 \%$ (moderate heterogeneity) for RD. We note that this failure is most likely due to differences in methods between the two studies. Chawla 2011 used additional thermal care measures (plastic bag without drying below the neck) for infants at $<28$ weeks' gestation, which was considered to be part of routine thermal care practices. Brennan 1996, the earlier study, did not employ such additional thermal care measures.
Hypothermia on admission to the NICU (core body temperature < 35.0 $0^{\circ} \mathrm{C}$ ) (Outcome 13.4)

Chawla 2011 also reported hypothermia as a core body temperature (axillary) $<35^{\circ} \mathrm{C}$ on admission to the NICU. Brennan 1996 presented individual core body temperatures as continuous outcomes; therefore we derived data for moderate hypothermia as defined by Chawla 2011. Two studies comprising 126 infants reported hypothermia as core body temperature (axillary) $<35^{\circ} \mathrm{C}$ on admission to the NICU (Brennan 1996; Chawla 2011). Each individual study showed no significant difference in effect for thermal mattress and routine care for infants of birth weight $\leq 1500$ grams.

Outcome 13.4.1: For infants of birth weight $\leq 1500$ grams, a thermal mattress significantly reduced risk of hypothermia (core body temperature (axillary) $<35^{\circ} \mathrm{C}$ ) on admission to the NICU (typical RR $0.18,95 \% \mathrm{Cl} 0.05$ to 0.65 ; typical RD $-0.18,95 \% \mathrm{Cl}-0.29$ to -0.07 ; two studies; $N=126$ ) (Analysis 13.4). Six infants would have to receive a thermal mattress to prevent one infant from becoming hypothermic (NNTB 6, 95\% $\mathrm{Cl} 4$ to 14 ).

However, the test for homogeneity failed with an $\mathrm{I}^{2}$ value of $0 \%$ for RR but $84 \%$ for RD (high heterogeneity). We note that this failure most likely is due to differences in methods between the two studies. Chawla 2011 used additional thermal care measures (plastic bag without drying below the neck) for infants at $<28$ weeks' gestation, which was considered to be part of routine thermal care practices. Brennan 1996, the earlier study, did not employ such additional thermal care measures.

\section{Outside the normothermic range on admission to the NICU or up to two hours after birth (Outcome 13.5)}

We derived this outcome for one study in terms of incidence of core body temperature (axillary) outside the normothermic range in intervention and control groups (thermal mattress and routine care) on admission to the NICU (Brennan 1996). Investigators defined normothermia as core body temperature (axillary) within the range $36.5^{\circ} \mathrm{C}$ to $37.5^{\circ} \mathrm{C}$. We were unable to derive this information from Chawla 2011 because investigators defined hypothermia as core body temperature (axillary) $<36^{\circ} \mathrm{C}$ on admission to the NICU. The was not a prespecified outcome at the review protocol stage.

Outcome 13.5.1: For infants of birth weight $\leq 1500$ grams, a thermal mattress significantly reduced risk of being outside the normothermic range (core body temperature (axillary) $36.5^{\circ} \mathrm{C}$ to $37.5^{\circ} \mathrm{C}$ ) on admission to the NICU (RR $0.30,95 \% \mathrm{Cl} 0.11$ to 0.83 ; RD $-0.58,95 \% \mathrm{Cl}-0.91$ to -0.26 ; one study; $\mathrm{N}=24$ ) (Analysis 13.5 ). Two infants would have to receive a thermal mattress to prevent one infant from being outside the normothermic range (NNTB 2, 95\% Cl 1 to 4$)$.

\section{Secondary outcomes}

Hyperthermia on admission to the NICU (core body temperature > $37.5^{\circ} \mathrm{C}$ ) (Outcome 13.6)

Two studies comprising 126 infants reported this outcome in terms of incidence of hyperthermia in intervention and control groups (thermal mattress and routine care) (Brennan 1996; Chawla 2011). Chawla 2011 defined hyperthermia as core body temperature (axillary) $>37.5^{\circ} \mathrm{C}$ on admission to the NICU. Brennan 1996 presented individual core body temperatures as continuous 
outcomes, so we derived data for hyperthermia as defined by Chawla 2011. Individual data from Brennan 1996 show no infants in the thermal mattress group or in the routine care group had a core body temperature (axillary) $>37.5^{\circ} \mathrm{C}$ on admission to the NICU. Each individual study showed no significant difference in effect for thermal mattress and routine care among infants with birth weight $\leq 1500$ grams.

Outcome 13.6.1: For infants of birth weight $\leq 1500$ grams, data show no statistically significant difference in risk of hyperthermia (core body temperature (axillary) $>37.5^{\circ} \mathrm{C}$ ) on admission to the NICU between infants who received a thermal mattress and those given routine care (RR 4.63, 95\% Cl 0.23 to 94.10 ; RD $0.03,95 \% \mathrm{Cl}$ -0.03 to 0.09 ; two studies; $N=126$ ) (Analysis 13.6).

The test for homogeneity passed with an $I^{2}$ value of $0 \%$ for RD.

\section{Mortality (Outcome 13.7)}

One study reported this outcome in terms of incidence of mortality in the intervention and control groups (thermal mattress and routine care) (Chawla 2011). Investigators did not provide a clear definition of the time frame for this outcome measure.

Outcome 13.7.1: For infants at < 32 weeks' gestation, data show no statistically significant difference in risk of mortality between infants who received a thermal mattress and those given routine care (RR $0.31,95 \% \mathrm{Cl} 0.01$ to $7.40 ; \mathrm{RD}-0.02,95 \% \mathrm{Cl}-0.07$ to 0.03 ; one study; $\mathrm{N}=102$ ) (Analysis 13.7).

\section{Major brain injury (intraventricular haemorrhage (IVH)) (Outcome 13.8)}

One study reported this outcome in terms of incidence of IVH (grade $\mathbb{I I}$ or $\mathbb{N}$ ) and of cystic periventricular leukomalacia on head ultrasonography in intervention and control groups (thermal mattress and routine care) (Chawla 2011). These data are presented as two separate outcomes.

Outcome 13.8.1: For infants at $<32$ weeks' gestation, data show no statistically significant difference in risk of IVH (grade $\mathbb{I}$ or $\mathbb{N}$ ) between infants who received a thermal mattress and those given routine care (RR 4.62, $95 \% \mathrm{Cl} 0.56$ to $38.19 ; \mathrm{RD} 0.07,95 \% \mathrm{Cl}-0.01$ to 0.16 ; one study; $\mathrm{N}=102$ ) (Analysis 13.8).

\section{Major brain injury (cystic periventricular leukomalacia) (Outcome 13.9)}

One study reported this outcome in terms of the incidence of IVH (grade $\mathbb{I I}$ or $\mathbb{N}$ ) and as cystic periventricular leukomalacia on head ultrasonography in intervention and control groups (thermal mattress and routine care) (Chawla 2011). These data are presented as two separate outcomes.

Outcome 13.9.1: For infants at $<32$ weeks' gestation, data show no statistically significant difference in risk of cystic periventricular leukomalacia between infants who received a thermal mattress and those given routine care ( $\mathrm{RR} 1.39,95 \% \mathrm{Cl} 0.24$ to 7.95 ; RD 0.02, $95 \% \mathrm{Cl}-0.07$ to 0.10 ; one study; $\mathrm{N}=102$ ) (Analysis 13.9).

\section{Bronchopulmonary dysplasia (BPD) (Outcome 13.10)}

One study reported this outcome in terms of incidence of the requirement for oxygen supplementation at 36 weeks' postmenstrual age in intervention and control groups (thermal mattress and routine care) (Chawla 2011). The was not a prespecified outcome at the review protocol stage.

Outcome 13.10.1: For infants at $<32$ weeks' gestation, data show no statistically significant difference in risk of BPD between infants who received a thermal mattress and those given routine care (RR $1.34,95 \% \mathrm{Cl} 0.69$ to 2.61 ; RD $0.08,95 \% \mathrm{Cl}-0.09$ to 0.25 ; one study; $\mathrm{N}=102$ ) (Analysis 13.10).

\section{Duration of hospital stay (days) (Outcome 13.11)}

One study reported this outcome in terms of duration of hospital stay (Chawla 2011).

Outcome 13.11.1: For infants at $<32$ weeks' gestation, data show no statistically significant differences between interventions (thermal mattress vs routine care) for duration of stay in hospital (MD -5.00 days, $95 \% \mathrm{Cl}-17.27$ to 7.27 ; one study; $\mathrm{N}=102$ ) (Analysis 13.11).

\section{Duration of ventilation (days) (Outcome 13.12)}

One study reported this outcome in terms of duration of ventilation (Chawla 2011).

Outcome 13.12.1: For infants at $<32$ weeks' gestation, data show no statistically significant differences between interventions (thermal mattress vs routine care) for duration of ventilation (MD -4.00 days, $95 \% \mathrm{Cl}-12.67$ to 4.67 ; one study; $\mathrm{N}=102$ ) (Analysis 13.12).

\section{Duration of oxygen requirement (days) (Outcome 13.13)}

One study reported this outcome in terms of duration of oxygen requirement (Chawla 2011). This was not a prespecified outcome at the review protocol stage.

Outcome 13.13.1: For infants at $<32$ weeks' gestation, data show no statistically significant differences between interventions (thermal mattress vs routine care) for duration of oxygen requirement (MD -7.00 days, $95 \% \mathrm{Cl}-19.66$ to 5.66; one study; $\mathrm{N}=$ 102) (Analysis 13.13).

\section{Hypoglycaemia (initial blood glucose $<40 \mathrm{mg} / \mathrm{dL}$ or $<2.2 \mathrm{mmol} / \mathrm{L}$ ) (Outcome 13.14)}

One study reported this outcome in terms of incidence of initial blood glucose $<40 \mathrm{mg} / \mathrm{dL}$ or $<2.2 \mathrm{mmol} / \mathrm{L}$ in intervention and control groups (thermal mattress and routine care) (Chawla 2011). This was not the prespecified definition of hypoglycaemia at the review protocol stage.

Outcome 13.14.1: For infants at $<32$ weeks' gestation, data show no statistically significant differences in risk of hypoglycaemia between infants who received a thermal mattress and those given routine care (RR $1.02,95 \% \mathrm{Cl} 0.47$ to 2.18 ; $\mathrm{RD} 0.00,95 \% \mathrm{Cl}-0.15$ to 0.16 ; one study; $\mathrm{N}=102$ ) (Analysis 13.14).

\section{Metabolic acidosis (Outcome 13.15)}

One study reported this outcome in terms of incidence of $\mathrm{pH}<7.15$ with base excess $\geq 10 \mathrm{mmol} / \mathrm{L}$ on initial blood gas in intervention and control groups (thermal mattress and routine care) (Chawla 2011).

Outcome 13.15.1: For infants at $<32$ weeks' gestation, data show no statistically significant differences in risk of metabolic acidosis 
between infants who received a thermal mattress and those given routine care (RR $0.31,95 \% \mathrm{Cl} 0.03$ to $2.86 ; \mathrm{RD}-0.04,95 \% \mathrm{Cl}-0.12$ to 0.03 ; one study; $\mathrm{n}=102$ ) (Analysis 13.15).

\section{Necrotising enterocolitis (NEC) (Outcome 13.16)}

One study reported this outcome in terms of incidence of NEC > stage 2 as defined by Bell's staging in intervention and control groups (thermal mattress and routine care) (Bell 1978; Chawla 2011).

Outcome 13.16.1: For infants at $<32$ weeks' gestation, data show no statistically significant differences in risk of NEC > stage 2 between infants who received a thermal mattress and those given routine care (RR $0.64,95 \% \mathrm{Cl} 0.33$ to 1.23 ; $\mathrm{RD}-0.12,95 \% \mathrm{Cl}-0.29$ to 0.05; one study; $\mathrm{N}=102$ ) (Analysis 13.16).

\section{Sepsis (Outcome 13.17)}

One study reported this outcome in terms of incidence of positive culture from sterile site such as blood or spinal fluid and intention to treat with antibiotics for seven days or longer in the intervention and control groups (thermal mattress and routine care) (Chawla 2011). This was not a prespecified outcome at the review protocol stage.

Outcome 13.17.1: For infants at $<32$ weeks' gestation, data show no statistically significant differences in risk of sepsis between infants who received a thermal mattress and those given routine care (RR 0.92, $95 \% \mathrm{Cl} 0.48$ to 1.79 ; $\mathrm{RD}-0.02,95 \% \mathrm{Cl}-0.19$ to 0.15 ; one study; $\mathrm{N}=102$ ) (Analysis 13.17).

\section{Other secondary outcomes}

No included studies reported the following secondary outcome measures (as predefined at the review protocol stage) for this comparison group: respiratory distress syndrome, surfactant given at any time, intubation in the delivery room, requirement for ventilation, PDA, chronic lung disease, or acute renal failure.

\section{Adverse occurrences due to the intervention}

Brennan 1996 reported that the intervention did not at any time interfere with care of the infants. Chawla 2011 reported that no infants had any local skin reaction, and that use of the thermal mattress was well received by delivery room personnel, with no respondent noting any interference with resuscitation.

No included studies reported the following secondary outcome measures (adverse occurrences) (as predefined at the review protocol stage) for this comparison group: burns, maceration, antibiotics, fluid problems, or negative psychological outcomes.

\section{Combinations of interventions category}

\section{Thermal mattress versus plastic wrap or bag (Comparison 14)}

Two studies comprising 77 infants compared thermal mattress versus plastic wrap or bag. Mathew 2012 compared the Transwarmer mattress versus polyvinyl bag for infants at $\leq 28$ weeks' gestation. Simon 2011 compared the Infatherm mattress versus polyethylene wrap (Neowrap) for infants at $\geq 24$ and $\leq 28$ weeks' gestation and birth weight $\leq 1250$ grams. These infants were grouped together in the gestational age of $\leq 28$ weeks subgroup for meta-analysis and are referred to as such in the text.

\section{Primary outcomes}

Core body temperature $\left({ }^{\circ} \mathrm{C}\right)$ of the infant taken on admission to the NICU or up to two hours after birth (Outcome 14.1)

Two studies comprising 77 infants reported this outcome in terms of core body temperature in ${ }^{\circ} \mathrm{C}$ (axillary) on admission to the NICU (Mathew 2012; Simon 2011). Each individual study showed no significant difference in effect for the thermal mattress versus plastic wrap or bag for infants at $\leq 28$ weeks' gestation. However, Mathew 2012 is tending towards favouring plastic bag, and Simon 2011 is showing borderline significance favouring thermal mattress.

Outcome 14.1.1. For infants at $\leq 28$ weeks' gestation, data show no statistically significant differences between interventions (thermal mattress and plastic bag or wrap) for core body temperature in ${ }^{\circ} \mathrm{C}$ on admission to the NICU (MD $0.18^{\circ} \mathrm{C}, 95 \% \mathrm{Cl}-0.18$ to 0.54 ; two studies; $\mathrm{N}=77$ ) (Analysis 14.1).

The test for homogeneity failed with an $\mathrm{I}^{2}$ value of $68 \%$ (moderate heterogeneity). The most plausible reason for this heterogeneity may be variability of use between practitioners when the intervention was polyethylene wrap as opposed to thermal mattress, as in Simon 2011; in some cases, displacement of the wrap could have resulted in heat loss, meaning that performance bias cannot be ruled out.

\section{Hypothermia on admission to the NICU (core body temperature < $36.5^{\circ} \mathrm{C}$, or skin temperature $<36^{\circ} \mathrm{C}$ ) (Outcome 14.2)}

Simon 2011, in addition to reporting core body temperature on admission to the NICU as a continuous variable, provided data in a dichotomous format in terms of incidence of hypothermia in intervention and comparison groups (thermal mattress and plastic wrap or bag). Investigators defined hypothermia as a core body temperature (axillary) $<36.5^{\circ} \mathrm{C}$ on admission to the NICU.

Outcome 14.2.1: For infants at $\geq 24$ and $\leq 28$ weeks' gestation with birth weight $\leq 1250$ grams, data show no significant differences in risk of hypothermia between infants who received thermal mattress and those given plastic wrap (RR $0.60,95 \% \mathrm{Cl} 0.32$ to 1.15 ; $\mathrm{RD}-0.27,95 \% \mathrm{Cl}-0.59$ to 0.04 ; one study; $\mathrm{N}=36$ ) (Analysis 14.2 ).

\section{Outside the normothermic range on admission to the NICU or up to} two hours after birth (Outcome 14.3)

We were able to derive data for the normothermic range from the definitions of hypothermia (core body temperature (axillary) $<36.5^{\circ} \mathrm{C}$ ) and hyperthermia (core body temperature (axillary) $>37.5^{\circ} \mathrm{C}$ ) on admission to the NICU provided in Simon 2011. Investigators defined normothermic range as core body temperature (axillary) $36.5^{\circ} \mathrm{C}$ to $37.5^{\circ} \mathrm{C}$ on admission to the $\mathrm{NICU}$. They reported this outcome as the incidence of core body temperature outside the normothermic range on admission to the NICU in both intervention and comparison groups (thermal mattress and plastic wrap). This was not a prespecified outcome at the review protocol stage.

Outcome 14.3.1: For infants at $\geq 24$ and $\leq 28$ weeks' gestation with birth weight $\leq 1250$ grams, data show no significant differences in risk of being outside the normothermic range (core body temperature (axillary) $36.5^{\circ} \mathrm{C}$ to $37.5^{\circ} \mathrm{C}$ ) on admission to the NICU between infants who received thermal mattress and those given plastic wrap (RR $0.69,95 \% \mathrm{Cl} 0.38$ to 1.24 ; RD $-0.21,95 \% \mathrm{Cl}-0.53$ to 0.10 ; one study; $\mathrm{N}=36$ ) (Analysis 14.3). 


\section{Secondary outcomes}

\section{Hyperthermia on admission to the NICU (core body temperature >} $37.5^{\circ} \mathrm{C}$ ) (Outcome 14.4)

Simon 2011, in addition to reporting core body temperature on admission to the NICU as a continuous variable, provided data in a dichotomous format in terms of incidence of hyperthermia in intervention and comparison groups (thermal mattress and plastic wrap). Investigators defined hyperthermia as a core body temperature (axillary) $>37.5^{\circ} \mathrm{C}$ on admission to the NICU.

Outcome 14.4.1: For infants at $\geq 24$ and $\leq 28$ weeks' gestation with birth weight $\leq 1250$ grams, data show no significant differences in risk of hyperthermia between infants who received thermal mattress and those given plastic wrap (RR 3.33, 95\% Cl 0.14 to 76.75 ; RD 0.06, 95\% Cl -0.09 to 0.20; one study; $\mathrm{N}=36$ ) (Analysis 14.4).

\section{Brain injury}

Two studies comprising 77 infants reported this outcome in terms of incidence of grade I or II IVH - in Simon 2011 - and grade III or N IVH - in Mathew 2012 and Simon 2011 - among intervention and comparison groups (thermal mattress and plastic wrap or bag). These data are presented separately. For the outcome incidence differences in effect for thermal mattress versus plastic wrap or bag for infants at $\leq 28$ weeks' gestation.

\section{Brain injury (IVH grade | or II ) (Outcome 14.5)} birth weight $\leq 1250$ grams, data show no significant differences in risk of IVH (grade I or II) between infants who received thermal mattress and those given plastic wrap (RR 0.45, 95\% Cl 0.10 to 2.01; $\mathrm{RD}-0.15,95 \% \mathrm{Cl}-0.40$ to 0.10 ; one study; $\mathrm{n}=36$ ) (Analysis 14.5 ).

\section{Major brain injury (IVH grade III or IV) (Outcome 14.6)}

Outcome 14.6.1: For infants at $\leq 28$ weeks' gestation, data show no significant differences in risk of IVH (grade $\mathbb{I}$ or $\mathbb{V}$ ) between infants who received thermal mattress and those given plastic wrap or bag (typical RR 0.91, 95\% Cl 0.31 to 2.71 ; typical RD $-0.01,95 \% \mathrm{Cl}-0.17$ to 0.14 ; two studies; $\mathrm{N}=77$ ) (Analysis 14.6). The test for homogeneity passed with an $\mathrm{I}^{2}$ value of $20 \%$ for RR and $19 \%$ for RD.

\section{Mortality (death before discharge) (Outcome 14.7)}

Two studies comprising 77 infants reported this outcome in terms of incidence of death before hospital discharge among intervention and comparison groups (thermal mattress and plastic wrap or bag) (Mathew 2012; Simon 2011). Each individual study showed no significant differences in effect for thermal mattress versus plastic wrap or bag for infants at $\leq 28$ weeks' gestation.

Outcome 14.7.1: For infants at $\leq 28$ weeks' gestation, data show no significant differences in risk of death before discharge between infants who received thermal mattress and those given plastic wrap or bag (typical RR $1.10,95 \% \mathrm{Cl} 0.39$ to 3.17 ; typical RD $0.01,95 \%$ $\mathrm{Cl}-0.14$ to 0.16 ; two studies; $\mathrm{N}=77$ ) (Analysis 14.7). The test for homogeneity passed with an $\mathrm{I}^{2}$ value of $0 \%$ for RR and RD.

\section{Bronchopulmonary dysplasia (BPD) (Outcome 14.8)}

One study reported this outcome in terms of incidence of BPD among intervention and comparison groups (thermal mattress and plastic wrap) (Simon 2011). Investigators did not provide a clear of grade III or IV IVH, each individual study showed no significant

Outcome 14.5.1: For infants at $\geq 24$ and $\leq 28$ weeks' gestation with

definition of BPD. This was not a prespecified outcome at the review protocol stage.

Outcome 14.8.1: For infants at $\geq 24$ and $\leq 28$ weeks' gestation with birth weight $\leq 1250$ grams, data show no significant differences in risk of BPD before discharge between infants who received thermal mattress and those given plastic wrap (RR $0.75,95 \% \mathrm{Cl} 0.40$ to 1.37 ; $\mathrm{RD}-0.16,95 \% \mathrm{Cl}-0.48$ to 0.16 ; one study; $\mathrm{N}=36$ ) (Analysis 14.8).

\section{Hypotension (Outcome 14.9)}

One study reported this outcome in terms of mean blood pressure less than gestational age in weeks during the first 24 hours of life and received treatment with fluid bolus, pressors, or both in intervention and active comparison groups (thermal mattress and plastic bag) (Mathew 2012).

Outcome 14.9.1: For infants at $\leq 28$ weeks' gestation, data show no significant differences in risk of hypotension in the first 24 hours of life between infants who received thermal mattress and those given plastic bag (RR 1.50, 95\% Cl 0.71 to 3.17 ; RD $0.17,95 \% \mathrm{Cl}-0.13$ to 0.46; one study; $\mathrm{N}=41$ ) (Analysis 14.9).

\section{Necrotising enterocolitis (NEC) (Outcome 14.10)}

Two studies comprising 77 infants reported this outcome in terms of incidence of NEC among intervention and comparison groups (thermal mattress and plastic wrap or bag) (Mathew 2012; Simon 2011). Investigators provided no clear definitions. Each individual study showed no significant differences in effect for thermal mattress and plastic wrap or bag.

Outcome 14.10.1: For infants at $\leq 28$ weeks' gestation, data show no significant differences in risk of NEC between infants who received thermal mattress and those given plastic wrap or bag (typical RR 1.92, 95\% Cl 0.61 to 5.97; typical RD $0.09,95 \% \mathrm{Cl}-0.06$ to 0.25 ; two studies; $\mathrm{N}=77$ ) (Analysis 14.10). The test for homogeneity passed with an $I^{2}$ value of $0 \%$ for RR and RD.

\section{Patent ductus arteriosus}

One study reported this outcome in terms of overall incidence of PDA among those treated with medication only and those treated with ligation in the intervention and comparison groups (thermal mattress and plastic wrap) (Simon 2011).

\section{Overall patent ductus arteriosus (PDA) (Outcome 14.11)}

Outcome 14.11.1: For infants at $\geq 24$ and $\leq 28$ weeks' gestation with birth weight $\leq 1250$ grams, data show no significant differences in risk of PDA between infants who received thermal mattress and those given plastic wrap (RR $1.76,95 \% \mathrm{Cl} 0.88$ to 3.49 ; RD $0.28,95 \%$ $\mathrm{Cl}-0.04$ to 0.59 ; one study; $\mathrm{N}=36$ ) (Analysis 14.11).

\section{Patent ductus arteriosus (PDA) treated with medication only (Outcome 14.12)}

Outcome 14.12.1: For infants at $\geq 24$ and $\leq 28$ weeks' gestation with birth weight $\leq 1250$ grams, data show no significant differences in risk of PDA treated with medication only between infants who received thermal mattress and those given plastic wrap (RR 1.44 $95 \% \mathrm{Cl} 0.69$ to 3.01 ; RD $0.16,95 \% \mathrm{Cl}-0.16$ to 0.48 ; one study; $\mathrm{N}=36$ ) (Analysis 14.12). 
Patent ductus arteriosus (PDA) treated with ligation (Outcome 14.13)

Outcome 14.13.1: For infants at $\geq 24$ and $\leq 28$ weeks' gestation with birth weight $\leq 1250$ grams, data show no significant differences in risk of PDA with ligation treatment between infants who received thermal mattress and those given plastic wrap (RR 5.56, 95\% Cl 0.29 to 108.16 ; RD $0.12,95 \% \mathrm{Cl}-0.06$ to 0.29 ; one study; $\mathrm{N}=36$ ) (Analysis 14.13).

\section{Retinopathy of prematurity (ROP)}

Simon 2011 reported this outcome in terms of overall incidence of ROP (all grades); Mathew 2012 reported incidence of ROP with laser therapy among intervention and comparison groups (thermal mattress and plastic wrap or bag). This was not a prespecified outcome at the review protocol stage.

\section{Retinopathy of prematurity (ROP) all grades (Outcome 14.14)}

Outcome 14.14.1: For infants at $\geq 24$ and $\leq 28$ weeks' gestation with birth weight $\leq 1250$ grams, data show no significant differences in risk of ROP (all grades) between infants who received thermal mattress and those given plastic wrap (RR $0.48,95 \% \mathrm{Cl} 0.15$ to 1.56 ; $\mathrm{RD}-0.19,95 \% \mathrm{Cl}-0.47$ to 0.09 ; one study; $\mathrm{N}=36$ ) (Analysis 14.14).

Retinopathy of prematurity (ROP) treated with laser therapy (Outcome 14.15)

Outcome 14.15.1: For infants at $\leq 28$ weeks' gestation, data show no significant differences in risk of ROP with laser therapy between infants who received thermal mattress and those given plastic bag (RR $0.53,95 \% \mathrm{Cl} 0.05$ to 5.35 ; RD $-0.05,95 \% \mathrm{Cl}-0.20$ to 0.11 ; one study; $\mathrm{N}=41$ ) (Analysis 14.15).

\section{Spontaneous intestinal perforation (SIP) (Outcome 14.16)}

One study reported this outcome in terms of incidence of SIP among intervention and comparison groups (thermal mattress and plastic bag) (Mathew 2012). Investigators provided no clear definition. This was not a prespecified outcome at the review protocol stage.

Outcome 14.16.1: For infants at $\leq 28$ weeks' gestation, data show no significant differences in risk of SIP between infants who received thermal mattress and those given plastic bag (RR 0.53 , $95 \% \mathrm{Cl} 0.11$ to 2.56 ; RD $-0.09,95 \% \mathrm{Cl}-0.30$ to 0.12 ; one study; $\mathrm{N}=$ 41) (Analysis 14.16).

\section{Worst base deficit in first 24 hours of life (Outcome 14.17)}

One study reported this outcome in terms of lowest $\mathrm{pH}$ and highest base deficit in the first 24 hours after birth (Mathew 2012).

Outcome 14.17.1: For infants at $\leq 28$ weeks' gestation, data show no statistically significant differences between interventions (thermal mattress and plastic bag) for worst base deficit in the first 24 hours after birth (MD $-1.70 \mathrm{mEq} / \mathrm{L}, 95 \% \mathrm{Cl}-3.99$ to 0.59 ; one study; $\mathrm{N}=41$ ) (Analysis 14.17).

\section{Worst pH in first 24 hours of life (Outcome 14.18)}

One study reported this outcome in terms of worst $\mathrm{pH}$ in the first 24 hours after birth (Mathew 2012). This was not a prespecified outcome at the review protocol stage.

Outcome 14.18.1: For infants at $\leq 28$ weeks' gestation, data show a statistically significant difference in worst $\mathrm{pH}$ in the first 24 hours after birth favouring the thermal mattress group when compared with the plastic bag group with intervention provided immediately after birth in the delivery room (MD $-0.09,95 \% \mathrm{Cl}-0.13$ to -0.05 ; one study; $\mathrm{N}=41$ ) (Analysis 14.18).

\section{Other secondary outcomes}

None of the included studies reported the following secondary outcome measures (as prespecified at the review protocol stage) for this comparison group: hypoglycaemia, respiratory distress syndrome, surfactant given at any time, intubation in the delivery room, requirement for ventilation, duration of ventilation, length of stay, chronic lung disease, or acute renal failure.

\section{Adverse occurrences due to the intervention}

Simon 2011 concluded that mattresses were easier to use than polyethylene wrap; however, economic factors may influence the decision to use one or the other, depending on financial circumstances. The polyethylene sheets used were almost seven times cheaper than the transwarming mattresses ( $\$ 2.36$ vs $\$ 14.52$ each). Staff experienced minor difficulties with the polyethylene wrap during resuscitation, which resulted in displacement and possible loss of heat. Infatherm mattresses did not interfere with resuscitation even in difficult cases.

Mathew 2012 reported that no instances occurred in which the infant received accidental burns as a result of being in contact with the thermal mattress; however, investigators expressed concerns with respect to access to the infant for monitoring and intervention in the vinyl bag group, making the thermal mattress more practical, particularly for probe placement for monitoring pulse oximetry.

None of the included studies reported the following secondary outcome measures (adverse occurrences) (as predefined at the review protocol stage) for this comparison group: events due to the intervention (e.g. maceration, skin or systemic infection, antibiotics, fluid problems, negative psychological outcomes).

\section{Plastic bag and thermal mattress versus plastic bag only (Comparison 15)}

Two studies comprising 119 infants compared plastic bag + thermal mattress versus plastic bag (Leslie 2007; McCarthy 2013). Although Leslie 2007 reported outcomes for infants at $<29$ weeks' gestation, these findings were grouped with those of the subgroup of infants at $<28$ weeks' gestation group for meta-analysis and are referred to as such in the text.

\section{Primary outcomes}

Core body temperature $\left({ }^{\circ} \mathrm{C}\right)$ of the infant taken on admission to the NICU or up to two hours after birth (Outcome 15.1)

Two studies comprising 119 infants reported this outcome in terms of core body temperature in ${ }^{\circ} \mathrm{C}$ on admission to the NICU in intervention and active comparator groups (plastic bag + thermal mattress and plastic bag only) (Leslie 2007; McCarthy 2013). Data show core body temperature in ${ }^{\circ} \mathrm{C}$ rectal - for McCarthy 2013 - and axillary - for Leslie 2007. Overall, Leslie 2007 showed no significant differences in effect for plastic bag + thermal mattress and plastic bag only for infants at $<29$ weeks' gestation, whereas McCarthy 2013 showed a significant effect in favour of the plastic bag + mattress intervention for infants at $<31$ weeks' gestation and for the subgroup at $<28$ weeks' gestation. 
Outcome 15.1.1: Overall, for infants at $<31$ weeks' gestation, data show a statistically significant difference for core body temperature on admission to the NICU favouring the plastic bag + thermal mattress intervention when compared with plastic bag only immediately after birth in the delivery room (MD $0.37^{\circ} \mathrm{C}, 95 \%$ $\mathrm{Cl} 0.09$ to 0.66 ; two studies; $\mathrm{N}=119$ ) (Analysis 15.1).

The overall test for homogeneity passed with an $\mathrm{I}^{2}$ value of $0 \%$ and for subgroup differences with an $\mathrm{I}^{2}$ value of $19.3 \%$.

Outcome 15.1.2: For infants at $<28$ weeks' gestation, data show a statistically significant difference for core body temperature on admission to the NICU favouring the plastic bag + thermal mattress intervention when compared with plastic bag only immediately after birth in the delivery room $\left(\mathrm{MD} 0.57^{\circ} \mathrm{C}, 95 \% \mathrm{Cl} 0.20\right.$ to 0.94 ; two studies; $\mathrm{N}=76$ ) (Analysis 15.1 ). The test for homogeneity passed with an $I^{2}$ value of $43 \%$ (low heterogeneity).

Outcome 15.1.3: For infants at 28 to 30 weeks' gestation, data show no statistically significant differences between interventions (plastic bag + thermal mattress and plastic bag only) for core body temperature on admission to the NICU (MD $0.10^{\circ} \mathrm{C}, 95 \% \mathrm{Cl}-0.36$ to 0.56; one study; $\mathrm{N}=43$ ) (Analysis 15.1).

\section{Hypothermia on admission to the NICU (core body temperature < $36.5^{\circ} \mathrm{C}$, or skin temperature $<36^{\circ} \mathrm{C}$ ) (Outcome 15.2)}

Leslie 2007 and McCarthy 2013, in addition to reporting core body temperature on admission to the NICU as a continuous variable, provided data in a dichotomous format in terms of incidence of hypothermia seen as core body temperature (rectal) $<36.5^{\circ} \mathrm{C}-$ in McCarthy 2013 - and hypothermia seen as core body temperature (axillary) $<36.5^{\circ} \mathrm{C}$ - in Leslie 2007 - in the intervention and comparison groups (plastic bag + thermal mattress and plastic bag only). Each individual study showed no significant differences in effect for plastic bag + thermal mattress and plastic bag only.

Outcome 15.2.1: Overall, for infants at $<31$ weeks' gestation, data show no significant differences in risk of hypothermia between infants who received plastic bag + thermal mattress and those given plastic bag only (typical RR $0.93,95 \% \mathrm{Cl} 0.45$ to 1.90 ; typical RD $-0.01,95 \% \mathrm{Cl}-0.15$ to 0.12 ; two studies; $\mathrm{N}=119$ ) (Analysis 15.2). The overall test for homogeneity failed with an $I^{2}$ value of 55\% (moderate heterogeneity) for RR and 65\% (moderate heterogeneity) for RD, but passed for subgroup differences with an $I^{2}$ value of $16.8 \%$ for RR and $37 \%$ (low heterogeneity) for RD.

Outcome 15.2.2: For infants at $<28$ weeks' gestation, data show no significant differences in risk of hypothermia between infants who received plastic bag + thermal mattress and those given plastic bag only (typical RR $0.66,95 \% \mathrm{Cl} 0.29$ to 1.46 ; typical RD $-0.10,95 \% \mathrm{Cl}$ -0.28 to 0.08 ; two studies; $N=76$ ) (Analysis 15.2). The overall test for homogeneity passed with an $\mathrm{I}^{2}$ value of $0 \%$ for RR and $14 \%$ for RD.

Outcome 15.2.3: For infants at 28 to 30 weeks' gestation, data show no significant differences in risk of hypothermia between infants who received plastic bag + thermal mattress and those given plastic bag only (RR 3.82, $95 \% \mathrm{Cl} 0.46$ to 31.43 ; RD $0.13,95 \% \mathrm{Cl}-0.05$ to 0.32 ; one study; $\mathrm{N}=43$ ) (Analysis 15.2).

\section{Outside the normothermic range on admission to the NICU or up to} two hours after birth (Outcome 15.3)

McCarthy 2013, in addition to reporting core body temperature on admission to the NICU as a continuous variable, provided data in a dichotomous format in terms of incidence of normothermia (core body temperature (rectal) $36.5^{\circ} \mathrm{C}$ to $37.5^{\circ} \mathrm{C}$ ) in intervention and comparison groups (plastic bag + thermal mattress and plastic bag only). Leslie 2007 provided data for incidence of hypothermia and hyperthermia; therefore for the purposes of meta-analysis, we defined normothermia as core body temperature (axillary) $36.5^{\circ} \mathrm{C}$ to $38^{\circ} \mathrm{C}$. We meta-analysed the incidence of core body temperature outside the defined normothermic range. Overall, Leslie 2007 showed no significant differences in effect for plastic bag + thermal mattress and plastic bag only among infants at < 29 weeks' gestation, whereas McCarthy 2013 showed a significant effect in favour of the comparator (plastic bag only) for infants at < 31 weeks' gestation, and for the subgroup at $<28$ weeks' gestation. This was not a prespecified outcome at the review protocol stage.

Outcome 15.3.1: Overall, for infants at $<31$ weeks' gestation, data show no significant differences in the risk of being outside the normothermic range between those who received plastic bag + thermal mattress and those given plastic bag only (typical RR 1.46, $95 \% \mathrm{Cl} 0.94$ to 2.27 ; typical RD $0.16,95 \% \mathrm{Cl}-0.00$ to 0.33 ; two studies; $\mathrm{N}=119$ ) (Analysis 15.3). The overall test for homogeneity failed with an $I^{2}$ value of $87 \%$ (high heterogeneity) for RR and $88 \%$ (high heterogeneity) for RD, but passed for subgroup differences with an $I^{2}$ value of $0 \%$ for RR and for RD.

Outcome 15.3.2: For infants at $<28$ weeks' gestation, data show no significant differences in the risk of being outside the normothermic range between infants who received plastic bag + thermal mattress and those given plastic bag only (typical RR $1.23,95 \% \mathrm{Cl} 0.69$ to 2.18 ; typical RD $0.08,95 \% \mathrm{Cl}-0.12$ to 0.29 ; two studies; $\mathrm{N}=76$ ) (Analysis 15.4). The test for homogeneity failed with an $\mathrm{I}^{2}$ value of $83 \%$ (high heterogeneity) for RR and 89\% (high heterogeneity) for RD.

Outcome 15.3.3: For infants at 28 to 30 weeks' gestation, data show no significant differences (borderline) in risk of being outside the normothermic range between infants who received plastic bag + thermal mattress and those given plastic bag only (RR $1.91,95 \% \mathrm{CI}$ 0.96 to 3.78 ; $\mathrm{RD} 0.30,95 \% \mathrm{Cl} 0.02$ to 0.59 ; one study; $\mathrm{N}=43$ ) (Analysis 15.3).

\section{Hyperthermia on admission to the NICU (core body temperature > $37.5^{\circ} \mathrm{C}$ ) (Outcome 15.4 )}

Leslie 2007 and McCarthy 2013, in addition to reporting core body temperature on admission to the NICU as a continuous variable, provided data in a dichotomous format in terms of incidence of hyperthermia defined as core body temperature (rectal) $>37.5^{\circ} \mathrm{C}$ by McCarthy 2013, and as core body temperature (axillary) > $38^{\circ} \mathrm{C}$ by Leslie 2007 , among intervention and comparison groups (plastic bag + thermal mattress and plastic bag only). Overall, Leslie 2007 showed no significant differences in effect for plastic bag + thermal mattress and plastic bag only among infants at $<29$ weeks' gestation, whereas McCarthy 2013 showed a significant effect in favour of the comparator (plastic bag only) for infants at $<31$ weeks' gestation.

Outcome 15.4.1: Overall, for infants at $<31$ weeks' gestation, plastic bag + thermal mattress significantly increased risk of hyperthermia on admission to the NICU when compared with plastic bag only (typical RR $2.15,95 \% \mathrm{Cl} 1.07$ to 4.32; typical RD $0.18,95 \% \mathrm{Cl} 0.03$ to 0.32 ; two studies; $\mathrm{N}=119$ ) (Analysis 15.4). For every six infants receiving plastic bag and thermal mattress, one infant would be hyperthermic on admission to the NICU (NNTH 
$6,95 \% \mathrm{Cl} 3$ to 33). The overall test for homogeneity passed for RR with an $I^{2}$ value of $16 \%$, and for subgroup differences with an $I^{2}$ value of $0 \%$ for both RR and RD, but failed for RD with an $I^{2}$ value of $76 \%$ (moderate heterogeneity). This may be attributable to differences in the definition of hyperthermia, which requires a higher temperature in Leslie $2007\left(>38^{\circ} \mathrm{C}\right)$.

Outcome 15.4.2: For infants at $<28$ weeks' gestation, data show no significant differences in risk of hyperthermia between infants who received plastic bag + thermal mattress and those given plastic bag only (typical RR $2.99,95 \% \mathrm{Cl} 0.93$ to 9.65; typical RD $0.18,95 \% \mathrm{Cl} 0.02$ to 0.34 ; two studies; $\mathrm{N}=76$ ) (Analysis 15.4). The test for homogeneity failed for RR with an $\mathrm{I}^{2}$ value of $68 \%$ (moderate heterogeneity), and for RD with an $\mathrm{I}^{2}$ value of $87 \%$ (high heterogeneity).

Note: Computational errors can occur when one or both arms of a study have a zero count. RevMan software compensates by adding 0.5 to each cell in a two-by-two table.

Outcome 15.4.3: For infants at 28 to 30 weeks' gestation, data show no significant differences in risk of hyperthermia between infants who received plastic bag + thermal mattress and those given plastic bag only (RR 1.59, $95 \% \mathrm{Cl} 0.70$ to $3.60 ; \mathrm{RD} 0.17,95 \% \mathrm{Cl}-0.12$ to 0.45 ; one study; $\mathrm{N}=43$ ) (Analysis 15.4).

\section{Major brain injury: abnormal cranial ultrasonography (Outcome 15.5)}

One study reported this outcome in terms of incidence of abnormal cranial ultrasonography (IVH $\geq$ grade $\mathbb{I}$, periventricular leukomalacia, or post-haemorrhagic hydrocephalus) in the intervention and comparison groups (plastic bag + thermal mattress and plastic bag only) (McCarthy 2013). This was not a prespecified outcome at the review protocol stage.

Outcome 15.5.1: For infants at $<31$ weeks' gestation, data show no significant differences in risk of abnormal cranial ultrasonography between infants who received plastic bag + thermal mattress and those given plastic bag only (RR $0.32,95 \% \mathrm{Cl} 0.03$ to 2.89 ; RD -0.06 , $95 \% \mathrm{Cl}-0.17$ to 0.05 ; one study; $\mathrm{N}=72$ ).

\section{Mortality (death within hospital stay) (Outcome 15.6)}

Two studies comprising 119 infants reported this outcome in terms of incidence of death before hospital discharge in intervention and comparison groups (plastic bag + thermal mattress and plastic bag only) (Leslie 2007; McCarthy 2013). Each individual study showed no significant differences in effect for plastic bag + thermal mattress and plastic bag only among infants at $<31$ weeks' gestation.

Outcome 15.6.1: For infants at $<31$ weeks' gestation, data show no significant differences in risk of death before hospital discharge between infants who received plastic bag + thermal mattress and those given plastic bag only (RR $0.73,95 \% \mathrm{Cl} 0.32$ to 1.66 ; RD -0.05 , $95 \% \mathrm{Cl}-0.18$ to 0.08 ; two studies; $\mathrm{N}=119$ ). The test for homogeneity passed with an $\mathrm{I}^{2}$ value of $0 \%$ for RR and RD.

\section{Chronic lung disease (Outcome 15.7)}

One study reported this outcome in terms of the incidence of requirement for oxygen supplementation on the date of 36 weeks' adjusted gestation in intervention and comparison groups (plastic bag + thermal mattress and plastic bag only) (McCarthy 2013).
Outcome 15.7.1: For infants at < 31 weeks' gestation, data show no significant differences in risk of chronic lung disease between infants who received plastic bag + thermal mattress and those given plastic bag only (RR 1.73, 95\% $\mathrm{Cl} 0.72$ to 4.18 ; RD $0.13,95 \% \mathrm{Cl}-0.07$ to 0.32 ; one study; $\mathrm{N}=72$ ).

\section{Coagulation support (Outcome 15.8)}

One study reported this outcome in terms of incidence of the use of platelets or fresh frozen plasma or cryoprecipitate in intervention and comparison groups (plastic bag + thermal mattress and plastic bag only) (Leslie 2007). This was not a prespecified outcome at the review protocol stage.

Outcome 15.8.1: For infants at $<29$ weeks' gestation, data show no significant differences in risk of coagulation support between infants who received plastic bag + thermal mattress and those given plastic bag only (RR 1.04, 95\% Cl 0.30 to 3.69; RD $0.01,95 \% \mathrm{Cl}-0.21$ to 0.22 ; one study; $\mathrm{N}=47$ ).

\section{Inotrope use (Outcome 15.9)}

Two studies comprising 119 infants reported this outcome in terms of incidence of inotrope use (dopamine or dobutamine or hydrocortisone) in intervention and comparison groups (plastic bag + thermal mattress and plastic bag only) (Leslie 2007; McCarthy 2013). This was not a prespecified outcome at the review protocol stage. Each individual study showed no significant difference in effect for the plastic bag + thermal mattress and plastic bag only among infants at $<31$ weeks' gestation.

Outcome 15.9.1: For infants at $<31$ weeks' gestation, data show no significant differences in risk of Inotrope use between infants who received plastic bag + thermal mattress and those given plastic bag only (RR $1.14,95 \% \mathrm{Cl} 0.61$ to $2.11 ; \mathrm{RD} 0.03,95 \% \mathrm{Cl}-0.12$ to 0.19 ; two studies; $\mathrm{N}=119)$. The test for homogeneity passed with an $\mathrm{I}^{2}$ value of $0 \%$ for RR and RD.

\section{Intubated during admission (Outcome 15.10)}

One study reported this outcome in terms of incidence of intubation during admission in the intervention and comparison groups (plastic bag + thermal mattress and plastic bag only) (McCarthy 2013). This was not a prespecified outcome at the review protocol stage.

Outcome 15.10.1: For infants at $<31$ weeks' gestation, data show no significant differences in risk of intubation during admission between infants who received plastic bag + thermal mattress and those given plastic bag only (RR $0.98,95 \% \mathrm{Cl} 0.73$ to 1.32 ; RD -0.01 , $95 \% \mathrm{Cl}-0.22$ to 0.20 ; one study; $\mathrm{N}=72$ ).

\section{Greater than or equal to one dose of surfactant during admission (Outcome 15.11)}

One study reported this outcome in terms of incidence of requirement for one or more doses of surfactant during admission in intervention and comparison groups (plastic bag + thermal mattress and plastic bag only) (McCarthy 2013). This was not a prespecified outcome at the review protocol stage.

Outcome 15.11.1: For infants at $<31$ weeks' gestation, data show no significant differences in risk of receiving one or more doses of surfactant during admission between infants who received plastic bag + thermal mattress and those given plastic bag only (RR 0.99 , $95 \% \mathrm{Cl} 0.70$ to 1.38 ; RD $-0.01,95 \% \mathrm{Cl}-0.23$ to 0.21 ; one study; $\mathrm{N}=72$ ). 


\section{Other secondary outcomes}

None of the included studies reported the following secondary outcome measures (as predefined at the review protocol stage) for this comparison group: hypoglycaemia, respiratory distress syndrome, surfactant given at any time, intubation in the delivery room, requirement for ventilation, duration of ventilation, length of stay, severe metabolic acidosis, PDA, NEC, or acute renal failure.

\section{Adverse occurrences due to the intervention}

McCarthy 2013 reported that wrapping in plastic bags did not present many problems for the neonatal team, and that once infants were placed in plastic bags and the bags were sealed, they remained sealed until arrival to the neonatal unit. Pulse oximeters were used in all cases, and the infant's heart rate was auscultated through the bag with no difficulty.

None of the included studies reported the following secondary outcome measures (adverse occurrences) (as predefined at the review protocol stage) for this comparison group: burns, maceration, antibiotics, fluid problems, or negative psychological outcomes.

\section{DISCUSSION}

Hypothermia (body temperature below normal) on admission to neonatal units continues to be a persistent problem worldwide across all climates. "A naked newborn exposed to an environmental temperature of $23^{\circ} \mathrm{C}$ suffers the same heat loss as a naked adult at $0^{\circ} \mathrm{C}^{\prime \prime}$ (WHO 1997), and this is negatively correlated with gestation and birth weight. Therefore, early intervention in the delivery room is crucial for minimisation of heat loss during resuscitation, stabilisation, and transport to the neonatal intensive care unit (NICU), particularly for the least mature, smallest, and sickest infants. National and international agencies responsible for development of neonatal resuscitation guidelines face a challenging role while research evidence is emerging/not yet conclusive, but long-term safety data are not yet available. In 2006, the ILCOR 2006 consensus statement first recommended that plastic bags or plastic wrapping under radiant heat should be considered as a standard technique to maintain temperature. Since that time, recommendations have continued to evolve as research evidence has become available, and these thermal care practices have been adopted to varying degrees (El-Naggar 2012; Godhamgaonkar 2011; Smith 2016). Consequently, for this review update, we have expanded our inclusion criteria to reflect these changes in clinical practice. We remain focussed on interventions to prevent hypothermia applied immediately at birth apart from 'routine' care (as defined in our original protocol) but have also included randomised and quasi-randomised trials comparing any other single/combination of intervention(s) designed for prevention of hypothermia in preterm and/or low birth weight infants and applied within 10 minutes after birth in the delivery room that otherwise would have been excluded.

\section{Summary of main results}

Twenty-five studies across 15 comparison groups met the inclusion criteria (2481 randomly assigned participants; 2433 participants completing the studies). Interventions were categorised into three main groups: barriers to heat loss (18 studies - 11 comparison groups), external heat sources (three studies - two comparison groups), and combinations of interventions (four studies - two comparison groups). We judged the quality of evidence for the main comparison group (plastic wraps or bags vs routine care) to be moderate across key clinical outcomes. For the remaining comparison groups, we derived evidence from one or two studies with small sample sizes, thereby limiting our ability to draw firm conclusions.

\section{Barriers to heat loss category}

Core body temperature $\left({ }^{\circ} \mathrm{C}\right)$ on admission to the NICU or up to two hours after birth (plastic wraps or bags vs routine care): Thirteen studies comprising 1633 infants reported core body temperature in ${ }^{\circ} \mathrm{C}$ (rectal or axillary) on admission to the NICU or up to two hours after birth (Cardona Torres 2012; Chantaroj 2011; Farhadi 2012; Gathwala 2010; Knobel 2005; Leadford 2013; Reilly 2015; Rohana 2011; Smith 2013; Talakoub 2015; Trevisanuto 2010; Vohra 1999; Vohra 2004). Overall, plastic wraps or bags were effective in reducing heat loss for infants at $<37$ weeks' gestation when compared with infants who received routine care immediately after birth in the delivery room. Each individual study showed a significant effect in favour of the intervention for core body temperature on admission to the NICU or up to two hours after birth across gestations, with the exception of a small study that showed no significant effect for the subgroup of infants at $\geq$ 28 and $\leq 31$ weeks' gestation (Vohra 1999). Findings indicate that the effect is greater among the most immature infants. Data show a substantial level of heterogeneity within each subgroup of infants. Possible causes may lie within variations in methods used across studies, particularly with respect to "routine care", or in baseline hypothermia rates, as previously discussed. Heat loss prevention observed, despite these variations in how the standard of care was implemented between sites, demonstrates that findings are robust and generalisable across centres. A funnel plot analysis for core body temperature on admission to the NICU suggests possible publication bias with respect to smaller non-effect studies that have not been published. Plastic wraps or bags seem to prevent rather than delay the postnatal fall in temperature with significant effects in favour of plastic wraps and bags at 1 hour, 90 minutes, and 2 hours after birth; at post stabilisation; and at 30 minutes, 1 hour, 90 minutes, and 2 hours after the initial NICU admission temperature was taken.

Hypothermia on admission to NICU or up to two hours after birth (plastic wraps or bags vs routine care): Ten studies comprising 1417 infants reported the incidence of hypothermia in intervention and control groups (plastic wrap or bag and routine care) (Cardona Torres 2012; Chantaroj 2011; Farhadi 2012; Knobel 2005; Leadford 2013; Reilly 2015; Rohana 2011; Trevisanuto 2010; Vohra 1999; Vohra 2004). Overall, plastic wraps or bags were effective in reducing risk of hypothermia for all infants at $<37$ weeks' gestation when compared with those given routine care immediately after birth in the delivery room. Four infants would have to be wrapped in plastic to prevent one infant from becoming hypothermic on admission to the NICU. Most individual studies showed a significant effect in favour of the intervention (plastic wrap or bag), with the exception of Chantaroj 2011, which reported no differences in effect among infants at $<28$ weeks' gestation; however few infants were included in this subgroup. The small Vohra 1999 and Cardona Torres 2012 studies showed borderline significant effect in favour of the plastic wrap or bag group. Reductions in risk of hypothermia varied across gestational age groups, with $33 \%$ overall, $30 \%$ for infants at $<28$ weeks' gestation, and $83 \%$ for infants at 28 to 32 weeks' gestation. 
Hyperthermia on admission to NICU or up to two hours after birth (plastic wraps or bags vs routine care): Twelve studies comprising 1523 infants reported the incidence of hyperthermia in intervention and control groups (plastic wrap or bag and routine care) (Cardona Torres 2012; Chantaroj 2011; Farhadi 2012; Gathwala 2010; Knobel 2005; Leadford 2013; Reilly 2015; Smith 2013; Talakoub 2015; Trevisanuto 2010; Vohra 2004; Vohra 1999). Each individual study showed no significant differences in risk of hyperthermia, with the exception of the large pragmatic 'real-world' multi-centre study, which showed that plastic wrap significantly increased the risk of being hyperthermic (core body temperature $\geq 37.5^{\circ} \mathrm{C}$ ) on admission to the NICU and at post stabilisation for infants at $\geq 24$ and $<28$ weeks' gestation (Reilly 2015). Overall, plastic wraps or bags significantly increased the risk of hyperthermia on admission to the NICU for infants at $<37$ weeks' gestation when compared with those given routine care, with highest risk seen among infants at $<28$ weeks' gestation. Twenty-five infants would have to be wrapped in plastic to have one infant hyperthermic on admission to the NICU. Five studies (Chantaroj 2011; Gathwala 2010; Leadford 2013; Talakoub 2015; Vohra 1999) included in the meta-analysis reported no occurrence of hyperthermia in either group, and one further study reported that two infants in the wrap group were hyperthermic within the first 12 hours of life (Rohana 2011). Reilly 2015 reported that the risk of hyperthermia did not appear to be related to delivery room temperature.

Core body temperature outside the normothermic range on admission to the NICU or up to two hours after birth (plastic wraps or bags vs routine care): To balance reduced risk of hypothermia with increased risk of hyperthermia, we metaanalysed data for the outcome measure 'incidence of core body temperature outside normothermic range on admission to NICU or up to two hours after birth'. This outcome was reported by or was derived from definitions of hypothermia and hyperthermia provided by five studies comprising 1048 infants (Chantaroj 2011; Farhadi 2012; Leadford 2013; Reilly 2015; Trevisanuto 2010). Investigators defined normothermia as core body temperature (rectal or axillary) $36.5^{\circ} \mathrm{C}$ to $37.5^{\circ} \mathrm{C}$ (Chantaroj 2011; Farhadi 2012); core body temperature (axillary) $36.5^{\circ} \mathrm{C}$ to $37.4^{\circ} \mathrm{C}$ (baseline) (Reilly 2015); core body temperature (axillary) $36.4^{\circ} \mathrm{C}$ to $37.5^{\circ} \mathrm{C}$ (Trevisanuto 2010); and core body temperature (axillary) $36.5^{\circ} \mathrm{C}$ to $37.5^{\circ} \mathrm{C}$ at one hour after birth (Leadford 2013). Most individual studies showed a significant effect in favour of the intervention (plastic wrap or bag group), with the exception of Chantaroj 2011, which showed no differences in effect among infants at $<28$ weeks' gestation; however the number of infants in this subgroup was very small. Trevisanuto 2010 showed a borderline significant effect in favour of the plastic wrap or bag group for infants at $<29$ weeks' gestation. Overall, for infants at $<37$ weeks' gestation, plastic wraps or bags significantly reduced the risk of having a core body temperature outside the normothermic range on admission to the NICU. Overall, five infants would have to be wrapped in plastic to prevent one infant from having a core body temperature outside the normothermic range, increasing to seven for infants at $<28$ weeks' gestation.

Mortality - death within hospital stay or at six months' corrected gestation (plastic wraps or bags vs routine care): Ten studies comprising 1447 infants provided insufficient evidence to suggest that plastic wraps or bags significantly reduce risk of death during hospital stay (or at six months' corrected age) for all gestations.

Morbidity (plastic wraps or bags vs routine care): For infants at $\geq 24$ and $<28$ weeks' gestation, plastic wraps or bags significantly reduced the risk of pulmonary haemorrhage during hospital stay. Data show no evidence of a significant difference for any of the following reported secondary outcomes: incidence of: brain injury (major brain injury), bronchopulmonary dysplasia (BPD), gastrointestinal perforation, intubation in the delivery room, steroids for BPD, necrotising enterocolitis (NEC), patent ductus arteriosus (PDA), pneumothorax, requirement for bubble continuous positive airway pressure (CPAP) or ventilation, respiratory distress syndrome, ROP, and sepsis (late or early), nor for arterial oxygen saturation, bicarbonate, first blood gas $\mathrm{pH}$, blood glucose concentration, median or mean duration of hospitalisation, median duration of CPAP, mean duration of oxygen therapy, and median duration of ventilation.

Emerging evidence based on two or fewer studies (plastic wraps or bags or caps vs routine care): Plastic wraps were also effective in reducing heat losses (without an increase in risk of hyperthermia) on admission to the NICU for infants at $<37$ weeks' gestation with birth weight $\leq 2500$ grams undergoing interhospital transport from multiple maternity homes and hospitals to a tertiary care regional referral NICU when compared with those given routine care (one study comprising 96 infants; Bhavsar 2015). Four infants would have to be wrapped in plastic to prevent one from becoming hypothermic. Plastic wraps were also effective in reducing the risk of hypoglycaemia within two hours of birth when compared with routine care (Bhavsar 2015). Data show no significant differences in the following reported secondary outcomes: base excess, blood gas $\mathrm{pH}$, mean duration of oxygen therapy, blood glucose level, and severe metabolic acidosis.

One study comprising 60 infants compared plastic bag with previous drying versus routine care as opposed to most of the studies in Comparison 1 (plastic wrap or bag vs routine care), in which infants were placed in plastic wraps or bags while still wet (Cardona Torres 2012). For infants at $\geq 28$ and $<37$ weeks' gestation with birth weight $\geq 1000$ and $\leq 2499$ grams, data show significant effects favouring the plastic wrap or bag with previous drying at 30 minutes, 1 hour, 90 minutes, and 2 hours after birth. No infants were hyperthermic in the plastic wrap or bag with previous drying groups nor among those receiving routine care. Data show no significant differences in any of the reported secondary outcomes: median glucose concentration at 120 minutes after birth, heart rate, respiratory rate, or blood pressure.

Plastic caps were also effective in reducing heat losses for the younger group of infants at $<29$ weeks' gestation compared with routine care (one study comprising 64 infants; Trevisanuto 2010). Infants in the routine care group were placed in prewarmed towels after drying (no head covering). Two infants would have to wear a plastic cap to prevent one infant from becoming hypothermic, and to prevent one infant from having a core body temperature outside the normothermic range (core body temperature $36.4^{\circ} \mathrm{C}$ to $37.5^{\circ} \mathrm{C}$ ) on admission to the NICU. No infants had hyperthermia in the plastic cap group nor among those receiving routine care. Data show no significant differences in reported secondary outcomes: major brain injury, mortality, bicarbonate, blood gas $\mathrm{pH}$, first serum glucose concentration, and intubation in the delivery room. 
Two studies comprising 122 infants- Talakoub 2015; Tescon-delos Santos 2012 - compared plastic bags + plastic caps versus routine care for infants at $\geq 28$ and $\leq 32$ weeks' gestation (Talakoub 2015), nor for infants at $\geq 28$ and $\leq 36$ weeks' gestation (Tescon-delos Santos 2012). Overall, the combination of plastic bags + plastic caps was effective in reducing heat losses on admission to the NICU compared with routine care. Heterogeneity was high owing to differences in gestational age across studies. The combination of plastic bags + plastic caps was not effective in reducing heat losses for more mature infants at $\geq 28$ and $\leq 36$ weeks' gestation up to one hour after the initial NICU admission temperature was taken (Tescon-delos Santos 2012), but this combination was effective for less mature infants at one and two hours after the initial NICU admission temperature was taken (Talakoub 2015). Data show no significant differences in risk of hyperthermia for infants at $\geq 28$ and $\leq 36$ weeks' gestation on admission to the NICU (Tescondelos Santos 2012). Researchers provided limited reporting of prespecified secondary outcomes for this comparison group and no evidence of a significant difference for any of the reported secondary outcome measures - hyponatraemia or weight (grams) on the fifth day of life - nor for adverse occurrences.

Emerging evidence based on two or fewer studies (stockinette cap vs routine care): When the barrier to heat loss was a stockinette cap (one study comprising 40 infants; Roberts 1981), data show a borderline statistically significant difference in temperature on admission to the NICU in favour of the intervention group for infants at $\geq 32$ and $\leq 36$ weeks' gestation with a birth weight $<2000$ grams, but no difference for infants at $\geq 2000$ grams. This finding is consistent with those reported by Greer 1988, which compared various head coverings under radiant warmers for infants $>2500$ grams. When head coverings were applied within one minute of birth, infants wearing stockinettes had lower mean core body temperature at 5,15 , and 30 minutes after delivery as compared with the hatless group or the group wearing an insulated fabric bonnet. As a result, researchers did not recommend stockinettes for use in conjunction with a radiant warmer (Greer 1988).

Emerging evidence based on two or fewer studies (barrier vs barrier): Plastic bags with previous drying were shown to be comparable to plastic bags without previous drying for infants at $\geq$ 28 and $<37$ weeks' gestation with birth weight $\geq 1000$ and $\leq 2499$ grams (one study comprising 60 infants; Cardona Torres 2012). Data show no evidence of a significant difference for any of the reported secondary outcomes of hyperthermia or glucose concentration at 120 minutes of life, heart rate, respiratory rate, or blood pressure.

When plastic caps were compared with plastic wraps (no cap) for infants at < 29 weeks' gestation (one study comprising 64 infants; Trevisanuto 2010), data show no significant differences between the two interventions for core body temperature on admission to the NICU and a borderline significant difference at one hour after the initial admission temperature was taken. However, use of oximetry signified opening of the bag during placement of the sensor, which could have reduced the temperature protective effect. Data show no significant differences for reported secondary outcomes: hyperthermia on admission to the NICU, major brain injury, mortality, bicarbonate concentration, blood gas $\mathrm{pH}$, first serum glucose concentration, and intubation at birth.

Caglar 2014 (one study comprising 59 infants) demonstrated that plastic bags were more effective in reducing heat losses than plastic wraps at 20 minutes, 40 minutes, and 1 hour after birth among infants at $\leq 32$ weeks' gestation. However, data show no significant differences between the two interventions in risk of hypothermia within one hour after birth. Investigators reported no additional secondary outcomes.

Doglioni 2014 (one study comprising 100 infants) compared plastic total body wraps (body + head) versus plastic body wraps (head uncovered) for infants at $<29$ weeks' gestation. Data show no significant differences between the two interventions for core body temperature on admission to the NICU and at one hour after admission. Investigators reported no significant differences in reported secondary outcomes: hyperthermia, mortality (death before discharge), major brain injury, BPD, and NEC.

For infants at $\geq 28$ and $\leq 32$ weeks' gestation, the combination of plastic bags + plastic hats was shown to be more effective in reducing heat losses on admission to the NICU and at one and two hours after admission when compared with plastic wraps + cotton hats (one study comprising 64 infants; Talakoub 2015). No hyperthermic infants were included in either group. Investigators reported no additional secondary outcomes. A recent randomised controlled trial (RCT) conducted in Malaysia compared the polyethylene cap 'NeoCap' versus a cotton cap as an adjunct to polyethylene occlusive body wrap (without drying) for 80 infants at 24 to 34 weeks' gestation (Shafie 2017). This study reported no significant differences in mean NICU admission (axillary) temperatures $\left(35.3^{\circ} \mathrm{C}\right.$ vs $\left.35.1^{\circ} \mathrm{C} ; \mathrm{P}=0.36\right)$; however, mean poststabilisation temperature (after respiratory support, peripheral lines, and cardiorespiratory monitor probes had been secured) was significantly higher in the NeoCap group $\left(36.0^{\circ} \mathrm{C}\right.$ vs $35.5^{\circ} \mathrm{C}$; $P=0.01$ ). Rates of admission hypothermia were high in both groups (axillary temperature $<36.5^{\circ} \mathrm{C}$ ) at $92.5 \%$ (NeoCap group) versus $100 \%$ (control group). The mean ambient delivery room temperature was $23.3^{\circ} \mathrm{C}$ throughout this study and may have been a contributing factor.

\section{External heat sources category}

Emerging evidence based on two or fewer studies (skin-to-skin care (SSC) vs routine care): SSC was shown to be effective in reducing risk of hypothermia when compared with conventional incubator care for infants with birth weight $\geq 1200$ and $\leq 2199$ grams (one study comprising 126 infants; Bergman 2004). Two infants would have to receive SSC to prevent one infant from becoming hypothermic (skin temperature $<35.5^{\circ} \mathrm{C}$ ). No evidence showed a significant difference for the reported secondary outcome hypoglycaemia.

Emerging evidence based on two or fewer studies (thermal mattress vs routine care): Two studies comprising 126 infants compared thermal mattress versus routine care (Brennan 1996; Chawla 2011). Thermal (transwarmer) mattress significantly kept infants $\leq 1500$ grams warmer and reduced the incidence of hypothermia on admission to the NICU with no significant difference in risk of hyperthermia. Three infants would have to receive a thermal mattress to prevent one infant from being hypothermic (core body temperature $<36^{\circ} \mathrm{C}$ ) on admission to the NICU. Thermal (transwarmer) mattress also significantly reduced risk of a core body temperature outside the normothermic range $\left(36.5^{\circ} \mathrm{C}\right.$ to $\left.37.5^{\circ} \mathrm{C}\right)$ on admission to the NICU. Two infants would have to receive a thermal mattress to prevent one infant from having a temperature outside the normothermic range. Data show 
a high level of heterogeneity between studies in this comparison group; the most likely reason for this is that the two studies used different methods. Chawla 2011 used additional thermal care measures (plastic bag without drying below the neck) for infants at $<28$ weeks' gestation; this was considered to be part of routine thermal care practices. However, Brennan 1996 - the earlier study - did not employ such additional thermal care measures. Data show no evidence of a significant difference for any of the reported secondary outcomes: mortality, major brain injury, BPD, mean duration of: hospital stay, ventilation or oxygen requirement, incidence of hypoglycaemia, metabolic acidosis, NEC, or sepsis.

\section{Combination of interventions category}

Emerging evidence based on two or fewer studies (thermal mattress vs plastic wraps or bags): Two studies comprising 77 infants compared thermal mattresses versus plastic wraps or bags for infants at $\leq 28$ weeks' gestation. Each individual study showed no significant difference in effect for core body temperature, incidence of hypothermia, hyperthermia, and core body temperature outside the normothermic range on admission to the NICU. Mathew 2012 tended towards favouring plastic bags, whereas Simon 2011 showed borderline significance favouring thermal mattresses for core body temperature on admission to the NICU. The most plausible reason for the observed moderate heterogeneity lies with Simon 2011, in which variability of use between practitioners when the intervention was polyethylene wrap and, in some cases, displacement of the wrap could have resulted in heat loss. Data show no significant differences for reported secondary outcomes: brain injury, major brain injury, mortality, BPD, hypotension, NEC, PDA, ROP, spontaneous intestinal perforation, worst base deficit, and worst $\mathrm{pH}$ in the first 24 hours of life.

Emerging evidence based on two or fewer studies (plastic bags and thermal mattresses vs plastic bags): Two studies comprising 119 infants compared plastic bag + thermal mattress versus plastic bag (Leslie 2007; McCarthy 2013). Investigators reported that plastic bags in combination with thermal mattresses were shown to keep infants warmer on admission to the NICU when compared with plastic bags alone for infants at $<31$ weeks' gestation (Leslie 2007; McCarthy 2013), and greater effect size was seen for infants at < 28 weeks' gestation. However, overall, data show no significant difference in risk of hypothermia (core body temperature $<36.5^{\circ} \mathrm{C}$ ) but an overall significant increased risk of hyperthermia associated with the simultaneous combination of plastic bag and thermal mattress. For every six infants receiving plastic bag + thermal mattress, one infant would be hyperthermic on admission to the NICU for infants at $<31$ weeks' gestation. Researchers found no significant difference in risk of having a core body temperature outside the normothermic range on admission to the NICU nor for reported secondary outcomes: major brain injury, mortality, chronic lung disease, coagulation support, inotrope use, intubation during admission, and one or more doses of surfactant during admission.

\section{Overall for all included interventions designed to prevent heat loss at birth}

Despite variation in interventions applied; definitions of 'routine care' and definitions of hypothermia, hyperthermia, and normothermia; and groups of infants included, a similar pattern emerged across all studies, with infants in the intervention group significantly warmer (or showing a non-significant trend in that direction) than infants receiving 'routine care'. Evidence of moderate quality shows that plastic wraps or bags keep preterm infants warmer and lead to higher temperatures on admission to neonatal units with less hypothermia and temperatures outside the range of normothermia. Extremely preterm infants ( $<28$ weeks' gestation) appeared to benefit the most. Other interventions such as thermal mattresses, skin-to-skin care, and plastic caps also reduce hypothermia risk when compared with routine care, but these findings are based on two or fewer studies with small sample sizes. One small study found that stockinette caps were not more effective when compared with routine care in infants at 32 to 36 weeks' gestation.

Hyperthermia in the newborn may be caused by intrapartum maternal fever or, as our evidence suggests, by use of interventions to reduce hypothermia in the delivery room. Animal and human studies suggest that hyperthermia is harmful to the newborn brain. In infants at $\leq 6$ hours' postnatal age and at $\geq 36$ weeks' gestation with moderate or severe hypoxic-ischaemic encephalopathy, elevated temperatures were associated with increased odds of death or moderate/severe disability at 18 to 22 months on secondary data analysis of the NICHD whole body cooling trial (Laptook 2008a). Lyu 2015, in a retrospective observational study of 9833 preterm infants at $<33$ weeks' gestation in the Canadian Neonatal Network, demonstrated a U-shaped relationship between admission temperature and poor clinical outcome. The lowest rates of adverse outcomes were associated with temperatures between $36.5^{\circ} \mathrm{C}$ and $37.2^{\circ} \mathrm{C}$. Hyperthermia has also been shown to worsen ventilator-induced lung injury and inflammation in preterm lambs (Ball 2010). Therefore, iatrogenic hyperthermia should be avoided, particularly in infants at risk of neurological injury. Caution must be taken to avert iatrogenic hyperthermia, particularly when multiple interventions are used simultaneously immediately after birth in the delivery room (e.g. plastic bags + thermal mattresses).

Many observational studies (as discussed in the Background section) have demonstrated increased mortality among preterm hypothermic infants compared with those who maintain normothermia, yet evidence is insufficient to suggest that interventions to prevent hypothermia reduce risk of in-hospital mortality across all comparison groups included in this review. In addition, researchers have provided limited evidence of benefit and no evidence of harm for most short-term morbidity outcomes known to be associated with being hypothermic, including major brain injury, BPD, ROP, NEC, and nosocomial infection. Limitations include lack of power to detect effects of these interventions on morbidity and mortality across most comparison groups. Data show significant differences for only two morbidity outcomes. For infants at $\geq 24$ and $<28$ weeks' gestation, plastic wraps or bags significantly reduced risk of pulmonary haemorrhage during hospital stay when compared with routine care (moderate-quality evidence). Plastic wraps were also more effective in reducing risk of hypoglycaemia within two hours of birth when compared with routine care for infants at $<37$ weeks' gestation with birth weight $\leq 2500$ grams undergoing interhospital transport in one small study. Further investigation is needed to explore the pathways from admission hypothermia to mortality, and to ascertain whether hypothermia is a step in the causal pathway (Laptook 2008), possibly via late sepsis (Laptook 2007); alternatively, hypothermia may be a marker for illness and poorer outcome by association rather than for causality. 
No reported adverse events across all studies, such as skin maceration or infection, were attributable to the intervention, nor did plastic bags, plastic caps, skin-to-skin care, or thermal mattresses interfere with resuscitation practices. However, mixed reports have discussed plastic wraps with regard to interference with resuscitation and placing of umbilical lines and probes. Problems appear to be related to displacement of plastic wraps.

Although the interventions studied in this review offer some shortterm benefit regarding heat loss prevention in vulnerable preterm and/or low birth weight infants, their effect on morbidity and their long-term safety remain largely unknown; however, the harmful effects of hypothermia are well documented, and the findings of this review demonstrate that hypothermia can be prevented. Further information on effects of plastic wraps on long-term neurodevelopmental outcomes will become available upon full publication of follow-up data from the Heat Loss Prevention (HeLP) study (Reilly 2015). Therefore, monitoring (for both benefits and risks of potential adverse events) should continue in neonatal units where such interventions are adopted as routine practice, because wide variation in clinical practice has been reported. In addition, clarification of 'normal' temperatures for these populations of infants is essential, as are better data correlating axillary versus rectal versus other temperature-taking sites. The multi-centre HeLP study will yield important prospective data on 'normal' temperatures in this population, including correlation of axillary and rectal temperatures.

The 2015 neonatal resuscitation guidance provided by the American Heart Association (AHA), the European Resuscitation Council (ERC), and the International Liaison Committee on Resuscitation (ILCOR) states that for all gestational ages, admission temperature of newly born, non-asphyxiated infants is a strong predictor of mortality and morbidity and should be recorded as an outcome predictor and as a key quality indicator (Perlman 2015). Fastman 2014 conducted a small qualitative study in the USA to explore clinicians' perspectives on barriers to management of hypothermia among low birth weight infants in the delivery room, during transport, and upon arrival to the NICU. A multidisciplinary team approach to neonatal resuscitation, thermal care, and overall delivery room management, coupled with integration of temperature monitoring into quality and safety programmes, was considered an important step in addressing this long-standing problem. Paradoxically, hypothermia is a greater problem in lowerincome countries, where climates are generally warmer. Four studies were conducted in lower-middle-income countries: India (Bhavsar 2015; Gathwala 2010), Zambia (Leadford 2013), and the Phillippines (Tescon-delos Santos 2012); and seven in uppermiddle-income countries: South Africa (Bergman 2004), Mexico (Cardona Torres 2012), Thailand (Chantaroj 2011), Turkey (Caglar 2014), Iran (Farhadi 2012; Talakoub 2015), and Malaysia (Rohana 2011). Plastic wraps (food grade polyethylene, clean but not sterile) used in the HeLP study cost approximately CAD 157 for 5000 wraps, which, when calculated per infant, equates to approximately (USD) 3 cents each (Reilly 2015). Therefore, these may be an affordable option for preterm infants in low-resource settings. Another low-cost option would be skin-to-skin care; however, only one study fulfilled our inclusion criteria, because in most studies, participants were term infants, or skin-to-skin care was not initiated immediately at birth (Bergman 2004).

\section{Overall completeness and applicability of evidence}

All included studies fulfilled our strict PICO (population, intervention, control, and outcomes) criteria. The primary outcome in one included trial was all-cause mortality; all other studies were powered for our primary outcome of temperature on admission to the NICU or for hypothermia. Investigators applied all interventions immediately after birth in the delivery room. Participants were categorised by gestation (all preterm) in 17 studies, by birth weight (all low birth weight) in two studies, and by both gestation and birth weight inclusion criteria in six studies. Four studies were conducted in lower-middle-income countries and seven in uppermiddle-income countries; all other studies took place in highincome countries. Available information showed that all studies used a combination of external heat sources (radiant warmer/ drop light, warmer table, transport incubator) and caps as part of routine thermal care (control group and/or intervention group). In two studies, researchers attempted to keep ambient delivery room temperature at $26^{\circ} \mathrm{C}$ for preterm births. Ranges of routine delivery room ambient temperatures included a cool $20^{\circ} \mathrm{C}$ to $21^{\circ} \mathrm{C}$ to a warm $26^{\circ} \mathrm{C}$ to $28^{\circ} \mathrm{C}$. Four studies reported the main outcome measure (temperature on admission to the NICU or up to two hours after birth) as a continuous variable, one study as a dichotomous variable (derived from skin temperature ${ }^{\circ} \mathrm{C}$ ), and the remaining studies as both a continuous and a dichotomous variable. Overall, 18 studies reported core body temperature as axillary ${ }^{\circ} \mathrm{C}$, four studies as rectal ${ }^{\circ} \mathrm{C}$, and two studies as both axillary and rectal ${ }^{\circ} \mathrm{C}$; one study reported skin temperature. Definitions of hypothermia were not consistent across studies. Researchers provided limited reporting of prespecified secondary outcomes in earlier studies, but more recent studies reported important secondary outcomes such as mortality, major brain injury, bronchopulmonary dysplasia (BPD), necrotising enterocolitis (NEC), retinopathy of prematurity (ROP), sepsis, and adverse events, particularly hyperthermia on admission to the NICU. Definitions of morbidity outcomes were also variable across studies. Future studies would benefit from using standardised definitions. This would reduce heterogeneity and risk of reporting bias, thereby facilitating meta-analyses (Webbe 2017). Core outcomes in Neonatology (COIN) are currently under development as part of the Core Outcome Measures in Effectiveness Trials initiative (COMET) (Webbe 2017).

In summary, the literature included in our review was clearly applicable to our primary review objective - "To assess the efficacy and safety of interventions, designed for prevention of hypothermia in preterm and/or low birth weight infants, and applied within 10 minutes after birth in the delivery room, compared with routine thermal care or any other single/ combination of intervention(s) also designed for prevention of hypothermia in preterm and/or low birth weight infants, and applied within 10 minutes after birth in the delivery room". However, reporting of secondary outcomes was limited across studies, with many outcomes reported for just one study within comparison groups; therefore imprecision is a major concern. In addition, these studies were not powered for all secondary outcomes. We addressed publication bias for each outcome when we were able to include more than 10 studies in the meta-analysis.

\section{Quality of the evidence}

Overall, for all included studies, across both domains for selection bias, we considered 12 studies ( $48 \%$ ) to be at low risk (i.e. adequate sequence generation and allocation concealment). For $92 \%$ of 
included studies, we noted no attempt to blind participants and healthcare personnel to the intervention (unclear but unlikely in two studies). Outcomes were objective and so were less likely to be biased than subjective outcome measures. In addition, secondary outcome data for key morbidities were assessed throughout the neonatal stay, when personnel were less likely to be aware of the original allocation group. Only three studies reported any attempt to blind outcome assessors or the data analysis team to the intervention; therefore, potential detection biases cannot be ruled out. However, for our primary outcome measure - core body or skin temperature - we conducted a simple linear regression analysis using the temperature time series (birth to 120 minutes) for one intervention and control group reported in Cardona Torres 2012. We found a significant linear trend $(P<0.029)$ and widening of the mean temperature difference between control and intervention groups (polyethylene bag without previous drying). This pattern of results suggests that detection bias was unlikely. No studies reported incomplete outcome data $\geq 20 \%$. Therefore risk of attrition bias was low. All infants randomised in most (64\%) studies completed the trial. We did not have access to study protocols for 16 of our included studies; therefore our assessment of the potential risk of reporting bias was limited. In all, 14 studies (56\%) appeared to be free from any additional sources of bias, and one had unclear risk of bias (McCarthy 2013).

Two studies reported issues with displacement of plastic wrap, which may have resulted in heat loss (Simon 2011; Smith 2013). This may have influenced the findings for comparison group 14 (plastic wrap or bag vs thermal mattress), which showed no significant differences in effect between the two interventions (Simon 2011). This bias is unlikely to change the conclusions for comparison group 1 (plastic wrap or bag vs routine care), which already demonstrated a highly significant difference in effect favouring plastic bag or wrap (Smith 2013). Some reported differences in infant characteristics (birth weight or gestation) could have influenced the effect size in favour of the intervention (Bhavsar 2015; Vohra 1999), or in favour of the control (Cardona Torres 2012). One study reported a higher incidence of swaddling in the control group but showed a statistically significant difference in effect for the comparison group plastic wrap and plastic cap versus routine care in favour of the intervention (Tescon-delos Santos 2012). For skin-to-skin versus routine care (comparison group 12), Bergman 2004 found a risk of selection bias in that many preterm infants were born without the nurse researcher present. Further issues included higher delivery room temperatures in the intervention group for stockinette cap versus routine care (comparison group 11); despite this, data show no evidence of effect. Knobel 2005 attempted to maintain delivery room temperature at $26^{\circ} \mathrm{C}$ but reported a wide range of temperatures. Post hoc analyses controlling for delivery room temperature showed that mean admission temperatures in the intervention group (plastic wrap or bag) were $0.6^{\circ} \mathrm{C}$ higher than in the routine care group. Leslie 2007 compared plastic bag + thermal mattress versus plastic bag only (comparison 15) and found no differences between the two interventions; however, this study was underpowered and risk of bias cannot be ruled out, leading to unreliable findings. In addition, Reilly 2015 reported several protocol violations among treatment groups, including delayed application of the wrap, wrap opening during the study period, and early removal of the wrap. Despite these violations, investigators reported significantly higher mean baseline and poststabilisation temperatures for the wrap group. Further violations included use of adjunct heat sources in small numbers of infants in both wrap and non-wrap groups, which was unlikely to affect findings.

We used the Grading of Recommendations Assessment, Development and Evaluation (GRADE) approach, as outlined in the GRADE Handbook (Schünemann 2013), to assess the quality of evidence for the following (clinically relevant) outcomes for our main comparison group: core body temperature on admission to the NICU or up to two hours after birth; hypothermia on admission to the NICU - core body temperature $<36.5^{\circ} \mathrm{C}$ or skin temperature $<36^{\circ} \mathrm{C}$; core body temperature $\left({ }^{\circ} \mathrm{C}\right)$ one hour after the initial NICU admission temperature was taken; hyperthermia on admission to the NICU - core body temperature $>37.5^{\circ} \mathrm{C}$; major brain injury during hospital stay; pulmonary haemorrhage during hospital stay; and mortality (death within hospital stay or at six months' corrected gestation). For all outcomes presented, we assessed the quality of the evidence as moderate: "We are moderately confident in the effect estimate: The true effect is likely to be close to the estimate of the effect, but there is a possibility that it is substantially different." We chose to not downgrade any of our outcome measures for performance or detection bias, as discussed above. We addressed issues of publication bias and heterogeneity by funnel plot inspection and sensitivity analysis when we included 10 or more studies in the meta-analysis.

- Moderate-quality evidence shows that plastic wraps or bags applied immediately at birth in the delivery room significantly reduced heat losses (13 studies; 1633 infants) and reduced hypothermia ( 10 studies; 1417 infants) on admission to the NICU and at one hour after the initial NICU temperature was taken (six studies; 373 infants) for infants at $<37$ weeks' gestation when compared with those given routine care; as defined by any of the following routine practices: providing a warm delivery room at a minimum of $25^{\circ} \mathrm{C}$, drying the infant immediately after birth, removing any wet blankets and wrapping in a prewarmed blanket, prewarming any contact surfaces, avoiding draughts, and, in developed countries (The World Bank 2016), using radiant warmers or incubators. We downgraded this evidence by one level for publication bias (core body temperature/ hypothermia on admission to NICU) and for inconsistency (core body temperature one hour after the initial NICU temperature was taken).

- Moderate-quality evidence shows that plastic wraps or bags applied immediately at birth in the delivery room significantly increased risk of hyperthermia on admission to the NICU (12 studies; 1523) for infants at $<37$ weeks' gestation when compared with those given routine care. We downgraded this evidence by one level for imprecision because conclusions were based on 50 incidences of hyperthermia in total. These data were sufficient to show significance but with wide $95 \%$ confidence intervals (Cls).

- Moderate-quality evidence reveals no evidence of effect on major brain injury during hospital stay for plastic wraps or bags applied immediately at birth in the delivery room for infants at $<37$ weeks' gestation when compared with those given routine care. No studies in this comparison group were powered for this outcome. We downgraded this evidence by one level for imprecision, as total numbers of episodes (or events) were below optimal information size according to guidance.

- Moderate-quality evidence reveals no evidence of effect on mortality (death during hospital stay or at six months' corrected gestation if still in hospital) (10 studies; 1447 infants) for plastic 
wraps or bags applied immediately at birth in the delivery room for infants at $<37$ weeks' gestation when compared with those given routine care. We downgraded this evidence by one level for publication bias. Nine out of ten studies were not powered for secondary outcomes. Reilly 2015 was powered for all-cause mortality as the primary outcome. The 2015 AHA, ERC, and ILCOR neonatal resuscitation guidance reported evidence from 36 observational studies showing increased risk of mortality associated with hypothermia at admission to the NICU (Perlman 2015). We upgraded this low-quality evidence to moderate quality owing to effect size, dose-effect relationship, and single direction of evidence, according to the GRADE approach. Perlman 2015 meta-analysed data from Reilly 2015,Vohra 1999, and Vohra 2004 and assessed the evidence as low quality, downgraded for indirectness and imprecision. However, we did not downgrade our meta-analyses of 10 RCTs for imprecision, although numbers were below Office of Information Services (OIS) guidance, with $95 \%$ Cls for RRs attaining just $\pm 25 \%$ of best estimate.

- Moderate-quality evidence shows that plastic wraps or bags applied immediately at birth in the delivery room significantly reduced risk of pulmonary haemorrhage during hospital stay for infants at $<28$ weeks' gestation compared with those given routine care. We downgraded this evidence by one level for imprecision because the evidence came from only one study (Reilly 2015).

The remaining comparison groups comprised a single study (groups 2, 3, 4, 6, 7, 8, 9, 10, 11, and 12) and a maximum of two studies across outcomes (group 5, 13, 14, and 15), which limits our ability to draw firm conclusions.

\section{Potential biases in the review process}

The strength of this review lies in the fact that review authors undertook a comprehensive literature search for both published and unpublished studies (up to 2013), although thereafter, publication bias cannot be ruled out. We also searched clinical trials registries to identify ongoing trials. We contacted trial authors for clarification pertaining to methodological and subgroup data. In addition, we agreed on strict inclusion criteria for PICO to ensure that the review was focused on interventions provided to prevent hypothermia for preterm and/or low birth weight infants applied within 10 minutes of birth rather than those given to term infants or over a longer period. We made every effort to minimise bias at all stages of the review process, in line with Cochrane methods.

\section{Agreements and disagreements with other studies or reviews}

\section{Plastic barriers to heat loss}

Two recent systematic reviews assessed the efficacy and safety of plastic coverings for reducing heat loss, mortality, and morbidity in preterm infants (Li 2016; Oatley 2016). Oatley 2016 also included full-term neonates. Literature search end dates were August 2015 for Li 2016 and January 2015 for Oatley 2016, and the systematic reviews included RCTs and quasi-RCTs (11 studies; $N=1601$ ) (Li 2016), as well as both observational studies and RCTs (22 studies) (Oatley 2016). Li 2016 included findings of the multi-centre HeLP study (Reilly 2015), but for Oatley 2016, this information fell outside the search parameters. Both reviews are in agreement with our findings and provide similar evidence in support of the effectiveness of plastic coverings in reducing heat losses and preventing hypothermia compared with routine thermal care across a range of gestational ages. Reductions in risk of hypothermia of $21 \%$ to $46 \%$ provided in Oatley 2016 and of $21 \%$ to $71 \%$ as reported in Li 2016 are slightly below the range of our pooled data of $33 \%$ overall ( $<37$ weeks' gestation), $30 \%$ for infants at $<28$ weeks' gestation, and $83 \%$ for infants at $\geq 28$ weeks' gestation (upper-middle-income settings). Data show no significant reductions in neonatal mortality or morbidity. Li 2016 reported a significantly higher incidence of hyperthermia in the plastic wrap group compared with the control group (typical RR 2.55, 95\% Cl 1.56 to 4.15; eight studies; $N=1438$ ), which was readily resolved within one to two hours after unwrapping. However, based on the results of eight RCTs, Oatley 2016 suggested that risk of hyperthermia is low $(<5 \%)$, that no evidence suggested increased risk with concurrent use of an incubator, and that in all reported cases neonates returned to normothermia when wrapping was removed. As mentioned previously, we did not include data from Reilly 2015. Our findings are consistent with those of Li 2016 but show greater risk with respect to hyperthermia (RR 3.91, 95\% Cl 2.05 to 7.44; 12 studies, $N=1523$ ). For Reilly 2015 , we meta-analysed data for baseline temperature rather than for poststabilisation temperature (RR $2.55,95 \% \mathrm{Cl} 1.56$ to 4.15 vs RR $2.35,95 \% \mathrm{Cl} 1.38$ to 4.00 ). Implications for research included the need for further study to assess the role of plastic coverings outside the hospital setting and inclusion of longer follow-up periods to assess the impact of this intervention on mortality and neurodevelopmental outcomes. Further studies to assess effectiveness of plastic wrap compared with skin-to-skin care (SSC) immediately after birth would inform practice in low-resource settings. No studies for this comparison group fulfilled our inclusion criteria. The World Health Organization addressed the question "Should these plastic wraps/caps be used instead of kangaroo mother care immediately after birth in a subgroup of preterm infants?" (WHO 2015). Evidence presented in Oatley 2016 (no study compared plastic wraps vs kangaroo mother care (KMC)) led WHO to conclude that evidence on the effectiveness of plastic bags/wraps in providing thermal care for preterm newborns immediately at birth was insufficient. However, during stabilisation and transfer of these infants to specialised neonatal wards, wrapping in plastic bags/wraps may be considered an alternative method of preventing hypothermia.

\section{External heat sources}

Only one small RCT comparing SSC versus routine care fulfilled the inclusion criteria for this review and showed that SSC was effective in reducing the risk of hypothermia when compared with conventional incubator care for infants with birth weight between 1200 and 2199 grams (Bergman 2004). Conde-Agudelo 2016, recently conducted a Cochrane Review (literature search end date June 2016) to determine whether evidence is available to support use of KMC in low birth weight infants as an alternative to conventional neonatal care before or after the initial period of stabilisation; similarly only one study evaluated KMC before stabilisation (Worku 2005). We excluded this study from our review because the intervention was not applied immediately at birth, within 10 minutes, in the delivery room, and the mean age of participants at enrolment was approximately 10 hours. The review authors were unable to draw any conclusions regarding the effectiveness of KMC in non-stabilised low birth weight infants and highlighted the need for rigorous trials to assess the effectiveness of early-onset continuous KMC for non-stabilised or relatively 
stabilised low birth weight infants in low-income countries. Clearly, studies to date have primarily focussed on healthy term infants or poststabilisation preterm and/or low birth weight infants. Of 86 studies included in a recent systematic review (RCTs and observational studies) documenting initiation time of SSC, only seven $(8 \%)$ were initiated immediately after birth, and $41(48 \%)$ had stability criteria for initiation (Boundy 2016). A recent prospective cohort of 90 moderately preterm infants of 32 to 34 weeks' gestation in Norway assessed the feasibility and safety of early SSC in the delivery room after vaginal birth compared with immediate transfer to the NICU in a conventional incubator (Kristoffersen 2016). Data show no statistically significant temperature differences between the two groups at 30 minutes after birth. However, this study was at risk of bias owing to differences in measurement sites for temperature (SSC (rectal) and incubator (axillary)), and the study was underpowered. Nevertheless, investigators implemented SSC immediately after birth with no additional resources, and this approach appeared to be both feasible and safe for this group of infants.

\section{AUTHORS' CONCLUSIONS}

\section{Implications for practice}

Hypothermia remains a worldwide problem, especially for small infants and those born preterm. Across all gestations, admission temperature is a strong predictor of mortality and morbidity (Perlman 2015). Any additional intervention designed to prevent hypothermia in very preterm and/or low birth weight infants, and applied within 10 minutes after birth in the delivery room, compared with routine thermal care is beneficial in practice. Moderate-quality evidence shows that low-cost plastic wraps or bags keep preterm infants warmer and lead to higher temperatures on admission to neonatal units with less hypothermia and fewer temperatures outside the range of normothermia. Extremely preterm infants appear to benefit the most. Further emerging evidence suggests that plastic caps, thermal mattresses, and skin-to-skin care also reduce risk of hypothermia across a range of gestations and birth weights when compared with routine care. Caution must be taken to avoid iatrogenic hyperthermia, particularly when multiple interventions are used simultaneously (e.g. plastic bags + thermal mattresses). Many observational studies demonstrate increased mortality in preterm hypothermic infants compared with those who maintain normothermia, yet evidence is insufficient to suggest that these interventions reduce risk of inhospital (or at six months' corrected gestation) mortality across all comparison groups.
Limited evidence suggests benefit and no evidence suggests harm for most short-term morbidity outcomes known to be associated with being hypothermic, including major brain injury, bronchopulmonary dysplasia, retinopathy of prematurity, necrotising enterocolitis, and nosocomial infection. Therefore, hypothermia may be a marker for illness and poorer outcomes by association rather than for causality. Limitations include small numbers of studies; small sample sizes; and variations in methods and definitions used for hypothermia, hyperthermia, normothermia, routine care, and morbidity; coupled with lack of power to detect effects of these interventions on morbidity and mortality across most comparison groups.

Overall, for the main comparison (plastic wraps or bags vs routine care), we are moderately confident that results and our conclusions are reliable. Across the remaining comparison groups, evidence is insufficient to allow firm judgements.

Preterm thermal care strategies should be tailored to the individual setting with consideration of the environment, available resources (both structural and financial), and baseline hypothermia rates. These strategies should be collaborative, multi-disciplinary, standardised, evidence-based thermal management approaches provided before delivery and throughout neonatal intensive care unit (NICU) admission. Adherence to thermal care guidelines should be audited by integrating performance monitoring into practice, and by continuing to record core body temperature on admission to the NICU as a measure of quality of care and as a predictor of outcomes.

\section{Implications for research}

Future studies assessing the efficacy of interventions designed to maintain normothermia at birth should be adequately powered to detect rarer short-term morbidity outcomes and all-cause mortality, and should focus on longer-term outcomes, particularly neurodevelopmental outcomes. These large-scale studies should use standardised morbidity definitions.

\section{ACKNOWLEDGEMENTS}

Diarmuid Kennedy, Angela Thompson, Alex Mcllroy, and Patricia Watt, Faculty Librarians Medicine and Health Sciences, Queen's University Belfast; Mike Stevenson, Consultant in Medical Statistics; Nuala Livingston, Cochrane Editorial Unit (London); Roger Soll, Colleen Ovelman, and Yolanda Brosseau, Cochrane Neonatal Review Group (CNRG); Suzanne Sweeney, Texas Woman's University Library, Texas Woman's University. 


\section{R E F E R E N C E S}

\section{References to studies included in this review}

Bergman 2004 \{published data only\}

Bergman NJ, Linley LL, Fawcus SR. Randomized controlled trial of skin-to-skin contact from birth versus conventional incubator for physiological stabilization in 1200- to 2199-gram newborns. Acta Paediatrica 2004;93(6):779-85. [PUBMED: 15244227]

\section{Bhavsar 2015 \{published data only\}}

Bhavsar SR, Kabra NS, Avasthi BS, Sharma RS, Dash SW, Tali SH, et al. Efficacy and safety of polyethylene wrap in preventing hypothermia in preterm and low birth weight neonates during transport: a randomised controlled trial. Perinatology 2015;16(1):23-30.

\section{Brennan 1996 \{published data only\}}

Brennan AB. Effect of sodium acetate transport mattresses on admission temperatures of infants $<=1500$ grams [dissertation]. Gainesville (FL): University of Florida, 1996.

\section{Caglar 2014 \{published and unpublished data\}}

Caglar S, Gozen D, Ince Z. Heat Loss Prevention (Help) after birth in preterm infants using vinyl isolation bag or polyethylene wrap. Journal of Obstetric, Gynecologic, and Neonatal Nursing 2014;43(2):216-23. [DOI: 10.1111/1552-6909.12291]

\section{Cardona Torres 2012 \{published data only\}}

Cardona Torres LM, Amador Licona N, Garcia Campos ML, Guizar-Mendoza JM. Polyethylene wrap for thermoregulation in the preterm infant: a randomised trial. Indian Pediatrics 2012;49(2):129-32. [PUBMED: 21992867]

Chantaroj 2011 \{published data only\}

Chantaroj S, Techasatid W. Effect of polyethylene bag to prevent heat loss in preterm infants at birth: a randomised controlled trial. Journal of the Medical Association of Thailand 2011;94(Suppl 7):S32-7. [PUBMED: 22619904]

\section{Chawla 2011 \{published data only\}}

Chawla S, Amaram A, Gopal SP, Natarajan G. Safety and efficacy of Trans-warmer mattress for preterm neonates: results of a randomised controlled trial. Journal of Perinatology 2011;31(12):780-4. [DOI: 10.1038/jp.2011.33]

\section{Doglioni 2014 \{published data only\}}

Doglioni N, Cavallin F, Mardegan V, Palatron S, Filippone M, Vecchiato $L$, et al. Total body polyethylene wraps for preventing hypothermia in preterm infants: a randomised trial. Journal of Pediatrics 2014;165(2):261-6.e1. [DOI: 10.1016/ j.jpeds.2014.04.010; PUBMED: 24837862]

\section{Farhadi 2012 \{published and unpublished data\}}

Farhadi R, Naderi M, Rahmani Z, Ghafari V, Khalilian A. Effect of "ZIPKIF" plastic bag on prevention of hypothermia in preterm infants: a randomised controlled trial. Journal of Mazandaran University of Medical Sciences 2012;22(93):19-26.
Gathwala 2010 \{published data only\}

Gathwala G, Singh G, Kunal, Agrawal N. Safety and efficacy of vinyl bags in prevention of hypothermia of preterm neonates at birth. Indian Journal of Public Health 2010;54(1):24-6. [DOI: 10.4103/0019-557X.70543]

\section{Knobel 2005 \{published data only\}}

Knobel RB, Wimmer JE, Holbert D. Heat loss prevention for preterm infants in the delivery room. Journal of Perinatology 2005;25(5):304-8. [DOI: 10.1038/sj.jp.7211289]

Leadford 2013 \{published data only\}

Leadford AE, Warren JB, Manasyan A, Chomba E, Salas AA, Schelonka RL, et al. Plastic bags for prevention of hypothermia in preterm and low birth weight infants. Pediatrics 2013; Vol. 132, issue 1:e128-34. [DOI: 10.1542/peds.2012-2030]

Leslie 2007 \{published data only\}

Leslie A, Wardle SP, Budge H, Marlow N, Brocklehurst P. [Randomised controlled trial of gel warming mattresses to prevent hypothermia during resuscitation at birth of premature infants]. Neonatal Society 2007 Autumn Meeting. London: Neonatal Society, 2007.

\section{Mathew 2012 \{published data only\}}

Mathew B, Lakshminrusimha S, Sengupta S, Carrion V. Randomized controlled trial of vinyl bags versus thermal mattress to prevent hypothermia in extremely low gestational age infants. American Journal of Perinatology 2013;30(4):317-22. [DOI: $10.1055 / \mathrm{s}-0032-1324700$ ]

\section{McCarthy 2013 \{published data only\}}

McCarthy LK, Molloy EJ, Twomey AR, Murphy JF, O'Donnell CP. $A$ randomised trial of exothermic mattresses for preterm newborns in polyethylene bags. Pediatrics 2013;132(1):e135-41. [DOI: 10.1542/peds.2013-0279]

\section{Reilly 2015 \{published and unpublished data\}}

Reilly MC, Vohra S, Dunn M, Ferrelli K, Rac VE, Vincer M, et al. The Vermont Oxford Network (VON) heat loss prevention (HELP) trial. Proceedings of the Pediatric Academic Societies' Annual Meeting; 2012 April 28 - May 1; Boston (MA). The Woodlands (TX): Pediatric Academic Societies, 2012.

* Reilly MC, Vohra S, Rac VE, Dunn M, Ferrelli K, Kiss A, et al. Vermont Oxford Network Heat Loss Prevention (HeLP) Trial Study Group. Randomized trial of occlusive wrap for heat loss prevention in preterm infants. Journal of Pediatrics 2015;166(2):262-8. [DOI: 10.1016/j.jpeds.2014.09.068]

Vohra S, Reilly M, Rac VE, Bhaloo Z, Zayack D, Wimmer J, et al. Study protocol for multicentre randomised controlled trial of HeLP (Heat Loss Prevention) in the delivery room. Contemporary Clinical Trials 2013;36(1):54-60. [DOI: 10.1016/ j.cct.2013.06.001; PUBMED: 23770234] 
Roberts 1981 \{published data only\}

Roberts JR. Use of a stockinette cap on premature infants after delivery [dissertation]. Denton (TX): Texas Women's University, 1981.

\section{Rohana 2011 \{published data only\}}

Rohana J, Khairina W, Boo NY, Shareena I. Reducing hypothermia in preterm infants with polyethylene wrap. Pediatrics International 2011;53(4):468-74. [DOI: 10.1111/ j.1442-200X.2010.03295.x]

\section{Simon 2011 \{published data only\}}

Simon P, Dannaway D, Bright B, Krous L, Wlodaver A, Burks B, et al. Thermal defence of extremely low gestational age newborns (EGLANS) during resuscitation: exothermic mattress vs polyethylene wrap. Journal of Perinatology 2011;31(1):33-7.

\section{Smith 2013 \{published and unpublished data\}}

Smith J, Usher K, Alcock G, Buettner P. Application of plastic wrap to improve temperatures in infants born less than 30 weeks gestation: a randomised controlled trial. Neonatal Network 2013; Vol. 32, issue 4:235-45. [DOI: 10.1891/0730-0832.32.4.235]

\section{Talakoub 2015 \{published data only\}}

Talakoub S, Shahbazifard Z, Armanian AM, Ghazavi Z. Effect of two polyethylene covers in prevention of hypothermia among premature neonates. Iranian Journal of Nursing and Midwifery Research 2015;20(3):322-6. [PUBMED: 26120331]

Tescon-delos Santos 2012 \{published and unpublished data\} Tecson-delos Santos CM, Uy ME, Uy H, Sto Nino OV. [Polyethylene plastic wrap and cap for thermoregulation of preterm infants 28 to 36 weeks old admitted to the neonatal intensive care unit: a randomised controlled trial]. Journal of Paediatrics and Child Health. 2012; Vol. 48 (Suppl 1):154. [DOI: 10.1111/j.1440-1754.2012.02412.x]

\section{Trevisanuto 2010 \{published and unpublished data\}}

Trevisanuto D, Dogioni N, Cavallin MS, Parotto M, Micaglio M, Zanardo V. Heat loss prevention in very preterm infants in delivery rooms: a prospective, randomised controlled trial of polyethylene caps. Journal of Pediatrics 2010;156(6):914-7. [DOI: 10.1016/j.jpeds.2009.12.021]

\section{Vohra 1999 \{published data only\}}

Vohra S, Frent G, Campbell V, Abbott M, Whyte R. Effect of polyethylene occlusive skin wrapping on heat loss in very low birth weight infants at delivery: a randomised trial. Journal of Pediatrics 1999;134(5):547-51. [PUBMED: 10228287]

\section{Vohra 2004 \{published data only\}}

Vohra S, Roberts R, Zhang B, Janes M, Schmidt B. Heat loss prevention (HELP) in the delivery room: a randomised controlled trial of polyethylene occlusive skin wrapping in very preterm infants. Journal of Pediatrics 2004;145(6):750-3. [DOI: 10.1016/j.jpeds.2004.07.036]

\section{References to studies excluded from this review}

Acharya 2014 \{published data only\}

Acharya N, Singh RR, Bhatta NK, Poudel P. Randomized control trial of Kangaroo Mother Care in low birth weight babies at a tertiary level hospital. Journal of Nepal Paediatric Society 2014;34(1):18-23.

\section{Ammari 2009 \{published data only\}}

Ammari A, Schulze KF, Ohira-Kist K, Kashyap S, Fifer WP, Myers MM, et al. Effects of body position on thermal, cardiorespiratory and metabolic activity in low birthweight infants. Early Human Development 2009;85(8):497-501. [DOI: 10.1016/j.earlhumdev.2009.04.005]

\section{Anderson 2003 \{published data only\}}

Anderson GC, Chiu SH, Dombrowski MA, Swinth JY, Albert JM, Wada N. Mother-newborn contact in a randomised trial of kangaroo (skin-to- skin) care. Journal of Obstetric, Gynecologic, and Neonatal Nursing 2003;32(5):604-11. [PUBMED: 14565739]

\section{Andrade 2005 \{published data only\}}

Andrade I, Guedes Z. Suckling of the premature newborn child: comparison between the Kangaroo Mother method with traditional care [Succao do recem-nascido prematuro: comparacao do metodo Mae-Canguro com os cuidados tradicionais]. Revista Brasileria de Saude Materno Infantil 2005;5(1):61-9. [DOI: 10.1590/S1519-38292005000100008]

\section{Ballesteros 2012 \{published data only\}}

Ballesteros RM, Agulay EA, Matias AAD, Mendoza SL. Kangaroo mother care for low birth weight infants <2000g: a randomised controlled trial. Journal of Paediatrics and Child Health. 2012; Vol. 48 Suppl 1:83.

\section{Baum 1968 \{published data only\}}

Baum JD, Scopes JW. The silver swaddler. Device for preventing hypothermia in the newborn. Lancet 1968;1(7544):672-3. [PUBMED: 4170656]

\section{Beiranvand 2014 \{published data only\}}

Beiranvand S, Valizadeh F, Hosseinabadi R, Pournia Y. The effects of skin-to-skin contact on temperature and breastfeeding successfulness in full-term newborns after cesarean delivery. International Journal of Pediatrics 2014;2014:846486. [DOI: 10.1155/2014/846486]

Bell 1983 \{published data only\}

Bell EF, Rios GR. A double-walled incubator alters the partition of body heat loss of premature infants. Pediatric Research 1983;17(2):135-40. [DOI: 10.1203/00006450-198302000-00011]

\section{Bergstrom 2005 \{published data only\}}

Bergstrom A, Byaruhanga R, Okong P. The impact of newborn bathing on the prevalence of neonatal hypothermia in Uganda: a randomised, controlled trial. Acta Paediatrica 2005;94(10):1462-7. [PUBMED: 16299878]

Besch 1971 \{published data only\}

Besch NJ, Perlstein PH, Edwards NK, Keenan WJ, Sutherland JM. The transparent baby bag. A shield against heat loss. New 
England Journal of Medicine 1971;284(3):121-4. [DOI: 10.1056/ NEJM197101212840302]

Bhat 2015 \{published data only\}

Bhat SR, Meng NF, Kumar K, Nagesh KN, Kawale A, Bhutani VK. Keeping babies warm: a non-inferiority trial of a conductive thermal mattress. Archives of Disease in Childhood. Fetal and Neonatal Edition 2015;100(4):F309-12. [PUBMED: 25791056]

\section{Bier 1996 \{published data only\}}

Bier JA, Ferguson AE, Morales Y, Liebling JA, Archer D, Oh W, et al. Comparison of skin-to-skin contact with standard contact in low-birth-weight infants who are breast-fed. Archives of Pediatrics \& Adolescent Medicine 1996;150(12):1265-9. [PUBMED: 8953998]

\section{Boo 2007a \{published data only\}}

Boo NY, Jamli FM. Short duration of skin-to-skin contact: effects on growth and breastfeeding. Journal of Paediatrics and Child Health 2007;43(12):831-6. [DOI: 10.1111/ j.1440-1754.2007.01198.x]

\section{Boo 2007b \{published data only\}}

Boo NY, Chew EL. A randomised control trial of cling film for prevention of hypothermia in term infants during phototherapy. Singapore Medical Journal 2006;47(9):757-62. [PUBMED: 16924356]

\section{Brice 1981 \{published data only\}}

Brice JE, Rutter N, Hull D. Reduction of skin water loss in the newborn II. Clinical trial of two methods in very low birthweight babies. Archives of Disease in Childhood 1981;56(9):673-5. [PUBMED: 7294869]

\section{Bystrova 2003 \{published data only\}}

Bystrova K, Widstrom AM, Matthiesen AS, Ransjo-Arvidson, Wells-Nystrom B, Wassberg C, et al. Skin-to-skin contact may reduce negative consequences of "the stress of being born": a study on temperature in newborn infants, subjected to different ward routines in St. Petersburg. Acta Paediatrica 2003;92(3):320-6. [PUBMED: 12725547]

\section{Bystrova 2007 \{published data only\}}

Bystrova K, Matthiesen AS, Vorontsov I, Widstrom AM, RansjoArvidson AB, Uvnas-Moberg K. Maternal axillary and breast temperature after giving birth: effects of delivery ward practices and relation to infant temperature. Birth 2007;34(4):291-300. [DOI: 10.1111/j.1523-536X.2007.00187.x]

Carfoot 2005 \{published data only\}

Carfoot S, Williamson P, Dickson R. A randomised controlled trial in the north of England examining the effects of skin-toskin care on breast feeding. Midwifery 2005;21(1):71-9. [DOI: 10.1016/j.midw.2004.09.002]

\section{Cattaneo 1998 \{published data only\}}

Cattaneo A, Davanzo R, Worku B, Surjono A, Escheverria M, Bedri A, et al. Kangaroo mother care for low birthweight infants: a randomised controlled trial in different settings. Acta Paediatrica 1998;87(9):976-85. [PUBMED: 9764894]
Chaput 1979 \{published data only\}

Chaput de Saintonge DM, Cross KW, Shathorn MK, Lewis SR, Stothers JK. Hats for the newborn infant. British Medical Journal 1979;2(6190):570-1. [PUBMED: 387172]

\section{Charpak 1997 \{published data only\}}

Charpak N, Ruiz-Pelaez JG, Figueroa de CZ, Charpak Y. Kangaroo mother versus traditional care for newborn infants $</=2000$ grams: a randomised, controlled trial. Pediatrics 1997;100(4):682-8. [PUBMED: 9310525]

\section{Cheah 2000 \{published data only\}}

Cheah FC, Boo NY. Risk factors associated with neonatal hypothermia during cleaning of newborn infants in labour rooms. Journal of Tropical Pediatrics 2000;46(1):46-50. [PUBMED: 10730042]

\section{Christensson 1992 \{published data only\}}

Christensson K, Siles C, Moreno L, Belaustequi A, De La Fuente P, Lagercrantz $\mathrm{H}$, et al. Temperature, metabolic adaptation and crying in healthy full-term newborns cared for skin-to-skin or in a cot. Acta Paediatrica 1992;81(6-7):488-93. [PUBMED: 1392359]

Christensson 1995 \{published data only\}

Christensson K, Cabrera T, Christensson E, Uvnas Moberg K, Winberg J. Separation distress call in the human neonate in the absence of maternal body contact. Acta Paediatrica 1995;84(5):468-73. [PUBMED: 7633137]

Christensson 1996 \{published data only\}

Christensson K. Fathers can effectively achieve heat conservation in healthy newborn infants. Acta Paediatrica 1996;85(11):1354-60. [PUBMED: 8955466]

\section{Christensson 1998 \{published data only\}}

Christensson K, Bhat GJ, Amadi BC, Eriksson B, Hojer B. Randomised study of skin-to-skin versus incubator care for rewarming low-risk hypothermic neonates. Lancet 1998;352(9134):1115. [DOI: 10.1016/S0140-6736(98)00028-2]

\section{Chwo 1999 \{published data only\}}

Chwo MJ. Early kangaroo care for 34-36 week preterm infants: effects on temperature, weight, behavior, and acuity [dissertation]. Clevland $(\mathrm{OH})$ : Case Western Reserve University, 1999.

\section{Chwo 2002 \{published data only\}}

Chwo MJ, Anderson GC, Good M, Dowling DA, Shaiu SH, Cho DM. A randomised controlled trial of early kangaroo care for preterm infants: effects on temperature, weight, behaviour and acuity. Journal of Nursing Research 2002;10(2):129-42. [PUBMED: 12119598]

Coles 1979 \{published data only\} Coles EC, Valman HB. Hats for the newborn infant. British Medical Journal 1979;2(6192):734-5. [PUBMED: 509089 ]

CTRI/2011/11/002125 \{published data only\}

CTRI/2011/11/002125. A randomised, open-label, active controlled study to determine the safety and efficacy of the rural-at-home Embrace ${ }^{T M}$ infant warmer (REIW) in maintaining 
thermo-stability $\left(36.5^{\circ} \mathrm{C}\right.$ to $\left.37.5^{\circ} \mathrm{C}\right)$, of low-birth-weight (LBW) neonates when used in a home setting. http://apps.who.int/ trialsearch/Trial2.aspx?TrialID=CTRI/2011/11/002125 (first received 14 November 2011).

\section{Dahm 1972 \{published data only\}}

Dahm LS, James LS. Newborn temperature and calculated heat loss in the delivery room. Pediatrics 1972;49(4):504-13. [PUBMED: 5013414]

\section{Darmstadt 2007 \{published data only\}}

Dramstadt GL, Kumar V, Yadav R, Singh V, Singh P, Mohanty S, et al. Introduction of community-based skin-to-skin care in rural Uttar Pradesh, India. Journal of Perinatology 2006;26(10):597-604.

\section{Darmstadt 2008 \{published data only\}}

Dramstadt GL, Saha SK, Ahmed AS, Ahmed S, Chowdhury MA, Law PA, et al. Effect of skin barrier therapy on neonatal mortality rates in preterm infants Bangladesh: a randomised, controlled, clinical trial. Pediatrics 2008;121(3):522-9. [DOI: 10.1542/peds.2007-0213]

\section{Day 1964 \{published data only\}}

Day RL, Caliguiri L, Kamenski C, Ehrlich F. Body temperature and survival of premature infants. Pediatrics 1964;34:171-81. [PUBMED: 14211077]

\section{Dehghani 2015 \{published data only\}}

Dehghani K, Movahed ZP, Dehghani H, Nasiriani, K. A randomised controlled trial of kangaroo mother care versus conventional method on vital signs and arterial oxygen saturation rate in newborns who were hospitalized in neonatal intensive care unit. Journal of Clinical Neonatology 2015;4(1):26-31. [DOI: 10.4103/2249-4847.151163]

\section{De Ocampo 2012 \{published data only\}}

De Ocampo FS, Esterlita UM. A randomised controlled trial of kangaroo mother care (KMC) versus conventional care in increasing the rate of weight gain among low birthweight (LBW) neonates. Journal of Paediatrics and Child Health 2012;48:74-5.

Dodd 2003 \{published data only\}

Dodd VL. Effects of kangaroo care in preterm infants [dissertation]. Storrs (CT): The University of Connecticut, 2003.

\section{Duryea 2016 \{published data only\}}

Duryea EL, Nelson DB, Wyckoff MH, Grant EN, Tao W, Sadana N, et al. The impact of ambient operating room temperature on neonatal and maternal hypothermia and associated morbidities: a randomised controlled trial. American Journal of Obstetrics and Gynecology 2016;214(4):505.e1-7. [DOI: 10.1016/ j.ajog.2016.01.190]

\section{Erlandsson 2007 \{published data only\}}

Erlandsson K, Dsilna A, Fagerberg I, Christensson K. Skinto-skin care with the father after cesarean birth and its effect on newborn crying and prefeeding behavior. Birth 2007;34(2):105-14. [DOI: 10.1111/j.1523-536X.2007.00162.x]
Fallis 2006 \{published data only\}

Fallis WM, Hamelin K, Symonds J, Wang X. Maternal and newborn outcomes related to maternal warming during cesarean delivery. Journal of Obstetric, Gynecologic and Neonatal Nursing 2006;35(3):324-31. [DOI: 10.1111/ j.1552-6909.2006.00052.x]

\section{Fardig 1980 \{published data only\}}

Fardig JA. A comparison of skin-to-skin contact and radiant heaters in promoting neonatal thermoregulation. Journal of Nurse-Midwifery 1980;25(1):19-28. [PUBMED: 6898112]

Ferber 2004 \{published data only\}

Ferber SG, Makhoul IR. The effect of skin-to-skin contact (kangaroo care) shortly after birth on the neurobehavioral responses of the term newborn: a randomised, controlled trial. Pediatrics 2004;113(4):858-65. [PUBMED: 15060238]

\section{Gabriel 2010 \{published data only\}}

Marin Gabriel MA, Llana Martin I, Lopez Escobar A, Fernandez Villalba E, Romero Blanco I, Touza Pol P. Randomized controlled trial of early skin-to-skin contact: effects on the mother and the newborn. Acta Paediatrica 2010;99(11):1630-4. [DOI: 10.1111/ j.1651-2227.2009.01597.x]

Gao 2010 \{published data only\}

Gao F, Chen JL, Zhou CF, Jin DY. The effect of mother-infant skin-to-skin contact shortly after birth on newborn behaviours. Chinese Journal of Nursing 2010;45:1061-4.

\section{Gathwala 2008 \{published data only\}}

Gathwala G, Singh B, Balhara B. KMC facilitates mother baby attachment in low birth weight infants. Indian Journal of Pediatrics 2008;75(1):43-7. [PUBMED: 18245934]

\section{Ghavane 2012 \{published data only\}}

Ghavane S, Murki S, Subramanian S, Gaddam P, Kandraju H, Thumalla S. Kangaroo mother care in kangaroo ward for improving the growth and breastfeeding outcomes when reaching term gestational age in very low birth weight infants. Acta Paediatrica 2012;101(12):e545-9. [DOI: 10.1111/apa.12023]

\section{Gouchon 2010 \{published data only\}}

Gouchon S, Gregori D, Picotto A, Patrucco G, Nangeroni M, Di Giulio P. Skin-to skin contact after cesarean delivery: an experimental study. Nursing Research 2010;59(2):78-84. [DOI: 10.1097/NNR.0b013e3181d1a8bc]

\section{Gray 2004 \{published data only\}}

Gray PH, Paterson S, Finch G, Hayes M. Cot-nursing using a heated, water-filled mattress and incubator care: a randomised clinical trial. Acta Paediatrica 2004;93(3):350-5. [PUBMED: 15124838]

\section{Green-Abate 1994 \{published data only\}}

Green-Abate C, Tafari N, Rao MR, Yu K, Clemens JD. Comparison of heated water-filled mattress and spaceheated room with infant incubator in providing warmth to low birthweight newborns. International Journal of Epidemiology 1994;23(6):1226-33. 
Greer 1988 \{published data only\}

Greer PS. Head coverings for newborns under radiant warmers. Journal of Obstetric, Gynecological and Neonatal Nursing 1988;17(4):265-71. [DOI: 10.1111/j.1552-6909.1988.tb00438.x]

\section{Grover 1994 \{published data only\}}

Grover G, Berkowitz CD, Lewis RJ, Thompson M, Berry L, Seidel J. The effects of bundling on infant temperature. Pediatrics 1994;94(5):669-73. [PUBMED: 7936893]

\section{Gulezian 1980 \{published data only\}}

Gulezian GZ. Effect of skin-to-skin contact on transitional newborn infants' temperature [dissertation]. Chicago (IL): University of Illinois at the Medical Center, 1980.

\section{Harrison 2004 \{published data only\}}

Harrison M. Kangaroo mother care:an innovative way of care for infants [dissertation]. Hattiesburg (MS): University of Southern Mississippi, 2004.

\section{Heaman 2012 \{published data only\}}

Heaman MI, Martens PJ, Brownell MD, Chartier MJ, Helewa ME, Derksen SA, et al. Predictors of inadequate prenatal care: a population-based study in Manitoba, Canada. Journal of Paediatrics and Child Health 2012;48(Suppl 1):8-81. [DOI: 10.1111/j.1440-1754.2012.02411.x]

\section{Hellin Martinez 2000 \{published data only\}}

Heillin Martinez MJ, Lopez Terol E, Perez Lopez M, Vidagany Escrig I. Maintain the temperature of a healthy newborn infant. Revista Rol de Enfermeria 2000;23(1):63-5.

Hobbs 1975 \{published data only\}

Hobbs JF, MacKuanying N, Eidelman I, Schneider KM. A new transparent insulating infant gown to maintain thermal stability. Pediatric Research 1975;9:366 [Abstract 658].

\section{Holzman 1985 \{published data only\}}

Holzman IR. A method to maintain infant temperature. American Journal of Diseases of Children 1985;139(4):390-2. [PUBMED: 3976629]

\section{Horn 2002 \{published data only\}}

Horn EP, Schroeder F, Gottschalk A, Sessler DI, Hiltmeyer N, Standl T, et al. Active warming during cesarean delivery. Anesthesia and Analgesia 2002;94(2):409-14. [PUBMED: 11812709]

\section{Horn 2014 \{published data only\}}

Horn EP, Bein B, Steinfath M, Ramaker K, Buchloh B, Hocker J. The incidence and prevention of hypothermia in newborn bonding after cesarean delivery: a randomised controlled trial. Anesthesia and Analgesia 2014;118(5):997-1002. [DOI: 10.1213/ ANE.0000000000000160]

\section{Hsu 2015 \{published data only\}}

Hsu KH, Chiang MC. A randomised trial of using thermal blanket to improve thermoregulation among preterm infants. Archives of Disease in Childhood. 5th Congress of the European Academy of Paediatric Societies 2014;99(Suppl 2):A195-6. [DOI: 10.1136/ archdischild-2014-307384.526]
Hsu KH, Chiang MC, Lin SW, Lin JJ, Wang YC, Lien R. Thermal blanket to improve thermoregulation in preterm infants: a randomized controlled trial. Pediatric Critical Care Medicine 2015;16(7):637-43. [DOI: 10.1097/PCC.0000000000000447; PUBMED: 25901548]

\section{Huang 2002 \{published data only\}}

Haung L, Chwo M, Chu D, Chang Y. Effects of very early kangaroo care on infants' extrauterine adaptation. Hu Li za Zhi [Journal of Nursing] 2002;49(5):37-51.

\section{Huang 2006 \{published data only\}}

Huang YY, Huang CY, Lin SM, Wu SC. Effect of very early kangaroo care on extrauterine temperature adaptation in newborn infants with hypothermia problems. Hu Li za Zhi [Journal of Nursing] 2006;53(4):41-8. [PUBMED: 16874601]

\section{Jia 2012 \{published data only\}}

Jia YS, Lin ZL, Lv H, Li YM, Green R, Lin J. Effect of delivery room temperature on the admission temperature of premature infants: a randomised controlled trial. Journal of Perinatology 2013;33(4):264-7. [DOI: 10.1038/jp.2012.100]

\section{Johanson 1992 \{published data only\}}

Johanson RB, Spencer SA, Rolfe P, Jones P, Malla DS. Effect of post-delivery care on neonatal body temperature. Acta Paediatrica 1992;81(11):859-63. [PUBMED: 1467605]

Kadam 2005 \{published data only\}

Kadam S, Binoy S, Kanbur W, Mondkar JA, Fernandez A. Feasibility of kangaroo mother care in Mumbai. Indian Journal of Pediatrics 2005;72(1):35-8. [PUBMED: 15684446]

\section{Kaushal 2005 \{published data only\}}

Kaushal M, Agarwal R, Aggarwal R, Singal A, Upadhyay M, Sirnivas V, et al. Cling wrap, an innovative intervention for temperature maintenance and reduction of insensible water loss in very low-birthweight babies nursed under radiant warmers: a randomised, controlled trial. Annals of Tropical Paediatrics 2005;25(2):111-8. [DOI: 10.1179/146532805X45700]

\section{Kong 2011 \{published data only\}}

Kong YS, Medhurst A, Cheong JLY, Kotsanas D, Jolley D. The effect of incubator humidity on the body temperature of infants born at 28 weeks' gestation or less: a randomised controlled trial. Neonatal, Paediatric and Child Health Nursing 2011;14(2):14-22.

\section{Kumar 2008 \{published data only\}}

Kumar V, Mohanty S, Kumar A, Misra RP, Santosham S, Baqui AH, et al. The Saksham Study Group. Effect of community-based behaviour change management on neonatal mortality in Shivgarh, Uttar Pradesh, India: a clusterrandomised controlled trial. Lancet 2008;372(9644):1151-62. [DOI: 10.1016/S0140-6736(08)61483-X]

\section{Legault 1993 \{published data only\}}

Legault M, Goulet C. Comparative study of two methods of holding premature infants: the kangaroo method versus traditional method [Etude comparative de deux methodes de sortie du premature: methode kangourou versus methode 
traditionnelle]. Canadian Journal of Nursing Research 1993;25(4):67-80. [PUBMED: 10603808]

\section{Lim 2004 \{published data only\}}

Lim NL. Prevention of neonatal hypothermia. Proceedings of the 13th Congress of the Federation-of-Asia-and-OceaniaPerinatal-Societies (FAOPS); April 14-18, 2004; Kuala Lumpur (Malaysia). Bologna (Italy): Medimond International Proceedings Division, 2004.

\section{Loring 2012 \{published data only\}}

Loring C, Gregory K, Gargan B, LeBlanc V, Lundgren D, Reilly J, et al. Tub bathing improves thermoregulation of the late preterm infant. Journal of Obstetric, Gynecologic, and Neonatal Nursing 2012;41(2):171-9. [DOI: 10.1111/j.1552-6909.2011.01332.x]

\section{Ludington-Hoe 1994 \{published data only\}}

Ludington-Hoe SM, Thompson C, Swinth J, Hadeed AJ, Anderson GC. Kangaroo care: research results and practice implications and guidelines. Neonatal Network 1994;13(1):19-27. [PUBMED: 8114658]

\section{Ludington-Hoe 2000 \{published data only\}}

Ludington-Hoe SM, Nguyen N, Swinth JY, Satyshur RD. Kangaroo care compared to incubators in maintaining body warmth in preterm infants. Biological Research for Nursing 2000;2(1):60-73.

\section{Ludington-Hoe 2004 \{published data only\}}

Ludington-Hoe SM, Anderson GC, Swinth JY, Thompson C, Hadeed AJ. Randomized controlled trial of kangaroo care: cardiorespiratory and thermal effects on healthy preterm infants. Neonatal Network 2004;23(3):39-48. [DOI: 10.1891/0730-0832.23.3.39]

\section{Marks 1985 \{published data only\}}

Marks KH, Devenyi AG, Bello ME, Nardis EE, Seaton JF, Ultman JS. Thermal head wrap for infants. Journal of Pediatrics 1985;107(6):956-9. [PUBMED: 4067755]

\section{Mazurek 1999 \{published data only\}}

Mazurek T, Mikiel-Kostyra K, Mazur J, Wieczorek P, Radwanska B, Pachuta-Weigier L. Influence of immediate newborn care on infant adaptation to the environment [Wplyw postepowania z noworodkiem bezpoSrednio po porodzie na cechy jego adaptacji do Srodowiska]. Medycyna Wieku Rozwojowego 1999;3(2):215-24. [PUBMED: 10910653]

\section{Medves 2004 \{published data only\}}

Medves JM, O'Brien B. Effect of bather and location of first bath on maintaining thermal stability in newborns. Journal of Obstetric, Gynecologic and Neonatal Nursing 2004;33(2):175-82. [PUBMED: 15095796]

\section{Meyer 2001 \{published data only\}}

Meyer MP, Payton MJ, Salmon A, Hutchinson C, de Klerk A. A clinical comparison of radiant warmer and incubator care for preterm infants from birth to 1800 grams. Pediatrics 2001;108(2):395-401. [PUBMED: 11483805]

\section{Meyer 2007 \{published data only\}}

Meyer MP, Bold GT. Admission temperatures following radiant warmer or incubator transport for preterm infants $<28$ weeks: a randomised study. Archives of Disease in Childhood Fetal and Neonatal Edition 2007;92(4):F295-7. [DOI: 10.1136/ adc.2006.107128]

Meyer 2015 \{published and unpublished data\}

ACTRN12609000694213. Giving humidified gases to preterm infants from birth [Effect of humidified and non humidified $T$ piece resuscitation on admission temperatures in preterm infants $<32$ weeks: a randomised controlled trial]. http://apps.who.int/trialsearch/Trial2.aspx? TrialID=ACTRN12609000694213 (first received 11 January 2010).

* Meyer MP, Hou D, Ishrar NN, Dito I, te Pas AB. Initial respiratory support with cold, dry gas versus heated humidified gas and admission temperature of preterm infants. Journal of Pediatrics 2015;166(2):245-50. [DOI: 10.1016/j.jpeds.2014.09.049]

Miles 2006 \{published data only\}

Miles R, Cowan F, Glover V, Stevenson J, Modi N. A controlled trial of skin-to-skin contact in extremely preterm infants. Early Human Development 2006;82(7):447-55. [DOI: 10.1016/ j.earlhumdev.2005.11.008]

\section{Monterosso 1999 \{published data only\}}

Monterosso L, Percival P, Cole J, Evans SF. Effect of nappy liners on temperature stability in very preterm infants. Journal of Paediatrics and Child Health 1999;35(4):363-6. [PUBMED: 10457293]

\section{Moore 2007 \{published data only\}}

Moore ER, Anderson GC. Randomized controlled trial of very early mother-infant skin-to-skin contact and breastfeeding status. Journal of Midwifery \& Women's Health 2007;52(2):116-25. [DOI: 10.1016/j.jmwh.2006.12.002]

Nagai 2010 \{published data only\}

Nagai S, Andrianarimanana D, Rabesandratana NH, Yonemoto N, Nakayama T, Mori R. Earlier versus later continuous kangaroo mother care (KMC) for stable low-birthweight infants: a randomised controlled trial. Acta Paediatrica 2010;99(6):827-35. [DOI: 10.1111/j.1651-2227.2009.01676.x]

Nako 2000 \{published data only\}

Nako Y, Harigaya A, Tomomasa T, Morikawa A, Amada M, Kijima C, et al. Effects of bathing immediately after birth on early neonatal adaptation and morbidity: a prospective randomised comparative study. Pediatrics International 2000;42(5):517-22. [PUBMED: 11059542]

\section{NCT00531492 \{published data only\}}

NCT00531492. Efficacy of Continuous Skin to Skin Care (Technique Kangaroo: TK) After Birth for Low Birth Weight (LBW) Infants and Their Mothers in Developing Countries (EtudeTK) [Randomized controlled trial of continuous skin to skin care (technique kangourou: TK) for low birth weight (LBW) infants and their mothers at University Hospital Center of Mahajanga (Centre Hospitalier Universitaire de Mahajanga: 
CHUM), Madagascar]. clinicaltrials.gov/ct2/show/NCT00531492 (17 September 2007).

\section{NCT01604421 \{published data only\}}

NCT01604421. Evaluation of Use of Plastic Bags to Prevent Neonatal Hypothermia - Part II [Randomized Evaluation of the Use of Plastic Bags to Prevent Neonatal Hypothermia in Developing Countries - Part II]. clinicaltrials.gov/ct2/show/ NCT01604421 (21 May 2012).

\section{NCT01604434 \{published data only\}}

NCT01604434. Evaluation of Use of Plastic Bags to Prevent Neonatal Hypothermia - Part III [Randomized evaluation of the use of plastic bags to prevent neonatal hypothermia in developing countries - Part III . clinicaltrials.gov/ct2/show/ NCT01604434 (21 May 2012).

\section{NCT01604447 \{published data only\}}

NCT01604447. Evaluation of Use of Plastic Bags to Prevent Neonatal Hypothermia - Part IV [Randomized evaluation of the use of plastic bags to prevent neonatal hypothermia in developing countries - Part $\mathbb{V}$ ] . clinicaltrials.gov/ct2/show/ NCT01604447 (21 May 2012).

\section{NCT01604460 \{published data only\}}

NCT01604460. Evaluation of Use of Plastic Bags to Prevent Neonatal Hypothermia - Part V [Randomized evaluation of the use of plastic bags to prevent neonatal hypothermia in developing countries - Part V ] . clinicaltrials.gov/ct2/show/ NCT01604460 (21 May 2012).

\section{NCT01776281 \{published data only\}}

NCT01776281. Effects of kangaroo mother care among low birthweight (LBW) and preterm infants (KMC) [Effects of Kangaroo Mother Care Among LBW and Preterm Infants:A Randomized Control Trial in Karachi]. clinicaltrials.gov/ct2/ show/NCT01776281 (22 January 2013).

\section{New 2012 \{published data only\}}

New K, Flint A, Bogossian F, East C, Davies MW. Transferring preterm infants from incubators to open cots at $1600 \mathrm{~g}$ : a multicentre randomised controlled trial. Archives of Disease in Childhood. Fetal and Neonatal Edition 2012;97(2):F88-92. [DOI: 10.1136/adc.2011.213587]

Nimbalker 2014 \{published data only\}

Nimbalkar SM, Patel VK, Patel DV, Nimbalkar AS, Seith A, Phatak A. Effect of early skin-to-skin contact following normal delivery on incidence of hypothermia in neonates more than 1800g: randomised control trial. Journal of Perinatology 2014;34(5):364-8. [DOI: 10.1038/jp.2014.15]

\section{Nopper 1996 \{published data only\}}

Nopper AJ, Horri KA, Sookdeo-Drost S, Wang TH, Mancini AJ, Lane AT. Topical ointment therapy benefits premature infants. Journal of Pediatrics 1996;128(5 Pt 1):660-9. [PUBMED: 8627439]

\section{Nuntnarumit 2004 \{published data only\}}

Nuntnarumit P, Deesomchok A. Efficacy of polyethylene occlusive skin wrapping with plastic bag in preventing hypothermia in infants less than 32 weeks' gestational age at delivery. Proceedings of the Pediatric Academic Societies' Annual Meeting; 2004 May 1-4; San Francisco, (CA). The Woodlands (TX): Pediatric Academic Societies, 2004

Nuntnarumit 2013 \{published data only\}

Nuntmarumit P, Swatesutipun B, Udomsubpayakul U, Thanacharoenpipat $P$. A randomised controlled trial of plastic drape for prevention of hypothermia during umbilical catheterization. American Journal of Perinatology 2013;30(10):839-42. [DOI: 10.1055/s-0033-1333670]

Omene 1978 \{published data only\}

Omene JA, Diejomaoh FM, Faal M, Diakparomre MA, Obiaya M. Heat loss in Nigerian newborn infants in the delivery room. International Journal of Gynaecology and Obstetrics 1978-1979;16(4):300-2. [PUBMED: 35391]

Pattinson 2005 \{published data only\}

Pattinson RC, Arsalo I, Bergh AM, Malan AF, Patrick M, Phillips N. Implementation of kangaroo mother care: a randomised trial of two outreach strategies. Acta Paediatrica 2005;94(7):924-7. [PUBMED: 10.1080/08035250510028399]

Punnahitananda 2008 \{published data only\}

Punnahitananda S, Thaithumyanon P. Efficacy of polyethylene plastic wrap for the prevention of hypothermia during immediate postnatal period in preterm infants: a randomised controlled trial. Proceedings of the Pediatric Academic Societies' Meeting; 2008 May 2-6, 2008; Honolulu (Hawaii). The Woodlands (TX): Pediatric Academic Societies, 2008:EPAS2008:634458.10.

Punthmatharith 2001 \{published data only\}

Punthmatharith B. Randomized controlled trial of early kangaroo (skin-to-skin) care: effects on maternal feelings, maternal-infant interaction and breastfeeding success in Thailand [dissertation]. Clevland $(\mathrm{OH})$ : Case Western Reserve University, 2001.

Raman 1992 \{published data only\}

Raman S, Shahla A. Temperature drop in normal term newborn infants born at the University Hospital, Kuala Lumpar. Australian and New Zealand Journal of Obstetrics and Gynaecology 1992;32(2):117-9.

\section{Ramanathan 2001 \{published data only\}}

Ramanathan K, Paul VK, Deorari AK, Taneja U, George G. Kangaroo mother care in very low birth weight infants. Indian Journal of Pediatrics 2001;68(11):1019-23. [PUBMED: 11770234]

Ramani 2015 \{published data only\}

Ramani M, Choe EA, Major M, Newton R, Carlo W. Randomised trial of skin-to-skin contact to prevent hypothermia in term neonates. Journal of Investigative Medicine. 2015; Vol. 63:418.

Roberts 2000 \{published data only\}

Roberts KL, Paynter C, McEwan B. A comparison of kangaroo mother care and conventional cuddling care. Neonatal Network 2000;19(4):31-5. [DOI: 10.1891/0730-0832.19.4.31] 
Rojas 2001 \{published data only\}

Rojas MA, Kaplan M, Mayes L, Quevedo ME, Foster LB, Sherwonit E, et al. Traditional holding (TH) and skin-to-skin care (SSC) for newborn infants $<=1500$ grams. A randomised controlled trial. Pediatric Research 2001;49:360A.

Rojas 2003 \{published data only\}

Rojas MA, Kaplan M, Quevedo M, Sherwonit E, Foster LB, Ehrenkranz RA, et al. Somatic growth of preterm infants during skin-to-skin care versus traditional holding: a randomised, controlled trial. Journal of Developmental and Behavioral Pediatrics 2003;24(3):163-8. [PUBMED: 12806228]

\section{Ruiz 1998 \{published data only\}}

Ruiz JG. Kangaroo mother versus 'traditional' care for newborn infants $<=2000$ grams: a randomised control trial. Journal of Clinical Epidemiology 1998;51(Suppl 1):S12. [DOI: http:// dx.doi.org/10.1016/S0895-4356(98)90036-1]

\section{Sankaranarayanan 2005 \{published data only\}}

Sankaranarayanan K, Mondkar JA, Chauhan MM, Mascarenhas BM, Mainkar AR, Salvi RY. Oil massage in neonates: an open randomised controlled study of coconut versus mineral oil. Indian Pediatrics 2005;42(9):877-84. [PUBMED: 16208048]

\section{Sarman 1989 \{published data only\}}

Sarman I, Tunell R. Providing warmth for preterm babies by a heated, water filled mattress. Archives of Disease in Childhood 1989;64(1 Spec No):29-33. [PUBMED: 2923482]

\section{Sarman 1992 \{published data only\}}

Sarman I. Thermal responses and heart rates of low-birthweight premature babies during daily care on a heated, waterfilled mattress. Acta Paediatrica 1992;81(1):15-20. [PUBMED: 1600297]

\section{Short 1998 \{published data only\}}

Short MA. A comparison of temperature in VLBW infants swaddled versus unswaddled in a double-walled incubator in skin control mode. Neonatal Network 1998;17(3):25-31. [PUBMED: 9601347]

\section{Sloan 1994 \{published data only\}}

Sloan NL, Camacho WL, Rojas EP, Stern C, Maternidad Isidro Ayora Study Team. Kangaroo mother method: randomised controlled trial of an alternative method of care for stabilised low-birthweight infants. The Lancet 1994;344(8925):782-5. [PUBMED: 7916073]

\section{Sloan 2008 \{published data only\}}

Sloan NL, Ahmed S, Mitra SN, Choudhury N, Chowdhury M, Rob U, et al. Community-based kangaroo mother care to prevent neonatal and infant mortality: a randomised, controlled cluster trial. Pediatrics 2008;121(5):e1047-59. [DOI: 10.1542/ peds.2007-0076]

\section{Srivastava 2014 \{published data only\}}

Srivastava S, Gupta A, Bhatnagar A, Dutta S. Effect of very early skin to skin contact on success at breastfeeding and preventing early hypothermia in neonates. Indian Journal of Public Health 2014;58(1):22-6. [DOI: 10.4103/0019-557X.128160]

\section{Stirparo 2013 \{published data only\}}

Stirparo S, Farcomeni A, Laudani A, Capogna G. Maintaining neonatal normothermia during WHO recommended skin-toskin contact in the setting of cesarean section under regional anesthesia. Open Journal of Anesthesiology 2013;3:186-8. [DOI: 10.4236/ojanes.2013.33043]

\section{St John 2011 \{published data only\}}

St John LT, Christie LH, Burchfield J, Wyckoff MH. Effectiveness of plastic lined hats for prevention of hypothermia in premature newborns in the delivery room. Proceedings of the Pediatric Academic Societies and Asian Society for Pediatric Research Joint Meeting; 2011; Apr 30 - May 3 2011; Denver (CO). Woodlands (TX): Pediatric Academic Societies, 2011.

\section{Subramanian 2012 \{published data only\}}

Subramanian S, Gavvhane S, Murki S, Gaddam P. Effect of kangaroo mother care (KMC) in comparison with conventional method of care $(\mathrm{CMC})$ on growth and breastfeeding in very low birthweight (VLBW) infants at 40 weeks of gestational age: a randomised trial. Proceedings of the Pediatric Academic Societies' Annual Meeting; 2012 April 28 - May 1; Boston (MA). The Woodlands (TX): Pediatric Academic Societies, 2012.

\section{Suman 2008 \{published data only\}}

Suman RP, Udani R, Nanavanti R. Kangaroo mother care low birth weight infants: a randomised controlled trial. Indian Pediatrics 2008;45(1):17-23. [PUBMED: 18250500]

\section{Swarnkar 2016 \{published data only\}}

Swarnkar K, Vagha J. Effect of kangaroo mother care on growth and morbidity pattern in low birth weight infants. Journal of Krishna Institute of Medical Sciences University 2016;5(1):91-9.

\section{Syfrett 1993 \{published data only\}}

Syfrett BE. Very early and virtually continuous kangaroo care for 34 to 36 week gestation preterm infants: effects on temperature, breastfeeding, supplementation, and weight [dissertation]. Tallahassee (FL): University of Florida, 1993.

\section{Trevisanuto 2009 \{published data only\}}

Trevisanuto D, Fasson A, Doglioni N, Biason S, Micaglio M, Zanardo V. A new device (cicogna) for transferring the neonate in the delivery room setting: a randomised, controlled trial. Journal of Maternal-Fetal and Neonatal Medicine 2009;22(2):148-51.

\section{Tsogt 2005 \{published data only\}}

Tsogt B, Maniseki-Holland S, Pollock J, Blair P, Fleming P. The development of thermoregulation in a harsh environment: a prospective controlled study of the effects of swaddling on infants' thermal balance in a Mongolian winter. Proceedings of the Neonatal Societies Meeting; 2005 June 30 - July 1; Bristol (UK). London (UK): The Neonatal Societies, 2005.

\section{Tsogt 2016 \{published data only\}}

Tsogt B, Manaseki-Holland S, Pollock J, Blair PS, Flemming P. Thermoregulatory effects of swaddling in Mongolia: a randomised controlled study. Archives of Diseases in Childhood 2016;101(2):152-60. [DOI: 10.1136/archdischild-2014-307908] 
Vaidya 2005 \{published data only\}

Vaidya K, Sharma A, Dhungel S. Effect of early mother-baby close contact over the duration of exclusive breastfeeding. Nepal Medical College Journal 2005;7(2):138-40. [PUBMED: 16519083]

van den Bosch 1990 \{published data only\}

van den Bosch CA, Bullough CH. Effect of early suckling on term neonates' core body temperature. Annals of Tropical Paediatrics 1990;10(4):347-53. [PUBMED: 1708961]

\section{van den Bosch 1996 \{published data only\}}

van den Bosch CA, Nhlane C, Kazembe P. Trial of polyethylene tobacco-wrap in prevention of hypothermia in neonates less than 1500 grams. Tropical Doctor 1996;26(1):26-8.

\section{Villalon 1992 \{published data only\}}

Villalon HU, Alvarez PC, Barria EH, Caneleo DH, Carrillo LM, Duran SG, et al. Effect of early skin-to-skin contact on temperature regulation, heart rate, and respiratory rate in healthy, full term newborns [Contacto percoz piel a piel: efecto sobre los parametros fisiologicos en las cuatro horas posteriores al parto en recien nacidos de termino sanos]. Revista Chilena de Pediatria 1992;63(3):140-4.

\section{Whitelaw 1988 \{published data only\}}

Whitelaw A, Heisterkamp G, Sleath K, Acolet D, Richards M. Skin to skin contact for very low birthweight infants and their mothers. Archives of Disease in Childhood 1988;63(11):1377-81. [PUBMED: 3060024 ]

\section{Worku 2005 \{published data only\}}

Worku B, Kassie A. Kangaroo mother care: a randomised controlled trial on effectiveness of early kangaroo mother care for the low birthweight infants in Addis Ababa, Ethiopia. Journal of Tropical Pediatrics 2005;51(2):93-7. [10.1093/tropej/fmh085]

\section{Yeh 1980 \{published data only\}}

Yeh TF, Voora S, Lilien LD, Matwynshym J, Srinivasan G, Pildes RS. Oxygen consumption and insensible water loss in premature infants in single-versus double-walled incubators. Journal of Pediatrics 1980;97(6):967-71. [PUBMED: 6777480]

\section{Yentur 2009 \{published data only\}}

Yentur EA, Topcu I, Ekici Z, Ozturk T, Keles GT, Civi M. The effect of epidural and general anesthesia on newborn rectal temperature at elective cesarean section. Brazilian Journal of Medical and Biological Research 2009;42(9):863-7. [PUBMED: 19738991]

\section{Yokoyama 2009 \{published data only\}}

Yokoyama K, Suzuki M, Shimada Y, Matsushima T, Bito H, Sakamoto A. Effect of administration of pre-warmed intravenous fluids on the frequency of hypothermia following spinal anesthesia for Cesarean delivery. Journal of Clinical Anesthesia 2009;21(4):242-8. [DOI: 10.1016/ j.jclinane.2008.12.010]

\section{References to studies awaiting assessment}

Ahmed 2013 \{published data only\}

Anmed BT, Hussein M, Monir H. Effect of plastic bag (vinyl bags) on prevention of hypothermia in preterm infants. Medical Journal of Cairo University 2013;81(2):169-73.

\section{Castro 2007 \{published data only\}}

Castro MM, Repeto M, Cancela MJ, Latof M, Hernandez C, Bustos R. Clinical experience in the use of polyethylene bags to reduce hypothermia in newborns less than $1000 \mathrm{~g}$ [Experiencia clinica en la utilizacion de bolsa de polietileno para disminuir la hipotermia en el recien nacido menor de 1000 gramos]. Archivos de Pediatria del Uruguay 2007;78(2):110-4.

Nimbalkar 2015 \{published data only\}

Nimbalkar SM, Khanna AK, Patel DV, Nimbalkar AS, Phatak AG. Comparison of polythene occlusive skin wrapping with routine cloth wrapping in reducing heat loss during transportation in preterm neonates (<34 weeks) after delivery: randomised control trial. Proceedings of the Pediatric Academic Societies' Annual Meeting; 2015 April 25-28; San Diego, (CA). The Woodlands (TX): Pediatric Academic Societies, 2015.

\section{Shafie 2017 \{published data only\}}

Shafie H, Syed Z, Adli A, Shareena I, Rohanna J. Polyethylene versus cotton cap as an adjunct to body wrap in preterm infants. Pediatrics International 2017;59:776-80. [DOI: 10.1111/ ped.13285]

\section{References to ongoing studies \\ CRTI/2014/11/005200 \{published data only\}}

CRTI/2014/11/005200. Prevention of hypothermia during transport of newborns more than or equal to $1.5 \mathrm{KG}$ using EMBRACE [Embrace vs conventional care during transport of newborn more than or equal to 1500 grams: a randomised controlled trial]. http://ctri.nic.in/Clinicaltrials/pmaindet2.php? trialid $=10416 \&$ EncHid=\&userName=embrace (first received 13 November 2014).

\section{CTRI/2016/02/006673 \{published data only\}}

CTRI/2016/02/006673. A study to evaluate the effectiveness of adding portable warmer bed called Embrace Thermopod or polythene bag to standard care for maintaining the temperature of low birth weight babies soon after birth and during transport from the delivery room to nursery [An open label randomised trial comparing standard thermal care with EMBRACE thermopod or polythene bag to prevent hypothermia soon after birth in low birth weight babies POEMS TRIAL]. http://apps.who.int/trialsearch/Trial2.aspx? TrialID=CTRI/2016/02/006673 (first received 12 January 2015).

\section{ISRCTN13184012 \{published data only\}}

ISRCTN13184012. Prevention of hypothermia, in the immediate neonatal period, in infants with gestational age $\leq 28$ weeks: a comparative study of the effectiveness of two models of polyethylene bags. http://www.isrctn.com/ ISRCTN13184012 (first received 16 February 2016). [DOI: 10.1186/ISRCTN13184012] 


\section{NCT01604317 \{published data only\}}

NCT01604317. Randomized evaluation of the use of plastic bags to prevent neonatal hypothermia in developing countries - part I . clinicaltrials.gov/ct2/show/NCT01604317 (first received 21 May 2012).

\section{NCT02189746 \{published data only\}}

NCT02189746. Kangaroo mother care to prevent hypothermia in preterm infants [A randomized trial of kangaroo mother care to prevent neonatal hypothermia - trials $1 \mathrm{~A} \& 1 \mathrm{~B}]$. https:// www.clinicaltrials.gov/ct2/show/NCT02189746?term=Prevent +AND+hypothermia+AND+infant\&rank=1 (first received 11 July 2014).

\section{NCT02250079 \{published data only\}}

NCT02250079. Polyethylene body bags as an alternative to radiant heat lamp during the neonatal adaptation in infants older than 29 weeks. [Features and Differences Between Neonates Undergoing Body Bag With Polyethylene and Conventional Drying During Neonatal Adaptation in Maternal and Child Headquarters Hospital and the Victoria Hospital Engativá, Bogotá, 2013]. http://clinicaltrials.gov/ct2/show/ record/NCT02250079 (first received 8 September 2014).

NCT02311972 \{published data only\}

NCT02311972. Temperature monitoring with innerSense temperature sensor/feeding tube after birth through stabilisation in VLBW infants. http://www.clinicaltrials.gov/ct2/ show/NCT02311972 (first received 04 December 2016).

\section{Additional references}

\section{Abd-El Hamid 2012}

Abd-El Hamid S, Badr-El Din MM, Dabous NI, Saad KM. Effect of the use of a polyethylene wrap on the morbidity and mortality of very low birthweight infants in Alexandria University Children's Hospital. Journal of the Egyptian Public Health Association 2012;87(5-6):104-8. [DOI: 10.1097/01.EPX.0000421565.24496.d9]

\section{Adamson 1965}

Adamson SK Jr, Gandy GM, James LS. The influence of therma factors upon oxygen consumption of the newborn human infant. Journal of Pediatrics 1965;66:495-508. [PUBMED: 14264308]

\section{Adamson 1965a}

Adamson SK Jr, Towell ME. Thermal homeostasis in the fetus and newborn. Anesthesiology 1965;26:531-48. [PUBMED: 14313463]

\section{Ali 2012}

Ali R, Mirza R, Qadir M, Ahmed S, Bhatti Z, Demas S. Neonatal hypothermia among hospitalized high risk newborns in a developing country. Pakistan Journal of Medical Sciences 2012;28(1):49-53.

\section{Almeida 2009}

Almedia PG, Chandley J, Davis J, Harrigan RC. Use of heated gel mattress and its impact on admission temperature of very low birth-weight infants. Advances in Neonatal Care 2009;9(1):34-9. [DOI: 10.1097/01.ANC.0000346094.28110.11]

\section{Arrindell 2012}

Arrindell EL, Dhanireddy R, Talati A. Admission hypothermia reduction and its impact on morbidity. Proceedings of the Pediatric Academic Societies' Annual Meeting; 2012 April 28May 1; Boston (MA). Pediatric Academic Societies: Marathon Multimedia LLC [www.pas.meeting.org/], 2012.

\section{Bailey 2000}

Bailey J, Rose P. Temperature measurement in the preterm infant: a literature review. Journal of Neonatal Nursing 2000;6(1):28-32.

\section{Ball 2010}

Ball M, Hillman NH, Kallapur SG, Polglase GR, Jobe AH, Pillow JJ. Body temperature effects on lung injury in ventilated preterm lambs. Resuscitation 2010;81:749-54.

\section{Baumgart 1981}

Baumgart S, Engle WD, Fox WW, Polin RA. Effect of heat shielding on convective and evaporative heat losses and on radiant heat transfer in the premature infant. Journal of Pediatrics 1981;99(6):948-56. [PUBMED: 7310591]

\section{Bell 1978}

Bell MJ, Ternberg JL, Feigin RD, Keating JP, Marshall R, Barton L, et al. Neonatal necrotizing enterocolitis. Therapeutic decisions based on clinical staging. Annals of Surgery 1978;187(1):1-7. [PUBMED: 413500]

\section{Bell 1980}

Bell EF, Weinstein MR, Oh W. Heat balance in premature infants: comparative effects of convectively heated incubator and radiant warmer, with and without plastic heat shield. Journal of Pediatrics 1980;96(3 Pt 1):460-5. [PUBMED: 7359242]

\section{Bhatt 2007}

Bhatt DR, White R, Martin G, Van Marter LJ, Finer N, Goldsmith JP, et al. Transitional hypothermia in preterm newborns. Journal of Perinatology 2007;27(Suppl 2):S45-7. [DOI: 10.1038/sj.jp.7211842]

\section{Bloom 1994}

Bloom RS, Cropley C, the AHA/AAP Neonatal Resuscitation Steering Committee, editors. Textbook of Neonatal Resuscitation. 3rd Edition. Dallas (TX): American Heart Association, 1994:2-9.

\section{Boo 2013}

Boo NY, Cheah IGS. Admission hypothermia among VLBW infants in Malaysian NICUs. Journal of Tropical Pediatrics 2013;59(6):447-52

\section{Borse 1997}

Borse N, Deodhar J, Pandit AN. Effects of thermal environment on neonatal thermoregulation. Indian Pediatrics 1997;34(8):718-20. [PUBMED: 9492401] 


\section{Boundy 2016}

Boundy EO, Dastjerdi R, Spiegelman D, Fawzi WW, Missmer SA, Lieberman E, et al. Kangaroo mother care and neonatal outcomes: a meta-analysis. Pediatrics 2016;137(1):1-16. [DOI: 10.1542/peds.2015-2238]

\section{Boutron 2008}

Boutron I, Moher D, Altman DG, Schultz KF, Ravard P, CONSORT Group. Extending the CONSORT statement to randomised trials of nonpharmacologic treatment: explanation and elaboration. Annals of Internal Medicine 2008;148(4):295-309. [PUBMED: 18283207]

\section{Bredemeyer 2005}

Bredemeyer S, Reid S, Wallace M. Thermal management of preterm births. Journal of Advanced Nursing 2005;52(5):482-9. [DOI: 10.1111/j.1365-2648.2005.03616.x]

\section{Britton 1980}

Britton GR. Early mother-infant contact and infant temperature stabilization. JOGN Nursing; Journal of Obstetric, Gynecologic, and Neonatal Nursing 1980;9(2):84-6. [PUBMED: 6900787]

\section{Bruck 1961}

Bruck K. Temperature regulation in the newborn infant. Biology of the Neonate 1961;3:65-119. [DOI: 10.1159/000239810]

\section{Brun 1997}

Brun C, Stokvad P, Alsbjorn BF. Burn wounds after resuscitation of a newborn girl [Brandsar efter genoplivning af en nyfodt pige]. Ugestrift for Laeger 1997;159(44):6531-2. [PUBMED: 9411974]

\section{Buczkowski-Bickmann 1992}

Buczkowski-Bickmann MK. Thermoregulation in the neonate and the consequences of hypothermia. CRNA: The Clinical Forum for Nurse Anesthetists 1992;3(2):77-82. [PUBMED: 1483148]

\section{Caldas 2017}

Caldas JPS, Millen FC, Camargo JF, Castro PAC, Camilo ALDF, Marba STM. Effectiveness of a measure program to prevent admission hypothermia in very low-birth weight preterm infants. Jornal de Pediatria 6 September 2017 [Epub ahead of print]. [DOI: 10.1016/j.jped.2017.06.016]

\section{Capobianco 1980}

Capobianco JA. Keeping the newborn warm: how to safeguard the infant against life-threatening heat loss. Nursing 1980;10(5):64-7. [PUBMED: 6899898]

\section{Carmichael 2007}

Carmichael A, McCullough S, Kempley ST. Critical dependence of acetate thermal mattress on gel activation temperature. Archives of Disease in Childhood Fetal and Neonatal Edition 2007;92(1):F44-5. [DOI: 10.1136/adc.2006.096297]

\section{Carroll 2010}

Carroll PD, Nankervis CA, Giannone PJ, Cordero L. Use of polyethylene bags in extremely low birthweight infant resuscitation for the prevention of hypothermia. Journal of Reproductive Medicine 2010;55(1-2):9-13. [PUBMED: 20337201]

\section{Chadd 1972}

Chadd MA, Gray OP. Hypothermia and coagulation defects in the newborn. Archives of Disease in Childhood 1972;47(255):819-21. [PUBMED: 5086515]

\section{Chang 2015}

Chang HY, Sung YH, Wang SM, Lung HL, Chang JH, Hsu CH, et al. Short and long term outcomes in very low birth weight infants with admission hypothermia. PLoS ONE 2015;10(7):e0131976. [PUBMED: 10.1371/journal.pone.0131976]

\section{Chitty 2013}

Chitty H, Wyllie J. Importance of maintaining the newly born temperature in the normal range from delivery to admission. Seminars in Fetal and Neonatal Medicine 2013;18(6):362-8. [DOI: 10.1016/j.siny.2013.08.002]

\section{Christensson 1988}

Christensson K, Ransjo-Arvidson AB, Kakoma C, Lungu F, Darkwah G, Chikamata D, et al. Midwifery care routines and prevention of heat loss in the newborn: a study in Zambia. Journal of Tropical Pediatrics 1988;34(5):208-12. [PUBMED: 3199487]

\section{Conde-Agudelo 2016}

Conde-Agudelo A, Diaz-Rossello JL. Kangaroo mother care to reduce morbidity and mortality in low birthweight infants. Cochrane Database of Systematic Reviews 2016, Issue 8. [DOI: 10.1002/14651858.CD002771.pub4]

\section{Cordaro 2012}

Cordaro T, Gibbons Phalen A, Zukowsky K. Hypothermia and occlusive skin wrap in the low birthweight premature infant: an evidentiary review. Newborn and Infant Nursing Reviews 2012;12(2):78-85. [DOI: 10.1053/j.nainr.2012.03.001]

\section{Costeloe 2000}

Costeloe K, Hennessy E, Gibson AT, Marlow N, Wilkinson AR. The EPICure study: outcomes to discharge from hospital for infants born at the threshold of viability. Pediatrics 2000;106(4):659-71. [PUBMED: 11015506]

\section{Davis 1980}

Davis $V$. The structure and function of brown adipose tissue in the neonate. JOGN Nursing; Journal of Obstetric, Gynecologic, and Neonatal Nursing 1980;9(6):368-72. [PUBMED: 6906468]

\section{de Almeida 2014}

de Almeida MF, Guinsburg R, Sancho GA, Rosa IRM, Lamy ZC, Martinez FE, et al. Brazillian Network on Neonatal Research. Hypothermia and early neonatal mortality in preterm infants. Journal of Pediatrics 2014;164(2):271-5.e1. [DOI: 10.1016/ j.jpeds.2013.09.049]

\section{DeMauro 2013}

DeMauro SB, Douglas E, Karp K, Schmidt B, Patel J, Kronberger $A$, et al. Improving delivery room management for very preterm infants. Pediatrics 2013;132(4):e1018-25. [DOI: 10.1542/peds.2013-0686] 


\section{Du 1969}

Du JN, Oliver TK Jr. The baby in the delivery room. A suitable microenvironment. Journal of the American Medical Association 1969;207(8):1502-4. [PUBMED: 5818277]

\section{El-Naggar 2012}

El-Naggar W, McNamara PJ. Delivery room resuscitation of preterm infants in Canada: current practice and views of neonatologists at level III centers. Journal of Perinatology 2012;32(7):491-7. [DOI: 10.1038/jp.2011.128]

\section{Elliott 1957}

Elliott RI, Mann TP. Neonatal cold injury due to accidental exposure to cold. Lancet 1957;272(6962):229-34. [PUBMED: 13399181]

\section{Fastman 2014}

Fastman BR, Howell EA, Holzman I, Kleiman LC. Current perspectives on temperature management and hypothermia in low birth weight infants. Newborn and Infant Nursing Reviews 2014;14(2):50-5.

\section{Gandy 1964}

Gandy GM, Adamsons K Jr, Cunningham N, Silverman WA, James LS. Thermal environment and acid-base homeostasis in human infants during the first few hours of life. Journal of Clinical Investigation 1964;43:751-8. [DOI: 10.1172/JCI104959]

\section{Glass 1968}

Glass L, Silverman WA, Sinclair JC. Effects of the thermal environment on cold resistance and growth of small infants after the first week of life. Pediatrics 1968;41(6):1033-46. [PUBMED: 5652915]

\section{Godhamgaonkar 2011}

Godhamgaonkar A, Parmar V, Kyte E, Sarwar M. The use of polyethylene bags for delivery-room thermoregulation in premature babies across England. Archives Diseases in Childhood Fetal and Neonatal Edition 2011;96 Suppl 1:Fa36. [DOI: 10.1136/archdischild.2011.300164.65]

\section{GRADEpro GDT [Computer program]}

GRADE Working Group, McMaster University (developed by Evidence Prime), 2015. Available from gradepro.org. GRADEpro GDT. Version accessed 30 June 2016. Hamilton (ON): GRADE Working Group, McMaster University (developed by Evidence Prime), 2015. Available from gradepro.org.

\section{Hammarlund 1979}

Hammarlund K, Sedin G. Transepidermal water loss in newborn infants. III. Relation to gestational age. Acta Paediatrica Scandinavica 1979;68(6):795-801. [PUBMED: 539401]

\section{Hammarlund 1980}

Hammarlund K, Nilsson GE, Oberg PA, Sedin G. Transepidermal water loss in newborn infants. V. Evaporation from the skin and heat exchange during the first hours of life. Acta Paediatrica Scandinavica 1980;69(3):385-92. [DOI: 7376866]

\section{Harer 2017}

Harer MW, Vergales B, Cady T, Early A, Chisholm C, Swanson JR. Implementation of a multidisciplinary guideline improves preterm infant admission temperatures. Journal of Perinatology 2017;37:1242-7. [DOI: 10.1038/.jp.2017.112]

\section{Harned 1970}

Harned HS Jr, Herrington RT, Ferreiro Jl. The effects of immersion and temperature on respiration in newborn lambs. Pediatrics 1970;45(4):598-605. [PUBMED: 5438162]

\section{Hey 1970}

Hey EN, Katz G. The optimal thermal environment for naked babies. Archives of Disease in Childhood 1970;45(241):328-34. [PUBMED: 5427846]

\section{Hey 1975}

Hey E. Thermal neutrality. British Medical Bulletin 1975;31(1):69-74. [PUBMED: 1181001]

\section{Higgins 2003}

Higgins JPT, Thompson SG, Deeks JJ, Altman GD. Measuring inconsistency in meta-analyses. BMJ 2003;327(7414):557-60. [DOI: 10.1136/bmj.327.7414.557]

\section{Higgins 2011}

Higgins JP, Green S, editor(s). Cochrane Handbook for Systematic Reviews of Interventions Version 5.1.0 (updated March 2011). The Cochrane Collaboration, 2011. Available from handbook.cochrane.org.

\section{Ho 2007}

Ho QT, Lee HC, Rhine WD. The impact of a quality improvement project to reduce hypothermia in premature newborns. Proceedings of the Pediatric Academic Societies' Annual Meeting; 2007 May 5-8; Toronto (Canada). The Woodlands (TX): Pediatric Academic Socities, 2007.

\section{Ibrahim 2009}

Ibrahim CP, Yoxall CW. Use of plastic bags to prevent hypothermia at birth in preterm infants - do they work at lower gestations?. Acta Paediatrica 2009;98(2):256-60. [DOI: 10.1111/ j.1651-2227.2008.01076.x]

\section{Ibrahim 2010}

Ibrahim CP, Yoxall CW. Use of self heating gel mattresses eliminates admission hypothermia in infants born below 28 weeks gestation. European Journal of Pediatrics 2010;169(7):795-9. [DOI: 10.1007/s00431-009-1113-y]

\section{ILCOR 2006}

The International Liaison Committee on Resuscitation. The International Liaison Committee on Resuscitation (ILCOR) consensus on science with treatment recommendations for pediatric and neonatal patients: neonatal resuscitation. Pediatrics 2006;117(5):e978-88. [DOI: 10.1542/peds.2006-0350]

\section{Jaleel 2011}

Jaleel SA, McDermott C, McCallion N. Incidence of hypothermia in low birthweight infants admitted to the neonatal intensive 
care unit between 2002 and 2009. Pediatric Research 2010;68:479-80. [DOI: 10.1203/00006450-201011001-00961]

\section{Kaplan 2009}

Kaplan HC, Breig R, Meek-Silvers D, Hallett-Voss C, Corcoran N, Falciglia $\mathrm{H}$, et al. Intervention to reduce delivery room cold stress in very low birthweight infants. Proceedings of the Pediatric Academic Societies' Annual Meeting; 2009 May 2-5; Baltimore (MD). The Woodlands (TX): Pediatric Academic Societies, 2009.

\section{Karlsson 2012}

Karlsson v, Heinemann AB, Sjors G, Nykvist KH, Agren J. Early skin-to-skin care in extremely preterm infants: thermal balance and care environment. Journal of Pediatrics 2012;161(3):422-6. [DOI: 10.1016/j.jpeds.2012.02.034]

\section{Kattwinkel 2010}

Kattwinkel J, Perlman JM, Aziz K, Colby C, Fairchild K, Gallagher J, et al. Part 15: neonatal resuscitation: 2010 American Heary Association guidelines for cardiopulmonary resuscitation and emergency cardiovascular care. Circulation 2010;122(18 Suppl 3):S090-19. [DOI: 10.1161/ CIRCULATIONAHA.110.971119]

\section{Knobel 2005a}

Knobel RB, Vohra S, Lehmann CU. Heat loss prevention in the delivery room for preterm infants: a national survey of newborn intensive care units. Journal of Perinatology 2005;25(8):514-8. [DOI: 10.1038/sj.jp.7211344]

\section{Knobel-Dail 2015}

Knobel-Dail. Preventing hypothermia in preterm infants: a program of research. Rwanda Journal Series F: Medicine and Health Sciences 2015;2(2):57-61. [10.4314/rj.v2i2.10F]

\section{Kristoffersen 2016}

Kristoffersen L, Stoen R, Hansen LF, Wilhelmsen J, Bergseng H. Skin-to-skin care after birth for moderately preterm infants. Journal of Obstetric, Gynecologic and Neonatal Nursing 2016;45(3):339-45. [DOI: 10.1016/j.jogn.2016.02.007]

\section{Kumar 2009}

Kumar V, Shearer JC, Kumar A, Darmstadt GL. Neonatal hypothermia in low resource settings: a review. Journal of Perinatology 2009;29(6):401-12. [DOI: 10.1038/jp.2008.233]

\section{Kumar 2012}

Kumar R, Joseph T, Kamat M, Khilfeh M, Jackson P, Pyati S. Impact of interventions to prevent hypothermia at birth in subgroups of $\leq 1500 \mathrm{~g}$ very low birthweight (VLBW) infants. Proceedings of the Pediatric Academic Societies' Annual Meeting; 2012 April 28-May 1; Boston (MA). The Woodlands (TX): Pediatric Academic Societies, 2012.

\section{L'Herault 2001}

L'Herault J, Petroff L, Jeffrey J. The effectiveness of a thermal mattress in stabilizing and maintaining body temperature during the transport of very low-birth weight newborns. Applied Nursing Research 2001;14(4):210-9. [PUBMED: 11699024]

\section{Lang 2004}

Lang N, Bromiker R, Arad I. The effect of wool versus cotton head covering and length of stay with mother following delivery on infant temperature. International Journal of Nursing Studies 2004;41(8):843-6. [DOI: 10.1016/j.ijnurstu.2004.03.010]

\section{Laptook 2007}

Laptook A, Salhab W, Bhaskar B, the Neonatal Research Nerwork. Admission temperature of low birth weight infants: predictors and associated morbidities. Pediatrics 2007;119(3):e643-9. [DOI: 10.1542/peds.2006-0943]

\section{Laptook 2008}

Laptook AR, Watinson M. Temperature management in the delivery room. Seminars in Fetal \& Neonatal Medicine 2008;13(6):383-91. [DOI: 10.1016/j.siny.2008.04.003]

\section{Laptook 2008a}

Laptook A, Tyson J, Shankaran S, McDonald S, Ehrenkranz R, Fanaroff $A$, et al. Elevated temperature after hypoxic ischaemic encephalopathy: a risk factor for adverse outcome. Pediatrics 2008;122(3):491-9.

\section{LeBlanc 1991}

LeBlanc MH. Thermoregulation: incubators, radiant warmers, artificial skins and body hoods. Clinics in Perinatology 1991;18(3):403-22. [PUBMED: 1934849]

\section{Lee 2000}

Lee SK, McMillan DD, Ohlsson A, Pendray M, Synnes A, Whyte R, et al. Variations in practice and outcomes in the Canadian NICU Network 1996-1997. Pediatrics 2000;106(5):1070-9. [PUBMED: 11061777]

\section{Lee 2008}

Lee HC, Ho QT, Rhine WD. A quality improvement project to improve admission temperatures in very low birthweight infants. Journal of Perinatology 2008;28(11):754-8. [DOI: 10.1038/.jp.2008.92]

\section{Lenclen 2002}

Lenclen R, Mazraani M, Jugie M, Couderc S, Hoenn C, Carbajal R, et al. Use of a polyethylene bag: a way to improve the thermal environment of the premature newborn at the delivery room [Utilisation d'un sac en polyethylene: un moyen d'ameliorer l'environnement thermique du premature en salle de naissance]. Archives de Pediatrie 2002;9(3):238-44. [PUBMED: 11938534]

\section{Lewis 2011}

Lewis DA, Sanders LP, Brockopp DY. The effect of three nursing interventions on thermoregulation in low birthweight infants. Neonatal Network 2011;30(3):160-4. [DOI: 10.1891/0730-0832.30.3.160]

\section{Li 2016}

Li S, Guo P, Zou Q, He F, Xu F, Tan L. Efficacy and safety of plastic wrap for prevention of hypothermia after birth and during NICU in preterm infants: a systematic review and meta-analysis. PloS One 2016;11(6):e0156960. [DOI: 10.1371/journal.pone.0156960] 


\section{Lunze 2013}

Lunze K, Bloom DE, Jamison DT, Hamer DH. The global burden of neonatal hypothermia: systematic review of a major challenge for newborn survival. BMC Medicine 2013;11(24):1-11. [DOI: 10.1186/1741-7015-11-24]

\section{Lyu 2015}

Lyu Y, Shah PS, Ye XY, Warre R, Piedboeuf B, Deshpandey A, et al. Canadian Neonatal Network. Association between admission temperature and mortality and major morbidity in preterm infants born at fewer than 33 weeks' gestation. JAMA Pediatrics 2015;169(4):e150277. [DOI: 10.1001/jamapediatrics.2015.0277]

\section{Manai 2013}

Manai M, Jegatheesan P, DeSandre G, Song D, Showalter L, Govindaswami B. Elimination of admission hypothermia in preterm very low-birth-weight infants by standardization of delivery room management. Permanente Journal 2013;17(3):8-13. [DOI: 10.7812/TPP/12-130]

\section{Mathew 2008}

Mathew B, Lakshminrusimha S, Carrion V. Vinyl bags versus thermal mattress to prevent hypothermia in extremely low birth weight (ELBW) infants. Pediatric Academic Societies' Annual Meeting. May 2-6 2008, Honolulu, Hawaii. Pediatric Academic Societies, 2008.

\section{McCall 2014}

McCall E, Alderdice F, Halliday H, Johnston L, Vohra S. Challenges of minimizing heat loss at birth: a narrative overview of evidence-based thermal care interventions. Newborn and Infant Nursing Reviews 2014;14(2):56-63. [DOI: 10.1053/ j.nainr.2014.03.008]

\section{McCarthy 2011}

McCarthy LK, O'Donnell CPF. Warming preterm infants in the delivery room: polyethylene bags, exothermic mattresses or both?. Acta Paediatrica 2011;100(12):1534-7. [DOI: 10.1111/ j.1651-2227.2011.02375.x]

\section{McCarthy 2012}

McCarthy LK, Henset CC, O'Donnell CPF. In vitro effect of exothermic mattresses on temperature in the delivery room. Resuscitation 2012;83(10):e201-2. [DOI: 10.1016/ j.resuscitation.2012.07.004]

\section{McGrory 2017}

McGrory L, Owen LS, Thio M, Dawson JA, Rafferty AR, Malhotra A, et al. A randomised trial of conditioned or unconditioned gases for stabilising preterm infants at birth. Journal of Pediatrics 26 October 2017 [Epub ahead of print]. [DOI: 10.1016/j.jpeds.2017.09.006; PUBMED: 29106924]

\section{Miller 2011}

Miller SS, Lee HC, Gould JB. Hypothermia in very low birthweight infants: distribution, risk factors and outcomes. Journal of Perinatology 2011;31(Suppl 1):S49-56. [DOI: 10.1038/ jp.2010.177]

\section{Moore 2016}

Moore ER, Anderson GC, Bergman N, Dowswell T. Early skinto-skin contact for mothers and their healthy newborn infants. Cochrane Database of Systematic Reviews 2016, Issue 11. [DOI: 10.1002/14651858.CD003519.pub4]

\section{Mullany 2010}

Mullany LC, Katz J, Khatry SK, LeClerq S, Dramstadt GL. Neonatal hypothermia and associated risk factors among newborns of southern Nepal. BMC Medicine 2010;8(43):1-13. [DOI: 10.1186/1741-7015-8-43; PUBMED: 20615216]

\section{NCT00603837}

NCT00603837. Warming mattress versus polyethylene wrapping to prevent hypothermia in preterm newborns [Thermal Defense of Extremely Low Gestational Age Newborns (ELGANs) During Resuscitation: Exothermic Mattresses vs. Polyethylene Wrap]. clinicaltrials.gov/ct2/show/NCT00603837 (first received 19 December 2007).

\section{Newton 2003}

Newton T, Watkinson M. Preventing hypothermia at birth in preterm babies: at a cost of overheating some? Archives of Disease in Childhood. Fetal and Neonatal Edition 2003;88(3):F256. [PUBMED: 12719405]

\section{NNAP 2016}

UK National Neonatal Audit Programme. National Neonatal Audit Programme. 2016 Annual Report on 2015 Data. London: Royal College of Paediatrics and Child Health, 2016.

\section{NNAP 2017}

UK National Neonatal Audit Programme. National Neonatal Audit Programme. 2017 Annual Report on 2016 Data. London: Royal College of Paediatrics and Child Health, 2017.

\section{Oatley 2016}

Oatley HK, Blencowe H, Lawn JE. The effect of coverings, including plastic bags and wraps, on mortality and morbidity in preterm and full-term neonates. Journal of Perinatology 2016;36(Suppl 1):S83-9. [DOI: 10.1038/jp.2016.35]

\section{Oliver 1965}

Oliver TK Jr. Temperature regulation and heat production in the newborn. Pediatric Clinics of North America 1965;12:765-79. [PUBMED: 14312836]

\section{Papile 1978}

Papile LA, Burstein J, Burstein R, Koffler H. Incidence and evolution of subependymal and intraventricular haemorrhage: a study of infants with birth weights less than $1500 \mathrm{gm}$. Journal of Pediatrics 1978;92(4):529-34. [PUBMED: 305471]

\section{Pearlman 2012}

Pearlman SA, Vellanki H, Dean B, Search T, Baldwin R, Paul DA. Rapid cycle quality improvement leads to increased admission temperature in preterm infants. Proceedings of the Pediatric Academic Societies' Annual Meeting; 2012 April 28-May 1; Boston (MA). The Woodlands (TX): Pediatric Academic Societies, 2012. 


\section{Perlman 2015}

Perlman JM, Wyllie J, Kattwinkel J, Wyckoff MH, Aziz K, Guinsburg R, et al. Neonatal Resuscitation Chapter Collaborators. Part 7: Neonatal Resuscitation: 2015 International Consensus on Cardiopulmonary Resuscitation and Emergency Cardiovascular Care Science With Treatment Recommendations. Circulation 2015;132(16 Suppl 1):S204-41. [DOI: $10.1161 /$ CIR.0000000000000276]

\section{Phillips 2013}

Phillips R. The sacred hour: uninterrupted skin-to-skin contact immediately after birth. Newborn and Infant Nursing Reviews 2013;13(2):67-72.

\section{Pinheiro 2011}

Pinheiro JM, Boynton S, Furdon SA, Dugan R, Reu-Donlon C. Use of chemical warming packs during delivery room resuscitation is associated with decreased rates of hypothermia in very low-birth-weight neonates. Advances in Neonatal Care 2011;11(5):357-62. [DOI: 10.1097/ANC.0b013e318229aa8f]

\section{Pinheiro 2014}

Pinheiro JMB, Furdon SA, Boynton S, Dugan R, ReuDonlon $C$, Jensen S. Decreasing hypothermia during delivery room stabilization of preterm neonates. Pediatrics 2014;133(1):e218-26. [DOI: 10.1542/peds.2013-1293]

\section{Pomerance 1974}

Pomerance JJ, Madore C, Gluck L. Effect of temperature on survival of infants with RDS. Pediatric Research 1974;8:449. [DOI: 10.1203/00006450-197404000-00656]

\section{ResearchGate 2016 [Computer program]}

ResearchGate. ResearchGate. https://www.researchgate.net/. Version accessed 30 June 2016. ResearchGate, 2008-2016.

\section{RevMan 2014 [Computer program]}

Nordic Cochrane Centre, The Cochrane Collaboration. Review Manager 5 (RevMan 5). Version 5.3. Copenhagen: Nordic Cochrane Centre, The Cochrane Collaboration, 2014.

\section{Russo 2014}

Russo A, McCready M, Torres L, Theuriere C, Venturini S, Spaight $M$, et al. Reducing hypothermia in preterm infants following delivery. Pediatrics 2014;133(4):e1055-62. [DOI: 10.1542/peds.2013-2544]

\section{Rutter 2000}

Rutter N. Clinical consequences of an immature barrier. Seminars in Neonatology 2000;5(4):281-7. [DOI: 10.1053/ siny.2000.0014]

\section{Schünemann 2013}

Schünemann H, Brożek J, Guyatt G, Oxman A, editors. GRADE Working Group. GRADE Handbook for Grading Quality of Evidence and Strength of Recommendations. https:// gdt.gradepro.org/app/handbook/handbook.html. Updated October 2013.

\section{Scopes 1963}

Scopes JW, Tizard JP. The effect of intravenous noradrenaline on the oxygen consumption of new-born mammals. Journal of Physiology 1963;165:305-26. [PUBMED: 13992492]

\section{Shearman 2012}

Shearman AD, Hou D, Dunster KR, Jardine L. Heating of gases during neonatal resuscitation: a bench study. Resuscitation 2012;83(3):369-73.

\section{Shennan 1988}

Shennan AT, Dunn MS, Ohlsson A, Lennox K, Hoskins EM. Abnormal pulmonary outcomes in preterm infants: prediction from oxygen requirement in the neonatal period. Pediatrics 1988;82(4):527-32. [PUBMED: 3174313]

\section{Silverman 1958}

Silverman WA, Fertig JW, Berger AP. The influence of the thermal environment upon the survival of newly born premature infants. Pediatrics 1958;22(5):876-86. [PUBMED: 13600915]

\section{Silvestri 2012}

Silvestri JM, Trivedi S, Spanier Mingolelli SR, Patel AL. Prevention of hypothermia through Quality Improvement (QI) of resuscitation practices for very low birthweight (VLBW) infants. Proceedings of the Pediatric Academic Societies' Annual Meeting; 2012 April 28-May 1; Boston (MA). The Woodlands (TX): Pediatric Academic Societies, 2012.

\section{Smales 1978}

Smales ORC, Kime R. Thermoregulation in babies immediately after birth. Archives of Disease in Childhood 1978;53(1):58-61. [PUBMED: 626520]

\section{Smith 2004}

Smith LS. Temperature monitoring in newborns: a comparison of thermometry and measurement sites. Journal of Neonatal Nursing 2004;10(5):6-14.

\section{Smith 2013a}

Smith J, Alcock G, Usher K. Temperature measurement in the preterm and term neonate: a review of the literature. Neonatal Network 2013;32(1):16-25. [DOI: 10.1891/0730-0832.32.1.16]

\section{Smith 2016}

Smith J. Current practices on prevention of hypothermia and temperature taking in the preterm and term infant: a survey. Journal of Neonatal Nursing 2016;22(5):206-12. [DOI: 10.1016/ j.jnn.2016.04.001]

\section{Soll 2008}

Soll RF. Heat loss prevention in neonates. Journal of Perinatology 2008;28(Suppl 1):S57-9. [PUBMED: 10.1038/ jp.2008.51]

\section{Stapleton 1987}

Stapleton FB, Jones DP, Green RS. Acute renal failure in neonates: incidence, etiology and outcome. Pediatric Nephrology 1987;1(3):314-20. [PUBMED: 3153295] 


\section{Stephenson 1970}

Stephenson JM, Du JN, Oliver TK Jr. The effect of cooling on blood gas tensions in newborn infants. Journal of Pediatrics 1970;76(6):848-52. [PUBMED: 5444577]

\section{Stern 1970}

Stern L. The newborn infant and his thermal environment. Current Problems in Pediatrics 1970;1(1):1-29.

\section{Tafari 1973}

Tafari N, Olsson EE. Neonatal cold injury in the tropics. Ethiopian Medical Journal 1973;11(1):57-65.

\section{te Pas 2010}

te Pas AB, Lopriore E, Dito I, Morley CJ, Walther FJ. Humidified and heated air during stabilization at birth improves temperature in preterm infants. Pediatrics 2010;125(6):e1427-32. [DOI: 10.1542/peds.2009-2656]

\section{The World Bank 2013}

The World Bank. How we classify countries. http:// data.worldbank.org/about/county-classifications (accessed 3 July 2013).

\section{The World Bank 2016}

The World Bank. How does the world bank classify countries? https://datahelpdesk.worldbank.org/knowledgebase/ articles/378834-how-does-the-world-bank-classify-countries (accessed 22 November 2016).

\section{Thomas 1994}

Thomas K. Thermoregulation in neonates. Neonatal Network 1994;13(2):15-25. [PUBMED: 8139518]

\section{Vohra 2004 [pers comm]}

Vohra S. Multi centered randomised controlled trial of heat loss prevention (HeLP) in delivery room [personal communication] Email to: E McCall 08 July 2004 and September 2007.

\section{VON 2017}

Vermont Oxford Network. Vermont Oxford Network Database of Very Low Birth Weight Infants Born in 2016. Burlington, VT: Vermont Oxford Network, 2017.

\section{VON 2017a}

Vermont Oxford Network. NICU by the numbers: despite decreases, nearly 4 in 10 infants are cold when admitted to NICU. https://public.vtoxford.org/wp-content/uploads/2017/09/ NICU-by-the-Numbers_4-in-10-Infants-are-Cold.pdf (accessed: 12 December 2017).

\section{Webbe 2017}

Webbe J, Sinha I, Gale C. Core Outcomes Sets. Archives of Diseases in Childhood Education and Practice 2017 Jun 30 [Epub ahead of print]. [DOI: 10.1136/archdischild-2016-312117]

\section{WHO 1997}

Department of Reproductive Health and Research (RHR), World Health Organisation. Thermal protection of the newborn: a practical guide (WHO/RHT/MSM/97.2). Geneva: World Health Organisation, 1997.

\section{WHO 2015}

World Health Organisation. WHO recommendations on interventions to improve preterm birth outcomes. http:// apps.who.int/iris/bitstream/10665/183037/1/9789241508988_ eng.pdf. Geneva: WHO, 2015.

\section{Wilson 2016}

Wilson E, Maier RF, Norman M, Misselwitz B, Howell EA, Zeitlin J, Effective Perinatal Intensive Care in Europe (EPICE) Research Group. Admission hypothermia in very preterm infants and neonatal mortality and morbidity. Journal of Pediatrics 2016;175:61-7. [10.1016/j.jpeds.2016.04.016]

\section{Wyllie 2015}

Wyllie J, Bruinenberg J, Roehr C, Rudiger M, Trevisanuto D, Urlesberger B. European Resuscitation Council Guidelines for Resuscitation 2015. Section 7. Resuscitation and support of transition of babies at birth. Resuscitation 2015;95:249-63. [DOI: 10.1016/j.resuscitation.2015.07.029]

\section{Yip 2017}

Yip WY, Quek BH, Fong MCW, Thilagamangai, Ong SSG, Lim BG, et al. A quality improvement project to reduce hypothermia in preterm infants on admission to the neonatal intensive care unit. International Journal for Quality in Health Care 2017;29(7):922-8. [DOI: 10.1093/intqhc/mzx131; PUBMED: 29045653]

\section{Yu 1984}

Yu VHY, Joseph R, Bajuk B, Orgill A, Astbury J. Perinatal risk factors for necrotizing enterocolitis. Archives of Disease in Childhood 1984;59:430-4.

\section{References to other published versions of this review McCall 2005}

McCall EM, Alderdice FA, Halliday HL, Jenkins JG, Vohra S. Interventions to prevent hypothermia at birth in preterm and/ or low birthweight babies. Cochrane Database of Systematic Reviews 2005, Issue 1. [DOI: 10.1002/14651858.CD004210.pub2]

\section{McCall 2008}

McCall EM, Alderdice FA, Halliday HL, Jenkins JG, Vohra S. Interventions to prevent hypothermia at birth in preterm and/ or low birthweight infants. Cochrane Database of Systematic Reviews 2008, Issue 1. [DOI: 10.1002/14651858.CD004210.pub3]

\section{McCall 2010}

McCall EM, Alderdice F, Halliday HL, Jenkins JG, Vohra S. Interventions to prevent hypothermia at birth in preterm and/ or low birthweight infants. Cochrane Database of Systematic Reviews 2010, Issue 3. [DOI: 10.1002/14651858.CD004210.pub4]

* Indicates the major publication for the study 
CHARACTERISTICS OF STUDIES

Characteristics of included studies [ordered by study ID]

Bergman 2004

Methods

Randomised

Multi-centre (2)

Blinding of randomisation:

- Generation of allocation sequence: adequate (computer minimisation method)

- Allocation concealment: unclear ("... a computer minimisation method determined allocation in a concealed manner"; ".. second hospital... minimisation factors and subsequent allocation by the computer were exchanged by mobile telephone, with no delays")

Blinding of intervention: participants: unfeasible; healthcare providers/personnel: unfeasible

Blinding of outcome measurement: yes; after the pilot phase, the principal investigator and data analysis teams were blinded. A statistician, who was blinded to the allocation, performed statistical analyses.

Complete follow-up: no (31 of 35 infants randomised completed the study: 1 infant was excluded after 2 hours, when the mother required a caesarean section for a second twin; 3 further infants were 'bailed out' within the first 3 hours, requiring additional respiratory support, and were moved to the neonatal intensive care)

Room temperature, maternal temperature, and incubator temperature were recorded.

Ethical approval was obtained.

Informed consent was obtained.

Participants

Infants were delivered at birth weight $\geq 1200$ and $\leq 2199$ grams.

Thirty-five infants were randomised: intervention group $(n=21)$, control group $(n=14) ; 31$ completed the study: intervention group $(n=18)$, control group $(n=13)$.

Setting: 2 secondary level referral hospitals in Cape Town treating public patients referred from Midwife Obstetric Units, South Africa

Country income grouping: upper-middle

Exclusion criteria:

Mothers:

- Delivered outside the unit

- Had a caesarean section

- Too severely ill to be able to look after themselves or their infants

- Known to have positive HIV status

- Giving their babies up for adoption

Infants:

- Birth weight < 1200 grams or $>2199$ grams

- Apgar score $<6$ at 5 minutes

- Congenital malformations detected at birth

Intervention group (skin-to-skin care) characteristics:

Mothers:

- Gravidity mean (1.9)

-Parity mean (1.8)

- Race 'African' (8)

- Race 'Coloured' (12) 
- Smoking in pregnancy (7)

-Alcohol use (6)

- Opiate in labour (9)

- Oxytocin in labour (3)

- Hypertension (3)

- Antepartum steroids (5)

Infants:

- Male (12)

- Resuscitation (4)

- Birth weight (grams) mean (SD) 1813 (260)

- Gestation (weeks) mean (SD) 34.2 (1.9)

-Appropriate-for-gestation (13)

- Small-for-gestation (7)

Control group (conventional care) characteristics:

Mothers:

- Gravidity mean (1.9)

- Parity mean (1.6)

- Race 'African' (4)

- Race 'Coloured' (10)

- Smoking in pregnancy (4)

-Alcohol use (2)

- Opiate in labour (6)

- Oxytocin in labour (1)

- Hypertension (1)

- Antepartum steroids (3)

Infants:

- Male (7)

- Resuscitation baby (1)

- Birth weight (grams) mean (SD) 1866 (258)

- Gestation (weeks) mean (SD) 35.3 (1.9)

-Appropriate-for-gestation (10)

- Small-for-gestation (5)

All infants were delivered onto a theatre cloth on the mother's abdomen/chest, dried gently, and assessed. They were then placed naked on the mother's naked chest and covered with double-layered cotton cloth. The bed was placed in semi-Fowler's position. 
Bergman 2004 (Continued)

Intervention group:

The infant remained in skin-to-skin contact, was secured to the mother's chest; hips were flexed and placed in a 'frog position'; arms were also flexed.

Control group:

The infant was immediately transferred to a prewarmed servo-controlled closed incubator, which remained with the mother in the delivery ward for the first hour. If the infant temperature was $<36^{\circ} \mathrm{C}$, a cap and booties were applied, along with a heat shield placed over the infant. If this was insufficient, a sheet of plastic was framed over the foot end of the heat shield and the outlet of the warm air funnelled over the infant. The medical officer was responsible for decisions regarding requirement for intensive or high care and subsequent 'bail-out' with no further research recordings.

Outcomes

Infant stability in terms of a set of predetermined physiological parameters. Observations for heart rate, respiratory rate, oxygen saturation, and temperature were recorded at 5-minute intervals in the first hour, then at 15-minute intervals. Continuous observations for apnoea and signs of respiratory distress; blood sugar measured at 1, 3, and 6 hours

Primary outcomes:

- Neonatal intensive care admissions

- Number of exceeded parameters

- Composite stabilisation score (SCRIP) (1 to 6 hours)

- Composite stabilisation score (SCRIP) (sixth hour)

Secondary outcomes:

- Skin temperature remaining $<35.5^{\circ} \mathrm{C}$ for 2 consecutive readings

- Heart rate $<100$ or $>180$ beats per minute for 2 consecutive readings

- Apnoea longer than 20 seconds

- Oxygen saturation below $87 \%$ for 2 consecutive recordings, despite supplementation with nasal prong oxygen, $\mathrm{FiO}_{2}$ up to 0.6 and CPAP up to $5 \mathrm{~cm}$ water

- Blood glucose $<2.6 \mathrm{mmol} / \mathrm{L}$ reading confirmed by laboratory

Notes

Recruitment for this study was terminated after interim analysis was carried out, on the basis of significant results.

Labour ward temperatures were constant throughout the study, and incubators were prewarmed, with a mean temperature reading of $36^{\circ} \mathrm{C}$ at commencement.

\section{Risk of bias}

Bias Authors' judgement Support for judgement

Random sequence genera- Low risk tion (selection bias)
Quote: "a computer minimisation method determined allocation in a concealed manner"; ".. second hospital... minimisation factors and subsequent allocation by the computer were exchanged by mobile telephone, with no delays"

Allocation concealment Unclear risk Information is insufficient to assess risk of bias.
(selection bias)

$\begin{array}{ll}\begin{array}{l}\text { Blinding of participants } \\ \text { and personnel (perfor- } \\ \text { mance bias) }\end{array} & \text { High risk } \\ \text { All outcomes } & \begin{array}{l}\text { Blinding of participants: unfeasible } \\ \text { Quote: "it was obviously not possible to blind the participants or the nurse re- } \\ \text { searchers to the intervention" } \\ \text { Comment: Review authors agreed that it was unfeasible to blind participants } \\ \text { and healthcare providers/personnel to the intervention. }\end{array}\end{array}$


Bergman 2004 (Continued)

Blinding of outcome as- Low risk Blinding of outcome measurement: yes; after the pilot phase, the principal insessment (detection bias) All outcomes

Incomplete outcome data Low risk
(attrition bias)

All outcomes

\section{vestigator and data analysis teams were blinded. A statistician, blinded to the} allocation, performed statistical analyses.
Thirty-one of 35 (11\% loss to follow-up) infants randomised completed the study. Three infants and 1 infant were lost to follow-up in the intervention and control groups, respectively. One infant was excluded after 2 hours when the mother required a caesarean section for a second twin, 3 further infants 'bailed out' within the first 3 hours, requiring additional respiratory support, and were moved to the neonatal intensive care.

Selective reporting (re- Unclear risk Information is insufficient to assess risk of bias.
porting bias)
porting bias)

Other bias High risk

Quote: "The possibility of selection bias exists: Many prematurely born infants delivered unexpectedly (without a nurse researcher available), and the aetiology of such premature delivery may differ from those studied"

Bhavsar 2015

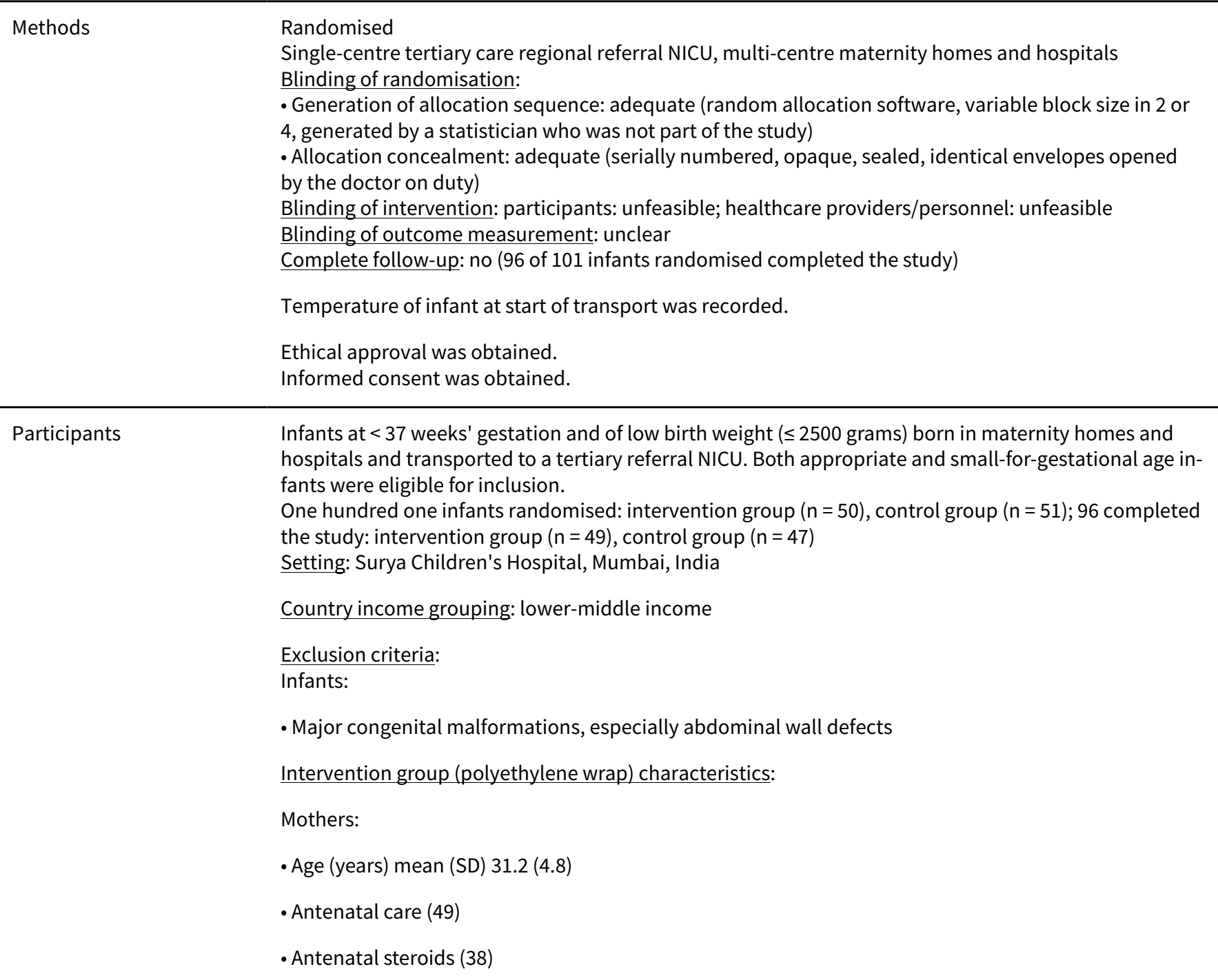


Bhavsar 2015 (Continued)

Infants:

- Male (23)

- Female (26)

• Birth weight (grams) mean (SD) 1686 (446)

- Gestation (weeks) mean (SD) 32.6 (2.4)

- Mode of delivery: vaginal (6), caesarean (43), multiple birth (25)

- Temperature at start of transport $\left({ }^{\circ} \mathrm{C}\right)$ mean (SD) $35.6(0.73)$

- Transfer time to NICU (minutes) mean (SD) 75.6 (35.1)

Control group (conventional care) characteristics:

Mothers:

- Age (years) mean (SD) 31.1 (4.5)

- Antenatal care (47)

- Antenatal steroids (34)

Infants:

- Male (29)

- Female (18)

- Birth weight (grams) mean (SD) 1470 (446)

- Gestation (weeks) mean (SD) 31.9 (2.8)

- Mode of delivery: vaginal (9), caesarean (38), multiple birth (20)

- Temperature at start of transport $\left({ }^{\circ} \mathrm{C}\right)$ mean (SD) $35.4(0.87)$

- Transfer time to NICU (minutes) mean (SD) 66.1 (29.1)

- Sterile transparent non-permeable material used for covering operative surfaces (DISPOSIT, RD Hygiene Products, Mumbai, Maharashtra, India)

All infants were delivered in maternity homes and were resuscitated on radiant warmers preset at maximal heat output. Resuscitation was carried out as per NRP 2010 guidelines.

Intervention group:

The infant was covered with polyethylene wrap from head to toe + a sterile cloth within 10 minutes of delivery following drying and resuscitation. Auscultation was performed over the polyethylene wrap, and if umbilical access was required, a hole was cut in the wrap.

Control group:

The infant was covered with sterile cloth only. Infants in both groups were transported to the Surya Children's Hospital NICU in an ambulance equipped with transport incubator after recording of axillary temperature. Following admission to the NICU, infants were transferred to a prewarmed servo-controlled open-care system (gestation-appropriate neutral thermal environment). Axillary temperature was taken before the infants were unwrapped. 
Secondary outcomes:

- Core body temperature difference ${ }^{\circ} \mathrm{C}$ (axillary) from delivery room to admission

- Hypothermia (core body temperature (axillary) $<35^{\circ} \mathrm{C}$ )

- Hypoglycaemia (blood glucose level $<40 \mathrm{mg} / \mathrm{dL}$ within 2 hours of birth)

- Severe metabolic acidosis ( $\mathrm{pH}<7.20$ and/or base deficit $>10 \mathrm{mmol} / \mathrm{L}$ ) within the first 3 days of life

- Requirement for oxygen and duration of requirement of oxygen (days)

- Hyperthermia (admission temperature $>37.5^{\circ} \mathrm{C}$ ) at time of admission to the NICU

Notes

Infants in the intervention group were heavier than those in the control group: birth weight (grams) mean (SD) 1686 (446) vs mean (SD) 1470 (446) $(\mathrm{P}<0.05)$.

Trial authors were contacted for clarification on the definition of hypothermia and hyperthermia and for secondary outcome data for severe metabolic acidosis. Infomation was provided by Dr. Nandkishor Kabra (25/10/2016).

\section{Risk of bias}

\begin{tabular}{lll}
\hline Bias & Authors' judgement & Support for judgement \\
\hline $\begin{array}{l}\text { Random sequence genera- } \\
\text { tion (selection bias) }\end{array}$ & Low risk & $\begin{array}{l}\text { Quote: "The random number sequence was generated in variable block size } \\
\text { in either } 2 \text { or } 4 \text { using random allocation software.......by a statistician, who was } \\
\text { not part of the study" }\end{array}$ \\
\hline $\begin{array}{l}\text { Allocation concealment } \\
\text { (selection bias) }\end{array}$ & Low risk & $\begin{array}{l}\text { Quote: "The random courses were kept in serially numbered, opaque, sealed, } \\
\text { and identical envelopes....opened by the doctor on duty" }\end{array}$
\end{tabular}

Blinding of participants High risk and personnel (performance bias)

All outcomes

\section{Blinding of participants: unfeasible}

Blinding of healthcare providers/personnel: unfeasible

Comment: Review authors agreed that it was unfeasible to blind participants and healthcare providers/personnel to the intervention.

Quote: "Blinding could not be performed as the caregivers involved in the care of the neonate at the time of transfer were aware of the group allocation"

\begin{tabular}{|c|c|c|}
\hline $\begin{array}{l}\text { Blinding of outcome as- } \\
\text { sessment (detection bias) } \\
\text { All outcomes }\end{array}$ & High risk & Blinding of outcome measurement: no \\
\hline $\begin{array}{l}\text { Incomplete outcome data } \\
\text { (attrition bias) } \\
\text { All outcomes }\end{array}$ & Low risk & $\begin{array}{l}\text { Ninety-six of } 101 \text { ( } 5 \% \text { loss to follow-up) infants randomised completed the } \\
\text { study. } \\
\text { Quote: "Temperature measurement at admission was missed in one neonate } \\
\text { from polyethylene wrap group and three neonates in control group" }\end{array}$ \\
\hline $\begin{array}{l}\text { Selective reporting (re- } \\
\text { porting bias) }\end{array}$ & Unclear risk & $\begin{array}{l}\text { This trial was registered retrospectively; therefore selective reporting } \\
\text { bias cannot be assessed. http://ctri.nic.in/Clinicaltrials/showallp.php? } \\
\text { mid1=8277\&EncHid=\&userName=Bhavsar } \\
\text { CTRI/2014/02/004415 }\end{array}$ \\
\hline Other bias & High risk & $\begin{array}{l}\text { Infants in the intervention group were heavier than those in the control group: } \\
\text { birth weight (grams) mean (SD) } 1686(446) \text { vs mean (SD) } 1470(446)(P<0.05) \text {. } \\
\text { This could have influenced the effect size. Consent was refused for } 35 \text { infants } \\
\text { assessed for eligibility. }\end{array}$ \\
\hline
\end{tabular}




\section{Methods}

Randomised

Single-centre

Blinding of randomisation:

- Generation of allocation sequence: adequate (lot drawing - pulling cards from an envelope in which

24 cards had been placed. Equal numbers of cards had "mattress" or "no mattress" written on them)

- Allocation concealment: unclear

Blinding of intervention: participants: unfeasible; healthcare providers/personnel: unfeasible

Blinding of outcome measurement: unclear

Complete follow-up: yes (all 24 infants randomised completed the study)

Gestational age, birth weight, and time temperature taken after birth were recorded.

The proposal for the study underwent full review and approval by the Institutional Review Board of the University of Florida, Health Science Centre.

Informed parental consent before delivery

Participants

Infants delivered at birth weight $\leq 1500$ grams

Twenty-four infants randomised: intervention group $(n=12)$, control group $(n=12)$

Setting: large teaching hospital with level 3 NICUs, North-Central Florida, USA

Country income grouping: high

Exclusion criteria:

Mothers:

- Maternal temperature at delivery was $>38.0^{\circ} \mathrm{C}$

Infants:

- Obvious neural tube defect

- Omphalocoele, gastroschisis, exstrophy of the bladder, cloacal exstrophy, or any other open lesion that would cause greater than normal heat loss at delivery

- Infant had a sacral teratoma or any other lesion that would prevent the infant from being positioned and evaluated in the normal manner.

All infant characteristics: gestation range (weeks) 24 to 32, birth weight range (grams) 531 to 1498 Intervention group (transwarmer mattress) characteristics:

Infants:

- Gestation (weeks) mean 27.8, birth weight (grams) mean 1033, time axillary temperature taken (minutes) after birth mean 18

Control group (conventional care) characteristics:

Infants:

- Gestation (weeks) mean 27.3, birth weight (grams) mean 1027, time axillary temperature taken (minutes) after birth mean 19

Interventions

Transwarmer Infant Transport Mattress (Prism Technologies, San Antonio, Texas, USA). These mattresses are filled with a gel of sodium acetate, water, and thickeners. Once activated, they heat to approximately $40^{\circ} \mathrm{C}$ for 2 hours.

Intervention group:

The NICU nurse activated the mattress when delivery was imminent and placed it onto the radiant warmer surface with blankets on top of it; the newborn infant was immediately placed onto the blankets and dried, and the blankets removed, then was placed directly onto the warming mattress. 
Brennan 1996 (Continued)

\section{Control group:}

Infants received the same care as the intervention group but without the warming mattress. Both groups were evaluated and resuscitated according to the Neonatal Resuscitation Program and were transported to the NICU on the radiant warmer surface. Infants in the intervention group remained on the mattress until admission temperatures were taken in the NICU.

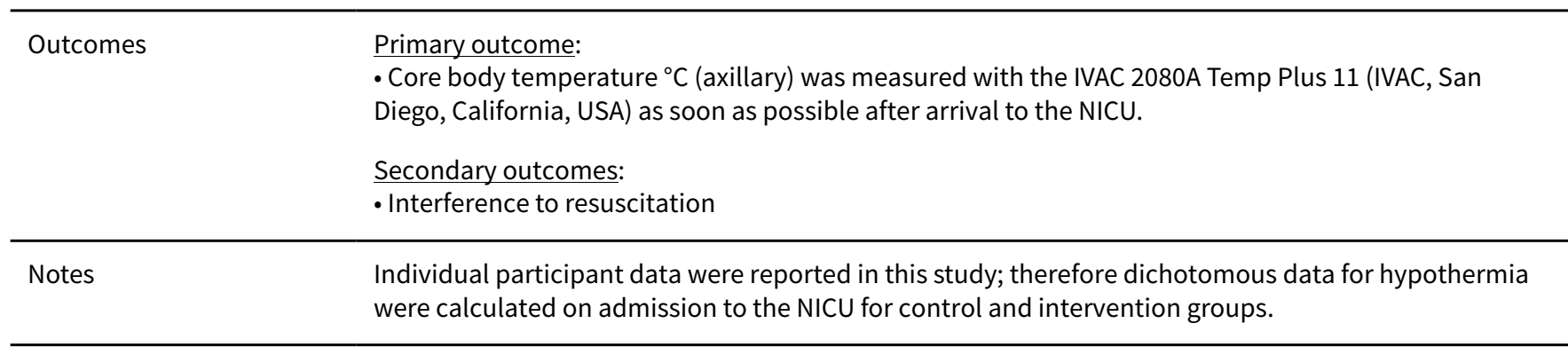

\section{Risk of bias}

Bias Authors' judgement Support for judgement

Random sequence genera- Low risk tion (selection bias)
Quote: "Random assignment was made by pulling a card from an envelope in which 24 cards had been placed. Twelve cards had the word "mattress" written on them, and 12 had "no mattress" written on them"

\begin{tabular}{lll}
\hline $\begin{array}{l}\text { Allocation concealment } \\
\text { (selection bias) }\end{array}$ & Unclear risk & Information is insufficient to assess risk of bias. \\
\hline $\begin{array}{l}\text { Blinding of participants } \\
\begin{array}{l}\text { and personnel (perfor- } \\
\text { mance bias) }\end{array}\end{array}$ & High risk & Blinding of participants: unfeasible \\
All outcomes & Blinding of healthcare providers/personnel: unfeasible \\
& $\begin{array}{l}\text { Comment: Review authors agreed that it was unfeasible to blind participants } \\
\text { and healthcare providers/personnel to the intervention. }\end{array}$ \\
\hline
\end{tabular}

Blinding of outcome as- Unclear risk
sessment (detection bias) All outcomes

\begin{abstract}
Blinding of outcome measurement: unclear
Quote: "The babies in the experimental group remained on the transport mattress until the admission temperatures were taken in the NICU. Admission temperatures were taken and recorded by the nurses admitting the infants. If the baby was in the experimental group, the warm mattress was discarded once the admission temperature was taken"
\end{abstract}

Comment: The trial author did not state whether or not outcome assessors were blinded to the intervention. It is unlikely that outcome assessors were blinded for the primary outcome measure. Information is insufficient to assess whether this was the case for subsequent secondary outcome measures.

Incomplete outcome data Low risk Complete follow-up: All infants randomised completed the study.
(attrition bias)

All outcomes

Selective reporting (re- Unclear risk Information is insufficient to assess risk of bias.
porting bias)

Other bias Low risk


Caglar 2014

\section{Methods}

Randomised

Single-centre

Blinding of randomisation:

- Generation of allocation sequence: adequate (computer-based random number generator - personal communication with trial author)

- Allocation concealment: adequate (sealed, opaque envelopes were opened just before birth - personal communication with trial author)

Blinding of intervention: participants: unfeasible; healthcare providers/personnel: unfeasible Blinding of outcome measurement: unclear

Complete follow-up: yes (all 59 infants randomised completed the study)

Transfer time to the NICU was recorded.

The proposal for the study was approved by the Istanbul Faculty of Medicine, Istanbul University Ethics Committee.

Written informed consent was obtained.
Infants at $\leq 32$ weeks' gestation

Fifty-nine infants randomised: intervention group $(n=22)$, comparison group $(n=37)$

Setting: Istanbul University Hospital, Istanbul, Turkey

Country income grouping: upper-middle

Inclusion criteria:

Mothers:

- Body temperature $<38^{\circ} \mathrm{C}$

Infants:

- No congenital anomaly (neural tube defect, omphalocoele, gastroschisis, or other open lesions)

- Initial axillary body temperature $\geq 36.4^{\circ} \mathrm{C}$

Intervention group (vinyl bag) characteristics:

Infants:

- Gestation (weeks) mean (SD) 29.27 (1.80)

- Birth weight (grams) mean (SD) 1183 (520)

- Gender: male (9)

- Mode of delivery: 'caesarean section' (21)

-Ventilatory support: intermittent mandatory ventilation (15), continuous positive airway pressure (4), oxygen hood (3),

- Surfactant intake (10)

-Apgar score @ 1 minute mean (SD) 5.8 (2.5)

-Apgar score @ 5 minutes mean (SD) 7.5 (2.0)

- Umbilical cord gas pH mean (SD) 7.27 (0.05) 
- Transport time delivery room to NICU (minutes) mean (SD) 16.09 (6.03)

- Mortality (3)

Comparison group (polyethylene wrap) characteristics:

Infants:

- Gestation (weeks) mean (SD) 28.64 (2.05)

- Birth weight (grams) mean (SD) 1079 (336)

- Gender: male (23)

- Mode of delivery: 'caesarean section' (32)

- Ventilatory support: intermittent mandatory ventilation (27), continuous positive airway pressure (8), oxygen hood (2)

- Surfactant intake (14)

•Apgar score @ 1 minute mean (SD) 5.3 (2.3)

-Apgar score @ 5 minutes mean (SD) 7.6 (1.6)

- Umbilical cord gas pH mean (SD) 7.30 (0.07)

- Blood sugar at delivery (mg/dL) mean (SD) 62.3 (29.8)

- Transport time delivery room to NICU (minutes) mean (SD) 20.67 (9.94)

- Mortality (1)

Intervention group:

Infants were placed in vinyl isolation bags so that the entire body was covered up to the neck.

Comparison group: plastic wrap:

Infants were wrapped in transparent and non-sterile stretch film (made of polyethylene intended for food conservation) to cover the entire body up to the neck. In both groups, infants were treated with the standard care procedure as recommended by WHO. The heads of infants were dried and covered immediately after delivery, and wet towels were replaced. Infants were placed under a preheated radiant warmer, and the delivery room temperature was between $21^{\circ} \mathrm{C}$ and $22^{\circ} \mathrm{C}$. Infants were resuscitated under a radiant warmer, then were transported to the NICU in a transport incubator at $35^{\circ} \mathrm{C}$, where they were transferred to another prewarmed $\left(35^{\circ} \mathrm{C}\right)$ incubator. An IV catheter and/or an umbilical catheter were inserted into the infants in the NICU while they were still inside the bag or wrapped. Infants' body temperature was measured at 4 time points. At the end of 1 hour, infants were removed from the bag or wrap and were placed in preheated incubators. All core body temperatures ${ }^{\circ} \mathrm{C}$ (axillary) were recorded with a Chicco digital thermometer (product code: 00.069703.400.000; made in Italy). The axillary temperature was read after the digital thermometer had been in place for at least 15 seconds.

Outcomes Primary outcomes:

- Core body temperature ${ }^{\circ} \mathrm{C}$ (axillary) immediately after birth and 20 minutes, 40 minutes, and 60 minutes after birth

- Normothermia (core body temperature (axillary) $36.5^{\circ} \mathrm{C}$ to $37.5^{\circ} \mathrm{C}$ )

- Cold stress (core body temperature (axillary) $36^{\circ} \mathrm{C}$ to $36.4^{\circ} \mathrm{C}$ )

- Moderate hypothermia (core body temperature (axillary) $32^{\circ} \mathrm{C}$ to $35.9^{\circ} \mathrm{C}$ ) 
Caglar 2014 (Continued)

- Severe hypothermia (core body temperature (axillary) $<32^{\circ} \mathrm{C}$ )

Notes Mortality was reported as a baseline characteristic with no significant differences between the 2 groups. The timing of mortality is unclear.

Delivery room ambient temperature was between $21^{\circ} \mathrm{C}$ and $22^{\circ} \mathrm{C}$.

\section{Risk of bias}

\begin{tabular}{|c|c|c|}
\hline Bias & Authors' judgement & Support for judgement \\
\hline $\begin{array}{l}\text { Random sequence genera- } \\
\text { tion (selection bias) }\end{array}$ & Low risk & $\begin{array}{l}\text { Quote: "The groups were appointed by a computer-based random number } \\
\text { generator" (personal communication with Seda Calgar, } 30 \text { September 2016) }\end{array}$ \\
\hline $\begin{array}{l}\text { Allocation concealment } \\
\text { (selection bias) }\end{array}$ & Low risk & $\begin{array}{l}\text { Quote: "Randomisation of consented infants was carried out in the delivery } \\
\text { room by opening sealed opaque envelopes just prior to birth" (personal com- } \\
\text { munication with Seda Calgar, } 10 \text { April 2016) }\end{array}$ \\
\hline \multirow{4}{*}{$\begin{array}{l}\text { Blinding of participants } \\
\text { and personnel (perfor- } \\
\text { mance bias) } \\
\text { All outcomes }\end{array}$} & \multirow[t]{4}{*}{ High risk } & Blinding of participants: unfeasible \\
\hline & & Blinding of healthcare providers/personal: unfeasible \\
\hline & & $\begin{array}{l}\text { Comment: Review authors agree that it was unfeasible to blind participants } \\
\text { and healthcare providers/personnel to the intervention. }\end{array}$ \\
\hline & & $\begin{array}{l}\text { Quote: "Due to the nature of the intervention, it was not possible to blind per- } \\
\text { sonnel to the treatment allocation" (personal communication with Seda Cal- } \\
\text { gar, } 10 \text { April 2016) }\end{array}$ \\
\hline
\end{tabular}

\begin{tabular}{lll}
\hline $\begin{array}{l}\text { Blinding of outcome as- } \\
\text { sessment (detection bias) } \\
\text { All outcomes }\end{array}$ & Unclear risk & Blinding of outcome assessment: unclear \\
\hline $\begin{array}{l}\text { Incomplete outcome data } \\
\text { (attrition bias) } \\
\text { All outcomes }\end{array}$ & Low risk & Complete follow-up: All infants randomised completed the study \\
\hline $\begin{array}{l}\text { Selective reporting (re- } \\
\text { porting bias) }\end{array}$ & Unclear risk & $\begin{array}{l}\text { Information is insufficient to assess risk of bias. No published protocol (PhD } \\
\text { thesis) }\end{array}$ \\
\hline $\begin{array}{l}\text { Other bias } \\
\text { Low risk }\end{array}$ & $\begin{array}{l}\text { During the study period, 173 babies satisfied the criteria for inclusion. Howev- } \\
\text { er, only 59 infants were enrolled. One hundred fourteen infants (65.9\%) were } \\
\text { excluded because the researcher was not able to arrive in time for the delivery } \\
\text { owing to "urban challenges". The study sample was limited to 59, which result- } \\
\text { ed in unequal numbers of infants in intervention ( }=22) \text { and control groups }(\mathrm{n} \\
=37) \text { The researcher was unable to make it to all deliveries in time. It appears } \\
\text { that this inability was random (personal communication with Seda Calgar, 14 } \\
\text { October 2016). }\end{array}$ \\
\hline
\end{tabular}

Cardona Torres 2012

$\begin{array}{ll}\text { Rethods } & \text { Randomised } \\ & \text { Single-centre } \\ & \text { Blinding of randomisation: } \\ \text { - Generation of allocation sequence: unclear } & \text { - Allocation concealment: unclear } \\ & \text { Blinding of intervention: participants: unfeasible; healthcare providers/personnel: unfeasible } \\ & \text { Blinding of outcome measurement: unclear }\end{array}$


Cardona Torres 2012 (Continued)

Complete follow-up: no (90 of 99 infants randomised completed the study; reasons for loss to follow-up were not documented)

The incubator temperature was recorded at baseline and every 15 minutes until 2 hours after birth.

The proposal for the study was approved by the Institutional Ethics Committee, Instituto Mexicano del Seguro Social, Hospital General de Zona No. 4, Celaya, Mexico.

Informed written consent was obtained.

Participants
Infants delivered at $\geq 28$ and $<37$ weeks' gestation, with birth weight $\geq 1000$ and $\leq 2499$ grams Ninety-nine infants randomised: intervention group $1(n=35)$, intervention group $2(n=33)$, control group $(n=31) ; 90$ infants completed the study: intervention group $1(n=30)$, intervention group $2(n=$ 30), control group $(n=30)$

Setting: General Hospital in Celaya, Mexico

Country income grouping: upper-middle

Exclusion criteria:

Mothers:

- None specified

Infants:

- Suffering loss of cutaneous integrity

- Suspected congenital heart disease (based on inspection and auscultation findings such as presence of cyanosis and abnormal heart rate, rhythm, and regularity, and heart sounds)

Intervention group 1: (polyethylene bag with previous drying) characteristics:

Mothers:

- Complications of pregnancy: gestational diabetes mellitus (0), pre-eclampsia/eclampsia (2), premature rupture of membranes (6), other complications (3)

Infants:

- Sex (female/male) (15/15)

- Gestation (weeks) mean (SD) 35.2 (1.8)

- Birth weight (grams) mean (SD) 2101 (319)

- Small-for-gestation (6)

-Apgar score @ 5 minutes median (IQR) 9.0 (7 to 9)

- Mode of delivery: eutocic (9), distocic (1), elective caesarean (9), urgent caesarean (11)

- Glucose at birth (mg/dL) median (IQR) 66.0 (49.0 to 77.0$)$

Intervention group 2: (polyethylene bag without previous drying) characteristics:

Mothers:

- Complications of pregnancy: gestational diabetes mellitus (1), pre-eclampsia/eclampsia (6), premature rupture of membranes (4), other complications (3)

Infants:

- Sex (female/male) (15/15)

- Gestation (weeks) mean (SD) 34.5 (2.6)

- Birth weight (grams) mean (SD) 1934 (456) 
Cardona Torres 2012 (Continued)

- Small-for-gestation (8)

-Apgar score @ 5 minutes median (IQR) 9.0 (7 to 9)

- Mode of delivery: eutocic (8), distocic (1), elective caesarean (9), urgent caesarean (12)

- Glucose at birth (mg/dL) median (IQR) 69.5 (56.2 to 81.2)

Control group (conventional care) characteristics:

Mothers:

- Complications of pregnancy: gestational diabetes mellitus (0), pre-eclampsia/eclampsia (3), premature rupture of membranes (2), other complications (3)

Infants:

- Sex (female/male) (14/16)

- Gestation (weeks) mean (SD) 35.6 (1.7)

- Birth weight (grams) mean (SD) 2173 (275)

- Small-for-gestation (9)

-Apgar score @ 5 minutes median (IQR) 9.0 (5 to 9)

- Mode of delivery: eutocic (4), distocic (1), elective caesarean (14), urgent caesarean (11)

- Glucose at birth (mg/dL) median (IQR) 70 (56.0 to 98.0)

Polyethylene bag with and without previous drying:

Intervention group 1: polyethylene bag with previous drying

Infants were dried similarly to the control group and wrapped with a polyethylene bag. The polyethylene bag was removed 2 hours after birth.

Intervention group 2: polyethylene bag without previous drying

Infants were wrapped with a polyethylene bag without previous drying. Only the head was dried. The polyethylene bag was removed 2 hours after birth.

Control group: conventional care. Infants were routinely dried with a sterile preheated field (heated under radiant warmer for 10 minutes at $36.5^{\circ} \mathrm{C}$ ), according to the International Guidelines for Neonatal Resuscitation, and wrapped traditionally in another preheated field. Immediately after birth, infants in all groups were placed under a radiant warmer (Blosson, Series 900, it Marks Fisher and Paykel). All infants were placed in a single-walled incubator (Medix TR 306).

\section{Outcomes}

\section{Primary outcomes:}

- Time to achieve mean core body temperature (axillary) of $36.5^{\circ} \mathrm{C}$

- Core body temperature ${ }^{\circ} \mathrm{C}$ (axillary) every 15 minutes until 2 hours after birth

- Hypothermia: definition not provided

\section{Secondary outcomes:}

- Capillary glucose levels $(\mathrm{mg} / \mathrm{dL})$ at 120 minutes of birth measured with an Optium Xceed monitor

- Blood pressure measured via the flushing technique with a sphygmomanometer at 15 minutes, 1 hour, and 2 hours after birth

- Interference to resuscitation 
Cardona Torres 2012 (Continued)

Notes

Temperature of the incubator at 120 minutes was significantly higher in the control group than in the intervention group (polyethylene bag with previous drying).

Trial author was contacted for further clarification regarding methodological quality and further details of the intervention - no response has been received to date (May 2013).

Some additional information was obtained from http://clinicaltrials.gov/ct2/show/record/ NCT01236599.

\section{Risk of bias}

\begin{tabular}{lll}
\hline Bias & Authors' judgement & Support for judgement \\
\hline $\begin{array}{l}\text { Random sequence genera- } \\
\text { tion (selection bias) }\end{array}$ & Unclear risk & Information is insufficient to assess risk of bias. \\
\hline $\begin{array}{l}\text { Allocation concealment } \\
\text { (selection bias) }\end{array}$ & Unclear risk & Information is insufficient to assess risk of bias. \\
\hline $\begin{array}{l}\text { Blinding of participants } \\
\text { and personnel (perfor- } \\
\text { mance bias) }\end{array}$ & High risk & Blinding of participants: unfeasible \\
All outcomes & $\begin{array}{l}\text { Blinding of healthcare providers/personnel: unfeasible } \\
\text { Comment: Review authors agreed that it was unfeasible to blind participants } \\
\text { and healthcare providers/personnel to the intervention. }\end{array}$ \\
& $\begin{array}{l}\text { Comment: This study was described as "open label" and is available at http:// } \\
\text { clinicaltrials.gov/ct2/show/record/NCT01236599. }\end{array}$
\end{tabular}

\begin{tabular}{|c|c|c|}
\hline $\begin{array}{l}\text { Blinding of outcome as- } \\
\text { sessment (detection bias) } \\
\text { All outcomes }\end{array}$ & Unclear risk & $\begin{array}{l}\text { Blinding of outcome measurement: unclear } \\
\text { Information is insufficient to assess risk of bias. }\end{array}$ \\
\hline $\begin{array}{l}\text { Incomplete outcome data } \\
\text { (attrition bias) } \\
\text { All outcomes }\end{array}$ & Low risk & $\begin{array}{l}\text { Ninety of } 99 \text { ( } 9 \% \text { loss to follow-up) infants randomised completed the study } \\
\text { (intervention group } 1: 5 \text { infants lost to follow-up; intervention group } 2: 3 \text { in- } \\
\text { fants lost to follow-up; control group: } 1 \text { infant lost to follow-up). Reasons for } \\
\text { loss to follow-up were not documented. }\end{array}$ \\
\hline $\begin{array}{l}\text { Selective reporting (re- } \\
\text { porting bias) }\end{array}$ & Low risk & $\begin{array}{l}\text { All study outcomes detailed at the time of trial registration were reported in } \\
\text { the published manuscript as per } \\
\text { http://clinicaltrials.gov/ct2/show/record/NCT01236599. }\end{array}$ \\
\hline Other bias & High risk & $\begin{array}{l}\text { Infants in the intervention group (polyethylene bag without previous drying) } \\
\text { were significantly smaller than those in the control group. This could be a con- } \\
\text { sequence of poor randomisation. }\end{array}$ \\
\hline
\end{tabular}

Chantaroj 2011

\begin{tabular}{ll}
\hline Methods & Randomised \\
Single-centre \\
Blinding of randomisation: \\
- Generation of allocation sequence: adequate, computer-generated random sequence. Infants were \\
stratified by gestation (at < 28 weeks or $\geq 28$ weeks) using block random allocation (4) \\
- Allocation concealment: unclear, sealed opaque envelopes \\
Blinding of intervention: participants: unfeasible; healthcare providers/personnel: unfeasible \\
Blinding of outcome measurement: unclear \\
Complete follow-up: yes (all 38 infants randomised completed the study) \\
\hline
\end{tabular}


Chantaroj 2011 (Continued)

Maternal temperatures, delivery room temperatures, transport incubator temperatures, and nursery temperatures were recorded.

The proposal for the study was approved by the institutional ethical committee, Thammasat University: Code: MTU-P-1-82/52.

Parental consent was sought from mothers before delivery.

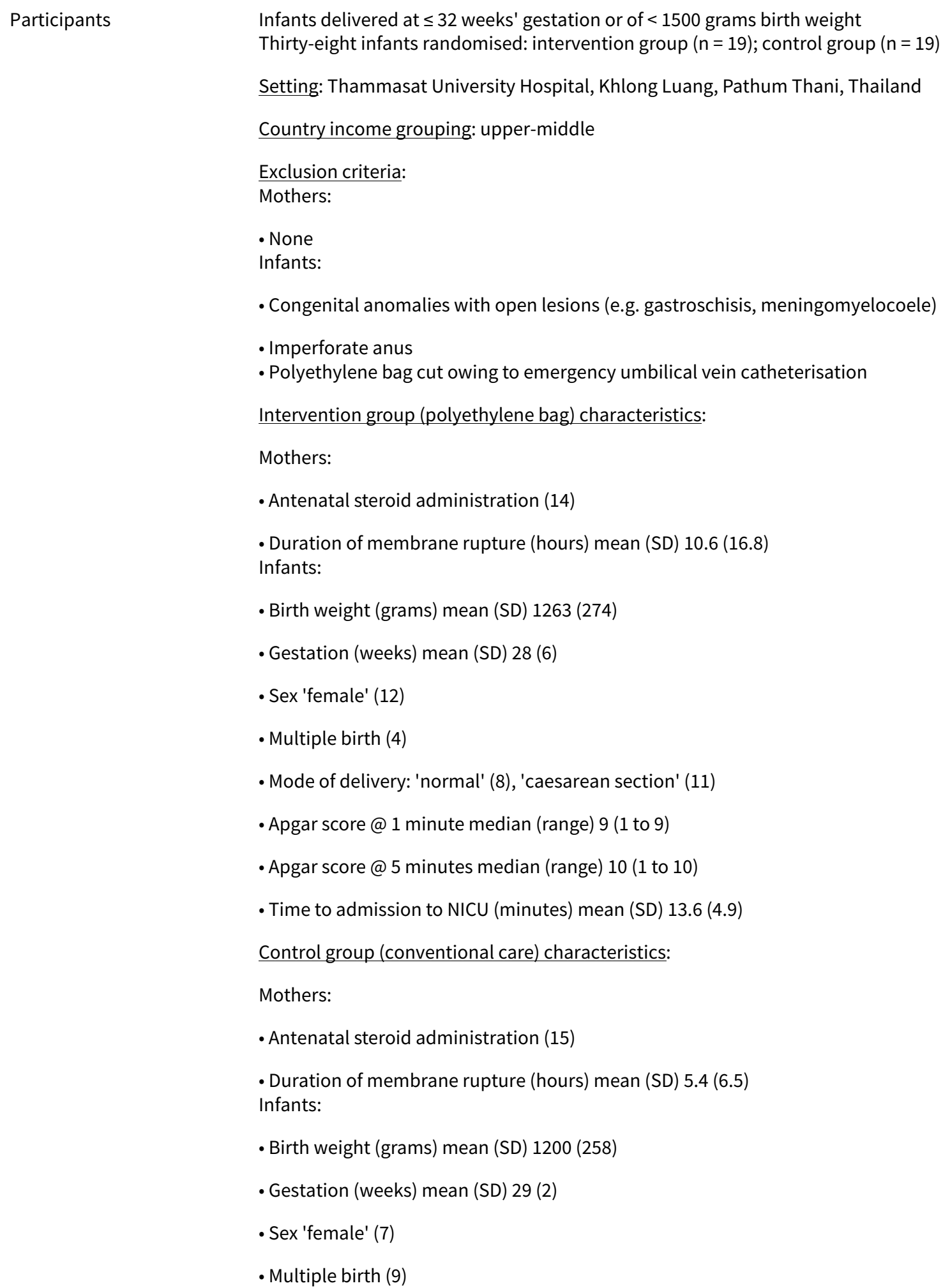


Chantaroj 2011 (Continued)

- Mode of delivery: 'normal' (3), 'caesarean section' (16)

-Apgar score @ 1 minute median (range) 7 (2 to 10)

- Apgar score @ 5 minutes median (range) 10 (2 to 10)

- Time to admission to NICU (minutes) mean (range) 16.2 (6.7)

Interventions

Polyethylene bag - $27 \mathrm{~cm}$ by $28 \mathrm{~cm}$

Infants in both groups were stabilised under a radiant warmer.

Intervention group:

A polyethylene bag was opened under the radiant warmer, and the infant was placed in the bag from the shoulders down without drying. Only the head was dried, and a yarn hat was placed on the infant before transfer to the NICU. Airway management and vital sign observations were carried out through the transparent bag. Infants were covered with warm blankets and were transferred to the neonatal unit in prewarmed transport incubators. Upon arrival to the NICU, infants were placed under radiant warmers, and the polyethylene bags were removed.

\section{Control group:}

Infants were routinely dried under a radiant warmer, and a yarn hat was placed on the infant before transfer to the NICU. Airway management and vital sign observations were carried out in accordance with the International Guidelines for Neonatal Resuscitation. After initial stabilisation, infants were covered with a polyvinyl wrap according to standard care in this hospital before transfer to the NICU. Infants in both groups were covered with warm blankets and were transferred to the neonatal unit in prewarmed transport incubators.

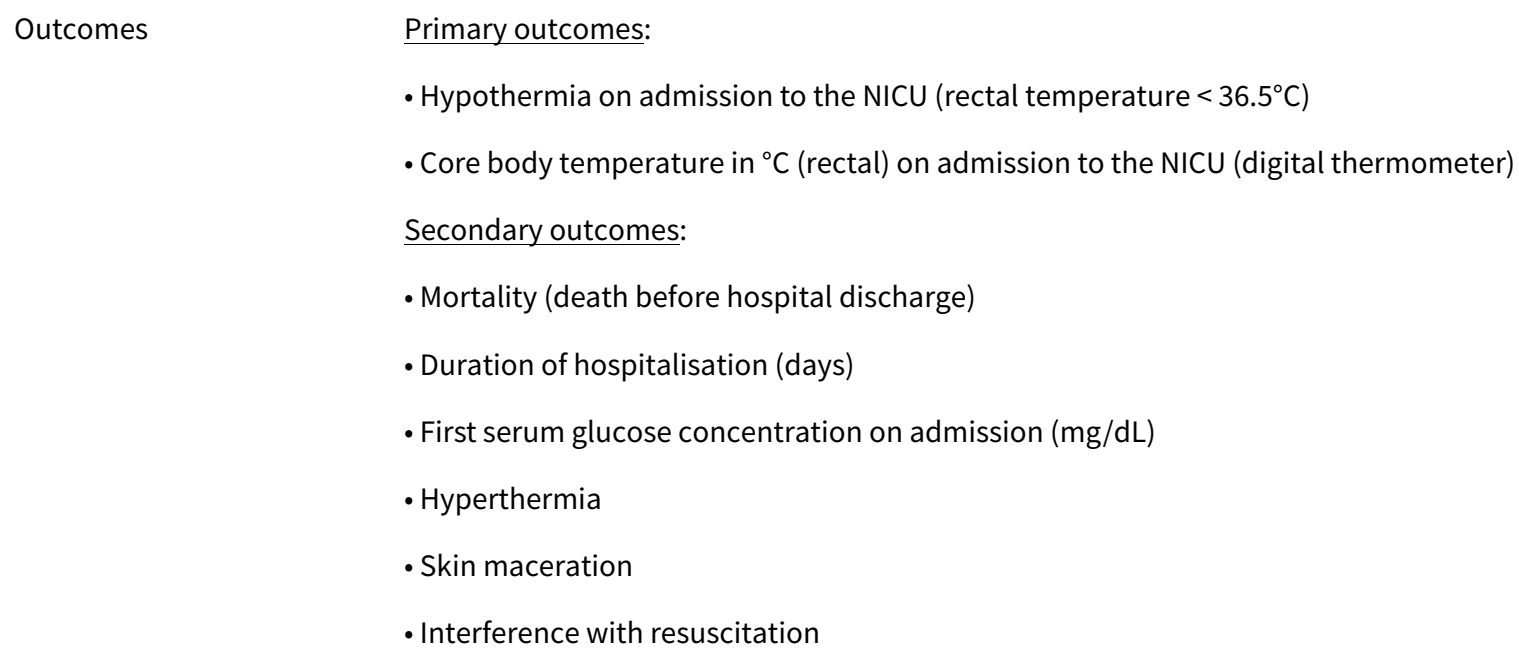
control group:median (range) 25.0 ( 25.0 to 26.0 ). The study aimed to keep the delivery room temperature at $26^{\circ} \mathrm{C}$ for every preterm delivery.

\section{Risk of bias}

\begin{tabular}{lll}
\hline Bias & Authors' judgement & Support for judgement \\
\hline $\begin{array}{l}\text { Random sequence genera- } \\
\text { tion (selection bias) }\end{array}$ & Low risk & $\begin{array}{l}\text { Quote: "The infants were stratified by gestational age }(<28 \text { weeks or } \geq 28 \\
\text { weeks). A block of } 4 \text { randomisations was used to ensure a balance of infants in } \\
\text { each allocation" }\end{array}$ \\
& $\begin{array}{l}\text { Quote: (from correspondence) "We used the computer to generate the random } \\
\text { sequence" }\end{array}$
\end{tabular}


Chantaroj 2011 (Continued)

Allocation concealment High risk Quote: "Randomisation was carried out in the delivery room by opening (selection bias) (opaque-sealed) envelopes just prior to birth"

Comment: Study authors did not explicitly report that the sealed opaque envelopes were sequentially numbered.

$\begin{array}{ll}\begin{array}{l}\text { Blinding of participants } \\ \text { and personnel (perfor- }\end{array} & \text { High risk } \\ \begin{array}{l}\text { mance bias) } \\ \text { All outcomes }\end{array} & \begin{array}{l}\text { Blinding of intervention: participants: unfeasible } \\ \text { Comment: Review authors agreed that it was unfeasible to blind participants } \\ \text { and healthcare providers/personnel to the intervention. }\end{array}\end{array}$

\begin{tabular}{lll}
\hline Blinding of outcome as- & High risk & Blinding of outcome measurement: no \\
$\begin{array}{l}\text { sessment (detection bias) } \\
\text { All outcomes }\end{array}$ & $\begin{array}{l}\text { Quote: (from correspondence) "No blinding of participants, care providers and } \\
\text { outcome assessors" }\end{array}$
\end{tabular}

Incomplete outcome data Low risk Complete follow-up: All infants randomised completed the study.
(attrition bias)

All outcomes

\begin{tabular}{|c|c|c|}
\hline $\begin{array}{l}\text { Selective reporting (re- } \\
\text { porting bias) }\end{array}$ & Unclear risk & Information is insufficient to assess risk of bias. \\
\hline Other bias & Low risk & \\
\hline
\end{tabular}

\section{Chawla 2011}

Quasi-randomised
Single-centre
Blinding of randomisation:
- Generation of allocation sequence: inadequate, intervention used for predetermined 12th hour of the
day from 08:00 to 20:00 hours or from 20:00 to 08:00 hours on alternate months
- Allocation concealment: inadequate, as above
Blinding of intervention: participants: unfeasible; healthcare providers/personnel: unfeasible
Blinding of outcome measurement: no
Complete follow-up: yes (all 102 infants randomised completed the study)
Delivery room ambient temperatures $\left({ }^{\circ} \mathrm{C}\right)$ were recorded.
Study was approved by the Wayne State University Institutional Review Board, which authorised enrol-
ment of participants without previous informed parental consent. Parents were provided with a study
information sheet after delivery, and further medical record data extraction was performed if parental
verbal consent was given.

\section{Participants}

Infants at $<32$ weeks' gestation

One hundred two infants randomised: intervention group $(n=53)$; control group $(n=49)$

Setting: Hutzel Women's Hospital, Detroit, Michigan, USA

Country income grouping: high

Exclusion criteria:

Mothers:

- Maternal fever $\left(>38.2^{\circ} \mathrm{C}\right)$ just before delivery

Infants:

- Major congenital anomalies

- Those considered to be previable 
Mothers:

- Maternal age (years) mean (SD) 25.3 (6.0)

- Antenatal steroids (43)

- Temperature $\left({ }^{\circ} \mathrm{C}\right)$ before delivery mean (SD) $36.8(0.5)$

- Temperature $\left({ }^{\circ} \mathrm{C}\right)$ post delivery mean (SD) $36.3(0.7)$

Infants:

- Gestation (weeks) mean (SD) 28.7 (3.0)

- Birth weight (grams) mean (SD) 1151 (407)

- Chorioamnionitis (10)

- Race 'Black' (46)

- Mode of delivery: 'caesarean section' (22)

-Sex 'male' (26)

- Cord pH mean (SD) 7.28 (0.1)

-Apgar score @ 5 minutes mean (SD) 6 (2.2)

- Delivery room resuscitation (36)

Control group (conventional care) characteristics:

Mothers:

- Maternal age (years) mean (SD) 25.4 (5.7)

- Antenatal steroids (31)

- Temperature $\left({ }^{\circ} \mathrm{C}\right)$ before delivery mean (SD) $36.6(0.5)$

- Temperature $\left({ }^{\circ} \mathrm{C}\right)$ post delivery mean (SD) 36.4 (0.7)

Infants:

- Gestation (weeks) mean (SD) 28.7 (2.4)

• Birth weight (grams) mean (SD) 1175 (413)

- Chorioamnionitis (9)

- Race 'Black' (45)

- Mode of delivery: 'caesarean section' (20)

- Sex 'male' (23)

- Cord pH mean (SD) 7.25 (0.16)

-Apgar score @ 5 minutes mean (SD) 6 (2.2)

- Delivery room resuscitation (35) maximum temperature of $40^{\circ} \mathrm{C}$ within 60 seconds, with the effect lasting up to 2 hours. All neonatal fellows were trained on appropriate use of the TWM before commencement of the study. All infants received routine thermal care, including hats and radiant warmers, and were transported to the NICU in prewarmed incubators with temperatures set at $37^{\circ} \mathrm{C}$. Infants at $<28$ weeks' gestation were placed without drying in a reclosable plastic bag below the neck. All deliveries were attended by a neonatal 
fellow, along with at least 2 paediatric residents and a respiratory therapist and in some cases a neonatal nurse or nurse practitioner.

Intervention group:

Infants were received onto the TWM, which was activated before delivery, and were covered with a blanket and placed on the resuscitation warmer. The infant remained on the TWM from the delivery room until the time of first temperature in the NICU. Axillary temperature $\left({ }^{\circ} \mathrm{C}\right)$ was measured in the delivery room before the infant was placed onto the transport isolette and on arrival to the NICU.

Control group:

Same as intervention group without use of the TWM

- Core body temperature in ${ }^{\circ} \mathrm{C}$ (axillary) on admission to the NICU, measured with a digital thermometer (Temp-Plus II, Signature Emergency Products, LLC, Boothwyn, Pennsylvania, USA)

- Moderate hypothermia: core body (axillary) temperature $<36{ }^{\circ} \mathrm{C}$ on admission to the NICU

Secondary outcomes:

- Mortality

- Need for delivery room resuscitation

- Hypoglycaemia (initial blood glucose $<40 \mathrm{mg} / \mathrm{dL}$ or $<2.2 \mathrm{mmol} / \mathrm{L}$ )

- Metabolic acidosis ( $\mathrm{pH}<7.15$ with base excess $\geq 10$ on initial blood gas)

- Necrotising enterocolitis $\geq$ stage 2 (Bell 1978)

- Intraventricular haemorrhage grade III or IV (Papile 1978)

- Bronchopulmonary dysplasia (oxygen need at 36 weeks' postmenstrual age)

- Sepsis (positive culture from sterile site such as blood or spinal fluid and intention to treat with antibiotics for $\geq 7$ days)

- Cystic periventricular leukomalacia on head ultrasonography

- Duration of oxygen administration

- Requirement for ventilation

- Duration of hospital stay (days)

- Hyperthermia: core body temperature (axillary) $>37.5^{\circ} \mathrm{C}$ on admission to the NICU

- Local skin reaction as noted by research personnel or bedside nurse soon after removal of the TWM

- Interference with resuscitation (yes/no response on questionnaire following each delivery)

\section{Risk of bias}


Chawla 2011 (Continued)

Random sequence genera- High risk Quote: "A quasi randomised trial was performed....Transwarmer infant transtion (selection bias) port mattress used for predetermined 12 hours of the day, either from 08:00 to 20:00 hours or 20:00 to 08:00 hours on alternate months"

\begin{tabular}{lll}
\hline $\begin{array}{l}\text { Allocation concealment } \\
\text { (selection bias) }\end{array}$ & High risk above
\end{tabular}

\begin{tabular}{ll}
\hline $\begin{array}{l}\text { Blinding of participants } \\
\text { and personnel (perfor- }\end{array}$ & High risk \\
$\begin{array}{l}\text { mance bias) } \\
\text { All outcomes }\end{array}$ & $\begin{array}{l}\text { Blinding of participants: unfeasible } \\
\text { Blinding of healthcare providers/personnel: unfeasible }\end{array}$ \\
& $\begin{array}{l}\text { Quote: "The study was not blinded due to the nature of the intervention" } \\
\text { Comment: Review authors agreed that it was unfeasible to blind participants } \\
\text { and healthcare providers/personnel to the intervention. }\end{array}$ \\
\hline
\end{tabular}

\begin{tabular}{lll}
\hline $\begin{array}{l}\text { Blinding of outcome as- } \\
\text { sessment (detection bias) } \\
\text { All outcomes }\end{array}$ & High risk & Blinding of outcome measurement: no \\
\hline $\begin{array}{l}\text { Incomplete outcome data } \\
\text { (attrition bias) }\end{array}$ & Low risk & Complete follow-up: All infants randomised completed the study. \\
All outcomes & $\begin{array}{l}\text { Comment: } 27 \text { infants did not have their temperature measured in the delivery } \\
\text { room owing to unavailability of the thermometer; however, this outcome mea- } \\
\text { sure was not used in the review meta-analyses. }\end{array}$
\end{tabular}

\begin{tabular}{|c|c|c|}
\hline $\begin{array}{l}\text { Selective reporting (re- } \\
\text { porting bias) }\end{array}$ & Unclear risk & Information is insufficient to assess risk of bias. \\
\hline Other bias & Low risk & \\
\hline
\end{tabular}

\section{Doglioni 2014}

\begin{tabular}{|c|c|}
\hline \multirow[t]{11}{*}{ Methods } & Randomised; infants of multiple births randomised as individuals \\
\hline & Multi-centre (3) \\
\hline & Blinding of randomisation: \\
\hline & - Generation of allocation sequence: adequate, computer-generated randomised sequence \\
\hline & $\begin{array}{l}\text {-Allocation concealment: adequate, double-enclosed, opaque, sealed, and sequentially numbered en- } \\
\text { velopes prepared at the University Hospital of Padova, then distributed to participating centres. Infants } \\
\text { of multiple births were randomised as individuals. }\end{array}$ \\
\hline & Blinding of intervention: participants/healthcare providers/personnel: unfeasible \\
\hline & Blinding of outcome measurement: no \\
\hline & Complete follow-up: yes (all 100 infants randomised completed the study) \\
\hline & $\begin{array}{l}\text { Study protocol was approved by the Ethical Committee for Human Investigation at each participating } \\
\text { centre. }\end{array}$ \\
\hline & Written Informed consent was obtained. \\
\hline & Delivery room/operating room temperature in ${ }^{\circ} \mathrm{C}$ and time to NICU admission (minutes) were recorded. \\
\hline
\end{tabular}


Doglioni 2014 (Continued)

One hundred infants randomised: intervention group $(n=50)$; control group $(n=50)$

Setting: Three level 3 hospitals in Veneto region of Italy (Department of Women's and Children's Health, Medical School, University of Padua; Azienda Ospedaliera di Padova, Padua, San Bortolo Hospital, Vicenza and Camposampiero Hospital, Camposampiero)

Country income grouping: high

Exclusion criteria:

-Infants with congenital anomalies with open lesions (e.g. gastroschisis, meningomyelocoele)

- Infants whose delivery was not attended by the neonatal team

Intervention group $(\mathrm{n}=50)$ characteristics:

Mothers:

- Age (years) mean (SD) $33.6(5.8)$

- Temperature $\left({ }^{\circ} \mathrm{C}\right)$ mean (SD) $36.6(0.5)$

- Completed antenatal steroids (38)

- Vaginal delivery (7)

- Caesarean section (43)

- Multiple birth (10)

Infants:

- Gestation (weeks) mean (SD) 25.7 (1.7)

- Birth weight (grams) mean (SD) 789 (214)

- Head circumference (cm) mean (SD) 24.0 (2.1)

- Body length (cm) mean (SD) 32.7 (2.9)

- Small-for-gestational age (9)

- Male/female (28/22)

-Apgar score @ 1 minute median (IQR) 6 (5 to 7)

-Apgar score @ 5 minutes median (IQR) 8 (7 to 8)

- Intubated in delivery room/operating room (26)

Control group $(n=50)$ characteristics:

Mothers:

- Age (years) mean (SD) 33.1 (5.6)

-Antenatal steroids (41)

- Temperature $\left({ }^{\circ} \mathrm{C}\right)$ mean (SD) $36.7(0.6)$

- Vaginal delivery (6)

- Caesarean section (44)

- Multiple birth (15)

Infants: 
Doglioni 2014 (Continued)

- Gestation (weeks) mean (SD) 25.8 (1.7)

- Birth weight (grams) mean (SD) 770 (245)

- Head circumference (cm) mean (SD) 3.8 (2.3)

- Body length (cm) mean (SD) 32.5 (3.7)

- Small-for-gestational age (12)

- Male/female (27/23)

-Apgar score @ 1 minute median (IQR) 6 (3 to 7)

-Apgar score @ 5 minutes median (IQR) 8 (6 to 8)

- Intubated in delivery room/operating room (39)

Interventions

Total body wrap: polyethylene bags (Vedovato SNC; Camposampiero, Padova, Italy, isolation transport bag $60 \mathrm{~cm}$ by $40 \mathrm{~cm}$ )

Intervention group:

Prewarmed bags were cut on 1 side (cut similar to a sleeping bag). The infant's wet body and head (face exposed) were covered with the bag quickly after birth.

\section{Comparison group:}

Infant was placed into prewarmed polyethylene bag (cut similar to a sleeping bag) while wet. Only the head was dried and left uncovered. All infants were cared for in the delivery room with radiant warmers (PANDA i-RES; GE Healthcare, Laurel, Maryland, USA), which were turned on approximately 10 minutes before birth (manual control $85 \%$ power output). Polyethylene bags were placed on radiant warmer before delivery. Infant stabilisation and resuscitation in delivery room were carried out as clinically indicated in line with the International Liaison Committee on Resuscitation Guidelines (Kattwinkel 2010). The gases used to provide respiratory support in the delivery room were neither heated nor humidified. Umbilical cord clamping was not delayed for any infant in either group. Immediately after birth, a hole provided access for oximetry sensor (Radical-7; Masimo, Irvine, California, USA). Infants in both groups were transferred to the NICU in a transport incubator set to maximum air temperature $\left(35^{\circ} \mathrm{C}\right.$ to $37^{\circ} \mathrm{C}$ ) (Ginevri; Tecnologie Biomediche, Cecchina, Rome, Italy; or Draeger 5400; Draegerwerk, Lubeck, Germany). At NICU admission, all infants were placed in a double-walled incubator (8000 SC; Drager Medical, Lubeck, Germany) maintained at $35^{\circ} \mathrm{C}$ and $70 \%$ humidity. The wrap was removed, and axillary temperature was taken. Mothers' axillary temperature was measured within 30 minutes of delivery. Delivery or operating room temperature was recorded (wall-mounted thermometer). All core body temperatures in ${ }^{\circ} \mathrm{C}$ (axillary) were recorded via a digital thermometer (C202; Terumo, Tokyo, Japan).

Outcomes

\section{Primary outcomes:}

- Core body temperature in ${ }^{\circ} \mathrm{C}$ (axillary) on admission to NICU (immediately after wrap removal)

- Hypothermia (mild: core body temperature (axillary) $36.0^{\circ} \mathrm{C}$ to $36.4^{\circ} \mathrm{C}$; moderate: core body temperature (axillary) $32.0^{\circ} \mathrm{C}$ to $35.9^{\circ} \mathrm{C}$ ) on admission to NICU

- Hyperthermia (mild: core body temperature (axillary) $>37.5^{\circ} \mathrm{C}$; moderate: core body temperature (axillary) $>38^{\circ} \mathrm{C}$ ) on admission to NICU

\section{Secondary outcomes:}

- Core body temperature in ${ }^{\circ} \mathrm{C}$ (axillary) 1 hour after NICU admission

- Mortality before hospital discharge

- Bronchopulmonary dysplasia (oxygen dependency at 36 weeks' post conception)

- Major brain injury (sonographic evidence of IVH with ventricular dilatation, parenchymal haemorrhagic infarction, or periventricular leukomalacia) 
Doglioni 2014 (Continued)

- Blood gas analysis

- Serum glucose concentration $(\mathrm{mg} / \mathrm{dL})$ on NICU admission

- Necrotising enterocolitis

Notes

Delivery or operating room ambient temperature $\left({ }^{\circ} \mathrm{C}\right)$ mean $(\mathrm{SD})$ was $23.9(0.7)$ and $24.1(0.7)(\mathrm{P}=0.31)$ in the intervention and control groups, respectively.

Blood gas analysis (base excess) and serum glucose concentration on NICU admission were used in regression analysis.

\section{Risk of bias}

\begin{tabular}{lll}
\hline Bias & Authors' judgement & Support for judgement \\
\hline $\begin{array}{l}\text { Random sequence genera- } \\
\text { tion (selection bias) }\end{array}$ & Low risk & $\begin{array}{l}\text { Quote: "Eligible infants were assigned to the total body wrap or control group } \\
\text { at 1:1 for each participating centre according to a computer-generated, ran- } \\
\text { domised sequence" }\end{array}$ \\
\hline $\begin{array}{l}\text { Allocation concealment } \\
\text { (selection bias) }\end{array}$ & Low risk & $\begin{array}{l}\text { Quote: "The randomised allocation was concealed in double-enclosed, } \\
\text { opaque, sealed, and sequentially numbers prepared at University Hospital of } \\
\text { Padua and then distributed to participating centres...... In the delivery or oper- } \\
\text { ating room, the next sequential randomised envelope was opened only when } \\
\text { the infant was deemed eligible by the attending neonatologist" }\end{array}$ \\
\hline
\end{tabular}

\begin{tabular}{ll}
\hline $\begin{array}{l}\text { Blinding of participants } \\
\text { and personnel (perfor- }\end{array}$ & High risk \\
$\begin{array}{l}\text { mance bias) } \\
\text { All outcomes }\end{array}$ & $\begin{array}{l}\text { Blinding of participants: unfeasible } \\
\text { Blinding of healthcare providers/personnel: unfeasible }\end{array}$ \\
& $\begin{array}{l}\text { Comment: Review authors agreed that it was unfeasible to blind participants } \\
\text { and healthcare providers/personnel to the intervention. }\end{array}$
\end{tabular}

\begin{tabular}{|c|c|c|}
\hline $\begin{array}{l}\text { Blinding of outcome as- } \\
\text { sessment (detection bias) } \\
\text { All outcomes }\end{array}$ & High risk & Blinding of outcome assessment: no \\
\hline $\begin{array}{l}\text { Incomplete outcome data } \\
\text { (attrition bias) } \\
\text { All outcomes }\end{array}$ & Low risk & Complete follow-up: All infants randomised completed the study. \\
\hline $\begin{array}{l}\text { Selective reporting (re- } \\
\text { porting bias) }\end{array}$ & Low risk & $\begin{array}{l}\text { All study outcomes detailed at the time of trial registration were reported in } \\
\text { the published manuscript as per } \\
\text { https://clinicaltrials.gov/ct2/show/NCT01671241. }\end{array}$ \\
\hline Other bias & Low risk & $\begin{array}{l}\text { Seven infants assessed for eligibility were excluded because the neonatal team } \\
\text { involved in the study was not present at the delivery ( } 5 \text { infants) and parents } \\
\text { declined to participate ( } 2 \text { infants). Rate of intubation was significantly higher } \\
\text { in the control group; however, delivery/operating room temperature and time } \\
\text { to NICU admission were similar in both groups. }\end{array}$ \\
\hline
\end{tabular}

\section{Farhadi 2012}

$\begin{array}{ll}\text { Methods } & \text { Randomised } \\ & \text { Single-centre } \\ & \text { Blinding of randomisation: } \\ & \text { - Generation of allocation sequence: adequate, random number tables (block randomisation) }\end{array}$


Farhadi 2012 (Continued)

- Allocation concealment: unclear, sealed opaque envelopes

Blinding of intervention: participants: unfeasible; healthcare providers/personnel: unfeasible

Blinding of outcome measurement: unclear

Complete follow-up: yes (all 40 infants randomised completed the study)

Delivery room temperature was recorded.

The proposal for the study was approved by the Research Ethics Committee in the Mazandaran University of Medical Science, Sari, Iran.

Participants

Inborn infants at $\leq 32$ weeks' gestation born alive in the maternity or operating room

Forty infants randomised: intervention group $(n=20)$; control group $(n=20)$

Setting: Imam Khomeini Educational and Medical Hospital, Sari, Iran

Country income grouping: upper-middle

Exclusion criteria:

Mothers:

- Maternal high fever

Infants:

- Neural tube defects

- Distinct inherited disorders

- Inherited skin diseases such as epidermolysis bullosa

-Abdominal wall disorders: omphalocoele, gastroschisis

$\bullet<23$ weeks' gestation or birth weight $<400$ grams

-Small-for-gestation

Intervention group (Zipkif plastic food bags) characteristics:

Mothers:

- None

Infants:

- Gestation (weeks) mean (SD) 28.8 (2.8)

-Sex 'male' (11)

• Birth weight (grams) mean (SD) 1278 (518)

- Mode of delivery: 'vaginal' (5)

-Apgar @ 1 minute median (6)

-Apgar@ 5 minutes median (7)

Control group (conventional care) characteristics:

Mothers:

- None

Infants:

- Gestation (weeks) mean (SD) 29.2 (2.3)

- Sex 'male' (10)

- Birth weight (grams) mean (SD) 1190 (492)

- Mode of delivery: 'vaginal' (5) 
Farhadi 2012 (Continued)

-Apgar@ 1 minute median (6)

-Apgar @ 5 minutes median (8)

Interventions $\quad$ Zipkif plastic food bag - manufactured by TechnoSip Inc., New York, New York, USA; heat-resistant microwave bags made from polyethylene $40 \mathrm{~cm}$ by $25 \mathrm{~cm}$ and cut out from upper neck area

Intervention group:

Immediately after birth and umbilical cord cutting, infants were placed in a prewarmed plastic bag up to the neck (head positioned out of the bag dried and covered with a cap) and posterior areas were immediately closed. Resuscitation continued until the infant reached normal status. Body temperature in ${ }^{\circ} \mathrm{C}$ (axillary) upon admission was measured with a digital thermometer (TD12-Exotherm, Maxi-Med Medical Supply, Simi Valley, California, USA) via a small hole. All other initial examinations were carried out on the surface of the bag. In an emergency for which the umbilical vein catheter was required or pulse oximeter small holes were installed in the plastic bag, infant was removed from the plastic bag and transferred to the maternity ward.

Control group:

Infants were dried conventionally with warm blankets. After stabilisation, both groups were transferred to the maternity ward in a portable incubator set at $35^{\circ} \mathrm{C}$, where they were placed under a radiant warmer.

Primary outcomes:
O Core body temperature in ${ }^{\circ} \mathrm{C}$ (axillary) on admission to NICU
$\cdot$ Hypothermia: core body temperature (axillary) $<36.5^{\circ} \mathrm{C}$ on admission to NICU
$\cdot$ Core body temperature in ${ }^{\circ} \mathrm{C}$ (axillary) 1 hour after stabilisation
Secondary outcomes:
$\cdot$ Hyperthermia: core body temperature (axillary) $>37.5^{\circ} \mathrm{C}$ on admission to NICU
$\cdot$ Arterial oxygen saturation on admission to NICU
$\cdot$ Blood sugar (mean) at 1 hours after birth measured with a glucometer
$\cdot$ Intraventricular haemorrhage (brain sonography on third day after birth - all grades)
$\cdot$ Mortality within 3 days of life
$\cdot$ Resuscitation time in seconds (mean)

This manuscript was published in Persian and subsequently was translated into the English language by Flex Language Services Ltd., Belfast.

\section{Risk of bias}

Bias Authors' judgement Support for judgement

$\begin{array}{lll}\begin{array}{l}\text { Random sequence genera- } \\ \text { tion (selection bias) }\end{array} & \text { Low risk } & \begin{array}{l}\text { Quote: (from correspondence) "We used random allocation from random } \\ \text { number tables as a concurrent block randomisation in parallel groups..." }\end{array}\end{array}$

Allocation concealment High risk
(selection bias)
(selection bias)
Quote: (from correspondence) "We used sealed opaque envelopes which immediately before the delivery the midwife delivered it to resident who was responsible for trial" 
Farhadi 2012 (Continued)

Comment: Study authors did not explicitly report that the sealed opaque envelopes were sequentially numbered. We noted some difficulties pertaining to translation of the randomisation method, so it may be likely that sequential numbering was carried out.

\begin{tabular}{|c|c|c|}
\hline $\begin{array}{l}\text { Blinding of participants } \\
\text { and personnel (perfor- } \\
\text { mance bias) }\end{array}$ & High risk & $\begin{array}{l}\text { Blinding of participants: unfeasible } \\
\text { Blinding of healthcare providers/personnel: unfeasible }\end{array}$ \\
\hline All outcomes & & $\begin{array}{l}\text { Comment: Review authors agreed that it was unfeasible to blind participants } \\
\text { and healthcare providers/personnel to the intervention. }\end{array}$ \\
\hline
\end{tabular}

\begin{tabular}{|c|c|c|}
\hline $\begin{array}{l}\text { Blinding of outcome as- } \\
\text { sessment (detection bias) }\end{array}$ & Unclear risk & $\begin{array}{l}\text { Blinding of outcome measurement: unclear } \\
\text { Comment: Information is insufficient to assess risk of bias. }\end{array}$ \\
\hline
\end{tabular}

\begin{tabular}{|c|c|c|}
\hline $\begin{array}{l}\text { Incomplete outcome data } \\
\text { (attrition bias) } \\
\text { All outcomes }\end{array}$ & Low risk & Complete follow-up: All infants randomised completed the study. \\
\hline $\begin{array}{l}\text { Selective reporting (re- } \\
\text { porting bias) }\end{array}$ & Low risk & $\begin{array}{l}\text { All study outcomes detailed at the time of trial registration were reported in } \\
\text { the published manuscript as per }\end{array}$ \\
\hline
\end{tabular}

Other bias Low risk

\section{Gathwala 2010}

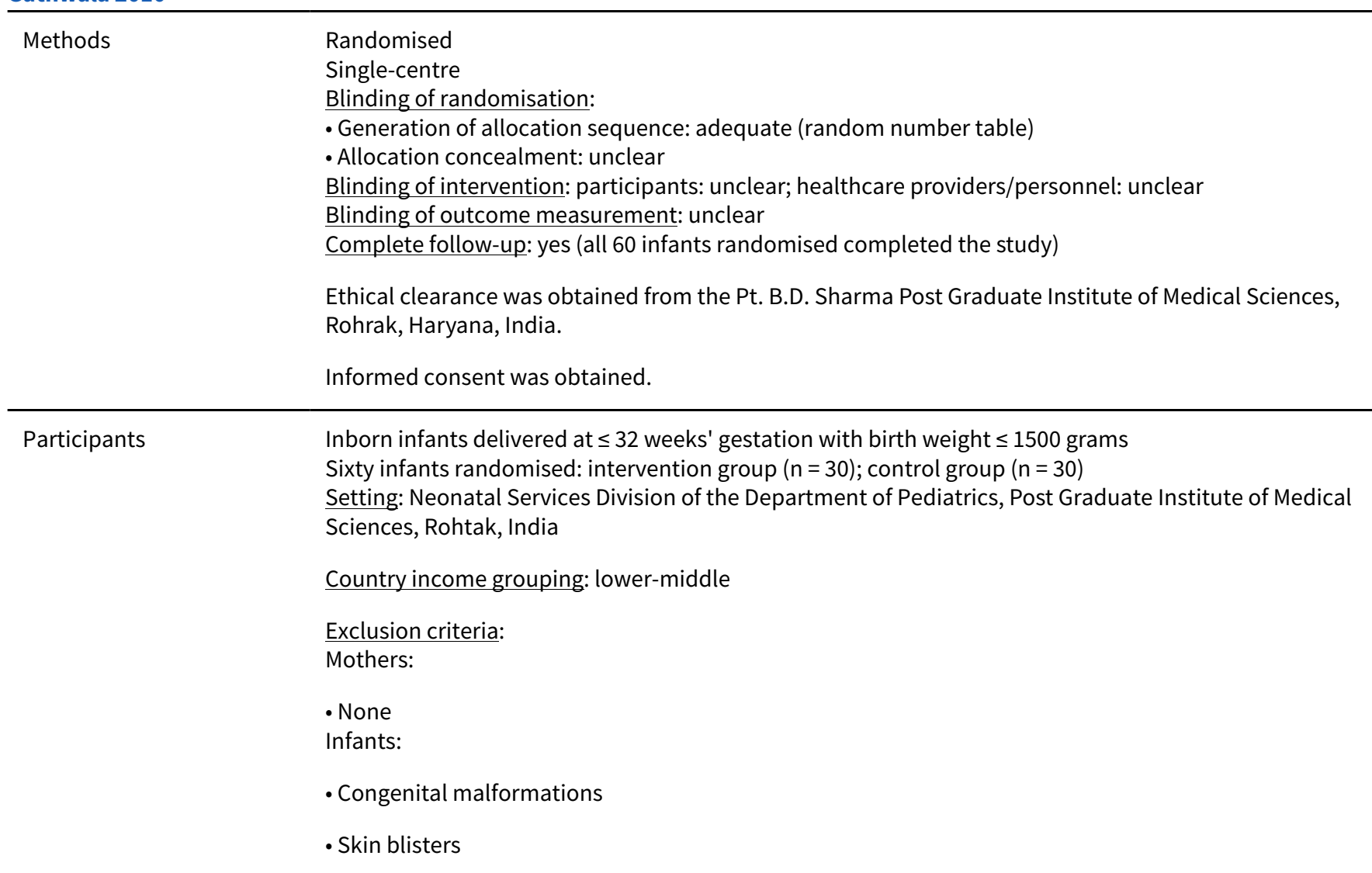


- Parental refusal to consent

Intervention group (vinyl bag) characteristics:

Mothers:

- Maternal age (years) mean (SD) 20.93 (2.99)

- Booked (18)

Infants:

- Gestation (weeks) mean (SD) 29.93 (1.08)

- Birth weight (kg) mean (SD) 1.12 (0.19)

-Apgar score @1 minute mean (SD) 6.13 (1.35)

•Apgar score @ 5 minutes mean (SD) 7.76 (1.13)

- Mode of delivery: 'emergency caesarean section' (2)

- Cord pH mean (SD) 7.24 (0.11)

Control group (conventional care) characteristics:

Mothers:

- Maternal age (years) mean (SD) 20.43 (2.06)

- Booked (9)

Infants:

- Gestation (weeks) mean (SD) 29.46 (1.33)

- Birth weight (kg) mean (SD) 1.11 (0.23)

•Apgar score @1 minute mean (SD) 5.66 (1.78)

•Apgar score @ 5 minutes mean (SD) 7.63 (0.92)

- Mode of delivery: 'emergency caesarean section' (1)

- Cord pH mean (SD) 7.22 (0.123)

Intervention group:

Infants were placed in vinyl bags up to the neck; the head was covered with a cap after drying immediately following delivery, and the infants was resuscitated under a radiant warmer. Ascultation was carried out over the bag, and a hole was made if umbilical access was needed.

Control group:

Infants were resuscitated by conventional drying under a radiant warmer. Both groups were moved to the NICU in a prewarmed transport incubator (HM-NeoTR, Hill-Med, Inc., Miami, Florida, USA) set at a temperature of $35^{\circ} \mathrm{C}$ and were placed in an open care warmer, where axillary and rectal temperature was measured by thermometer immediately after the vinyl bag was removed after 1 hour.

Outcomes

Primary outcomes:

- Core body temperature in ${ }^{\circ} \mathrm{C}$ (rectal and axillary) on admission to NICU

- Core body temperature in ${ }^{\circ} \mathrm{C}$ (rectal and axillary) 1 hour after admission to NICU

Secondary outcomes: 
Gathwala 2010 (Continued)

- Hyperthermia

Notes

Delivery room ambient temperatures not reported. Cap material used in intervention group not known, and not reported whether cap was used in the control group. Trial author contacted for further information, but no reply received (May 2013)

\section{Risk of bias}

\begin{tabular}{lll}
\hline Bias & Authors' judgement & Support for judgement \\
\hline $\begin{array}{l}\text { Random sequence genera- } \\
\text { tion (selection bias) }\end{array}$ & Low risk & $\begin{array}{l}\text { Quote: "......were randomised to either the study group (vinyl bag group) or the } \\
\text { control group) using a random number table" }\end{array}$ \\
\hline $\begin{array}{l}\text { Allocation concealment } \\
\text { (selection bias) }\end{array}$ & Unclear risk & Information is insufficient to assess risk of bias. \\
\hline $\begin{array}{l}\text { Blinding of participants } \\
\text { and personnel (perfor- } \\
\text { mance bias) }\end{array}$ & High risk & Blinding of participants: unfeasible \\
All outcomes & Blinding of healthcare providers/personnel: unfeasible \\
& $\begin{array}{l}\text { Comment: Review authors agreed that it was unfeasible to blind participants } \\
\text { and healthcare providers/personnel to the intervention. }\end{array}$ \\
\hline
\end{tabular}

\begin{tabular}{|c|c|c|}
\hline $\begin{array}{l}\text { Blinding of outcome as- } \\
\text { sessment (detection bias) }\end{array}$ & Unclear risk & Blinding of outcome measurement: unclear \\
\hline All outcomes & & issess risk of bias. \\
\hline
\end{tabular}

Incomplete outcome data Low risk Complete follow-up: All infants randomised completed the study.

(attrition bias)

All outcomes

Selective reporting (re- Unclear risk Information is insufficient to assess risk of bias.
porting bias)

porting bias)

Other bias Low risk

\section{Knobel 2005}

\begin{tabular}{ll}
\hline Rethods & Randomised \\
Single-centre \\
Blinding of randomisation: \\
- Generation of allocation sequence: adequate (tossing of sealed, opaque envelopes containing control \\
vs treatment cards) \\
• Allocation concealment: adequate (sealed, opaque envelopes, sequentially numbered) \\
Blinding of intervention: participants: unfeasible; healthcare providers/personnel: unfeasible \\
Blinding of outcome measurement: unclear \\
Complete follow-up: yes (all 88 infants randomised completed the study) \\
Delivery to admission time and delivery room environmental temperatures were recorded. \\
Parental consent was sought if the mother was expected to deliver before completing 29 weeks' gesta- \\
tion. \\
Infants delivered at <29 weeks' gestation \\
Eighty-eight infants randomised: intervention group ( $\mathrm{n}=41)$; control group ( $\mathrm{n}=47$ ) \\
Setting: Pitt County Memorial Hospital, Greenville, North Carolina, USA \\
Country income grouping: high \\
\hline Participants
\end{tabular}


Exclusion criteria:

- Congenital anomalies with open lesions (e.g. gastroschisis, meningomyelocoele)

- Resuscitation not undertaken because of previability

- Meconium staining of amniotic fluid

- Subsequent assessment indicated that infant had gestation of $\geq 29$ completed weeks.

Intervention group (polyurethane bag) characteristics:

- Birth weight (grams) mean (SD) 918 (259)

- Gestation (weeks) mean (SD) 26.5 (1.4)

- Delivery to admission time (minutes) mean (SD) 16.0 (6.6)

- Modified SNAPPE 11 score mean (SD) 29.6 (17.5)

Control group (conventional care) characteristics:

- Birth weight (grams) mean (SD) 850 (253)

- Gestation (weeks) mean (SD) 26.1 (1.4)

- Delivery to admission time (minutes) mean (SD) 15.9 (5.1)

- Modified SNAPPE 11 score mean (SD) 30.9 (19.1)

Interventions

Polyurethane bag - measured 19" by 18" (DeRoyal REF30-5510; DeRoyal Industries, Powell, Tennessee, USA)

Intervention group:

A polyurethane bag was placed on a radiant warmer bed before delivery. Infants were placed immediately into a polyurethane bag, while still wet, up to their necks, and the head and face were dried. Infants were resuscitated according to the guidelines for Neonatal Resuscitation.

Control group:

Infants received usual care in accordance with these guidelines without being placed in polyurethane bags. Both groups were taken to the neonatal intensive care unit on a warmer table (with the heat off) with warm blankets on top of the infant. Standard practice at the time of the study also included use of a knit hat for all infants. On arrival to the NICU, infants were placed under radiant warmers and bags were removed. Rectal temperatures were taken immediately with a digital thermometer (IVAC TempPlus II Electronic Thermometer, Model 2080, Alaris Medical Systems, San Diego, California, USA). After removal of bags, both groups received care according to usual NICU routines and attending staff preferences.

Outcomes Primary outcomes:

- Core body temperature in ${ }^{\circ} \mathrm{C}$ (rectal) on admission to the NICU

- Hypothermia on admission to the NICU: core body temperature (rectal) $<36.4^{\circ} \mathrm{C}$

Secondary outcomes:

- Mortality (death before hospital discharge)

- Major brain injury (sonographic evidence of IVH with ventricular dilatation, parenchymal haemor-

rhagic infarction, or periventricular leukomalacia)

- Duration of supplemental oxygen therapy (days)

- Duration of hospitalisation (days)

- Hyperthermia

- Interference with assessment and resuscitation 
Knobel 2005 (Continued)

Notes

Investigators attempted to prewarm delivery rooms to approximately $26^{\circ} \mathrm{C}$ for all deliveries at $<29$ weeks' gestation. Delivery room environmental temperatures were recorded via wall-mounted mercury thermometers positioned at eye level next to delivery room doors.

\section{Risk of bias}

\begin{tabular}{lll}
\hline Bias & Authors' judgement & Support for judgement \\
\hline $\begin{array}{l}\text { Random sequence genera- } \\
\text { tion (selection bias) }\end{array}$ & Low risk & $\begin{array}{l}\text { Quote: (from correspondence) "The actual control versus treatment card was } \\
\text { inserted in blank envelopes, the envelopes were tossed to mix them up, them } \\
\text { stacked and numbered" }\end{array}$ \\
\hline $\begin{array}{l}\text { Allocation concealment } \\
\text { (selection bias) }\end{array}$ & Low risk & $\begin{array}{l}\text { Quote: "Randomisation of consented infants was carried out in the delivery } \\
\text { room by opening sealed, opaque envelopes just prior to birth" }\end{array}$ \\
& $\begin{array}{l}\text { Quote: (from correspondence) "Although we did not write it in the publication, } \\
\text { the envelopes were numbered sequentially and stacked in a box" }\end{array}$
\end{tabular}

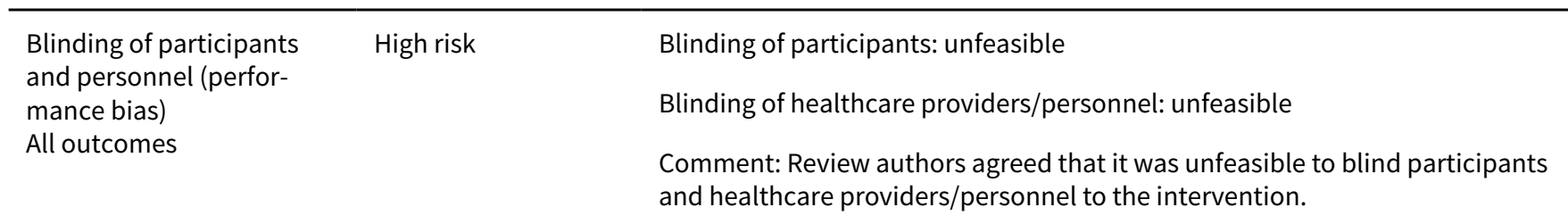

\begin{tabular}{|c|c|c|}
\hline $\begin{array}{l}\text { Blinding of outcome as- } \\
\text { sessment (detection bias) } \\
\text { All outcomes }\end{array}$ & Unclear risk & $\begin{array}{l}\text { Blinding of outcome measurement: unclear } \\
\text { Information is insufficient to assess risk of bias. }\end{array}$ \\
\hline $\begin{array}{l}\text { Incomplete outcome data } \\
\text { (attrition bias) } \\
\text { All outcomes }\end{array}$ & Low risk & Complete follow-up: All infants randomised completed the study. \\
\hline $\begin{array}{l}\text { Selective reporting (re- } \\
\text { porting bias) }\end{array}$ & Unclear risk & Information is insufficient to assess risk of bias. \\
\hline Other bias & High risk & $\begin{array}{l}\text { This study attempted to maintain delivery room temperature at } 26^{\circ} \mathrm{C} \text { for all } \\
\text { preterm deliveries, but actual temperatures ranged from } 18.9^{\circ} \mathrm{C} \text { to } 31.1^{\circ} \mathrm{C} \text {. On } \\
\text { post hoc analysis, warmer delivery room temperatures were associated with } \\
\text { higher admission temperatures, but only the subgroup of infants delivered } \\
\text { in warm rooms and placed in plastic bags had a mean temperature }>36.4^{\circ} \mathrm{C} \text {. } \\
\text { However, data show no significant differences between intervention and con- } \\
\text { trol groups in mean delivery room temperature. After controlling for delivery } \\
\text { suite temperature, mean admission temperature in the intervention group } \\
\text { was still } 0.6^{\circ} \mathrm{C} \text { higher than in the control group. }\end{array}$ \\
\hline
\end{tabular}

Leadford 2013

Randomised; twins and higher-order multiples randomised individually
Single-centre
Blinding of randomisation:
• Generation of allocation sequence: adequate, random number generator
• Allocation concealment: adequate, " blinded" sealed numbered envelopes
Blinding of intervention: participants: unfeasible; healthcare providers/personnel: unfeasible
Blinding of outcome measurement: no
Complete follow-up: yes (all 104 infants randomised completed the study)


Leadford 2013 (Continued)

The proposal for the study was approved by the institutional review boards at the University of Alabama at Birmingham, Oregon Health and Science University and University Teaching Hospital, Luska,

Zambia.

Informed written consent was obtained.

Participants

Inborn infants at $\geq 26$ and $<37$ weeks' gestation or with birth weight $\geq 1000$ and $\leq 2500$ grams

Infants were admitted to the NICU if birth weight was $<1400$ grams and infants had respiratory distress or other abnormal signs needing treatment or observation.

One hundred four infants randomised: intervention group $(n=49)$; control group $(n=55)$

Setting: Tertiary University Teaching Hospital, Luaska, Zambia

Country income grouping: lower-middle

Exclusion criteria:

Mothers:

- None

Infants:

- Abdominal wall defect

- Myelomeningocoele

- Other major congenital anomaly

- Obvious skin disorders

Intervention group (plastic bag) characteristics:

Infants:

- Gestation (weeks) median (IQR) 34 (32 to 36)

- Gestation (weeks) < $32(10)$

- Sex 'male' (27)

- Birth weight (kg) mean (SD) 2.20 (0.56)

- Mode of delivery: 'vaginal' (42)

- NICU admission (14)

- Hypothermia at 10 minutes (41)

Control group (conventional care) characteristics:

Infants:

- Gestation (weeks) median (IQR) 34 (31 to 36)

- Gestation (weeks) < 32 (14)

- Sex 'male' (28)

- Birth weight $(\mathrm{kg})$ mean (SD) 2.11 (0.52)

- Mode of delivery: 'vaginal' (51)

- NICU admission (9)

- Hypothermia at 10 minutes (45) 


\section{Intervention group:}

Infants received standard care (as in control group) except they were placed into a plastic bag covering the trunk and extremities (within 10 minutes of birth) after brief drying on the mother's abdomen during cord cutting. Infants remained in the plastic bag for at least 1 hour after birth. The bag was removed at 1 hour if the core temperature was within the normal range $\left(36.5^{\circ} \mathrm{C}\right.$ to $\left.37.5^{\circ} \mathrm{C}\right)$. Infants not achieving normothermia remained in the plastic bag until this was reached.

\section{Control group:}

Infants were placed on the mother's abdomen immediately after delivery, then were dried with blankets. If further resuscitation was needed, this was carried out in a small nursery in the labor and delivery unit, where a radiant warmer was available. Infants delivered by caesarean section were dried and stimulated under a radiant warmer in the operating room and were transferred to the nursery in the labour and delivery unit. World Health Organization (WHO) Essential Newborn Care Resuscitation protocols were followed. Infants were transferred to the nursery, where they were weighed, wrapped in terry cloth and a large fleece blanket (supplied by the family), and received a hat and were placed under a radiant warmer or in an open crib. Core body temperatures in ${ }^{\circ} \mathrm{C}$ (axillary) were taken with a digital thermometer.

Outcomes

Primary outcomes:

- Normothermia: core body temperature (axillary) $\left(36.5^{\circ} \mathrm{C}\right.$ to $\left.37.5^{\circ} \mathrm{C}\right) 1$ hour after birth

- Core body temperature in ${ }^{\circ} \mathrm{C}$ (axillary) at 1 hour after birth

- Hypothermia: core body temperature (axillary) $<36.5^{\circ} \mathrm{C} 1$ hour after birth

Secondary outcomes:

- Hyperthermia: core body temperature (axillary) $>38.0^{\circ} \mathrm{C}$ at 1 hour after birth

- Hypotension on admission to NICU

- Hypoglycaemia on admission to NICU

- Seizures during first 24 hours after birth

- Respiratory distress syndrome

- Bronchopulmonary dysplasia

- Pneumothorax

- Sepsis

- Major brain injury (IVH grade II or N or periventricular leukomalacia)

- Necrotising enterocolitis

- Bowel perforation

- Pulmonary haemorrhage

- Mortality (death before discharge) ficult to maintain with open doors and windows. Control of environmental temperature is lacking in the delivery room and in resuscitation areas.

Limitations: inaccuracy of pregnancy dating in low-resource setting; high proportion of infants $>2500$ grams birth weight; possibility that term infants were included in the study; no control of ambient temperatures in the delivery room and in resuscitation areas 
Leadford 2013 (Continued)

Risk of bias

\begin{tabular}{|c|c|c|}
\hline Bias & Authors' judgement & Support for judgement \\
\hline $\begin{array}{l}\text { Random sequence genera- } \\
\text { tion (selection bias) }\end{array}$ & Low risk & $\begin{array}{l}\text { Quote: "Randomisation was blinded and done using sealed numbered en- } \\
\text { velopes assigned by a random number generator" }\end{array}$ \\
\hline \multirow[t]{2}{*}{$\begin{array}{l}\text { Allocation concealment } \\
\text { (selection bias) }\end{array}$} & Low risk & $\begin{array}{l}\text { Quote: "Randomisation was blinded and done using sealed numbered en- } \\
\text { velopes assigned by a random number generator" }\end{array}$ \\
\hline & & $\begin{array}{l}\text { Comment: Review authors agreed that sealed numbered envelopes were mos } \\
\text { likely to be opaque. }\end{array}$ \\
\hline \multirow{3}{*}{$\begin{array}{l}\text { Blinding of participants } \\
\text { and personnel (perfor- } \\
\text { mance bias) } \\
\text { All outcomes }\end{array}$} & High risk & Blinding of participants: unfeasible \\
\hline & & Blinding of healthcare providers/personnel: unfeasible \\
\hline & & $\begin{array}{l}\text { Comment: Review authors agreed that it was unfeasible to blind participants } \\
\text { and healthcare providers/personnel to the intervention. }\end{array}$ \\
\hline $\begin{array}{l}\text { Blinding of outcome as- } \\
\text { sessment (detection bias) } \\
\text { All outcomes }\end{array}$ & High risk & Blinding of outcome measurement: no \\
\hline $\begin{array}{l}\text { Incomplete outcome data } \\
\text { (attrition bias) } \\
\text { All outcomes }\end{array}$ & Low risk & Complete follow-up: All infants randomised completed the study. \\
\hline \multirow[t]{2}{*}{$\begin{array}{l}\text { Selective reporting (re- } \\
\text { porting bias) }\end{array}$} & Low risk & $\begin{array}{l}\text { Most study outcomes detailed at the time of trial registration were reported in } \\
\text { the published manuscript as per }\end{array}$ \\
\hline & & http://clinicaltrials.gov/ct2/show/NCT01403623. \\
\hline Other bias & Low risk & \\
\hline
\end{tabular}

\section{Leslie 2007}

Randomised
Single-centre
Blinding of randomisation:
- Generation of allocation sequence: adequate (computer-generated random sequence in blocks of
varying size: 2 gestation strata $\leq 26$ weeks and $>26$ weeks)
- Allocation concealment: adequate (sequentially numbered, opaque, sealed (tamper-evident) mat-
tress boxes (containing ready to use new mattresses or a previously activated mattress that could not
be reactivated), in accordance with allocation). Clinicians attending deliveries took the top box from
the study pile. Each numbered box was opened in the delivery room to reveal the study allocation.
Blinding of intervention: participants: unfeasible; healthcare providers/personnel: unfeasible
Blinding of outcome measurement: no
Complete follow-up: yes (all 47 infants randomised completed the study)

The proposal for the study was approved by the local research ethics committee.

Written informed consent was obtained. 
Leslie 2007 (Continued)

Forty-seven infants randomised: intervention group $(n=23)$; control group $(n=24)$

Setting: Nottingham University Hospitals NHS Trust, Nottingham, United Kingdom

Country income grouping: high

Exclusion criteria:

Infants:

- Major congenital abnormality

Intervention group characteristics:

- Birth weight (grams) median (range) 840 (530 to 1240)

- Gestation (weeks) median (range) 26 (23 to 28 )

Control group characteristics:

- Birth weight (grams) median (range) 805 (560 to 1320)

- Gestation (weeks) median (range) 26 (23 to 28 )

Interventions

Sodium acetate gel warming mattress (Transwarmer; Prism Technologies Group, San Antonio, Texas, USA)

All infants received standard thermal care at birth. Infants were immediately placed in a plastic bag and were stabilised under a radiant warmer prewarmed on full power. A wool hat was placed on the head, and stabilised infants were transferred to the adjacent NICU under the radiant warmer. Infants remained in the plastic bag until placed in their warm and humidified incubator in the NICU.

Intervention group (mattress + plastic bag):

The transwarmer mattress was activated in accordance with the manufacturer's instructions and was placed on the resuscitaire, woven side up, and with a thin cotton sheet or drape between the mattress and the infant.

\section{Comparison group (plastic bag routine care):}

Received same care as intervention group without use of the gel mattress. Admission temperatures were measured by the NICU nurse using Suretemp electronic thermometer (Welch Allyn Ltd., Aylesbury, UK).

\begin{tabular}{|c|c|}
\hline Outcomes & $\begin{array}{l}\text { Primary outcomes: } \\
\text { - Core body temperature in }{ }^{\circ} \mathrm{C} \text { (axillary) on admission to NICU } \\
\text { - Hypothermia on admission to NICU (core body temperature (axillary) }<36.5^{\circ} \mathrm{C} \text { ) } \\
\text { Secondary outcomes: } \\
\text { - Hyperthermia on admission to NICU (core body temperature (axillary) }>38.0^{\circ} \mathrm{C} \text { ) } \\
\text { - Use of inotropes (dopamine, dobutamine, hydrocortisone) } \\
\text { - Coagulation support (use of platelets, fresh frozen plasma (FFP), and cryoprecipitate) } \\
\text { - Mortality }\end{array}$ \\
\hline Notes & $\begin{array}{l}\text { Trial authors (Dr. Stephen Wardle \& Dr. Andrew Leslie) were contacted ( } 07 \text { July 2016) via email and pro- } \\
\text { vided clarification and additional methodological details, as well as primary and secondary outcome } \\
\text { data. }\end{array}$ \\
\hline
\end{tabular}


Leslie 2007 (Continued)

Risk of bias

\begin{tabular}{|c|c|c|}
\hline Bias & Authors' judgement & Support for judgement \\
\hline $\begin{array}{l}\text { Random sequence genera- } \\
\text { tion (selection bias) }\end{array}$ & Low risk & $\begin{array}{l}\text { Computer-generated random sequence: } 2 \text { gestation strata: } \leq 26 \text { weeks and }> \\
26 \text { weeks }\end{array}$ \\
\hline $\begin{array}{l}\text { Allocation concealment } \\
\text { (selection bias) }\end{array}$ & Low risk & $\begin{array}{l}\text { Quote "..blinded study pack" (sealed, opaque, sequentially numbered mat- } \\
\text { tress boxes) }\end{array}$ \\
\hline \multirow{3}{*}{$\begin{array}{l}\text { Blinding of participants } \\
\text { and personnel (perfor- } \\
\text { mance bias) } \\
\text { All outcomes }\end{array}$} & \multirow[t]{3}{*}{ High risk } & Blinding of participants: unfeasible \\
\hline & & Blinding of healthcare providers/personnel: unfeasible \\
\hline & & $\begin{array}{l}\text { Comment: Review authors agreed that it was unfeasible to blind participants } \\
\text { and healthcare providers/personnel to the intervention. }\end{array}$ \\
\hline $\begin{array}{l}\text { Blinding of outcome as- } \\
\text { sessment (detection bias) } \\
\text { All outcomes }\end{array}$ & High risk & Blinding of outcome measurement: no \\
\hline $\begin{array}{l}\text { Incomplete outcome data } \\
\text { (attrition bias) } \\
\text { All outcomes }\end{array}$ & Low risk & Complete follow-up: All infants randomised completed the study. \\
\hline $\begin{array}{l}\text { Selective reporting (re- } \\
\text { porting bias) }\end{array}$ & Unclear risk & Information is insufficient to assess risk of bias. \\
\hline Other bias & High risk & $\begin{array}{l}\text { Study was underpowered (powered to detect a difference in mean tempera- } \\
\text { ture (intervention and control) of } 0.8^{\circ} \mathrm{C} \text { ). }\end{array}$ \\
\hline
\end{tabular}

\section{Mathew 2012}

Randomised
Single-centre
Blinding of randomisation:
- Generation of allocation sequence: adequate (computer-generated random sequence: blocks of 4 (2
gestation strata from 23 to $26(0)$ weeks and from $26(1)$ to $28(0)$ weeks)
• Allocation concealment: adequate (sealed, opaque, sequentially numbered envelopes)
Blinding of intervention: participants: unfeasible; healthcare providers/personnel: unfeasible
Blinding of outcome measurement: unclear
Complete follow-up: yes (all 41 infants randomised completed the study)
Transfer time to the NICU was recorded.
The proposal for the study was approved by the Children and Youth Institutional Review Board of the
University at Buffalo.
Written informed consent was obtained from mothers admitted to the labor and delivery unit.

Participants

Infants at $\geq 23$ and $\leq 28$ weeks' gestation

Forty-one infants randomised: intervention group $(n=21)$, control group $(n=20)$

Setting: Women and Children's Hospital at Buffalo, Buffalo, New York, USA

Country income grouping: high

Exclusion criteria:

Mothers: 
- Pyrexia before onset of labour

- Suspected chorioamnionitis

Infants:

- Blistering skin disorders

-Abdominal wall defects

- Neural tube defects, or structural abnormality of the central nervous system

Intervention group (vinyl bag) characteristics:

Mothers:

- Antenatal steroid administration (17)

Infants:

- Gestation (weeks) mean (SD) 26 (1.3)

- Sex 'female' (12)

- Birth weight (grams) mean (SD) 768 (162)

-Apgar score @ 1 minute median (IQR) 6 (2)

-Apgar score @ 5 minutes median (IQR) 8 (0)

- Mode of delivery: 'vaginal' (4)

- 'Caesarean section' (17)

- Multiple birth (7)

- Cord pH mean (SD) 7.30 (0.07)

- Cord base excess (mEqL) mean (SD) -1.6 (2.6)

Active comparator group (thermal mattress) characteristics:

Mothers:

- Antenatal steroid administration (13)

Infants:

- Gestational (weeks) mean (SD) 26 (1.2)

- Sex 'female' (11)

• Birth weight (grams) mean (SD) 766 (186)

-Apgar score @ 1 minute median (IQR) 5.5 (2)

-Apgar score @ 5 minutes median (IQR) 8 (1)

- Mode of delivery: 'vaginal' (5), 'caesarean section' (15), multiple birth (10)

- Cord pH mean (SD) $7.33(0.06)$

- Cord base excess (mEqL) mean (SD) -0.94 (1.6) Laurel, Maryland, USA) during resuscitation and stabilisation. Resuscitation was performed according 
to neonatal resuscitation programme guidelines. Infants were transported to the NICU in prewarmed Air Shields TI 100 Transport Incubators (Himm Rom Airshields, Hatboro, Pennsylvania, USA) set at $35^{\circ} \mathrm{C}$.

Intervention group (thermal mattress):

Infants were placed on the preactivated Transwarmer Mattress after drying.

Comparison group (polyvinyl bag):

Infants were placed into the Vi-Drape Isolation Bags (Medical Concepts Development, St. Paul, Minnesota, USA) below the neckline immediately after birth without drying, and the bag was secured loosely around the neck with straps. The head was dried and covered with a hat. Auscultation was carried out over the bag, and a hole was cut in the bag if umbilical access was required.

On arrival at the NICU, infants were weighed in the bag or on the mattress and were placed into prewarmed isolettes (Giraffe Omnibed; Ohmeda Medical/GE Healthcare, Laurel, Maryland, USA) adjusted to neutral thermal environment for gestational age of the infant. Axillary temperatures in ${ }^{\circ} \mathrm{C}$ were obtained after admission to the NICU via an electronic thermometer (Suretemp; Welch Allyn, San Diego, California, USA).

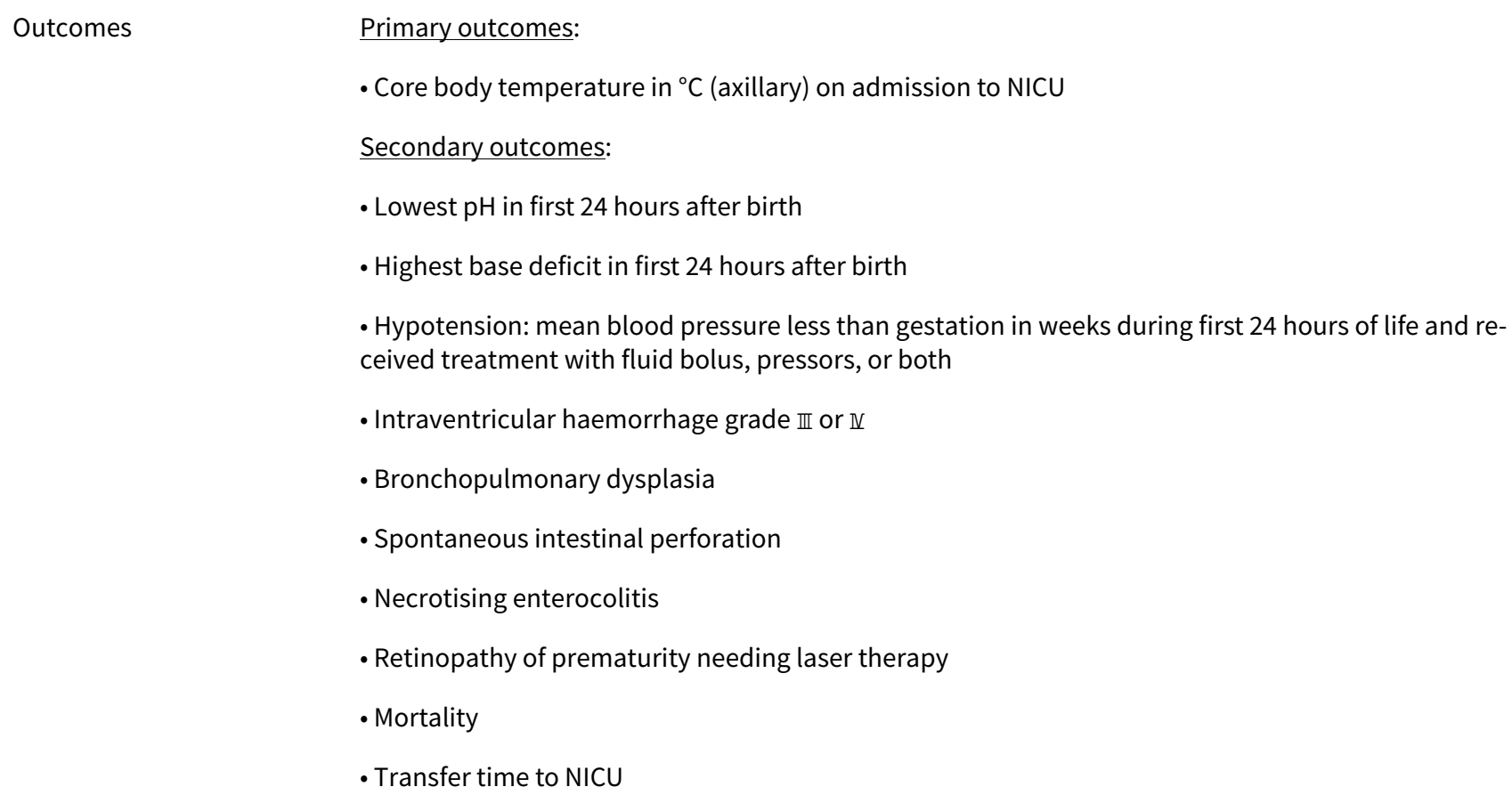
maintained between $21^{\circ} \mathrm{C}$ and $22^{\circ} \mathrm{C}$.

\section{Risk of bias}

Bias Authors' judgement Support for judgement

Random sequence genera- Low risk tion (selection bias)

Quote: "Infants were randomised into two gestational age strata: 23 to 26 (0) weeks and 26 (1) to $28(0)$ weeks. The block size was four infants...Randomisation was done according to a computer generated random sequence"

$\begin{array}{ll}\begin{array}{l}\text { Allocation concealment } \\ \text { (selection bias) }\end{array} & \text { Low risk } \\ & \begin{array}{l}\text { Quote: "Treatment was assigned using sealed, opaque envelopes that were } \\ \text { opened by the neonatology fellow prior to attending the delivery" }\end{array} \\ & \begin{array}{l}\text { Quote: (from correspondence) "...the sealed opaque envelopes were sequen- } \\ \text { tially numbered" }\end{array}\end{array}$


Mathew 2012 (Continued)

Blinding of participants and personnel (perfor-

High risk Blinding of participants: unfeasible mance bias)

Blinding of healthcare providers/personnel: unfeasible

All outcomes

Quote: "Due to the nature of the intervention, it was not possible to blind the personnel to the treatment allocation"

Comment: Review authors agreed that it was unfeasible to blind participants and healthcare providers/personnel to the intervention.

Blinding of outcome as- Unclear risk Blinding of outcome measurement: unclear

sessment (detection bias)

All outcomes

Incomplete outcome data Low risk Complete follow-up: All infants randomised completed the study.
(attrition bias)

All outcomes

Selective reporting (re- Unclear risk

porting bias)

Information is insufficient to assess risk of bias. Figures for BPD were not provided; however, no significant difference was reported in the text.

Other bias

Low risk

\section{McCarthy 2013}

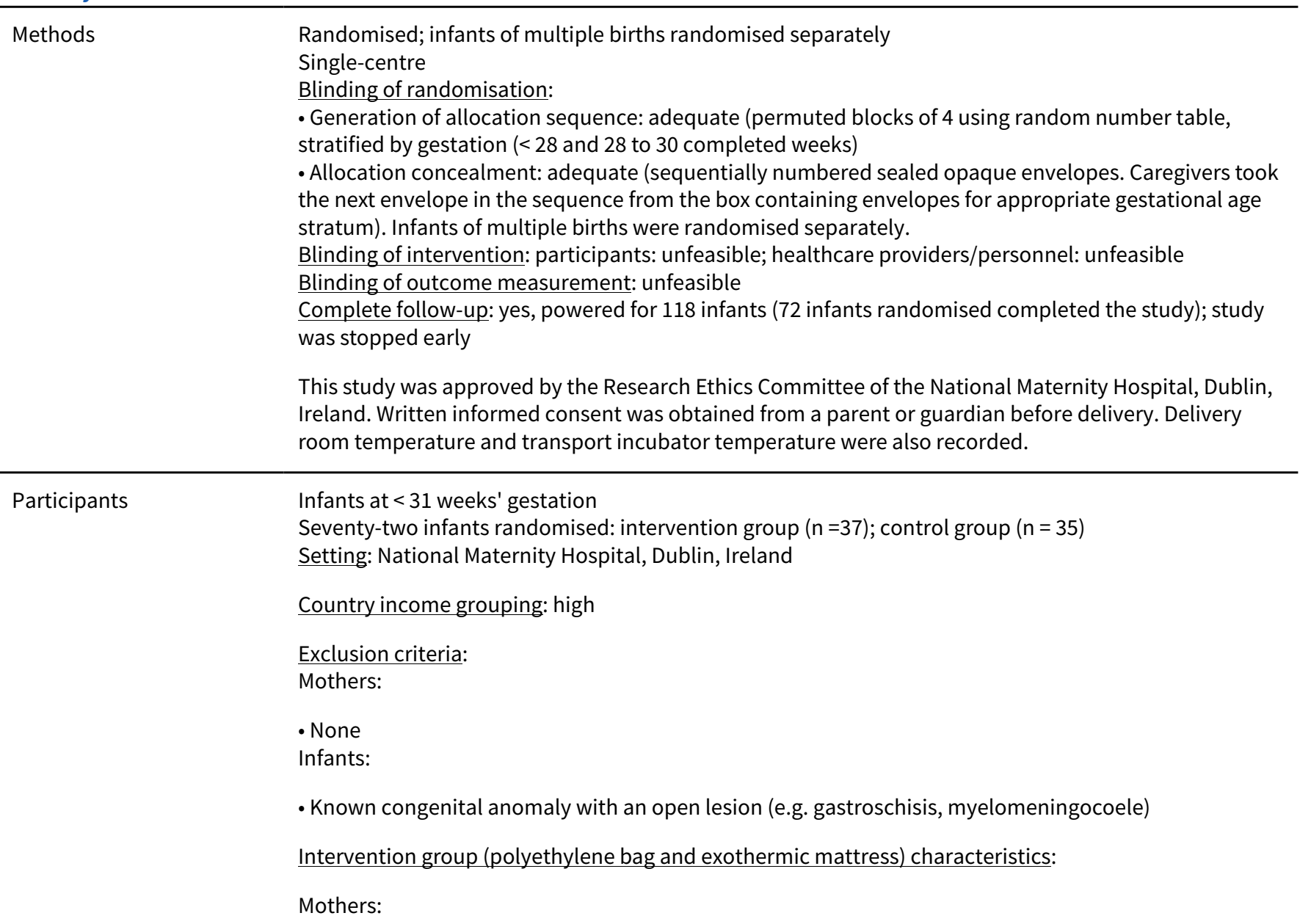


McCarthy 2013 (Continued)

\section{- None \\ Infants:}

- Gestation (weeks) mean (SD) 28 (2)

- Birth weight (grams) mean (SD) 1085 (360)

- Sex 'male' (22)

- Time (minutes) to NICU admission mean (SD) 24 (7)

- Antenatal steroid exposure (37)

- Caesarean delivery (22)

- Multiple birth (14)

- Regional anaesthesia (17)

- Maternal temperature $\left({ }^{\circ} \mathrm{C}\right)$ mean (SD) $36.7(0.5)$

- Maternal temperature $>37.7^{\circ} \mathrm{C}(4)$

- Respiratory support in delivery room (37)

- Intubated in delivery room (4)

Comparator (conventional care, polyethylene bag only) characteristics:

Mothers:

- None

Infants:

- Gestation (weeks) mean (SD) 28 (2)

- Birth weight (grams) mean (SD) 1194 (386)

- Sex 'male' (24)

- Time (minutes) to NICU admission mean (SD) 19 (7)

- Antenatal steroid exposure (35)

- Caesarean delivery (21)

- Multiple birth (12)

- Regional anaesthesia (19)

- Maternal temperature $\left({ }^{\circ} \mathrm{C}\right)$ mean (SD) $36.8(0.6)$

- Maternal temperature $>37.7^{\circ} \mathrm{C}(5)$

- Respiratory support in delivery room (35)

- Intubated in delivery room (6)

Polyethylene bag (food grade SuperValu sealable, food storage bag $26.5 \mathrm{~cm}$ by $35 \mathrm{~cm}$ ) and exothermic chemical mattress -Transwarmer Infant Transport Mattress; Cooper Surgical Inc., Trumbull, Connecticut, USA)

After birth, all infants were placed in resealable food grade polyethylene bags and were placed supine on a resuscitation cot (CosyCot Infant Warmer; Fisher and Paykel Healthcare, Auckland, NewZealand) under radiant heat. For all infants, a cotton towel and a cotton knit hat (Premier Gueard International, Guangzhou Fortunique, Guangdong Province, China) were placed before delivery on the resuscitation cot to warm under radiant heaters. Stabilisation was carried out in line with international guidelines. 
All infants were placed, without drying, head first into the polyethylene bag, so that the head protruded through a hole cut in the bottom of the bag. Once the pulse oximeter was applied to the infant's right wrist, the bag was sealed to enclose the infant's body. Only the head and face were dried, and the head was covered with a hat.

Intervention group (polyethylene bag and exothermic chemical mattress):

Infants were placed on top of an activated exothermic chemical mattress (activated at least 3 minutes before delivery and placed transparent side down under a warm towel) and remained on it during transfer in a prewarmed transport incubator. Rectal temperature was measured with a digital thermometer on admission to the NICU.

Comparison group (polyethylene bag):

Infants received no additional measures to provide heat and were placed on warmed towels on preheated resuscitaire in the same way. After stabilisation, all Infants were transferred in a prewarmed transport incubator wrapped in 3 warm blankets (Air-Shields TI-100; Soma Technology, Bloomfield, Connecticut, USA) set to maximum air temperature $\left(35^{\circ} \mathrm{C}\right.$ to $\left.37^{\circ} \mathrm{C}\right)$. Rectal temperature was measured with a digital thermometer (Vicks SmartTemp Digital Thermometer; Procter \& Gamble, Cincinnati, Ohio, USA) upon admission to the NICU. Infants were then placed in warm incubators, and the polyethylene bag and exothermic mattress were removed.

Outcomes

Primary outcomes:

- Normothermia on admission to NICU: core body temperature (rectal) $36.5^{\circ} \mathrm{C}$ to $37.5^{\circ} \mathrm{C}$.

Secondary outcomes:

- Core body temperature in ${ }^{\circ} \mathrm{C}$ (rectal) on admission to NICU

- Hyperthermia on admission to NICU: core body temperature (rectal) $>37.5^{\circ} \mathrm{C}$

- Hypothermia on admission to NICU: core body temperature (rectal) $<36.5^{\circ} \mathrm{C}$

- Core body temperature in ${ }^{\circ} \mathrm{C}$ (axillary) on admission to NICU

- Oxygen at 36 weeks' adjusted gestation

$\bullet \geq 1$ dose surfactant during admission

- Inotrope use

- Intubated during admission

- Chronic lung disease

- Abnormal cranial ultrasonography (IVH grade $\geq \mathbb{I I}$, periventricular leukomalacia, or post-hemorrhagic hydrocephalus)

- Mortality (death before discharge)

Notes

Delivery room ambient temperature in ${ }^{\circ} \mathrm{C}$ : plastic bag and exothermic mattress group: mean (SD) 22.9 (1.8); plastic bag group: mean (SD) $22.4(1.3)(P=0.193)$

Some additional information was obtained from http://www.controlled-trials.com/isrctn/pf/31707342.

Quote: "the external monitoring committee recommended stopping recruitment after analysing data from the first 59 infants, as there was a significant difference in the primary outcome between the groups"

\section{Risk of bias}


McCarthy 2013 (Continued)

Random sequence genera- Low risk tion (selection bias)
Quote: " Randomisation was stratified according to gestational age (at $<28$ weeks and 28 to 30 weeks inclusive). We generated the treatment allocation schedule in permuted blocks of four using random numbers table. It was stratified by gestational age"

Allocation concealment Low risk
(selection bias)

Quote: "Cards indicating treatment allocation "Bag \& Mattress" (PB+EM) or "Bag" (PB) were concealed in sequentially numbered, sealed opaque envelopes. A member of the neonatal team brought the next envelope in sequence from the appropriate stratum to the delivery room. The envelope was opened in the delivery room just before delivery to allow for timely activation of the EM for infants assigned to that group"

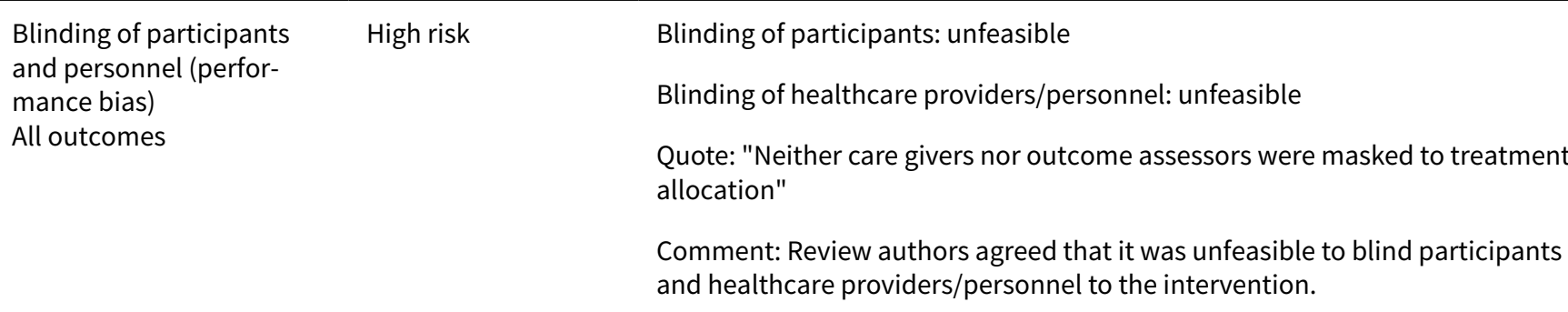

$\begin{array}{lll}\begin{array}{l}\text { Blinding of outcome as- } \\ \text { sessment (detection bias) }\end{array} & \text { High risk } & \text { Blinding of outcome measurement: no } \\ \text { All outcomes } & \begin{array}{l}\text { Quote: "Neither care givers nor outcome assessors were masked to treatment } \\ \text { allocation" }\end{array}\end{array}$

\begin{tabular}{ll}
\hline $\begin{array}{l}\text { Incomplete outcome data } \\
\text { (attrition bias) }\end{array}$ & Low risk \\
All outcomes & $\begin{array}{l}\text { Complete follow-up: All infants randomised completed the study. Twenty-one } \\
\text { infants were not enrolled: infant not resuscitated in DR (1), parents declined } \\
\text { (17), parents not approached (2), infant consented but not enrolled (1). }\end{array}$ \\
\hline
\end{tabular}

$\begin{array}{ll}\begin{array}{l}\text { Selective reporting (re- } \\ \text { porting bias) }\end{array} & \text { Most important clinical outcomes detailed at the time of trial registration } \\ & \text { were reported in the published manuscript as per http://apps.who.int/tri- } \\ \text { alsearch/Trial.aspx?TrialID=ISRCTN31707342. }\end{array}$

The following secondary outcomes were not reported in the manuscript: Apgar scores, chest compressions, and use of adrenaline and volume restriction in the DR, duration of oxygen therapy, oxygen at 28 days, sepsis (early and late onset), PDA, NEC, and hospital stay. This was most likely due to space limitations.

\begin{tabular}{ll}
\hline Other bias & Time to admission (minutes) was significantly longer in the polyethylene bag \\
and mattress group when compared with the polyethylene bag group. Analy- \\
ses of the data show a weak linear relationship between length of time from \\
birth to admission and hyperthermia. The longer an infant was in the delivery \\
room, the greater was the likelihood of hyperthermia in both groups.
\end{tabular}

\title{
Reilly 2015
}

\section{Methods}

\author{
Randomised; 2 gestational age strata: 24 (0) to 25 (6) and 26 (0) to 27 (6) weeks' gestation
}

\author{
Multi-centre (39 Vermont Oxford Network Centres), stratified by centre \\ Blinding of randomisation: \\ - Generation of allocation sequence: adequate (permuted block (4 or 6 ) randomisation lists using ran- \\ dom number-generating software; separate independent lists for each site and each gestational age \\ stratum) \\ - Allocation concealment: adequate (sealed, opaque, and sequentially numbered envelopes) \\ Blinding of intervention: participants: unfeasible
}


Blinding of healthcare providers/personnel: unfeasible

Blinding of outcome measurement: adequate ("blinding of outcome assessor")

Complete follow-up: no (801 of 813 infants randomised completed the study; in the wrap group, 4 infants were still-born and 1 infant was randomised in error; in the no wrap group, 4 infants were randomised in error and in 1 case consent was withdrawn)

Length of stay and cause of death were also recorded.

Ethical approval: preferred model of consent for participating centre

- Antenatal informed consent (families at risk of delivering eligible infants approached before delivery for study participation and a signed informed consent)

- Ethical approval for waiver consent (families approached and informed that their child is included in the study)

- Ethical approval for delayed consent (families approached when appropriate after birth and informed of the study; consent obtained for study participation)

- Each participating institution submitted a copy of its centre's initial Institutional Review Board approval and subsequent annual renewals to the Vermont Oxford Network (VON) HeLP Trial Coordinating Centre.

- Delivery room temperature, age of infant(s) when occlusive wrap was closed, age in minutes when occlusive wrap was removed, age in minutes on leaving the delivery room, axillary baseline temperature in ${ }^{\circ} \mathrm{C}$, age in minutes upon arrival to the NICU and/or post resuscitation, mode of transport from delivery room to NICU, length of stay (days), and cause of death were recorded.

- Standardised room thermometers were used.

Participants

Infants at $\geq 24$ and $\leq 27$ weeks' gestation and a firm decision before birth to provide full resuscitation measures

Eight hundred thirteen infants randomised: intervention group $(n=411)$, control group $(n=402) ; 801$ completed the study: intervention group $(n=404)$, control group $(n=397)$

Setting: multi-centre (Canada, USA, and UK)

Country income grouping: high

Exclusion criteria:

Mothers:

- None

Infants:

- Major congenital anomalies that are not covered by skin (e.g. gastroschisis, meningomyelocoele)

- Blistering skin conditions that preclude the use of occlusive wrap

Intervention group (polyethylene occlusive skin wrap) characteristics:

Maternal:

- Prenatal care (390)

- Antenatal steroids (375)

- Mode of delivery: 'vaginal' (123)

- Rupture of membranes $>24$ hours (113)

- Chorioamnionitis (79) 
- Education less than high school (76)

Infants:

- Birth weight (grams) mean (SD) 800 (205)

- Gestation (weeks) mean 25.6

- Gender 'male' (208)

• Race: 'Asian' (48), 'Black' (106), 'Native American' (0), 'White' (230), 'Other' (18), 'Hispanic' (47)

- Multiple birth (75)

Control group (standard care) characteristics:

Maternal:

- Prenatal care (382)

- Antenatal steroids (370)

- Mode of delivery: 'vaginal' (129)

- Rupture of membranes $>24$ hours (130)

- Chorioamnionitis (75)

- Maternal temperature $\left({ }^{\circ} \mathrm{C}\right.$ ) before delivery mean (range) 36.9 (34.6 to 39.5 )

- Education less than high school (84)

Infants:

- Birth weight (grams) mean (SD) 821 (199)

- Gestation (weeks) mean 26.0

- Gender 'male' (219)

• Race: 'Asian' (41), 'Black' (112), 'Native American' (2), 'White' (226), 'Other' (15), 'Hispanic' (42)

- Multiple birth (84)

Intervention group:

Infants were immediately placed under a radiant warmer (according to NRP guidelines) and on the wrap from the neck down. The temperature of the delivery room was recorded before these events. The infant's head was dried and covered with a hat, while the wrap was folded over the infant's body. Additional stabilisation was carried out according to usual practice (NRP guidelines). Use of adjunct warming devices was permitted. Method of transport from delivery room to the NICU was determined by usual care at participating centres. Infants were transported in the wrap.

\section{Control group:}

Standard care according to International Guidelines for Neonatal Resuscitation (NRP guidelines). Infants were immediately dried under radiant heat and were subsequently managed identically to the intervention group. Delivery room temperature was recorded before delivery, and once the infant was dried under radiant heat, the head was covered with a hat, while the wet towels were removed. Additional stabilisation was carried out according to usual practice (NRP guidelines). Method of transport from delivery room to NICU was determined by usual care at participating centres. 
Reilly 2015 (Continued)

\section{Outcomes}

\section{Primary outcome:}

- All-cause mortality at discharge or at 6 months' corrected gestation if the infant remained in hospital Secondary outcomes:

- Baseline core body temperature in ${ }^{\circ} \mathrm{C}$ (axillary) measured with the standard thermometer (Medline Digital Thermometer, Mundelein, Illinois, USA)

- Core body temperature in ${ }^{\circ} \mathrm{C}$ (axillary) post stabilisation measured with the standard thermometer (Medline Digital Thermometer, Mundelein, Illinois, USA)

- Apgar scores

- Blood gases

-Hypotension

- Hypoglycaemia

- Seizures during first 12 hours of life

- Patent ductus arteriosus

- Gastrointestinal perforation

- Respiratory distress syndrome

-Bronchopulmonary dysplasia

- Necrotising enterocolitis

- Intraventricular haemorrhage, periventricular leukomalacia

- Pulmonary haemorrhage

- Retinopathy of prematurity

- Sepsis

- Hearing loss at discharge from hospital or at 6 months' corrected gestation

- Pneumothorax

- Death and neurosensory disability measures at 18 to 24 months' corrected gestation
Delivery room temperature $\left({ }^{\circ} \mathrm{C}\right.$ ) mean (range) wrap group 23.5 (16.1 to 36); no wrap group 23.3 (15 to 32.2)

This multi-centre trial involved 39 centres in Canada, USA, and UK - all part of the Vermont Oxford Network.

This study has received funding from the Canadian Institute of Health Research (CIHR).

Quote: "Interim analyses for safety were performed at 25\% enrolment, and for safety and futility at 50\% enrolment of the projected sample size. The futility analysis determined that even if the full sample size were enrolled, the study would not achieve its goal of demonstrating a $25 \%$ relative risk reduction in the primary outcome. Consequently, the Data and Safety monitoring Board recommended halting enrolment, and the Heat Loss Prevention (HeLP) Trial Steering Committee closed enrolment 30 June 2010"

\section{Risk of bias}


Reilly 2015 (Continued)

Random sequence genera- Low risk tion (selection bias)
Quote: "Permuted block randomisation lists, using random-number generating software are produced. Variable block sizes of either four or six....Separate independent randomisation lists are created for each site and each gestational age strata..."

Allocation concealment
(selection bias)

Blinding of participants High risk
and personnel (performance bias)

All outcomes
Quote: "Randomisation occurs immediately prior to delivery. Treatment allocation is contained in a sealed, opaque, and sequentially numbered envelope"

\begin{tabular}{ll}
\hline $\begin{array}{l}\text { Blinding of outcome as- } \\
\text { sessment (detection bias) } \\
\text { All outcomes }\end{array}$ & Low risk \\
\hline
\end{tabular}

\begin{tabular}{|c|c|c|}
\hline $\begin{array}{l}\text { Incomplete outcome data } \\
\text { (attrition bias) } \\
\text { All outcomes }\end{array}$ & Low risk & $\begin{array}{l}\text { Complete follow-up: no, } 801 \text { of } 813 \text { ( } 1.5 \% \text { loss to follow-up) infants ran- } \\
\text { domised in the wrap group completed the study; } 3 \text { infants were still-born } \\
\text { and } 1 \text { infant was randomised in error; in the no wrap group, infants were ran- } \\
\text { domised in error and in } 1 \text { case consent was withdrawn. }\end{array}$ \\
\hline
\end{tabular}

\begin{tabular}{ll}
\hline $\begin{array}{l}\text { Selective reporting (re- } \\
\text { porting bias) }\end{array}$ & Most important study outcomes detailed at the time of trial registration were \\
& reported in the published manuscript at http://clinicaltrials.gov/ct2/show/ \\
& NCT00607464 and http://dx.doi.org/10.1016/j.cct.2013.06.001. Outcomes not \\
reported included hearing loss (failed hearing test), $\mathrm{pH} / \mathrm{base}$ deficit, blood & pressure/hypotension, blood glucose (hypoglycaemia), and seizures within \\
first 12 hours. The steering committee decided not to report all outcomes ow- & ing to space limitations. The manuscript focused on the primary outcome and \\
important secondary outcomes that were more global. Personal communica- & tion: Maureen O'Reilly via email (16 June 2017)
\end{tabular}

\begin{tabular}{ll}
\hline Other bias & Study was stopped at $50 \%$ enrolment. However, this trial is the largest RCT \\
evaluating short-term effects of wrapping infants immediately after birth (24 & to 27 weeks' gestation). This large pragmatic trial with 39 participating centres \\
was subject to several protocol violations in the treatment group (i.e. delayed & application of wrap, opening of the wrap during the study period, and early re- \\
moval of the wrap). Despite these violations, significantly greater baseline and & poststabilisation temperatures in the wrap group suggested that they may not \\
have greatly affected heat losses. In addition, use of adjunct heat source when & temperature was not known to be $<35.5^{\circ} \mathrm{C}$. Only 12 infants in the wrap group \\
and 17 in the no wrap group experienced this violation; therefore this was un- & likely to have impacted the significance of primary and secondary outcomes. \\
Additional limiting factor for consideration is the method of consent, which & may have led to some selection bias.
\end{tabular}

Roberts 1981

\begin{tabular}{ll}
\hline Methods & Randomised \\
& Single-centre \\
& Stratified randomisation by gestation 32 to 34 and 35 to 36 completed weeks \\
& Blinding of randomisation: \\
- Generation of allocation sequence: adequate, lot drawing - index cards utilised to pre-arranged ran- \\
domisation - random number tables \\
- Allocation concealment: unclear
\end{tabular}


Blinding of intervention: participants: unfeasible; healthcare providers/personnel: unfeasible

Blinding of outcome measurement: unclear

Complete follow-up: yes (all 40 infants randomised completed the study)

Delivery room, transport incubator, highest maternal temperatures, time elapsed from birth to onset of drying, and time elapsed from birth to arrival in nursery were recorded, and core body temperatures in ${ }^{\circ} \mathrm{C}$ (axillary) were recorded in the delivery room.

This study was approved by the Human Subject Review Committee of Texas Women's University.

Informed consent was obtained from the mother during early labour and before the mother received any medication..

Participants

Infants at $\geq 32$ and $\leq 36$ weeks' gestation delivered from 7 am until 5 pm Monday through Friday

Forty infants randomised: intervention group $(n=17)$, control group $(n=23)$

Setting: Parkland Memorial Hospital, Dallas, Texas, USA

Country income grouping: high

Criteria for exclusion:

Mothers:

- Maternal temperature $\geq 37.8^{\circ} \mathrm{C}$

Infants:

- Not appropriate birth weight for gestational age

-Apgar score @ 5 minutes $<7$

- Signs of central nervous system defect

- Infant dropped from study on development of sepsis

- Delivery outside of delivery room

Intervention group (stockinette cap) characteristics:

Infants:

- Sex 'male' (4), 'female' (13)

• Ethnicity: 'White' (6), 'Black' (5), 'Mexican-American' (6)

- Vaginal delivery (15)

- Caesarean section (2)

- Gestation (weeks) range 32 to 34 (5), 35 to 36 (12); gestation (weeks) mean 34.9

- Birth weight (grams) range 1420 to 2890; birth weight (grams) mean 2253.4

-Apgar score @ 5 minutes=9 (16)

- Infants transported in incubator (7)

- Temperature transport incubator range $31^{\circ} \mathrm{C}$ to $40^{\circ} \mathrm{C}$; mean $35.3^{\circ} \mathrm{C}$

- Highest maternal temperature range $36.6^{\circ} \mathrm{C}$ to $37.7^{\circ} \mathrm{C}$; mean $37.1^{\circ} \mathrm{C}$

Control group (conventional care) characteristics:

Infants:

- Sex 'male' (12), 'female' (11)

• Ethnicity: 'White' (8), 'Black' (13), 'Mexican-American' (2) 
Roberts 1981 (Continued)

- Vaginal delivery (18)

- Caesarean section (5)

- Gestation (weeks) 32 to 34 (4), 35 to 36 (19); gestation (weeks) mean 35.2

- Birth weight (grams) range 1360 to 2965; birth weight (grams) mean 2276.8

-Apgar score @ 5 minutes=9 $(22)$

- Infants transported in incubator (12)

- Temperature transport incubator range $33^{\circ} \mathrm{C}$ to $37^{\circ} \mathrm{C}$; mean $35.2^{\circ} \mathrm{C}$

- Highest maternal temperature range $36.2^{\circ} \mathrm{C}$ to $37.8^{\circ} \mathrm{C}$; mean $37.2^{\circ} \mathrm{C}$

Interventions

Stockinette cap - a sterile headpiece made of stockinette (a material used to protect skin under orthopaedic casts), which covers the forehead, the ears, underneath the occipital bone, and all the area above this plane

Intervention group:

Infants received a stockinette cap after delivery as soon as possible after being dried under a radiant warmer.

Control group:

Infants received the same treatment as the intervention group but did not receive a stockinette cap. All infants weighing < 2500 grams were transported to the nursery in a transport incubator. Core body temperatures in ${ }^{\circ} \mathrm{C}$ (axillary) were measured with IVAC electric thermometer Model 821 on admission to NICU.

Primary outcomes:
Outcomes

\section{Risk of bias}

\section{Bias}

Random sequence genera- Low risk tion (selection bias)

\section{Authors' judgement Support for judgement}

Quote: ".....60 index cards were utilised to pre-arrange randomisation ....The total expected population was divided into infants who were 32 to 34 weeks' gestational age and infants who were 35 to 36 weeks' gestational age. Four cards were selected from 32 to 34 week pile, two were designated control group and two were designated experimental group. The cards were numbered one through four according to the occurrence of the designated number in a table of random numbers. The 35 to 36 week pile was treated in a like manner .... This was continued until all sixty cards were processed"

\begin{tabular}{lll}
\hline $\begin{array}{l}\text { Allocation concealment } \\
\text { (selection bias) }\end{array}$ & Unclear risk & Information is insufficient to assess risk of bias. \\
\hline $\begin{array}{l}\text { Blinding of participants } \\
\begin{array}{l}\text { and personnel (perfor- } \\
\text { mance bias) }\end{array}\end{array}$ & High risk & Blinding of participants: unfeasible \\
\hline
\end{tabular}


Roberts 1981 (Continued)

All outcomes
Comment: Review authors agreed that it was unfeasible to blind participants and healthcare providers/personnel to the intervention.

\begin{tabular}{lll}
\hline Blinding of outcome as- & Unclear risk & Blinding of outcome measurement: unclear \\
sessment (detection bias) & & \\
All outcomes & Information is insufficient to assess risk of bias.
\end{tabular}

Incomplete outcome data Low risk Complete follow-up: All infants randomised completed the study.

(attrition bias)

All outcomes

\begin{tabular}{lll}
\hline $\begin{array}{l}\text { Selective reporting (re- } \\
\text { porting bias) }\end{array}$ & Unclear risk & Information is insufficient to assess risk of bias. \\
\hline Other bias & High risk & $\begin{array}{l}\text { For infants }<2000 \text { grams, mean delivery room temperatures were different be- } \\
\text { tween intervention and control groups. }\end{array}$ \\
\hline
\end{tabular}

Rohana 2011

Methods Randomised; for multiple pregnancies, each infant individually randomised

Single-centre

Blinding of randomisation: 5 gestational strata: 24 to 25,26 to 27,28 to 29,30 to 31 , and 32 to 33 com-

pleted weeks

- Generation of allocation sequence: unclear

- Allocation concealment: adequate, sealed, opaque sequentially numbered envelopes

Blinding of intervention: participants: unfeasible; healthcare providers/personnel: unfeasible

Blinding of outcome measurement: no

Complete follow-up: no (110 of 115 infants randomised completed the study). Reasons for loss to fol-

low-up were reported: protocol deviation ( 5 infants). Delivery room temperatures and interval between

the time when infants were wrapped and when their temperatures were measured was recorded. The proposal for the study was approved by the Universiti Kebangsaan Malaysia Medical Centre Scientific and Ethics Committees.

Written informed consent was obtained.

Participants

Infants at $\geq 24$ and $\leq 33$ weeks' gestation

One hundred fifteen Infants randomised: intervention group ( $n=55)$, control group $(n=60) ; 110$ of 115

completed the study: intervention group $(n=50)$, control group $(n=60)$ Two infants were not admitted

to the NICU.

Setting: Universiti Kebangsaan Medical Centre, Kauala Lumpar, Malaysia

Country income grouping: upper-middle

Exclusion criteria:

Mothers:

- None

Infants:

- Major congenital anomalies that were not covered by the skin (e.g. gastroschisis, meningomyelocoele)

- Delivery not attended by neonatal doctors

- Blistering skin conditions

Intervention group (plastic wrap) characteristics:

Mothers: 
Rohana 2011 (Continued)

- Maternal pyrexia (1)

Infants:

- Gestation (weeks) mean (SD) 29.7 (2.9)

- Sex 'male' (27)

- Multiple birth (9)

- Birth weight (grams) mean (SD) 1277 (462)

- Mode of delivery: 'caesarean section' (36)

-Apgar score @ 5 minutes median (IQR) 8.3 (8 to 9)

- Age (minimum) at admission to NICU mean (SD) 47.4 (16.4)

Control group (conventional care) characteristics:

Mothers:

- Maternal pyrexia (6)

Infants:

- Gestation (weeks) mean (SD) 29.8 (2.7)

-Sex 'male' (37)

- Multiple birth (20)

- Birth weight (grams) mean (SD) 1328 (459)

- Mode of delivery: 'caesarean section' (44)

- Apgar score @ 5 minutes median (IQR) 8.5 (8 to 9)

- Age (minimum) at admission to NICU mean (SD) 49.6 (19.8)

\section{Intervention group:}

The radiant warmer was set at 'full on' before the infant birth and remained at this setting until 15 minutes after birth, when it was changed to servo-controlled mode to maintain skin temperature of $36.5^{\circ} \mathrm{C}$. Immediately after birth, the infant was placed on the prewarmed polyethylene wrap under the radiant warmer. While one staff member folded the wrap loosely over the infant from the neck down, without drying the body, another team member dried and covered the infant's head with a cotton hat. A pulse oximeter was placed on the foot before the wrap was closed. Further resuscitation steps were carried out as per National Resuscitation Program guidelines. Umbilical access was maintained by leaving the end of the cord clamp protruding between the folds of the wrap. A servo-controlled skin temperature probe was placed by slightly parting the wrap and closing again once the probe was in place and was set to maintain a skin temperature of $36.5^{\circ} \mathrm{C}$. Wraps were removed just before the poststabilisation temperature was taken.

\section{Control group:}

Radiant warmer settings were as per intervention group. Infants were dried under a radiant warmer with dry warm towels. After the infant was dried, the infant received a cotton cap and wet linens were removed from the body. Further resuscitation steps were carried out as per National Resuscitation Program guidelines. Infants in both groups were transferred to the NICU in a preheated transport incubator (T1500 Globe-Trotter Neonatal Transport System, Telford, Pennsylvania, USA). Infants who did not require admission to the NICU were transferred to a station in the labour room, where axillary skin tem- 
Rohana 2011 (Continued)

perature was recorded before transfer to the postnatal ward. Core body temperature in ${ }^{\circ} \mathrm{C}$ (axillary) was measured with digital thermometer MDS9650B (Medline Industries, Inc., Northfield, Illinois, USA).

\begin{tabular}{|c|c|}
\hline Outcomes & $\begin{array}{l}\text { Primary outcomes: } \\
\text { - Baseline (admission to NICU) core body temperature in }{ }^{\circ} \mathrm{C} \text { (axillary) } \\
\text { - Baseline (admission to NICU) hypothermia: core body temperature (axillary) }<36.5^{\circ} \mathrm{C} \\
\text { - Core body temperature in }{ }^{\circ} \mathrm{C} \text { (axillary) post stabilisation in NICU } \\
\text { Secondary outcomes: } \\
\text { - Mortality before discharge } \\
\text { - Hyperthermia (core body temperature (axillary) }>37.5^{\circ} \mathrm{C} \text { ) during first } 12 \text { hours of life } \\
\text { - Intraventricular haemorrhage grade II or } \mathbb{N} \\
\text { - Ventilation (days) } \\
\text { - CPAP (days) } \\
\text { - Length of stay (days) } \\
\text { - Respiratory distress syndrome } \\
\text { - Patent ductus arteriosus } \\
\text { - Necrotising enterocolitis }\end{array}$ \\
\hline Notes & $\begin{array}{l}\text { Delivery room temperatures in }{ }^{\circ} \mathrm{C} \text { mean (SD) } 21.9 \text { (2.3) intervention group; mean (SD) } 22.0 \text { (2.1) control } \\
\text { group ( } P=0.76) \\
\text { Most emergency preterm deliveries (i.e. mothers who presented with established labour, hypertensive } \\
\text { crisis, or abruptio placentae) were excluded. }\end{array}$ \\
\hline
\end{tabular}

\section{Risk of bias}

\begin{tabular}{lll}
\hline Bias & Authors' judgement & Support for judgement \\
\hline $\begin{array}{l}\text { Random sequence genera- } \\
\text { tion (selection bias) }\end{array}$ & Unclear risk & $\begin{array}{l}\text { Quote: ".....the attending doctor selected a sealed randomised envelope of } \\
\text { the smallest serial number in the corresponding gestational strata. These en- } \\
\text { velopes were prepared at the beginning of the study in which equal numbers } \\
\text { of envelopes containing papers with randomisation group written on them } \\
\text { were prepared for each strata e.g. strata A: } 10 \text { envelopes with "wrap" and } 10 \\
\text { envelopes with "control" written on them were prepared. These opaque en- } \\
\text { velopes were sealed and subsequently tossed. After that the envelopes were } \\
\text { arranged one to } 20 \text { and sequentially numbered. For multiple pregnancies, } \\
\text { each infant was individually randomised" }\end{array}$
\end{tabular}

\begin{tabular}{lll}
\hline $\begin{array}{l}\text { Allocation concealment } \\
\text { (selection bias) }\end{array}$ & Low risk & $\begin{array}{l}\text { Quote: "The randomisation envelopes were sealed opaque and sequentially } \\
\text { numbered" }\end{array}$ \\
\hline $\begin{array}{l}\text { Blinding of participants } \\
\text { and personnel (perfor- } \\
\text { mance bias) }\end{array}$ & High risk & Blinding of participants: unfeasible \\
All outcomes & Blinding of healthcare providers/personnel: unfeasible \\
& $\begin{array}{l}\text { Comment: Review authors agreed that it was unfeasible to blind participants } \\
\text { and healthcare providers/personnel to the intervention. }\end{array}$
\end{tabular}

\begin{tabular}{lll}
\hline $\begin{array}{l}\text { Blinding of outcome as- } \\
\text { sessment (detection bias) }\end{array}$ & High risk $\quad$ Blinding of outcome measurement: no
\end{tabular}


Rohana 2011 (Continued)

All outcomes
Quote: (personal correspondence) "Blinding of outcome assessors unfeasible"

One hundred ten of 115 (4\% loss to follow-up) infants randomised completed the study.
Reasons for loss to follow-up were reported: protocol deviation for all 5 infants
Selective reporting (reporting bias)
Low risk (attrition bias)

All outcomes

Other bias Low risk

Most emergency preterm deliveries (i.e. mothers who presented with established labour, hypertensive crisis, or abruptio placentae) were excluded. Therefore, findings may not be generalisable to infants born in emergency deliveries.

Simon 2011

Randomised
Single centre
Blinding of randomisation:
- Generation of allocation sequence: adequate (computer-generated random assignment)
- Allocation concealment: unclear: sealed opaque envelopes, no mention whether sequentially num-
bered
Blinding of intervention: participants: unfeasible; healthcare providers/personnel: unfeasible
Blinding of outcome measurement: no
Complete follow-up: no (36 of 39 infants randomised completed the study). Reasons for loss to fol-
low-up were randomisation to wrong group (1 infant), unrecorded delivery room temperature (1 in-
fant), and birth weight > 1250 grams (1 infant). Maternal temperature and delivery room temperature,
isolette temperature, and time to admission were recorded.
The proposal for the study was approved by the institutional review board at the University of Okla-
homa Sciences Center, Oklahoma City, Oklahoma.
Informed consent was obtained from parents before delivery.

Participants

Infants at $\geq 24$ and $\leq 28$ weeks' gestation and birth weight $\leq 1250$ grams were delivered vaginally or by caesarean section.

Thirty-nine infants randomised: intervention group $(n=20)$, active comparator group $(n=19) ; 36$ completed study: intervention group $(n=17)$, active comparator group $(n=19)$

Setting: University of Oklahoma Health Sciences Centre, Oklahoma City, Oklahoma, USA

Country income grouping: high

Exclusion criteria:

Mothers:

- None

Infants:

- Major congenital anomalies with open skin lesions

Intervention group (thermal mattress) characteristics:

Mothers:

- Maternal oral temperature $\left({ }^{\circ} \mathrm{C}\right)$ mean (SD) $36.8(0.4)$

Infants:

- Birth weight (grams) mean (SD) 811.8 (176.4)

- Gestation (weeks) mean (SD) 26.0 (1.2) 
Simon 2011 (Continued)

\author{
- Sex 'male' (10) \\ - Race: 'African American' (7), 'Asian' (1), 'Latino' (3), 'White' (6) \\ -Apgar score @ 1 minute median (5) \\ -Apgar score @ 5 minutes median (6) \\ - CRIB score median (6) \\ - Delivery room temperature $\left({ }^{\circ} \mathrm{C}\right)$ mean (SD) 22.2 (2.9) \\ - Isolette temperature $\left({ }^{\circ} \mathrm{C}\right)$ mean $(\mathrm{SD}) 37.0(0.5)$ \\ - Time to admission (minutes) mean (SD) 22.1 (4.9) \\ Active comparator group (polyethylene wrap) characteristics: \\ Mothers: \\ - Maternal oral temperature $\left({ }^{\circ} \mathrm{C}\right)$ mean (SD) $36.6(0.4)$ \\ Infants: \\ - Birth weight (grams) mean (SD) 900.6 (247.9) \\ - Gestation (weeks) mean (SD) 25.9 (1.3) \\ - Sex 'male' (11) \\ • Race: 'African American' (4), 'Asian' (0), 'Latino' (1), 'White' (14) \\ -Apgar score @ 1 minute median (5) \\ -Apgar score @ 5 minutes median (6) \\ - CRIB score median (4) \\ - Delivery room temperature $\left({ }^{\circ} \mathrm{C}\right)$ mean (SD) 22.7 (2.8) \\ - Isolette temperature $\left({ }^{\circ} \mathrm{C}\right)$ mean (SD) $36.8(1.0)$ \\ - Time to admission (minutes) mean (SD) 22.0 (4.9)
}

Thermal mattress - Infatherm Children's Medical Ventures, Monroeville, Pennsylvania, USA. This transwarming device is composed of a bioxynylon pouch/bag with the surface that is in contact with the infant covered with an interwoven cloth backing that prevents damage to the infant's skin and is stored at room temperature (when activated, starting temperature $24^{\circ} \mathrm{C}$; heats to a maximum temperature of $40^{\circ} \mathrm{C}$ ). The bag is made up of sodium acetate (food grade), water, and thickeners.

Intervention group: thermal mattress

The mattress was activated when the obstetrical staff was ready to hand off the infant to the neonatal team. Infants were routinely dried under the radiant warmer.

Comparison group: polyethylene wrap (polyethylene wrap - Neowrap (Fisher and Paykel, Auckland, New Zealand) is a square sheet of transparent polyethylene $60 \mathrm{~cm}$ by $60 \mathrm{~cm}$ ).

Infants were received from the obstetrician in a prewarmed towel and were placed immediately onto the plastic wrap under a radiant warmer at $100 \%$ power. While one resuscitation team member cut the umbilical cord, another team member placed the pulse oximeter probe on the infant, and a third team member wrapped the infant. Only the head was dried before wrapping. With the infant placed supine on the wrap, each cephalad corner of the wrap was grasped and was brought across the neckline to the contralateral side and tucked under the infant. The caudad portion was then brought up toward the midline and was tucked around the infant. A prewarmed cotton/polyester knit hat was placed on all infants. Standard resuscitative measures as per the Neonatal Resuscitation Program were followed. Infants were transported to the NICU in a prewarmed incubator, then were placed in a servo-controlled 
Simon 2011 (Continued)

warming device. Axillary temperature was measured with a digital thermometer manufactured by Kerma Medica Products (Portsmouth, Virginia, USA; Model 110-KMP).

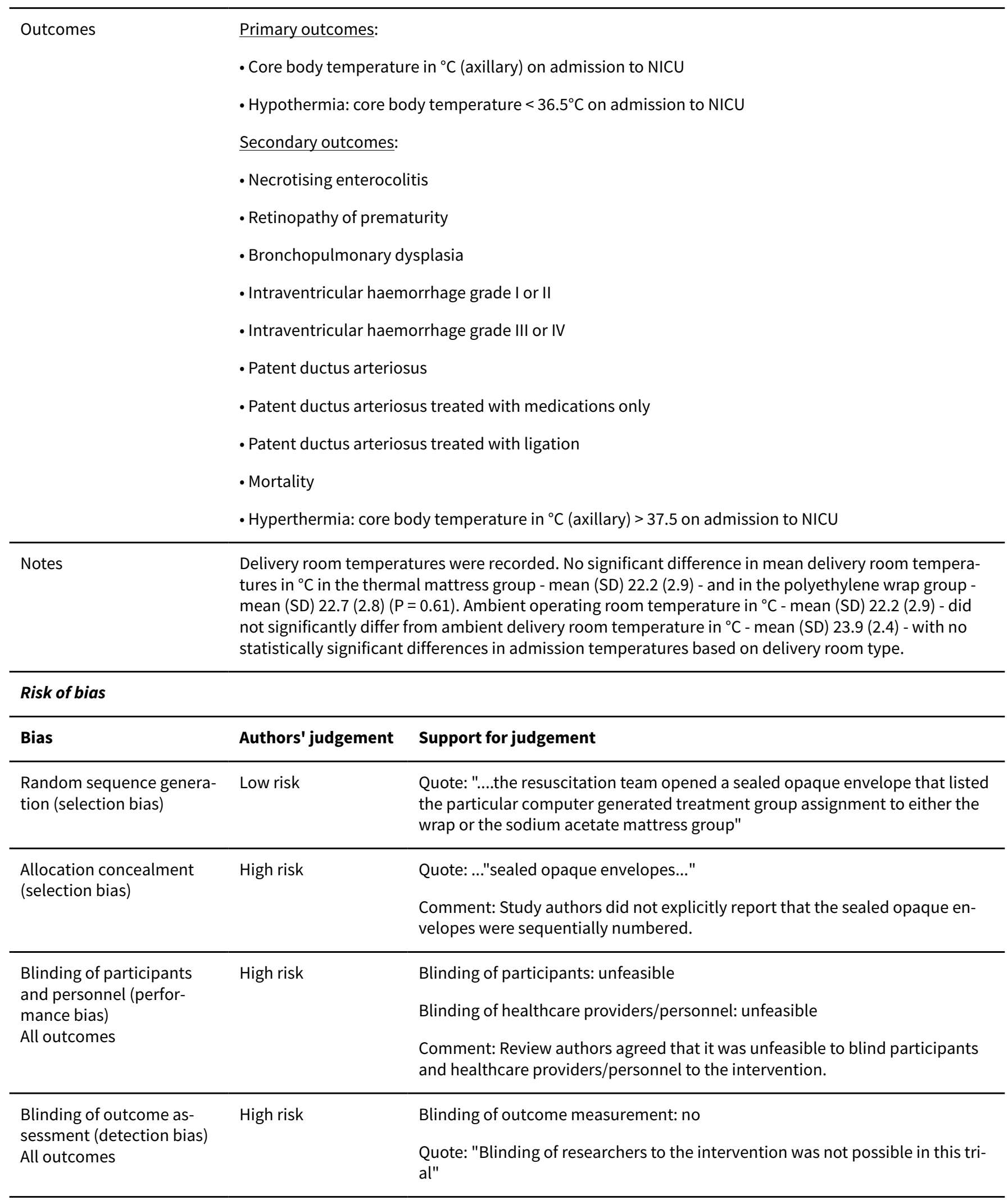


Simon 2011 (Continued)

Incomplete outcome data Low risk (attrition bias)

All outcomes
Thirty-six of 39 ( $8 \%$ loss to follow-up) infants randomised completed the study. Reasons for loss to follow-up were randomisation to wrong group (1 infant), unrecorded delivery room temperature (1 infant), and birth weight $>1250$ grams (1 infant). Intention-to-treat analysis including all patients initially enrolled produced similar results, which did not alter the final conclusion of the study.

$\begin{array}{ll}\begin{array}{l}\text { Selective reporting (re- } \\ \text { porting bias) }\end{array} & \text { Low risk } \\ & \text { tocol stage at the time of registration as per http://clinicaltrials.gov/ct2/show/ } \\ \text { NCT00603837. }\end{array}$

Other bias High risk

Comment: The researcher was not available in 141 of 148 cases excluded.

Quote: "...minor difficulties occurred with the polyethylene wrap during resuscitations ... brief opening of the wrap. In difficult resuscitations the wrap sometimes was displaced briefly when chest compressions were needed. In addition, auscultation of heart sounds was sometimes challenging ... Loss of heat may have occurred during these brief periods when the Neowrap was opened or displaced"

Comment: Trial authors also noted greater variability of use between practitioners with the wrap than with the mattress.

Smith 2013

Methods

Randomised; infants from multiple births randomised separately

Single-centre

Blinding of randomisation:

- Generation of allocation sequence: adequate (computer-generated randomisation list)

-Allocation concealment: inadequate (sealed opaque envelopes, no mention of whether these were sequentially numbered)

Blinding of intervention: participants: unclear; healthcare providers/personnel: unclear

Blinding of outcome measurement: unclear

Complete follow-up: no (92 of 95 infants randomised completed the study, 3 infants were excluded owing to missing data: intervention group (1), control group (2))

Baseline core body temperature in ${ }^{\circ} \mathrm{C}$ (axillary) was recorded.

This study was approved by the Townsville Health Service District Human Research Ethics Committee (HREC).

Informed parental consent was obtained before infant enrolment.

Participants

Infants at $<30$ weeks' gestation

Infants stratified into 2 groups. Group 1: $<27$ weeks' gestation; Group 2: 27 to 29 weeks' gestation

Ninety-five infants randomised: intervention group $(n=44)$, control group $(n=51), 92$ infants completed the study: intervention group $(n=43)$, control group $(n=49)$

Setting: tertiary neonatal referral centre at the Townsville Hospital in regional Queensland, Australia

Country income grouping: high 
Smith 2013 (Continued)

\section{Exclusion criteria:}

Infants:

- Congenital abnormalities with open lesions

- Considered to be non-viable

Intervention group (plastic wrap) characteristics:

- Birth weight (grams) mean (SD) 991 (299)

- Gestation (weeks) $<27(22)$

- Mode of delivery: 'spontaneous vaginal birth' (9), lower segment caesarean section (26), other (1), not recorded (7)

Control group (standard care) characteristics:

- Birth weight (grams) mean (SD) 982 (233)

- Gestation (weeks) < $27(26)$

- Mode of delivery: 'spontaneous vaginal birth' (13), lower segment caesarean section (28), other (1), not recorded (7)

Interventions

Plastic wrap (NeoWrap) - measured $60 \mathrm{~cm}$ by $60 \mathrm{~cm}$

Intervention group:

A clean wrap was placed under a preheated radiant warmer, and the infant was transferred immediately after birth without being dried; the Neowrap was placed from the neck down. The head was dried with a prewarmed towel, and a hat of soft woven fabric was applied. Upon arrival to the NICU, the infant remained in the wrap, was weighed, and was placed in a prewarmed incubator (in accordance with NICU guidance). The wrap was removed 2 hours after admission to the NICU.

\section{Control group:}

The infant was transferred to the prewarmed radiant warmer immediately after birth and was dried; wet towels were removed, and a hat of soft woven fabric was applied. Upon arrival to the NICU, the infant was weighed and was placed in a prewarmed incubator (in accordance with NICU guidance); a nappy was applied. In both groups, towels were prewarmed under the radiant warmer on full power before birth, and infants were transported to the NICU with use of the radiant warmer. Resuscitation was carried out according to guidelines of the American Academy of Pediatrics Neonatal Resuscitation Program. Continuous monitoring of skin temperature was carried out by placement of a skin probe in the right upper quadrant of the abdomen or back to detect hyperthermia. All core body temperatures in ${ }^{\circ} \mathrm{C}$ (axillary) were recorded with a BD digital thermometer device.

Outcomes

\section{Primary outcome:}

- Core body temperature in ${ }^{\circ} \mathrm{C}$ (axillary) on admission to the NICU (upon placement of infant in incubator)

\section{Secondary outcomes:}

- Core body temperature in ${ }^{\circ} \mathrm{C}$ (axillary) at $30,60,90$, and 120 minutes post admission to NICU

- Requirement for continuous positive airway pressure

- Requirement for ventilation

- Blood glucose level ( $\mathrm{mmol} / \mathrm{L})$

- Intraventricular haemorrhage, periventricular, or periventricular leukomalacia 
Smith 2013 (Continued)

$$
\text { - Early sepsis }
$$

- Late sepsis

$\cdot \mathrm{pH}$

- Mortality

Notes Baseline core body temperature in ${ }^{\circ} \mathrm{C}$ (axillary) after birth was slightly higher (not significant; $\mathrm{P}=0.074$ ) in the intervention (plastic wrap) group - mean (SD) 36.15 (0.85) - than in the control (conventional care) group - mean (SD) 35.81 (0.91). Trial authors highlighted that this could be attributed to timing of the baseline temperature measurement, which differed slightly in the intervention and control groups and may have impacted NICU admission temperatures.

\section{Risk of bias}

\begin{tabular}{lll}
\hline Bias & Authors' judgement & Support for judgement \\
\hline $\begin{array}{l}\text { Random sequence genera- } \\
\text { tion (selection bias) }\end{array}$ & Low risk & $\begin{array}{l}\text { Quote: "Randomisation was achieved by use of a computer generated ran- } \\
\text { domisation list. The list was stratified into two groups, group 1, infants }<27 \\
\text { weeks' gestation and group 2, infants 27 - 29 (6) weeks' gestation" }\end{array}$ \\
\hline
\end{tabular}

\begin{tabular}{ll}
\hline $\begin{array}{l}\text { Allocation concealment } \\
\text { (selection bias) }\end{array}$ & $\begin{array}{l}\text { Quote: "Randomisation concealment was adhered to by the use of a sealed } \\
\text { opaque envelope" }\end{array}$ \\
& $\begin{array}{l}\text { Comment: Study authors did not explicitly report that the sealed opaque en- } \\
\text { velopes were sequentially numbered. }\end{array}$
\end{tabular}

\begin{tabular}{|c|c|c|}
\hline $\begin{array}{l}\text { Blinding of participants } \\
\text { and personnel (perfor- } \\
\text { mance bias) }\end{array}$ & Unclear risk & $\begin{array}{l}\text { Blinding of participants: unclear } \\
\text { Blinding of healthcare providers/personnel: unclear }\end{array}$ \\
\hline
\end{tabular}

Unclear risk

Blinding of outcome as-
sessment (detection bias)
All outcomes

\section{Blinding of outcome assessment: unclear}

Comment: Information is insufficient to address risk of bias. However, owing to the nature of the intervention, it is unlikely that participants, healthcare providers/personnel, and outcome assessors were blinded to the intervention because infants had their skin temperature monitored at 30, 60, 90, and 120 minutes.

\begin{tabular}{lll}
\hline $\begin{array}{l}\text { Incomplete outcome data } \\
\text { (attrition bias) } \\
\text { All outcomes }\end{array}$ & Low risk & $\begin{array}{l}\text { Ninety-two of } 95 \text { (3\% loss to follow-up) infants randomised completed the } \\
\text { study; } 3 \text { infants were excluded owing to missing data: intervention group (1), } \\
\text { control group (2). }\end{array}$ \\
\hline $\begin{array}{l}\text { Selective reporting (re- } \\
\text { porting bias) }\end{array}$ & Low risk & $\begin{array}{l}\text { Comment: Only } 1 \text { secondary outcome measure was listed for this study at } \\
\text { protocol stage at the time of trial registration as per http://apps.who.int/tri- } \\
\text { alsearch/Trial.aspx?TriallD=ACTRN12608000212358. }\end{array}$ \\
\hline Other bias & High risk & $\begin{array}{l}\text { Comment: Trial authors reported that prolonged resuscitation, placement of } \\
\text { a nappy, and use of umbilical lines tended to 'disturb or dislodge' the wrap, } \\
\text { which could have caused heat loss and therefore underestimation of the effect } \\
\text { size. Time to arrival to the NICU from the delivery room also varied. }\end{array}$ \\
\hline
\end{tabular}

Talakoub 2015

Methods Randomised


Talakoub 2015 (Continued)

\author{
Multi-centre (2) \\ Blinding of randomisation:
}

- Generation of allocation sequence: adequate (drawing of lots to three 32-participant groups)

- Allocation concealment: unclear (insufficient information)

Blinding of intervention: participants: unclear; healthcare providers/personnel: unclear

Blinding of outcome measurement: unclear

Complete follow-up: no (96 of 98 infants randomised completed the study. One infant was lost owing to urination in the plastic bag, and one owing to a critical condition requiring resuscitation; this infant died)

Baseline core body temperature in ${ }^{\circ} \mathrm{C}$ (axillary) was recorded.

No ethical statement was provided (Masters Thesis project number 392529, Isfahan University of Medical Sciences, Isfahan, Iran).

Informed parental consent was obtained before infant enrolment.

Participants

Infants at $\geq 28$ and $\leq 32$ weeks' gestation

Ninety-eight infants randomised; 96 completed the study: intervention group $1(n=32)$, intervention group $2(n=32)$, and control group $(n=32)$.

Setting: Alzahra and Shahid Beheshti Hospitals, Isfahan, Iran

Country income grouping: upper-middle

Exclusion criteria:

Mothers:

- Fever

Infants:

- Neural tube defects

- Congenital obvious anomalies

- Congenital dermal diseases

-Abdominal wall defects

- Gestation $<28$ completed weeks and birth weight $<900$ grams (unclear)

Further exclusion criteria:

- Patients' parents' loss of interest in continuing with the study

- Neonates' expiration

- Neonates' urination in the polyethylene bag

- Infant having no vital signs 30 minutes after arrival to the NICU

Intervention group 1 (polyethylene bag and cotton hat) characteristics:

- No details of characteristics provided

Intervention group 1 (polyethylene bag and polyethylene hat) characteristics:

- No details of characteristics provided 
Talakoub 2015 (Continued)

\section{Control group (standard care) characteristics:}

- No details of characteristics provided

- Trial authors reported no significant differences in participants' demographic characteristics (gestational age, birth weight, transfer time, Apgar score, or delivery mode) between the 3 groups.

Interventions

Polyethylene bag $(25 \mathrm{~cm}$ by $40 \mathrm{~cm})$ and polyethylene bag + polyethylene hat

Intervention group 1: polyethylene bag and cotton hat. Immediately after birth, infants were placed in a prewarmed polyethylene bag up to the neck, without drying, under a radiant warmer. Their heads were covered by a cotton hat after drying.

Intervention group 2: polyethylene bag and polyethylene hat. Immediately after birth, infants were placed in a prewarmed polyethylene bag up to the neck, without drying, under a radiant warmer. Their heads were covered by a polyethylene prewarmed hat with no strips, without drying.

Control group: Infants were dried with a cloth and were placed under a radiant warmer. All infants were transferred from the labour room or operating room to the neonatal ward in a portable incubator set at $35^{\circ} \mathrm{C}$. After vital signs were checked, infants were placed under a radiant warmer. Axillary temperature was measured with a paediatric digital thermometer through a hole in the polyethylene cover. The polyethylene bag and/or hat remained on the infant for first hour. All core body temperatures in ${ }^{\circ} \mathrm{C}$ (axillary) were recorded with a paediatric digital thermometer (Omron, Japan).

\begin{tabular}{|c|c|c|}
\hline Outcomes & \multicolumn{2}{|c|}{$\begin{array}{l}\text { Primary outcomes: } \\
\text { - Core body temperature in }{ }^{\circ} \mathrm{C} \text { (axillary) on admission to NICU } \\
\text { - Core body temperature in }{ }^{\circ} \mathrm{C} \text { (axillary) at } 1 \text { hour and } 2 \text { hours after admission to NICU }\end{array}$} \\
\hline Notes & \multicolumn{2}{|c|}{ Trial author (Zohreh Ghazavi) contacted via email on 26 October 2016, but no reply received } \\
\hline \multicolumn{3}{|l|}{ Risk of bias } \\
\hline Bias & Authors' judgement & Support for judgement \\
\hline $\begin{array}{l}\text { Random sequence genera- } \\
\text { tion (selection bias) }\end{array}$ & Low risk & Quote: "...randomly allocated, by drawing of lots, to three 32-subject groups" \\
\hline $\begin{array}{l}\text { Allocation concealment } \\
\text { (selection bias) }\end{array}$ & Unclear risk & Information is insufficient to assess risk of bias. \\
\hline $\begin{array}{l}\text { Blinding of participants } \\
\text { and personnel (perfor- } \\
\text { mance bias) } \\
\text { All outcomes }\end{array}$ & Unclear risk & Information is insufficient to assess risk of bias. \\
\hline $\begin{array}{l}\text { Blinding of outcome as- } \\
\text { sessment (detection bias) } \\
\text { All outcomes }\end{array}$ & Unclear risk & Information is insufficient to assess risk of bias. \\
\hline $\begin{array}{l}\text { Incomplete outcome data } \\
\text { (attrition bias) } \\
\text { All outcomes }\end{array}$ & Low risk & $\begin{array}{l}\text { Ninety-six of } 98 \text { ( } 2 \% \text { loss to follow-up) infants randomised completed the } \\
\text { study. One infant was lost owing to urination in the plastic bag, and } 1 \text { owing to } \\
\text { a critical condition requiring resuscitation; this infant died. }\end{array}$ \\
\hline $\begin{array}{l}\text { Selective reporting (re- } \\
\text { porting bias) }\end{array}$ & Unclear risk & Information is insufficient to assess risk of bias. \\
\hline Other bias & Low risk & \\
\hline
\end{tabular}


Blinding of randomisation:

- Generation of allocation sequence: inadequate; when an infant fulfilling the criteria for inclusion was admitted to the delivery room, a ballot was selected from a sealed box containing a sheet of paper stating infant assignment to either plastic wrap and cap or routine care

- Allocation concealment: unclear (as above)

Blinding of intervention: participants: unfeasible; healthcare providers/personnel: unfeasible

Blinding of outcome measurement: yes; before entry into the NICU, the wrap was removed and the receiving nurse in charge, who was unaware of the intervention, recorded time of arrival and axillary $\left({ }^{\circ} \mathrm{C}\right)$ temperature

Complete follow-up: yes (all 58 infants randomised completed the study)

This study was approved by the ethics review board (Registration No. PED 2010-09-23-142).

Informed parental or guardian consent was obtained.

Participants

Infants at $\geq 28$ and $\leq 36$ weeks' gestation

Fifty-eight infants randomised: intervention group $(n=28)$; control group $(n=30)$

Setting: Tertiary Government Hospital, Manila, Philippines

Country income grouping: lower-middle

Exclusion criteria:

Mothers:

- None

Infants:

- Neural tube defects

- Omphalocoele, gastroschisis, exstrophy of the bladder, cloacal exstrophy, or any other open lesion that would cause greater than normal heat loss at delivery

- Sacral teratoma or other lesion that would prevent the infant from being positioned and evaluated in the normal manner

- Infants in whom resuscitation was not undertaken because of previability (i.e. lethal trisomy, anencephaly)

- Subsequent assessment indicating gestational age of $<28$ or $>36$ completed weeks

Intervention group (plastic body wrap (bag) and cap) characteristics:

Mothers:

- Maternal fever $>38^{\circ} \mathrm{C}$ at any time during admission (3)

Infants:

- Birth weight (grams) mean (SD) 1580.4 (467.7)

- Gestation (weeks) mean (SD) 33.2 (2.1) 
Tescon-delos Santos 2012 (Continued)

- Sex 'male' (19)

- Multiple birth (2)

- Mode of delivery: 'spontaneous vaginal birth' (14), 'caesarean section' (12)

- Small-for-gestational-age (10)

-Apgar @ 1 minute mean (SD) 7.8 (1.9)

-Apgar@ 5 minutes mean (SD) 8.5 (1.0)

- Transfer time (from delivery room to NICU) in hours mean (SD) 2.2 (1.8)

Control group (routine care) characteristics:

Mothers:

- Maternal fever $>38^{\circ} \mathrm{C}$ at any time during admission (4)

- Birth weight (grams) mean (SD) 1625.0 (446.0)

- Gestation (weeks) mean (SD) 33.6 (2.4)

- Sex 'male' (16)

- Multiple birth (3)

- Mode of delivery: 'spontaneous vaginal birth' (21), 'caesarean section' (7)

- Small-for-gestational-age (7)

•Apgar @ 1 minute mean (SD) 7.7 (1.7)

-Apgar @ 5 minutes mean (SD) 8.5 (0.8)

- Transfer time (from delivery room to NICU) in hours mean (SD) 2.2 (2.1)

Intervention group:

Infants received routine thermal care under a functioning droplight comprising drying immediately at birth and removing wet blankets and wrapping in dry blankets. Axillary temperature was taken at the fifth minute of life, and infants were wrapped with polyethylene plastic body wrap from the axilla down, with a corresponding polyethylene cap placed on the head worn up to the glabella within 5 minutes after delivery. The wrap was removed before entry into NICU premises.

\section{Control group:}

Infants received routine thermal care under a functioning droplight comprising drying immediately at birth and removing wet blankets and wrapping in prewarmed blankets. For both groups, in instances where hypothermia occurred $\left(<36.5^{\circ} \mathrm{C}\right)$ at the 60 th minute of life, infants were swaddled with additional prewarmed blankets. When a core body temperature $>37^{\circ} \mathrm{C}$ was recorded at any time, infants were unswaddled, or the wrap and cap were removed. If thermal burns developed, the wrap and cap were immediately removed. Axillary temperatures $\left({ }^{\circ} \mathrm{C}\right.$ ) were recorded at the fifth (baseline), 10th, 15th, 30th, and 60th minutes of life and upon NICU admission with a Fixotherm digital thermometer; a digital timer automatically sounded an alarm at the selected times.

- Core body temperatures in ${ }^{\circ} \mathrm{C}$ (axillary) at the 5th (baseline), 10th, 15th, 30th, and 60th minutes of life

Secondary outcomes: 
Tescon-delos Santos 2012 (Continued)

- Hyperthermia (core body temperature (axillary) $>37^{\circ} \mathrm{C}$ )

- Burns (injury to tissue caused by heat)

- Skin maceration (softening and breaking of the skin due to exposure to excessive moisture)

- Reported skin or systemic infection (caused by pathogenic organism) within first 5 days of birth

- Hyponatraemia (serum sodium concentration $<130 \mathrm{mmol} / \mathrm{L}$ )

- Hypernatraemia (serum sodium concentration > $150 \mathrm{mmol} / \mathrm{L}$ )

- Percentage weight loss from birth to fifth day of life

Notes

Ambient temperature in delivery room and NICU was considered to be within the range $26^{\circ} \mathrm{C}$ to $28^{\circ} \mathrm{C}$, although actual temperatures were not recorded.

\section{Risk of bias}

\begin{tabular}{lll}
\hline Bias & Authors' judgement & Support for judgement \\
\hline $\begin{array}{l}\text { Random sequence genera- } \\
\text { tion (selection bias) }\end{array}$ & High risk & $\begin{array}{l}\text { Quote: "....the resident catcher on duty picked a ballot from a sealed box con- } \\
\text { taining a sheet of paper which will either say wrap or no wrap" }\end{array}$ \\
\hline $\begin{array}{l}\text { Allocation concealment } \\
\text { (selection bias) }\end{array}$ & High risk & As above \\
\hline
\end{tabular}

Blinding of participants and personnel (perfor-

mance bias)

All outcomes

\section{High risk}

Blinding of participants: unfeasible

Blinding of healthcare providers/personnel: unfeasible

Comment: Review authors agreed that it was unfeasible to blind participants and healthcare providers/personnel to the intervention.

$\begin{array}{ll}\begin{array}{l}\text { Blinding of outcome as- } \\ \text { sessment (detection bias) }\end{array} & \text { Low risk } \\ \begin{array}{ll}\text { All outcomes } & \text { Blinding of outcome assessment: yes } \\ & \text { Quote: "Prior to entering the NICU premises, the wrap was removed by the res- } \\ \text { ident catcher on duty. The receiving nurse in charge, who was unaware of the } & \text { intervention, recorded the time of arrival at the NICU as well as the infant's ax- } \\ \text { illary temperature upon admission" }\end{array}\end{array}$

Incomplete outcome data Low risk Complete follow-up: All infants randomised completed the study.

(attrition bias)

All outcomes
Selective reporting (re- Unclear risk Information is insufficient to assess risk of bias. porting bias)
In both groups, additional swaddling with prewarmed blankets was carried out for infants who had a recorded axillary temperature $<36.5^{\circ} \mathrm{C}$, which prevented further hypothermia and potentially masked the true effect of the intervention. A higher incidence of further swaddling in the control group resulted in higher mean temperatures during the first 15 minutes of life. This additional swaddling may have been influenced by caregivers (not blinded to the intervention) being more attentive to the control group.

Methods Randomised


Trevisanuto 2010 (Continued)

\section{Single-centre \\ Blinding of randomisation:}

- Generation of allocation sequence: adequate (computer-generated randomisation sequence balanced in blocks of 6 participants). Multiple births were separately randomised.

- Allocation concealment: adequate (double-enclosed, opaque, sealed, and sequentially numbered envelopes)

Blinding of intervention: participants: unfeasible; healthcare providers/personnel: unfeasible Blinding of outcome measurement: no

Complete follow-up: yes (all 96 infants randomised completed the study)

This study was approved by the Azienda Ospedaliera-Padova Research Ethics Comittee. When possible, parental consent was obtained before delivery; remaining parents received a parent information form after initial stabilisation of the infant. Mother's axillary temperature was recorded within 30 minutes of delivery. Time from delivery to NICU admission (minutes) was recorded.

Participants

Inborn infants at $<29$ weeks' gestation

Ninety-six infants randomised: intervention group $1(n=32)$, intervention group $2(n=32)$, control group $(n=32)$

Setting: Pediatric Department, Medical School, University of Padua, Azienda Ospedaliera di Padova, Padua, Italy

Country income grouping: high

\section{Exclusion criteria:}

- Infants with congenital anomalies with open lesions (e.g. gastroschisis, meningomyelocoele)

- Infants whose delivery was not attended by the neonatal team

Intervention group 1 (polyethylene cap) characteristics:

Mothers:

- Ethnicity: 'White' (31), ethnicity 'Black ' (1)

- Age (years) mean (SD) 32 (7)

- Antenatal steroids (31)

- Temperature $\left({ }^{\circ} \mathrm{C}\right)$ mean (SD) $36.4(0.4)$

- Vaginal delivery (7)

- Caesarean section (25)

Infants:

- Gestation (weeks) mean (SD) 26.1 (1.4)

- Birth weight (grams) mean (SD) 834 (246)

- Female (18)

- Multiple birth (5)

-Apgar score @ 1 minute mean (SD) 5.9 (1.8)

-Apgar score @ 5 minutes mean (SD) 8.1 (1.0)

Intervention group 2 (polyethylene bag) characteristics:

Mothers: 
• Ethnicity: 'White' (30), 'Black' (2)

- Age (years) mean (SD) 33 (5)

-Antenatal steroids (30)

- Temperature $\left({ }^{\circ} \mathrm{C}\right)$ mean (SD) $36.4(0.5)$

- Vaginal delivery (8), caesarean section (24)

Infants:

- Gestation (weeks) mean (SD) 25.8 (1.5)

- Birth weight (grams) mean (SD) 800 (223)

- Female (19)

- Multiple birth (6)

-Apgar score @ 1 minute mean (SD) 5.3 (1.9)

-Apgar score @ 5 minutes mean (SD) 7.8 (0.8)

Control group (conventional care) characteristics:

Mothers:

• Ethnicity: 'White' (32), 'Black' (0)

- Age (years) mean (SD) 32 (4)

- Antenatal steroids (30)

- Temperature $\left({ }^{\circ} \mathrm{C}\right)$ mean (SD) $36.4(0.6)$

- Vaginal delivery (8)

- Caesarean section (24)

Infants:

- Gestation (weeks) mean (SD) 26.3 (1.0)

- Birth weight (grams) mean (SD) 813 (225)

- Female (16)

- Multiple birth (7)

- Apgar score @ 1 minute mean (SD) 5.1 (2.3)

-Apgar score @ 5 minutes mean (SD) 7.4 (1.5)

Intervention group 1: Polyethylene caps were placed on the radiant warmer bed before delivery. The head of the infant was covered with a polyethylene cap immediately after birth; only the head was dried.

Intervention group 2: Polyethylene bags (Vedovato SNC, Camposampiero, Padova, Italy; isolation transport bag $35 \mathrm{~cm}$ by $40 \mathrm{~cm}$ ) were placed on the radiant warmer bed before delivery. Infants were placed into the bag while still wet, up to the neck; only the head was dried (no head covering).

Control group: Infants were placed in prewarmed towels after drying (no head covering), according to International Guidelines for Neonatal Resuscitation. All infants were stabilised in the delivery room under radiant warmers (Babytherm 8004/8010; Drager Medizintechnik GmbH, Lubeck, Germany). A trans- 
Trevisanuto 2010 (Continued)

port incubator (MOG 500; Ginevri, Tecnologie Biomediche, Cecchina, Roma, Italy) was used to transfer all infants from the delivery room into the NICU, where they were placed in a double-walled incubator at $35^{\circ} \mathrm{C}$ temperature and $70 \%$ humidity (Drager Medical AG\&Co. KGaA, Lubeck, Germany). At this point, the cap or the wrap was removed. Core body temperature in ${ }^{\circ} \mathrm{C}$ (axillary) was measured with a digital thermometer (Terumo Digital Clinical Thermometer C202; Terumo Corporation, Tokio, Japan).

Primary outcomes:
Outcomes $\quad$ Core body temperature in ${ }^{\circ} \mathrm{C}$ (axillary) on admission to the NICU (immediately after cap and wrap re-
moval)
$\cdot$ Core body temperature in ${ }^{\circ} \mathrm{C}$ (axillary) 1 hour after admission to the NICU
$\cdot$ Hypothermia: core body temperature (axillary) $<36.4^{\circ} \mathrm{C}$ on admission to the NICU
Secondary outcomes:
$\cdot$ Mortality before hospital discharge
- Presence of major brain injury (sonographic evidence of IVH with ventricular dilatation, parenchymal
hemorrhagic infarction, or periventricular leukomalacia)
$\cdot$ Tracheal intubation at birth
$\cdot$ Delivery to admission time (minutes)
$\cdot$ Blood gas pH on NICU admission
$\cdot$ Bicarbonate on NICU admission (mmol/L)
$\cdot$ Serum glucose concentration on NICU admission (mmol/L)

Notes

Of 117 infants screened, 21 were excluded: neonatal team did not attend delivery $(n=13)$, parents refused to participate $(n=5)$, congenital malformations were noted $(n=3)$. Delivery to admission time was significantly shorter in the control group than in the plastic wrap group. This measure could be a potential indicator of how unwell an infant is and can be used to see if the team behaved differently towards intervention infants than control infants. If plastic wrap infants took longer to get to the NICU, these infants had a greater chance of being chilled and the plastic wrap measured estimate of treatment effect was likely to be a conservative one. Delivery room temperature was not monitored throughout the study. Delivery room temperature is normally approximately $24^{\circ} \mathrm{C}$.

\section{Risk of bias}

Bias Authors' judgement Support for judgement

\section{Random sequence genera- Low risk} tion (selection bias)
Quote: "....assigned to one of three study groups....according to a computer generated, randomised sequence balanced in blocks of six subjects"

\begin{tabular}{|c|c|c|}
\hline $\begin{array}{l}\text { Allocation concealment } \\
\text { (selection bias) }\end{array}$ & Low risk & $\begin{array}{l}\text { Quote: "The randomised allocation was concealed in double-enclosed, } \\
\text { opaque, sealed and sequentially numbered envelopes" }\end{array}$ \\
\hline \multirow{3}{*}{$\begin{array}{l}\text { Blinding of participants } \\
\text { and personnel (perfor- } \\
\text { mance bias) } \\
\text { All outcomes }\end{array}$} & \multirow[t]{3}{*}{ High risk } & Blinding of participants: unfeasible \\
\hline & & Blinding of healthcare providers/personnel: unfeasible \\
\hline & & $\begin{array}{l}\text { Comment: Review authors agreed that it was unfeasible to blind participants } \\
\text { and healthcare providers/personnel to the intervention. }\end{array}$ \\
\hline \multirow{2}{*}{$\begin{array}{l}\text { Blinding of outcome as- } \\
\text { sessment (detection bias) } \\
\text { All outcomes }\end{array}$} & \multirow[t]{2}{*}{ Unclear risk } & Blinding of outcome measure: unclear \\
\hline & & Information is insufficient to assess risk of bias. \\
\hline
\end{tabular}


Trevisanuto 2010 (Continued)

Incomplete outcome data Low risk Complete follow-up: All infants randomised completed the study. (attrition bias)

All outcomes

$\begin{array}{ll}\begin{array}{l}\text { Selective reporting (re- } \\ \text { porting bias) }\end{array} & \text { Low risk } \\ & \begin{array}{l}\text { All study outcomes detailed at the time of trial registration were report- } \\ \text { ed in the published manuscript as per http://clinicaltrials.gov/ct2/show/ } \\ \text { NCT00930917?term=NCT00930917\&rank=1. }\end{array}\end{array}$

Other bias Low risk

\title{
Vohra 1999
}

\section{Methods}

\author{
Randomised \\ Single-centre \\ Prognostic stratification was by gestation at 23 to 27 and 28 to 31 weeks. \\ Blinding of randomisation:
}

- Generation of allocation sequence: adequate (computer-generated random sequence)

-Allocation concealment: adequate (double-enclosed, opaque, sealed, and sequentially numbered envelopes)

Blinding of intervention: participants: unfeasible; healthcare providers/personnel: unfeasible

Blinding of outcome measurement: no

Complete follow-up: no (59 of 62 infants randomised completed the study; 1 infant was excluded as no rectal temperature was recorded, and 2 infants were excluded owing to an imperforate anus)

Delivery room, nursery temperatures, and other potential confounding variables (birth weight, maternal temperature, illnesses, medications, length of second stage of labour, length of time to arrival at nursery) were recorded.

Ethics approval was obtained.

No informed consent was obtained.

Institutional consent was given.

\section{Participants}

Infants delivered at $<32$ weeks' gestation

Sixty-two infants randomised: 59 completed the study: intervention group $(n=27)$, control group $(n=$ 32)

Setting: Grace Hospital (later, the IWK Grace Health Centre), Halifax, Nova Scotia, Canada

Country income grouping: high

Exclusion criteria:

- Major congenital anomalies with open lesions

- Infants whom the attending obstetrician considered "previable"

Intervention group (polyethylene wrap) characteristics (gestation $<28$ weeks):

- Birth weight (grams) mean (SD) 914 (163)

- Gestation (weeks) mean (SD) 26.1 (1.4)

-Apgar score @ 1 minute mean (SD) 5.0 (3.0)

-Apgar score @ 5 minutes mean (SD) 6.7 (1.7)

- Ruptured membranes (minutes) mean (SD) 186 (471)

- Length second stage (minutes) mean (SD) 26 (55)

Control group (conventional care) characteristics (gestation < 28 weeks): 
Vohra 1999 (Continued)

- Birth weight (grams) mean (SD) 742 (206)

- Gestation (weeks) mean (SD) 25.7 (1.5)

•Apgar score @ 1 minute mean (SD) 4.3 (2.1)

•Apgar score @ 5 minutes mean (SD) 7.1 (1.4)

- Ruptured membranes (minutes) mean (SD) 10 (21)

- Length second stage (minutes) mean (SD) 18 (24)

Intervention group (polyethylene wrap) characteristics (gestation $\geq 28$ weeks):

- Birth weight (grams) mean (SD) 1251 (282)

- Gestation (weeks) mean (SD) 29.6 (1.1)

-Apgar score @ 1 minute mean (SD) 6.5 (1.8)

-Apgar score @ 5 minutes mean (SD) 8.5 (1.1)

- Ruptured membranes (minutes) mean (SD) 105 (310)

- Length second stage (minutes) mean (SD) 15 (25)

Control group (conventional care) characteristics (gestation $\geq 28$ weeks):

- Birth weight (grams) mean (SD) 1265 (206)

- Gestation (weeks) mean (SD) 29.4 (1.5)

-Apgar score @ 1 minute mean (SD) 6.0 (2.1)

•Apgar score @ 5 minutes mean (SD) 8.0 (1.4)

- Ruptured membranes (minutes) mean (SD) 108 (21)

- Length second stage (minutes) mean (SD) 13 (24)

Interventions

Polyethylene bag - measured $20 \mathrm{~cm}$ by $50 \mathrm{~cm}$ and manufactured by Eastern Paper, a Division of EPC Industries

Intervention group:

A transparent polyethylene bag was opened at resuscitation under a radiant warmer, and the infant was placed on it from the shoulders down. Only the head of the infant was dried; the body was wrapped without drying.

Control group: Controls were managed with the protocol described by the Neonatal Resuscitation Program. Infants were routinely dried under the radiant warmer and were transferred to the neonatal unit in an incubator. Core body temperature in ${ }^{\circ} \mathrm{C}$ (rectal) was measured with a digital rectal thermometer.

Outcomes

Primary outcomes:

- Core body temperature in ${ }^{\circ} \mathrm{C}$ (rectal) on removal of the wrap on admission to NICU

- Hypothermia on admission to NICU

Secondary outcomes:

- Mortality

- Hyperthermia

- Infection

- Skin maceration

- Interference with resuscitation 
Vohra 1999 (Continued)

Notes

Forty-two infants who met the inclusion criteria were not enrolled in the study. Reasons were as follows: still-birth (1), gestation 34 weeks (2), failure to reach delivery in time (8), failure of staff to pick up envelope on the way to the delivery room (31). Non-enrolled infants did not differ significantly from those in the study population.

\section{Risk of bias}

\begin{tabular}{|c|c|c|}
\hline Bias & Authors' judgement & Support for judgement \\
\hline $\begin{array}{l}\text { Random sequence genera- } \\
\text { tion (selection bias) }\end{array}$ & Low risk & Quote: (from correspondence) "Computer generated randomisation list" \\
\hline $\begin{array}{l}\text { Allocation concealment } \\
\text { (selection bias) }\end{array}$ & Low risk & $\begin{array}{l}\text { Quote: "The randomisation allocation and instructions were prepared in dou- } \\
\text { ble-enclosed, opaque, sealed and sequentially numbered envelopes" }\end{array}$ \\
\hline \multirow{3}{*}{$\begin{array}{l}\text { Blinding of participants } \\
\text { and personnel (perfor- } \\
\text { mance bias) } \\
\text { All outcomes }\end{array}$} & \multirow[t]{3}{*}{ High risk } & Blinding of participants: unfeasible \\
\hline & & Blinding of caregivers: unfeasible \\
\hline & & $\begin{array}{l}\text { Comment: Review authors agreed that it was unfeasible to blind participants } \\
\text { and healthcare providers/personnel to the intervention. }\end{array}$ \\
\hline $\begin{array}{l}\text { Blinding of outcome as- } \\
\text { sessment (detection bias) } \\
\text { All outcomes }\end{array}$ & High risk & Blinding of outcome measurement: no \\
\hline $\begin{array}{l}\text { Incomplete outcome data } \\
\text { (attrition bias) } \\
\text { All outcomes }\end{array}$ & Low risk & $\begin{array}{l}\text { Fifty-nine of } 62 \text { infants ( } 5 \% \text { loss to follow-up) randomised completed the } \\
\text { study; } 1 \text { infant was excluded as no rectal temperature was recorded, and } 2 \text { in- } \\
\text { fants were excluded owing to an imperforate anus. }\end{array}$ \\
\hline $\begin{array}{l}\text { Selective reporting (re- } \\
\text { porting bias) }\end{array}$ & Unclear risk & Information is insufficient to assess risk of bias. \\
\hline Other bias & High risk & $\begin{array}{l}\text { Data show some imbalance in birth weight between study groups. For infants } \\
\text { with gestation < } 28 \text { completed weeks, birth weight was } 914 \text { grams (SD 163) for } \\
\text { the plastic wrap group and } 742 \text { grams (SD 206) for the non-wrap group. }\end{array}$ \\
\hline
\end{tabular}

Vohra 2004

Randomised
Single-centre
Blinding of randomisation:
- Generation of allocation sequence: adequate (computer-generated random sequence balanced in
blocks of 4 participants)
- Allocation concealment: adequate (double-enclosed, opaque, sealed, and sequentially numbered en
velopes)
Blinding of intervention: participants: unfeasible; healthcare providers/personnel: unfeasible
Blinding of outcome measurement: no
Complete follow-up: no (53 of 55 infants randomised completed the study; 2 infants died in the deliv-
ery room). Multiple eligible births were separately randomised. Institutional consent was given.

Participants

Infants at $<28$ weeks' gestation delivered with the birth attended by the neonatal team 55 infants randomised: intervention group $(n=28)$, control group $(n=27) ; 53$ completed the study: intervention group $(n=26)$, control group $(n=27)$

Setting: McMaster University Medical Centre, Hamilton, Ontario, Canada 
Vohra 2004 (Continued)

\section{Country income grouping: high}

Exclusion criteria:

- Neonatal team did not attend the delivery

- Major congenital anomalies that were not covered by skin (e.g. gastroschisis, meningomyelocoele)

- Blistering skin conditions

Intervention group (polyethylene wrap) characteristics:

- Female (14)

- Birth weight (grams) mean (SD) 858 (199)

- Gestation (weeks) mean (SD) 26 (1.5)

-Apgar @ 1 minute median (IQR) 6 (2 to 6)

- Apgar @ 5 minutes median (IQR) 6 (3 to 6)

Control group (conventional care) characteristics:

- Female (13)

- Birth weight (grams) mean (SD) 825 (270)

- Gestation (weeks) mean (SD) 26 (1.4)

-Apgar @ 1 minute median (IQR) 7 (6 to 8)

-Apgar @ 5 minutes median (IQR) 7 (7 to 8) Industries, Ltd.

Intervention group: Infants were placed on polyethylene and wrapped from the neck down; only the head was dried; they were stabilised under a radiant warmer

Control group: Infants were dried completely according to the International Guidelines for Neonatal Resuscitation and were stabilised under a radiant warmer. All infants were carried by 1 member of the neonatal team from the delivery suite to the neonatal unit and were placed in a single-walled incubator with $60 \%$ humidity.

- Hypothermia on admission to NICU

Secondary outcomes:

- Core body temperature in ${ }^{\circ} \mathrm{C}$ (rectal) taken 1 hour later

- Mortality - death (all causes) before discharge

- First blood gas pH

- Bicarbonate (mmol/L)

- First blood serum glucose (mmol/L) after NICU admission

- Hyperthermia

- Interference with resuscitation 
Vohra 2004 (Continued)

ence of congenital malformations (3). Baseline characteristics of excluded infants and their mothers

were not different from baseline characteristics of those included in the study.

\section{Risk of bias}

\begin{tabular}{lll}
\hline Bias & Authors' judgement & Support for judgement \\
\hline $\begin{array}{l}\text { Random sequence genera- } \\
\text { tion (selection bias) }\end{array}$ & Low risk & $\begin{array}{l}\text { Quote: "Eligible infants were assigned to polyethylene wrap group or the con- } \\
\text { trol group, according to a computer generated random sequence balanced in } \\
\text { blocks of four subjects" }\end{array}$ \\
\hline $\begin{array}{l}\text { Allocation concealment } \\
\text { (selection bias) }\end{array}$ & Low risk & $\begin{array}{l}\text { Quote: "The random allocation was concealed in double enclosed, opaque, } \\
\text { sealed and sequentially numbered envelopes" }\end{array}$ \\
\hline $\begin{array}{l}\text { Blinding of participants } \\
\text { and personnel (perfor- } \\
\text { mance bias) } \\
\text { All outcomes }\end{array}$ & High risk & Blinding of participants: unfeasible \\
& Blinding of caregivers: unfeasible \\
& $\begin{array}{l}\text { Comment: Review authors agreed that it was unfeasible to blind participants } \\
\text { and healthcare providers/personnel to the intervention. }\end{array}$
\end{tabular}

$\begin{array}{lll}\text { Blinding of outcome as- } & \text { High risk } & \text { Blinding of outcome measurement: no }\end{array}$

All outcomes

\begin{tabular}{|c|c|c|}
\hline $\begin{array}{l}\text { Incomplete outcome data } \\
\text { (attrition bias) }\end{array}$ & Low risk & $\begin{array}{l}\text { Fifty-three of } 55 \text { ( } 4 \% \text { loss to follow-up) infants randomised completed the } \\
\text { study; } 2 \text { infants died in the delivery room. }\end{array}$ \\
\hline
\end{tabular}

All outcomes study; 2 infants died in the delivery room.

Selective reporting (re- Unclear risk Information is insufficient to assess risk of bias.
porting bias)

Other bias Low risk

CPAP: continuous positive airway pressure.

CRIB: Clinical Risk Index for Babies.

$\mathrm{FiO}_{2}$ : fraction of inspired oxygen.

HIV: human immunodeficiency virus.

IQR: interquartile ratio.

NICU: neonatal intensive care unit.

NRP: Neonatal Resuscitation Program.

SCRIP: composite cardiorespiratory stabilisation score.

SD: standard deviation.

TWM: transwarmer mattress.

WHO: World Health Organization.

Characteristics of excluded studies [ordered by study ID]

\begin{tabular}{ll}
\hline Study & Reason for exclusion \\
\hline Acharya 2014 & $\begin{array}{l}\text { Intervention was not applied immediately at birth (within } 10 \text { minutes) in the delivery room. Stable } \\
\text { low birth weight infants }\end{array}$ \\
\hline Ammari 2009 & Intervention was not applied immediately at birth (within 10 minutes) in the delivery room. \\
\hline Anderson 2003 & No thermal outcome measures were reported. \\
\hline
\end{tabular}




\begin{tabular}{|c|c|}
\hline Study & Reason for exclusion \\
\hline Andrade 2005 & No thermal outcome measures were reported. \\
\hline Ballesteros 2012 & Intervention was not applied immediately at birth (within 10 minutes) in the delivery room. \\
\hline Baum 1968 & Participants had a birth weight $\geq 6 \mathrm{lb}$. \\
\hline Beiranvand 2014 & Participants were term infants (at 38 to 42 weeks' gestation) delivered via caesarean section. \\
\hline Bell 1983 & Intervention was not applied immediately at birth (within 10 minutes) in the delivery room. \\
\hline Bergstrom 2005 & $\begin{array}{l}\text { Participants were normal infants, with mean gestational age at delivery of } 38 \text { weeks in the interven- } \\
\text { tion group and } 38.4 \text { weeks in the control group. Intervention was not provided strictly for preven- } \\
\text { tion of hypothermia immediately at birth. }\end{array}$ \\
\hline Besch 1971 & Participants had a birth weight $>2000$ grams. \\
\hline Bhat 2015 & $\begin{array}{l}\text { Intervention was not applied immediately at birth (within } 10 \text { minutes) in the delivery room. Infants } \\
\text { were of postnatal age } 6 \text { hours to } 28 \text { days. }\end{array}$ \\
\hline Bier 1996 & Intervention was not applied immediately at birth (within 10 minutes) in the delivery room. \\
\hline Boo 2007a & $\begin{array}{l}\text { Intervention was not applied immediately at birth (within } 10 \text { minutes) in the delivery room. No } \\
\text { thermal outcome measures were reported. }\end{array}$ \\
\hline Boo 2007b & $\begin{array}{l}\text { Intervention was not applied immediately at birth (within } 10 \text { minutes) in the delivery room. Partici- } \\
\text { pants were normal term infants undergoing phototherapy. }\end{array}$ \\
\hline Brice 1981 & Intervention was not applied immediately at birth (within 10 minutes) in the delivery room. \\
\hline Bystrova 2003 & Participants had a birth weight $>2500$ grams. \\
\hline Bystrova 2007 & Participants were term. \\
\hline Carfoot 2005 & Participants were term. \\
\hline Cattaneo 1998 & Intervention was not applied immediately at birth (within 10 minutes) in the delivery room. \\
\hline Chaput 1979 & Participants had a birth weight > 2000 grams. \\
\hline Charpak 1997 & $\begin{array}{l}\text { Intervention was not applied immediately at birth (within } 10 \text { minutes) in the delivery room. No } \\
\text { thermal outcome measures were reported. }\end{array}$ \\
\hline Cheah 2000 & Participants were term. \\
\hline Christensson 1992 & Participants were term. \\
\hline Christensson 1995 & Participants were term. \\
\hline Christensson 1996 & Participants were term infants delivered by caesarean section. \\
\hline Christensson 1998 & Intervention was not applied immediately at birth (within 10 minutes) in the delivery room. \\
\hline Chwo 1999 & $\begin{array}{l}\text { Intervention was not applied immediately at birth (within } 10 \text { minutes) in the delivery room but on } \\
\text { the day following birth. }\end{array}$ \\
\hline
\end{tabular}




\begin{tabular}{ll}
\hline Study & Reason for exclusion \\
\hline Chwo 2002 & $\begin{array}{l}\text { Intervention was not applied immediately at birth (within } 10 \text { minutes) in the delivery room but on } \\
\text { the day following birth. }\end{array}$
\end{tabular}

\begin{tabular}{ll}
\hline Coles 1979 & Participants were term. \\
\hline CTRI/2011/11/002125 & $\begin{array}{l}\text { Intervention was not applied immediately at birth (within } 10 \text { minutes) in the delivery room. Mini- } \\
\text { mum participant age was } 6 \text { hours at the time of screening. }\end{array}$ \\
\hline
\end{tabular}

\begin{tabular}{ll}
\hline Dahm 1972 & Participants were term. \\
\hline Darmstadt 2007 & Intervention was not applied immediately at birth (within 10 minutes) in the delivery room. \\
\hline Darmstadt 2008 & $\begin{array}{l}\text { Primary outcome measure was rate of nosocomial infection. Hypothermia was reported as a mor- } \\
\text { bidity for neonates who died. }\end{array}$ \\
\hline Day 1964 & Intervention was not applied immediately at birth (within 10 minutes) in the delivery room. \\
\hline De Ocampo 2012 & $\begin{array}{l}\text { Participants were "stable" low birth weight infants; therefore it is unlikely that kangaroo mother } \\
\text { care was commenced immediately after birth. Trial author was contacted for clarification but no } \\
\text { response was received. }\end{array}$
\end{tabular}

\begin{tabular}{ll}
\hline Dehghani 2015 & $\begin{array}{l}\text { Participants were newborns hospitalised in the neonatal intensive care unit. Mean (SD) weeks' ges- } \\
\text { tation in the intervention group (kangaroo mother care) was } 34.48 \text { (2.42), and in the control group } \\
35.07 \text { (2.4). Intervention was not applied immediately at birth (within } 10 \text { minutes) in the delivery } \\
\text { room. Mean chronological age was approximately } 9 \text { days in both groups. }\end{array}$ \\
\hline
\end{tabular}

\begin{tabular}{ll}
\hline Dodd 2003 & No thermal outcome measures were reported. \\
\hline Duryea 2016 & $\begin{array}{l}\text { This study compared operating room temperature } 23^{\circ} \mathrm{C} \text { (intervention) vs regular operating room } \\
\text { temperature set at } 20^{\circ} \mathrm{C} \text { (control). A minimum operating room temperature of } 25^{\circ} \mathrm{C} \text { is considered } \\
\text { part of routine thermal care as defined by the criteria for considering studies for inclusion in this re- } \\
\text { view. Therefore operating room temperature of } 23^{\circ} \mathrm{C} \text { is not considered an additional intervention. }\end{array}$
\end{tabular}

$\begin{array}{ll}\text { Erlandsson } 2007 & \begin{array}{l}\text { Participants were delivered by caesarean section at term. No thermal outcome measures were re- } \\ \text { ported. }\end{array}\end{array}$

\begin{tabular}{ll}
\hline Fallis 2006 & Participants were term. Intervention was maternal warming during caesarean section. \\
\hline Fardig 1980 & Participants were term. \\
\hline
\end{tabular}

Ferber $2004 \quad$ Participants were term. Intervention was not applied immediately at birth (within 10 minutes) in the delivery room. No thermal outcome measures were reported.
Gabriel $2010 \quad$ Most participants were term singletons. Mean (SD) gestation (weeks): SSC group 38.9 (1.3), control group $39.1(1.2)$

\begin{tabular}{ll}
\hline Gao 2010 & Participants were term. \\
\hline Gathwala 2008 & $\begin{array}{l}\text { Intervention was not applied immediately at birth (within } 10 \text { minutes) in the delivery room. Kan- } \\
\text { garoo mother care was initiated at mean age of } 1.72 \text { days. No thermal outcome measures were re- } \\
\text { ported. }\end{array}$ \\
\hline
\end{tabular}

Ghavane 2012

Intervention was not applied immediately at birth (within 10 minutes) in the delivery room. Mean (SD) age of participants at randomisation was 14.1 (10.3) days vs 13.7 (10.2) days between groups.

Gouchon $2010 \quad$ Participants were term.




\begin{tabular}{ll}
\hline Study & Reason for exclusion \\
\hline Gray 2004 & $\begin{array}{l}\text { Intervention was not applied immediately at birth (within } 10 \text { minutes) in the delivery room. Partici- } \\
\text { pants were at least } 3 \text { days old. }\end{array}$ \\
\hline Green-Abate 1994 & $\begin{array}{l}\text { Intervention was not applied immediately at birth (within } 10 \text { minutes) in the delivery room. Partici- } \\
\text { pants were }<1 \text { week old. }\end{array}$
\end{tabular}

\begin{tabular}{|c|c|}
\hline Greer 1988 & Participants were term with birth weight $>2500$ grams. \\
\hline Grover 1994 & $\begin{array}{l}\text { Participants were term, and the intervention was not applied immediately at birth (within } 10 \text { min- } \\
\text { utes) in the delivery room. Participants were } 11 \text { to } 95 \text { days of age. }\end{array}$ \\
\hline Gulezian 1980 & Insufficient information - unable to obtain a copy of the abstract \\
\hline Harrison 2004 & Insufficient information - unable to obtain a copy of the abstract \\
\hline Heaman 2012 & Insufficient information. Trial author contacted but no response \\
\hline Hellin Martinez 2000 & Participants were term. \\
\hline Hobbs 1975 & Participants were term. \\
\hline Holzman 1985 & Participants were term. \\
\hline Horn 2002 & $\begin{array}{l}\text { Trial author was contacted for verification. Intervention was active warming during caesarean sec- } \\
\text { tion. }\end{array}$ \\
\hline Horn 2014 & $\begin{array}{l}\text { Participants were term infants delivered by elective caesarean section. Intervention was active } \\
\text { (forced-air skin surface) warming during caesarean section and bonding period. }\end{array}$ \\
\hline Hsu 2015 & $\begin{array}{l}\text { Intervention was not applied immediately at birth (within } 10 \text { minutes) in the delivery room. Partici- } \\
\text { pants received standard neonatal care and resuscitation in the delivery suite, and each infant was } \\
\text { transferred to the NICU in a transport incubator. Upon NICU admission, all infants were placed on } \\
\text { a prewarmed radiant warmer with target temperature } 37^{\circ} \mathrm{C} \text {. Intervention (thermal blanket) was ap- } \\
\text { plied at this time. }\end{array}$ \\
\hline
\end{tabular}

\begin{tabular}{ll}
\hline Huang 2002 & Participants had a birth weight $\geq 2500$ grams. \\
\hline Huang 2006 & $\begin{array}{l}\text { Participants were term. Intervention was not applied immediately at birth (within } 10 \text { minutes) in } \\
\text { the delivery room. }\end{array}$ \\
\hline Jia 2012 & $\begin{array}{l}\text { This study compared delivery room temperature } 24^{\circ} \mathrm{C} \text { to } 26^{\circ} \mathrm{C} \text { (intervention) vs regular delivery } \\
\text { room temperature set at } 20^{\circ} \mathrm{C} \text { to } 23^{\circ} \mathrm{C} \text { (control). A minimum delivery room temperature of } 25^{\circ} \mathrm{C} \text { is } \\
\text { considered part of routine thermal care as defined by the criteria for considering studies for inclu- } \\
\text { sion in this review. Therefore a delivery room temperature } 24^{\circ} \mathrm{C} \text { to } 26^{\circ} \mathrm{C} \text { is not considered an addi- } \\
\text { tional intervention. }\end{array}$ \\
\hline Johanson 1992 & $\begin{array}{l}\text { Participants were term with mean gestational age } 39.1 \text { weeks. Randomised controlled intervention } \\
\text { study vs prospective observational study }\end{array}$ \\
\hline Kadam 2005 & $\begin{array}{l}\text { Intervention was not applied immediately at birth (within } 10 \text { minutes) in the delivery room (as veri- } \\
\text { fied by trial author). }\end{array}$ \\
\hline Kaushal 2005 & \begin{tabular}{l} 
Intervention was not applied immediately at birth (within 10 minutes) in the delivery room. \\
\hline Kong 2011
\end{tabular} \\
\hline
\end{tabular}




\section{Study Reason for exclusion}

Kumar 2008

This cluster-randomised controlled efficacy trial looked at 3 community-based interventions that are outside the scope of this review.

Legault 1993

Intervention was not applied immediately at birth (within 10 minutes) in the delivery room. Participants were 1 week of age or older.

\begin{tabular}{ll}
\hline Lim 2004 & Unable to obtain a copy of the abstract \\
\hline Loring 2012 & $\begin{array}{l}\text { Intervention was not applied immediately at birth (within } 10 \text { minutes) in the delivery room. Partici- } \\
\text { pants were between } 24 \text { and } 36 \text { hours old. }\end{array}$ \\
\hline Ludington-Hoe 1994 & $\begin{array}{l}\text { Intervention was not applied immediately at birth (within } 10 \text { minutes) in the delivery room. Partic- } \\
\text { ipants in the intervention group averaged } 18 \text { days old, and control participants averaged } 13 \text { days } \\
\text { old. }\end{array}$
\end{tabular}

Ludington-Hoe $2000 \quad$ Participants had a postnatal age of 6 to 53 days on enrolment into the study; therefore the intervention was not applied immediately at birth (within 10 minutes) in the delivery room.

\begin{tabular}{ll}
\hline Ludington-Hoe 2004 & Intervention was not applied immediately at birth (within 10 minutes) in the delivery room. \\
\hline Marks 1985 & $\begin{array}{l}\text { Participants had a gestational age of } 36 \pm 1 \text { week. Intervention was not applied immediately at } \\
\text { birth (within } 10 \text { minutes) in the delivery room. Participants were } 14 \text { to } 68 \text { days old. }\end{array}$ \\
\hline Mazurek 1999 & Participants were term. \\
\hline Medves 2004 & Participants were term. \\
\hline Meyer 2001 & $\begin{array}{l}\text { Intervention was not applied immediately at birth (within } 10 \text { minutes) in the delivery room. Inter- } \\
\text { vention was applied on arrival to the NICU. }\end{array}$ \\
\hline Meyer 2007 & $\begin{array}{l}\text { This study compared plastic wrap plus radiant warmer vs plastic wrap plus incubator for transport } \\
\text { to the NICU. All infants were wrapped under a radiant warmer at birth, but it is unlikely that assign- } \\
\text { ment to incubator or radiant warmer took place within } 10 \text { minutes of birth for all infants. This com- } \\
\text { parison is not within the scope of this review. }\end{array}$
\end{tabular}

This randomised controlled trial assessed whether the addition of heated humidified gas at deliv-
ery and until neonatal unit arrival improved admission temperature of infants at $<32$ weeks' ges-
tation. On advice from Dr. Roger Soll (Neonatal Review Group), this intervention is considered be-
yond the scope of this review.

\begin{tabular}{ll}
\hline Miles 2006 & Intervention was not applied immediately at birth (within 10 minutes) in the delivery room. No \\
thermal outcome measures were reported.
\end{tabular}

\begin{tabular}{ll}
\hline Monterosso 1999 & $\begin{array}{l}\text { Intervention was not applied immediately at birth (within } 10 \text { minutes) in the delivery room. Partici- } \\
\text { pants were at least } 1 \text { week of age at time of study. }\end{array}$ \\
\hline
\end{tabular}

\begin{tabular}{ll}
\hline Moore 2007 & Participants were term. No thermal outcome measures were reported. \\
\hline Nagai 2010 & Intervention was not applied immediately at birth (within 10 minutes) in the delivery room. \\
\hline Nako 2000 & Participants were term and near term (at $>36$ weeks' gestation and $>2000$ grams birth weight). \\
\hline NCT00531492 & Intervention was not applied immediately at birth (within 10 minutes) in the delivery room. \\
\hline NCT01604421 & Intervention was not applied immediately at birth (within 10 minutes) in the delivery room.
\end{tabular}




\begin{tabular}{ll}
\hline Study & Reason for exclusion \\
\hline NCT01604434 & $\begin{array}{l}\text { Intervention was not applied immediately at birth (within } 10 \text { minutes) in the delivery room. Infants } \\
\text { entered the trial up to } 72 \text { hours after birth. Intervention was applied upon removal of the infant } \\
\text { from the incubator. }\end{array}$ \\
\hline
\end{tabular}

NCT01604447 Intervention was not applied immediately at birth (within 10 minutes) in the delivery room. Infants
entered the trial up to 72 hours after birth. Intervention was applied while infant was being placed
into an incubator.

\begin{tabular}{ll}
\hline NCT01604460 & Participants were term or had a birth weight $>2500$ grams. \\
\hline NCT01776281 & $\begin{array}{l}\text { Intervention was not applied immediately at birth (within } 10 \text { minutes) in the delivery room. Partici- } \\
\text { pants enrolled up to } 1 \text { week after birth. }\end{array}$
\end{tabular}

New 2012

Intervention was not applied immediately at birth (within 10 minutes) in the delivery room. Participants were at least 48 hours old.

Nimbalker 2014

Intervention was not applied immediately at birth (within 10 minutes) in the delivery room. Participants were stable newborns (term and late preterm). Intervention occurred 30 minutes to 1 hour after birth. Mean (SD) time to start skin-to-skin care in the intervention group was 43 (12.5) minutes after birth.

\begin{tabular}{ll} 
Nopper 1996 & $\begin{array}{l}\text { Intervention was not applied immediately at birth (within } 10 \text { minutes) in the delivery room. Partici- } \\
\text { pants enrolled within the first } 96 \text { hours after birth. }\end{array}$ \\
\hline Nuntnarumit 2004 & $\begin{array}{l}\text { This small RCT }(\mathrm{N}=33) \text { comparing polyethylene occlusive skin wrapping and plastic bag vs skin } \\
\text { wrapping alone (for infants at < } 33 \text { weeks' gestation) was originally presented in 2004; to date } \\
(2013), \text { a full manuscript has not been published in a peer-reviewed journal. On this basis, the re- } \\
\text { view team decided to exclude this study. }\end{array}$
\end{tabular}

\begin{tabular}{ll}
\hline Nuntnarumit 2013 & $\begin{array}{l}\text { Intervention was not applied immediately at birth (within } 10 \text { minutes) in the delivery room. This } \\
\text { study focussed on prevention of hypothermia by using a polyethylene plastic sheet to cover the } \\
\text { procedural field for infants requiring umbilical catheterisation. }\end{array}$
\end{tabular}

\begin{tabular}{ll}
\hline Omene 1978 & Participants were term. \\
\hline Pattinson 2005 & $\begin{array}{l}\text { Intervention was an educational package (with/without facilitation visits) on implementation of } \\
\text { kangaroo mother care. }\end{array}$ \\
\hline Punnahitananda 2008 & $\begin{array}{l}\text { This RCT conducted in Thailand comparing polyethylene wrap vs routine care for infants at } \leq 34 \\
\text { weeks' gestation has been excluded from this review update (2013). We were unable to contact the } \\
\text { trial author for further information regarding methodological quality, the definition of hypother- } \\
\text { mia, and data for rectal temperature on admission to the nursery for infants at } 31 \text { to } 34 \text { weeks' ges- } \\
\text { tation. }\end{array}$ \\
\hline
\end{tabular}

\begin{tabular}{ll}
\hline Punthmatharith 2001 & Thermal outcome measures were not reported. \\
\hline Raman 1992 & Participants were term. \\
\hline Ramanathan 2001 & $\begin{array}{l}\text { Intervention was not applied immediately at birth (within } 10 \text { minutes) in the delivery room. Ther- } \\
\text { mal outcome measures were not reported. }\end{array}$ \\
\hline Ramani 2015 & Participants were term (NCT02189750). \\
\hline Roberts 2000 & $\begin{array}{l}\text { Intervention was not applied immediately at birth (within } 10 \text { minutes) in the delivery room. Partici- } \\
\text { pants were born at } \geq 30 \text { weeks' gestation or corrected age. }\end{array}$ \\
\hline
\end{tabular}




\begin{tabular}{|c|c|}
\hline Study & Reason for exclusion \\
\hline Rojas 2001 & $\begin{array}{l}\text { Intervention was not applied immediately at birth (within } 10 \text { minutes) in the delivery room. Ther- } \\
\text { mal outcome measures were not reported. }\end{array}$ \\
\hline Rojas 2003 & $\begin{array}{l}\text { Intervention was not applied immediately at birth (within } 10 \text { minutes) in the delivery room. Ther- } \\
\text { mal outcome measures were not reported. }\end{array}$ \\
\hline Ruiz 1998 & $\begin{array}{l}\text { Intervention was not applied immediately at birth (within } 10 \text { minutes) in the delivery room. Ther- } \\
\text { mal outcome measures were not reported. }\end{array}$ \\
\hline Sankaranarayanan 2005 & Intervention was not applied immediately at birth (within 10 minutes) in the delivery room. \\
\hline Sarman 1989 & Intervention was not applied immediately at birth (within 10 minutes) in the delivery room. \\
\hline Sarman 1992 & $\begin{array}{l}\text { Intervention was not applied immediately at birth (within } 10 \text { minutes) in the delivery room. Partici- } \\
\text { pants were } 3 \text { to } 32 \text { days of age during the study. }\end{array}$ \\
\hline Short 1998 & $\begin{array}{l}\text { Intervention was not applied immediately at birth (within } 10 \text { minutes) in the delivery room. Partici- } \\
\text { pants were at } 32.9 \pm 15.9 \text { days of life. }\end{array}$ \\
\hline Sloan 1994 & $\begin{array}{l}\text { Intervention was not applied immediately at birth (within } 10 \text { minutes) in the delivery room. No } \\
\text { thermal outcome measures were reported. }\end{array}$ \\
\hline Sloan 2008 & $\begin{array}{l}\text { This cluster-randomised controlled trial looked at a community-based intervention by which com- } \\
\text { munity-based workers were taught to teach community-based kangaroo mother care, which is out- } \\
\text { side the scope of this review. }\end{array}$ \\
\hline Srivastava 2014 & Participants were term. \\
\hline St John 2011 & $\begin{array}{l}\text { This RCT ( } \mathrm{N}=130 \text { ), comparing plastic lined stockinette caps vs plain stockinette caps for infants at } \\
24 \text { to } 28 \text { weeks' gestation fulfilled the criteria for inclusion in this review. Additional standardised } \\
\text { thermal care protocols for both groups comprised plastic wrap, warmed blankets, and a sodium } \\
\text { acetate gel mattress. However, secondary outcome data showed that } 22 \% \text { ( } 14 \text { of } 65 \text { ) infants in the } \\
\text { intervention group had hypoglycaemia compared with } 6 \%(4 \text { of } 65 \text { ) in the control group, which rep- } \\
\text { resented a 4-fold increase. Methodological information as provided in the abstract was insufficient, } \\
\text { which could help to account for these findings. Trial authors were contacted for further informa- } \\
\text { tion, and no response was received within the time frame needed for this review; therefore, the re- } \\
\text { view team decided to exclude this study. }\end{array}$ \\
\hline
\end{tabular}

\begin{tabular}{ll}
\hline Stirparo 2013 & Participants were term. \\
\hline Subramanian 2012 & Intervention was not applied immediately at birth (within 10 minutes) in the delivery room. \\
\hline Suman 2008 & Intervention was not applied immediately at birth (within 10 minutes) in the delivery room. \\
\hline Swarnkar 2016 & $\begin{array}{l}\text { Participants were singleton, stable, low birth weight infants. Mean (SD) age (days) in the interven- } \\
\text { tion (kangaroo mother care) and control groups was 3.68 (1.26) and 3.34 (0.54), respectively. }\end{array}$
\end{tabular}

\begin{tabular}{ll}
\hline Syfrett 1993 & Intervention was not applied immediately at birth (within 10 minutes) in the delivery room. \\
\hline Trevisanuto 2009 & $\begin{array}{l}\text { Participants were mainly term infants delivered by caesarean section. Primary outcome measure } \\
\text { was time elapsed from cord clamping to positioning of the infant under the radiant warmer for in- } \\
\text { fants managed via the 'cicogna' system and those who received standard care. }\end{array}$ \\
\hline Tsogt 2005 & $\begin{array}{l}\text { Participants were term. Intervention was not applied immediately at birth (within } 10 \text { minutes) in } \\
\text { the delivery room. }\end{array}$
\end{tabular}




\begin{tabular}{ll}
\hline Study & Reason for exclusion \\
\hline Tsogt 2016 & Participants were healthy term infants (at $\geq 37$ weeks' gestation and birth weight $\geq 2500$ grams). \\
\hline Vaidya 2005 & $\begin{array}{l}\text { Participants were term. Intervention was not applied immediately at birth (within } 10 \text { minutes) in } \\
\text { the delivery room. No thermal outcomes were measured. }\end{array}$ \\
\hline van den Bosch 1990 & Participants were term. \\
\hline van den Bosch 1996 & $\begin{array}{l}\text { Intervention was not applied immediately at birth (within } 10 \text { minutes) in the delivery room. Inter- } \\
\text { vention was applied after 48 hours in the NICU. }\end{array}$ \\
\hline Villalon 1992 & Participants were term. \\
\hline Whitelaw 1988 & $\begin{array}{l}\text { Intervention was not applied immediately at birth (within } 10 \text { minutes) in the delivery room. Infants } \\
\text { entered the trial on average on day 16. No thermal outcomes were measured. }\end{array}$ \\
\hline Worku 2005 & Intervention was not applied immediately at birth (within 10 minutes) in the delivery room. \\
\hline Yeh 1980 & Intervention was not applied immediately at birth (within 10 minutes) in the delivery room. \\
\hline Yentur 2009 & Participants were term infants delivered by elective caesarean section. \\
\hline Yokoyama 2009 & Participants were term infants delivered by elective caesarean section. \\
\hline
\end{tabular}

NICU: neonatal intensive care unit.

$\mathrm{RCT}$ : randomised controlled trial.

SD: standard deviation.

SSC: skin-to-skin care.

Characteristics of studies awaiting assessment [ordered by study ID]

Ahmed 2013

\begin{tabular}{|c|c|}
\hline Methods & $\begin{array}{l}\text { Prospective evaluation of the safety and efficacy of vinyl bags in prevention of hypothermia during } \\
\text { resuscitation at birth in infants of low birth weight at } \leq 32 \text { completed weeks' gestation }\end{array}$ \\
\hline Participants & 50 inborn infants at $\leq 32$ completed weeks' gestation and $<1500$ grams birth weight \\
\hline Interventions & Vinyl bag \\
\hline Outcomes & $\begin{array}{l}\text { Primary outcomes: } \\
\text { - Core body temperature in }{ }^{\circ} \mathrm{C} \text { (axillary and rectal) on admission to NICU } \\
\text { - Core body temperature in }{ }^{\circ} \mathrm{C} \text { (axillary and rectal) } 1 \text { hour after admission to NICU } \\
\text { Secondary outcomes: } \\
\text { - Worst pH and base deficit in the first } 6 \text { hours } \\
\text { - Highest oxygen requirement in first } 24 \text { hours } \\
\text { - Blood glucose at birth and after } 2 \text { hours } \\
\text { - Mortality at } 30 \text { days }\end{array}$ \\
\hline
\end{tabular}


Ahmed 2013 (Continued)

Notes

Discrepencies regarding birth weight (< 1500 grams or $\leq 1500$ grams). Limited methodological information available. 'Randomised' is mentioned once in the abstract but nowhere else in the manuscript.

\section{Castro 2007}

\begin{tabular}{ll}
\hline Methods & $\begin{array}{l}\text { Prospective study following the implementation of an assistance protocol that includes use of } \\
\text { plastic bags in the labour room }\end{array}$
\end{tabular}
plastic bags in the labour room

\begin{tabular}{ll}
\hline Participants & 77 newborn infants of birth weight $<1000$ grams \\
\hline Interventions & Polyethylene bag \\
\hline Outcomes & Primary outcomes: \\
& $\cdot$ Core body temperature in ${ }^{\circ} \mathrm{C}$ (axillary) on admission to NICU \\
& $\cdot$ Hypothermia (axillary temperature $<36^{\circ} \mathrm{C}$ ) on admission to NICU \\
& Secondary outcomes: \\
& $\cdot$ Intraventricular haemorrhage (any and severe) \\
& $\cdot$ Early neonatal death \\
\hline Notes & Insufficient information to assess whether this study is a randomised controlled trial. Contact au- \\
thor email address is no longer valid.
\end{tabular}

Nimbalkar 2015

Methods Randomised controlled trial to determine the efficiency of polythene occlusive skin wrapping vs routine cloth wrapping during intrahospital transportation after birth on incidence of hypothermia in preterm neonates within 24 hours of life

\begin{tabular}{|c|c|}
\hline Participants & Preterm (< 34 weeks' gestation) \\
\hline Interventions & Polyethene occlusive skin wrapping \\
\hline \multirow[t]{4}{*}{ Outcomes } & Primary outcome: \\
\hline & $\begin{array}{l}\text { - Core body temperature in }{ }^{\circ} \mathrm{C} \text { (axillary) after resuscitation and every } 5 \text { minutes in the first hour and } \\
2,3,4,5,6,12 \text {, and } 24 \text { hours of life }\end{array}$ \\
\hline & Secondary outcome: \\
\hline & - Requirement for ventilatory support \\
\hline \multirow[t]{3}{*}{ Notes } & $\begin{array}{l}\text { This is an interim analyses of } 110 \text { participants in the clinical trial CTRI/2015/02/005520 http:// } \\
\text { ctri.nic.in. }\end{array}$ \\
\hline & Final sample size: 150 participants \\
\hline & $\begin{array}{l}\text { This study will be assessed on publication of the full manuscript, which currently is under review } \\
\text { (personal communication with Dr. S.M. Nimbalkar via email } 15 \text { September 2016). }\end{array}$ \\
\hline
\end{tabular}


Shafie 2017

\begin{tabular}{|c|c|}
\hline Methods & $\begin{array}{l}\text { Randomised controlled trial to determine whether polyethylene cap is more effective than cotton } \\
\text { cap as an adjunct to polyethylene occlusive body wrap in reducing hypothermia in preterm infants }\end{array}$ \\
\hline Participants & Preterm (24 to 34 weeks' gestation) \\
\hline Interventions & Polyethylene cap - 'NeoCap' \\
\hline Outcomes & $\begin{array}{l}\text { Primary outcomes: } \\
\text { - Admission hypothermia (axillary temperature }<36.5^{\circ} \mathrm{C} \text { ) } \\
\text { - Poststabilisation hypothermia (axillary temperature }<36.5^{\circ} \mathrm{C} \text { ) after respiratory support, peripher- } \\
\text { al lines, and cardiorespiratory monitor probes have been secured } \\
\text { Secondary outcomes: } \\
\text { - Mortality before } 36 \text { weeks' corrected age or before discharge } \\
\text { - Intraventricular haemorrhage grade II and above } \\
\text { - Necrotising enterocolitis (NEC) stage } 2 \mathrm{~B} \text { and above } \\
\text { - Patent ductus arteriosus (PDA) that required treatment } \\
\text { - Retinopathy of prematurity (ROP) } \\
\text { - Respiratory distress syndrome (RDS) that required exogenous surfactant } \\
\text { - Duration of respiratory assistance } \\
\text { - Hospital stay }\end{array}$ \\
\hline
\end{tabular}

NEC: necrotising enterocolitis.

NICU: neonatal intensive care unit.

PDA: patent ductus arteriosus.

ROP: retinopathy of prematurity.

$\mathrm{RCT}$ : randomised controlled trial.

RDS: respiratory distress syndrome.

Characteristics of ongoing studies [ordered by study ID]

\section{CRTI/2014/11/005200}

Trial name or title EMBRACE vs conventional care during transport of newborn more than or equal to 1500 grams: a randomised controlled trial

\begin{tabular}{ll}
\hline Methods & $\begin{array}{l}\text { Randomised controlled trial (RCT) of parallel design. Participant, investigator, outcome assessor, } \\
\text { and data entry operator blinded }\end{array}$ \\
\hline Participants & Participants are infants of birth weight $\geq 1500$ grams \\
\hline Interventions & Wrapping in EMBRACE compared with conventional care \\
\hline Outcomes & Primary outcome: \\
& $\bullet$ Hypothermia during transport of the neonate
\end{tabular}


CRTI/2014/11/005200 (Continued)

\section{Secondary outcome:}

- Hypothermia-associated morbidity (not specified)

\begin{tabular}{ll}
\hline Starting date & 20 November 2014 \\
\hline Contact information & Dr. Dipen Patel \\
& Associate Professor \\
& Department of Pediatrics \\
& Pramukhswaami Medical College \\
& Haramsad, Annand, Gujarat \\
& 388325, India \\
& Email: dipenvp@charutarhealth.org \\
\hline
\end{tabular}

Notes

\section{CTRI/2016/02/006673}

$\begin{array}{ll}\text { Trial name or title } & \begin{array}{l}\text { An open-label randomised controlled trial comparing standard thermal care with EMBRACE ther- } \\ \text { mopod or polyethylene bag to prevent hypothermia soon after birth in low birth weight babies - } \\ \text { POEMS TRIAL }\end{array}\end{array}$

\begin{tabular}{|c|c|}
\hline Methods & Randomised controlled trial (RCT), parallel-group, multiple-arm, open-label \\
\hline \multirow[t]{7}{*}{ Participants } & $\begin{array}{l}\text { Participants are low birth weight babies weighing } 1500 \text { to } 2500 \text { grams admitted to nursery from de- } \\
\text { livery room }\end{array}$ \\
\hline & Exclusions: \\
\hline & - Infants who require prolonged resuscitation for longer than 10 minutes \\
\hline & $\begin{array}{l}\text { - Major congenital anomalies (e.g. exomphalos major, gastroschisis, congenital diaphragmatic her- } \\
\text { nia) }\end{array}$ \\
\hline & - Exomphalos minor \\
\hline & - Congenital skin disorders (e.g. epidermolysis bullosa, congenital ichthyosis) \\
\hline & - Lack of parental consent \\
\hline \multirow[t]{3}{*}{ Interventions } & Intervention 1: polythene bag \\
\hline & Intervention 2: EMBRACE thermopod infant warmer \\
\hline & Control group: standard thermal care \\
\hline
\end{tabular}

Outcomes

Primary outcome:

- Axillary temperature on admission to the nursery

Secondary outcomes:

- Hypoglycaemia

- Respiratory distress 
CTRI/2016/02/006673 (Continued)

- Coagulopathy

- Sepsis

- Death at the time of nursery admission

\begin{tabular}{ll}
\hline Starting date & $12 / 01 / 2015$ \\
\hline Contact information & Dr. Niranjan Thomas \\
& Department of Neonatology 632004 \\
& Vellore, Tamil NADU \\
& India \\
& Email: niranjan@cmcvellore.ac.in \\
\hline
\end{tabular}

Notes

\section{ISRCTN13184012}

Trial name or title Prevention of hypothermia in the immediate neonatal period in infants with gestational age $\leq 28$ weeks: a comparative study of the effectiveness of two models of polyethylene bags

\begin{tabular}{|c|c|}
\hline Methods & Multi-centre, randomised controlled trial (RCT), parallel-design, open-label \\
\hline Participants & $\begin{array}{l}\text { Participants are newborn infants with gestational age between } 24 \text { weeks ( } 0 / 7 \mathrm{~d}) \text { and } 28 \text { weeks (0/7 } \\
\text { d) according to the best obstetrical estimate. } \\
\text { Exlusion criteria: } \\
\text { - Congenital anomalies not covered by skin (gastroschisis, myelomeningocoele) } \\
\text { - Skin lesions that prevent the skin covering with polyethylene bag } \\
\text { - Deceased in the delivery room }\end{array}$ \\
\hline Interventions & Neohelp bag vs usual, single-wall bags \\
\hline Outcomes & $\begin{array}{l}\text { Primary outcome: } \\
\text { - Skin temperature on admission to NICU } \\
\text { Secondary outcomes: measured until date of hospital discharge } \\
\text { - Metabolic acidosis } \\
\text { - Hypotension } \\
\text { - Hypoglycaemia } \\
\text { - Patent ductus arteriosus } \\
\text { - Necrotising enterocolitis } \\
\text { - Isolated gastrointestinal perforation } \\
\text { - Respiratory distress syndrome } \\
\text { - Pulmonary haemorrhage }\end{array}$ \\
\hline
\end{tabular}


ISRCTN13184012 (Continued)

- Pneumothorax

- Bronchopulmonary dysplasia

- Intraventricular haemorrhage

- Periventricular leukomalacia

- Retinopathy of prematurity

- Late proven blood culture sepsis

- Hospital stay

- Mortality

- Cause of death

\begin{tabular}{ll}
\hline Starting date & 28 March 2016 \\
\hline Contact information & Dr. Eduardo Fernandes \\
& Maternidade Dr. Alfredo Da Costa \\
& Rua Viriato \\
& Lisbon \\
& 1069-089 Lisbon \\
& Portugal \\
Email: no address given
\end{tabular}

Notes

\section{NCT01604317}

\section{Trial name or title}

Randomised evaluation of the use of plastic bags to prevent neonatal hypothermia in developing countries - part 1

\begin{tabular}{ll}
\hline Methods & Randomised controlled trial (RCT) of parallel design, open label \\
\hline Participants & Inborn infants with gestation at 29 to 36 completed weeks or birth weight 1400 to 2500 grams \\
Exclusion criteria: & Infants: \\
$\cdot$ Admitted to NICU \\
$\cdot$ Birth weight $<1400$ grams \\
$\cdot$ Abdominal wall defect or myelomeningocoele \\
$\cdot$ Major congenital anomalies \\
$\cdot$ Blistering skin conditions
\end{tabular}

Interventions

Experimental: infant's torso, upper and lower extremities, and a proportion of the head (face exposed) placed in a plastic bag during resuscitation 
NCT01604317 (Continued)

Active comparator: infant's lower extremities and torso placed in a plastic bag during resuscitation

Outcomes Core body temperature (axillary) $36.5^{\circ} \mathrm{C}$ to $37.5^{\circ} \mathrm{C}$, blood pressure, blood glucose, seizure, weight gain, respiratory distress, bronchopulmonary dysplasia, pneumothorax, sepsis, major brain injury, necrotising enterocolitis, pulmonary haemorrhage, death, hyperthermia, length of time in incubator

\begin{tabular}{ll}
\hline Starting date & June 2012 \\
\hline Contact information & Dr. Waldemar A. Carlo \\
& University of Alabama at Birmingham \\
& 1700 6th Avenue South: 176F Suite 9380 \\
& Birmingham, AL 35249-7335 \\
& USA \\
& Email: wcarlo@peds.uab.edu \\
\hline
\end{tabular}

Notes

NCT02189746

\begin{tabular}{|c|c|}
\hline Trial name or title & Kangaroo mother care to prevent hypothermia in preterm infants \\
\hline Methods & Randomised controlled trial (RCT) of parallel design, open label \\
\hline Participants & $\begin{array}{l}\text { Infants delivered in hospital with an estimated gestational age of } 32 \text { to } 366 / 7 \text { weeks } \\
\text { Exclusion criteria: } \\
\text { - Abdominal wall defect or myelomeningocoele } \\
\text { - Major congenital anomalies } \\
\text { - Blistering skin disorder }\end{array}$ \\
\hline Interventions & $\begin{array}{l}\text { Continuous kangaroo mother care to } 1 \text { hour after birth vs standard kangaroo mother care to } 1 \text { hour } \\
\text { after birth }\end{array}$ \\
\hline Outcomes & Axillary temperature $<36.0^{\circ} \mathrm{C}$ [Time frame: time of birth to 1 hour] \\
\hline Starting date & June 2014 \\
\hline \multirow[t]{6}{*}{ Contact information } & Dr. Waldemar A Carlo \\
\hline & University of Alabama at Birmingham \\
\hline & 1700 6th Avenue South: 176F Suite 9380 \\
\hline & Birmingham, AL 35249-7335 \\
\hline & USA \\
\hline & Email:wcarlo@peds.uab.edu \\
\hline
\end{tabular}

Notes 
NCT02250079

Trial name or title

Polyethylene body bags as an alternative to radiant heat lamp during the neonatal adaptation in infants older than 29 weeks

\begin{tabular}{|c|c|}
\hline Methods & Randomised controlled trial (RCT) of parallel design, open label \\
\hline \multirow[t]{6}{*}{ Participants } & Participants are inborn neonates at $>29$ weeks' gestation \\
\hline & Exclusion criteria: \\
\hline & -Abdominal wall defect \\
\hline & - Myelomeningocoele \\
\hline & - Major congenital anomaly \\
\hline & • Blistering skin disorder \\
\hline Interventions & Polyethylene bag vs conventional treatment \\
\hline \multirow[t]{5}{*}{ Outcomes } & Primary outcome: \\
\hline & - Temperature [Time frame: 10 minutes] \\
\hline & Secondary outcomes: \\
\hline & • Hypothermia [Time frame: 10 minutes] \\
\hline & • Hyperthermia [Time frame: 10 minutes] \\
\hline Starting date & June 2013 \\
\hline Contact information & Responsible party: Universidad Nacional de Colombia. No further information provided \\
\hline Notes & \\
\hline
\end{tabular}

\section{NCT02311972}

\section{Trial name or title}

Temperature monitoring with InnerSense esophogeal temperature sensor/feeding tube after birth through stabilisation in VLBW infants

\begin{tabular}{|c|c|}
\hline Methods & Randomised controlled trial (RCT) of parallel design, open label \\
\hline \multirow[t]{5}{*}{ Participants } & Participants are of very low birth weight (< 1500 grams). \\
\hline & Exclusion criteria: \\
\hline & - Parents did not consent before, or shortly after, delivery \\
\hline & - Major congenital anomalies including gastrointestinal or facial abnormalities \\
\hline & - Major cardiac anomalies \\
\hline Interventions & Philips InnerSense oesophageal temperature sensor/feeding tube vs standard of care feeding tube \\
\hline \multirow[t]{3}{*}{ Outcomes } & Primary outcomes: \\
\hline & - Axillary temperature on admission to NICU \\
\hline & - Axillary temperature at $1,4,8$, and 24 hours of infant life \\
\hline
\end{tabular}


NCT02311972 (Continued)

\section{Secondary outcomes:}

- Hypothermia (axillary temperature $<36.5^{\circ} \mathrm{C}$ ) on admission to NICU

- Hypothermia (axillary temperature $<36.5^{\circ} \mathrm{C}$ ) at $1,4,8$, and 24 hours of infant life

- Relationship of minute-to-minute temperatures, as measured by correlation coefficient within first 24 hours of infant life

- Caregiver satisfaction and ease of use

\begin{tabular}{ll}
\hline Starting date & November 2014 \\
\hline Contact information & Robin B. Dail, PhD, RN, FAAN \\
& Duke University \\
& School of Nursing \& School of Medicine \\
& Department of Pediatrics \\
& Durham, NC 27710 \\
& USA \\
Email@ robin.dail@duke.edu
\end{tabular}

\section{DATA AND ANALYSES}

\section{Comparison 1. Plastic wrap or bag versus routine care}

\begin{tabular}{|c|c|c|c|c|}
\hline Outcome or subgroup title & No. of studies & $\begin{array}{l}\text { No. of partici- } \\
\text { pants }\end{array}$ & Statistical method & Effect size \\
\hline $\begin{array}{l}1 \text { Core body temperature }\left({ }^{\circ} \mathrm{C}\right) \text { on ad- } \\
\text { mission to NICU or up to } 2 \text { hours after } \\
\text { birth }\end{array}$ & 13 & & $\begin{array}{l}\text { Mean Difference (IV, Fixed, } \\
95 \% \mathrm{CI})\end{array}$ & Subtotals only \\
\hline $\begin{array}{l}1.1 \text { All infants (<37 completed weeks' } \\
\text { gestation) }\end{array}$ & 13 & 1633 & $\begin{array}{l}\text { Mean Difference (IV, Fixed, } \\
95 \% \mathrm{CI})\end{array}$ & $0.58[0.50,0.66]$ \\
\hline $1.2<28$ completed weeks' gestation & 8 & 1171 & $\begin{array}{l}\text { Mean Difference (IV, Fixed, } \\
95 \% \mathrm{CI})\end{array}$ & $0.65[0.52,0.79]$ \\
\hline $1.3 \geq 28$ completed weeks' gestation & 4 & 200 & $\begin{array}{l}\text { Mean Difference (IV, Fixed, } \\
95 \% \mathrm{CI})\end{array}$ & $0.56[0.34,0.78]$ \\
\hline $\begin{array}{l}2 \text { Hypothermia on admission to } \mathrm{NICU} \text { : } \\
\text { core body temperature }<36.5^{\circ} \mathrm{C} \text { or } \\
\text { skin temperature }<36^{\circ} \mathrm{C}\end{array}$ & 10 & & $\begin{array}{l}\text { Risk Ratio (M-H, Fixed, 95\% } \\
\mathrm{Cl})\end{array}$ & Subtotals only \\
\hline
\end{tabular}




\begin{tabular}{|c|c|c|c|c|}
\hline Outcome or subgroup title & No. of studies & $\begin{array}{l}\text { No. of partici- } \\
\text { pants }\end{array}$ & Statistical method & Effect size \\
\hline $\begin{array}{l}2.1 \text { All infants (<37 completed weeks' } \\
\text { gestation) }\end{array}$ & 10 & 1417 & $\begin{array}{l}\text { Risk Ratio (M-H, Fixed, 95\% } \\
\mathrm{Cl})\end{array}$ & $0.67[0.62,0.72]$ \\
\hline $2.2<28$ completed weeks' gestation & 6 & 1029 & $\begin{array}{l}\text { Risk Ratio (M-H, Fixed, 95\% } \\
\mathrm{Cl})\end{array}$ & $0.70[0.65,0.77]$ \\
\hline $2.3 \geq 28$ completed weeks' gestation & 2 & 55 & $\begin{array}{l}\text { Risk Ratio (M-H, Fixed, 95\% } \\
\mathrm{Cl})\end{array}$ & $0.17[0.07,0.43]$ \\
\hline $\begin{array}{l}3 \text { Outside normothermic range on ad- } \\
\text { mission to NICU or up to } 2 \text { hours after } \\
\text { birth }\end{array}$ & 5 & & $\begin{array}{l}\text { Risk Ratio (M-H, Fixed, 95\% } \\
\mathrm{Cl})\end{array}$ & Subtotals only \\
\hline $\begin{array}{l}\text { 3.1 All infants (< } 37 \text { completed weeks' } \\
\text { gestation) }\end{array}$ & 5 & 1048 & $\begin{array}{l}\text { Risk Ratio (M-H, Fixed, 95\% } \\
\mathrm{Cl})\end{array}$ & $0.75[0.69,0.81]$ \\
\hline $3.2<28$ completed weeks' gestation & 3 & 871 & $\begin{array}{l}\text { Risk Ratio (M-H, Fixed, 95\% } \\
\mathrm{Cl})\end{array}$ & $0.81[0.75,0.88]$ \\
\hline $3.3 \geq 28$ completed weeks' gestation & 1 & 33 & $\begin{array}{l}\text { Risk Ratio (M-H, Fixed, 95\% } \\
\mathrm{Cl})\end{array}$ & $0.14[0.04,0.52]$ \\
\hline $\begin{array}{l}4 \text { Core body temperature }\left({ }^{\circ} \mathrm{C}\right) \text { at } 1 \\
\text { hour after birth }\end{array}$ & 1 & 60 & $\begin{array}{l}\text { Mean Difference (IV, Fixed, } \\
95 \% \mathrm{CI})\end{array}$ & $0.40[0.19,0.61]$ \\
\hline $\begin{array}{l}4.1 \text { All infants (< } 37 \text { completed weeks' } \\
\text { gestation) }\end{array}$ & 1 & 60 & $\begin{array}{l}\text { Mean Difference (IV, Fixed, } \\
95 \% \mathrm{Cl} \text { ) }\end{array}$ & $0.40[0.19,0.61]$ \\
\hline $\begin{array}{l}5 \text { Core body temperature }\left({ }^{\circ} \mathrm{C}\right) \text { at } 90 \\
\text { minutes after birth }\end{array}$ & 1 & 60 & $\begin{array}{l}\text { Mean Difference (IV, Fixed, } \\
95 \% \mathrm{Cl})\end{array}$ & $0.40[0.18,0.62]$ \\
\hline $\begin{array}{l}5.1 \text { All infants (< } 37 \text { completed weeks' } \\
\text { gestation) }\end{array}$ & 1 & 60 & $\begin{array}{l}\text { Mean Difference (IV, Fixed, } \\
95 \% \mathrm{Cl})\end{array}$ & $0.40[0.18,0.62]$ \\
\hline $\begin{array}{l}6 \text { Core body temperature }\left({ }^{\circ} \mathrm{C}\right) \text { at } 2 \\
\text { hours after birth }\end{array}$ & 1 & 60 & $\begin{array}{l}\text { Mean Difference (IV, Fixed, } \\
95 \% \mathrm{Cl})\end{array}$ & $0.30[0.13,0.47]$ \\
\hline $\begin{array}{l}6.1 \text { All infants (< } 37 \text { completed weeks' } \\
\text { gestation) }\end{array}$ & 1 & 60 & $\begin{array}{l}\text { Mean Difference (IV, Fixed, } \\
95 \% \mathrm{Cl} \text { ) }\end{array}$ & $0.30[0.13,0.47]$ \\
\hline $\begin{array}{l}7 \text { Core body temperature }\left({ }^{\circ} \mathrm{C}\right) \text { post } \\
\text { stabilisation }\end{array}$ & 2 & & $\begin{array}{l}\text { Mean Difference (IV, Fixed, } \\
95 \% \mathrm{Cl} \text { ) }\end{array}$ & Subtotals only \\
\hline $\begin{array}{l}7.1 \text { All infants (< } 37 \text { completed weeks' } \\
\text { gestation) }\end{array}$ & 2 & 911 & $\begin{array}{l}\text { Mean Difference (IV, Fixed, } \\
95 \% \mathrm{Cl})\end{array}$ & $0.43[0.22,0.64]$ \\
\hline $7.2<28$ completed weeks' gestation & 2 & 838 & $\begin{array}{l}\text { Mean Difference (IV, Fixed, } \\
95 \% \mathrm{Cl})\end{array}$ & $0.44[0.20,0.67]$ \\
\hline $7.3 \geq 28$ completed weeks' gestation & 1 & 73 & $\begin{array}{l}\text { Mean Difference (IV, Fixed, } \\
95 \% \mathrm{Cl})\end{array}$ & $0.40[-0.00,0.80]$ \\
\hline $\begin{array}{l}8 \text { Hypothermia post stabilisation: } \\
\text { core body temperature }<36.5^{\circ} \mathrm{C} \text { or } \\
\text { skin temperature }<36^{\circ} \mathrm{C}\end{array}$ & 2 & 841 & $\begin{array}{l}\text { Risk Ratio (M-H, Fixed, 95\% } \\
\mathrm{Cl})\end{array}$ & $0.76[0.65,0.88]$ \\
\hline
\end{tabular}




\begin{tabular}{|c|c|c|c|c|}
\hline Outcome or subgroup title & No. of studies & $\begin{array}{l}\text { No. of partici- } \\
\text { pants }\end{array}$ & Statistical method & Effect size \\
\hline $\begin{array}{l}8.1 \text { All infants ( } \leq 32 \text { completed weeks' } \\
\text { gestation) }\end{array}$ & 1 & 40 & $\begin{array}{l}\text { Risk Ratio (M-H, Fixed, 95\% } \\
\mathrm{Cl} \text { ) }\end{array}$ & $0.05[0.00,0.76]$ \\
\hline $8.2<28$ completed weeks' gestation & 1 & 801 & $\begin{array}{l}\text { Risk Ratio (M-H, Fixed, 95\% } \\
\mathrm{Cl})\end{array}$ & $0.79[0.69,0.92]$ \\
\hline $\begin{array}{l}9 \text { Outside normothermic range post } \\
\text { stabilisation }\end{array}$ & 2 & 841 & $\begin{array}{l}\text { Risk Ratio (M-H, Fixed, 95\% } \\
\mathrm{Cl})\end{array}$ & $0.88[0.78,1.00]$ \\
\hline $\begin{array}{l}9.1 \text { All infants ( } \leq 32 \text { completed weeks' } \\
\text { gestation) }\end{array}$ & 1 & 40 & $\begin{array}{l}\text { Risk Ratio (M-H, Fixed, 95\% } \\
\mathrm{Cl})\end{array}$ & $0.1[0.01,0.71]$ \\
\hline $9.2<28$ completed weeks' gestation & 1 & 801 & $\begin{array}{l}\text { Risk Ratio (M-H, Fixed, 95\% } \\
\mathrm{Cl})\end{array}$ & $0.92[0.81,1.04]$ \\
\hline $\begin{array}{l}10 \text { Core body temperature }\left({ }^{\circ} \mathrm{C}\right) 30 \\
\text { minutes after initial NICU admission } \\
\text { temperature was taken }\end{array}$ & 1 & 92 & $\begin{array}{l}\text { Mean Difference (IV, Fixed, } \\
95 \% \mathrm{CI})\end{array}$ & $0.57[0.27,0.87]$ \\
\hline $10.1 \leq 29$ completed weeks' gestation & 1 & 92 & $\begin{array}{l}\text { Mean Difference (IV, Fixed, } \\
95 \% \mathrm{CI})\end{array}$ & $0.57[0.27,0.87]$ \\
\hline $\begin{array}{l}11 \text { Core body temperature }\left({ }^{\circ} \mathrm{C}\right) 1 \text { hour } \\
\text { after initial NICU admission tempera- } \\
\text { ture was taken }\end{array}$ & 6 & & $\begin{array}{l}\text { Mean Difference (IV, Fixed, } \\
95 \% \mathrm{CI})\end{array}$ & Subtotals only \\
\hline $\begin{array}{l}11.1 \text { All infants (<37 completed } \\
\text { weeks' gestation) }\end{array}$ & 6 & 373 & $\begin{array}{l}\text { Mean Difference (IV, Fixed, } \\
95 \% \mathrm{CI})\end{array}$ & $0.36[0.25,0.47]$ \\
\hline $11.2<28$ completed weeks' gestation & 4 & 227 & $\begin{array}{l}\text { Mean Difference (IV, Fixed, } \\
95 \% \mathrm{CI})\end{array}$ & $0.49[0.33,0.66]$ \\
\hline $11.3 \geq 28$ completed weeks' gestation & 2 & 86 & $\begin{array}{l}\text { Mean Difference (IV, Fixed, } \\
95 \% \mathrm{CI})\end{array}$ & $0.72[0.48,0.96]$ \\
\hline $\begin{array}{l}12 \text { Core body temperature }\left({ }^{\circ} \mathrm{C}\right) 90 \\
\text { minutes after initial NICU admission } \\
\text { temperature was taken }\end{array}$ & 1 & 92 & $\begin{array}{l}\text { Mean Difference (IV, Fixed, } \\
95 \% \mathrm{CI})\end{array}$ & $0.56[0.27,0.85]$ \\
\hline $12.1 \leq 29$ completed weeks' gestation & 1 & 92 & $\begin{array}{l}\text { Mean Difference (IV, Fixed, } \\
95 \% \mathrm{CI})\end{array}$ & $0.56[0.27,0.85]$ \\
\hline $\begin{array}{l}13 \text { Core body temperature }\left({ }^{\circ} \mathrm{C}\right) 2 \\
\text { hours after initial NICU admission } \\
\text { temperature was taken }\end{array}$ & 2 & 156 & $\begin{array}{l}\text { Mean Difference (IV, Fixed, } \\
95 \% \mathrm{CI})\end{array}$ & $0.37[0.16,0.59]$ \\
\hline $13.1<28$ completed weeks' gestation & 1 & 92 & $\begin{array}{l}\text { Mean Difference (IV, Fixed, } \\
95 \% \mathrm{CI})\end{array}$ & $0.28[0.04,0.52]$ \\
\hline $13.2 \geq 28$ completed weeks' gestation & 1 & 64 & $\begin{array}{l}\text { Mean Difference (IV, Fixed, } \\
95 \% \mathrm{CI})\end{array}$ & $0.74[0.26,1.22]$ \\
\hline $\begin{array}{l}14 \text { Hyperthermia on admission to } \\
\text { NICU: core body temperature }>37.5^{\circ} \mathrm{C}\end{array}$ & 12 & & $\begin{array}{l}\text { Risk Ratio (M-H, Fixed, 95\% } \\
\mathrm{Cl} \text { ) }\end{array}$ & Subtotals only \\
\hline
\end{tabular}




\begin{tabular}{|c|c|c|c|c|}
\hline Outcome or subgroup title & No. of studies & $\begin{array}{l}\text { No. of partici- } \\
\text { pants }\end{array}$ & Statistical method & Effect size \\
\hline $\begin{array}{l}\text { 14.1 All infants (< } 37 \text { completed } \\
\text { weeks' gestation) }\end{array}$ & 12 & 1523 & $\begin{array}{l}\text { Risk Ratio (M-H, Fixed, 95\% } \\
\mathrm{Cl} \text { ) }\end{array}$ & $3.91[2.05,7.44]$ \\
\hline $14.2<28$ completed weeks' gestation & 7 & 1121 & $\begin{array}{l}\text { Risk Ratio (M-H, Fixed, 95\% } \\
\mathrm{Cl} \text { ) }\end{array}$ & $4.00[2.04,7.83]$ \\
\hline $14.3 \geq 28$ completed weeks' gestation & 3 & 138 & $\begin{array}{l}\text { Risk Ratio (M-H, Fixed, 95\% } \\
\mathrm{Cl})\end{array}$ & $0.0[0.0,0.0]$ \\
\hline $\begin{array}{l}15 \text { Hyperthermia post stabilisation: } \\
\text { core body temperature } \geq 37.5^{\circ} \mathrm{C}\end{array}$ & 1 & 801 & $\begin{array}{l}\text { Risk Ratio (M-H, Fixed, 95\% } \\
\text { Cl) }\end{array}$ & $2.35[1.38,4.00]$ \\
\hline $\begin{array}{l}15.1 \geq 24 \text { and }<28 \text { completed weeks' } \\
\text { gestation }\end{array}$ & 1 & 801 & $\begin{array}{l}\text { Risk Ratio (M-H, Fixed, 95\% } \\
\text { Cl) }\end{array}$ & $2.35[1.38,4.00]$ \\
\hline 16 Major brain injury & 5 & 1100 & $\begin{array}{l}\text { Risk Ratio (M-H, Fixed, 95\% } \\
\text { Cl) }\end{array}$ & $0.78[0.47,1.27]$ \\
\hline $\begin{array}{l}16.1 \text { All infants ( } \leq 33 \text { completed } \\
\text { weeks' gestation) }\end{array}$ & 1 & 110 & $\begin{array}{l}\text { Risk Ratio (M-H, Fixed, 95\% } \\
\text { Cl) }\end{array}$ & $0.3[0.03,2.60]$ \\
\hline $16.2<28$ completed weeks' gestation & 4 & 990 & $\begin{array}{l}\text { Risk Ratio (M-H, Fixed, 95\% } \\
\mathrm{Cl} \text { ) }\end{array}$ & $0.83[0.50,1.39]$ \\
\hline $\begin{array}{l}17 \text { Intraventicular haemorrhage (all } \\
\text { grades) }\end{array}$ & 3 & 880 & $\begin{array}{l}\text { Risk Ratio (M-H, Fixed, 95\% } \\
\text { Cl) }\end{array}$ & $0.92[0.77,1.09]$ \\
\hline $17.1<28$ completed weeks' gestation & 3 & 880 & $\begin{array}{l}\text { Risk Ratio (M-H, Fixed, 95\% } \\
\text { Cl) }\end{array}$ & $0.92[0.77,1.09]$ \\
\hline $\begin{array}{l}18 \text { Intraventricular haemorrhage } \\
\text { (grades III and IV) }\end{array}$ & 1 & 753 & $\begin{array}{l}\text { Risk Ratio (M-H, Fixed, 95\% } \\
\text { Cl) }\end{array}$ & $0.99[0.67,1.46]$ \\
\hline $\begin{array}{l}18.1 \geq 24 \text { and }<28 \text { completed weeks' } \\
\text { gestation }\end{array}$ & 1 & 753 & $\begin{array}{l}\text { Risk Ratio (M-H, Fixed, 95\% } \\
\mathrm{Cl})\end{array}$ & $0.99[0.67,1.46]$ \\
\hline $\begin{array}{l}19 \text { Mortality (death within hospital } \\
\text { stay or at } 6 \text { months' corrected gesta- } \\
\text { tion) }\end{array}$ & 10 & & $\begin{array}{l}\text { Risk Ratio (M-H, Fixed, 95\% } \\
\mathrm{Cl})\end{array}$ & Subtotals only \\
\hline $\begin{array}{l}19.1 \text { All infants (< } 37 \text { weeks' gesta- } \\
\text { tion) }\end{array}$ & 10 & 1447 & $\begin{array}{l}\text { Risk Ratio (M-H, Fixed, 95\% } \\
\mathrm{Cl})\end{array}$ & $0.91[0.73,1.15]$ \\
\hline $19.2<28$ completed weeks' gestation & 6 & 1114 & $\begin{array}{l}\text { Risk Ratio (M-H, Fixed, 95\% } \\
\mathrm{Cl})\end{array}$ & $0.92[0.72,1.18]$ \\
\hline $19.3 \geq 28$ completed weeks' gestation & 1 & 41 & $\begin{array}{l}\text { Risk Ratio (M-H, Fixed, 95\% } \\
\mathrm{Cl})\end{array}$ & $0.0[0.0,0.0]$ \\
\hline $\begin{array}{l}20 \text { Arterial oxygen saturation (per- } \\
\text { centage) }\end{array}$ & 1 & 40 & $\begin{array}{l}\text { Mean Difference (IV, Fixed, } \\
95 \% \mathrm{Cl})\end{array}$ & $-4.10[-14.00,7.80]$ \\
\hline $20.1 \leq 32$ completed weeks' gestation & 1 & 40 & $\begin{array}{l}\text { Mean Difference (IV, Fixed, } \\
95 \% \mathrm{Cl} \text { ) }\end{array}$ & $-4.10[-14.00,7.80]$ \\
\hline
\end{tabular}




\begin{tabular}{|c|c|c|c|c|}
\hline Outcome or subgroup title & No. of studies & $\begin{array}{l}\text { No. of partici- } \\
\text { pants }\end{array}$ & Statistical method & Effect size \\
\hline 21 Bicarbonate (mmol/L) & 2 & 117 & $\begin{array}{l}\text { Mean Difference (IV, Fixed, } \\
95 \% \mathrm{Cl} \text { ) }\end{array}$ & $0.31[-0.72,1.35]$ \\
\hline $21.1<28$ completed weeks' gestation & 2 & 117 & $\begin{array}{l}\text { Mean Difference (IV, Fixed, } \\
95 \% \mathrm{Cl} \text { ) }\end{array}$ & $0.31[-0.72,1.35]$ \\
\hline 22 Blood gas pH (first) & 2 & 117 & $\begin{array}{l}\text { Mean Difference (IV, Fixed, } \\
95 \% \mathrm{Cl} \text { ) }\end{array}$ & $0.01[-0.02,0.04]$ \\
\hline $22.1<28$ completed weeks' gestation & 2 & 117 & $\begin{array}{l}\text { Mean Difference (IV, Fixed, } \\
95 \% \mathrm{CI})\end{array}$ & $0.01[-0.02,0.04]$ \\
\hline 23 Blood gas $\mathrm{pH}<7.25$ & 1 & 84 & $\begin{array}{l}\text { Risk Ratio (M-H, Fixed, 95\% } \\
\mathrm{Cl})\end{array}$ & $0.81[0.55,1.20]$ \\
\hline $23.1 \leq 29$ completed weeks' gestation & 1 & 84 & $\begin{array}{l}\text { Risk Ratio (M-H, Fixed, 95\% } \\
\mathrm{Cl})\end{array}$ & $0.81[0.55,1.20]$ \\
\hline $\begin{array}{l}24 \text { Blood glucose concentration } \\
\text { (mmol/L) (first) }\end{array}$ & 4 & 195 & $\begin{array}{l}\text { Mean Difference (IV, Fixed, } \\
95 \% \mathrm{CI})\end{array}$ & $-0.14[-0.50,0.21]$ \\
\hline $\begin{array}{l}24.1 \text { All infants ( } \leq 32 \text { completed } \\
\text { weeks' gestation) }\end{array}$ & 2 & 78 & $\begin{array}{l}\text { Mean Difference (IV, Fixed, } \\
95 \% \mathrm{CI})\end{array}$ & $-0.62[-1.37,0.12]$ \\
\hline $24.2<28$ completed weeks' gestation & 2 & 117 & $\begin{array}{l}\text { Mean Difference (IV, Fixed, } \\
95 \% \mathrm{CI})\end{array}$ & $-0.00[-0.41,0.41]$ \\
\hline 25 Blood glucose $<2.6 \mathrm{mmol} / \mathrm{L}$ & 1 & 85 & $\begin{array}{l}\text { Risk Ratio (M-H, Fixed, 95\% } \\
\mathrm{Cl})\end{array}$ & $0.66[0.31,1.43]$ \\
\hline $25.1 \leq 29$ completed weeks' gestation & 1 & 85 & $\begin{array}{l}\text { Risk Ratio (M-H, Fixed, } 95 \% \\
\mathrm{Cl})\end{array}$ & $0.66[0.31,1.43]$ \\
\hline 26 Blood glucose $>6 \mathrm{mmol} / \mathrm{L}$ & 1 & 85 & $\begin{array}{l}\text { Risk Ratio (M-H, Fixed, 95\% } \\
\mathrm{Cl})\end{array}$ & $1.07[0.23,5.02]$ \\
\hline $26.1 \leq 29$ completed weeks' gestation & 1 & 85 & $\begin{array}{l}\text { Risk Ratio (M-H, Fixed, 95\% } \\
\mathrm{Cl})\end{array}$ & $1.07[0.23,5.02]$ \\
\hline $\begin{array}{l}27 \text { Blood glucose concentration } \\
\mathrm{mmol} / \mathrm{L} \text { at } 120 \text { minutes after birth }\end{array}$ & & & Other data & No numeric data \\
\hline $\begin{array}{l}27.1 \text { All infants (< } 37 \text { completed } \\
\text { weeks' gestation) }\end{array}$ & & & Other data & No numeric data \\
\hline $\begin{array}{l}28 \text { Bronchopulmonary dysplasia } \\
\text { (BPD) }\end{array}$ & 1 & 702 & $\begin{array}{l}\text { Risk Ratio (M-H, Fixed, 95\% } \\
\text { Cl) }\end{array}$ & $0.99[0.86,1.15]$ \\
\hline $\begin{array}{l}28.1 \geq 24 \text { and }<28 \text { completed weeks' } \\
\text { gestation }\end{array}$ & 1 & 702 & $\begin{array}{l}\text { Risk Ratio (M-H, Fixed, 95\% } \\
\mathrm{Cl})\end{array}$ & $0.99[0.86,1.15]$ \\
\hline 29 BPD steroids & 1 & 796 & $\begin{array}{l}\text { Risk Ratio (M-H, Fixed, 95\% } \\
\mathrm{Cl} \text { ) }\end{array}$ & $0.94[0.71,1.25]$ \\
\hline
\end{tabular}




\begin{tabular}{|c|c|c|c|c|}
\hline Outcome or subgroup title & No. of studies & $\begin{array}{l}\text { No. of partici- } \\
\text { pants }\end{array}$ & Statistical method & Effect size \\
\hline $\begin{array}{l}29.1 \geq 24 \text { and }<28 \text { completed weeks' } \\
\text { gestation }\end{array}$ & 1 & 796 & $\begin{array}{l}\text { Risk Ratio (M-H, Fixed, 95\% } \\
\mathrm{Cl})\end{array}$ & $0.94[0.71,1.25]$ \\
\hline 30 Duration of hospitalisation (days) & 2 & 126 & $\begin{array}{l}\text { Mean Difference (IV, Fixed, } \\
95 \% \mathrm{CI})\end{array}$ & $-6.35[-17.27,4.56]$ \\
\hline $\begin{array}{l}30.1 \text { All infants ( } \leq 32 \text { completed } \\
\text { weeks' gestation) }\end{array}$ & 1 & 38 & $\begin{array}{l}\text { Mean Difference (IV, Fixed, } \\
95 \% \mathrm{CI})\end{array}$ & $-7.50[-24.17,9.17]$ \\
\hline $30.2<28$ completed weeks' gestation & 1 & 88 & $\begin{array}{l}\text { Mean Difference (IV, Fixed, } \\
95 \% \mathrm{CI})\end{array}$ & $-5.49[-19.93,8.95]$ \\
\hline 31 Duration of hospitalisation (days) & & & Other data & No numeric data \\
\hline $\begin{array}{l}32 \text { Duration of continuous positive } \\
\text { airway pressure (CPAP) (days) }\end{array}$ & & & Other data & No numeric data \\
\hline 33 Duration of oxygen therapy (days) & 1 & 88 & $\begin{array}{l}\text { Mean Difference (IV, Fixed, } \\
95 \% \mathrm{CI})\end{array}$ & $\begin{array}{l}-6.51[-23.30 \\
10.28]\end{array}$ \\
\hline $33.1<29$ completed weeks' gestation & 1 & 88 & $\begin{array}{l}\text { Mean Difference (IV, Fixed, } \\
95 \% \mathrm{CI})\end{array}$ & $\begin{array}{l}-6.51[-23.30 \\
10.28]\end{array}$ \\
\hline 34 Duration of ventilation (days) & & & Other data & No numeric data \\
\hline 35 Gastrointestinal perforation & 1 & 795 & $\begin{array}{l}\text { Risk Ratio (M-H, Fixed, 95\% } \\
\mathrm{Cl})\end{array}$ & $1.25[0.68,2.28]$ \\
\hline $\begin{array}{l}35.1 \geq 24 \text { and }<28 \text { completed weeks' } \\
\text { gestation }\end{array}$ & 1 & 795 & $\begin{array}{l}\text { Risk Ratio (M-H, Fixed, 95\% } \\
\mathrm{Cl})\end{array}$ & $1.25[0.68,2.28]$ \\
\hline 36 Intubation in delivery room & 1 & 64 & $\begin{array}{l}\text { Risk Ratio (M-H, Fixed, 95\% } \\
\text { Cl) }\end{array}$ & $1.0[0.63,1.58]$ \\
\hline $36.1<29$ completed weeks' gestation & 1 & 64 & $\begin{array}{l}\text { Risk Ratio (M-H, Fixed, 95\% } \\
\mathrm{Cl})\end{array}$ & $1.0[0.63,1.58]$ \\
\hline 37 Necrotising enterocolitis (NEC) & 2 & 907 & $\begin{array}{l}\text { Risk Ratio (M-H, Fixed, 95\% } \\
\mathrm{Cl})\end{array}$ & $1.01[0.65,1.58]$ \\
\hline $\begin{array}{l}37.1 \text { All infants ( } \geq 24 \text { and } \leq 33 \text { com- } \\
\text { pleted weeks' gestation) }\end{array}$ & 1 & 110 & $\begin{array}{l}\text { Risk Ratio (M-H, Fixed, 95\% } \\
\text { Cl) }\end{array}$ & $5.98[0.29,121.75]$ \\
\hline $\begin{array}{l}37.2 \geq 24 \text { and }<28 \text { completed weeks' } \\
\text { gestation }\end{array}$ & 1 & 797 & $\begin{array}{l}\text { Risk Ratio (M-H, Fixed, 95\% } \\
\mathrm{Cl})\end{array}$ & $0.95[0.60,1.49]$ \\
\hline 38 Patent ductus arteriosus (PDA) & 2 & 905 & $\begin{array}{l}\text { Risk Ratio (M-H, Fixed, 95\% } \\
\mathrm{Cl})\end{array}$ & $0.90[0.78,1.03]$ \\
\hline $\begin{array}{l}38.1 \text { All infants ( } \geq 24 \text { and } \leq 33 \text { com- } \\
\text { pleted weeks' gestation) }\end{array}$ & 1 & 110 & $\begin{array}{l}\text { Risk Ratio (M-H, Fixed, 95\% } \\
\text { Cl) }\end{array}$ & $0.8[0.39,1.62]$ \\
\hline $\begin{array}{l}38.2 \geq 24 \text { and }<28 \text { completed weeks' } \\
\text { gestation }\end{array}$ & 1 & 795 & $\begin{array}{l}\text { Risk Ratio (M-H, Fixed, 95\% } \\
\mathrm{Cl})\end{array}$ & $0.90[0.79,1.04]$ \\
\hline
\end{tabular}




\begin{tabular}{|c|c|c|c|c|}
\hline Outcome or subgroup title & No. of studies & $\begin{array}{l}\text { No. of partici- } \\
\text { pants }\end{array}$ & Statistical method & Effect size \\
\hline 39 Pneumothorax & 1 & 796 & $\begin{array}{l}\text { Risk Ratio (M-H, Fixed, 95\% } \\
\mathrm{Cl})\end{array}$ & $0.98[0.56,1.69]$ \\
\hline $\begin{array}{l}39.1 \geq 24 \text { and }<28 \text { completed weeks' } \\
\text { gestation }\end{array}$ & 1 & 796 & $\begin{array}{l}\text { Risk Ratio (M-H, Fixed, 95\% } \\
\mathrm{Cl} \text { ) }\end{array}$ & $0.98[0.56,1.69]$ \\
\hline 40 Pulmonary haemorrhage & 1 & 796 & $\begin{array}{l}\text { Risk Ratio (M-H, Fixed, 95\% } \\
\mathrm{Cl} \text { ) }\end{array}$ & $0.60[0.38,0.95]$ \\
\hline $\begin{array}{l}40.1 \geq 24 \text { and }<28 \text { completed weeks' } \\
\text { gestation }\end{array}$ & 1 & 796 & $\begin{array}{l}\text { Risk Ratio (M-H, Fixed, 95\% } \\
\mathrm{Cl})\end{array}$ & $0.60[0.38,0.95]$ \\
\hline $\begin{array}{l}41 \text { Requirement for bubble continu- } \\
\text { ous positive airway pressure (BCPAP) }\end{array}$ & 1 & 92 & $\begin{array}{l}\text { Risk Ratio (M-H, Fixed, 95\% } \\
\mathrm{Cl})\end{array}$ & $0.97[0.81,1.18]$ \\
\hline $41.1 \leq 29$ completed weeks' gestation & 1 & 92 & $\begin{array}{l}\text { Risk Ratio (M-H, Fixed, 95\% } \\
\mathrm{Cl})\end{array}$ & $0.97[0.81,1.18]$ \\
\hline 42 Requirement for ventilation & 1 & 92 & $\begin{array}{l}\text { Risk Ratio (M-H, Fixed, 95\% } \\
\mathrm{Cl})\end{array}$ & $0.92[0.70,1.20]$ \\
\hline $42.1 \leq 29$ completed weeks' gestation & 1 & 92 & $\begin{array}{l}\text { Risk Ratio (M-H, Fixed, 95\% } \\
\mathrm{Cl})\end{array}$ & $0.92[0.70,1.20]$ \\
\hline $\begin{array}{l}43 \text { Respiratory distress syndrome } \\
\text { (RDS) }\end{array}$ & 2 & 910 & $\begin{array}{l}\text { Risk Ratio (M-H, Fixed, 95\% } \\
\mathrm{Cl})\end{array}$ & $1.01[0.97,1.06]$ \\
\hline $\begin{array}{l}43.1 \text { All infants ( } \geq 24 \text { and } \leq 33 \text { com- } \\
\text { pleted weeks' gestation) }\end{array}$ & 1 & 110 & $\begin{array}{l}\text { Risk Ratio (M-H, Fixed, 95\% } \\
\mathrm{Cl})\end{array}$ & $1.04[0.78,1.38]$ \\
\hline $\begin{array}{l}43.2 \geq 24 \text { and }<28 \text { completed weeks' } \\
\text { gestation }\end{array}$ & 1 & 800 & $\begin{array}{l}\text { Risk Ratio (M-H, Fixed, 95\% } \\
\mathrm{Cl})\end{array}$ & $1.01[0.97,1.05]$ \\
\hline 44 Retinopathy of prematurity (ROP) & 1 & 606 & $\begin{array}{l}\text { Risk Ratio (M-H, Fixed, 95\% } \\
\mathrm{Cl})\end{array}$ & $1.00[0.72,1.40]$ \\
\hline $\begin{array}{l}44.1 \geq 24 \text { and }<28 \text { completed weeks' } \\
\text { gestation }\end{array}$ & 1 & 606 & $\begin{array}{l}\text { Risk Ratio (M-H, Fixed, 95\% } \\
\mathrm{Cl})\end{array}$ & $1.00[0.72,1.40]$ \\
\hline 45 Sepsis (late) & 2 & 830 & $\begin{array}{l}\text { Risk Ratio (M-H, Fixed, 95\% } \\
\mathrm{Cl})\end{array}$ & $0.88[0.70,1.10]$ \\
\hline $45.1 \leq 29$ completed weeks' gestation & 2 & 830 & $\begin{array}{l}\text { Risk Ratio (M-H, Fixed, 95\% } \\
\mathrm{Cl})\end{array}$ & $0.88[0.70,1.10]$ \\
\hline 46 Sepsis (early) & 2 & 883 & $\begin{array}{l}\text { Risk Ratio (M-H, Fixed, 95\% } \\
\mathrm{Cl})\end{array}$ & $0.69[0.38,1.29]$ \\
\hline $46.1 \leq 29$ completed weeks' gestation & 2 & 883 & $\begin{array}{l}\text { Risk Ratio (M-H, Fixed, 95\% } \\
\mathrm{Cl})\end{array}$ & $0.69[0.38,1.29]$ \\
\hline
\end{tabular}


Analysis 1.1. Comparison 1 Plastic wrap or bag versus routine care, Outcome 1 Core body temperature $\left({ }^{\circ} \mathrm{C}\right)$ on admission to NICU or up to 2 hours after birth.

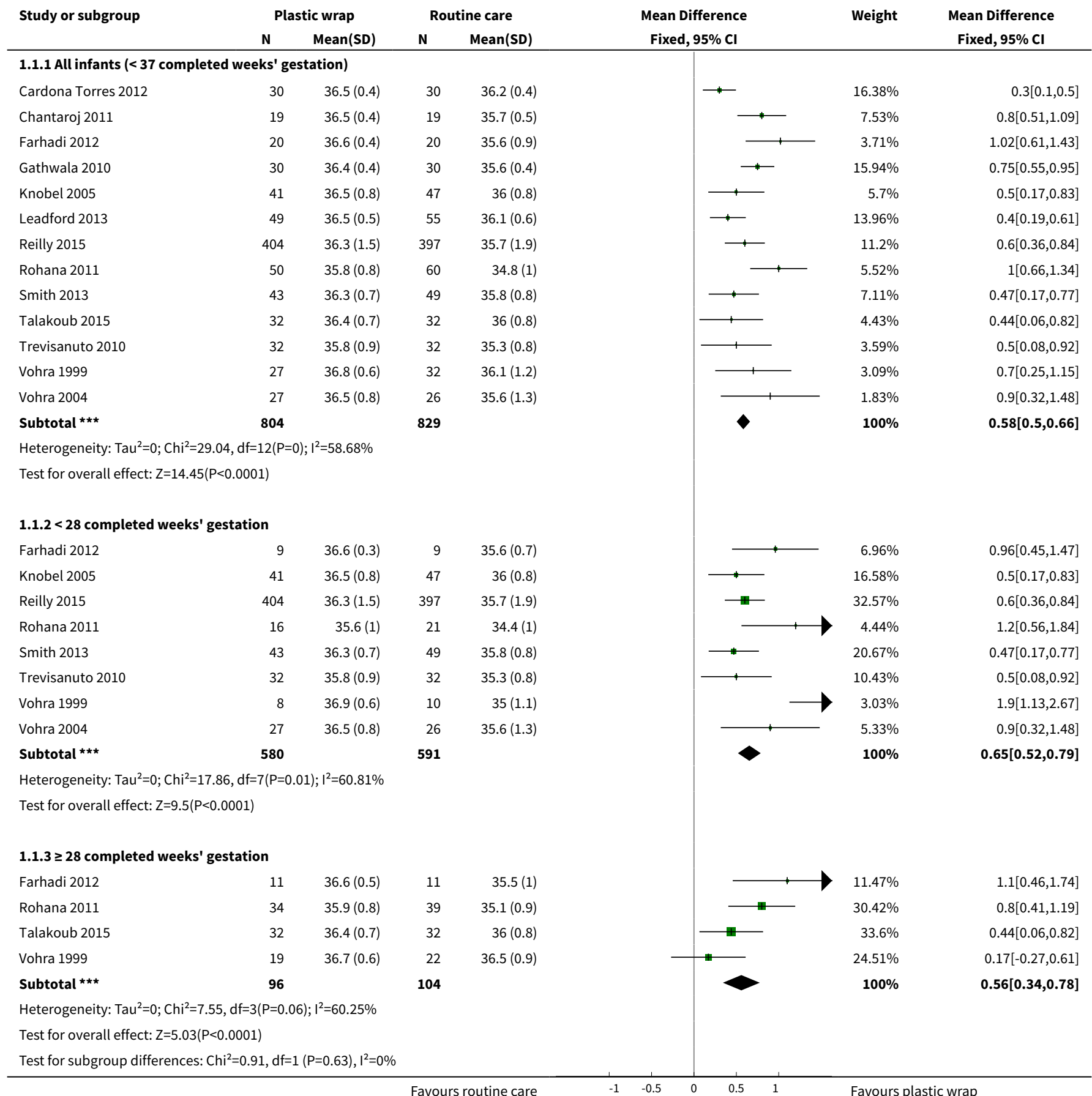


Analysis 1.2. Comparison 1 Plastic wrap or bag versus routine care, Outcome 2 Hypothermia on admission to NICU: core body temperature $<36.5^{\circ} \mathrm{C}$ or skin temperature $<36^{\circ} \mathrm{C}$.

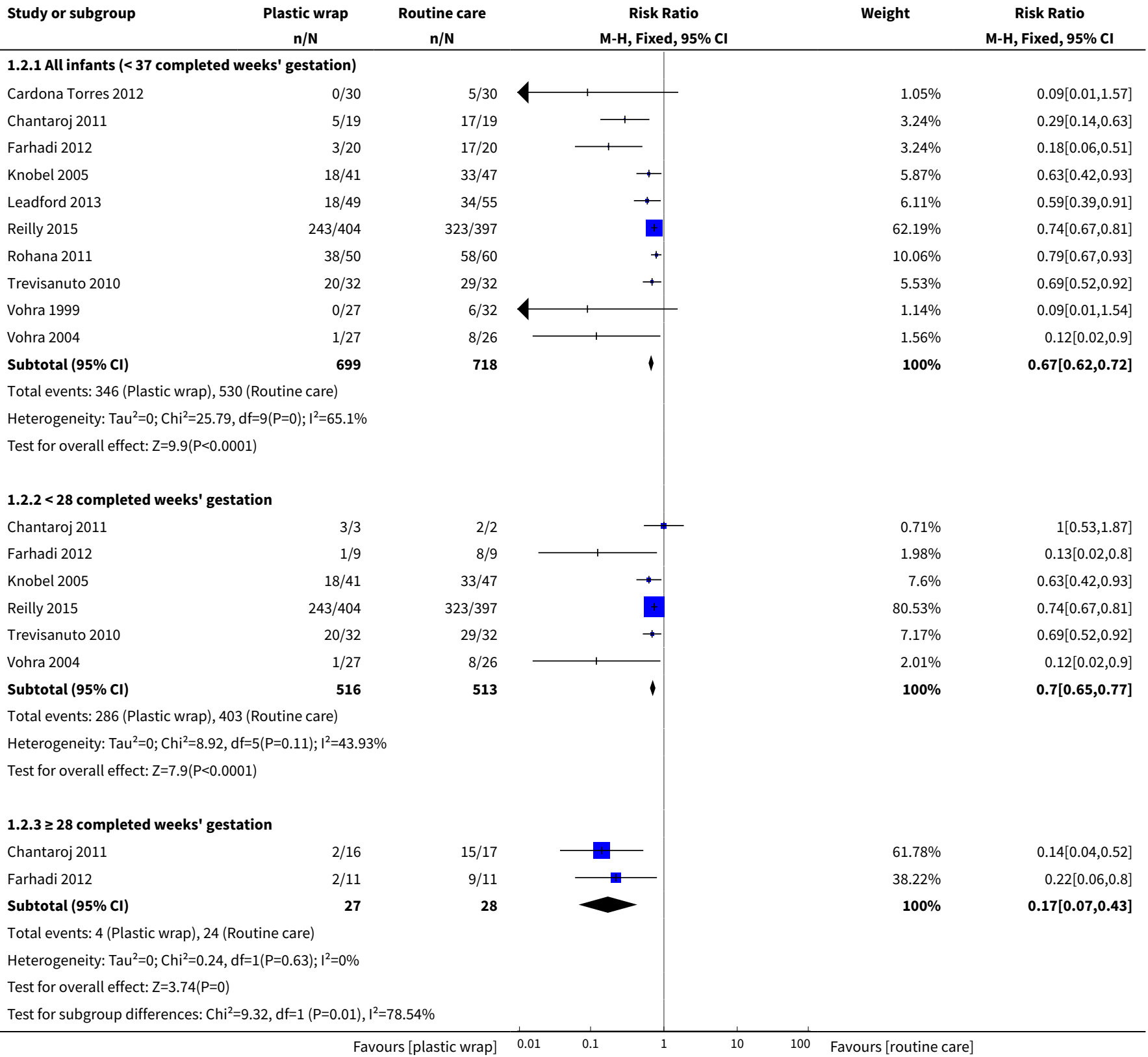

Analysis 1.3. Comparison 1 Plastic wrap or bag versus routine care, Outcome 3 Outside normothermic range on admission to NICU or up to $\mathbf{2}$ hours after birth.

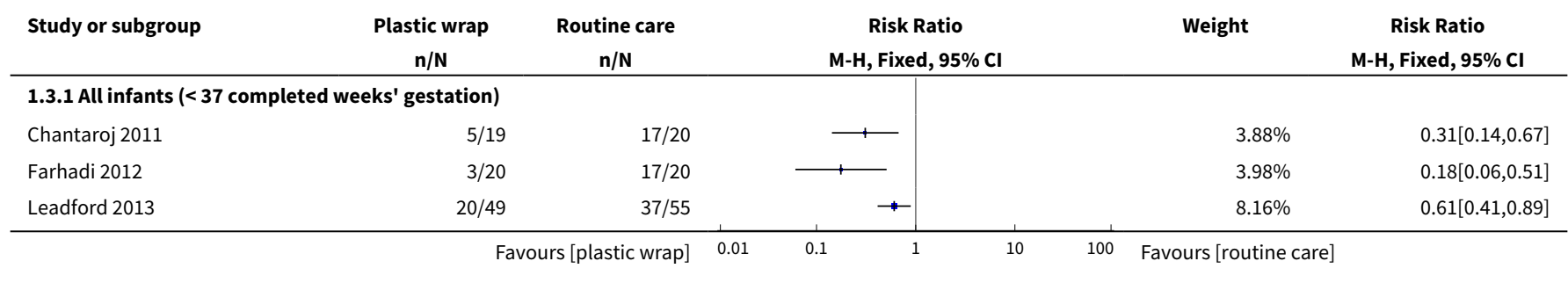




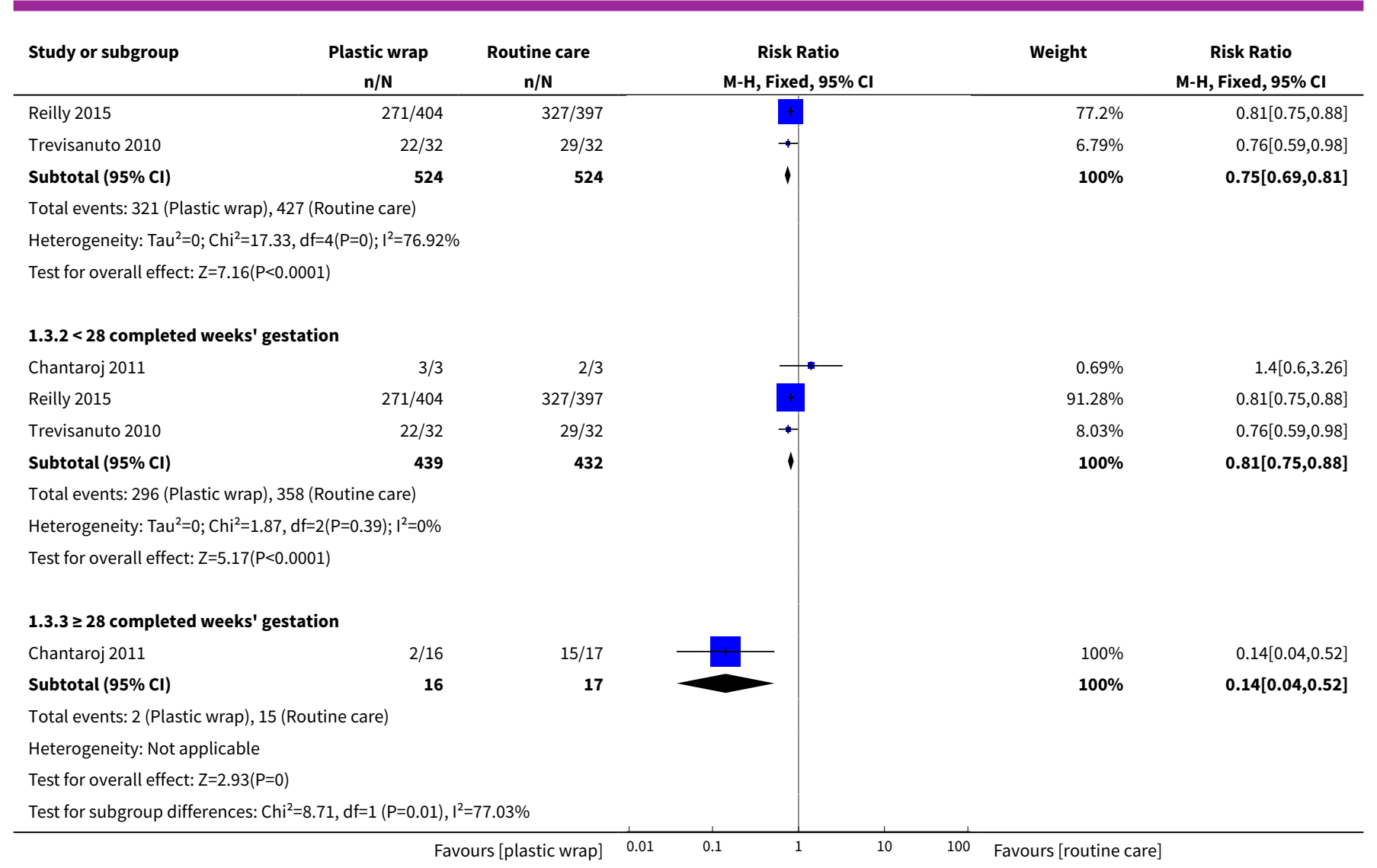

Analysis 1.4. Comparison 1 Plastic wrap or bag versus routine care, Outcome 4 Core body temperature $\left({ }^{\circ} \mathrm{C}\right)$ at 1 hour after birth.

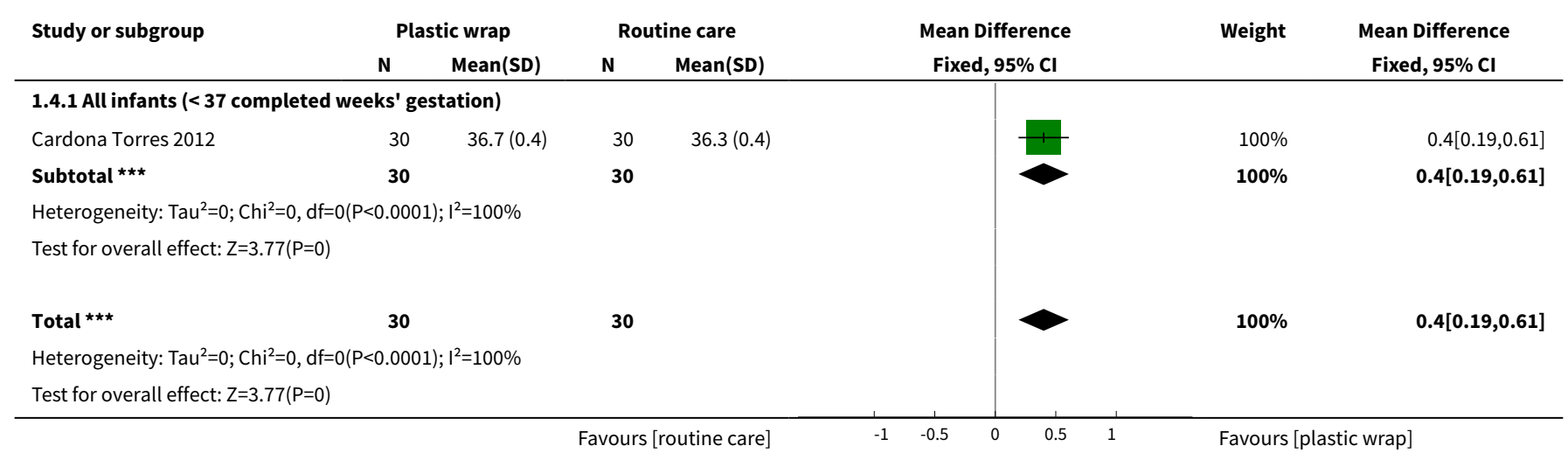

\section{Analysis 1.5. Comparison 1 Plastic wrap or bag versus routine care, Outcome 5 Core body temperature $\left({ }^{\circ} \mathrm{C}\right)$ at 90 minutes after birth.}

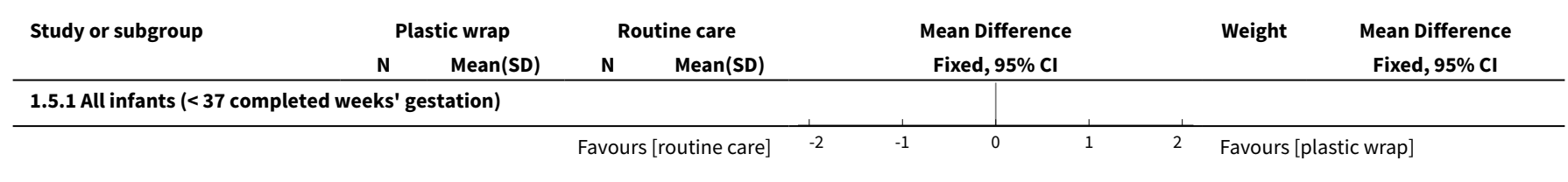




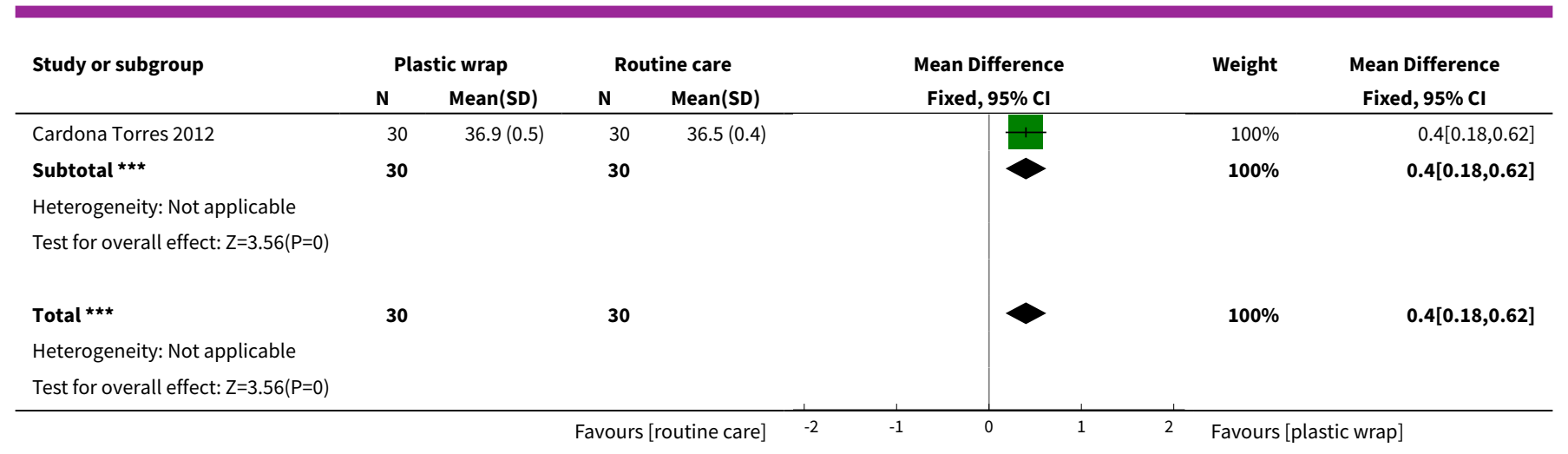

Analysis 1.6. Comparison 1 Plastic wrap or bag versus routine care, Outcome 6 Core body temperature $\left({ }^{\circ} \mathrm{C}\right)$ at 2 hours after birth.

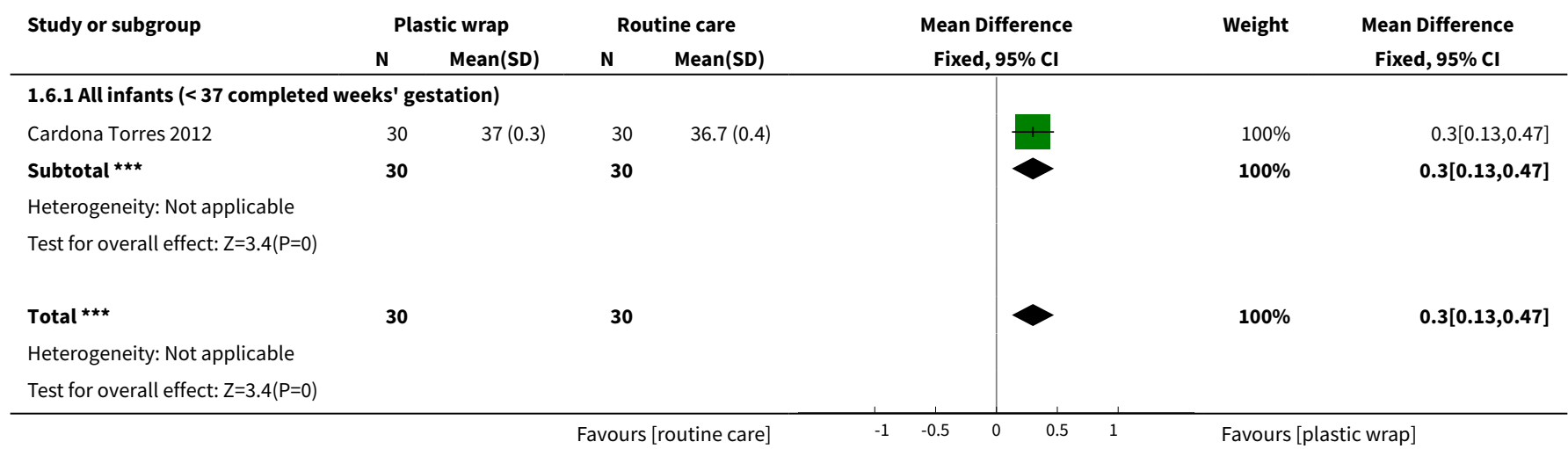

\section{Analysis 1.7. Comparison 1 Plastic wrap or bag versus routine care, Outcome 7 Core body temperature $\left({ }^{\circ} \mathrm{C}\right)$ post stabilisation.}

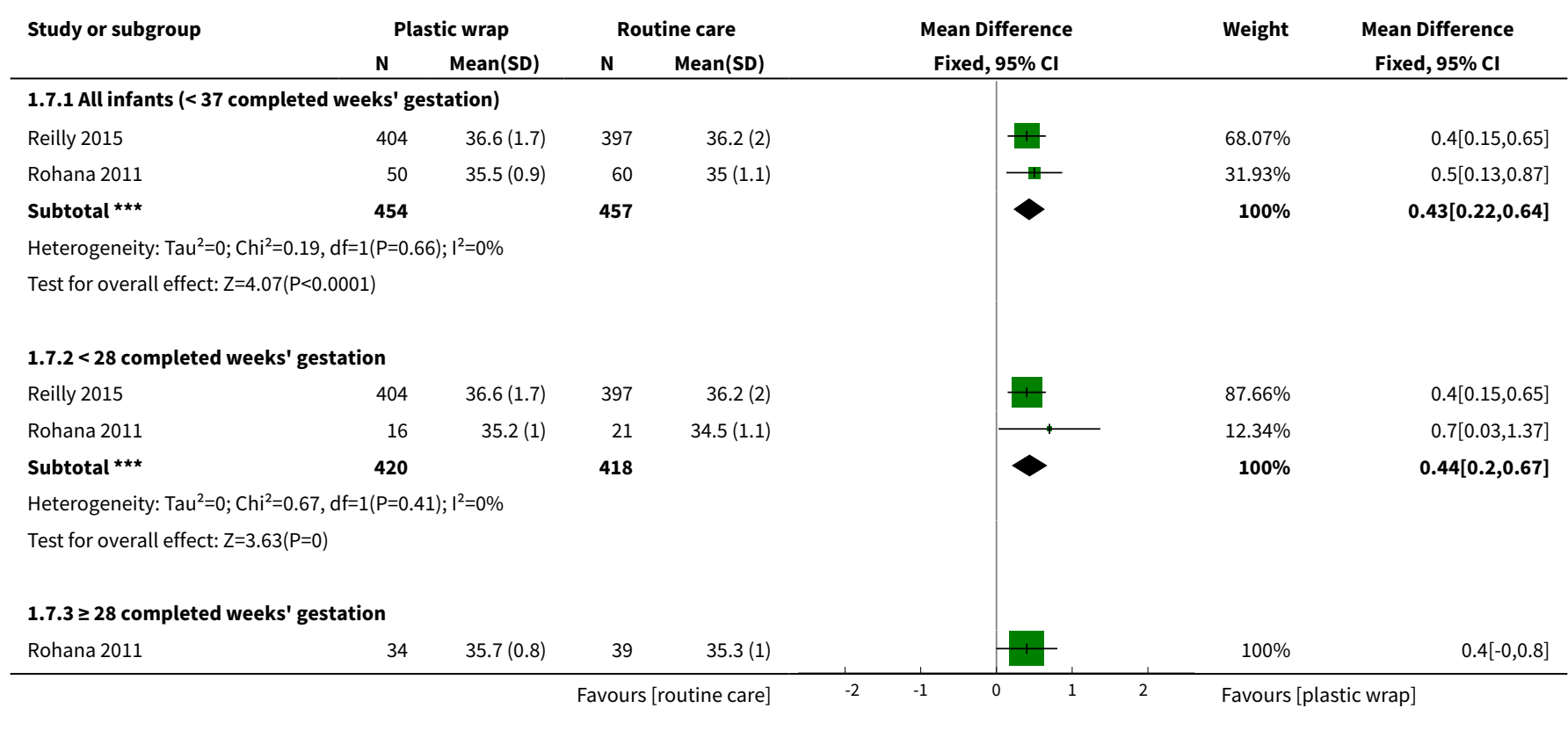




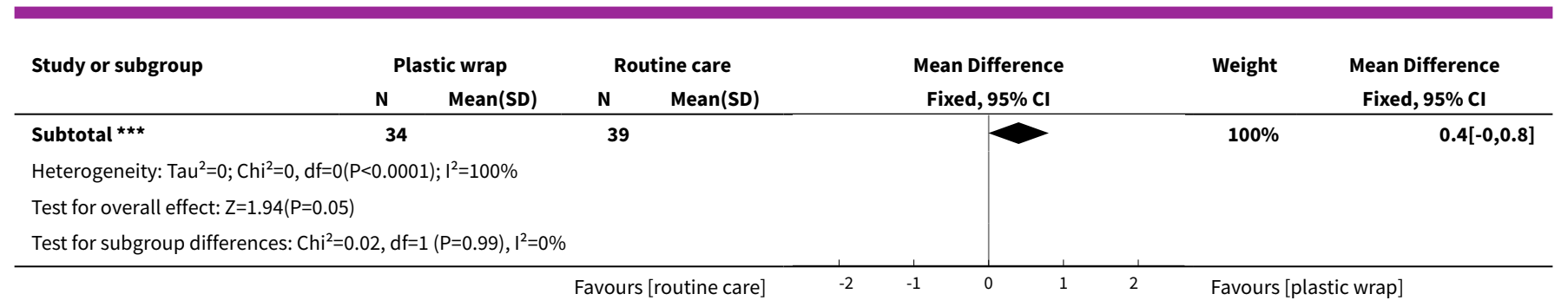

Analysis 1.8. Comparison 1 Plastic wrap or bag versus routine care, Outcome 8 Hypothermia post stabilisation: core body temperature $<36.5^{\circ} \mathrm{C}$ or skin temperature $<36^{\circ} \mathrm{C}$.

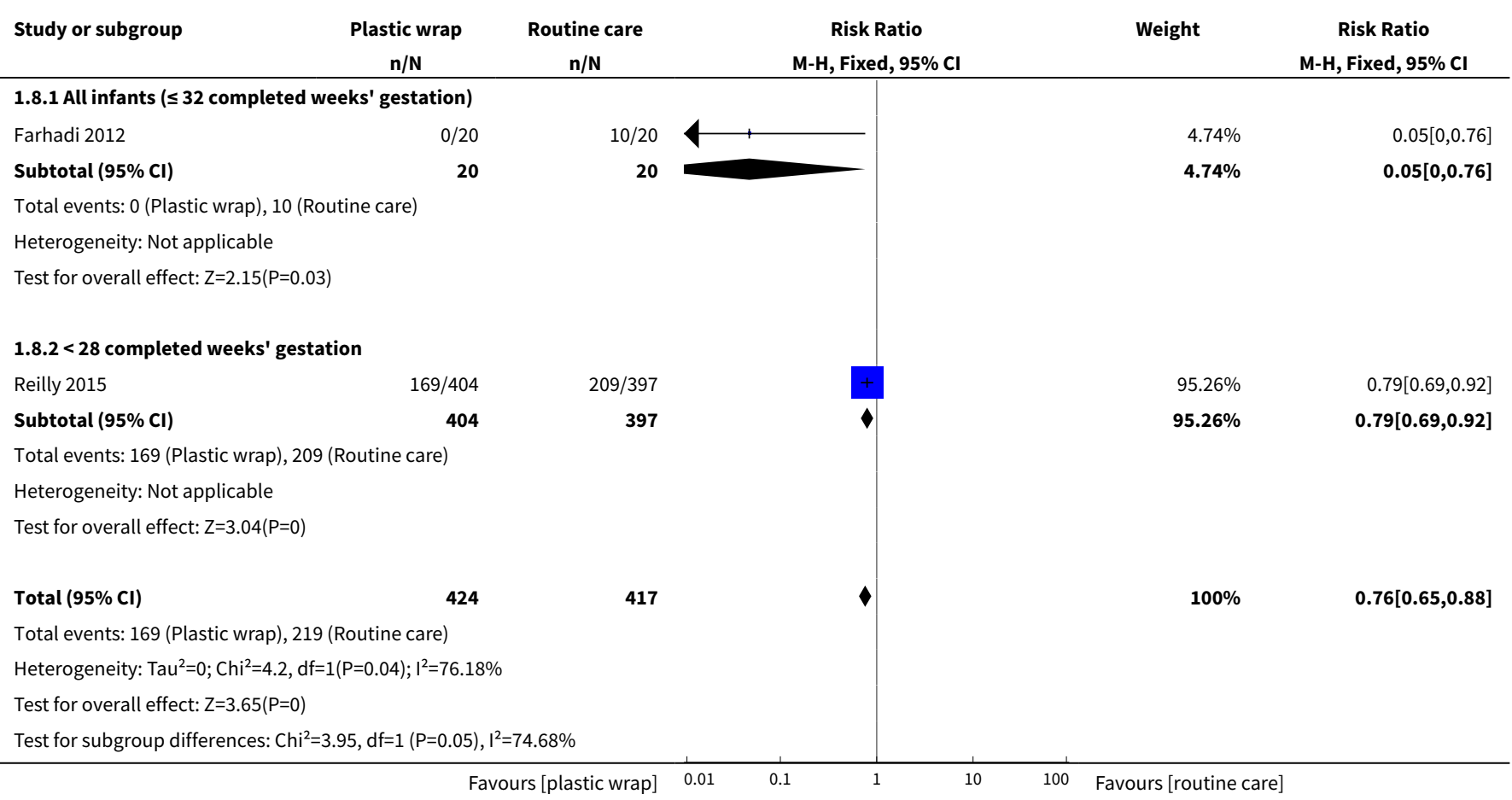

Analysis 1.9. Comparison 1 Plastic wrap or bag versus routine care, Outcome 9 Outside normothermic range post stabilisation.

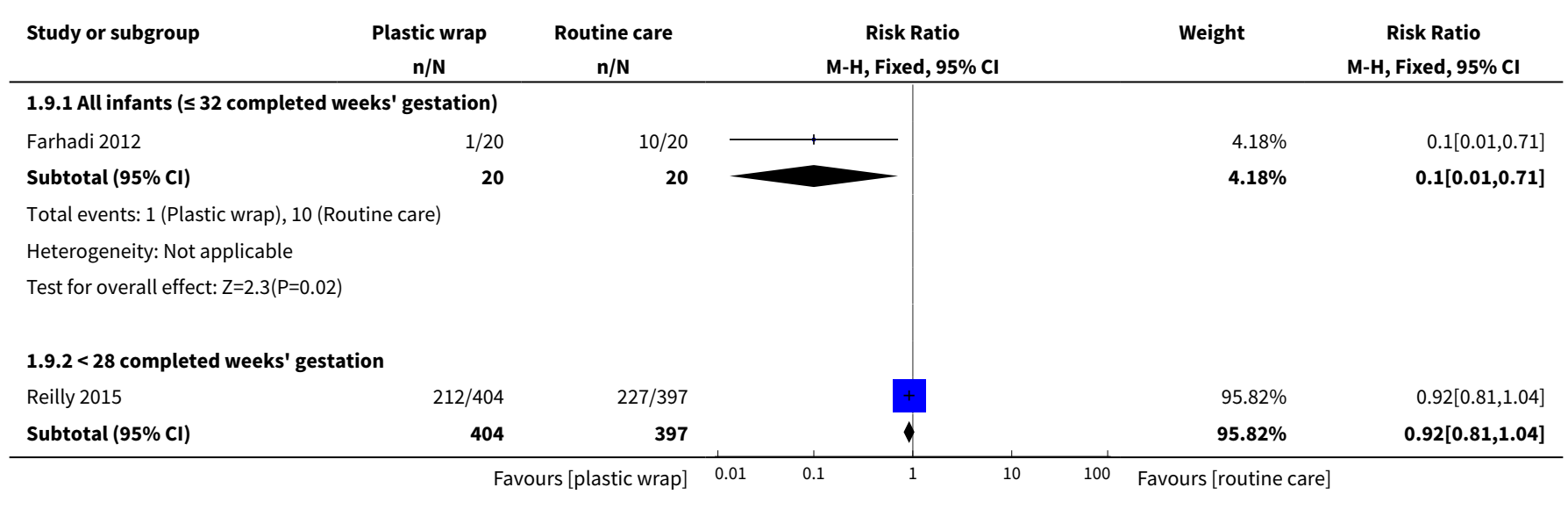




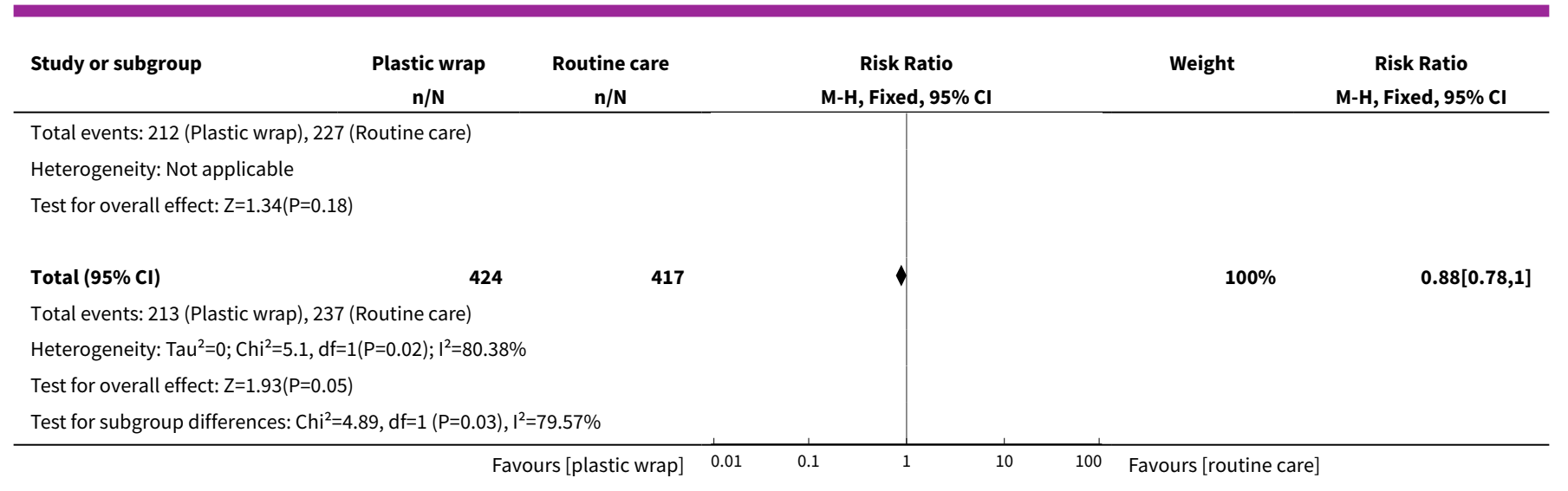

Analysis 1.10. Comparison 1 Plastic wrap or bag versus routine care, Outcome 10 Core body temperature $\left({ }^{\circ} \mathrm{C}\right) \mathbf{3 0}$ minutes after initial NICU admission temperature was taken.

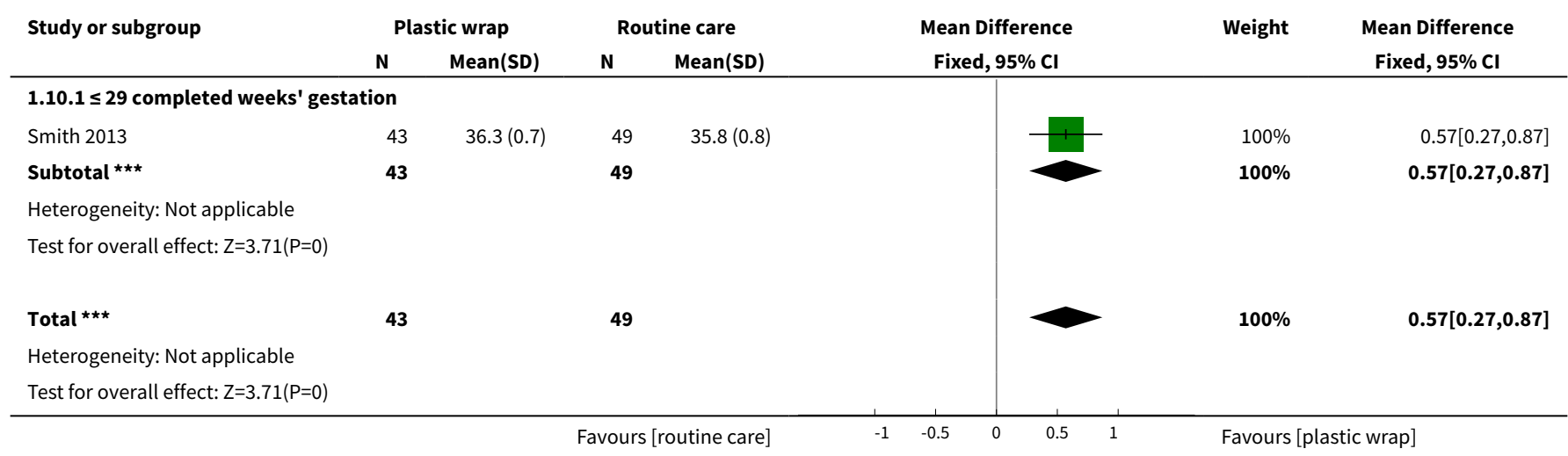

\section{Analysis 1.11. Comparison 1 Plastic wrap or bag versus routine care, Outcome 11 Core body temperature $\left({ }^{\circ} \mathrm{C}\right) \mathbf{1}$ hour after initial NICU admission temperature was taken.}

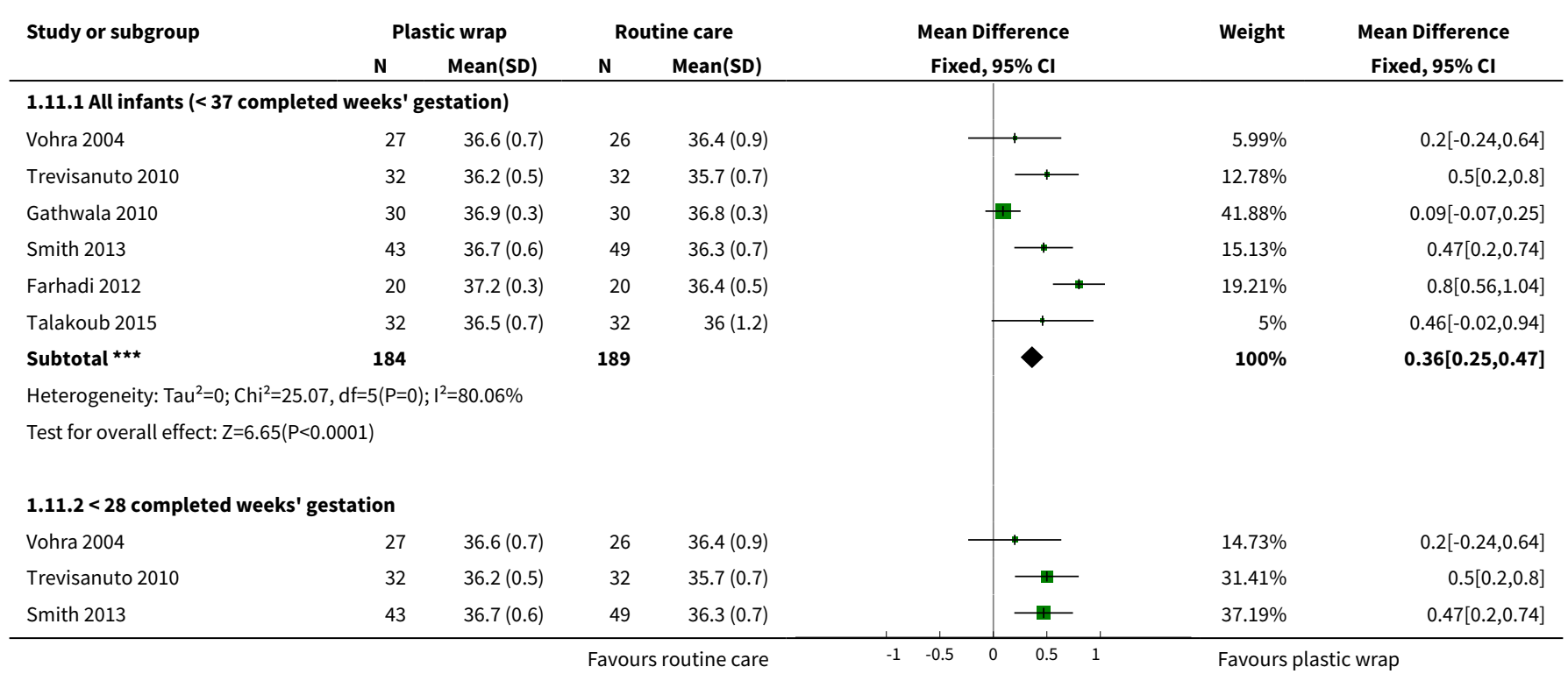




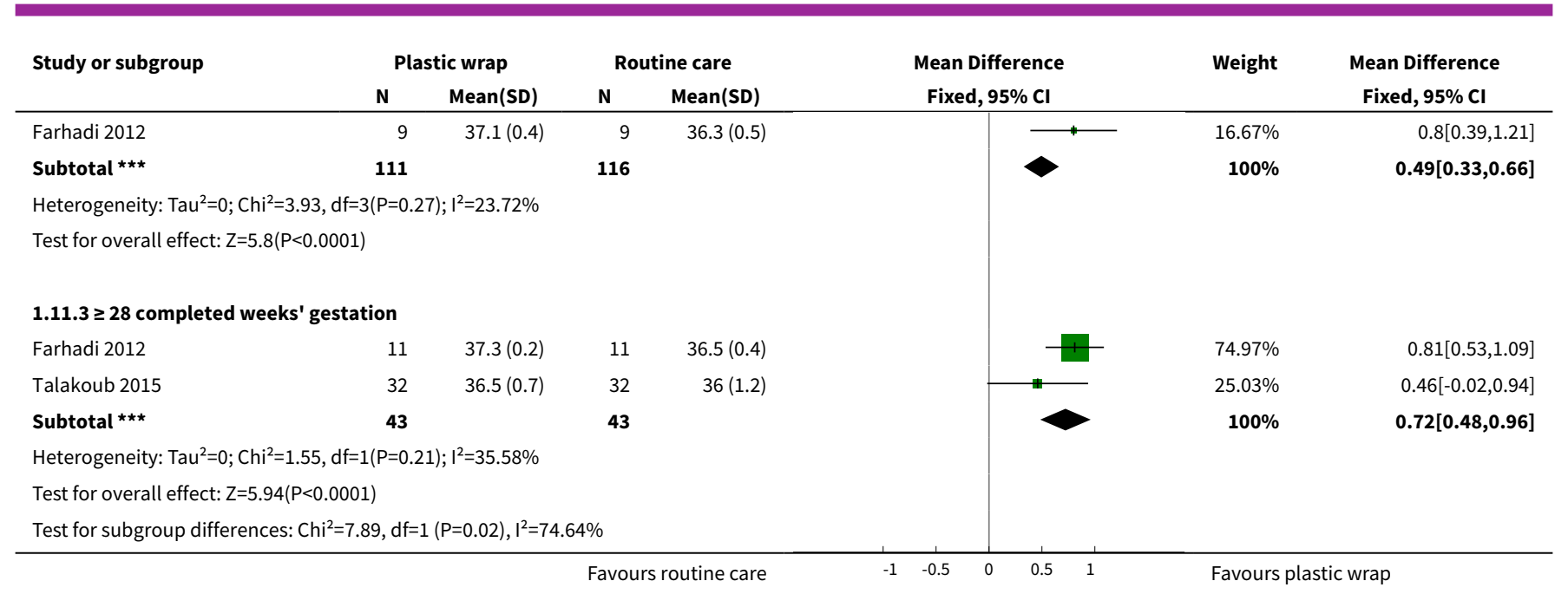

Analysis 1.12. Comparison 1 Plastic wrap or bag versus routine care, Outcome 12 Core body temperature $\left({ }^{\circ} \mathrm{C}\right) 90$ minutes after initial NICU admission temperature was taken.

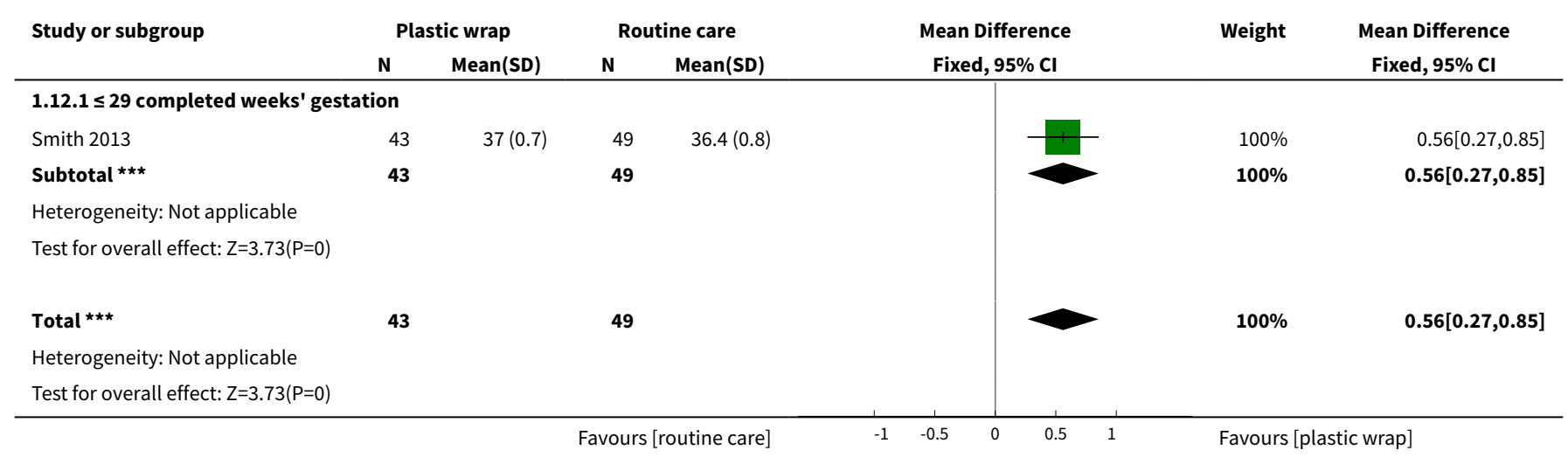

\section{Analysis 1.13. Comparison 1 Plastic wrap or bag versus routine care, Outcome 13 Core body temperature $\left({ }^{\circ} \mathrm{C}\right) \mathbf{2}$ hours after initial NICU admission temperature was taken.}

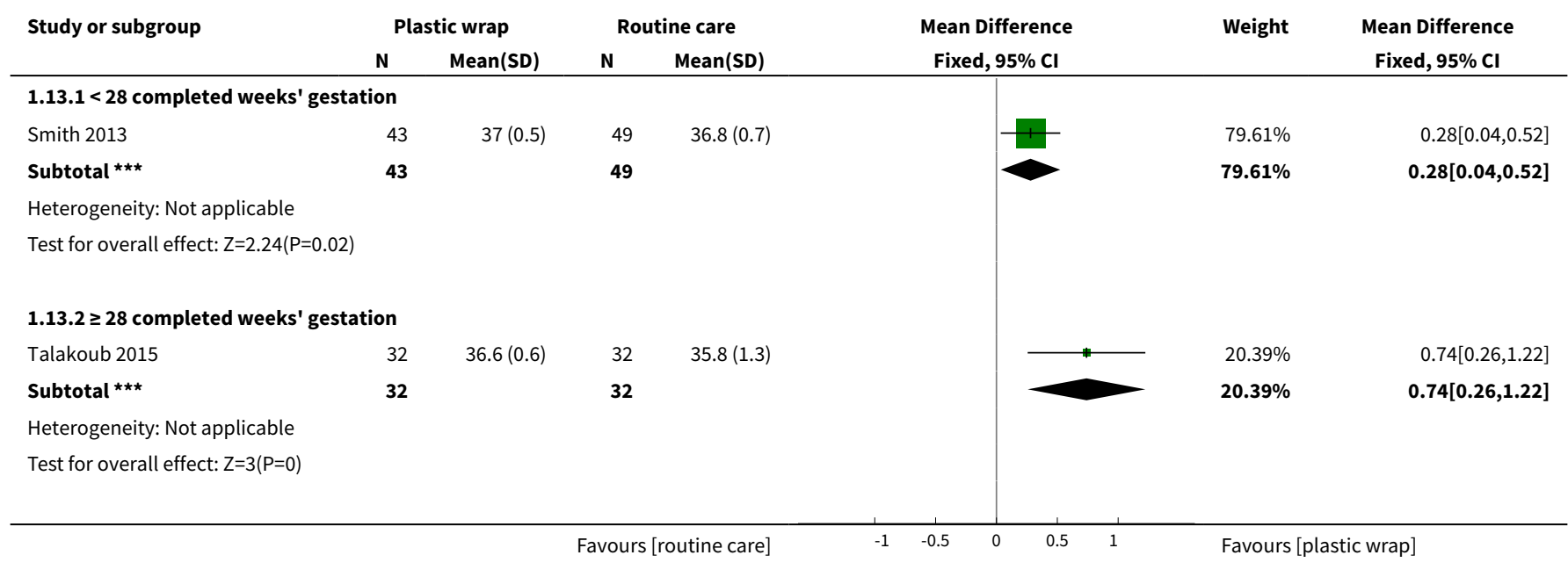




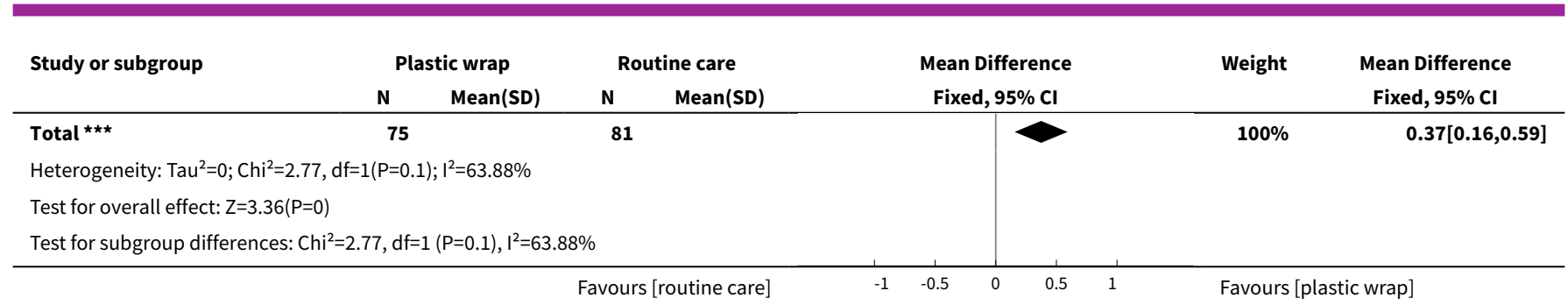

\section{Analysis 1.14. Comparison 1 Plastic wrap or bag versus routine care, Outcome 14 Hyperthermia on admission to NICU: core body temperature $>37.5^{\circ} \mathrm{C}$.}

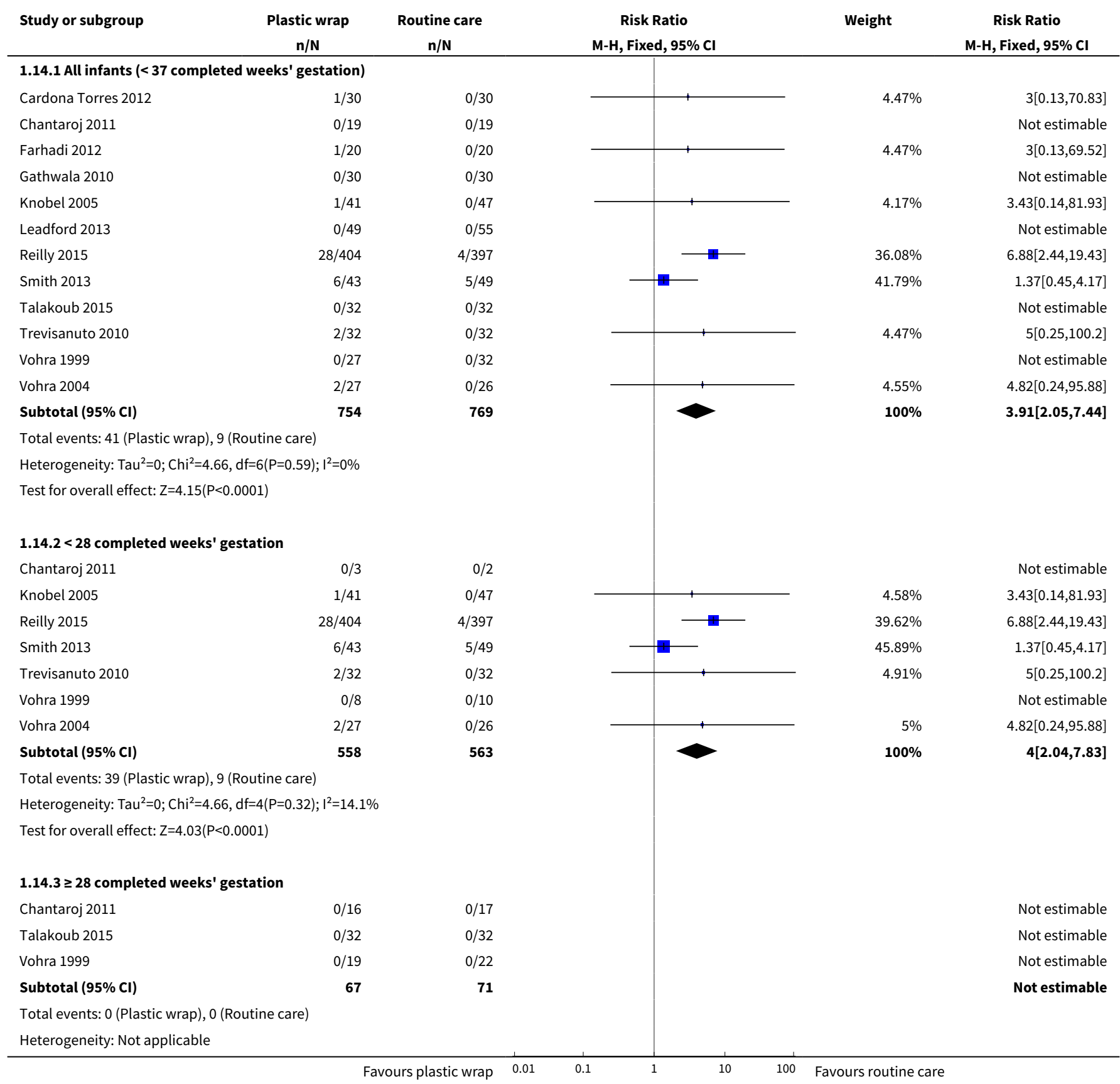




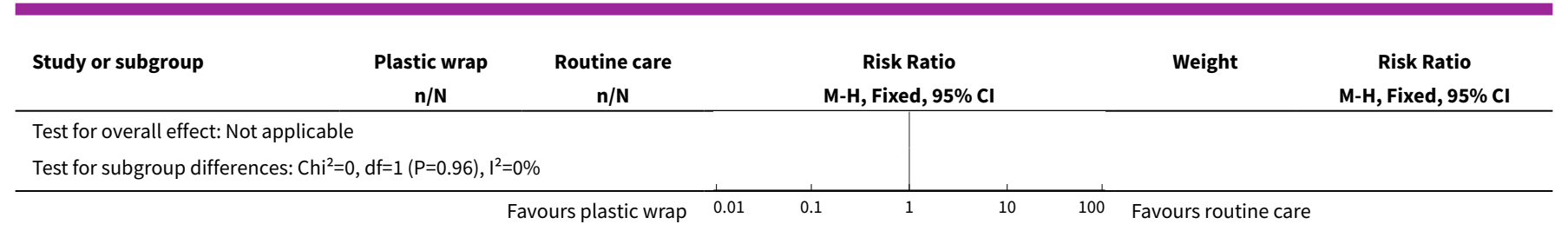

Analysis 1.15. Comparison 1 Plastic wrap or bag versus routine care, Outcome 15 Hyperthermia post stabilisation: core body temperature $\geq 37.5^{\circ} \mathrm{C}$.

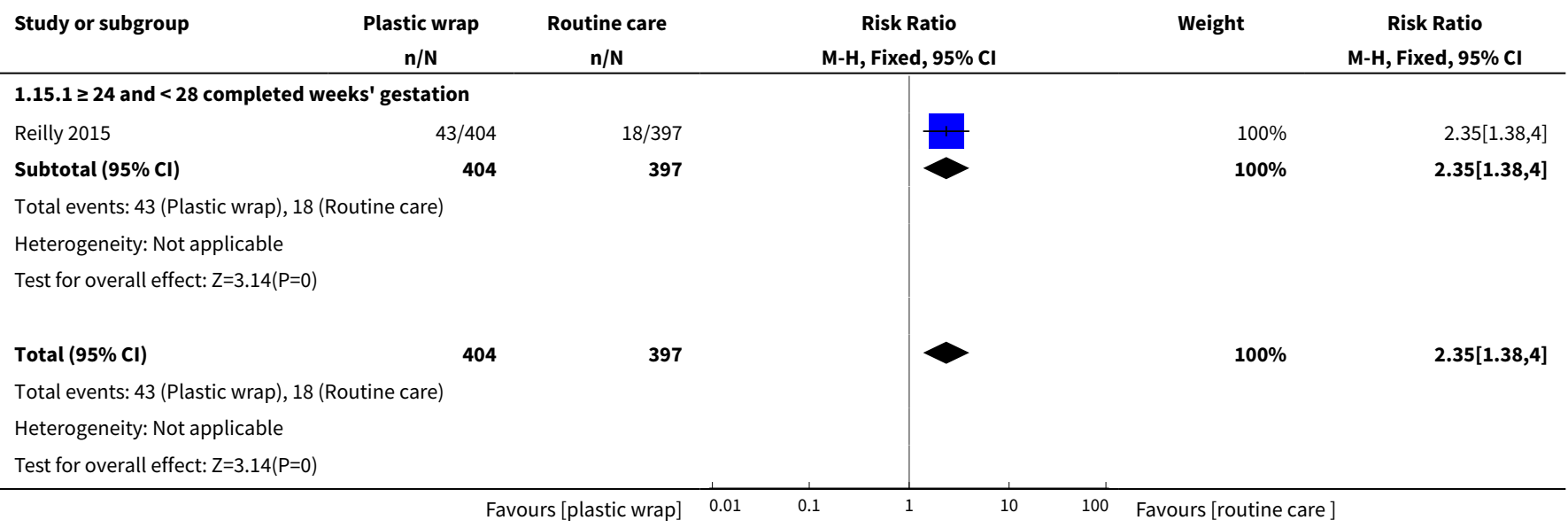

Analysis 1.16. Comparison 1 Plastic wrap or bag versus routine care, Outcome 16 Major brain injury.

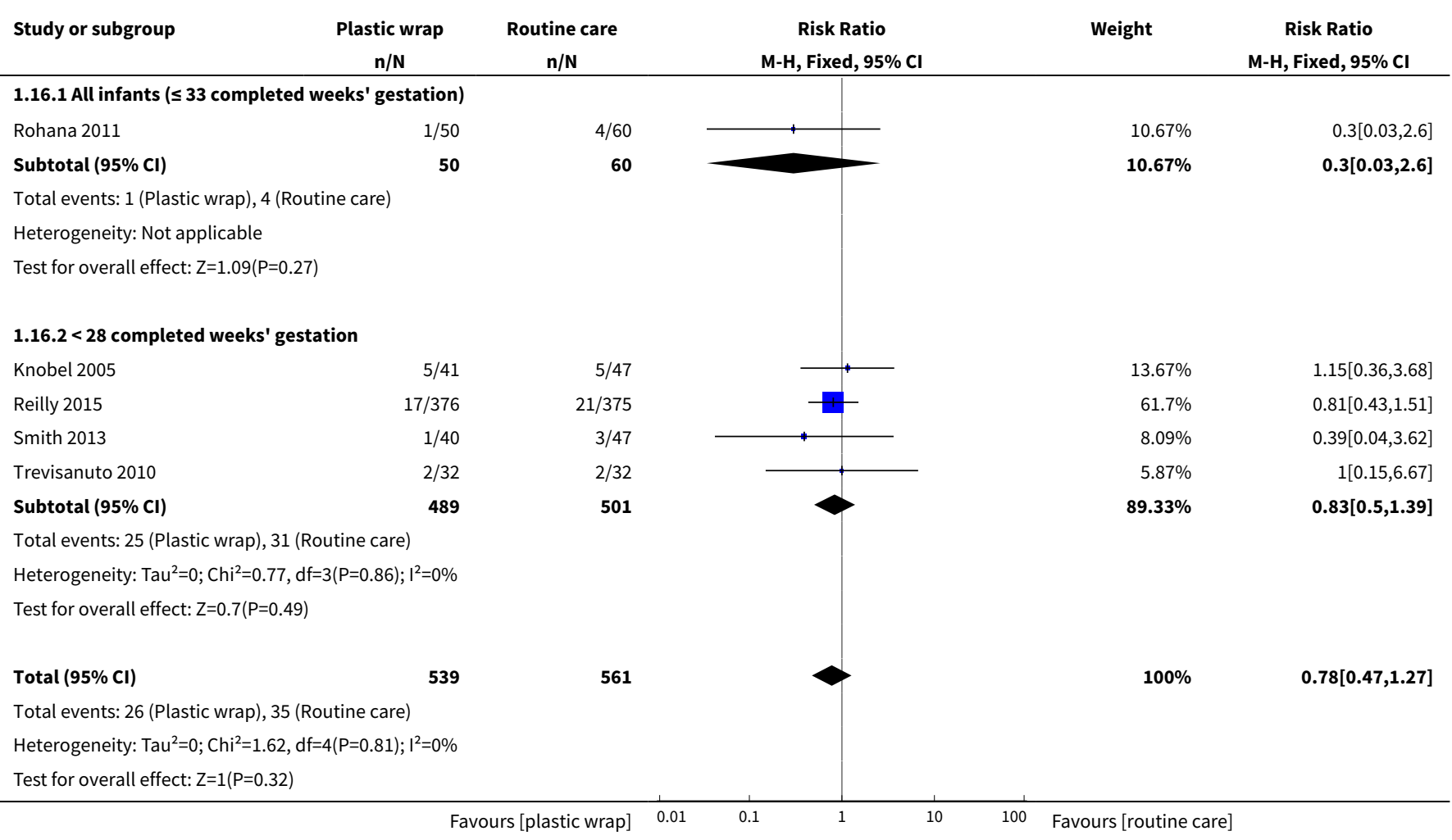




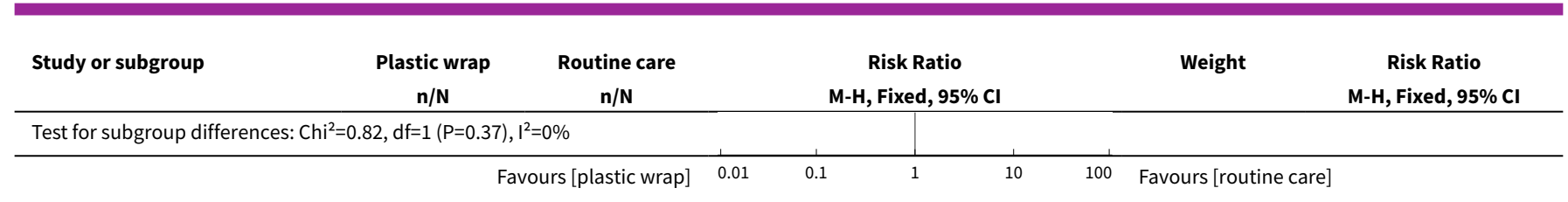

Analysis 1.17. Comparison 1 Plastic wrap or bag versus routine care, Outcome 17 Intraventicular haemorrhage (all grades).

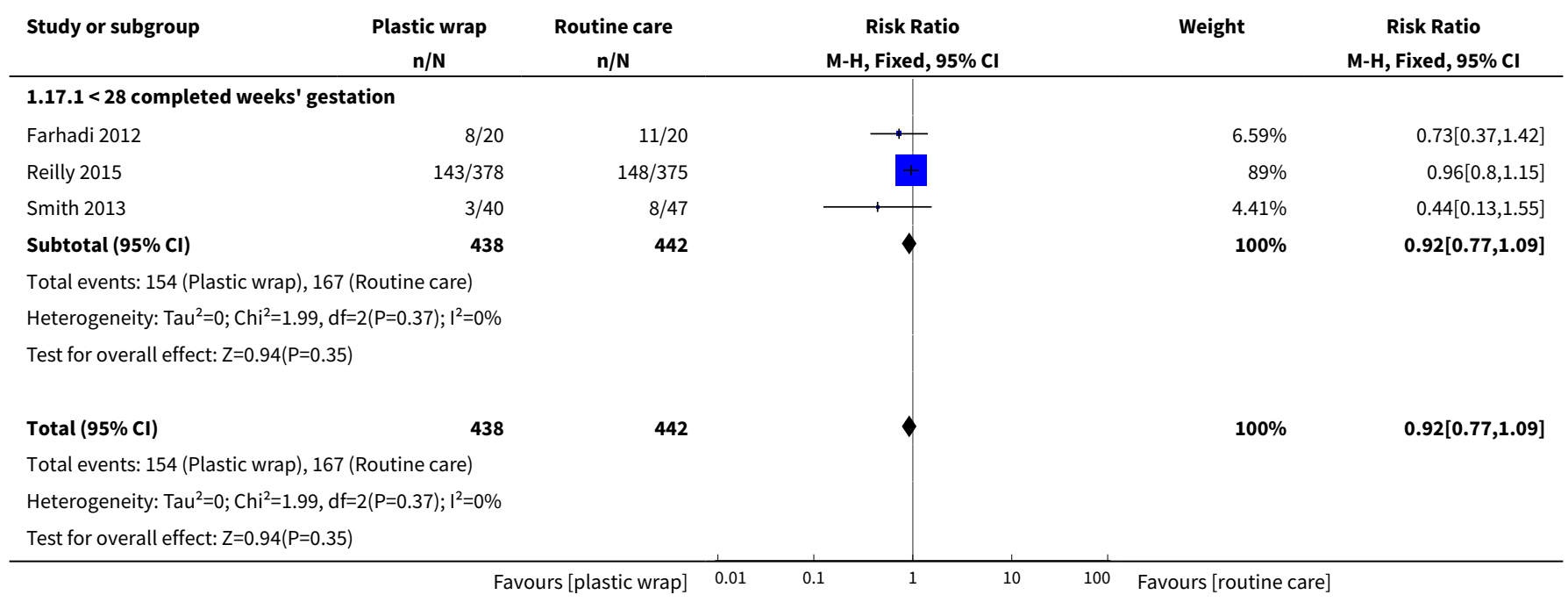

Analysis 1.18. Comparison 1 Plastic wrap or bag versus routine care, Outcome 18 Intraventricular haemorrhage (grades III and IV).

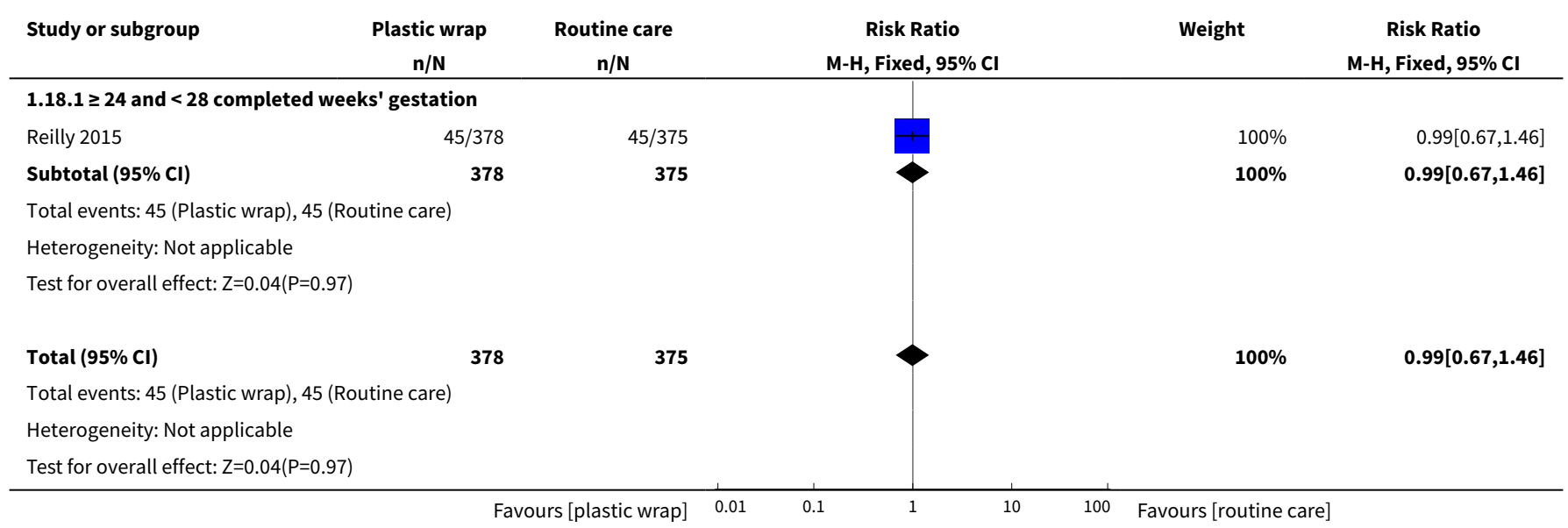


Analysis 1.19. Comparison 1 Plastic wrap or bag versus routine care, Outcome 19 Mortality (death within hospital stay or at 6 months' corrected gestation).

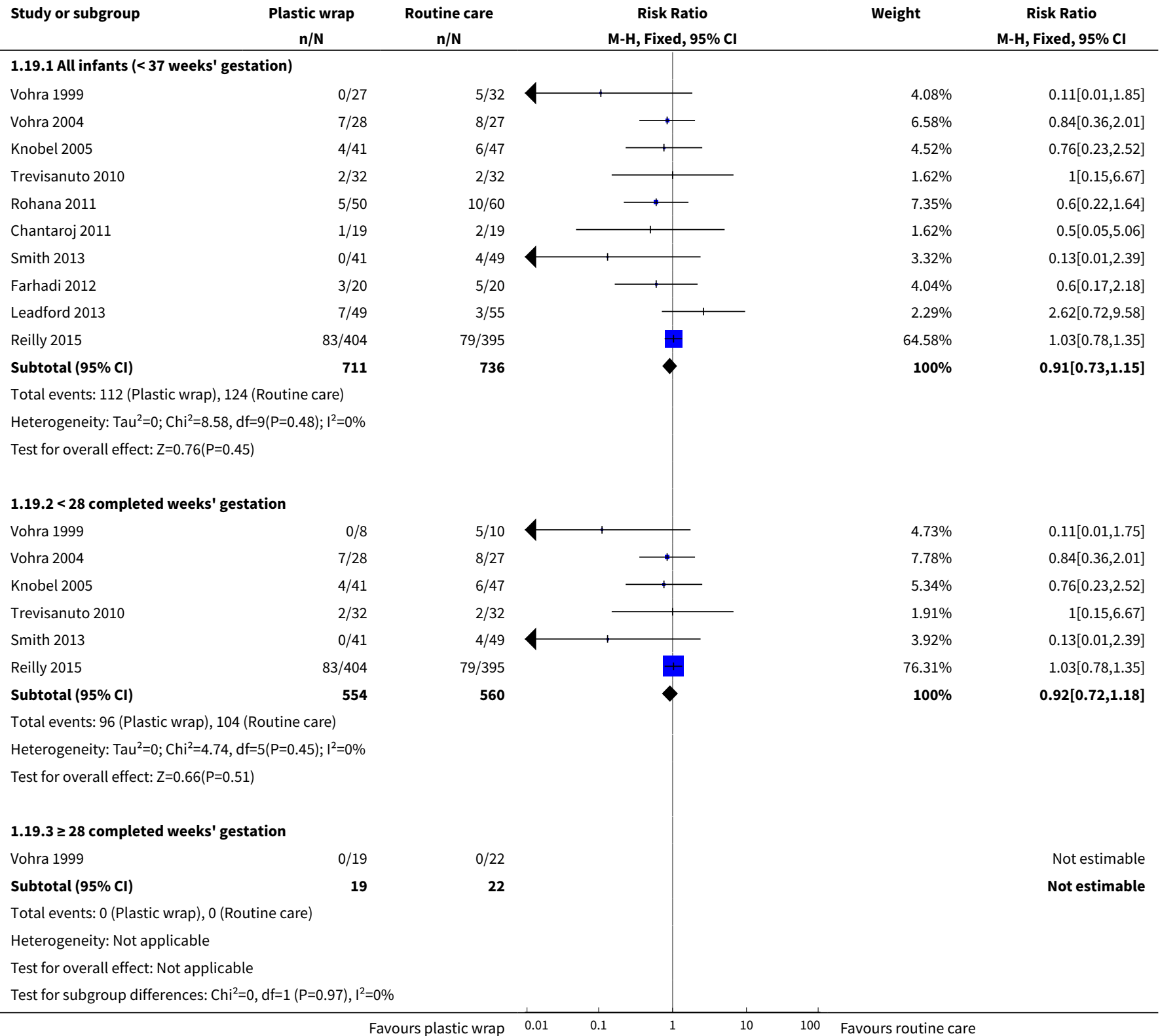

\section{Analysis 1.20. Comparison 1 Plastic wrap or bag versus routine} care, Outcome 20 Arterial oxygen saturation (percentage).

\begin{tabular}{|c|c|c|c|c|c|c|c|c|}
\hline \multirow[t]{2}{*}{ Study or subgroup } & \multicolumn{2}{|c|}{ Plastic wrap } & \multicolumn{2}{|c|}{ Routine care } & & \multirow{2}{*}{$\begin{array}{c}\text { Mean Difference } \\
\text { Fixed, } 95 \% \mathrm{Cl}\end{array}$} & \multirow[t]{2}{*}{ Weight } & \multirow{2}{*}{$\begin{array}{c}\text { Mean Difference } \\
\text { Fixed, } 95 \% \mathrm{Cl}\end{array}$} \\
\hline & $\mathbf{N}$ & Mean(SD) & $\mathbf{N}$ & Mean(SD) & & & & \\
\hline \multicolumn{9}{|c|}{$1.20 .1 \leq 32$ completed weeks' gestation } \\
\hline Farhadi 2012 & 20 & $77.9(21.4)$ & 20 & $82(16.7)$ & & & $100 \%$ & $-4.1[-16,7.8]$ \\
\hline Subtotal $* \star \star$ & 20 & & 20 & & & & $100 \%$ & $-4.1[-16,7.8]$ \\
\hline \multicolumn{9}{|c|}{ Heterogeneity: Not applicable } \\
\hline \multicolumn{9}{|c|}{ Test for overall effect: $Z=0.68(P=0.5)$} \\
\hline
\end{tabular}




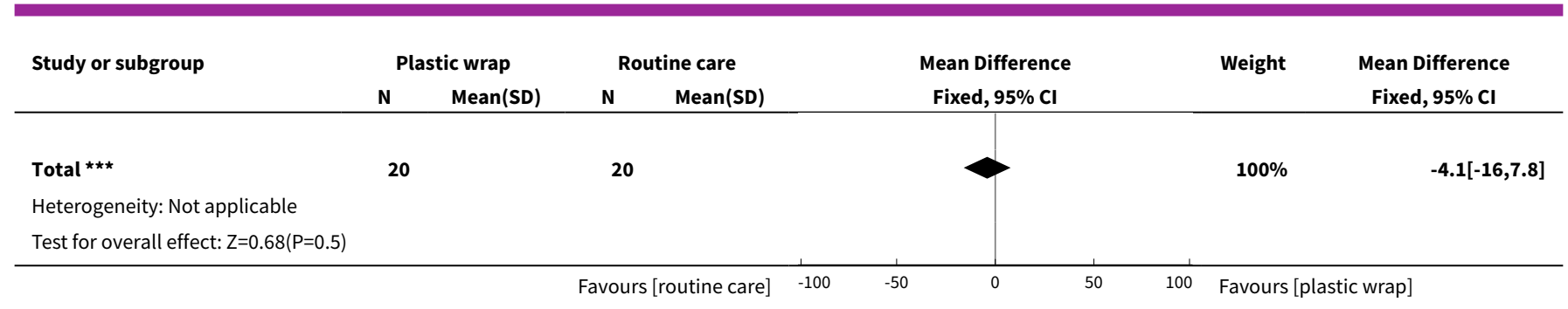

Analysis 1.21. Comparison 1 Plastic wrap or bag versus routine care, Outcome 21 Bicarbonate (mmol/L).

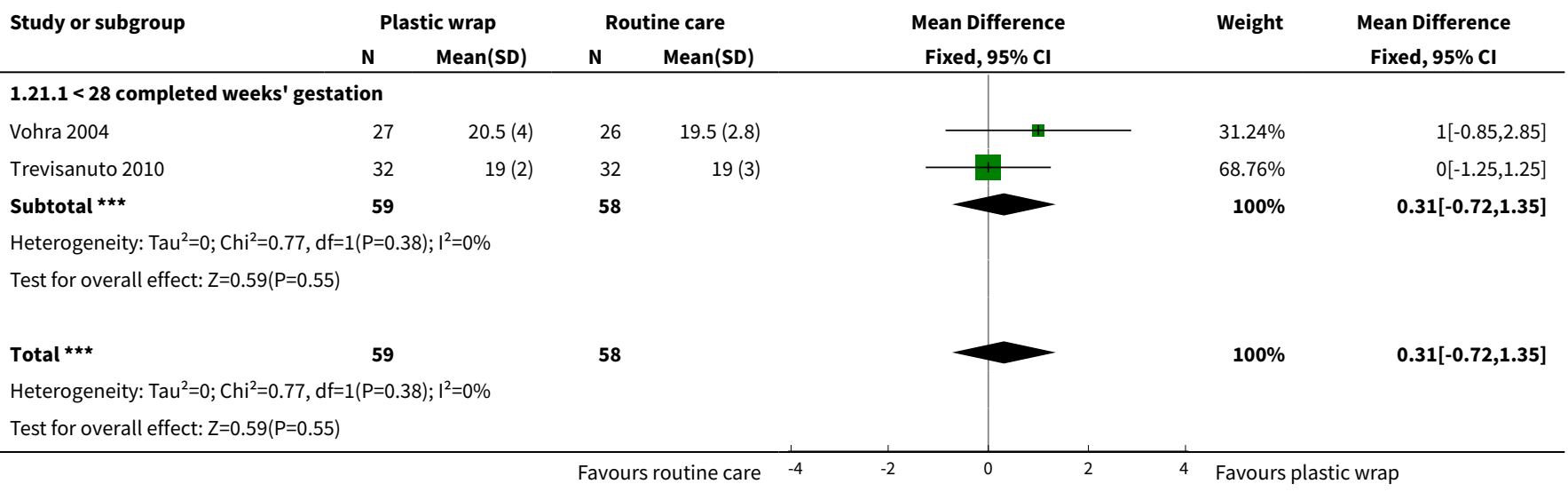

Analysis 1.22. Comparison 1 Plastic wrap or bag versus routine care, Outcome 22 Blood gas pH (first).

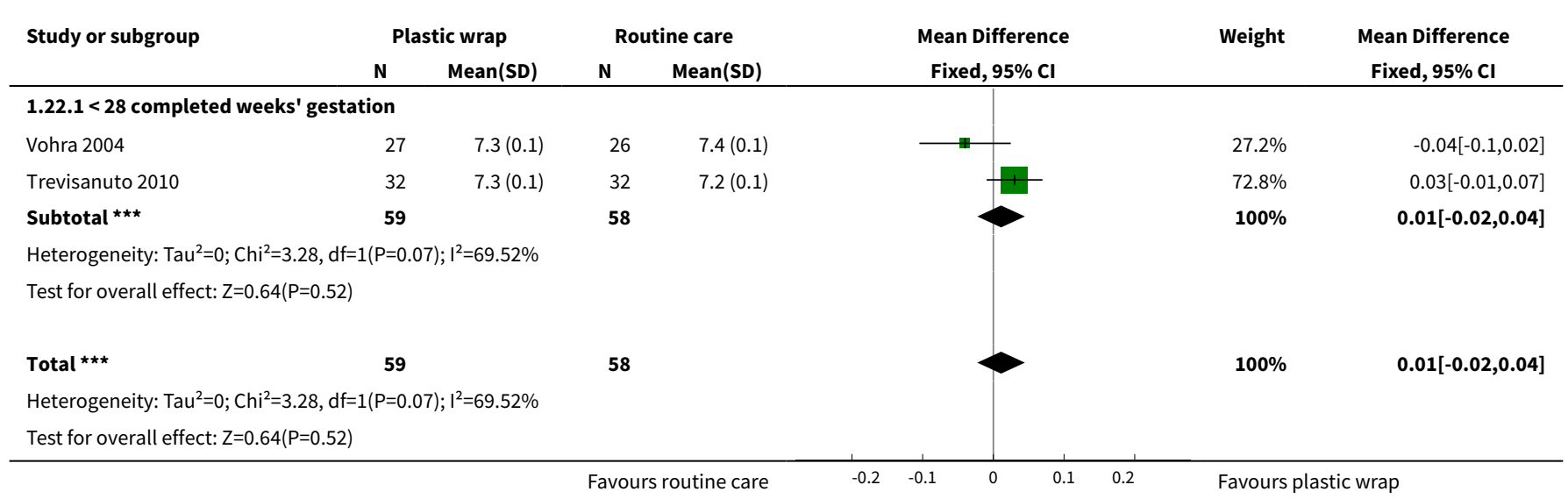

Analysis 1.23. Comparison 1 Plastic wrap or bag versus routine care, Outcome 23 Blood gas pH $<7.25$.

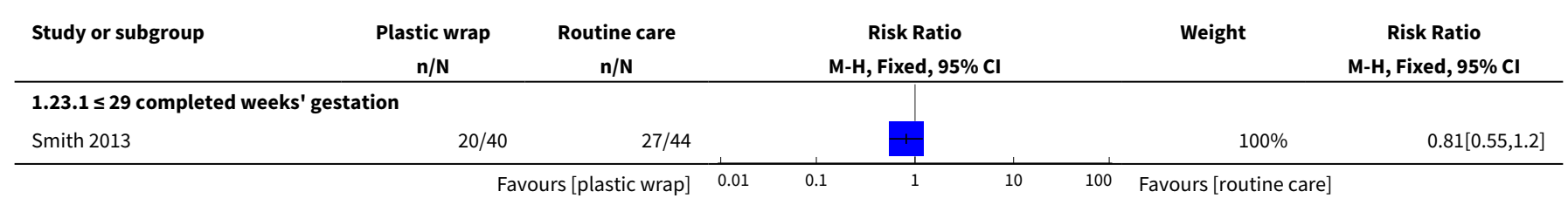




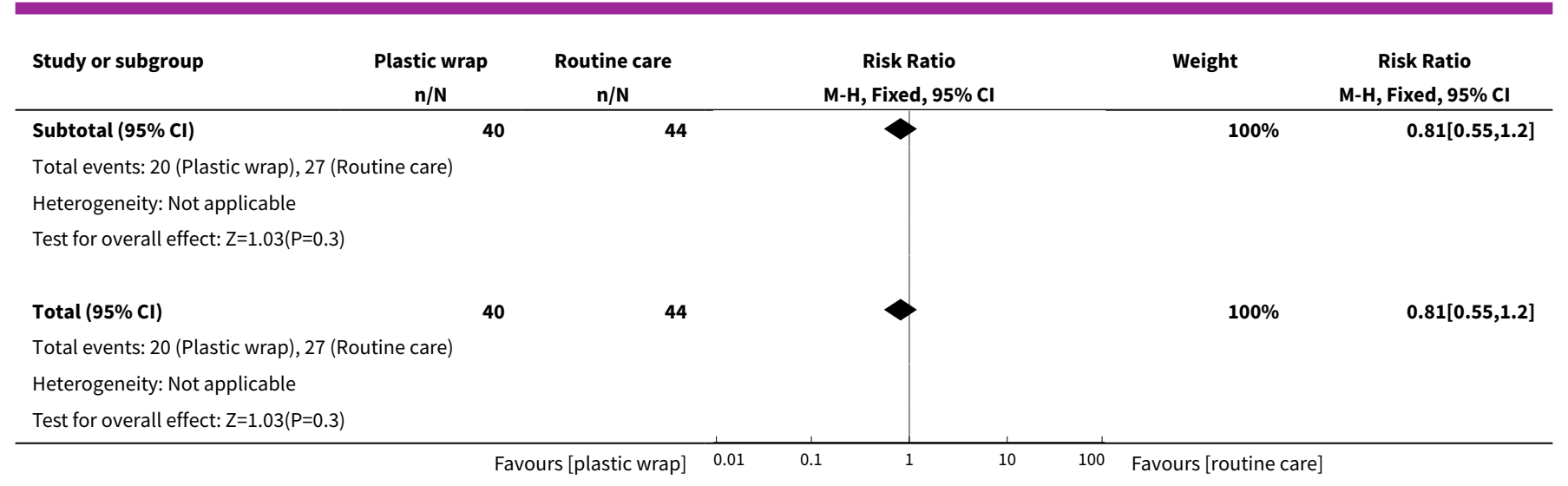

Analysis 1.24. Comparison 1 Plastic wrap or bag versus routine care, Outcome 24 Blood glucose concentration (mmol/L) (first).

\begin{tabular}{|c|c|c|c|c|c|c|c|}
\hline \multirow[t]{2}{*}{ Study or subgroup } & \multicolumn{2}{|c|}{ Plastic wrap } & \multicolumn{2}{|c|}{ Routine care } & \multirow{2}{*}{$\begin{array}{c}\text { Mean Difference } \\
\text { Fixed, } 95 \% \mathrm{Cl}\end{array}$} & \multirow[t]{2}{*}{ Weight } & \multirow{2}{*}{$\begin{array}{c}\text { Mean Difference } \\
\text { Fixed, } 95 \% \mathrm{Cl}\end{array}$} \\
\hline & $\mathbf{N}$ & Mean(SD) & $\mathbf{N}$ & Mean(SD) & & & \\
\hline \multicolumn{8}{|c|}{ 1.24.1 All infants ( $\leq 32$ completed weeks' gestation) } \\
\hline Chantaroj 2011 & 19 & $4.5(1.4)$ & 19 & $4.9(2)$ & & $10.8 \%$ & $-0.31[-1.4,0.78]$ \\
\hline Farhadi 2012 & 20 & $4(1.7)$ & 20 & $4.9(1.6)$ & & $12.18 \%$ & $-0.9[-1.92,0.12]$ \\
\hline 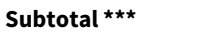 & 39 & & 39 & & & $22.98 \%$ & $-0.62[-1.37,0.12]$ \\
\hline \multicolumn{8}{|c|}{ Heterogeneity: Tau $^{2}=0 ; \mathrm{Chi}^{2}=0.6, \mathrm{df}=1(\mathrm{P}=0.44) ; \mathrm{I}^{2}=0 \%$} \\
\hline \multicolumn{8}{|c|}{ 1.24.2 $<28$ completed weeks' gestation } \\
\hline Vohra 2004 & 27 & $2.5(1.1)$ & 26 & $2.8(1.8)$ & & $19.59 \%$ & $-0.3[-1.11,0.51]$ \\
\hline Trevisanuto 2010 & 32 & $3.1(0.8)$ & 32 & $3(1.1)$ & 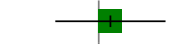 & $57.42 \%$ & $0.1[-0.37,0.57]$ \\
\hline Subtotal $* \star \star$ & 59 & & 58 & & & $77.02 \%$ & $-0[-0.41,0.41]$ \\
\hline \multicolumn{8}{|c|}{ Heterogeneity: Tau $^{2}=0 ; \mathrm{Chi}^{2}=0.7, \mathrm{df}=1(\mathrm{P}=0.4) ; \mathrm{I}^{2}=0 \%$} \\
\hline \multicolumn{8}{|c|}{ Test for overall effect: $\mathrm{Z}=0.01(\mathrm{P}=0.99)$} \\
\hline 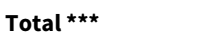 & 98 & & 97 & & & $100 \%$ & $-0.14[-0.5,0.21]$ \\
\hline \multicolumn{8}{|c|}{ Heterogeneity: $\mathrm{Tau}^{2}=0 ; \mathrm{Chi}^{2}=3.36, \mathrm{df}=3(\mathrm{P}=0.34) ; \mathrm{I}^{2}=10.73 \%$} \\
\hline \multicolumn{8}{|c|}{ Test for overall effect: $Z=0.79(P=0.43)$} \\
\hline Test for subgroup dif & $06, \mathrm{df}=$ & $P=0.15), I^{2}=5$ & & & & & \\
\hline
\end{tabular}

\section{Analysis 1.25. Comparison 1 Plastic wrap or bag versus routine care, Outcome $25 \mathrm{Blood}$ glucose $<2.6 \mathrm{mmol} / \mathrm{L}$.}

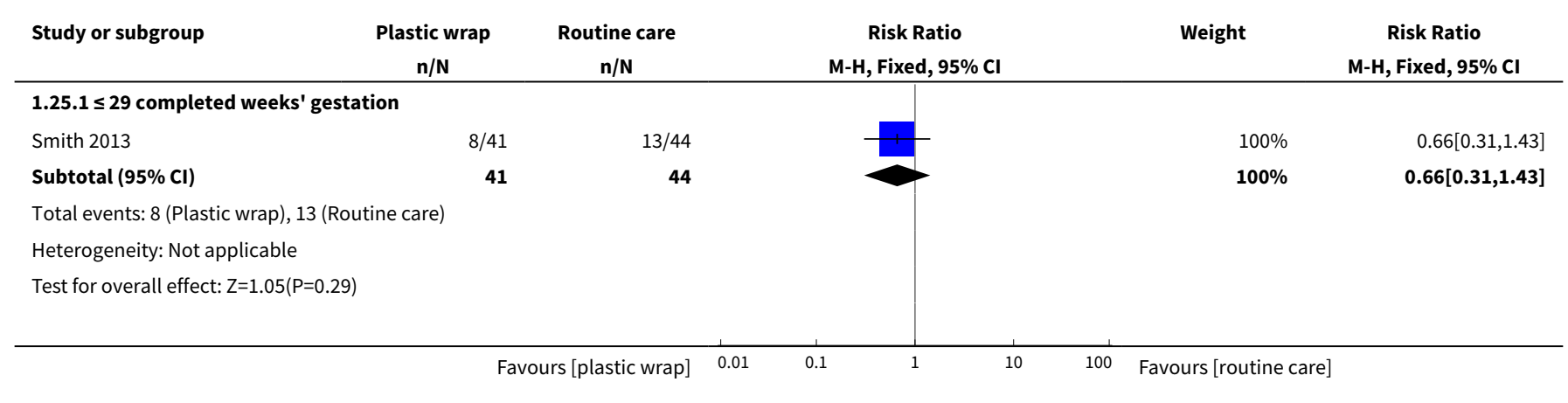




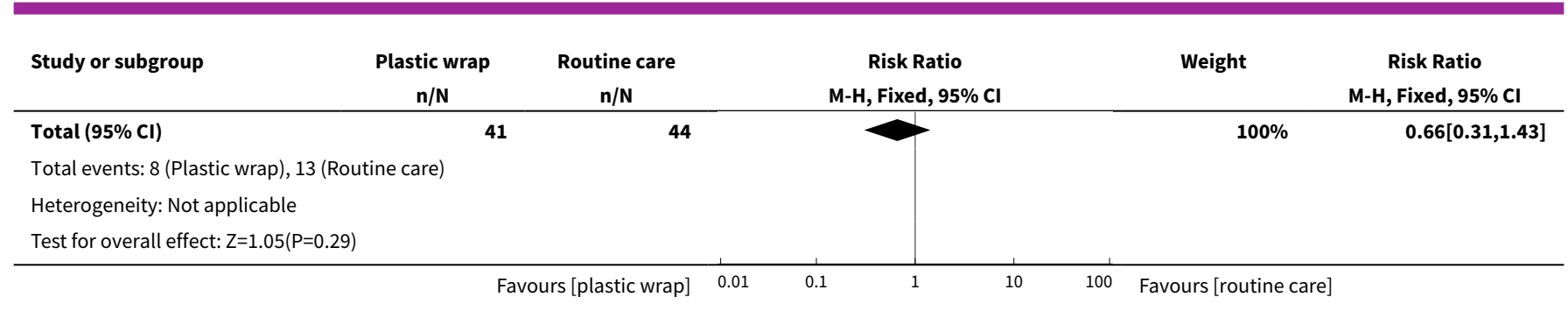

Analysis 1.26. Comparison 1 Plastic wrap or bag versus routine care, Outcome 26 Blood glucose $>6 \mathrm{mmol} / \mathrm{L}$.

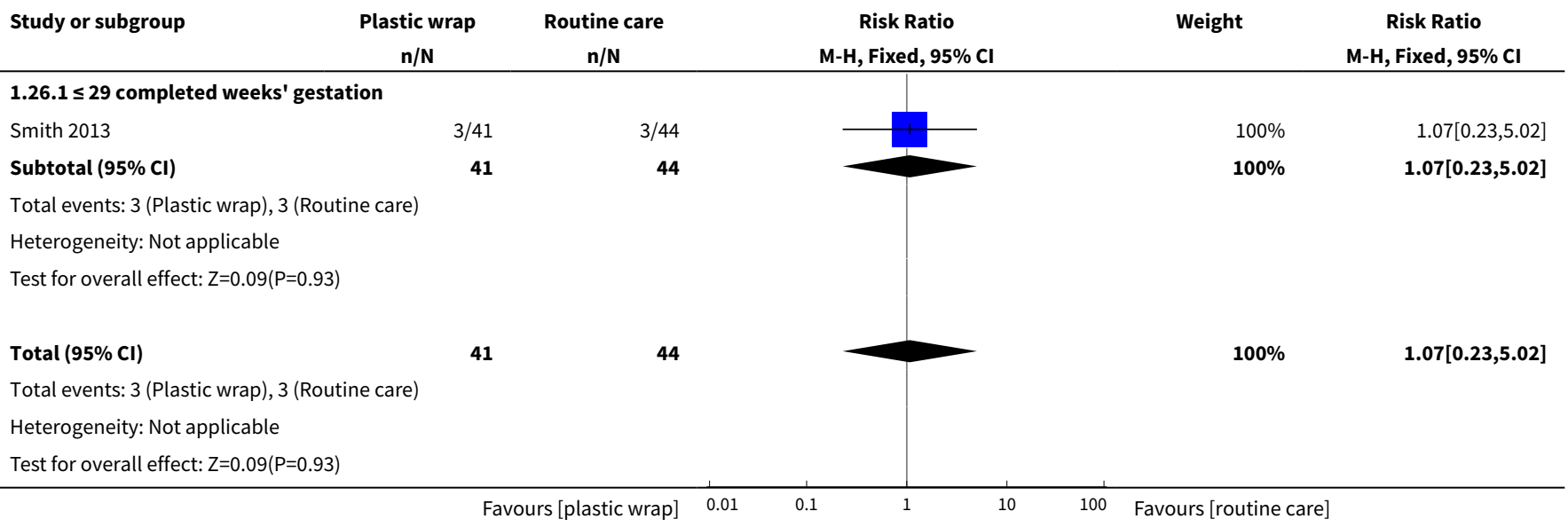

Analysis 1.27. Comparison 1 Plastic wrap or bag versus routine care, Outcome 27 Blood glucose concentration $\mathrm{mmol} / \mathrm{L}$ at 120 minutes after birth.

\begin{tabular}{|c|c|c|c|c|c|}
\hline \multicolumn{6}{|c|}{ Blood glucose concentration $\mathrm{mmol} / \mathrm{L}$ at 120 minutes after birth } \\
\hline Study & Group & $\mathbf{N}$ & Median & Interquartile range & $P$ value \\
\hline \multicolumn{6}{|c|}{ All infants ( $<37$ completed weeks' gestation) } \\
\hline Cardona Torres 2012 & $\begin{array}{l}\text { Plastic bag without dry- } \\
\text { ing }\end{array}$ & 30 & 5.4 & $3.2-8.8$ & $p>0.05$ \\
\hline Cardona Torres 2012 & Routine care & 30 & 3.6 & $2.7-4.4$ & \\
\hline
\end{tabular}

Analysis 1.28. Comparison 1 Plastic wrap or bag versus routine care, Outcome 28 Bronchopulmonary dysplasia (BPD).

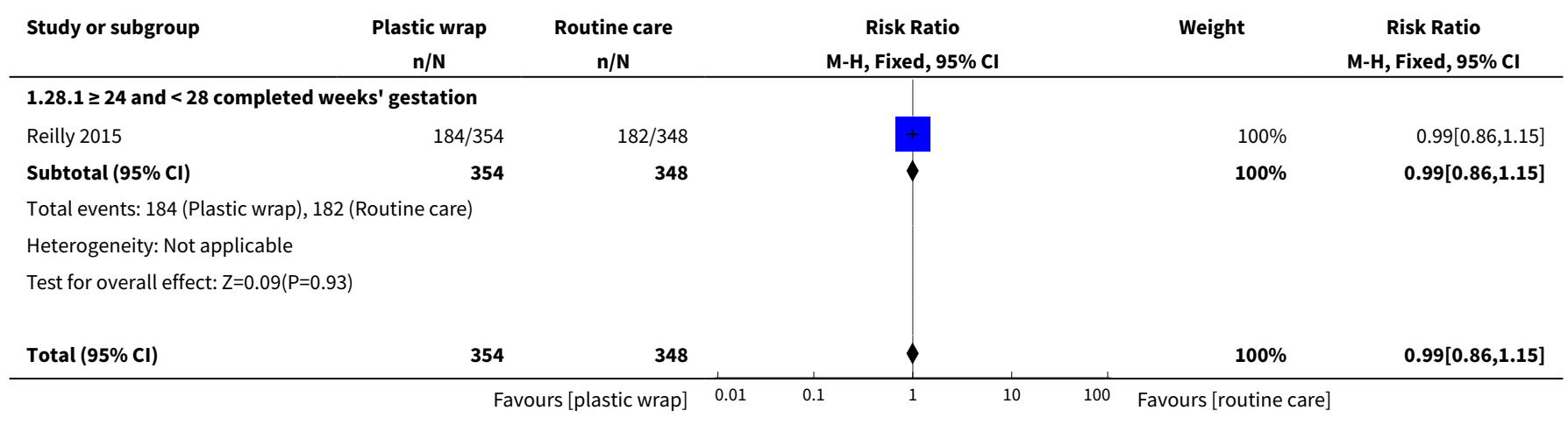




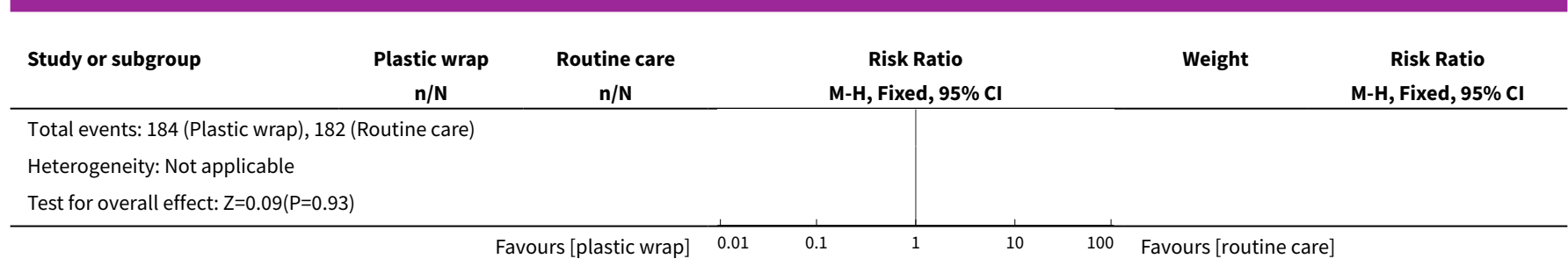

Analysis 1.29. Comparison 1 Plastic wrap or bag versus routine care, Outcome 29 BPD steroids.

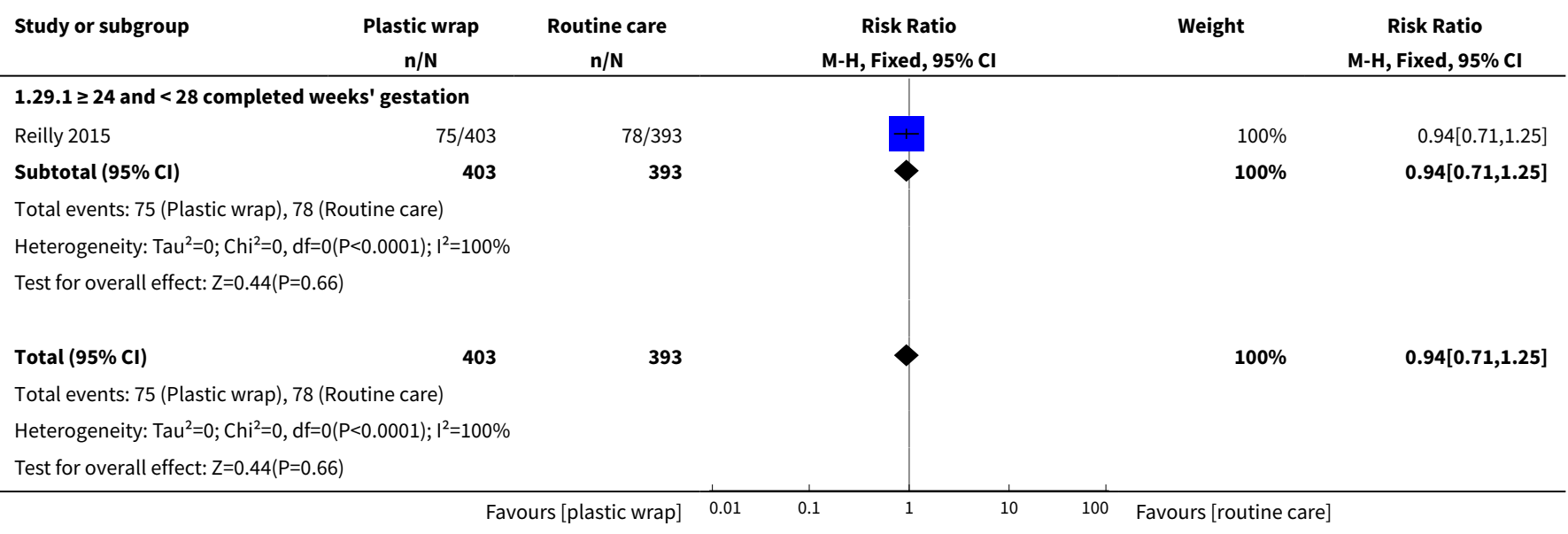

Analysis 1.30. Comparison 1 Plastic wrap or bag versus routine care, Outcome 30 Duration of hospitalisation (days).

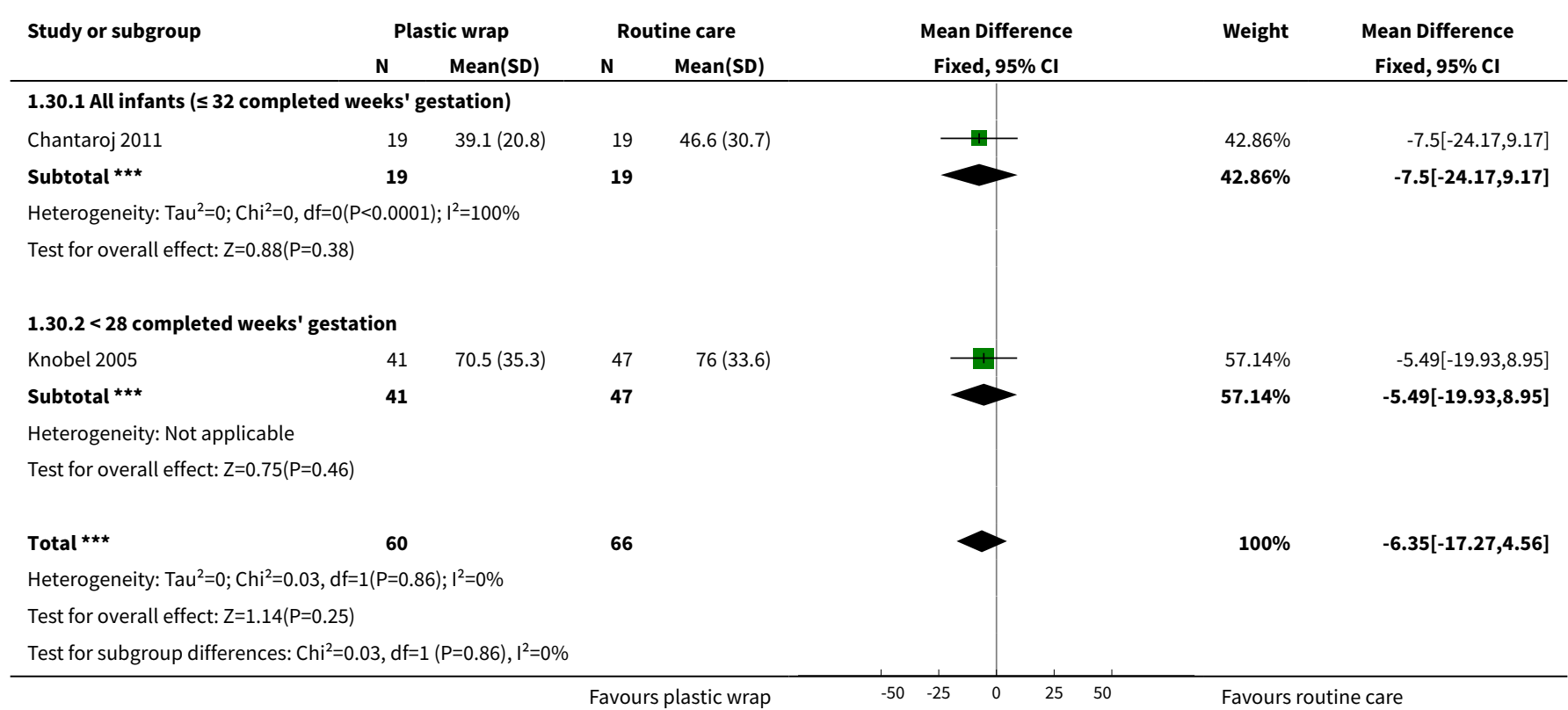


Analysis 1.31. Comparison 1 Plastic wrap or bag versus routine care, Outcome 31 Duration of hospitalisation (days).

\begin{tabular}{|c|c|c|c|c|c|}
\hline \multicolumn{6}{|c|}{ Duration of hospitalisation (days) } \\
\hline Study & Group & $\mathbf{N}$ & Median & IQR & P-value \\
\hline Rohana 2011 & $\begin{array}{l}\text { Intervention (polyethyl- } \\
\text { ene wrap) }\end{array}$ & 50 & 37 & 16.0 to 61.5 & 0.29 \\
\hline Rohana 2011 & Control (routine care) & 60 & 30 & 15.2 to 46.0 & \\
\hline
\end{tabular}

Analysis 1.32. Comparison 1 Plastic wrap or bag versus routine care, Outcome 32 Duration of continuous positive airway pressure (CPAP) (days).

\begin{tabular}{|c|c|c|c|c|c|}
\hline \multicolumn{6}{|c|}{ Duration of continuous positive airway pressure (CPAP) (days) } \\
\hline Study & Group & $\mathbf{N}$ & Median & IQR & P-value \\
\hline Rohana 2011 & $\begin{array}{l}\text { Intervention (polyethyl- } \\
\text { ene wrap) }\end{array}$ & 50 & 27.0 & 3.5 to 43.8 & 0.29 \\
\hline Rohana 2011 & Control (routine care) & 60 & 15.0 & 1 to 41.2 & \\
\hline
\end{tabular}

Analysis 1.33. Comparison 1 Plastic wrap or bag versus routine care, Outcome 33 Duration of oxygen therapy (days).

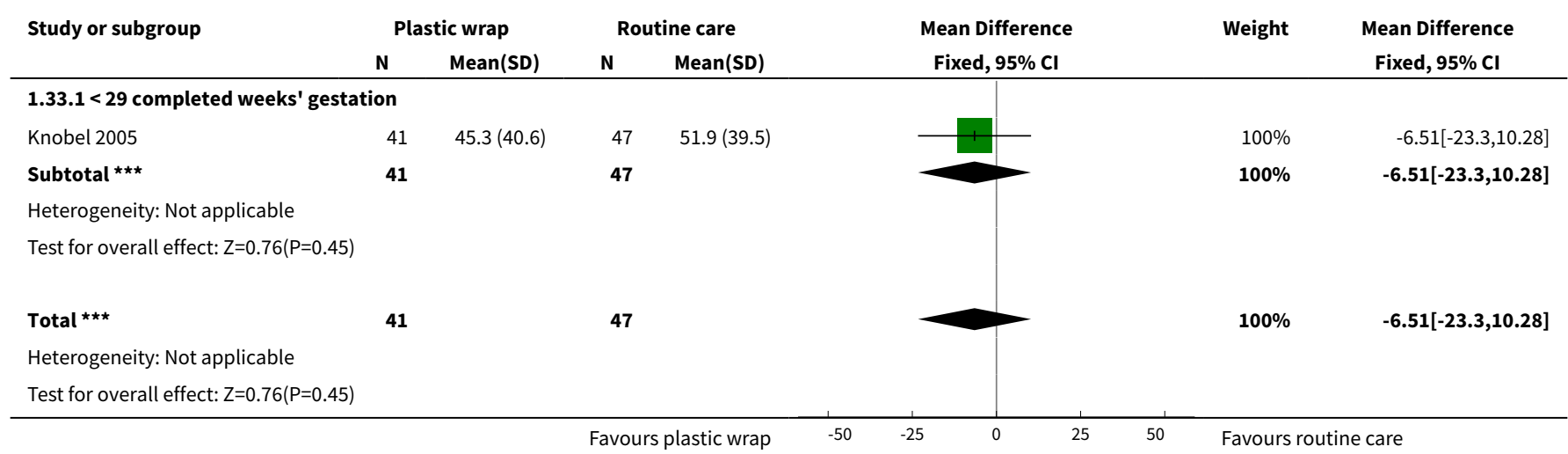

Analysis 1.34. Comparison 1 Plastic wrap or bag versus routine care, Outcome 34 Duration of ventilation (days).

\begin{tabular}{|c|c|c|c|c|c|}
\hline \multicolumn{6}{|c|}{ Duration of ventilation (days) } \\
\hline Study & Group & $\mathbf{N}$ & Median & IQR & P-value \\
\hline Rohana 2011 & $\begin{array}{l}\text { Intervention (polyethyl- } \\
\text { ene wrap) }\end{array}$ & 50 & 3.0 & 0 to 3 & 0.30 \\
\hline Rohana 2011 & Control (routine care) & 60 & 2.6 & 0 to 2 & \\
\hline
\end{tabular}

Analysis 1.35. Comparison 1 Plastic wrap or bag versus routine care, Outcome 35 Gastrointestinal perforation.

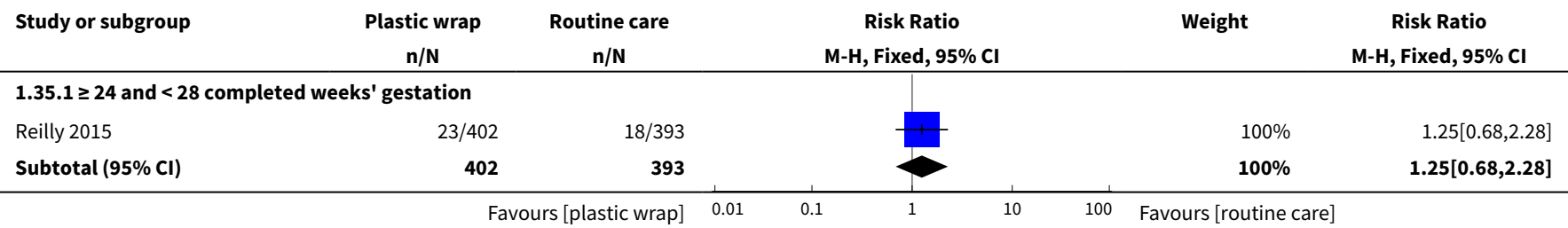




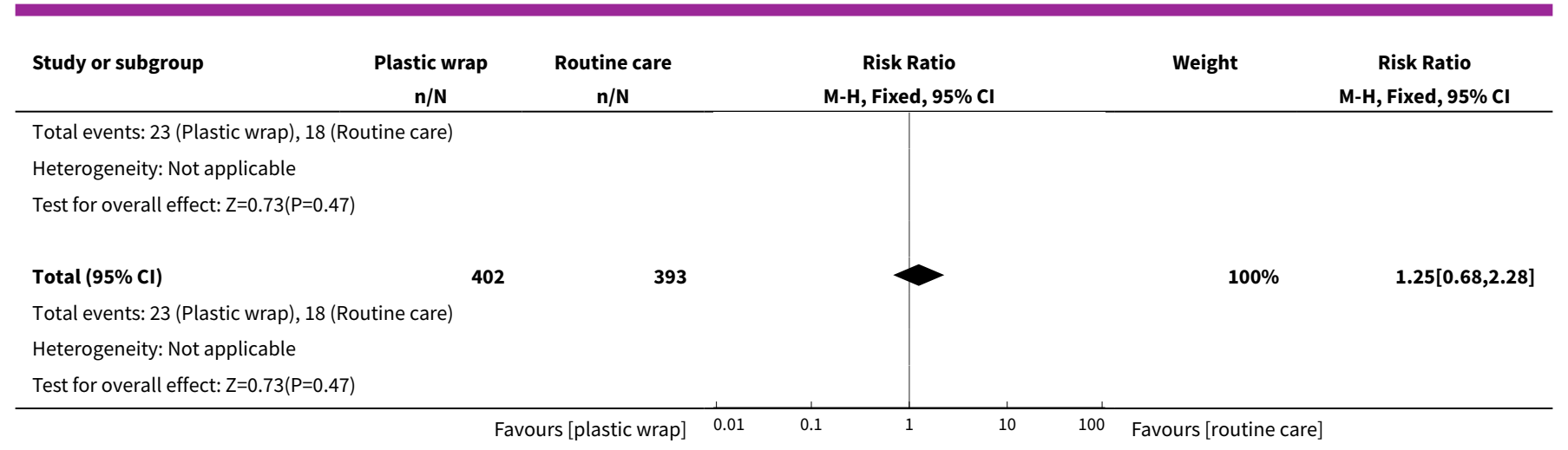

Analysis 1.36. Comparison 1 Plastic wrap or bag versus routine care, Outcome 36 Intubation in delivery room.

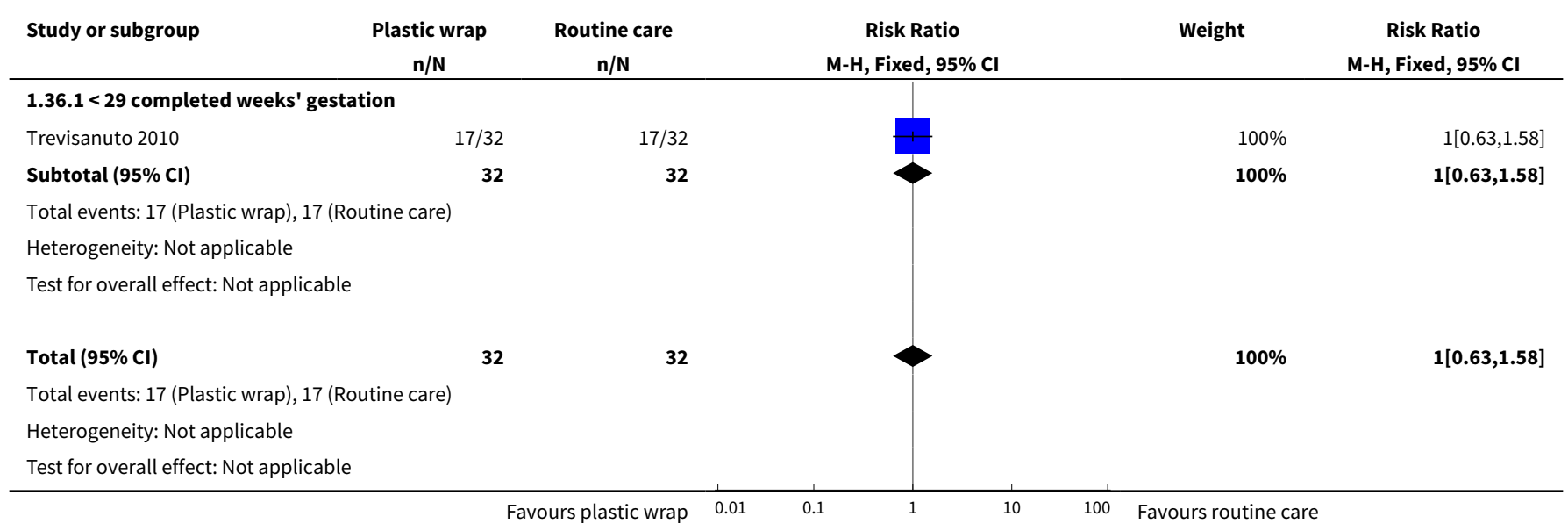

Analysis 1.37. Comparison 1 Plastic wrap or bag versus routine care, Outcome 37 Necrotising enterocolitis (NEC).

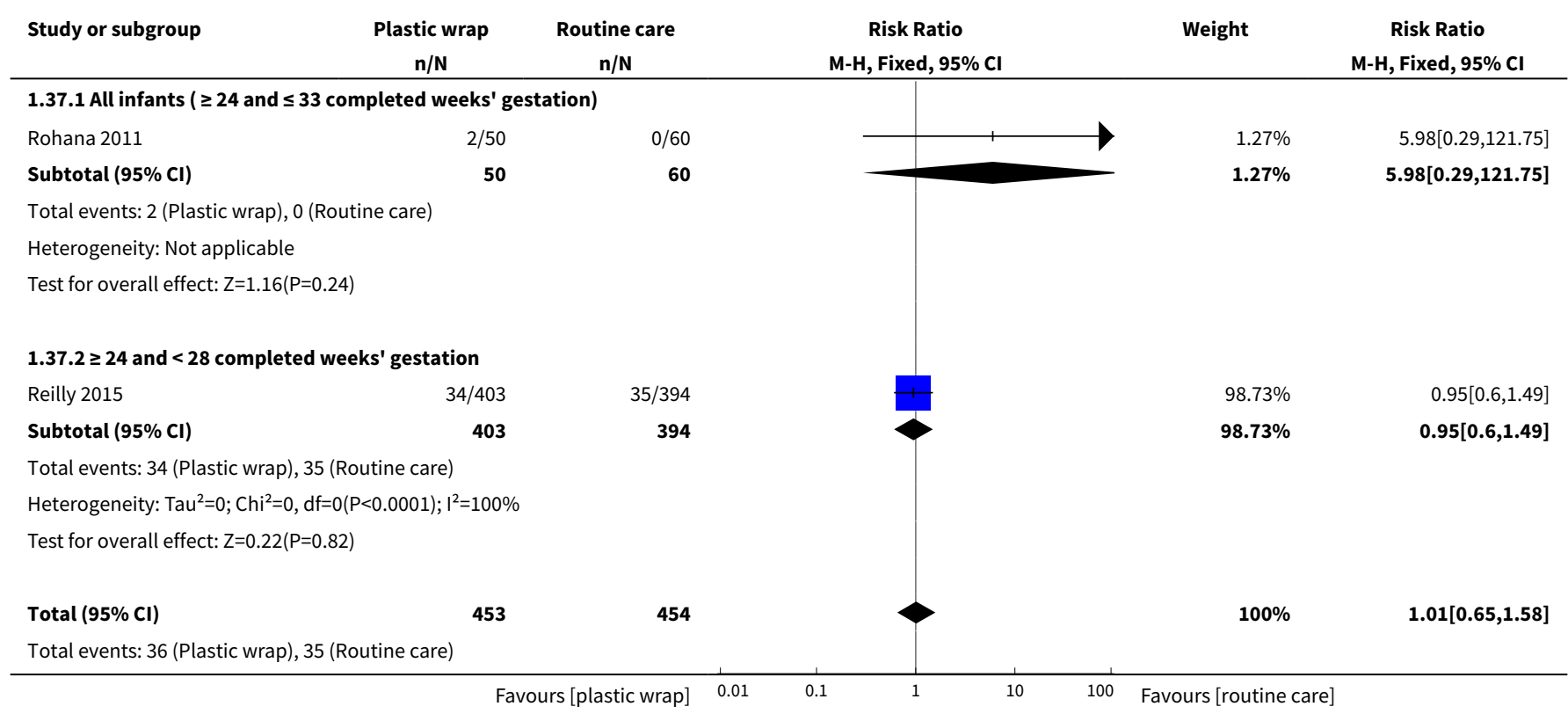




\begin{tabular}{|c|c|c|c|c|c|c|c|}
\hline Study or subgroup & $\begin{array}{l}\text { Plastic wrap } \\
\qquad \mathbf{n} / \mathbf{N}\end{array}$ & $\begin{array}{c}\text { Routine care } \\
\mathbf{n} / \mathbf{N}\end{array}$ & \multicolumn{3}{|c|}{$\begin{array}{c}\text { Risk Ratio } \\
\text { M-H, Fixed, } 95 \% \mathrm{Cl}\end{array}$} & Weight & $\begin{array}{c}\text { Risk Ratio } \\
\text { M-H, Fixed, 95\% C } \\
\end{array}$ \\
\hline \multicolumn{8}{|c|}{$\begin{array}{l}\text { Heterogeneity: } \text { Tau }^{2}=0 ; \mathrm{Chi}^{2}=1.41, \mathrm{df}=1(\mathrm{P}=0.23) ; \mathrm{I}^{2}=29.21 \% \\
\text { Test for overall effect: } \mathrm{Z}=0.06(\mathrm{P}=0.95)\end{array}$} \\
\hline
\end{tabular}

Analysis 1.38. Comparison 1 Plastic wrap or bag versus routine care, Outcome 38 Patent ductus arteriosus (PDA).

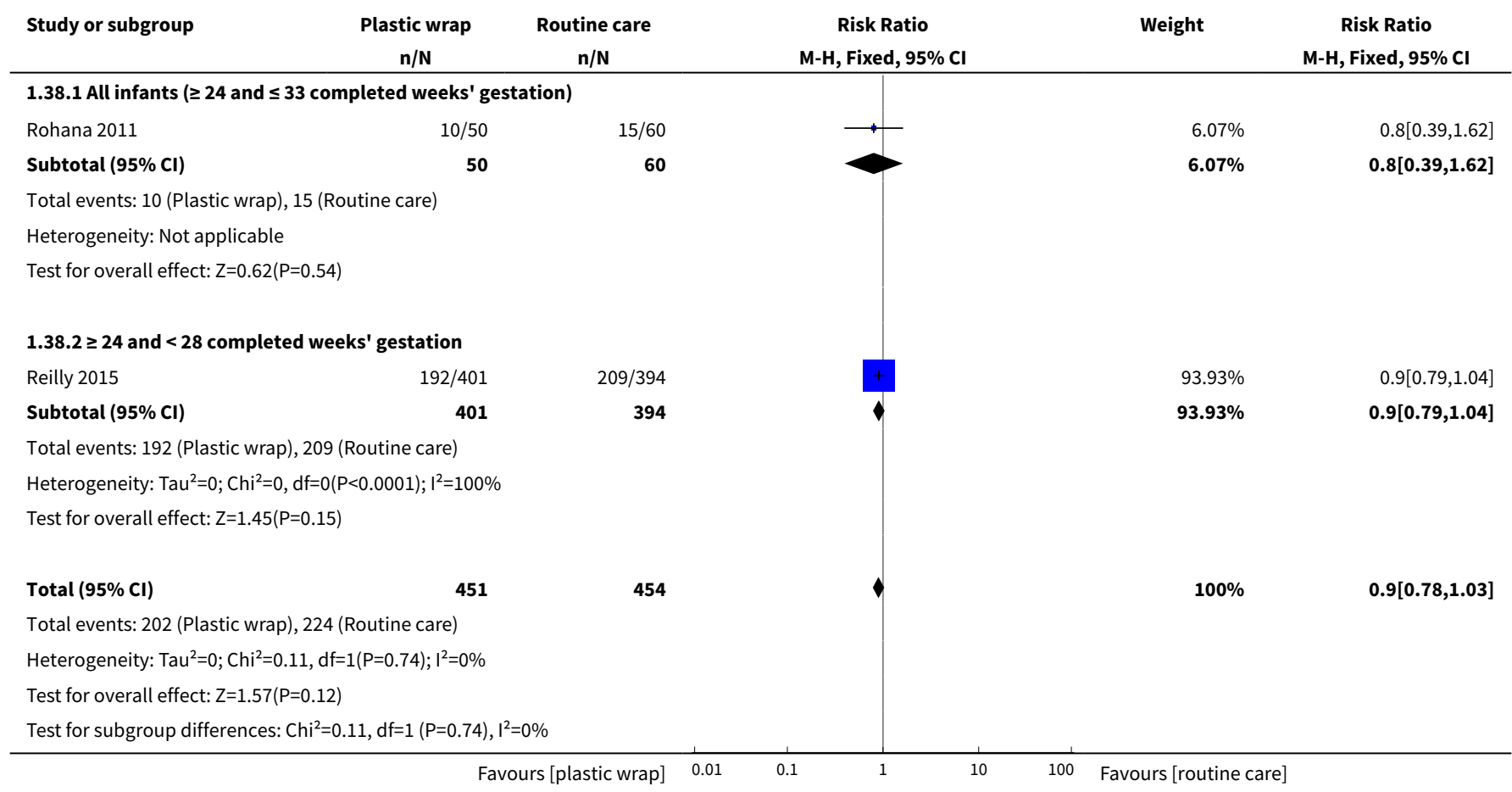

\section{Analysis 1.39. Comparison 1 Plastic wrap or bag versus routine care, Outcome 39 Pneumothorax.}

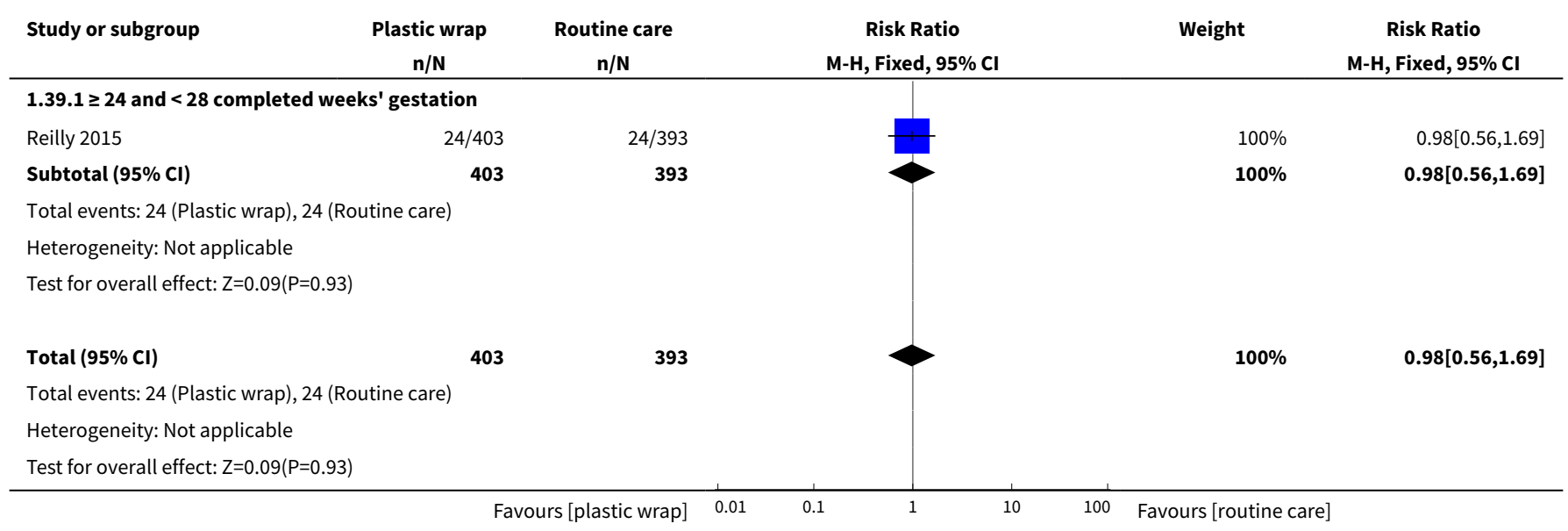


Analysis 1.40. Comparison 1 Plastic wrap or bag versus routine care, Outcome 40 Pulmonary haemorrhage.

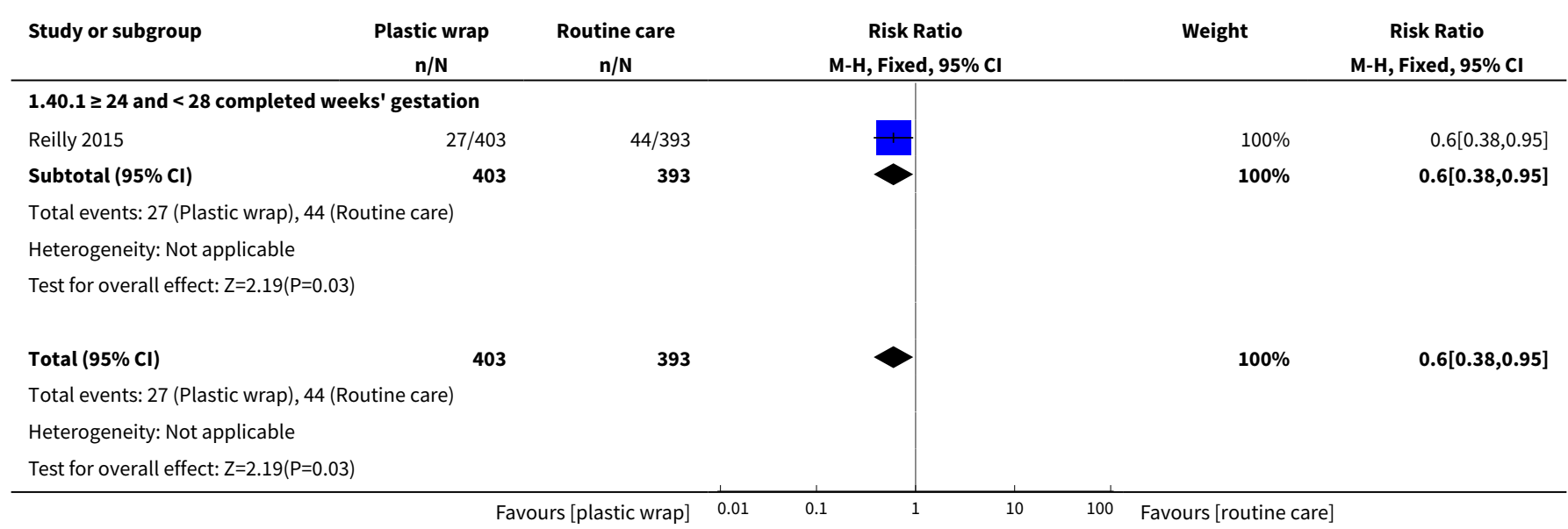

Analysis 1.41. Comparison 1 Plastic wrap or bag versus routine care, Outcome 41 Requirement for bubble continuous positive airway pressure (BCPAP).

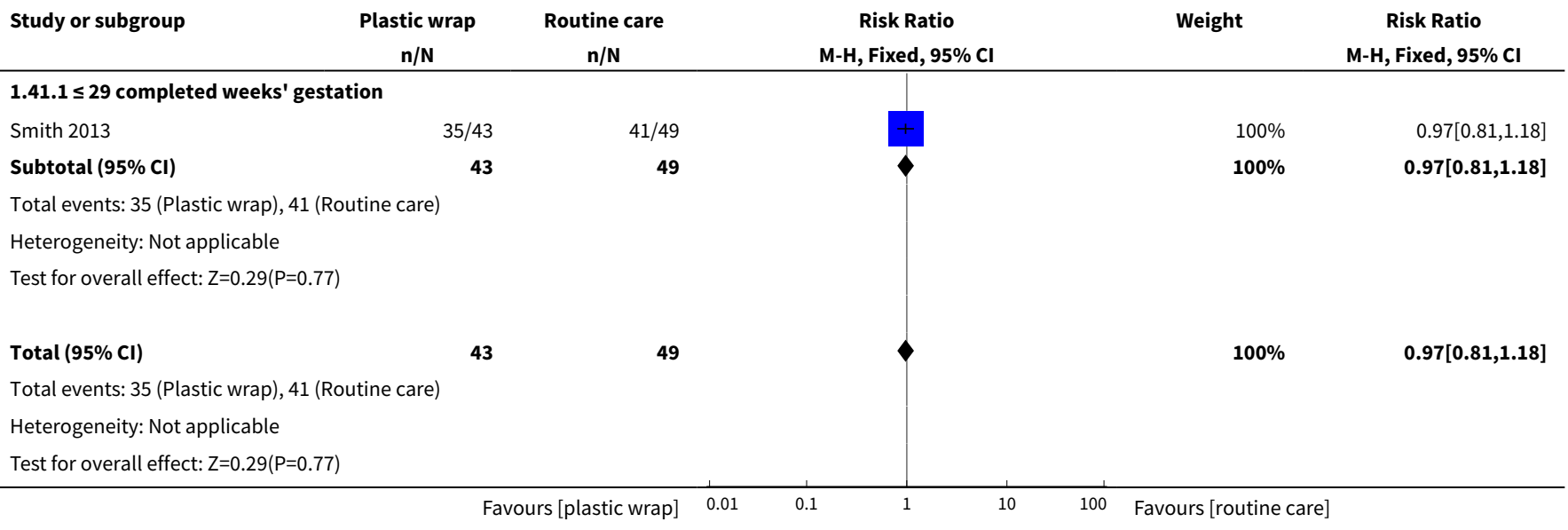

\section{Analysis 1.42. Comparison 1 Plastic wrap or bag versus routine care, Outcome 42 Requirement for ventilation.}

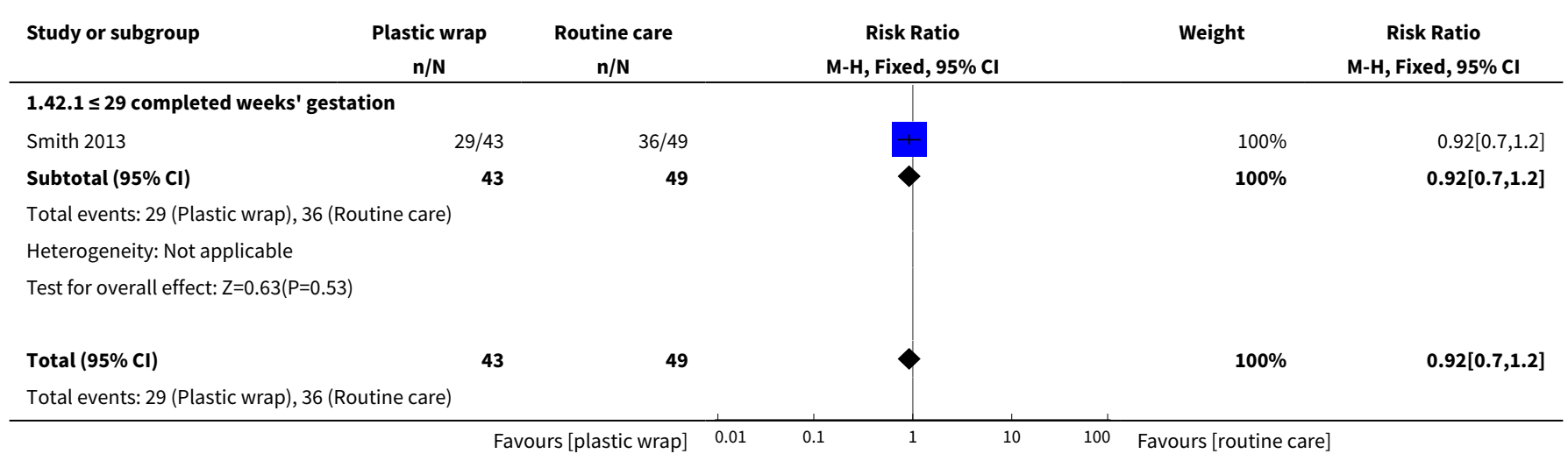




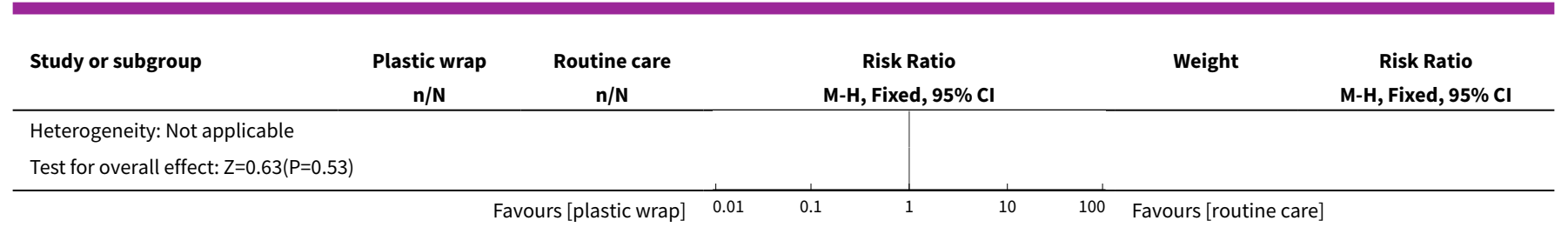

Analysis 1.43. Comparison 1 Plastic wrap or bag versus routine care, Outcome 43 Respiratory distress syndrome (RDS).

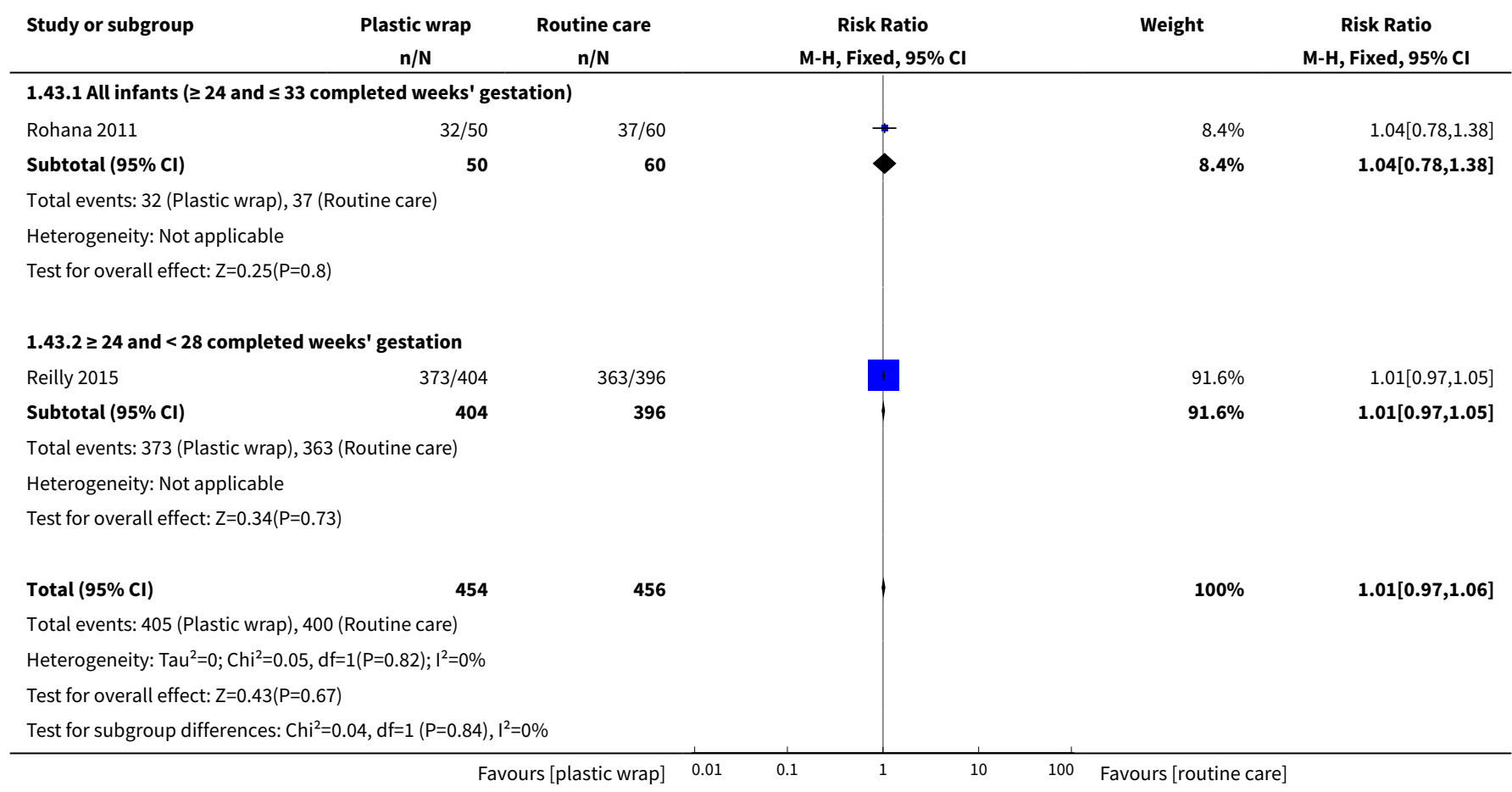

Analysis 1.44. Comparison 1 Plastic wrap or bag versus routine care, Outcome 44 Retinopathy of prematurity (ROP).

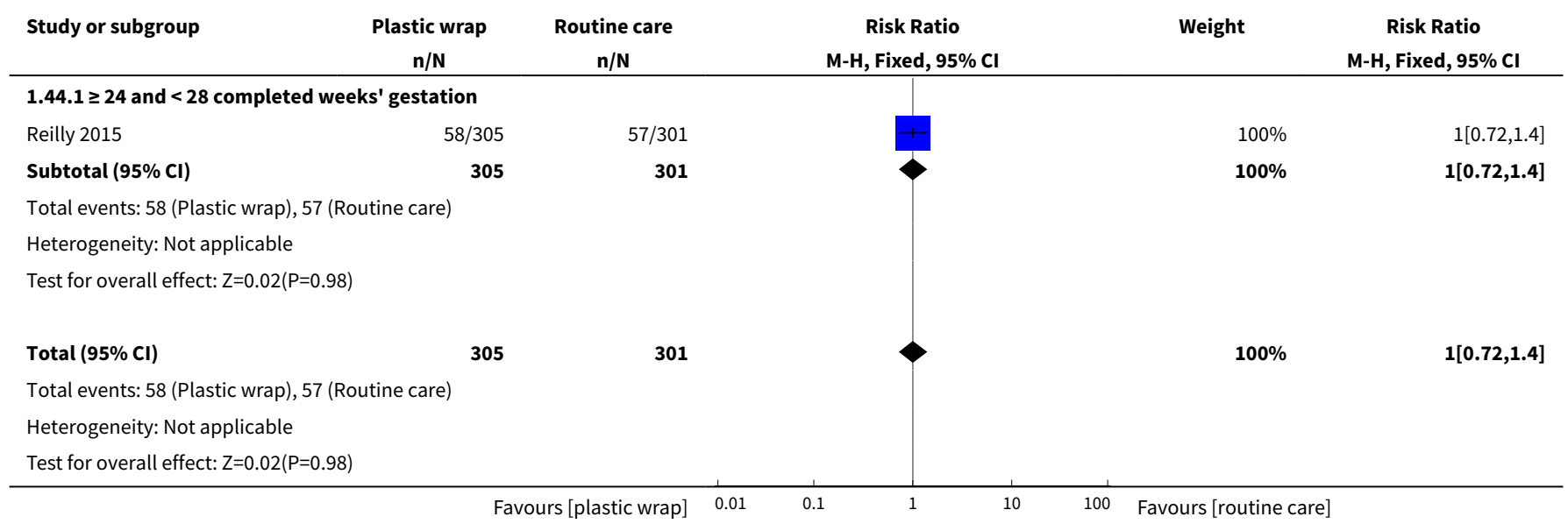


Analysis 1.45. Comparison 1 Plastic wrap or bag versus routine care, Outcome 45 Sepsis (late).

\begin{tabular}{|c|c|c|c|c|c|}
\hline Study or subgroup & $\begin{array}{c}\text { Plastic wrap } \\
\mathrm{n} / \mathrm{N}\end{array}$ & $\begin{array}{c}\text { Routine care } \\
\mathrm{n} / \mathrm{N}\end{array}$ & $\begin{array}{c}\text { Risk Ratio } \\
\text { M-H, Fixed, 95\% Cl }\end{array}$ & Weight & $\begin{array}{c}\text { Risk Ratio } \\
\text { M-H, Fixed, } 95 \% \mathrm{Cl} \\
\end{array}$ \\
\hline \multicolumn{6}{|c|}{ 1.45.1 $\leq 29$ completed weeks' gestation } \\
\hline Reilly 2015 & $78 / 373$ & $93 / 369$ & E & $82.69 \%$ & $0.83[0.64,1.08]$ \\
\hline Smith 2013 & $20 / 41$ & $21 / 47$ & $\rightarrow$ & $17.31 \%$ & $1.09[0.7,1.71]$ \\
\hline Subtotal $(95 \% \mathrm{Cl})$ & 414 & 416 & $\bullet$ & $100 \%$ & $0.88[0.7,1.1]$ \\
\hline \multicolumn{6}{|c|}{ Total events: 98 (Plastic wrap), 114 (Routine care) } \\
\hline \multicolumn{6}{|c|}{ Heterogeneity: $\operatorname{Tau}^{2}=0 ; \mathrm{Chi}^{2}=1.1, \mathrm{df}=1(\mathrm{P}=0.29) ; \mathrm{I}^{2}=8.92 \%$} \\
\hline \multicolumn{6}{|c|}{ Test for overall effect: $\mathrm{Z}=1.14(\mathrm{P}=0.25)$} \\
\hline Total $(95 \% \mathrm{Cl})$ & 414 & 416 & $\diamond$ & $100 \%$ & $0.88[0.7,1.1]$ \\
\hline \multicolumn{6}{|c|}{ Total events: 98 (Plastic wrap), 114 (Routine care) } \\
\hline \multicolumn{6}{|c|}{ Heterogeneity: $\operatorname{Tau}^{2}=0 ; \mathrm{Chi}^{2}=1.1, \mathrm{df}=1(\mathrm{P}=0.29) ; \mathrm{I}^{2}=8.92 \%$} \\
\hline Test for overall effec & & & & & \\
\hline
\end{tabular}

Analysis 1.46. Comparison 1 Plastic wrap or bag versus routine care, Outcome 46 Sepsis (early).

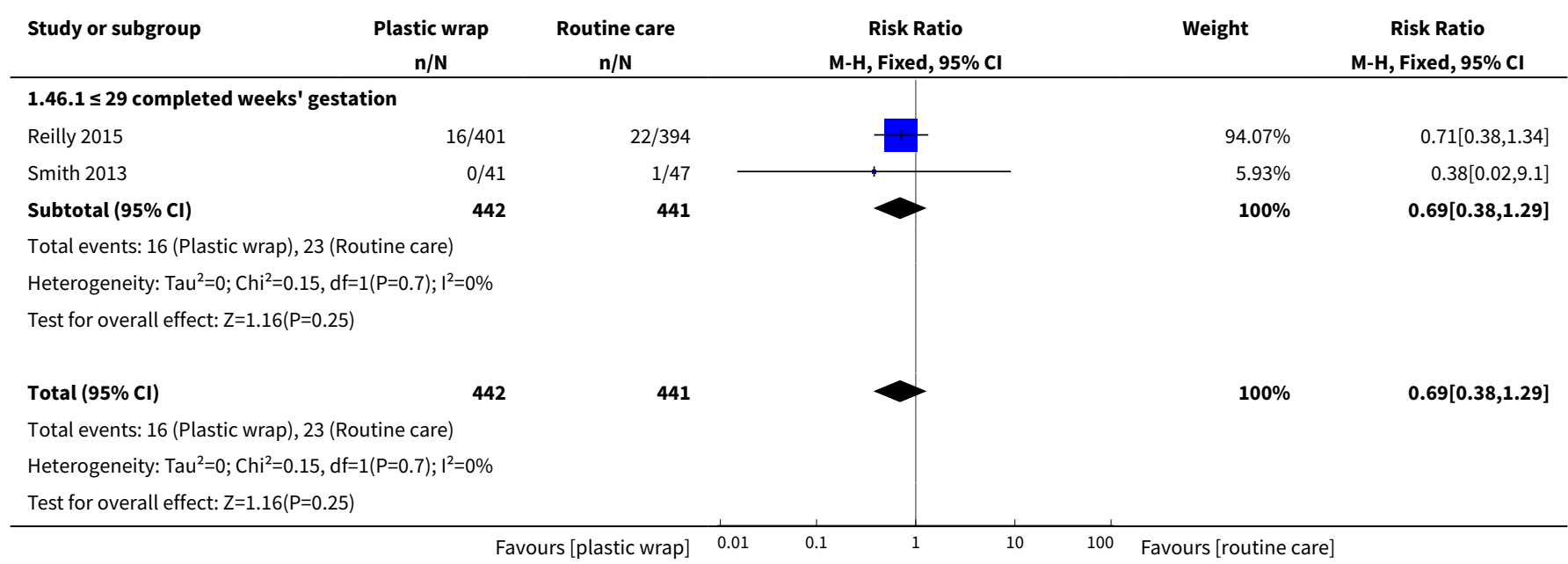

\section{Comparison 2. Plastic wrap versus routine care (interhospital neonatal transport)}

\begin{tabular}{lllll}
\hline Outcome or subgroup title & No. of studies & $\begin{array}{l}\text { No. of partici- } \\
\text { pants }\end{array}$ & Statistical method & Effect size \\
\hline $\begin{array}{l}1 \text { Core body temperature }\left({ }^{\circ} \mathrm{C}\right) \text { on admis- } \\
\text { sion to NICU or up to } 2 \text { hours after birth }\end{array}$ & 1 & 96 & $\begin{array}{l}\text { Mean Difference (IV, Fixed, } \\
95 \% \mathrm{Cl})\end{array}$ & $0.70[0.32,1.08]$ \\
\hline $\begin{array}{l}1.1<37 \text { completed weeks' gestation and } \\
\leq 2500 \text { grams birth weight }\end{array}$ & 1 & 96 & $\begin{array}{l}\text { Mean Difference (IV, Fixed, } \\
95 \% \mathrm{Cl})\end{array}$ & $0.70[0.32,1.08]$ \\
\hline
\end{tabular}




\begin{tabular}{|c|c|c|c|c|}
\hline Outcome or subgroup title & No. of studies & $\begin{array}{l}\text { No. of partici- } \\
\text { pants }\end{array}$ & Statistical method & Effect size \\
\hline $\begin{array}{l}2 \text { Hypothermia on admission to NICU: } \\
\text { core body temperature }<36.5^{\circ} \mathrm{C} \text { or skin } \\
\text { temperature }<36^{\circ} \mathrm{C}\end{array}$ & 1 & 96 & $\begin{array}{l}\text { Risk Ratio (M-H, Fixed, } \\
95 \% \mathrm{Cl})\end{array}$ & $0.48[0.27,0.84]$ \\
\hline $\begin{array}{l}2.1<37 \text { completed weeks' gestation and } \\
\leq 2500 \text { grams birth weight }\end{array}$ & 1 & 96 & $\begin{array}{l}\text { Risk Ratio (M-H, Fixed, } \\
95 \% \mathrm{Cl})\end{array}$ & $0.48[0.27,0.84]$ \\
\hline $\begin{array}{l}3 \text { Decrease in temperature (axillary }{ }^{\circ} \mathrm{C} \text { ) } \\
\text { from baseline before transport to NICU } \\
\text { admission }\end{array}$ & 1 & 96 & $\begin{array}{l}\text { Mean Difference (IV, Fixed, } \\
95 \% \mathrm{CI})\end{array}$ & $\begin{array}{l}-0.40[-0.61 \\
-0.19]\end{array}$ \\
\hline $\begin{array}{l}3.1<37 \text { completed weeks' gestation and } \\
\leq 2500 \text { grams birth weight }\end{array}$ & 1 & 96 & $\begin{array}{l}\text { Mean Difference (IV, Fixed, } \\
95 \% \mathrm{CI})\end{array}$ & $\begin{array}{l}-0.40[-0.61 \\
-0.19]\end{array}$ \\
\hline $\begin{array}{l}4 \text { Hyperthermia on admission to NICU: } \\
\text { core body temperature }>37.5^{\circ} \mathrm{C}\end{array}$ & 1 & 96 & $\begin{array}{l}\text { Risk Ratio (M-H, Fixed, } \\
95 \% \mathrm{Cl})\end{array}$ & $2.88[0.12,68.98]$ \\
\hline $\begin{array}{l}4.1<37 \text { completed weeks' gestation and } \\
\leq 2500 \text { grams birth weight }\end{array}$ & 1 & 96 & $\begin{array}{l}\text { Risk Ratio (M-H, Fixed, } \\
95 \% \mathrm{Cl})\end{array}$ & $2.88[0.12,68.98]$ \\
\hline 5 Base excess & 1 & 96 & $\begin{array}{l}\text { Mean Difference (IV, Fixed, } \\
95 \% \mathrm{CI} \text { ) }\end{array}$ & $0.60[-0.49,1.69]$ \\
\hline $\begin{array}{l}5.1<37 \text { completed weeks' gestation and } \\
\leq 2500 \text { grams birth weight }\end{array}$ & 1 & 96 & $\begin{array}{l}\text { Mean Difference (IV, Fixed, } \\
95 \% \mathrm{CI} \text { ) }\end{array}$ & $0.60[-0.49,1.69]$ \\
\hline 6 Blood gas $\mathrm{pH}$ & 1 & 96 & $\begin{array}{l}\text { Mean Difference (IV, Fixed, } \\
95 \% \mathrm{CI} \text { ) }\end{array}$ & $0.0[-0.02,0.02]$ \\
\hline $\begin{array}{l}6.1<37 \text { completed weeks' gestation and } \\
\leq 2500 \text { grams birth weight }\end{array}$ & 1 & 96 & $\begin{array}{l}\text { Mean Difference (IV, Fixed, } \\
95 \% \mathrm{CI})\end{array}$ & $0.0[-0.02,0.02]$ \\
\hline 7 Duration of oxygen therapy (days) & 1 & 96 & $\begin{array}{l}\text { Mean Difference (IV, Fixed, } \\
95 \% \mathrm{CI} \text { ) }\end{array}$ & $-2.39[-8.27,3.49]$ \\
\hline $\begin{array}{l}7.1<37 \text { completed weeks' gestation and } \\
\leq 2500 \text { grams birth weight }\end{array}$ & 1 & 96 & $\begin{array}{l}\text { Mean Difference (IV, Fixed, } \\
95 \% \mathrm{CI})\end{array}$ & $-2.39[-8.27,3.49]$ \\
\hline 8 Hemo glucose test & 1 & 96 & $\begin{array}{l}\text { Mean Difference (IV, Fixed, } \\
95 \% \mathrm{CI})\end{array}$ & $\begin{array}{l}7.20[-2.03 \\
16.43]\end{array}$ \\
\hline $\begin{array}{l}8.1<37 \text { completed weeks' gestation and } \\
\leq 2500 \text { grams birth weight }\end{array}$ & 1 & 96 & $\begin{array}{l}\text { Mean Difference (IV, Fixed, } \\
95 \% \mathrm{CI})\end{array}$ & $\begin{array}{l}7.20[-2.03 \\
16.43]\end{array}$ \\
\hline $\begin{array}{l}9 \text { Hypoglycaemia (blood glucose level < } \\
40 \mathrm{mg} / \mathrm{dL} \text { within } 2 \text { hours of birth) }\end{array}$ & 1 & 96 & $\begin{array}{l}\text { Risk Ratio (M-H, Fixed, } \\
95 \% \mathrm{Cl})\end{array}$ & $0.55[0.31,0.98]$ \\
\hline $\begin{array}{l}9.1<37 \text { completed weeks' gestation and } \\
\leq 2500 \text { grams birth weight }\end{array}$ & 1 & 96 & $\begin{array}{l}\text { Risk Ratio (M-H, Fixed, } \\
95 \% \mathrm{Cl})\end{array}$ & $0.55[0.31,0.98]$ \\
\hline 10 Severe metabolic acidosis & 1 & 96 & $\begin{array}{l}\text { Risk Ratio (M-H, Fixed, } \\
95 \% \mathrm{Cl})\end{array}$ & $1.15[0.38,3.52]$ \\
\hline $\begin{array}{l}10.1<37 \text { completed weeks' gestation and } \\
\leq 2500 \text { grams birth weight }\end{array}$ & 1 & 96 & $\begin{array}{l}\text { Risk Ratio (M-H, Fixed, } \\
95 \% \mathrm{Cl})\end{array}$ & $1.15[0.38,3.52]$ \\
\hline
\end{tabular}


Analysis 2.1. Comparison 2 Plastic wrap versus routine care (interhospital neonatal transport), Outcome 1 Core body temperature $\left({ }^{\circ} \mathrm{C}\right)$ on admission to NICU or up to 2 hours after birth.

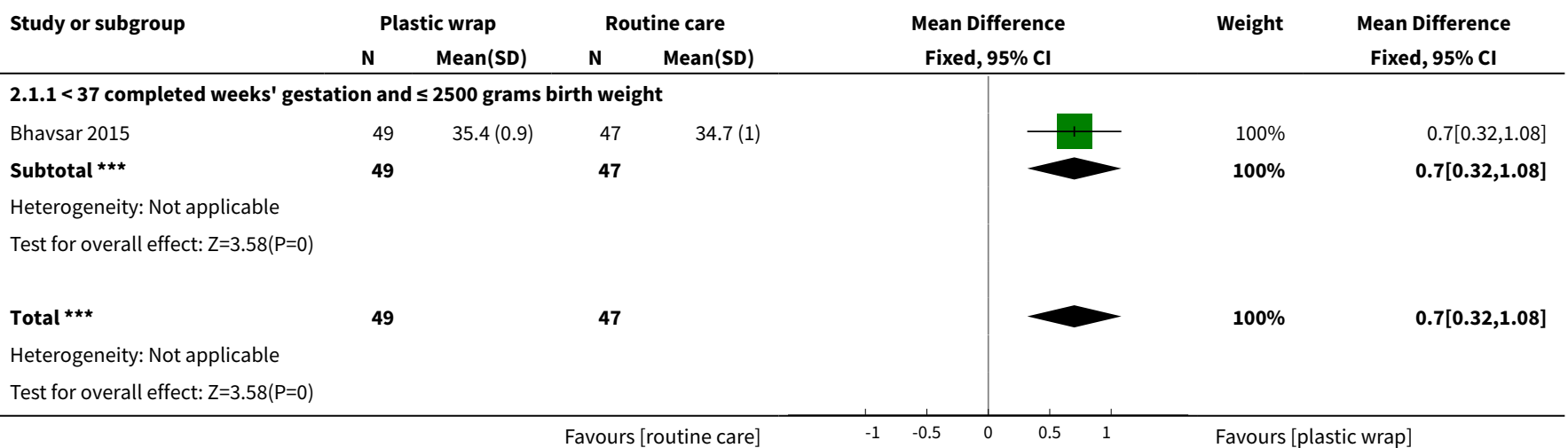

Analysis 2.2. Comparison 2 Plastic wrap versus routine care (interhospital neonatal transport), Outcome 2 Hypothermia on admission to NICU: core body temperature $<36.5^{\circ} \mathrm{C}$ or skin temperature $<36^{\circ} \mathrm{C}$.

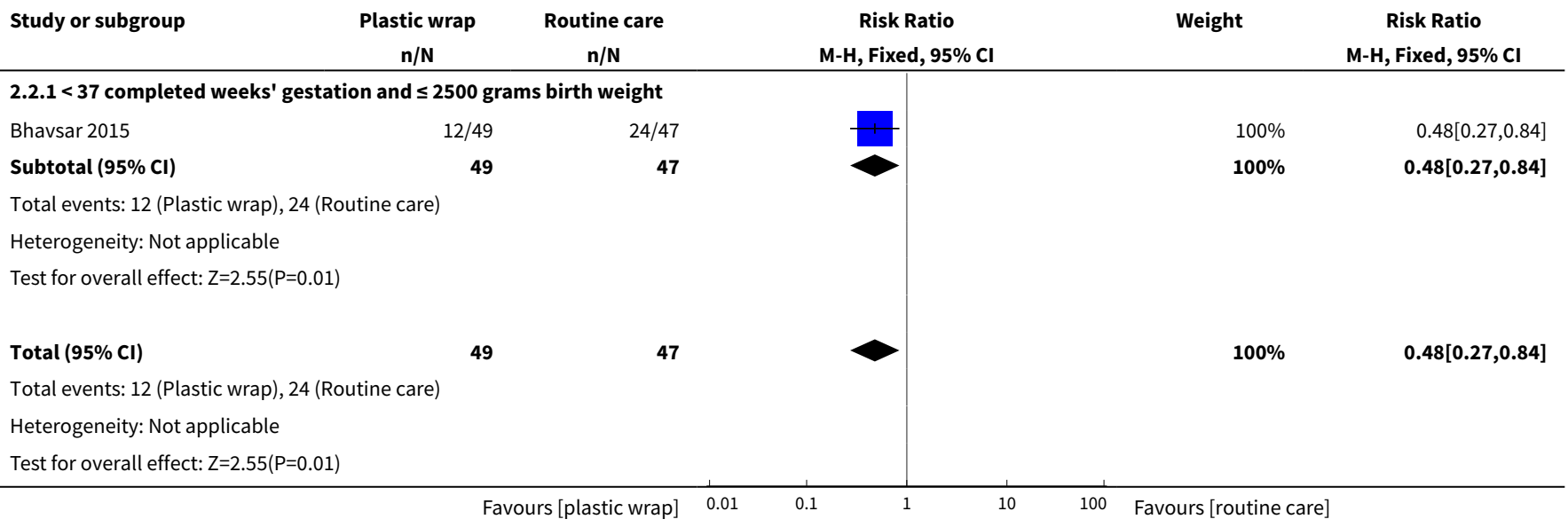

Analysis 2.3. Comparison 2 Plastic wrap versus routine care (interhospital neonatal transport), Outcome 3 Decrease in temperature $\left(\right.$ axillary ${ }^{\circ} \mathrm{C}$ ) from baseline before transport to NICU admission.

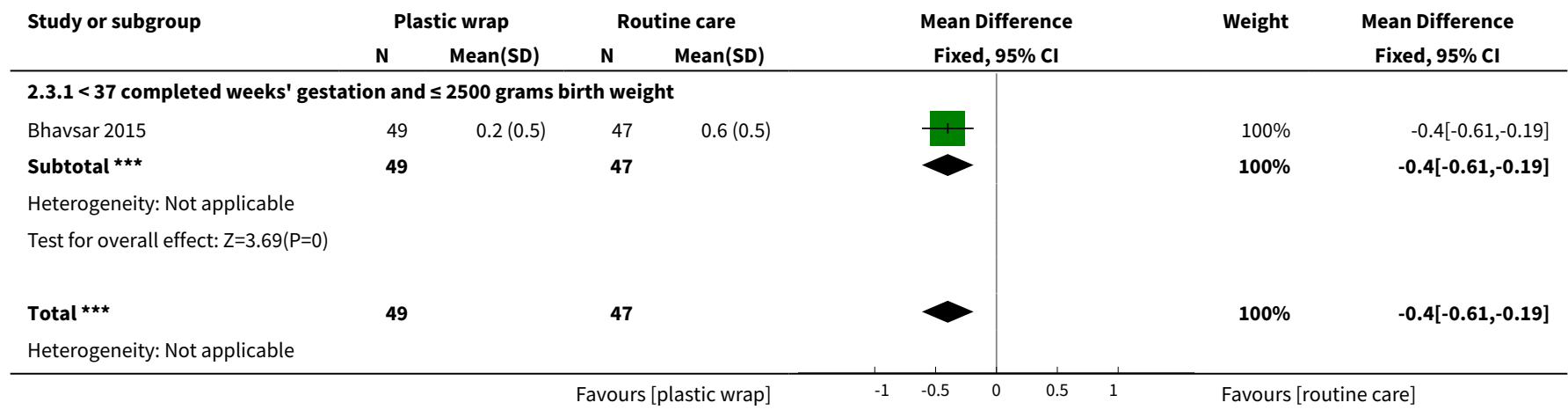




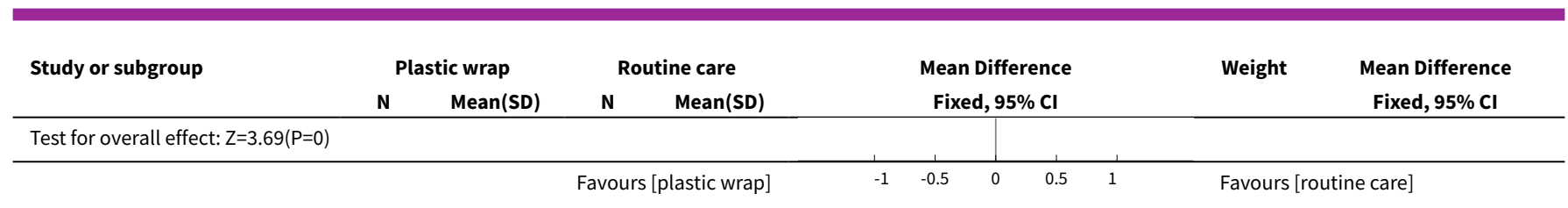

Analysis 2.4. Comparison 2 Plastic wrap versus routine care (interhospital neonatal transport), Outcome 4 Hyperthermia on admission to NICU: core body temperature $>37.5^{\circ} \mathrm{C}$.

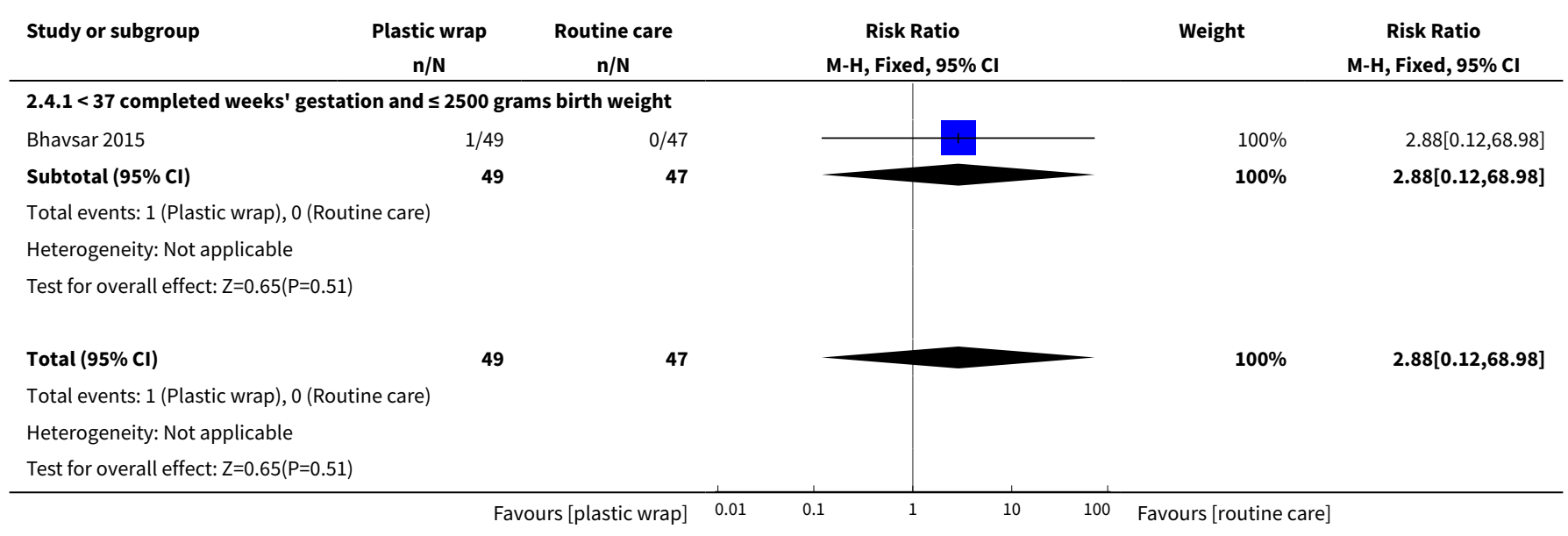

Analysis 2.5. Comparison 2 Plastic wrap versus routine care (interhospital neonatal transport), Outcome 5 Base excess.

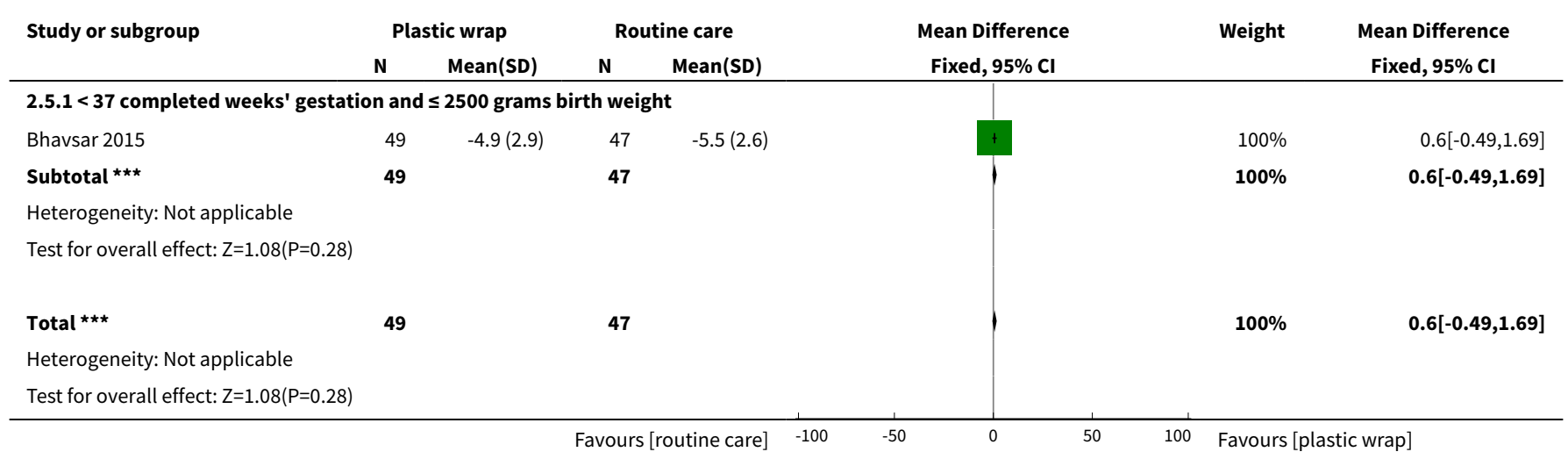

Analysis 2.6. Comparison 2 Plastic wrap versus routine care (interhospital neonatal transport), Outcome 6 Blood gas pH.

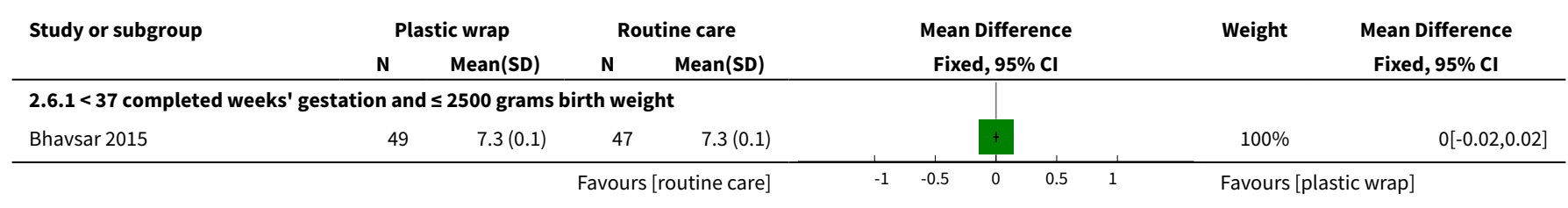




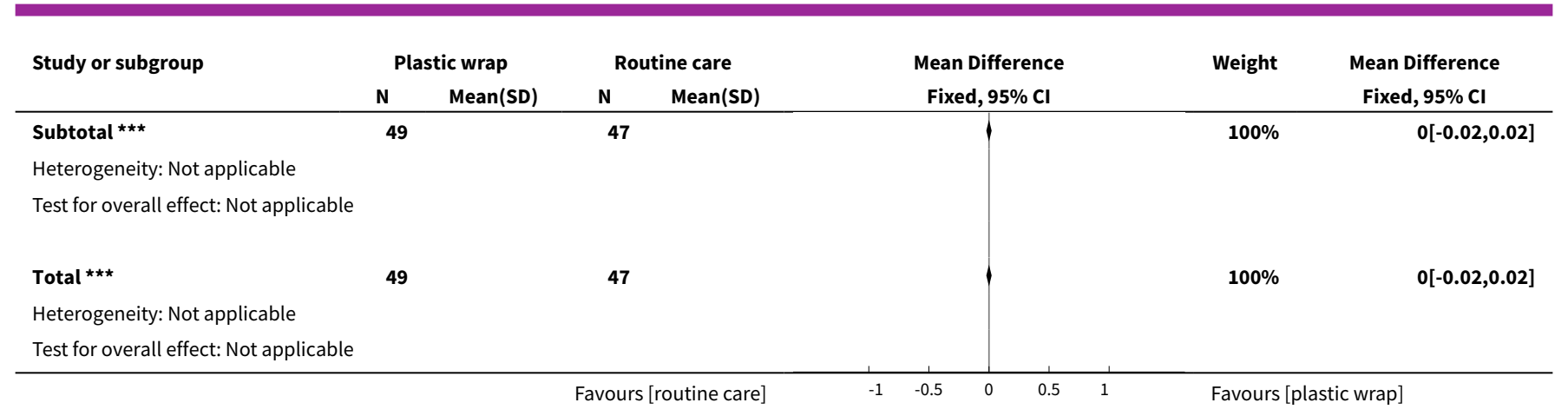

Analysis 2.7. Comparison 2 Plastic wrap versus routine care (interhospital neonatal transport), Outcome 7 Duration of oxygen therapy (days).

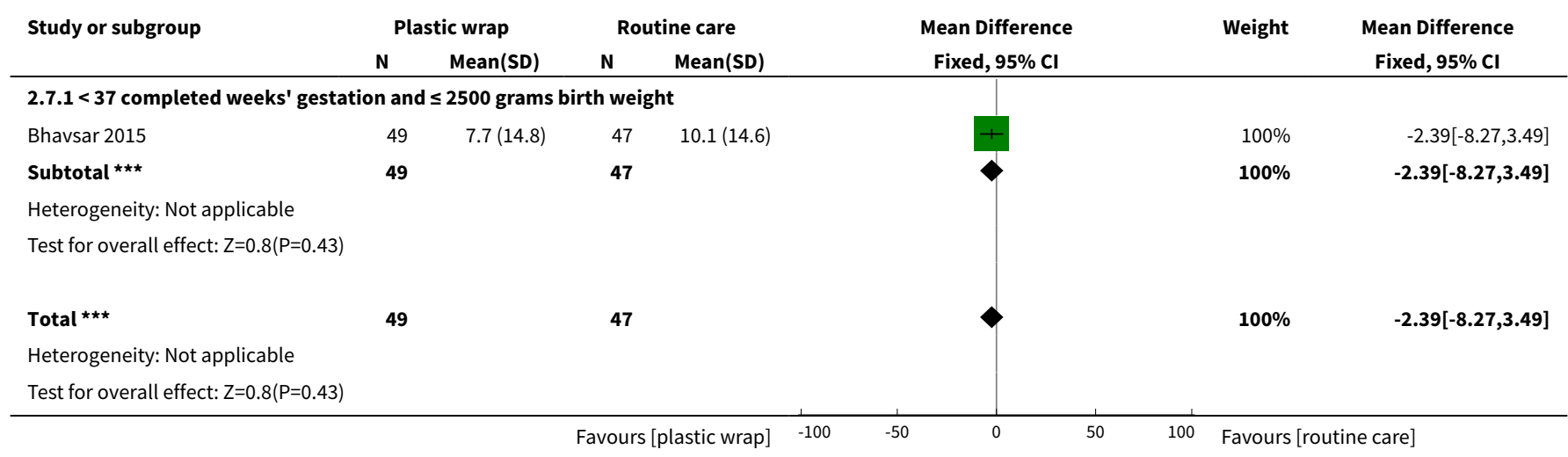

Analysis 2.8. Comparison 2 Plastic wrap versus routine care (interhospital neonatal transport), Outcome 8 Hemo glucose test.

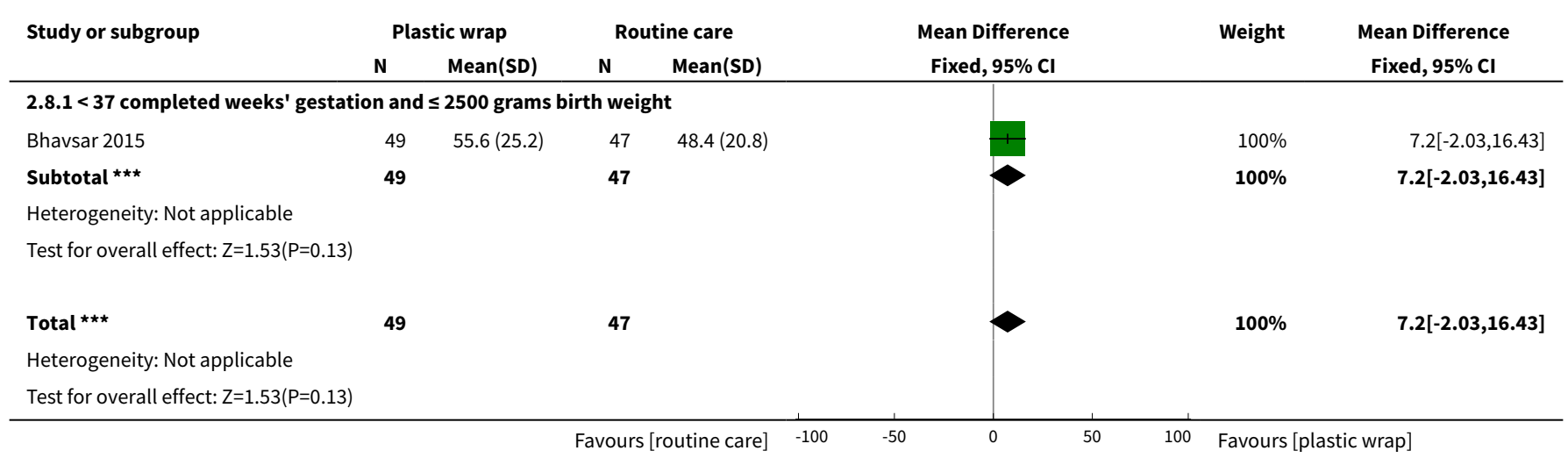


Analysis 2.9. Comparison 2 Plastic wrap versus routine care (interhospital neonatal transport), Outcome 9 Hypoglycaemia (blood glucose level $<40 \mathrm{mg} / \mathrm{dL}$ within 2 hours of birth).

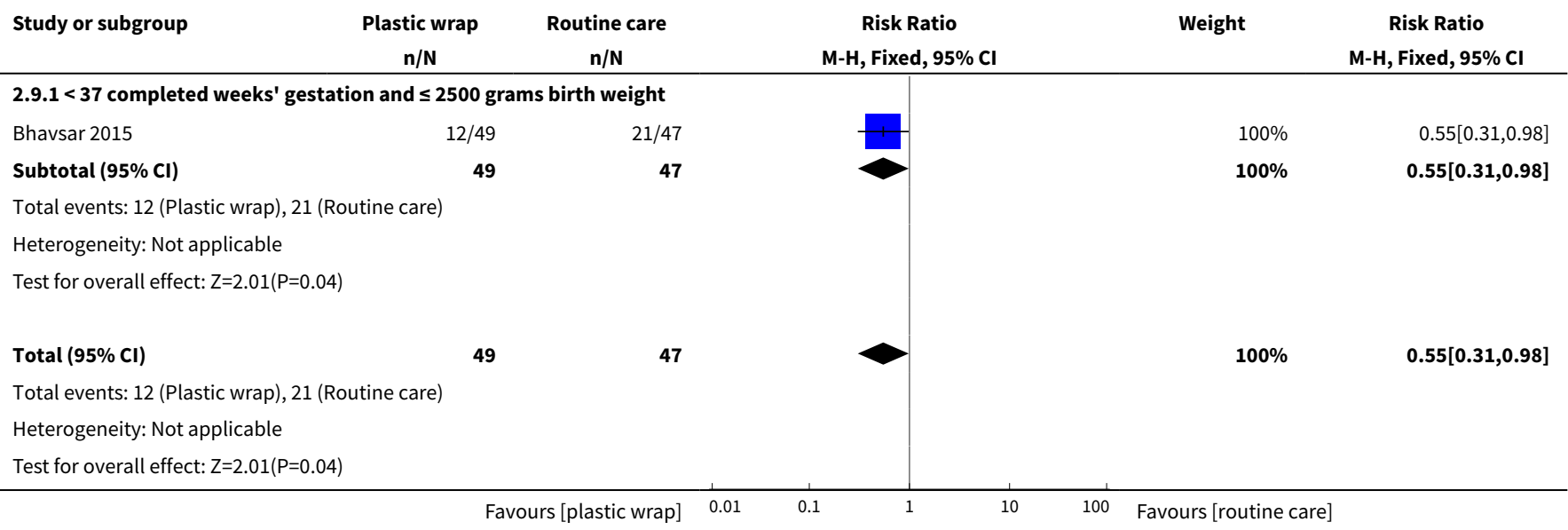

Analysis 2.10. Comparison 2 Plastic wrap versus routine care (interhospital neonatal transport), Outcome 10 Severe metabolic acidosis.

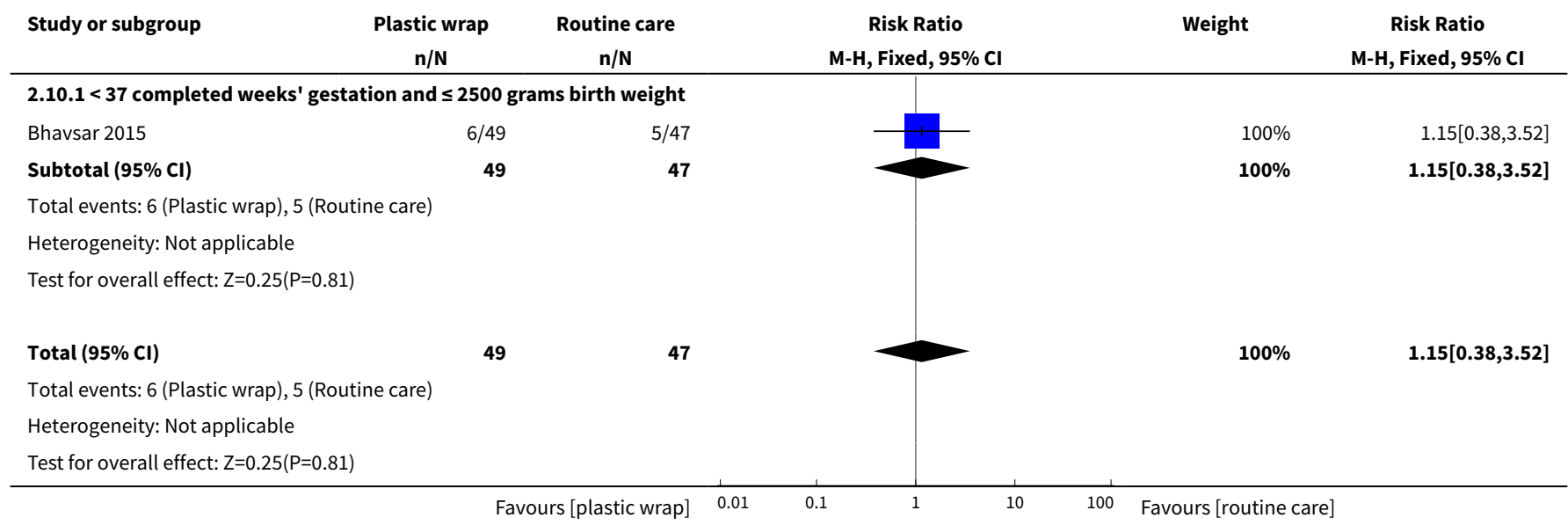

\section{Comparison 3. Plastic bag with previous drying versus routine care}

\begin{tabular}{lllll}
\hline Outcome or subgroup title & No. of studies & $\begin{array}{l}\text { No. of partici- } \\
\text { pants }\end{array}$ & Statistical method & Effect size \\
\hline $\begin{array}{l}1 \text { Core body temperature }\left({ }^{\circ} \mathrm{C}\right) 30 \text { minutes af- } \\
\text { ter birth }\end{array}$ & 1 & 60 & $\begin{array}{l}\text { Mean Difference }(\mathrm{IV}, \\
\text { Fixed, 95\% Cl) }\end{array}$ & $0.30[0.12,0.48]$ \\
\hline $\begin{array}{l}1.1(\geq 28 \text { and }<37 \text { completed weeks' ges- } \\
\begin{array}{l}\text { tation }+(\text { birth weight } \geq 1000 \text { grams and } \leq \\
2499 \text { grams) }\end{array}\end{array}$ & 1 & 60 & $\begin{array}{l}\text { Mean Difference (IV, } \\
\text { Fixed, 95\% Cl) }\end{array}$ & $0.30[0.12,0.48]$ \\
\hline $\begin{array}{l}2 \text { Core body temperature }\left({ }^{\circ} \mathrm{C}\right) 1 \text { hour after } \\
\text { birth }\end{array}$ & 1 & 60 & Mean Difference $(\mathrm{IV}$, & $0.30[0.11,0.49]$ \\
\hline
\end{tabular}




\begin{tabular}{|c|c|c|c|c|}
\hline Outcome or subgroup title & No. of studies & $\begin{array}{l}\text { No. of partici- } \\
\text { pants }\end{array}$ & Statistical method & Effect size \\
\hline $\begin{array}{l}2.1 \text { ( } \geq 28 \text { and }<37 \text { completed weeks' ges- } \\
\text { tation) }+ \text { (birth weight } \geq 1000 \text { grams and } \leq \\
2499 \text { grams) }\end{array}$ & 1 & 60 & $\begin{array}{l}\text { Mean Difference (IV, } \\
\text { Fixed, } 95 \% \mathrm{CI} \text { ) }\end{array}$ & $0.30[0.11,0.49]$ \\
\hline $\begin{array}{l}3 \text { Core body temperature }\left({ }^{\circ} \mathrm{C}\right) 90 \text { minutes af- } \\
\text { ter birth }\end{array}$ & 1 & 60 & $\begin{array}{l}\text { Mean Difference (IV, } \\
\text { Fixed, } 95 \% \mathrm{CI} \text { ) }\end{array}$ & $0.30[0.12,0.48]$ \\
\hline $\begin{array}{l}3.1 \text { ( } \geq 28 \text { and }<37 \text { completed weeks' ges- } \\
\text { tation) }+ \text { (birth weight } \geq 1000 \text { grams and } \leq \\
2499 \text { grams) }\end{array}$ & 1 & 60 & $\begin{array}{l}\text { Mean Difference (IV, } \\
\text { Fixed, } 95 \% \mathrm{CI} \text { ) }\end{array}$ & $0.30[0.12,0.48]$ \\
\hline $\begin{array}{l}4 \text { Core body temperature }\left({ }^{\circ} \mathrm{C}\right) 2 \text { hours after } \\
\text { birth }\end{array}$ & 1 & 60 & $\begin{array}{l}\text { Mean Difference (IV, } \\
\text { Fixed, } 95 \% \mathrm{CI} \text { ) }\end{array}$ & $0.40[0.24,0.56]$ \\
\hline $\begin{array}{l}4.1 \text { ( } \geq 28 \text { and }<37 \text { completed weeks' ges- } \\
\text { tation) }+ \text { (birth weight } \geq 1000 \text { grams and } \leq \\
2499 \text { grams) }\end{array}$ & 1 & 60 & $\begin{array}{l}\text { Mean Difference (IV, } \\
\text { Fixed, } 95 \% \mathrm{CI} \text { ) }\end{array}$ & $0.40[0.24,0.56]$ \\
\hline 5 Hypothermia & 1 & 60 & $\begin{array}{l}\text { Risk Ratio (M-H, Fixed, } \\
95 \% \mathrm{Cl})\end{array}$ & $0.09[0.01,1.57]$ \\
\hline $\begin{array}{l}5.1 \text { ( } \geq 28 \text { and }<37 \text { completed weeks' ges- } \\
\text { tation) }+ \text { (birth weight } \geq 1000 \text { grams and } \leq \\
2499 \text { grams) }\end{array}$ & 1 & 60 & $\begin{array}{l}\text { Risk Ratio }(\mathrm{M}-\mathrm{H}, \text { Fixed, } \\
95 \% \mathrm{Cl})\end{array}$ & $0.09[0.01,1.57]$ \\
\hline $\begin{array}{l}6 \text { Glucose concentration }(\mathrm{mmol} / \mathrm{L}) \text { at } 120 \\
\text { minutes after birth }\end{array}$ & & & Other data & No numeric data \\
\hline $\begin{array}{l}6.1 \text { ( } \geq 28 \text { and }<37 \text { completed weeks' ges- } \\
\text { tation) }+ \text { (birth weight } \geq 1000 \text { grams and } \leq \\
2499 \text { grams) }\end{array}$ & & & Other data & No numeric data \\
\hline
\end{tabular}

Analysis 3.1. Comparison 3 Plastic bag with previous drying versus routine care, Outcome 1 Core body temperature $\left({ }^{\circ} \mathrm{C}\right) 30$ minutes after birth.

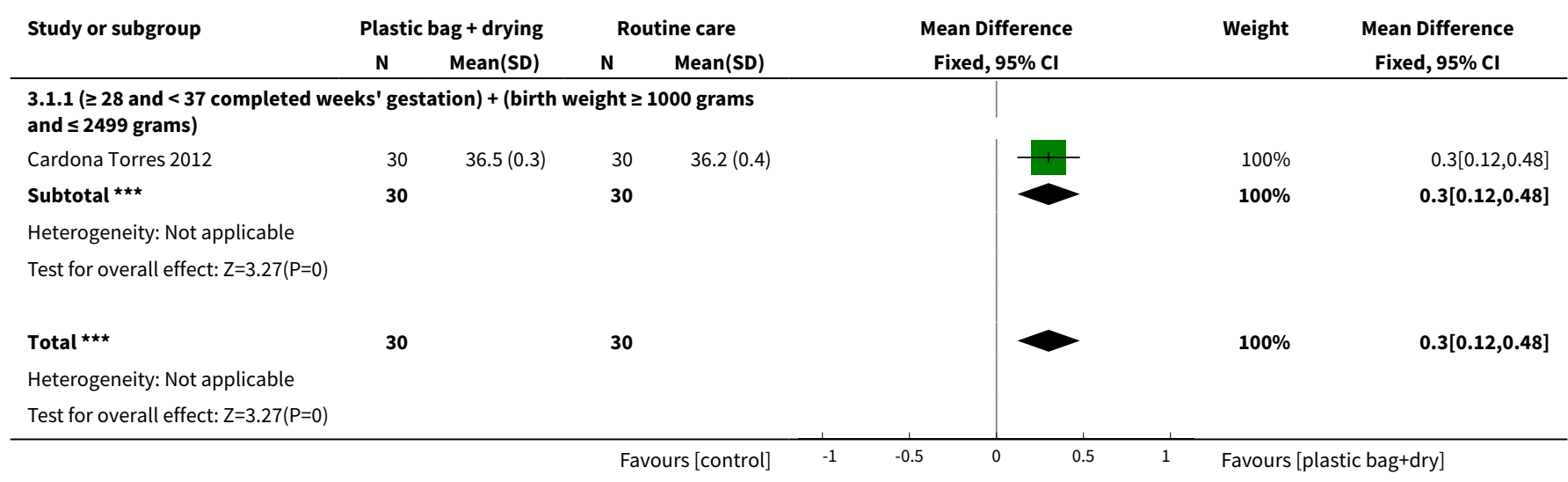


Analysis 3.2. Comparison 3 Plastic bag with previous drying versus routine care, Outcome 2 Core body temperature $\left({ }^{\circ} \mathrm{C}\right) 1$ hour after birth.

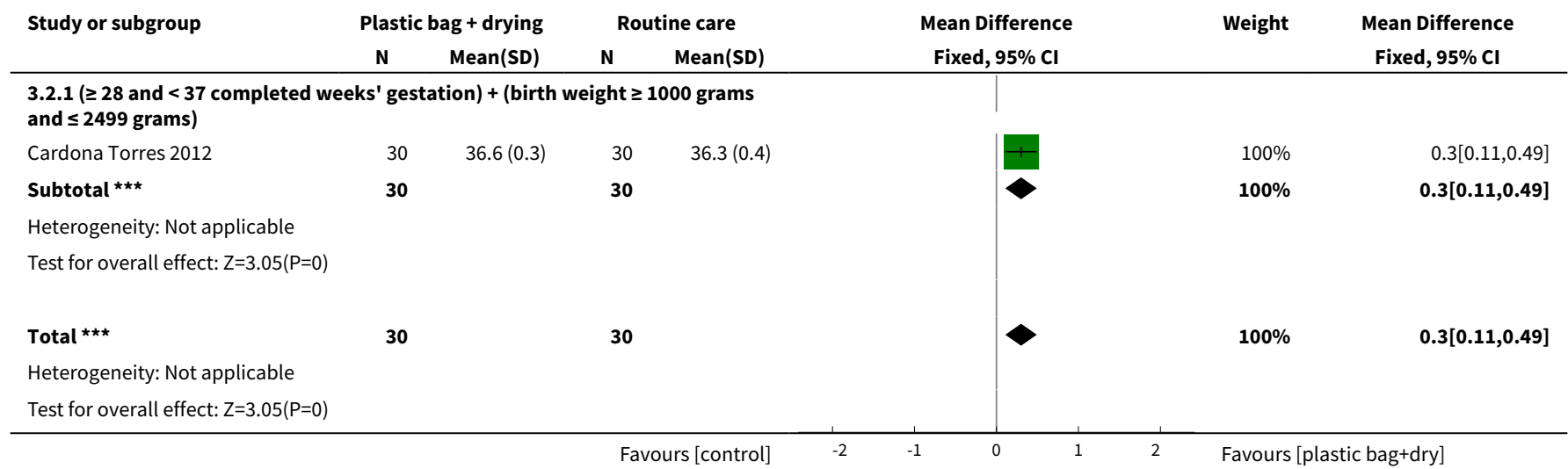

Analysis 3.3. Comparison 3 Plastic bag with previous drying versus routine care, Outcome 3 Core body temperature $\left({ }^{\circ} \mathrm{C}\right) 90$ minutes after birth.

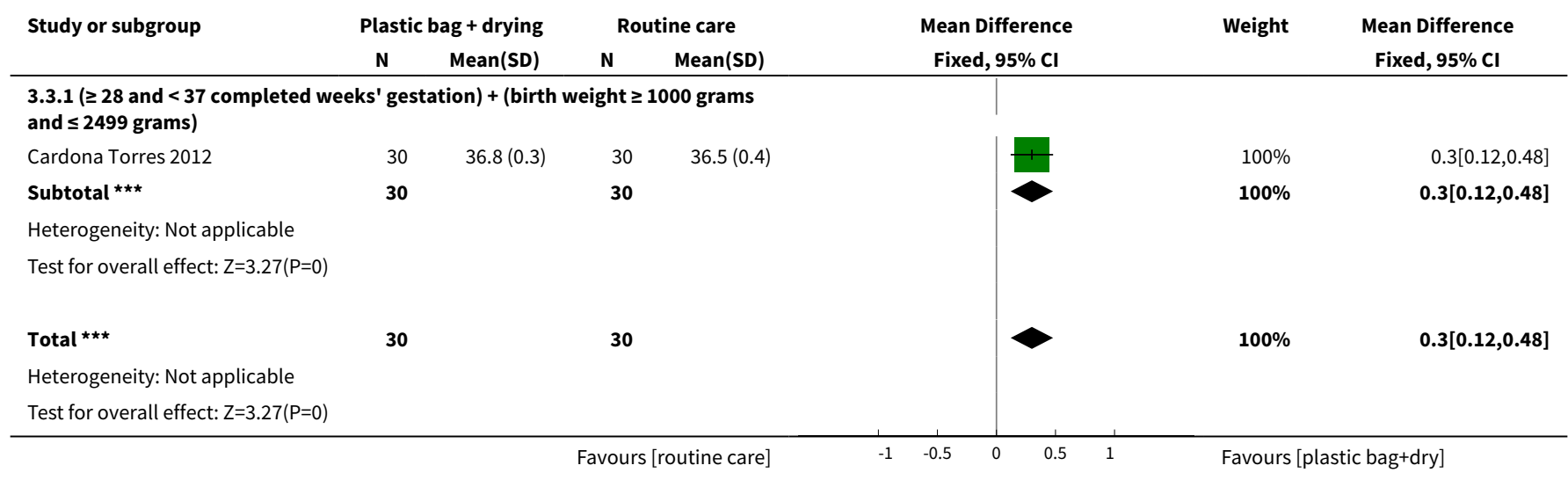

Analysis 3.4. Comparison 3 Plastic bag with previous drying versus routine care, Outcome 4 Core body temperature $\left({ }^{\circ} \mathrm{C}\right) 2$ hours after birth.

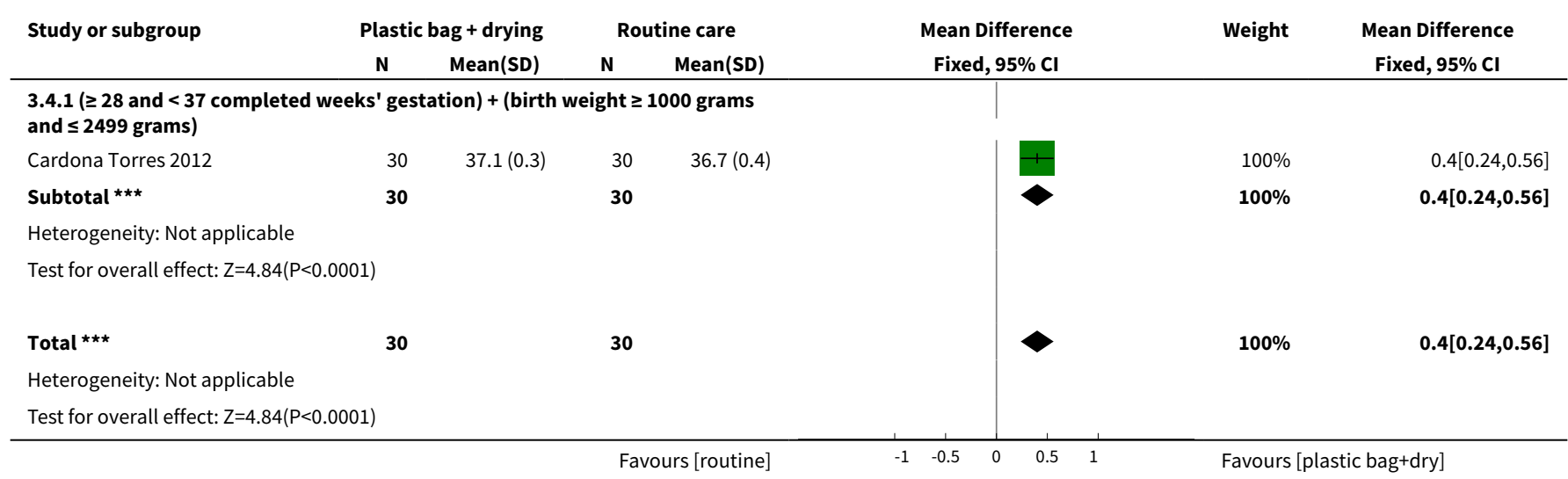


Analysis 3.5. Comparison 3 Plastic bag with previous drying versus routine care, Outcome 5 Hypothermia.

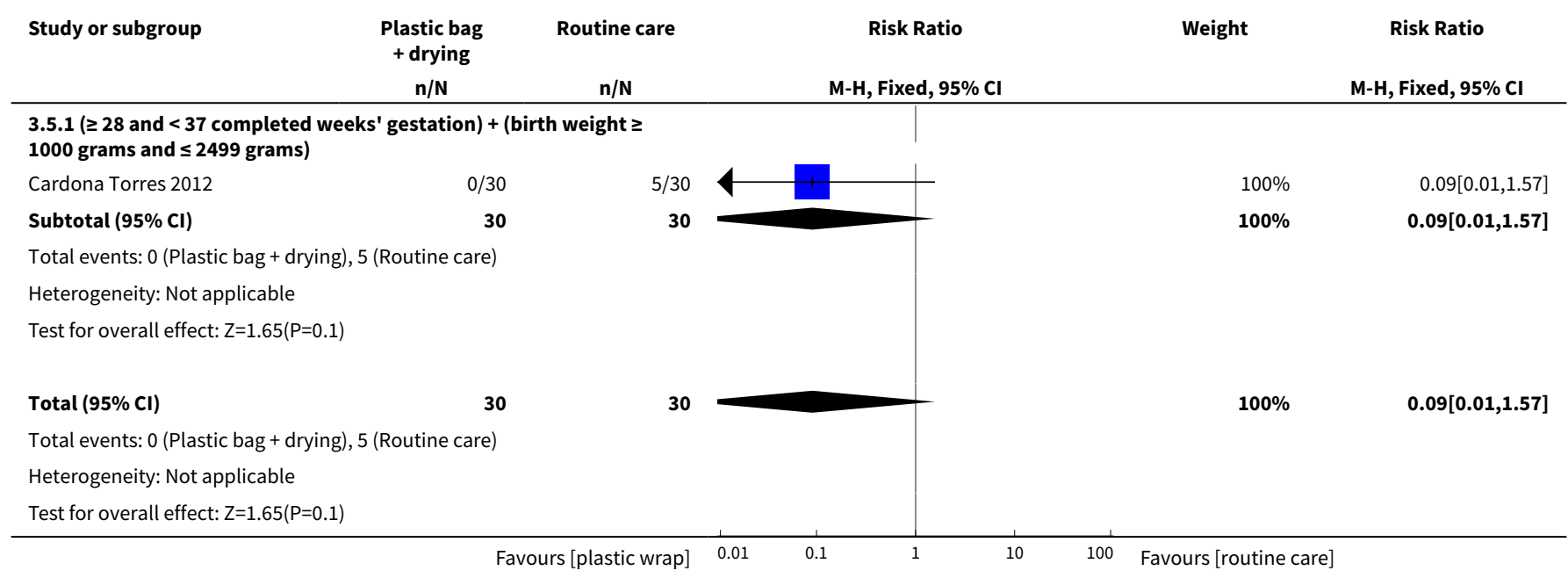

Analysis 3.6. Comparison 3 Plastic bag with previous drying versus routine care, Outcome 6 Glucose concentration $(\mathrm{mmol} / \mathrm{L})$ at 120 minutes after birth.

\begin{tabular}{|c|c|c|c|c|c|}
\hline \multicolumn{6}{|c|}{ Glucose concentration ( $\mathrm{mmol} / \mathrm{L}$ ) at 120 minutes after birth } \\
\hline Study & Group & $\mathbf{N}$ & Median & Interquartile range & $P$ value \\
\hline \multicolumn{6}{|c|}{ ( $\geq 28$ and $<37$ completed weeks' gestation) + (birth weight $\geq 1000$ grams and $\leq 2499$ grams) } \\
\hline Cardona Torres 2012 & Plastic bag with drying & 30 & 3.75 & $2.6-5.5$ & $p>0.05$ \\
\hline Cardona Torres 2012 & Control & 30 & 3.56 & $2.7-4.4$ & \\
\hline
\end{tabular}

\section{Comparison 4. Plastic cap versus routine care (no cap)}

\begin{tabular}{|c|c|c|c|c|}
\hline Outcome or subgroup title & No. of studies & $\begin{array}{l}\text { No. of partici- } \\
\text { pants }\end{array}$ & Statistical method & Effect size \\
\hline $\begin{array}{l}1 \text { Core body temperature }\left({ }^{\circ} \mathrm{C}\right) \text { on ad- } \\
\text { mission to NICU or up to } 2 \text { hours after } \\
\text { birth }\end{array}$ & 1 & 64 & $\begin{array}{l}\text { Mean Difference (IV, Fixed, } \\
95 \% \mathrm{CI} \text { ) }\end{array}$ & $0.80[0.41,1.19]$ \\
\hline $1.1<29$ completed weeks' gestation & 1 & 64 & $\begin{array}{l}\text { Mean Difference (IV, Fixed, } \\
95 \% \mathrm{CI})\end{array}$ & $0.80[0.41,1.19]$ \\
\hline $\begin{array}{l}2 \text { Hypothermia on admission to NICU: } \\
\text { core body temperature }<36.4^{\circ} \mathrm{C}\end{array}$ & 1 & 64 & $\begin{array}{l}\text { Risk Ratio (M-H, Fixed, 95\% } \\
\mathrm{Cl})\end{array}$ & $0.48[0.32,0.73]$ \\
\hline $2.1<29$ completed weeks' gestation & 1 & 64 & $\begin{array}{l}\text { Risk Ratio (M-H, Fixed, 95\% } \\
\mathrm{Cl})\end{array}$ & $0.48[0.32,0.73]$ \\
\hline $\begin{array}{l}3 \text { Outside normothermic range on ad- } \\
\text { mission to NICU or up to } 2 \text { hours after } \\
\text { birth }\end{array}$ & 1 & 64 & $\begin{array}{l}\text { Risk Ratio (M-H, Fixed, 95\% } \\
\mathrm{Cl} \text { ) }\end{array}$ & $0.48[0.32,0.73]$ \\
\hline $3.1<29$ completed weeks' gestation & 1 & 64 & $\begin{array}{l}\text { Risk Ratio (M-H, Fixed, 95\% } \\
\mathrm{Cl})\end{array}$ & $0.48[0.32,0.73]$ \\
\hline
\end{tabular}




\begin{tabular}{|c|c|c|c|c|}
\hline Outcome or subgroup title & No. of studies & $\begin{array}{l}\text { No. of partici- } \\
\text { pants }\end{array}$ & Statistical method & Effect size \\
\hline $\begin{array}{l}4 \text { Core body temperature }\left({ }^{\circ} \mathrm{C}\right) 1 \text { hour } \\
\text { after initial NICU admission tempera- } \\
\text { ture was taken }\end{array}$ & 1 & 64 & $\begin{array}{l}\text { Mean Difference (IV, Fixed, } \\
95 \% \mathrm{CI})\end{array}$ & $0.80[0.46,1.14]$ \\
\hline $4.1<29$ completed weeks' gestation & 1 & 64 & $\begin{array}{l}\text { Mean Difference (IV, Fixed, } \\
95 \% \mathrm{CI})\end{array}$ & $0.80[0.46,1.14]$ \\
\hline 5 Major brain injury & 1 & 64 & $\begin{array}{l}\text { Risk Ratio (M-H, Fixed, 95\% } \\
\mathrm{Cl})\end{array}$ & $1.5[0.27,8.38]$ \\
\hline $5.1<29$ completed weeks' gestation & 1 & 64 & $\begin{array}{l}\text { Risk Ratio (M-H, Fixed, 95\% } \\
\mathrm{Cl})\end{array}$ & $1.5[0.27,8.38]$ \\
\hline $\begin{array}{l}6 \text { Mortality (death within hospital } \\
\text { stay) }\end{array}$ & 1 & 64 & $\begin{array}{l}\text { Risk Ratio (M-H, Fixed, 95\% } \\
\mathrm{Cl})\end{array}$ & $1.5[0.27,8.38]$ \\
\hline $6.1<29$ completed weeks' gestation & 1 & 64 & $\begin{array}{l}\text { Risk Ratio (M-H, Fixed, 95\% } \\
\mathrm{Cl})\end{array}$ & $1.5[0.27,8.38]$ \\
\hline 7 Bicarbonate (mmol/L) & 1 & 64 & $\begin{array}{l}\text { Mean Difference (IV, Fixed, } \\
95 \% \mathrm{CI})\end{array}$ & $1.0[-0.25,2.25]$ \\
\hline $7.1<29$ completed weeks' gestation & 1 & 64 & $\begin{array}{l}\text { Mean Difference (IV, Fixed, } \\
95 \% \mathrm{CI} \text { ) }\end{array}$ & $1.0[-0.25,2.25]$ \\
\hline 8 Blood gas $\mathrm{pH}$ (first) & 1 & 64 & $\begin{array}{l}\text { Mean Difference (IV, Fixed, } \\
95 \% \mathrm{CI} \text { ) }\end{array}$ & $0.01[-0.03,0.05]$ \\
\hline $8.1<29$ completed weeks' gestation & 1 & 64 & $\begin{array}{l}\text { Mean Difference (IV, Fixed, } \\
95 \% \mathrm{CI})\end{array}$ & $0.01[-0.03,0.05]$ \\
\hline $\begin{array}{l}9 \text { First serum glucose concentration } \\
(\mathrm{mmol} / \mathrm{L}) \text { on admission to NICU }\end{array}$ & 1 & 64 & $\begin{array}{l}\text { Mean Difference (IV, Fixed, } \\
95 \% \mathrm{CI})\end{array}$ & $0.10[-0.42,0.62]$ \\
\hline $9.1<29$ completed weeks' gestation & 1 & 64 & $\begin{array}{l}\text { Mean Difference (IV, Fixed, } \\
95 \% \mathrm{CI} \text { ) }\end{array}$ & $0.10[-0.42,0.62]$ \\
\hline 10 Intubation at birth & 1 & 64 & $\begin{array}{l}\text { Risk Ratio (M-H, Fixed, 95\% } \\
\mathrm{Cl} \text { ) }\end{array}$ & $0.82[0.49,1.37]$ \\
\hline $10.1<29$ completed weeks' gestation & 1 & 64 & $\begin{array}{l}\text { Risk Ratio (M-H, Fixed, 95\% } \\
\mathrm{Cl} \text { ) }\end{array}$ & $0.82[0.49,1.37]$ \\
\hline
\end{tabular}

Analysis 4.1. Comparison 4 Plastic cap versus routine care (no cap), Outcome 1 Core body temperature $\left({ }^{\circ} \mathrm{C}\right)$ on admission to NICU or up to 2 hours after birth.

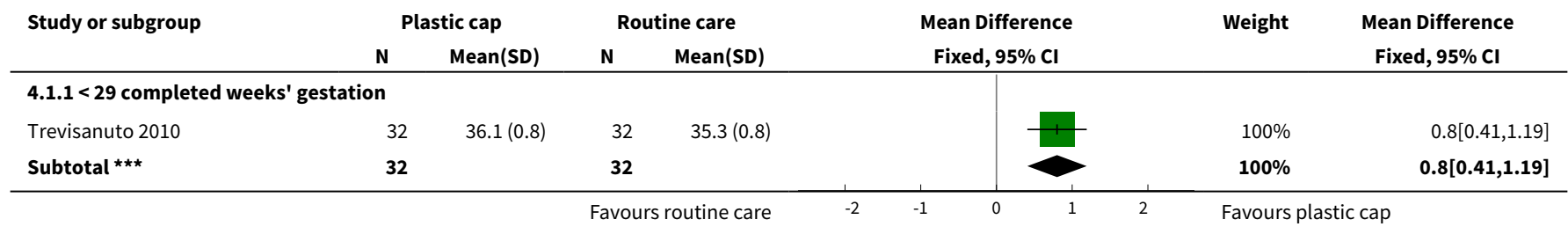




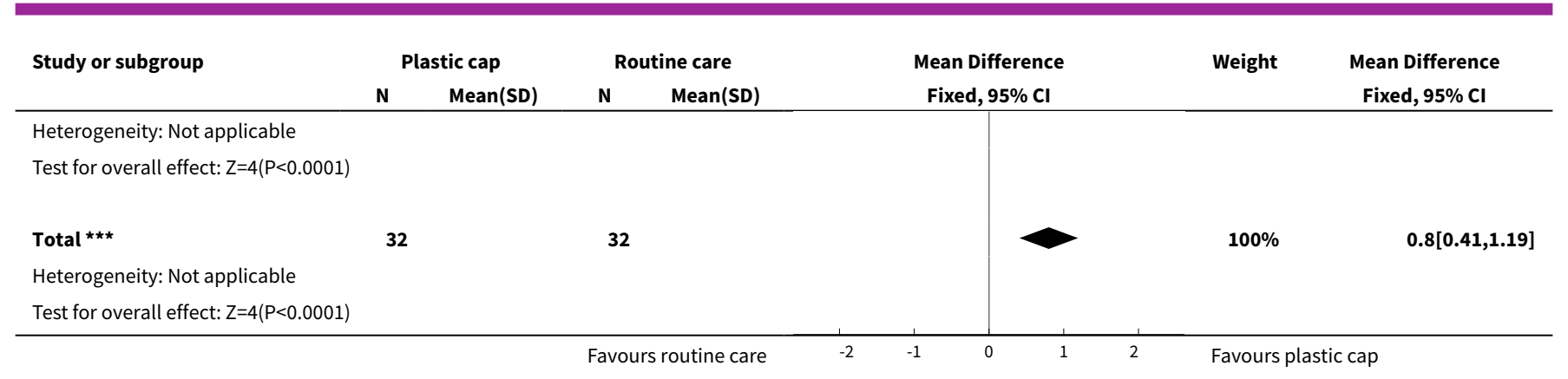

Analysis 4.2. Comparison 4 Plastic cap versus routine care (no cap), Outcome 2 Hypothermia on admission to NICU: core body temperature $<36.4^{\circ} \mathrm{C}$.

\begin{tabular}{|c|c|c|c|c|c|}
\hline Study or subgroup & $\begin{array}{c}\text { Plastic cap } \\
n / N \\
\end{array}$ & $\begin{array}{c}\text { Routine care } \\
\mathbf{n} / \mathbf{N} \\
\end{array}$ & $\begin{array}{c}\text { Risk Ratio } \\
\text { M-H, Fixed, 95\% Cl }\end{array}$ & Weight & $\begin{array}{c}\text { Risk Ratio } \\
\text { M-H, Fixed, 95\% Cl }\end{array}$ \\
\hline \multicolumn{6}{|c|}{ 4.2.1 < 29 completed weeks' gestation } \\
\hline Trevisanuto 2010 & $14 / 32$ & $29 / 32$ & & $100 \%$ & $0.48[0.32,0.73]$ \\
\hline Subtotal $(95 \% \mathrm{Cl})$ & 32 & 32 & & $100 \%$ & $0.48[0.32,0.73]$ \\
\hline \multicolumn{6}{|c|}{ Total events: 14 (Plastic cap), 29 (Routine care) } \\
\hline \multicolumn{6}{|c|}{ Heterogeneity: Not applicable } \\
\hline \multicolumn{6}{|c|}{ Test for overall effect: $Z=3.5(P=0)$} \\
\hline Total $(95 \% \mathrm{Cl})$ & 32 & 32 & & $100 \%$ & $0.48[0.32,0.73]$ \\
\hline \multicolumn{6}{|c|}{ Total events: 14 (Plastic cap), 29 (Routine care) } \\
\hline \multicolumn{6}{|c|}{ Heterogeneity: Not applicable } \\
\hline Test for overall effect & & & & & \\
\hline
\end{tabular}

Analysis 4.3. Comparison 4 Plastic cap versus routine care (no cap), Outcome 3 Outside normothermic range on admission to NICU or up to 2 hours after birth.

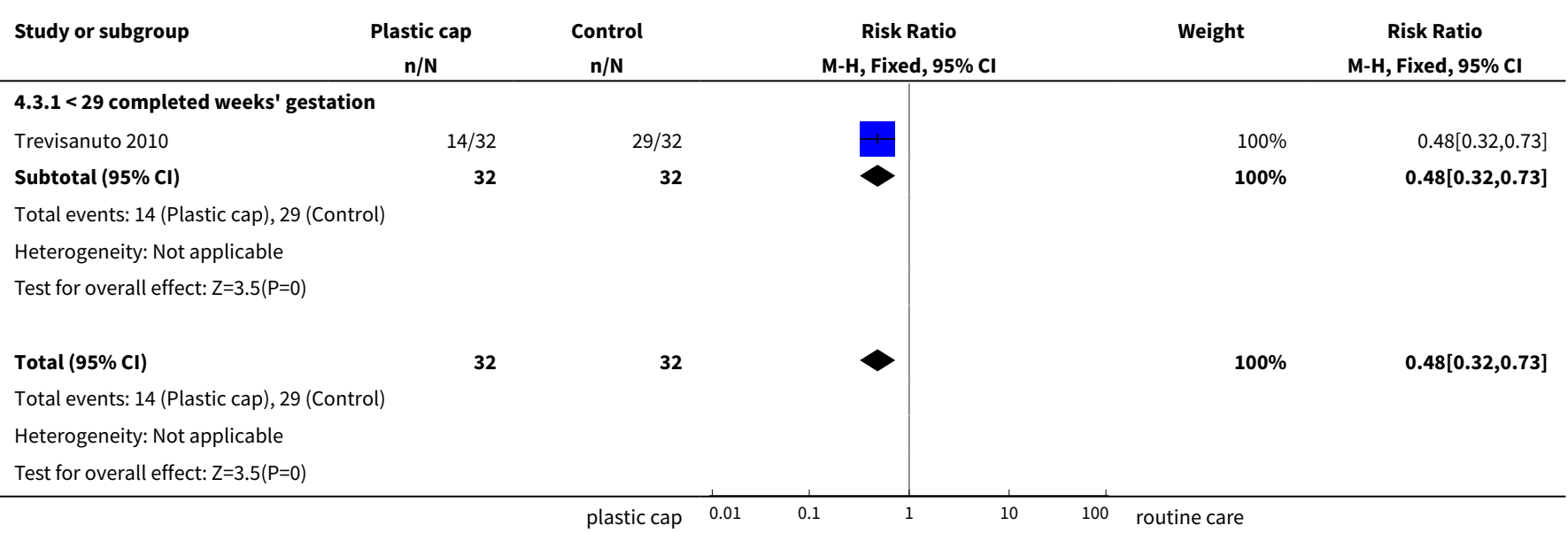


Analysis 4.4. Comparison 4 Plastic cap versus routine care (no cap), Outcome 4 Core body temperature $\left({ }^{\circ} \mathrm{C}\right) 1$ hour after initial NICU admission temperature was taken.

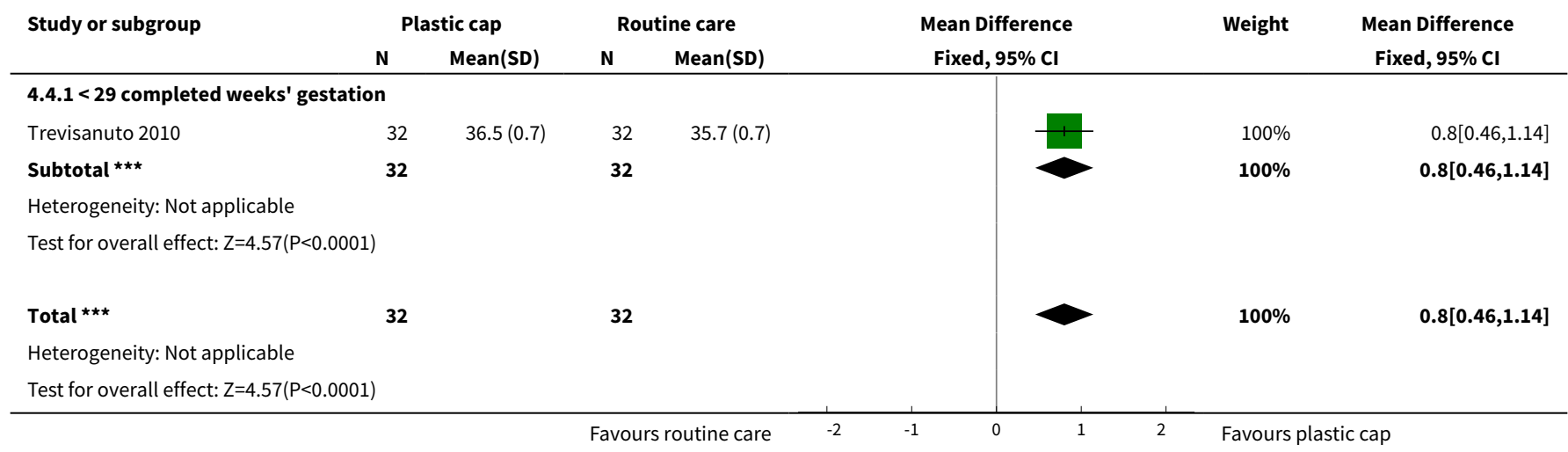

Analysis 4.5. Comparison 4 Plastic cap versus routine care (no cap), Outcome 5 Major brain injury.

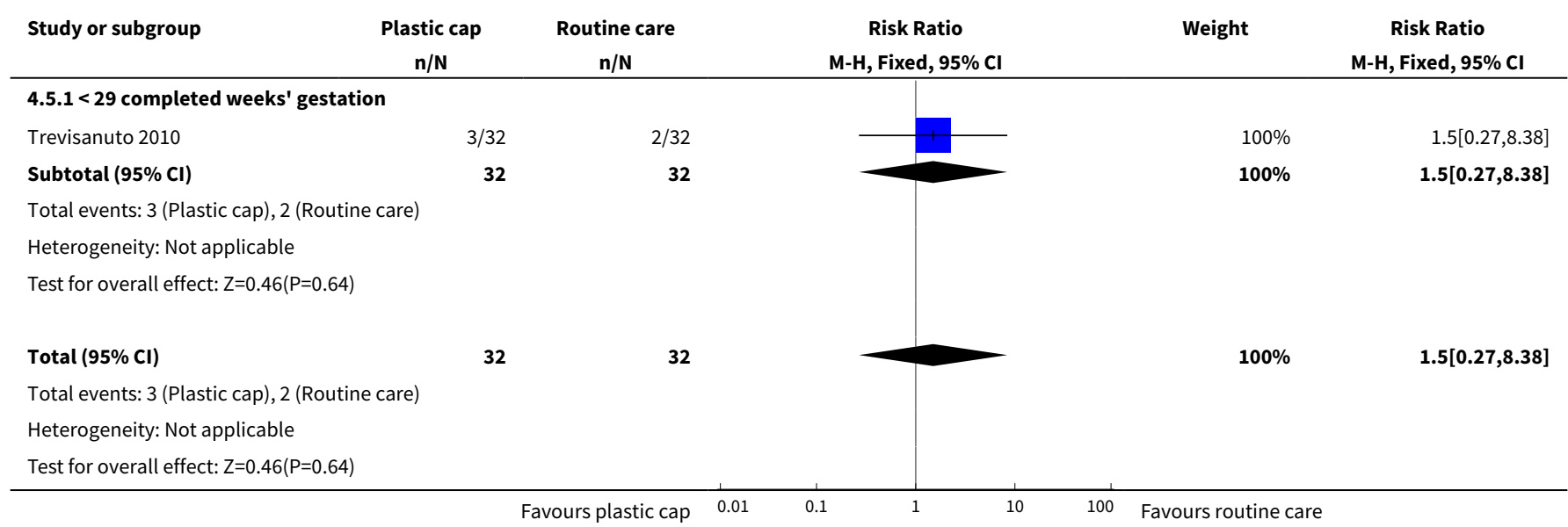

Analysis 4.6. Comparison 4 Plastic cap versus routine care (no cap), Outcome 6 Mortality (death within hospital stay).

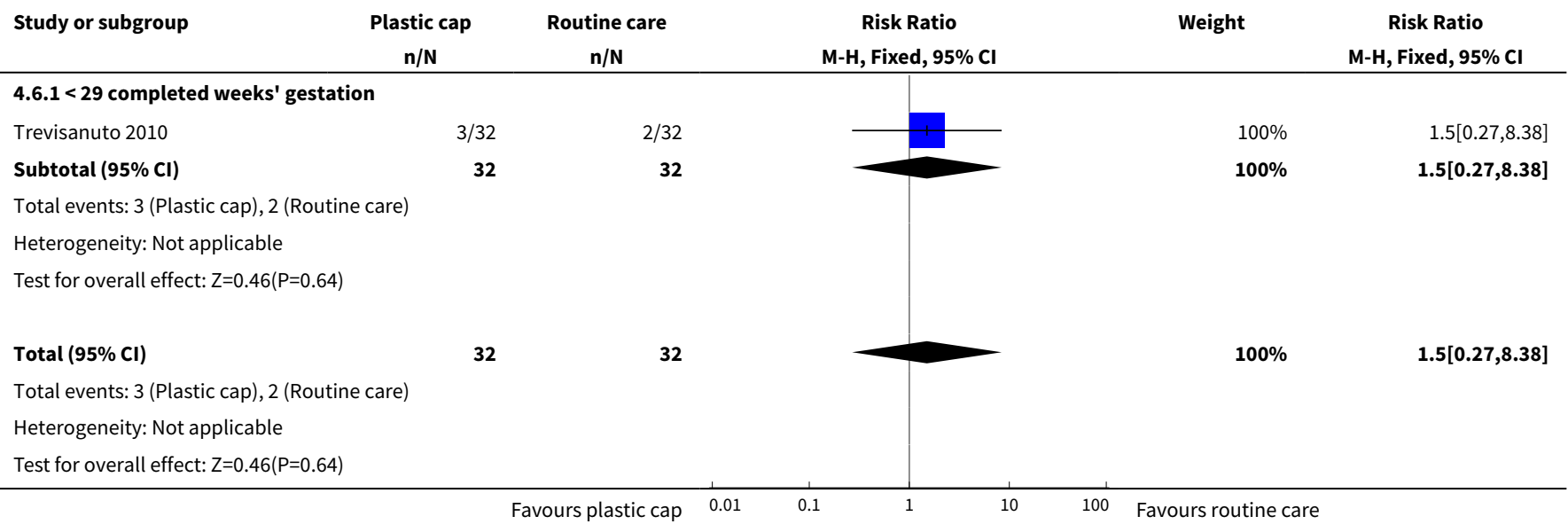




\section{Analysis 4.7. Comparison 4 Plastic cap versus routine care (no cap), Outcome 7 Bicarbonate (mmol/L).}

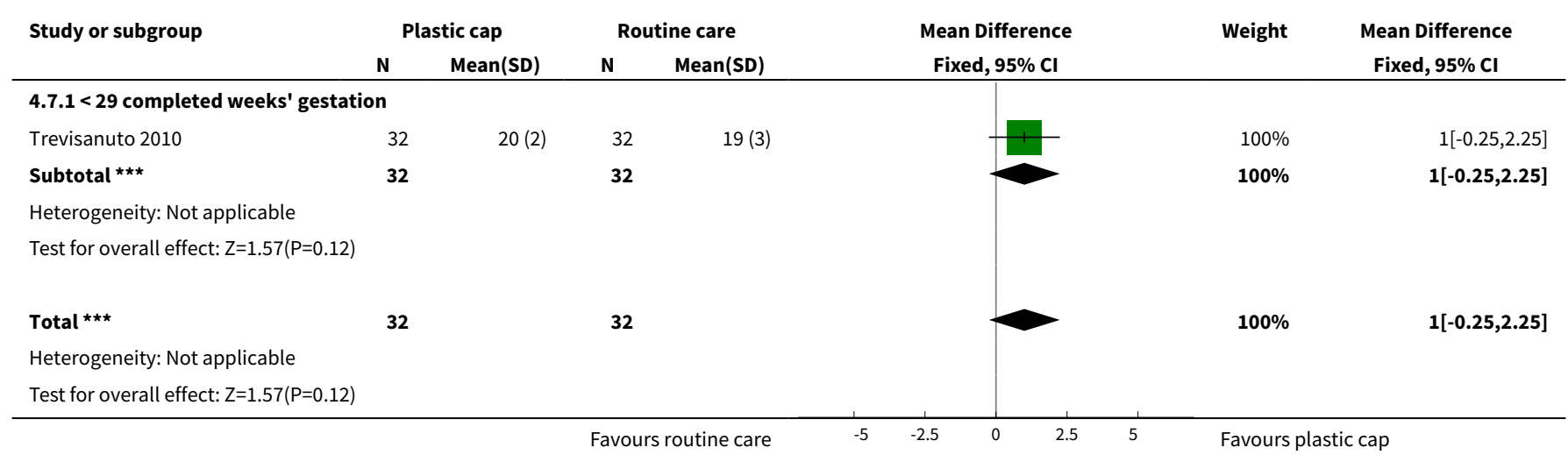

Analysis 4.8. Comparison 4 Plastic cap versus routine care (no cap), Outcome 8 Blood gas pH (first).

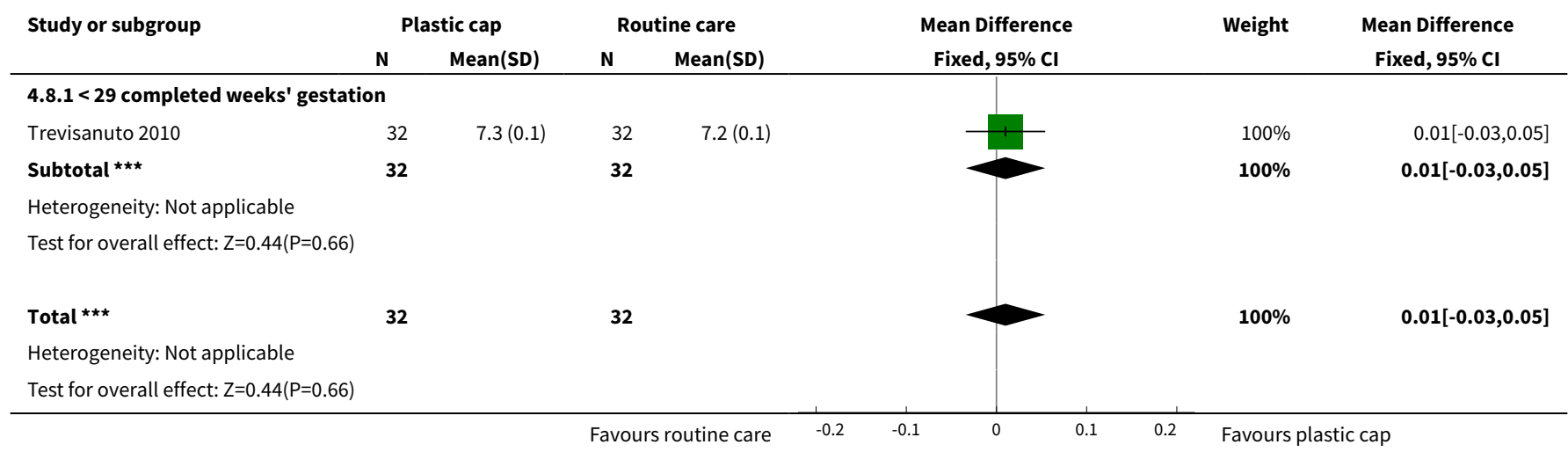

Analysis 4.9. Comparison 4 Plastic cap versus routine care (no cap), Outcome 9 First serum glucose concentration (mmol/L) on admission to NICU.

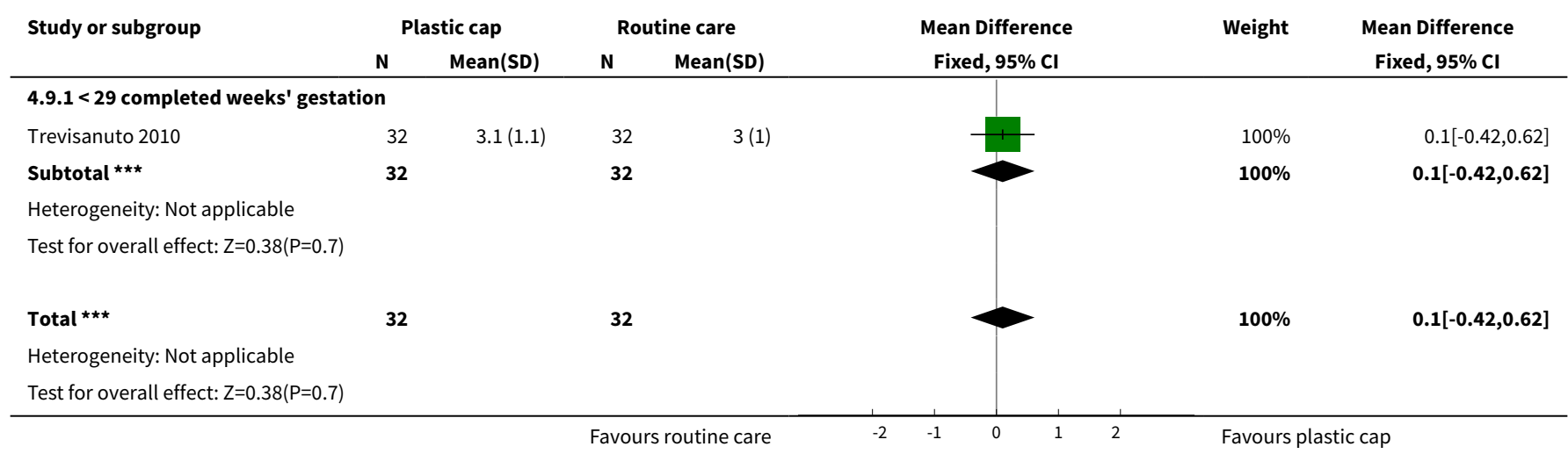


Analysis 4.10. Comparison 4 Plastic cap versus routine care (no cap), Outcome 10 Intubation at birth.

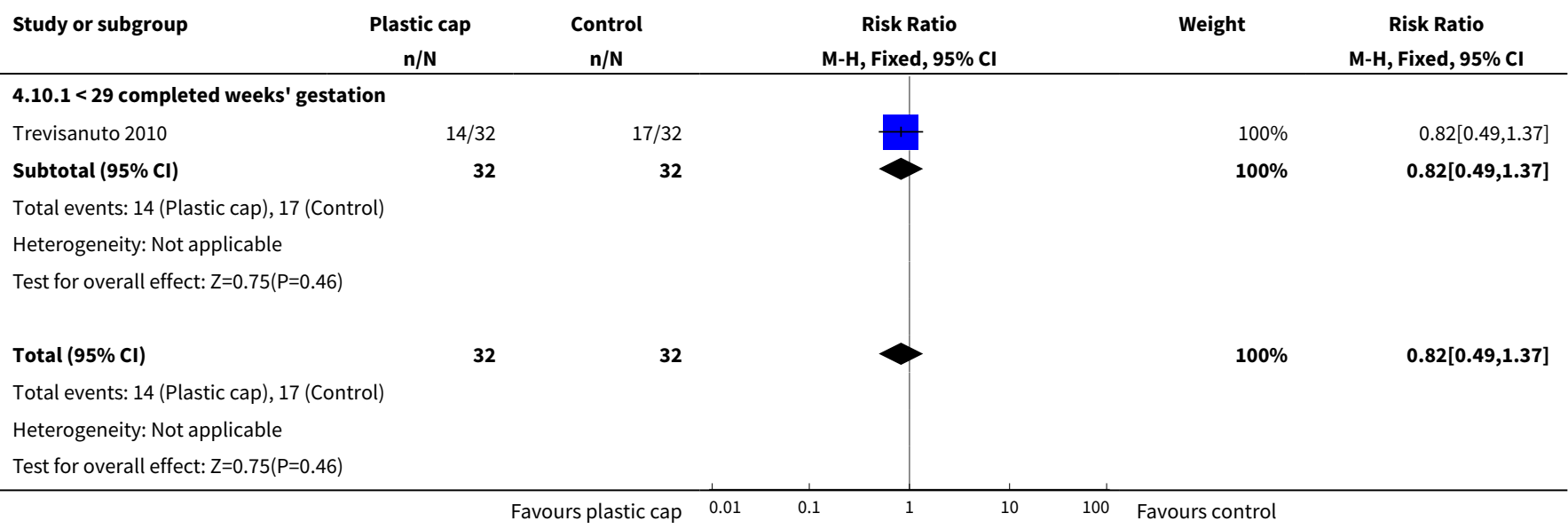

\section{Comparison 5. Plastic bag and plastic cap versus routine care}

\begin{tabular}{|c|c|c|c|c|}
\hline Outcome or subgroup title & No. of studies & $\begin{array}{l}\text { No. of partici- } \\
\text { pants }\end{array}$ & Statistical method & Effect size \\
\hline $\begin{array}{l}1 \text { Core body temperature }\left({ }^{\circ} \mathrm{C}\right) \text { on ad- } \\
\text { mission to NICU or up to } 2 \text { hours after } \\
\text { birth }\end{array}$ & 2 & 122 & $\begin{array}{l}\text { Mean Difference (IV, Fixed, } \\
95 \% \mathrm{CI})\end{array}$ & $0.32[0.15,0.50]$ \\
\hline $\begin{array}{l}1.1 \text { All infants (< } 37 \text { completed weeks' } \\
\text { gestation) }\end{array}$ & 2 & 122 & $\begin{array}{l}\text { Mean Difference (IV, Fixed, } \\
95 \% \mathrm{Cl} \text { ) }\end{array}$ & $0.32[0.15,0.50]$ \\
\hline $\begin{array}{l}2 \text { Core body temperature }\left({ }^{\circ} \mathrm{C}\right) \text { at 10th } \\
\text { minute of life }\end{array}$ & 1 & 58 & $\begin{array}{l}\text { Mean Difference (IV, Fixed, } \\
95 \% \mathrm{CI})\end{array}$ & $-0.27[-0.55,0.01]$ \\
\hline $\begin{array}{l}2.1 \text { All infants (< } 37 \text { completed weeks' } \\
\text { gestation) }\end{array}$ & 1 & 58 & $\begin{array}{l}\text { Mean Difference (IV, Fixed, } \\
95 \% \mathrm{Cl} \text { ) }\end{array}$ & $-0.27[-0.55,0.01]$ \\
\hline $\begin{array}{l}3 \text { Core body temperature }\left({ }^{\circ} \mathrm{C}\right) \text { at } 15 \text { th } \\
\text { minute of life }\end{array}$ & 1 & 58 & $\begin{array}{l}\text { Mean Difference (IV, Fixed, } \\
95 \% \mathrm{Cl})\end{array}$ & $-0.26[-0.56,0.05]$ \\
\hline $\begin{array}{l}3.1 \text { All infants (< } 37 \text { completed weeks' } \\
\text { gestation) }\end{array}$ & 1 & 58 & $\begin{array}{l}\text { Mean Difference (IV, Fixed, } \\
95 \% \mathrm{CI})\end{array}$ & $-0.26[-0.56,0.05]$ \\
\hline $\begin{array}{l}4 \text { Core body temperature }\left({ }^{\circ} \mathrm{C}\right) \text { at } 30 \text { th } \\
\text { minute of life }\end{array}$ & 1 & 58 & $\begin{array}{l}\text { Mean Difference (IV, Fixed, } \\
95 \% \mathrm{Cl})\end{array}$ & $0.06[-0.23,0.35]$ \\
\hline $\begin{array}{l}\text { 4.1 All infants (< } 37 \text { completed weeks' } \\
\text { gestation) }\end{array}$ & 1 & 58 & $\begin{array}{l}\text { Mean Difference (IV, Fixed, } \\
95 \% \mathrm{CI})\end{array}$ & $0.06[-0.23,0.35]$ \\
\hline $\begin{array}{l}5 \text { Core body temperature }\left({ }^{\circ} \mathrm{C}\right) \text { at } 1 \text { hour } \\
\text { of life }\end{array}$ & 1 & 58 & $\begin{array}{l}\text { Mean Difference (IV, Fixed, } \\
95 \% \mathrm{CI})\end{array}$ & $-0.02[-0.32,0.27]$ \\
\hline $\begin{array}{l}5.1 \text { All infants (< } 37 \text { completed weeks' } \\
\text { gestation) }\end{array}$ & 1 & 58 & $\begin{array}{l}\text { Mean Difference (IV, Fixed, } \\
95 \% \mathrm{Cl})\end{array}$ & $-0.02[-0.32,0.27]$ \\
\hline
\end{tabular}




\begin{tabular}{|c|c|c|c|c|}
\hline Outcome or subgroup title & No. of studies & $\begin{array}{l}\text { No. of partici- } \\
\text { pants }\end{array}$ & Statistical method & Effect size \\
\hline $\begin{array}{l}6 \text { Core body temperature }\left({ }^{\circ} \mathrm{C}\right) 1 \text { hour } \\
\text { after initial NICU admission tempera- } \\
\text { ture was taken }\end{array}$ & 1 & 64 & $\begin{array}{l}\text { Mean Difference (IV, Fixed, } \\
95 \% \mathrm{CI})\end{array}$ & $0.83[0.37,1.29]$ \\
\hline $\begin{array}{l}6.1 \geq 28 \text { and } \leq 32 \text { completed weeks' } \\
\text { gestation }\end{array}$ & 1 & 64 & $\begin{array}{l}\text { Mean Difference (IV, Fixed, } \\
95 \% \mathrm{Cl} \text { ) }\end{array}$ & $0.83[0.37,1.29]$ \\
\hline $\begin{array}{l}7 \text { Core body temperature }\left({ }^{\circ} \mathrm{C}\right) 2 \text { hours } \\
\text { after initial NICU admission tempera- } \\
\text { ture was taken }\end{array}$ & 1 & 64 & $\begin{array}{l}\text { Mean Difference (IV, Fixed, } \\
95 \% \mathrm{CI})\end{array}$ & $1.11[0.65,1.57]$ \\
\hline $\begin{array}{l}7.1 \geq 28 \text { and } \leq 32 \text { completed weeks' } \\
\text { gestation }\end{array}$ & 1 & 64 & $\begin{array}{l}\text { Mean Difference (IV, Fixed, } \\
95 \% \mathrm{Cl} \text { ) }\end{array}$ & $1.11[0.65,1.57]$ \\
\hline $\begin{array}{l}8 \text { Hyperthermia: core body tempera- } \\
\text { ture }>37.0^{\circ} \mathrm{C}\end{array}$ & 2 & 122 & $\begin{array}{l}\text { Risk Ratio (M-H, Fixed, 95\% } \\
\mathrm{Cl})\end{array}$ & $0.18[0.02,1.39]$ \\
\hline $\begin{array}{l}8.1 \text { All infants (< } 37 \text { completed weeks' } \\
\text { gestation) }\end{array}$ & 2 & 122 & $\begin{array}{l}\text { Risk Ratio (M-H, Fixed, 95\% } \\
\mathrm{Cl})\end{array}$ & $0.18[0.02,1.39]$ \\
\hline $\begin{array}{l}9 \text { Hyponatraemia (serum sodium con- } \\
\text { centration }<130 \mathrm{mmol} / \mathrm{L} \text { ) }\end{array}$ & 1 & 58 & $\begin{array}{l}\text { Risk Ratio (M-H, Fixed, 95\% } \\
\mathrm{Cl})\end{array}$ & $1.43[0.35,5.83]$ \\
\hline $\begin{array}{l}9.1 \text { All infants (< } 37 \text { completed weeks' } \\
\text { gestation) }\end{array}$ & 1 & 58 & $\begin{array}{l}\text { Risk Ratio (M-H, Fixed, 95\% } \\
\mathrm{Cl})\end{array}$ & $1.43[0.35,5.83]$ \\
\hline 10 Weight (grams) at fifth day of life & 1 & 58 & $\begin{array}{l}\text { Mean Difference (IV, Fixed, } \\
95 \% \mathrm{CI})\end{array}$ & $\begin{array}{l}-74.20[-301.63, \\
153.23]\end{array}$ \\
\hline $\begin{array}{l}\text { 10.1 All infants (< } 37 \text { completed weeks' } \\
\text { gestation) }\end{array}$ & 1 & 58 & $\begin{array}{l}\text { Mean Difference (IV, Fixed, } \\
95 \% \mathrm{CI} \text { ) }\end{array}$ & $\begin{array}{l}-74.20[-301.63, \\
153.23]\end{array}$ \\
\hline
\end{tabular}

\section{Analysis 5.1. Comparison 5 Plastic bag and plastic cap versus routine care, Outcome 1 Core body temperature $\left({ }^{\circ} \mathrm{C}\right)$ on admission to NICU or up to 2 hours after birth.}

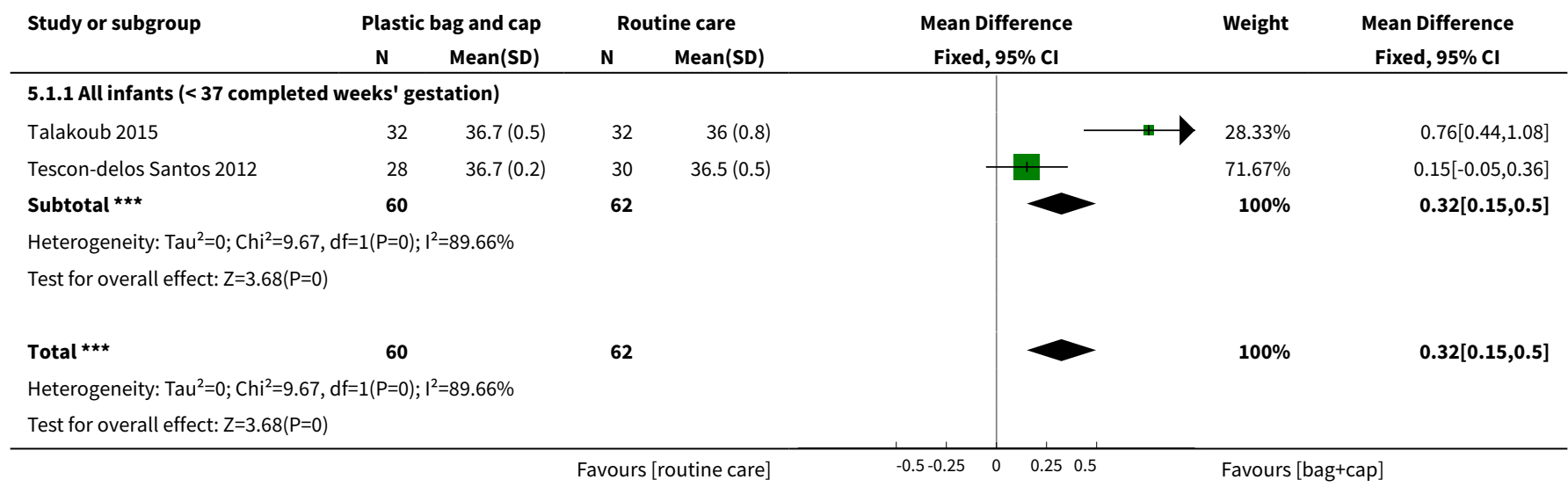


Analysis 5.2. Comparison 5 Plastic bag and plastic cap versus routine care, Outcome 2 Core body temperature $\left({ }^{\circ} \mathrm{C}\right)$ at 10 th minute of life.

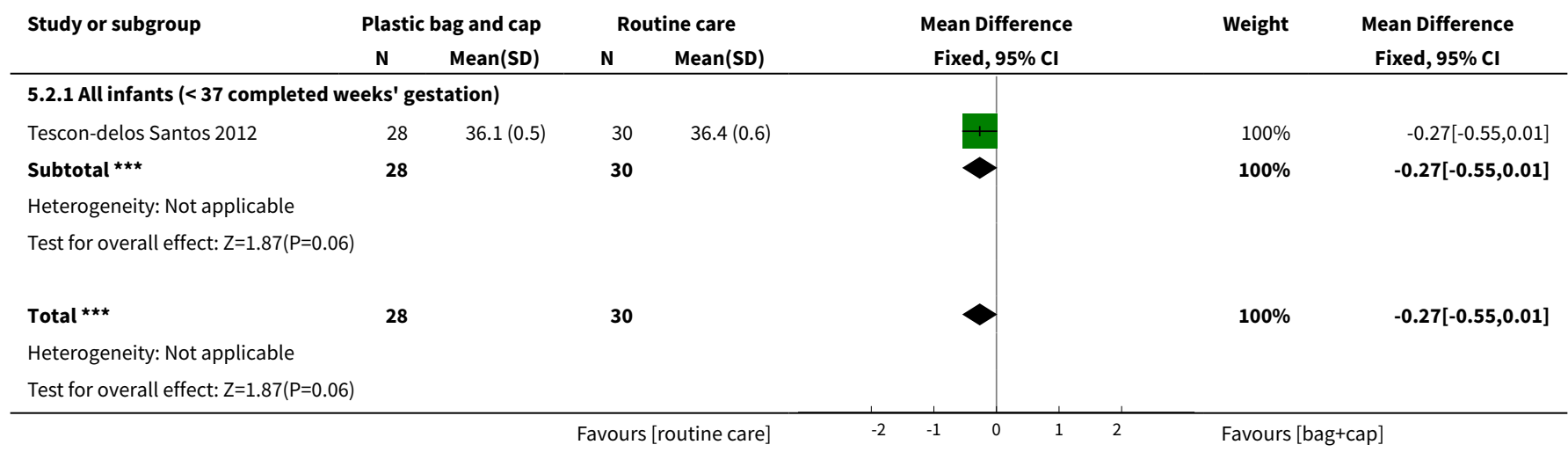

Analysis 5.3. Comparison 5 Plastic bag and plastic cap versus routine care, Outcome 3 Core body temperature $\left({ }^{\circ} \mathrm{C}\right)$ at 15 th minute of life.

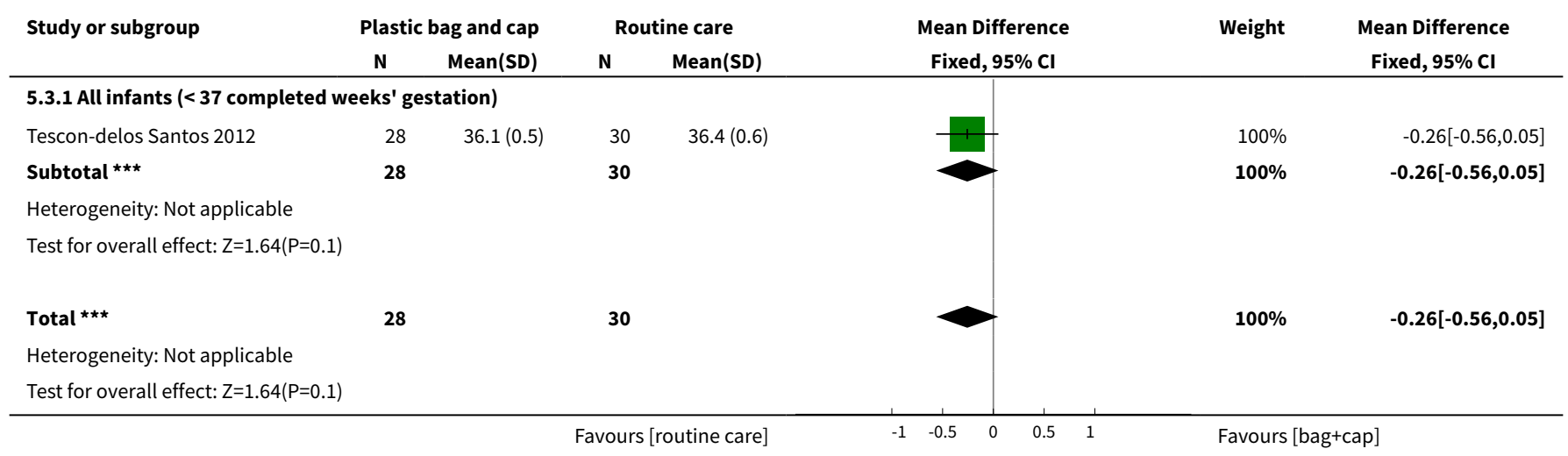

Analysis 5.4. Comparison 5 Plastic bag and plastic cap versus routine care, Outcome 4 Core body temperature $\left({ }^{\circ} \mathrm{C}\right)$ at 30th minute of life.

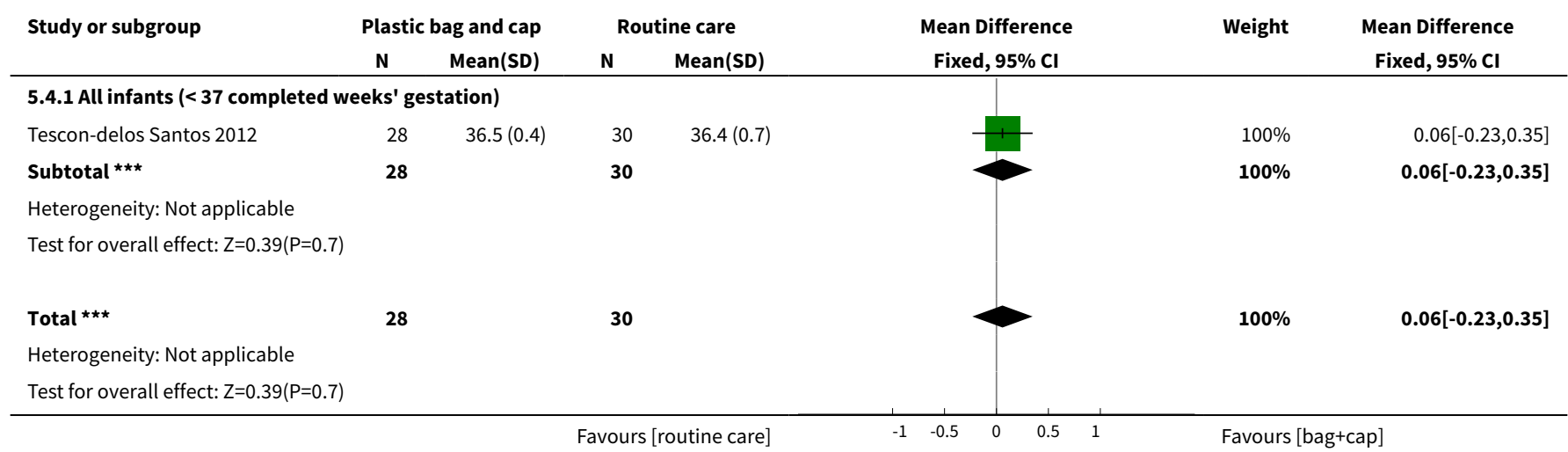


Analysis 5.5. Comparison 5 Plastic bag and plastic cap versus routine care, Outcome 5 Core body temperature $\left({ }^{\circ} \mathrm{C}\right)$ at 1 hour of life.

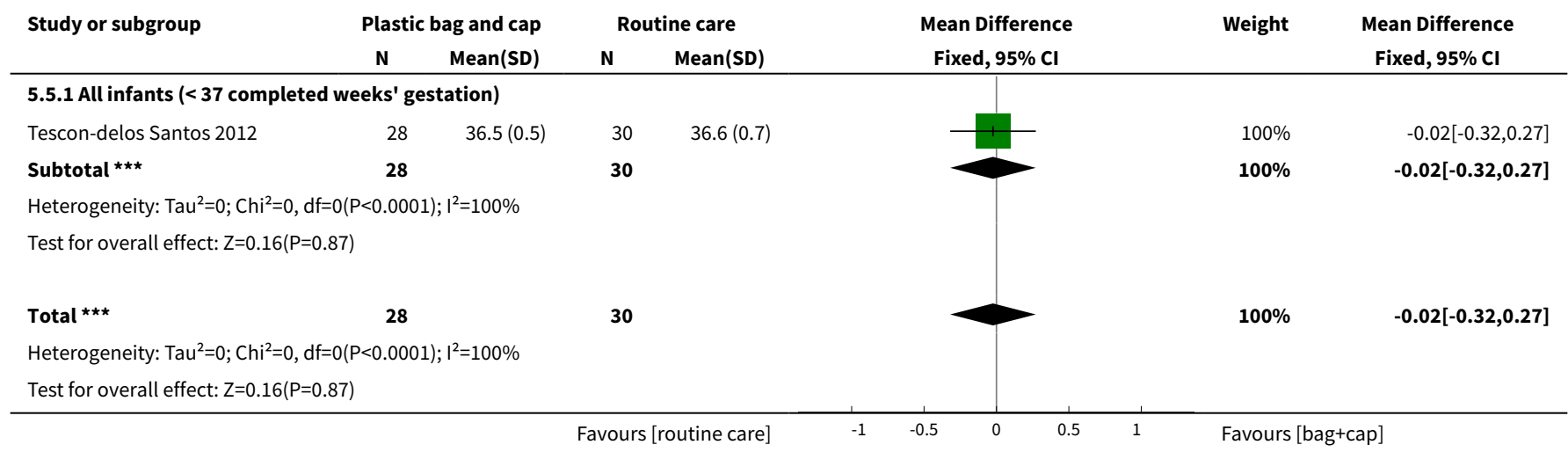

Analysis 5.6. Comparison 5 Plastic bag and plastic cap versus routine care, Outcome 6 Core body temperature $\left({ }^{\circ} \mathrm{C}\right) \mathbf{1}$ hour after initial NICU admission temperature was taken.

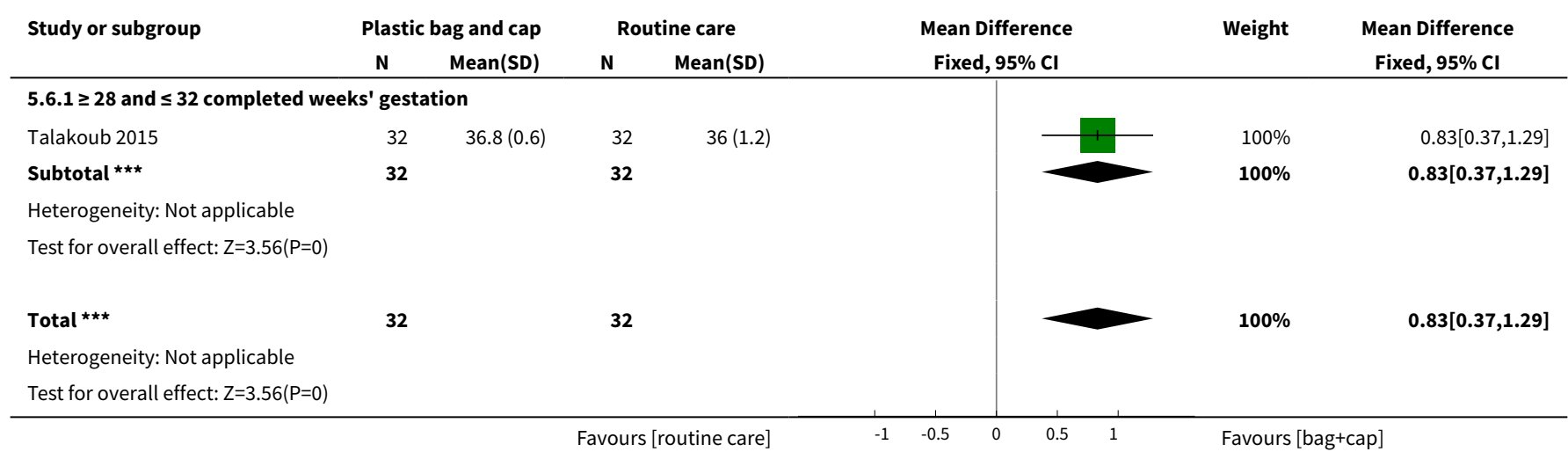

Analysis 5.7. Comparison 5 Plastic bag and plastic cap versus routine care, Outcome 7 Core body temperature $\left({ }^{\circ} \mathrm{C}\right) \mathbf{2}$ hours after initial NICU admission temperature was taken.

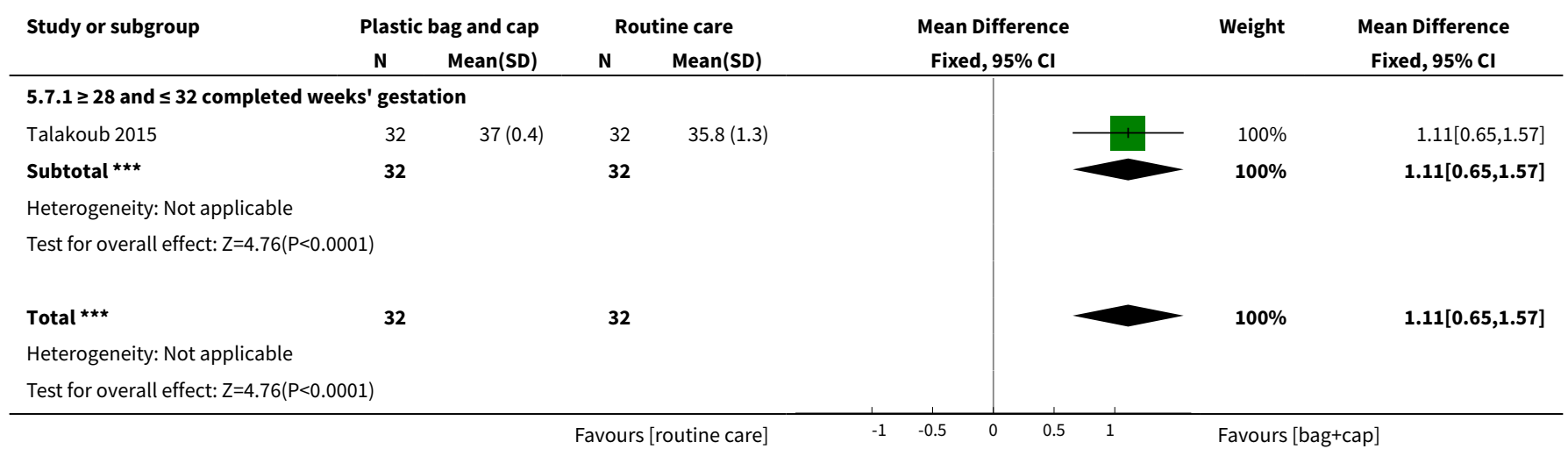


Analysis 5.8. Comparison 5 Plastic bag and plastic cap versus routine care, Outcome 8 Hyperthermia: core body temperature $>37.0^{\circ} \mathrm{C}$.

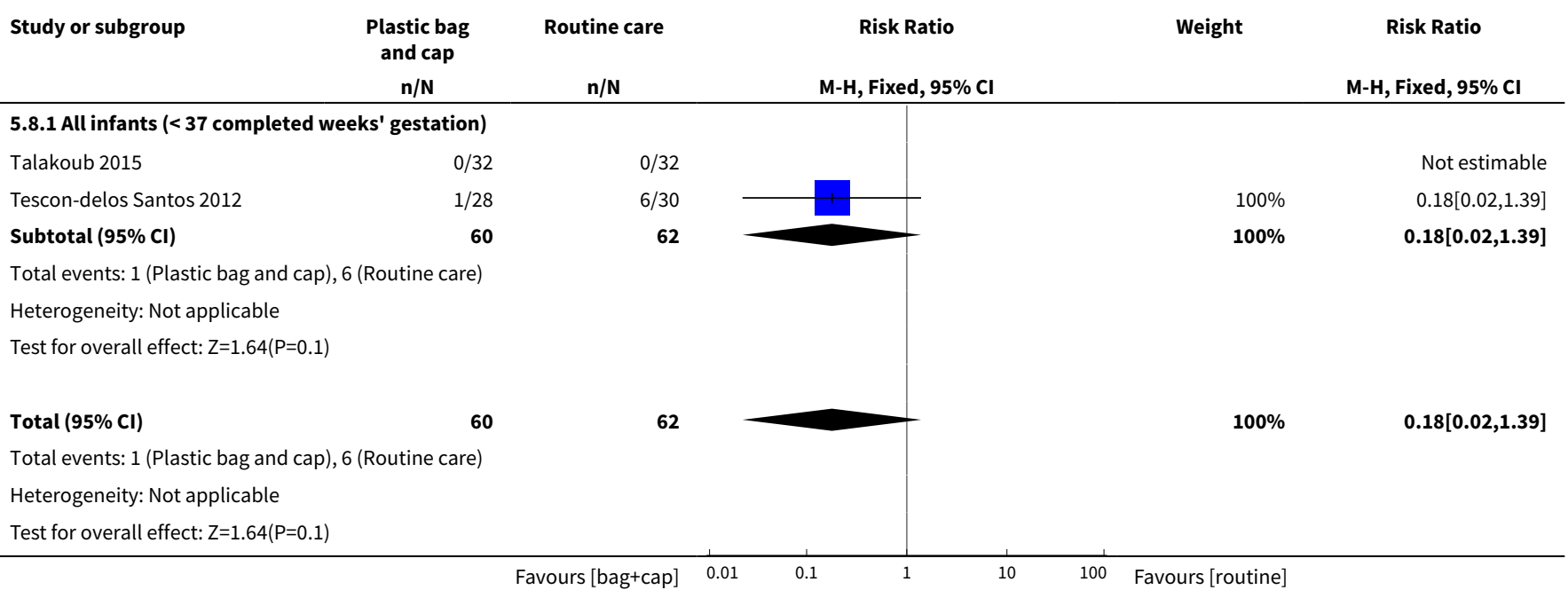

Analysis 5.9. Comparison 5 Plastic bag and plastic cap versus routine care, Outcome 9 Hyponatraemia (serum sodium concentration $<130 \mathrm{mmol} / \mathrm{L}$ ).

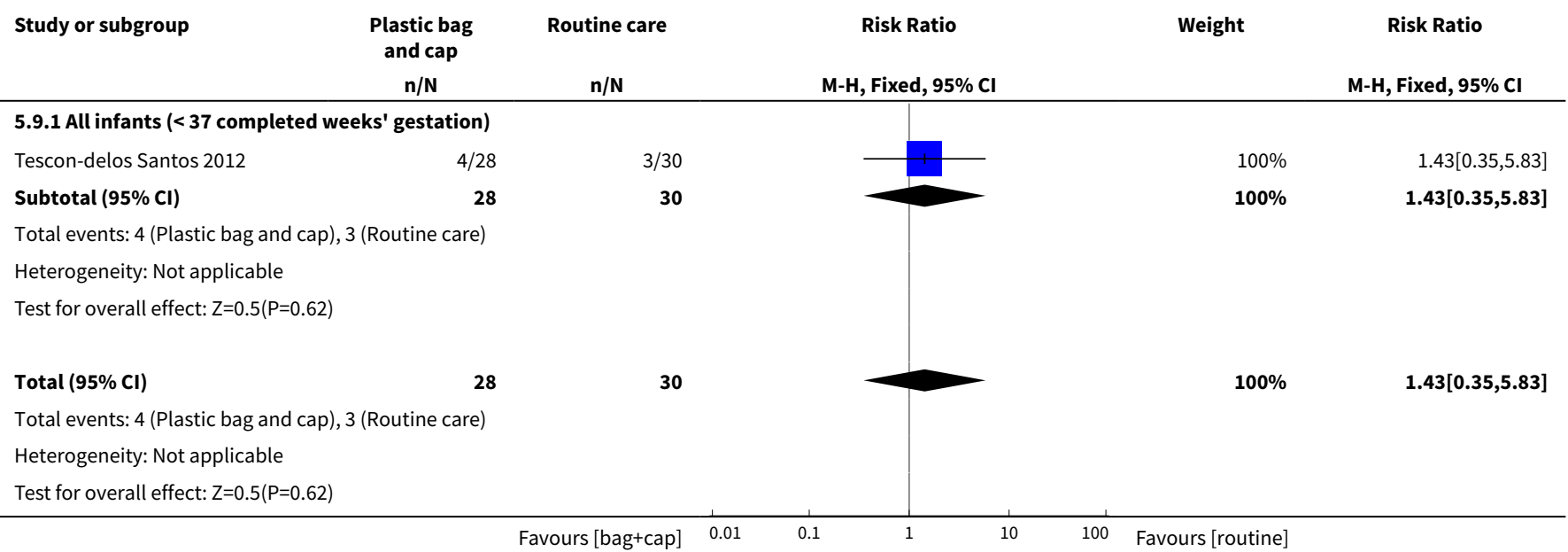

\section{Analysis 5.10. Comparison 5 Plastic bag and plastic cap versus} routine care, Outcome 10 Weight (grams) at fifth day of life.

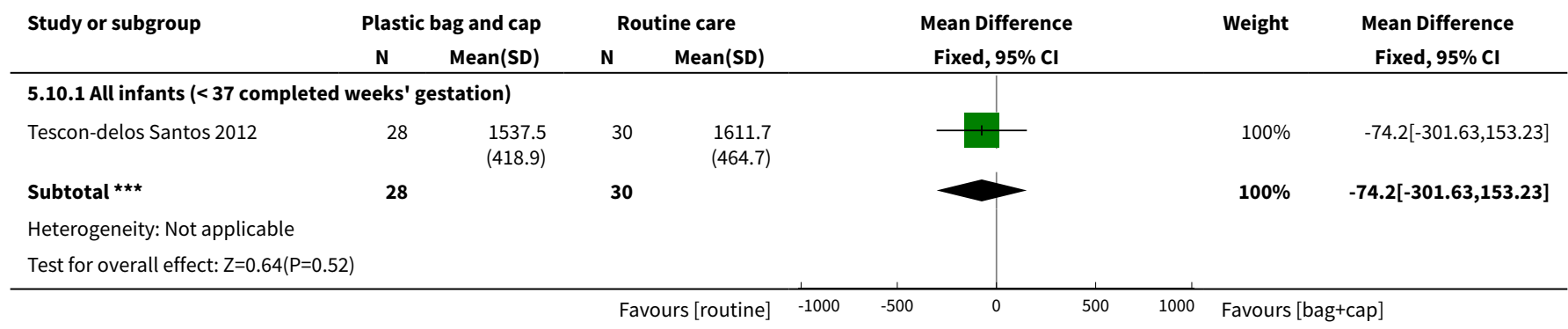




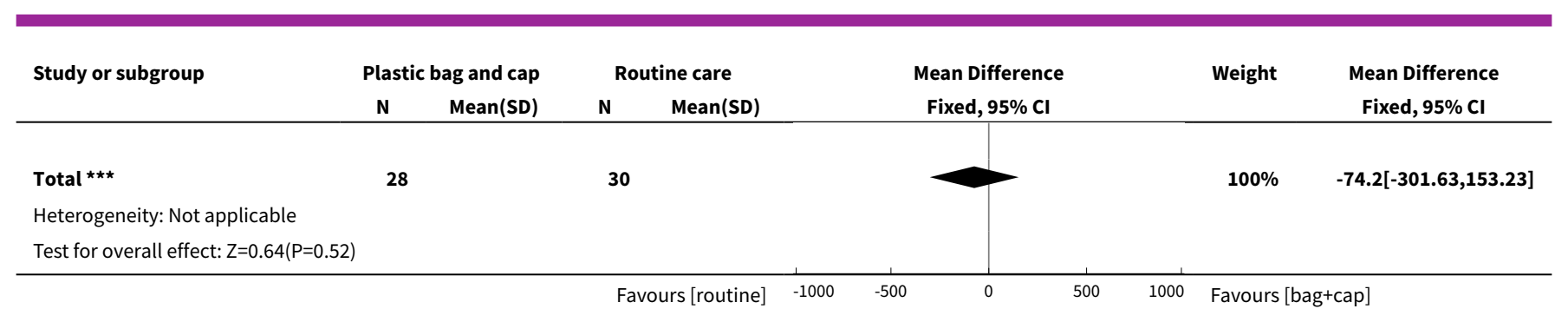

\section{Comparison 6. Plastic bag with previous drying versus plastic bag without previous drying}

\begin{tabular}{|c|c|c|c|c|}
\hline Outcome or subgroup title & No. of studies & $\begin{array}{l}\text { No. of partici- } \\
\text { pants }\end{array}$ & Statistical method & Effect size \\
\hline $\begin{array}{l}1 \text { Core body temperature }\left({ }^{\circ} \mathrm{C}\right) 30 \text { minutes af- } \\
\text { ter birth }\end{array}$ & 1 & 60 & $\begin{array}{l}\text { Mean Difference (IV, } \\
\text { Fixed, } 95 \% \mathrm{CI} \text { ) }\end{array}$ & $0.0[-0.17,0.17]$ \\
\hline $\begin{array}{l}1.1 \text { ( } \geq 28 \text { and }<37 \text { completed weeks' ges- } \\
\text { tation) }+ \text { (birth weight } \geq 1000 \text { grams and } \leq \\
2499 \text { grams) }\end{array}$ & 1 & 60 & $\begin{array}{l}\text { Mean Difference (IV, } \\
\text { Fixed, } 95 \% \mathrm{CI} \text { ) }\end{array}$ & $0.0[-0.17,0.17]$ \\
\hline $\begin{array}{l}2 \text { Core body temperature }\left({ }^{\circ} \mathrm{C}\right) 1 \text { hour after } \\
\text { birth }\end{array}$ & 1 & 60 & $\begin{array}{l}\text { Mean Difference (IV, } \\
\text { Fixed, } 95 \% \mathrm{CI} \text { ) }\end{array}$ & $-0.10[-0.28,0.08]$ \\
\hline $\begin{array}{l}2.1 \text { ( } \geq 28 \text { and }<37 \text { completed weeks' ges- } \\
\text { tation) }+ \text { (birth weight } \geq 1000 \text { grams and } \leq \\
2499 \text { grams) }\end{array}$ & 1 & 60 & $\begin{array}{l}\text { Mean Difference (IV, } \\
\text { Fixed, } 95 \% \mathrm{CI} \text { ) }\end{array}$ & $-0.10[-0.28,0.08]$ \\
\hline $\begin{array}{l}3 \text { Core body temperature }\left({ }^{\circ} \mathrm{C}\right) 90 \text { minutes af- } \\
\text { ter birth }\end{array}$ & 1 & 60 & $\begin{array}{l}\text { Mean Difference (IV, } \\
\text { Fixed, } 95 \% \mathrm{CI} \text { ) }\end{array}$ & $-0.10[-0.29,0.09]$ \\
\hline $\begin{array}{l}3.1 \text { ( } \geq 28 \text { and }<37 \text { completed weeks' ges- } \\
\text { tation })+(\text { birth weight } \geq 1000 \text { grams and } \leq \\
2499 \text { grams) }\end{array}$ & 1 & 60 & $\begin{array}{l}\text { Mean Difference (IV, } \\
\text { Fixed, } 95 \% \mathrm{CI} \text { ) }\end{array}$ & $-0.10[-0.29,0.09]$ \\
\hline $\begin{array}{l}4 \text { Core body temperature }\left({ }^{\circ} \mathrm{C}\right) 2 \text { hours after } \\
\text { birth }\end{array}$ & 1 & 60 & $\begin{array}{l}\text { Mean Difference (IV, } \\
\text { Fixed, } 95 \% \mathrm{CI} \text { ) }\end{array}$ & $0.10[-0.04,0.24]$ \\
\hline $\begin{array}{l}4.1 \text { ( } \geq 28 \text { and }<37 \text { completed weeks' ges- } \\
\text { tation })+(\text { birth weight } \geq 1000 \text { grams and } \leq \\
2499 \text { grams) }\end{array}$ & 1 & 60 & $\begin{array}{l}\text { Mean Difference (IV, } \\
\text { Fixed, } 95 \% \mathrm{CI} \text { ) }\end{array}$ & $0.10[-0.04,0.24]$ \\
\hline 5 Hyperthermia & 1 & 60 & $\begin{array}{l}\text { Risk Ratio (M-H, Fixed, } \\
95 \% \mathrm{Cl})\end{array}$ & $0.33[0.01,7.87]$ \\
\hline $\begin{array}{l}5.1 \text { ( } \geq 28 \text { and }<37 \text { completed weeks' ges- } \\
\text { tation) }+ \text { (birth weight } \geq 1000 \text { grams and } \leq \\
2499 \text { grams) }\end{array}$ & 1 & 60 & $\begin{array}{l}\text { Risk Ratio (M-H, Fixed, } \\
95 \% \mathrm{Cl})\end{array}$ & $0.33[0.01,7.87]$ \\
\hline $\begin{array}{l}6 \text { Glucose concentration }(\mathrm{mmol} / \mathrm{L}) \text { at } 2 \text { hours } \\
\text { after birth }\end{array}$ & & & Other data & No numeric data \\
\hline $\begin{array}{l}6.1 \text { ( } \geq 28 \text { and }<37 \text { completed weeks' ges- } \\
\text { tation) }+ \text { (birth weight } \geq 1000 \text { grams and } \leq \\
2499 \text { grams) }\end{array}$ & & & Other data & No numeric data \\
\hline
\end{tabular}


Analysis 6.1. Comparison 6 Plastic bag with previous drying versus plastic bag without previous drying, Outcome 1 Core body temperature $\left({ }^{\circ} \mathrm{C}\right) 30$ minutes after birth.

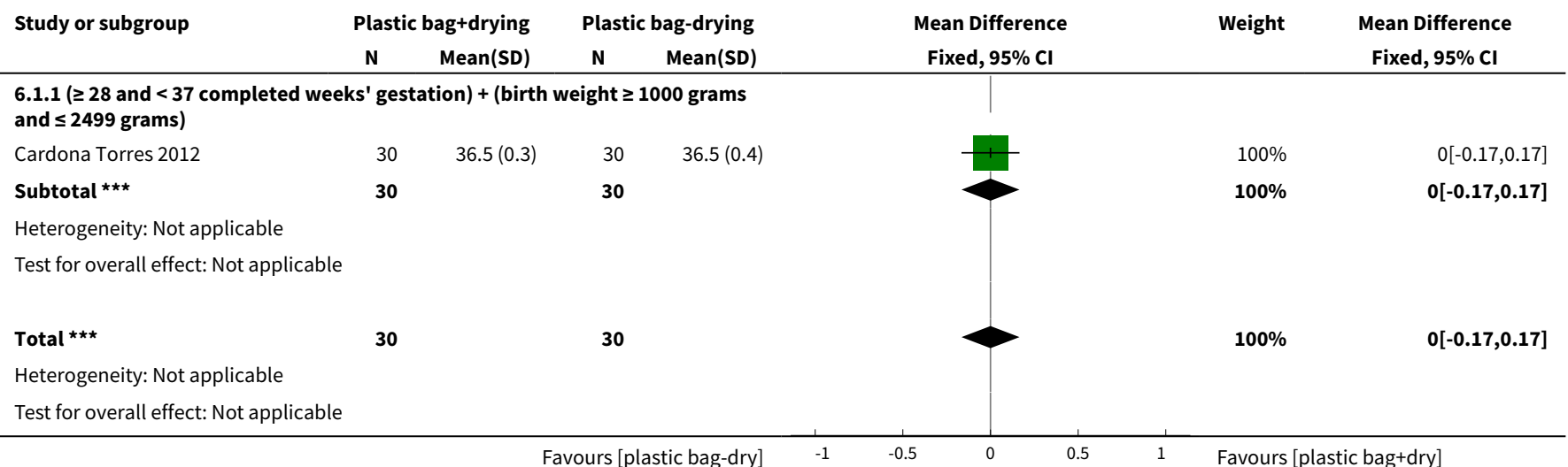

Analysis 6.2. Comparison 6 Plastic bag with previous drying versus plastic bag without previous drying, Outcome 2 Core body temperature $\left({ }^{\circ} \mathrm{C}\right) 1$ hour after birth.

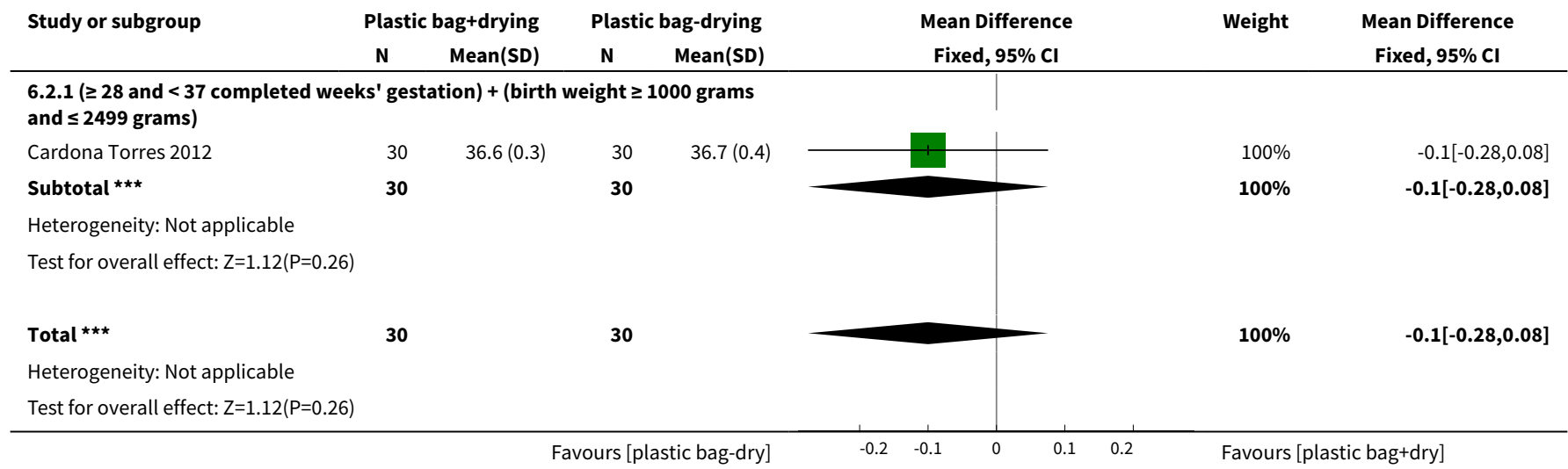

Analysis 6.3. Comparison 6 Plastic bag with previous drying versus plastic bag without previous drying, Outcome 3 Core body temperature $\left({ }^{\circ} \mathrm{C}\right) 90$ minutes after birth.

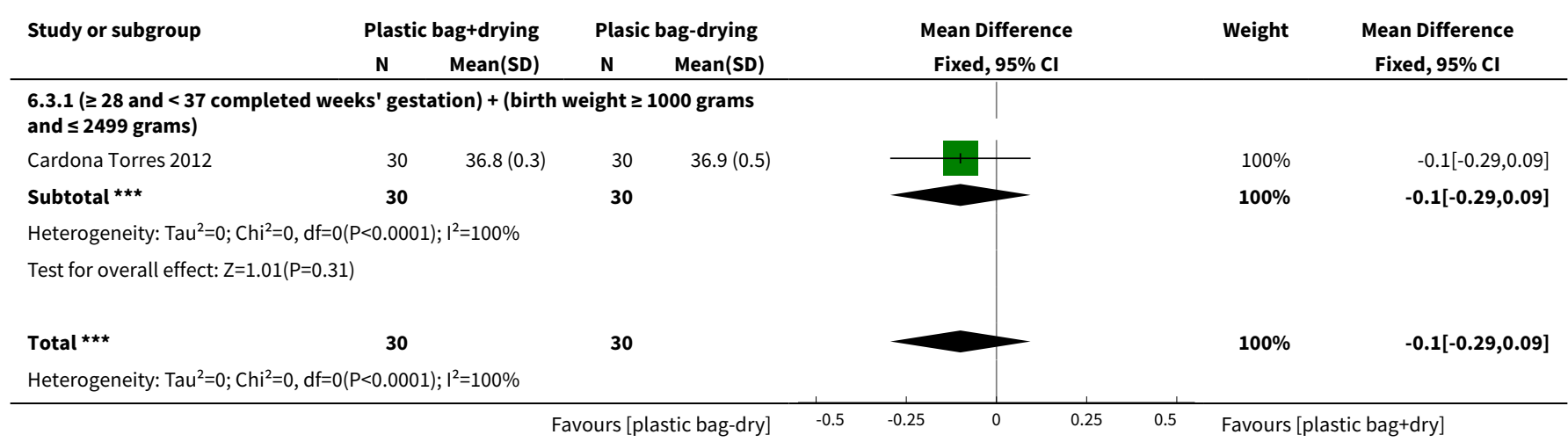




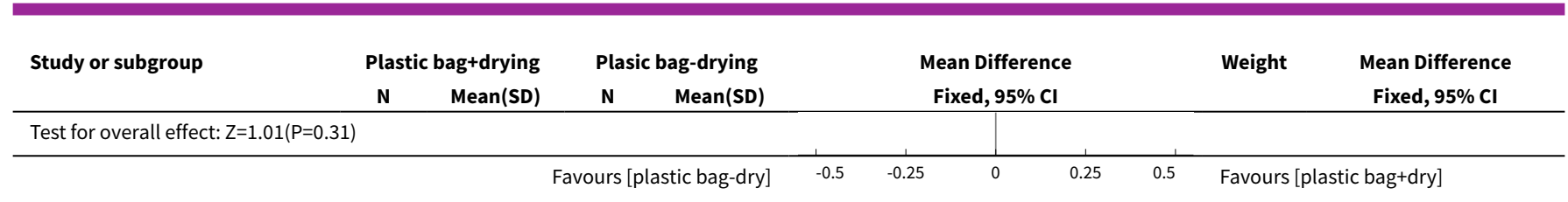

Analysis 6.4. Comparison 6 Plastic bag with previous drying versus plastic bag without previous drying, Outcome 4 Core body temperature $\left({ }^{\circ} \mathrm{C}\right) 2$ hours after birth.

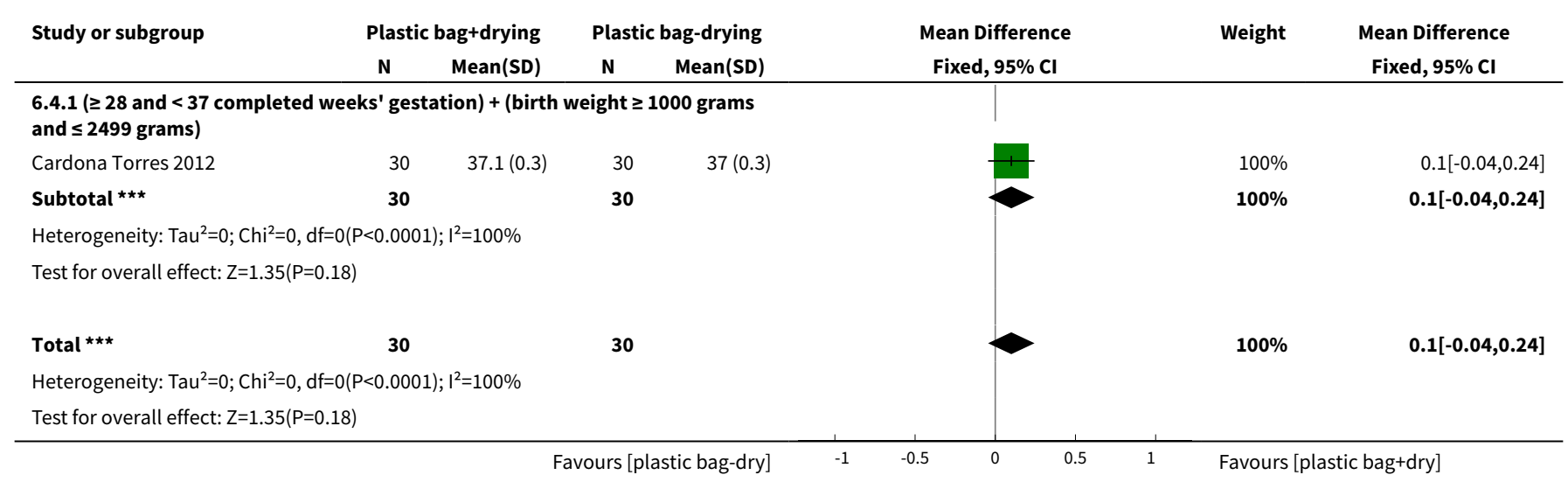

Analysis 6.5. Comparison 6 Plastic bag with previous drying versus plastic bag without previous drying, Outcome 5 Hyperthermia.

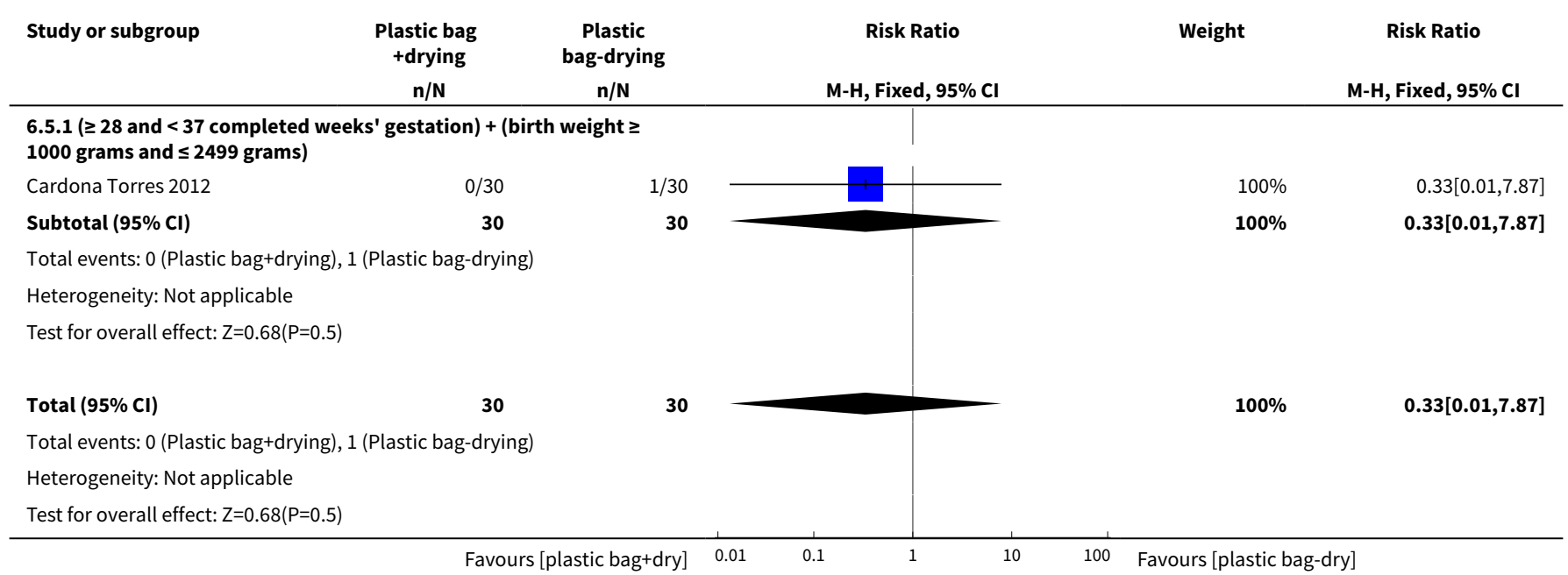

Analysis 6.6. Comparison 6 Plastic bag with previous drying versus plastic bag without previous drying, Outcome 6 Glucose concentration ( $\mathrm{mmol} / \mathrm{L}$ ) at 2 hours after birth.

\begin{tabular}{|c|c|c|c|c|c|}
\hline \multicolumn{6}{|c|}{ Glucose concentration ( $\mathrm{mmol} / \mathrm{L}$ ) at 2 hours after birth } \\
\hline Study & Group & $\mathbf{N}$ & Median & Interquartile range & P value \\
\hline \multicolumn{6}{|c|}{ ( $\geq 28$ and $<37$ completed weeks' gestation) + (birth weight $\geq 1000$ grams and $\leq 2499$ grams) } \\
\hline Cardona Torres 2012 & Plastic bag with drying & 30 & 3.8 & $2.6-5.5$ & $P>0.05$ \\
\hline
\end{tabular}




\begin{tabular}{cccccc} 
& \multicolumn{4}{c}{ Glucose concentration (mmol/L) at 2 hours after birth } \\
Study & Group & & N & Median & Interquartile range \\
\hline Cardona Torres 2012 & $\begin{array}{l}\text { Plasic bag without dry- } \\
\text { ing }\end{array}$ & 30 & 5.4 & $3.2-8.8$ \\
\hline
\end{tabular}

\section{Comparison 7. Plastic cap versus plastic bag}

\begin{tabular}{|c|c|c|c|c|}
\hline Outcome or subgroup title & No. of studies & $\begin{array}{l}\text { No. of partici- } \\
\text { pants }\end{array}$ & Statistical method & Effect size \\
\hline $\begin{array}{l}1 \text { Core body temperature }\left({ }^{\circ} \mathrm{C}\right) \text { on ad- } \\
\text { mission to NICU or up to } 2 \text { hours after } \\
\text { birth }\end{array}$ & 1 & 64 & $\begin{array}{l}\text { Mean Difference (IV, Fixed, } \\
95 \% \mathrm{CI})\end{array}$ & $0.30[-0.12,0.72]$ \\
\hline $1.1<29$ completed weeks' gestation & 1 & 64 & $\begin{array}{l}\text { Mean Difference (IV, Fixed, } \\
95 \% \mathrm{CI} \text { ) }\end{array}$ & $0.30[-0.12,0.72]$ \\
\hline $\begin{array}{l}2 \text { Hypothermia on admission to NICU: } \\
\text { core body temperature }<36.4^{\circ} \mathrm{C}\end{array}$ & 1 & 64 & $\begin{array}{l}\text { Risk Ratio (M-H, Fixed, 95\% } \\
\mathrm{Cl})\end{array}$ & $0.7[0.43,1.13]$ \\
\hline $2.1<29$ completed weeks' gestation & 1 & 64 & $\begin{array}{l}\text { Risk Ratio (M-H, Fixed, 95\% } \\
\text { Cl) }\end{array}$ & $0.7[0.43,1.13]$ \\
\hline $\begin{array}{l}3 \text { Outside normothermic range on ad- } \\
\text { mission to NICU or up to } 2 \text { hours after } \\
\text { birth }\end{array}$ & 1 & 64 & $\begin{array}{l}\text { Risk Ratio (M-H, Fixed, 95\% } \\
\mathrm{Cl})\end{array}$ & $0.64[0.40,1.01]$ \\
\hline $3.1<29$ completed weeks' gestation & 1 & 64 & $\begin{array}{l}\text { Risk Ratio (M-H, Fixed, 95\% } \\
\text { Cl) }\end{array}$ & $0.64[0.40,1.01]$ \\
\hline $\begin{array}{l}4 \text { Core body temperature }\left({ }^{\circ} \mathrm{C}\right) 1 \text { hour } \\
\text { after initial temperature on admis- } \\
\text { sion to NICU taken }\end{array}$ & 1 & 64 & $\begin{array}{l}\text { Mean Difference (IV, Fixed, } \\
95 \% \mathrm{CI})\end{array}$ & $0.30[0.00,0.60]$ \\
\hline $4.1<29$ completed weeks' gestation & 1 & 64 & $\begin{array}{l}\text { Mean Difference (IV, Fixed, } \\
95 \% \mathrm{CI})\end{array}$ & $0.30[0.00,0.60]$ \\
\hline $\begin{array}{l}5 \text { Hyperthermia on admission to } \\
\text { NICU: core body temperature }>37.5^{\circ} \mathrm{C}\end{array}$ & 1 & 64 & $\begin{array}{l}\text { Risk Ratio (M-H, Fixed, 95\% } \\
\mathrm{Cl})\end{array}$ & $0.2[0.01,4.01]$ \\
\hline $5.1<29$ completed weeks' gestation & 1 & 64 & $\begin{array}{l}\text { Risk Ratio (M-H, Fixed, 95\% } \\
\mathrm{Cl})\end{array}$ & $0.2[0.01,4.01]$ \\
\hline 6 Major brain injury & 1 & 64 & $\begin{array}{l}\text { Risk Ratio (M-H, Fixed, 95\% } \\
\mathrm{Cl})\end{array}$ & $1.5[0.27,8.38]$ \\
\hline $6.1<29$ completed weeks' gestation & 1 & 64 & $\begin{array}{l}\text { Risk Ratio (M-H, Fixed, 95\% } \\
\mathrm{Cl})\end{array}$ & $1.5[0.27,8.38]$ \\
\hline 7 Mortality (death at discharge) & 1 & 64 & $\begin{array}{l}\text { Risk Ratio (M-H, Fixed, 95\% } \\
\mathrm{Cl} \text { ) }\end{array}$ & $1.5[0.27,8.38]$ \\
\hline $7.1<29$ completed weeks' gestation & 1 & 64 & $\begin{array}{l}\text { Risk Ratio (M-H, Fixed, 95\% } \\
\mathrm{Cl})\end{array}$ & $1.5[0.27,8.38]$ \\
\hline $\begin{array}{l}8 \text { Bicarbonate concentration ( } \mathrm{mmol} / \\
\text { L) }\end{array}$ & 1 & 64 & $\begin{array}{l}\text { Mean Difference (IV, Fixed, } \\
95 \% \mathrm{CI} \text { ) }\end{array}$ & $1.0[0.02,1.98]$ \\
\hline
\end{tabular}




\begin{tabular}{|c|c|c|c|c|}
\hline Outcome or subgroup title & No. of studies & $\begin{array}{l}\text { No. of partici- } \\
\text { pants }\end{array}$ & Statistical method & Effect size \\
\hline $8.1<29$ completed weeks' gestation & 1 & 64 & $\begin{array}{l}\text { Mean Difference (IV, Fixed, } \\
95 \% \mathrm{Cl} \text { ) }\end{array}$ & $1.0[0.02,1.98]$ \\
\hline 9 Blood gas $\mathrm{pH}$ & 1 & 64 & $\begin{array}{l}\text { Mean Difference (IV, Fixed, } \\
95 \% \mathrm{Cl})\end{array}$ & $-0.02[-0.06,0.02]$ \\
\hline $9.1<29$ completed weeks' gestation & 1 & 64 & $\begin{array}{l}\text { Mean Difference (IV, Fixed, } \\
95 \% \mathrm{CI})\end{array}$ & $-0.02[-0.06,0.02]$ \\
\hline $\begin{array}{l}10 \text { First serum glucose concentration } \\
(\mathrm{mmol} / \mathrm{L}) \text { on admission to NICU }\end{array}$ & 1 & 64 & $\begin{array}{l}\text { Mean Difference (IV, Fixed, } \\
95 \% \mathrm{Cl} \text { ) }\end{array}$ & $0.0[-0.50,0.50]$ \\
\hline $10.1<29$ completed weeks' gestation & 1 & 64 & $\begin{array}{l}\text { Mean Difference (IV, Fixed, } \\
95 \% \mathrm{Cl} \text { ) }\end{array}$ & $0.0[-0.50,0.50]$ \\
\hline 11 Intubation at birth & 1 & 64 & $\begin{array}{l}\text { Risk Ratio (M-H, Fixed, 95\% } \\
\text { Cl) }\end{array}$ & $0.82[0.49,1.37]$ \\
\hline $11.1<29$ completed weeks' gestation & 1 & 64 & $\begin{array}{l}\text { Risk Ratio (M-H, Fixed, 95\% } \\
\mathrm{Cl} \text { ) }\end{array}$ & $0.82[0.49,1.37]$ \\
\hline
\end{tabular}

\section{Analysis 7.1. Comparison 7 Plastic cap versus plastic bag, Outcome 1 Core} body temperature $\left({ }^{\circ} \mathrm{C}\right)$ on admission to NICU or up to 2 hours after birth.

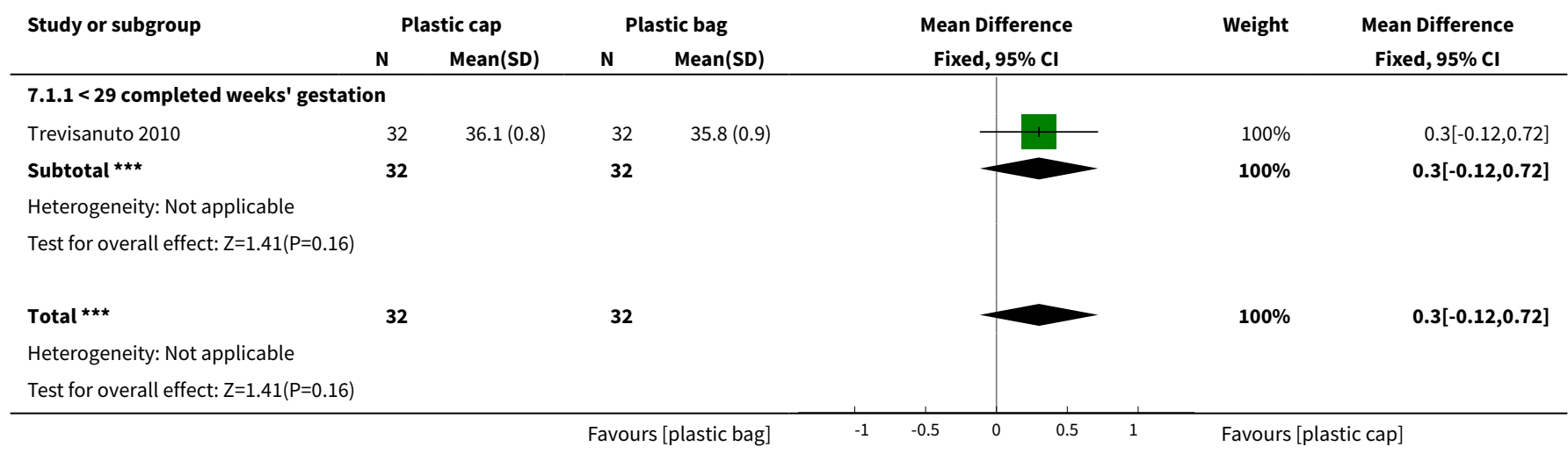

Analysis 7.2. Comparison 7 Plastic cap versus plastic bag, Outcome 2 Hypothermia on admission to NICU: core body temperature $<36.4^{\circ} \mathrm{C}$.

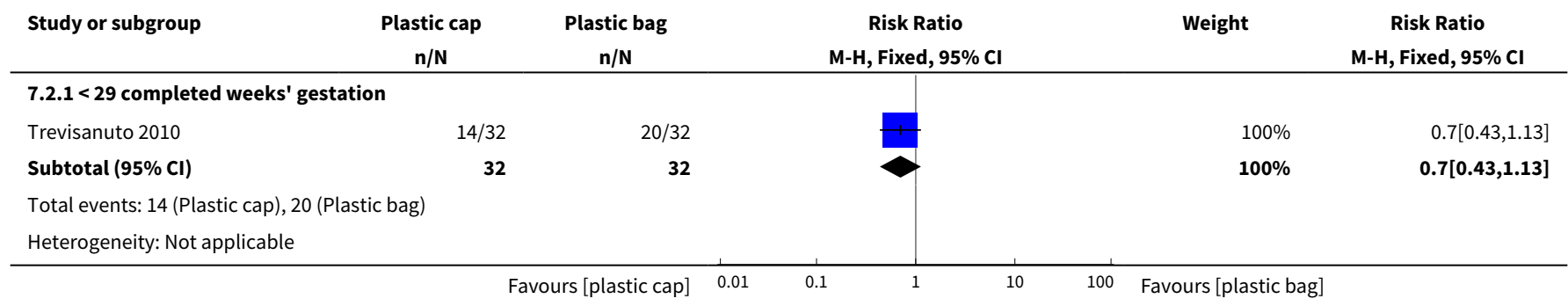




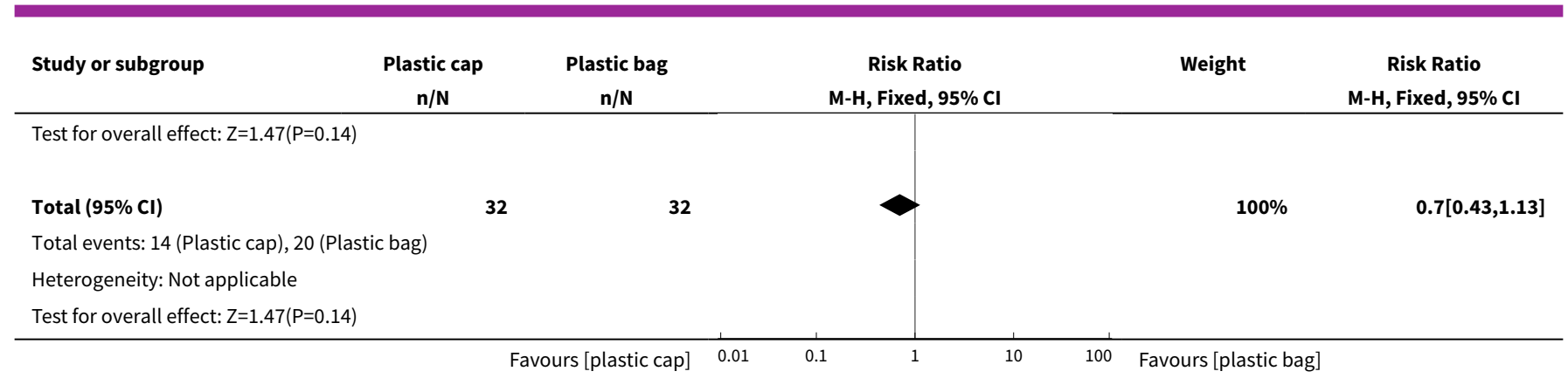

\section{Analysis 7.3. Comparison 7 Plastic cap versus plastic bag, Outcome 3 Outside} normothermic range on admission to NICU or up to 2 hours after birth.

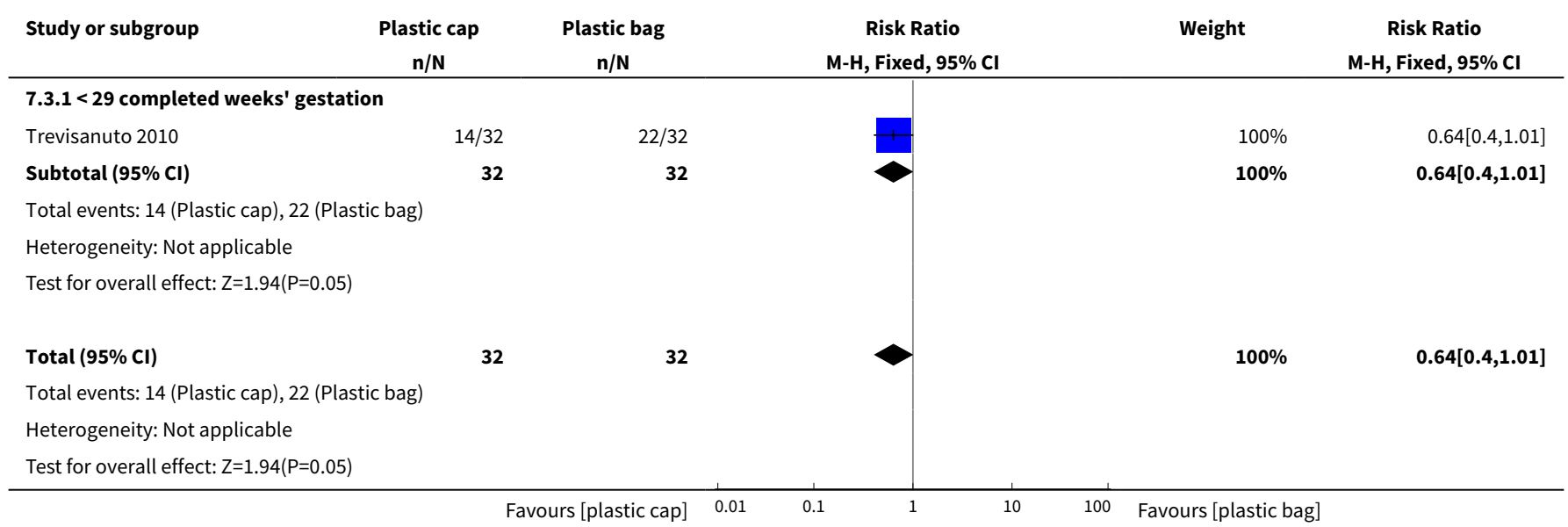

Analysis 7.4. Comparison 7 Plastic cap versus plastic bag, Outcome 4 Core body temperature $\left({ }^{\circ} \mathrm{C}\right) \mathbf{1}$ hour after initial temperature on admission to NICU taken.

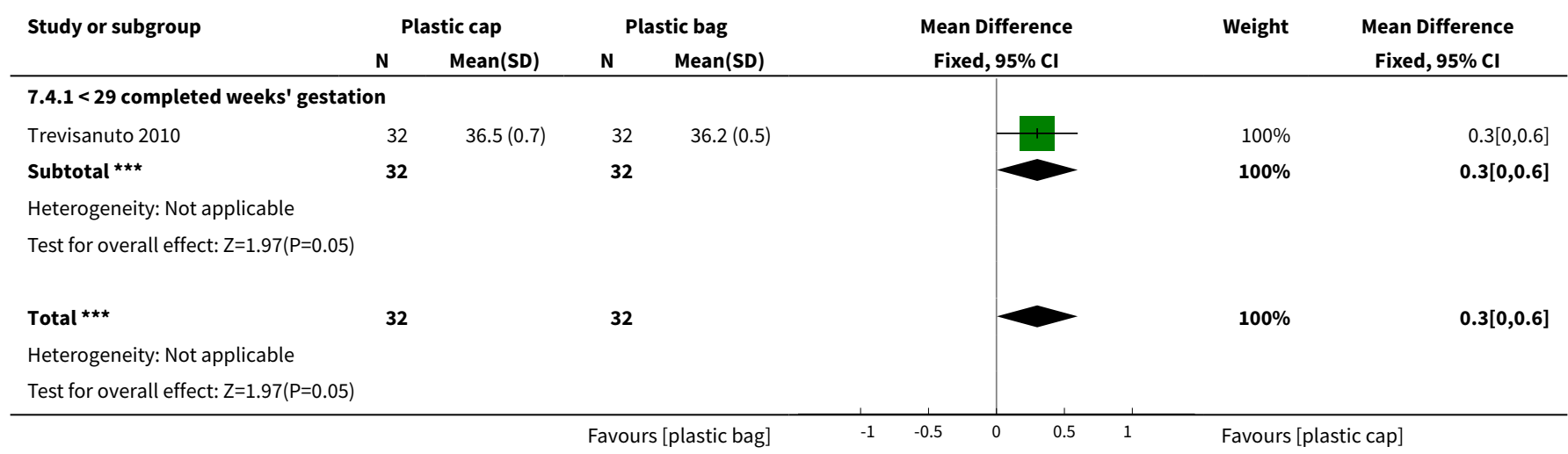


Analysis 7.5. Comparison 7 Plastic cap versus plastic bag, Outcome 5

Hyperthermia on admission to NICU: core body temperature $>37.5^{\circ} \mathrm{C}$.

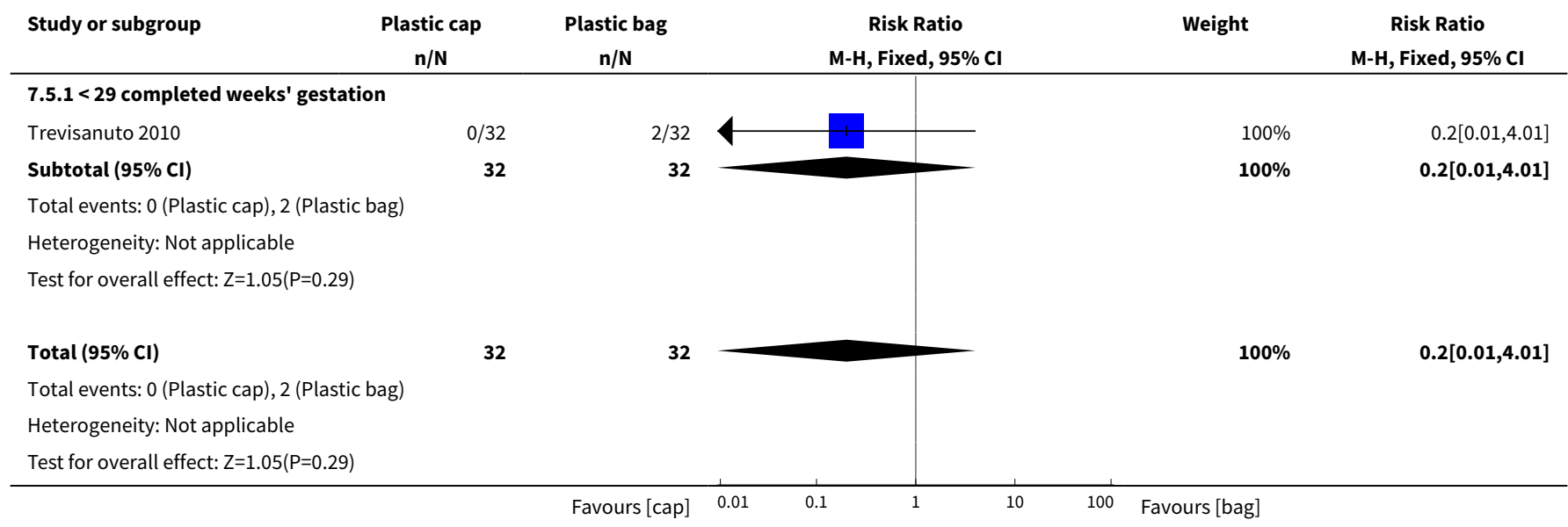

Analysis 7.6. Comparison 7 Plastic cap versus plastic bag, Outcome 6 Major brain injury.

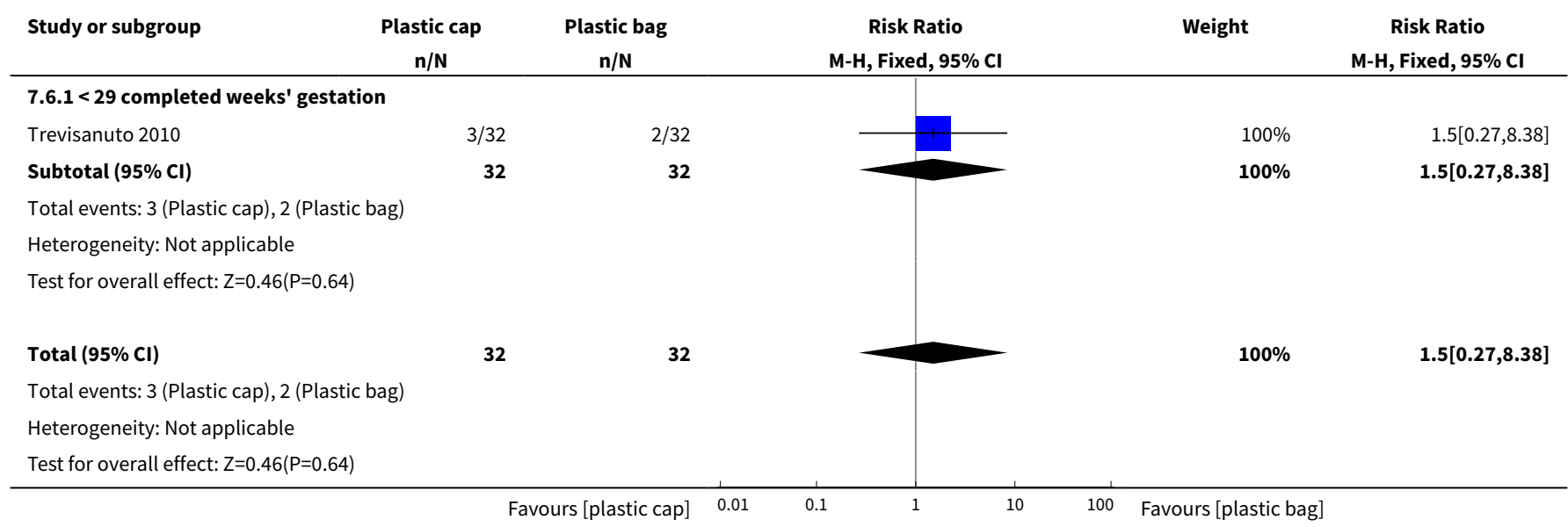

Analysis 7.7. Comparison 7 Plastic cap versus plastic bag, Outcome 7 Mortality (death at discharge).

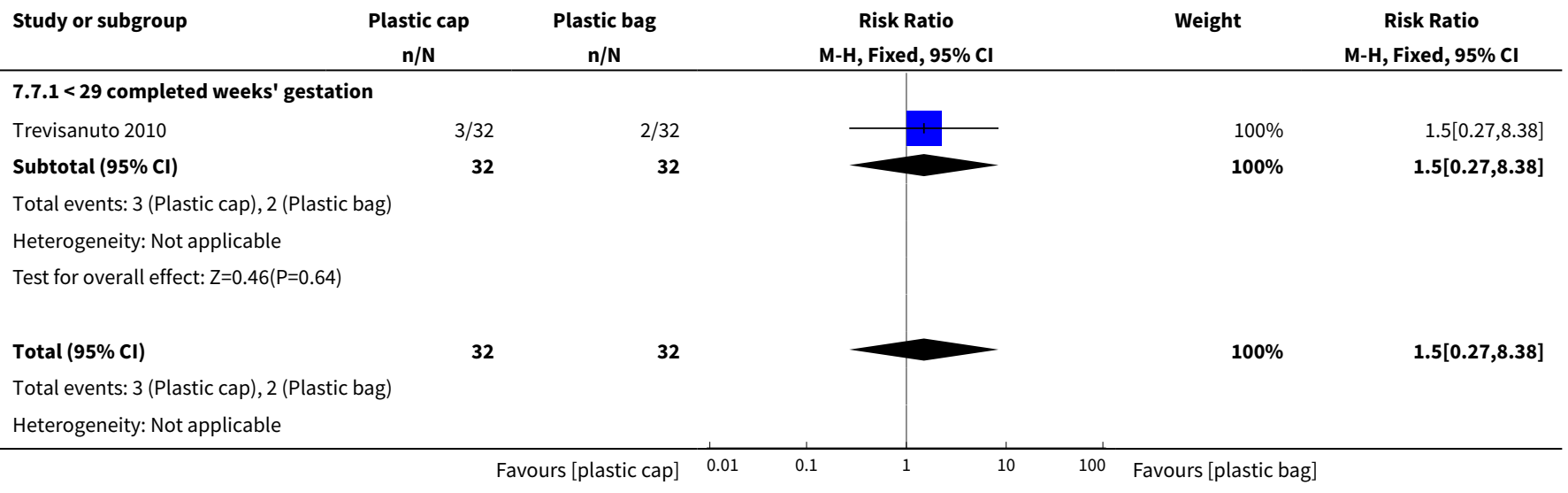




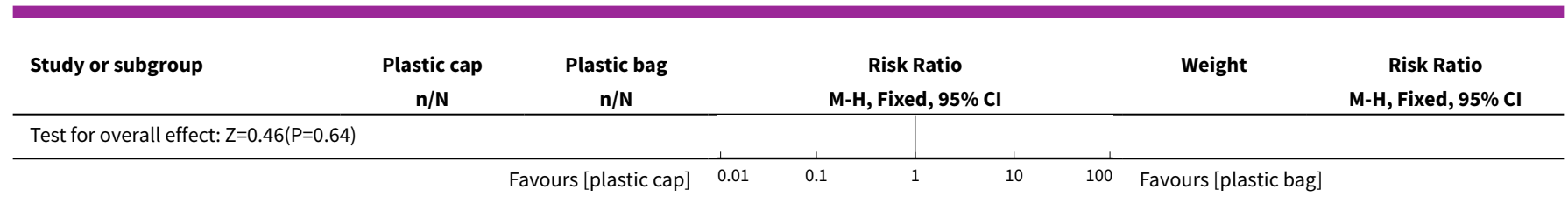

Analysis 7.8. Comparison 7 Plastic cap versus plastic bag, Outcome 8 Bicarbonate concentration (mmol/L).

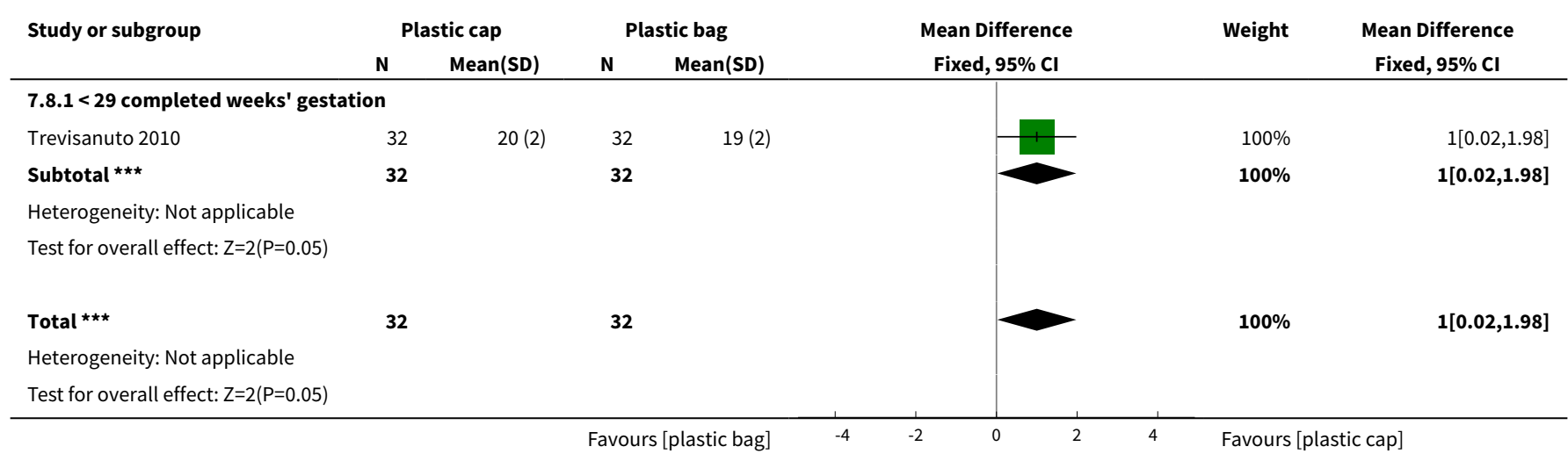

Analysis 7.9. Comparison 7 Plastic cap versus plastic bag, Outcome 9 Blood gas pH.

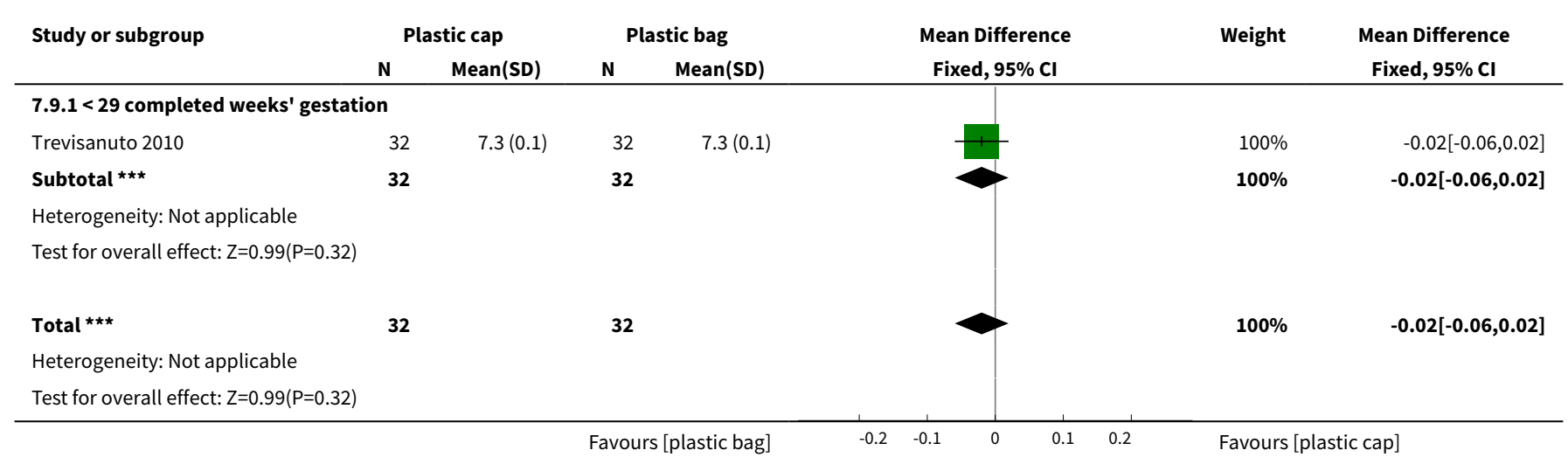

Analysis 7.10. Comparison 7 Plastic cap versus plastic bag, Outcome 10 First serum glucose concentration $(\mathrm{mmol} / \mathrm{L})$ on admission to NICU.

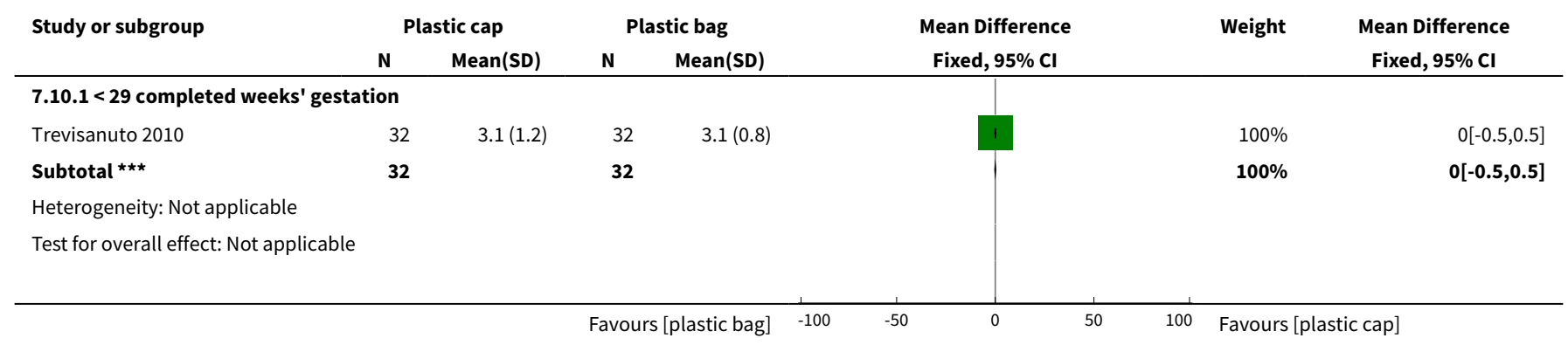




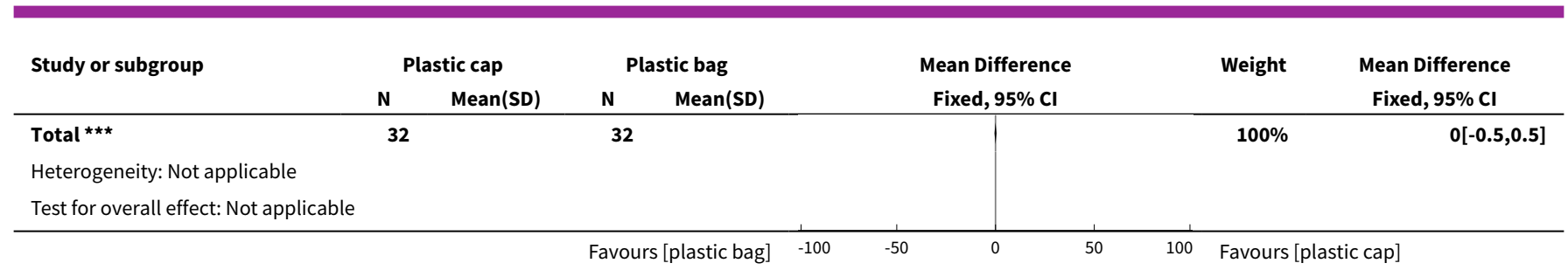

\section{Analysis 7.11. Comparison 7 Plastic cap versus plastic bag, Outcome 11 Intubation at birth.}

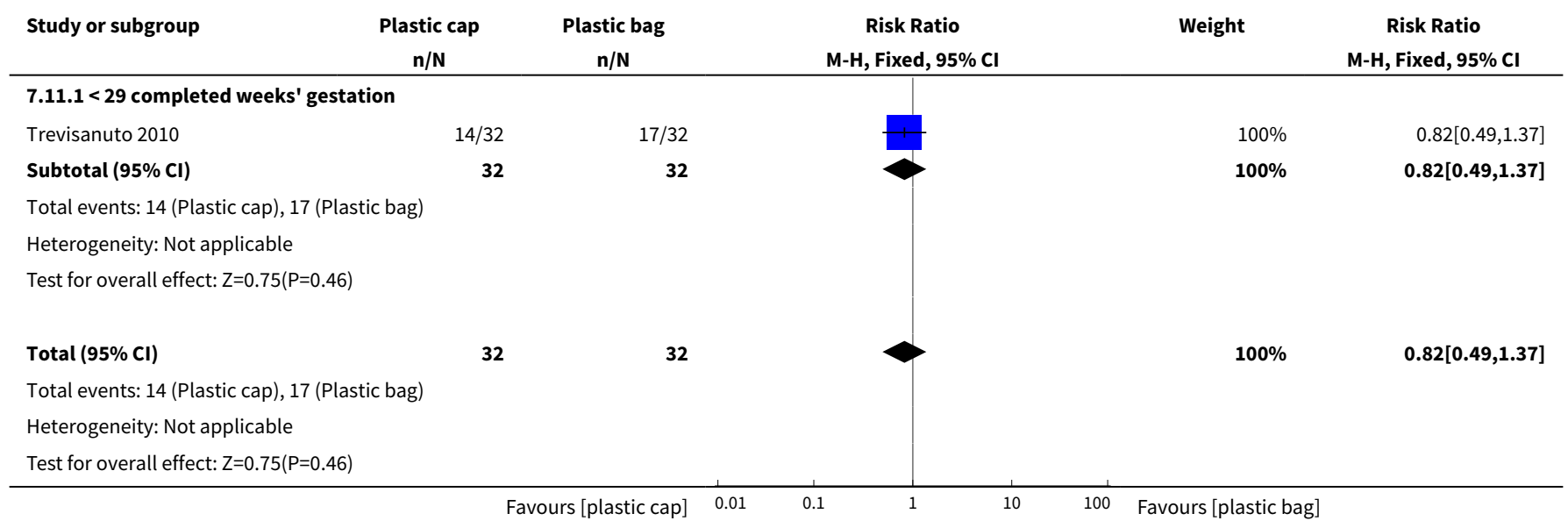

\section{Comparison 8. Plastic bag versus plastic wrap}

\begin{tabular}{|c|c|c|c|c|}
\hline Outcome or subgroup title & No. of studies & $\begin{array}{l}\text { No. of partici- } \\
\text { pants }\end{array}$ & Statistical method & Effect size \\
\hline $\begin{array}{l}1 \text { Core body temperature }\left({ }^{\circ} \mathrm{C}\right) 20 \mathrm{~min}- \\
\text { utes after birth }\end{array}$ & 1 & 59 & $\begin{array}{l}\text { Mean Difference (IV, Fixed, } \\
95 \% \mathrm{CI})\end{array}$ & $0.14[0.08,0.20]$ \\
\hline $1.1 \leq 32$ completed weeks' gestation & 1 & 59 & $\begin{array}{l}\text { Mean Difference (IV, Fixed, } \\
95 \% \mathrm{CI} \text { ) }\end{array}$ & $0.14[0.08,0.20]$ \\
\hline $\begin{array}{l}2 \text { Core body temperature }\left({ }^{\circ} \mathrm{C}\right) 40 \mathrm{~min}- \\
\text { utes after birth }\end{array}$ & 1 & 59 & $\begin{array}{l}\text { Mean Difference (IV, Fixed, } \\
95 \% \mathrm{CI} \text { ) }\end{array}$ & $0.30[0.24,0.36]$ \\
\hline $2.1 \leq 32$ completed weeks' gestation & 1 & 59 & $\begin{array}{l}\text { Mean Difference (IV, Fixed, } \\
95 \% \mathrm{CI})\end{array}$ & $0.30[0.24,0.36]$ \\
\hline $\begin{array}{l}3 \text { Core body temperature }\left({ }^{\circ} \mathrm{C}\right) 1 \text { hour af- } \\
\text { ter birth }\end{array}$ & 1 & 59 & $\begin{array}{l}\text { Mean Difference (IV, Fixed, } \\
95 \% \mathrm{CI})\end{array}$ & $0.35[0.29,0.41]$ \\
\hline $3.1 \leq 32$ completed weeks' gestation & 1 & 59 & $\begin{array}{l}\text { Mean Difference (IV, Fixed, } \\
95 \% \mathrm{CI})\end{array}$ & $0.35[0.29,0.41]$ \\
\hline $\begin{array}{l}4 \text { Decrease in core body temperature } \\
\left({ }^{\circ} \mathrm{C}\right) \text { during } 1 \text { hour after birth }\end{array}$ & 1 & 59 & $\begin{array}{l}\text { Mean Difference (IV, Fixed, } \\
95 \% \mathrm{CI})\end{array}$ & $\begin{array}{l}-1.34[-2.22 \\
-0.46]\end{array}$ \\
\hline
\end{tabular}




\begin{tabular}{|c|c|c|c|c|}
\hline Outcome or subgroup title & No. of studies & $\begin{array}{l}\text { No. of partici- } \\
\text { pants }\end{array}$ & Statistical method & Effect size \\
\hline $4.1 \leq 32$ completed weeks' gestation & 1 & 59 & $\begin{array}{l}\text { Mean Difference (IV, Fixed, } \\
95 \% \mathrm{CI})\end{array}$ & $\begin{array}{l}-1.34[-2.22 \\
-0.46]\end{array}$ \\
\hline $\begin{array}{l}5 \text { Hypothermia within } 1 \text { hour after birth: } \\
\text { core body temperature }<36.5^{\circ} \mathrm{C} \text { or skin } \\
\text { temperature }<36^{\circ} \mathrm{C}\end{array}$ & 1 & 59 & $\begin{array}{l}\text { Risk Ratio (M-H, Fixed, 95\% } \\
\mathrm{Cl} \text { ) }\end{array}$ & $0.84[0.63,1.12]$ \\
\hline $5.1 \leq 32$ completed weeks' gestation & 1 & 59 & $\begin{array}{l}\text { Risk Ratio (M-H, Fixed, 95\% } \\
\mathrm{Cl} \text { ) }\end{array}$ & $0.84[0.63,1.12]$ \\
\hline $\begin{array}{l}6 \text { Moderate hypothermia within } 1 \text { hour } \\
\text { after birth: core body temperature } 32^{\circ} \mathrm{C} \\
\text { to } 35.9^{\circ} \mathrm{C}\end{array}$ & 1 & 59 & $\begin{array}{l}\text { Risk Ratio (M-H, Fixed, 95\% } \\
\mathrm{Cl} \text { ) }\end{array}$ & $0.56[0.31,1.02]$ \\
\hline $6.1 \leq 32$ completed weeks' gestation & 1 & 59 & $\begin{array}{l}\text { Risk Ratio (M-H, Fixed, 95\% } \\
\mathrm{Cl} \text { ) }\end{array}$ & $0.56[0.31,1.02]$ \\
\hline $\begin{array}{l}7 \text { Outside normothermic range on ad- } \\
\text { mission to NICU or up to } 2 \text { hours after } \\
\text { birth }\end{array}$ & 1 & 59 & $\begin{array}{l}\text { Risk Ratio (M-H, Fixed, 95\% } \\
\mathrm{Cl} \text { ) }\end{array}$ & $0.84[0.63,1.12]$ \\
\hline $7.1 \leq 32$ completed weeks' gestation & 1 & 59 & $\begin{array}{l}\text { Risk Ratio (M-H, Fixed, 95\% } \\
\mathrm{Cl} \text { ) }\end{array}$ & $0.84[0.63,1.12]$ \\
\hline
\end{tabular}

Analysis 8.1. Comparison 8 Plastic bag versus plastic wrap, Outcome 1 Core body temperature $\left({ }^{\circ} \mathrm{C}\right) 20$ minutes after birth.

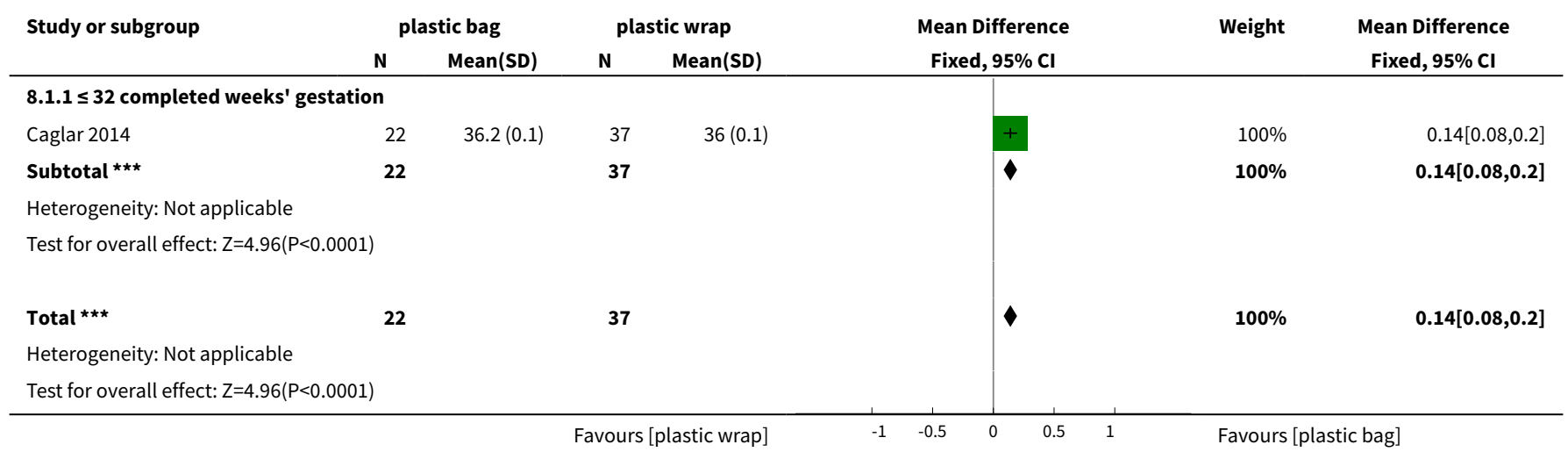

Analysis 8.2. Comparison 8 Plastic bag versus plastic wrap, Outcome 2 Core body temperature $\left({ }^{\circ} \mathrm{C}\right) \mathbf{4 0}$ minutes after birth.

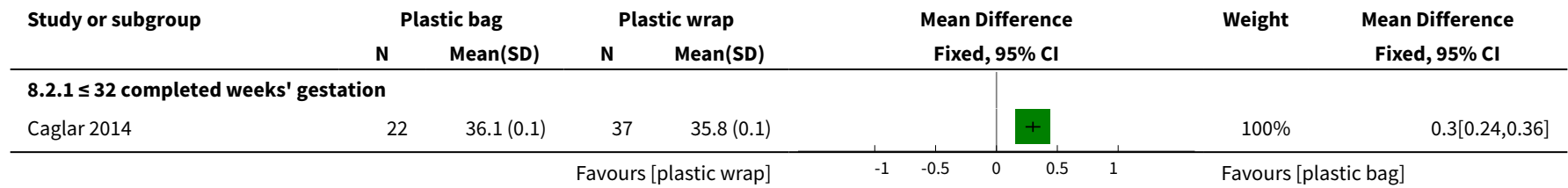




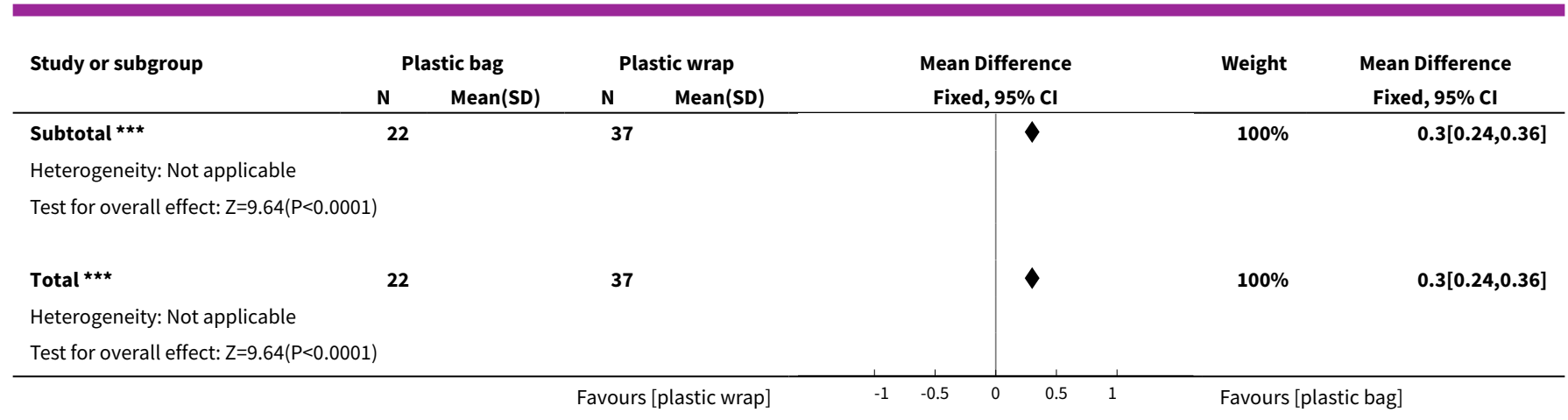

\section{Analysis 8.3. Comparison 8 Plastic bag versus plastic wrap, \\ Outcome 3 Core body temperature $\left({ }^{\circ} \mathrm{C}\right) 1$ hour after birth.}

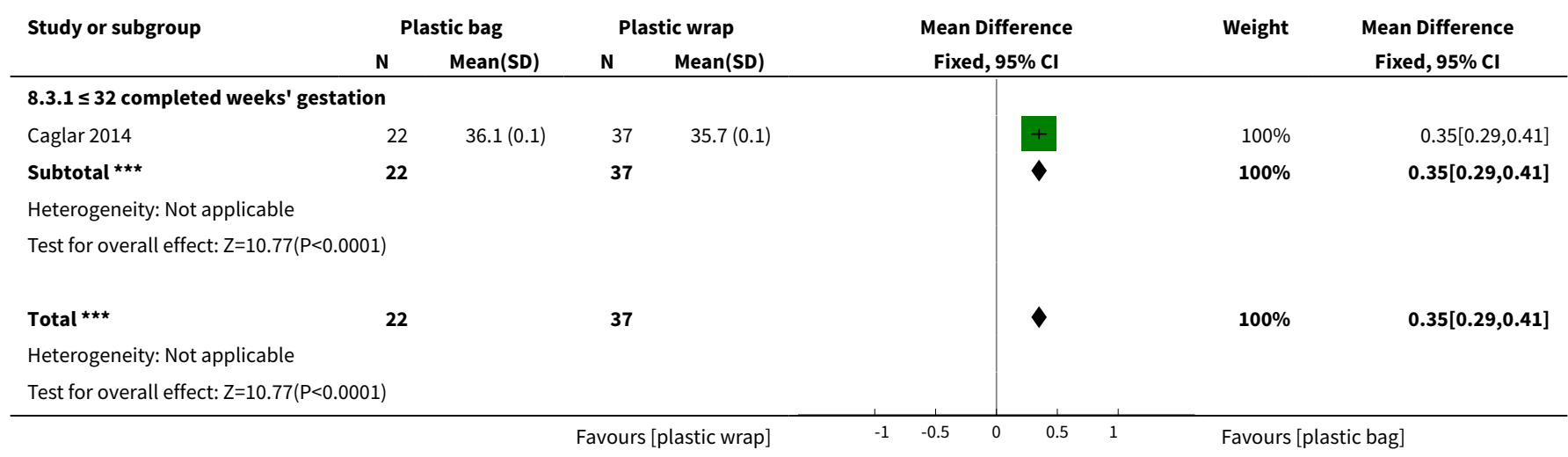

Analysis 8.4. Comparison 8 Plastic bag versus plastic wrap, Outcome

4 Decrease in core body temperature $\left({ }^{\circ} \mathrm{C}\right)$ during 1 hour after birth.

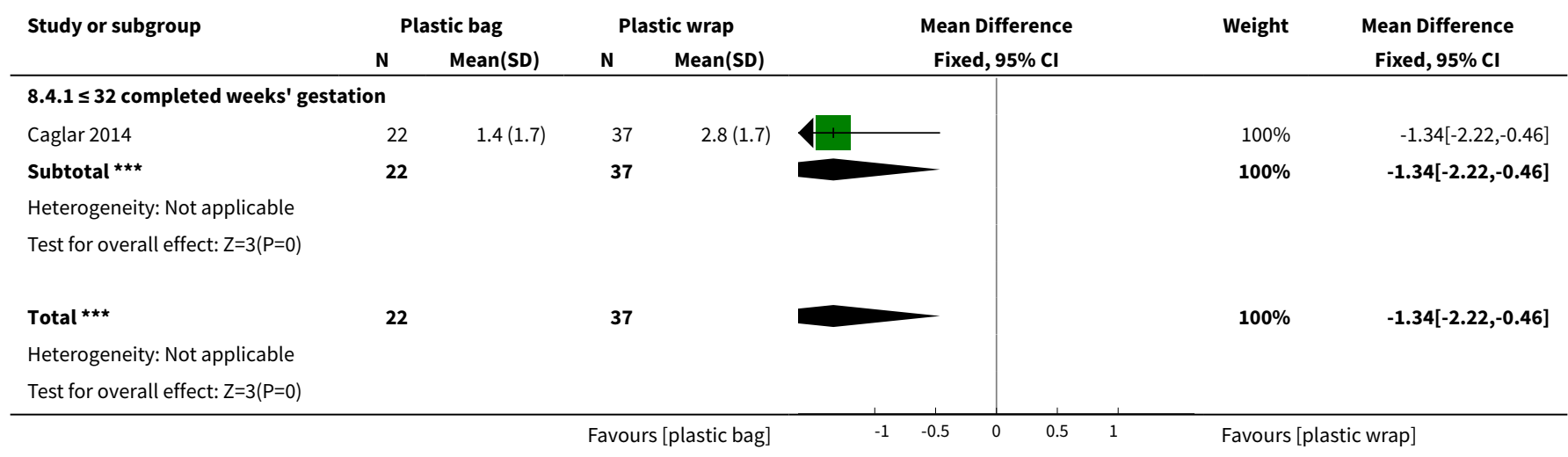


Analysis 8.5. Comparison 8 Plastic bag versus plastic wrap, Outcome 5 Hypothermia within 1 hour after birth: core body temperature $<36.5^{\circ} \mathrm{C}$ or skin temperature $<36^{\circ} \mathrm{C}$.

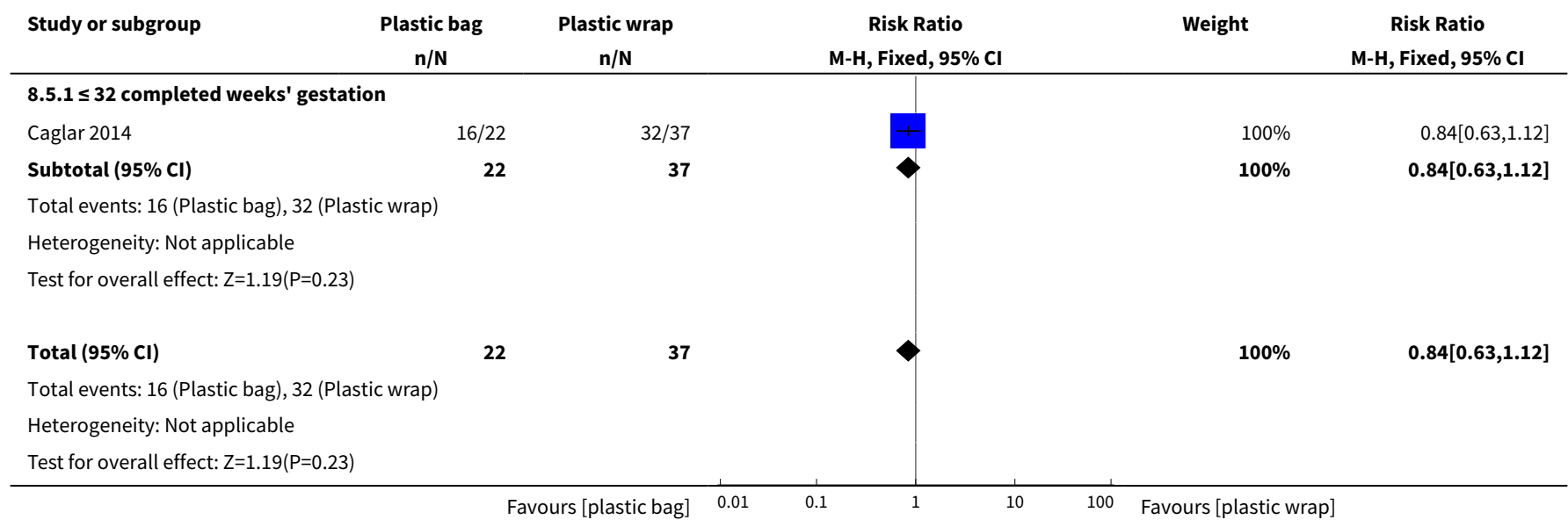

Analysis 8.6. Comparison 8 Plastic bag versus plastic wrap, Outcome 6 Moderate hypothermia within 1 hour after birth: core body temperature $32^{\circ} \mathrm{C}$ to $35.9^{\circ} \mathrm{C}$.

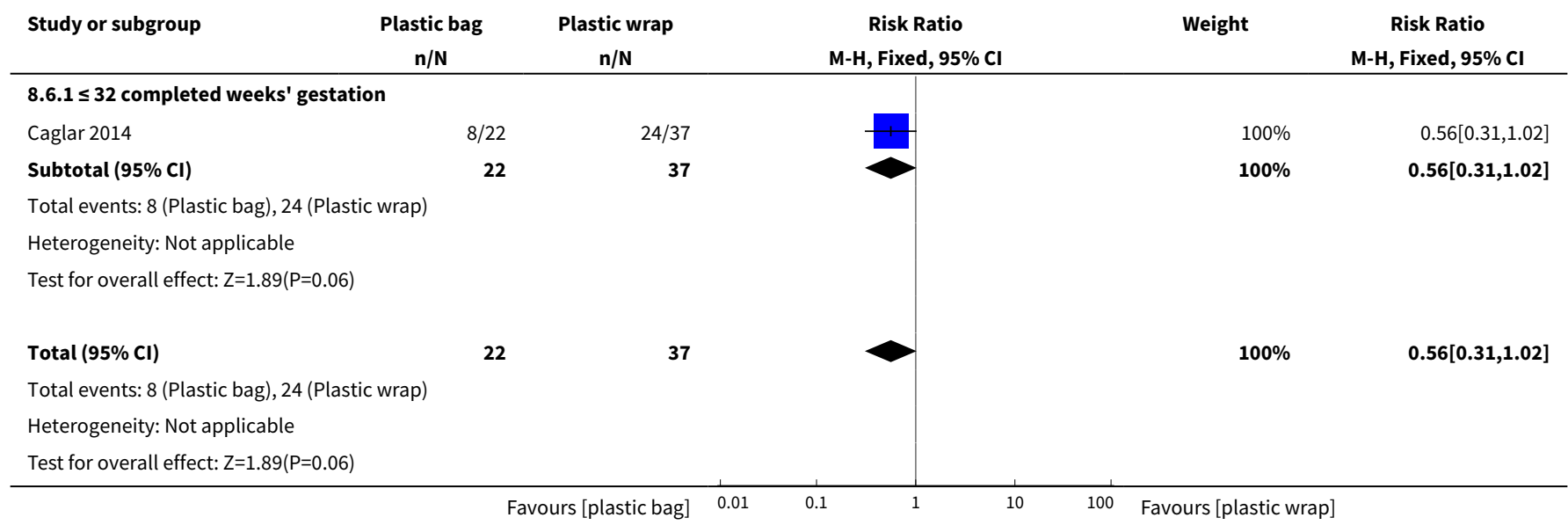

Analysis 8.7. Comparison 8 Plastic bag versus plastic wrap, Outcome 7 Outside normothermic range on admission to NICU or up to 2 hours after birth.

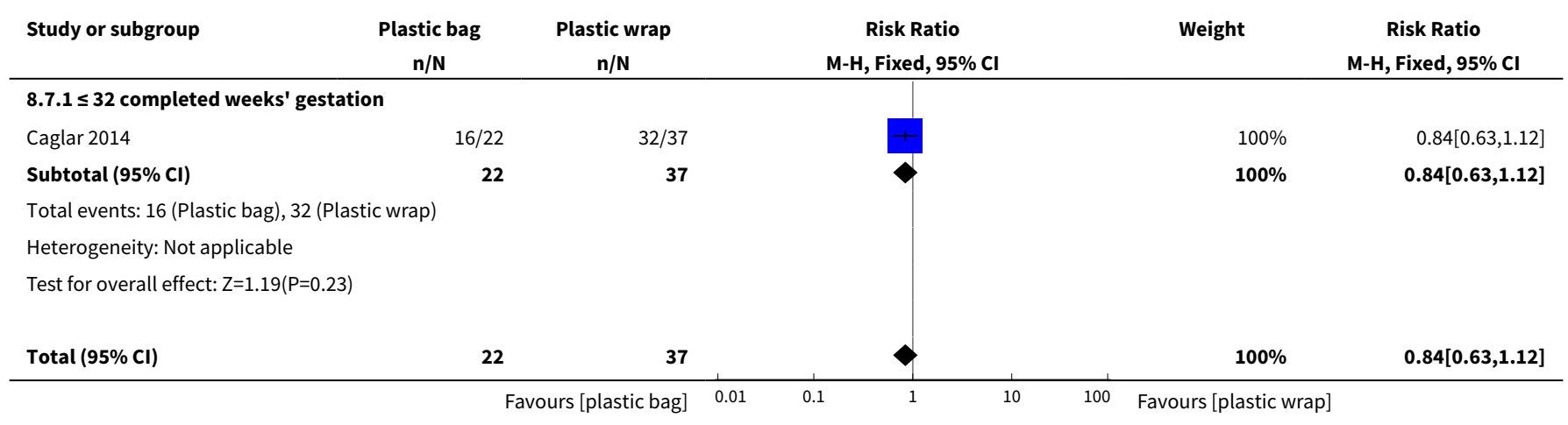




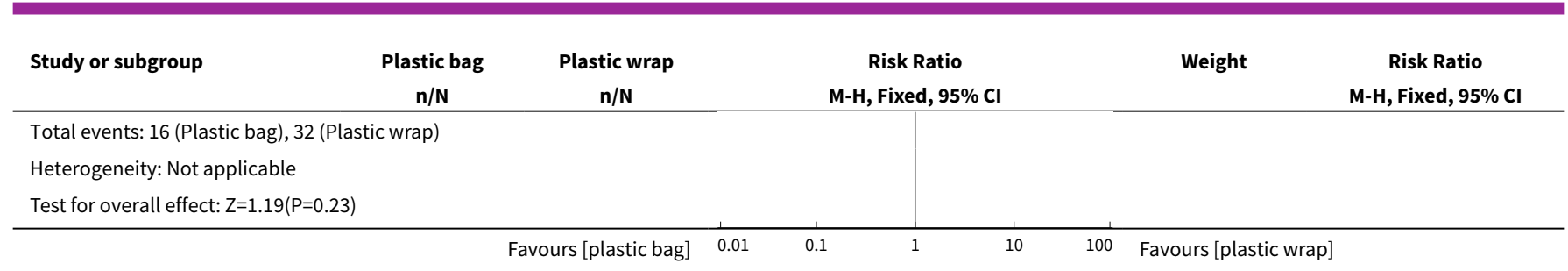

Comparison 9. Plastic total body wrap (body + head) versus plastic body wrap (head uncovered)

\begin{tabular}{|c|c|c|c|c|}
\hline Outcome or subgroup title & No. of studies & $\begin{array}{l}\text { No. of partici- } \\
\text { pants }\end{array}$ & Statistical method & Effect size \\
\hline $\begin{array}{l}1 \text { Core body temperature }\left({ }^{\circ} \mathrm{C}\right) \text { on ad- } \\
\text { mission to NICU }\end{array}$ & 1 & 100 & $\begin{array}{l}\text { Mean Difference (IV, Fixed, } \\
95 \% \mathrm{CI} \text { ) }\end{array}$ & $0.10[-0.18,0.38]$ \\
\hline $1.1<29$ completed weeks' gestation & 1 & 100 & $\begin{array}{l}\text { Mean Difference (IV, Fixed, } \\
95 \% \mathrm{CI} \text { ) }\end{array}$ & $0.10[-0.18,0.38]$ \\
\hline $\begin{array}{l}2 \text { Hypothermia on admission to NICU: } \\
\text { core body temperature }<36.5^{\circ} \mathrm{C}\end{array}$ & 1 & 100 & $\begin{array}{l}\text { Risk Ratio (M-H, Fixed, 95\% } \\
\mathrm{Cl} \text { ) }\end{array}$ & $0.87[0.55,1.37]$ \\
\hline $2.1<29$ completed weeks' gestation & 1 & 100 & $\begin{array}{l}\text { Risk Ratio (M-H, Fixed, 95\% } \\
\mathrm{Cl} \text { ) }\end{array}$ & $0.87[0.55,1.37]$ \\
\hline $\begin{array}{l}3 \text { Mild hypothermia on admission to } \\
\text { NICU: core body temperature } 36^{\circ} \mathrm{C} \text { to } \\
36.4^{\circ} \mathrm{C}\end{array}$ & 1 & 100 & $\begin{array}{l}\text { Risk Ratio (M-H, Fixed, 95\% } \\
\text { Cl) }\end{array}$ & $1.08[0.56,2.05]$ \\
\hline $3.1<29$ completed weeks' gestation & 1 & 100 & $\begin{array}{l}\text { Risk Ratio (M-H, Fixed, 95\% } \\
\mathrm{Cl})\end{array}$ & $1.08[0.56,2.05]$ \\
\hline $\begin{array}{l}4 \text { Moderate hypothermia on admis- } \\
\text { sion to NICU: core body temperature } \\
<32.0^{\circ} \mathrm{C} \text { to } 35.9^{\circ} \mathrm{C}\end{array}$ & 1 & 100 & $\begin{array}{l}\text { Risk Ratio (M-H, Fixed, 95\% } \\
\mathrm{Cl})\end{array}$ & $0.6[0.24,1.53]$ \\
\hline $4.1<29$ completed weeks' gestation & 1 & 100 & $\begin{array}{l}\text { Risk Ratio (M-H, Fixed, 95\% } \\
\mathrm{Cl})\end{array}$ & $0.6[0.24,1.53]$ \\
\hline $\begin{array}{l}5 \text { Outside normothermic range on ad- } \\
\text { mission to NICU or within } 2 \text { hours af- } \\
\text { ter birth }\end{array}$ & 1 & 100 & $\begin{array}{l}\text { Risk Ratio (M-H, Fixed, 95\% } \\
\mathrm{Cl})\end{array}$ & $0.88[0.59,1.32]$ \\
\hline $5.1<29$ completed weeks' gestation & 1 & 100 & $\begin{array}{l}\text { Risk Ratio (M-H, Fixed, 95\% } \\
\mathrm{Cl})\end{array}$ & $0.88[0.59,1.32]$ \\
\hline $\begin{array}{l}6 \text { Core body temperature }\left({ }^{\circ} \mathrm{C}\right) 1 \text { hour } \\
\text { after admission to NICU }\end{array}$ & 1 & 100 & $\begin{array}{l}\text { Mean Difference (IV, Fixed, } \\
95 \% \mathrm{CI})\end{array}$ & $-0.10[-0.47,0.27]$ \\
\hline $6.1<29$ completed weeks' gestation & 1 & 100 & $\begin{array}{l}\text { Mean Difference (IV, Fixed, } \\
95 \% \mathrm{CI})\end{array}$ & $-0.10[-0.47,0.27]$ \\
\hline $\begin{array}{l}7 \text { Hyperthermia on admission to } \\
\text { NICU: core body temperature }>37.5^{\circ} \mathrm{C}\end{array}$ & 1 & 100 & $\begin{array}{l}\text { Risk Ratio (M-H, Fixed, 95\% } \\
\text { Cl) }\end{array}$ & $1.0[0.21,4.72]$ \\
\hline
\end{tabular}




\begin{tabular}{|c|c|c|c|c|}
\hline Outcome or subgroup title & No. of studies & $\begin{array}{l}\text { No. of partici- } \\
\text { pants }\end{array}$ & Statistical method & Effect size \\
\hline $7.1<29$ completed weeks' gestation & 1 & 100 & $\begin{array}{l}\text { Risk Ratio (M-H, Fixed, 95\% } \\
\mathrm{Cl} \text { ) }\end{array}$ & $1.0[0.21,4.72]$ \\
\hline 8 Intraventricular haemorrhage & 1 & 100 & $\begin{array}{l}\text { Risk Ratio (M-H, Fixed, 95\% } \\
\mathrm{Cl} \text { ) }\end{array}$ & $0.8[0.34,1.86]$ \\
\hline $8.1<29$ completed weeks' gestation & 1 & 100 & $\begin{array}{l}\text { Risk Ratio (M-H, Fixed, 95\% } \\
\mathrm{Cl} \text { ) }\end{array}$ & $0.8[0.34,1.86]$ \\
\hline 9 Periventricular leukomalacia & 1 & 100 & $\begin{array}{l}\text { Risk Ratio (M-H, Fixed, 95\% } \\
\mathrm{Cl} \text { ) }\end{array}$ & $1.0[0.06,15.55]$ \\
\hline $9.1<29$ completed weeks' gestation & 1 & 100 & $\begin{array}{l}\text { Risk Ratio (M-H, Fixed, 95\% } \\
\mathrm{Cl} \text { ) }\end{array}$ & $1.0[0.06,15.55]$ \\
\hline 10 Mortality & 1 & 100 & $\begin{array}{l}\text { Risk Ratio (M-H, Fixed, 95\% } \\
\mathrm{Cl} \text { ) }\end{array}$ & $0.29[0.06,1.31]$ \\
\hline $10.1<29$ completed weeks' gestation & 1 & 100 & $\begin{array}{l}\text { Risk Ratio (M-H, Fixed, 95\% } \\
\mathrm{Cl})\end{array}$ & $0.29[0.06,1.31]$ \\
\hline $\begin{array}{l}11 \text { Bronchopulmonary dysplasia } \\
\text { (BPD) }\end{array}$ & 1 & 100 & $\begin{array}{l}\text { Risk Ratio (M-H, Fixed, 95\% } \\
\mathrm{Cl} \text { ) }\end{array}$ & $1.0[0.59,1.69]$ \\
\hline $11.1<29$ completed weeks' gestation & 1 & 100 & $\begin{array}{l}\text { Risk Ratio (M-H, Fixed, 95\% } \\
\mathrm{Cl} \text { ) }\end{array}$ & $1.0[0.59,1.69]$ \\
\hline 12 Necrotising enterocolitis & 1 & 100 & $\begin{array}{l}\text { Risk Ratio (M-H, Fixed, 95\% } \\
\mathrm{Cl} \text { ) }\end{array}$ & $1.0[0.41,2.46]$ \\
\hline $12.1<29$ completed weeks' gestation & 1 & 100 & $\begin{array}{l}\text { Risk Ratio (M-H, Fixed, 95\% } \\
\mathrm{Cl} \text { ) }\end{array}$ & $1.0[0.41,2.46]$ \\
\hline
\end{tabular}

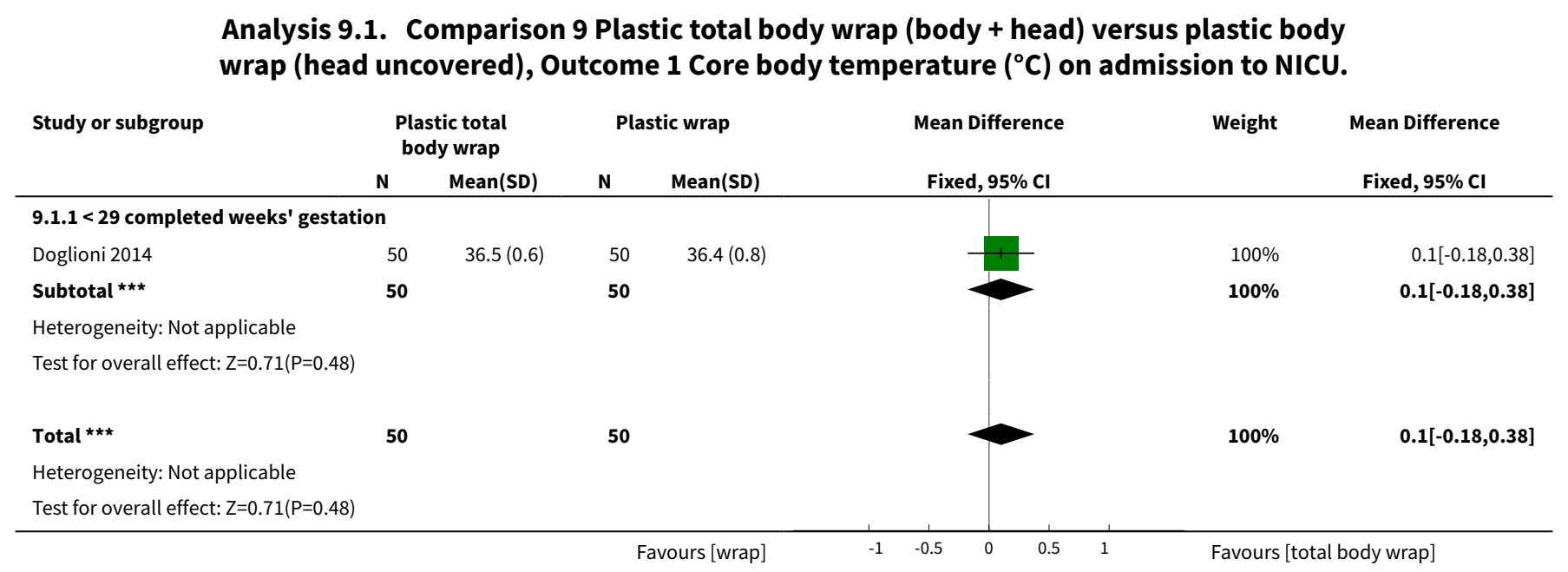


Analysis 9.2. Comparison 9 Plastic total body wrap (body + head) versus plastic body wrap (head uncovered), Outcome 2 Hypothermia on admission to NICU: core body temperature $<36.5^{\circ} \mathrm{C}$.

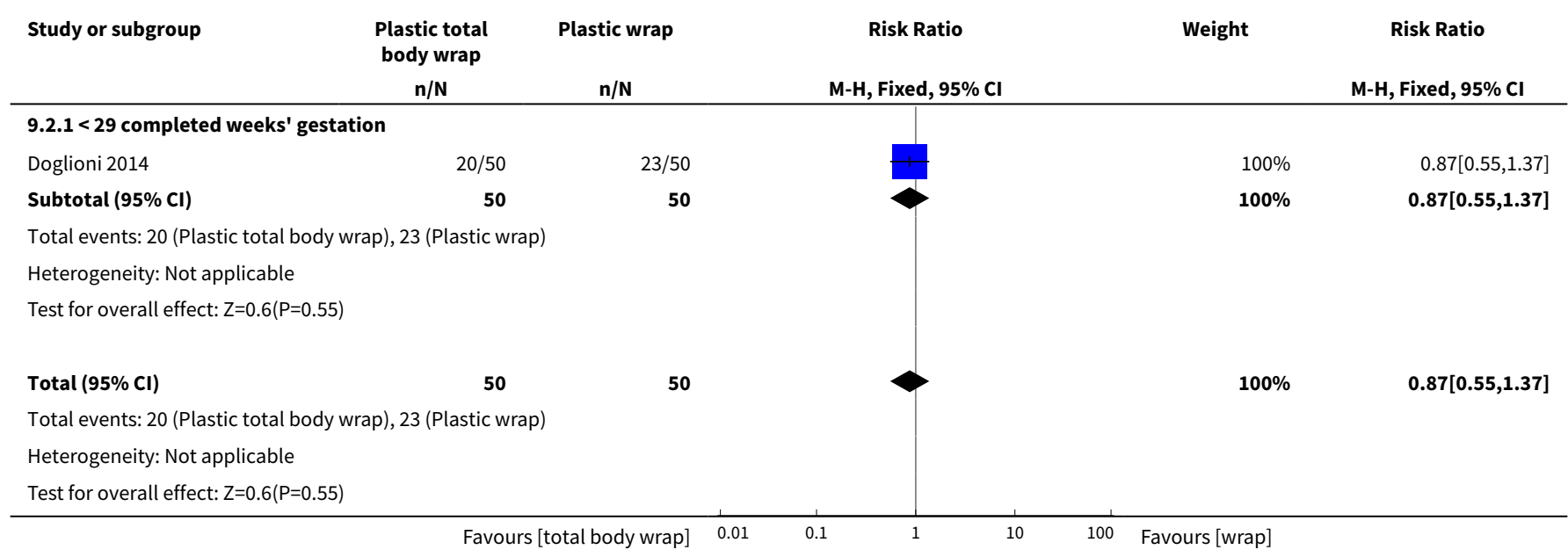

Analysis 9.3. Comparison 9 Plastic total body wrap (body + head) versus plastic body wrap (head uncovered), Outcome 3 Mild hypothermia on admission to NICU: core body temperature $36^{\circ} \mathrm{C}$ to $36.4^{\circ} \mathrm{C}$.

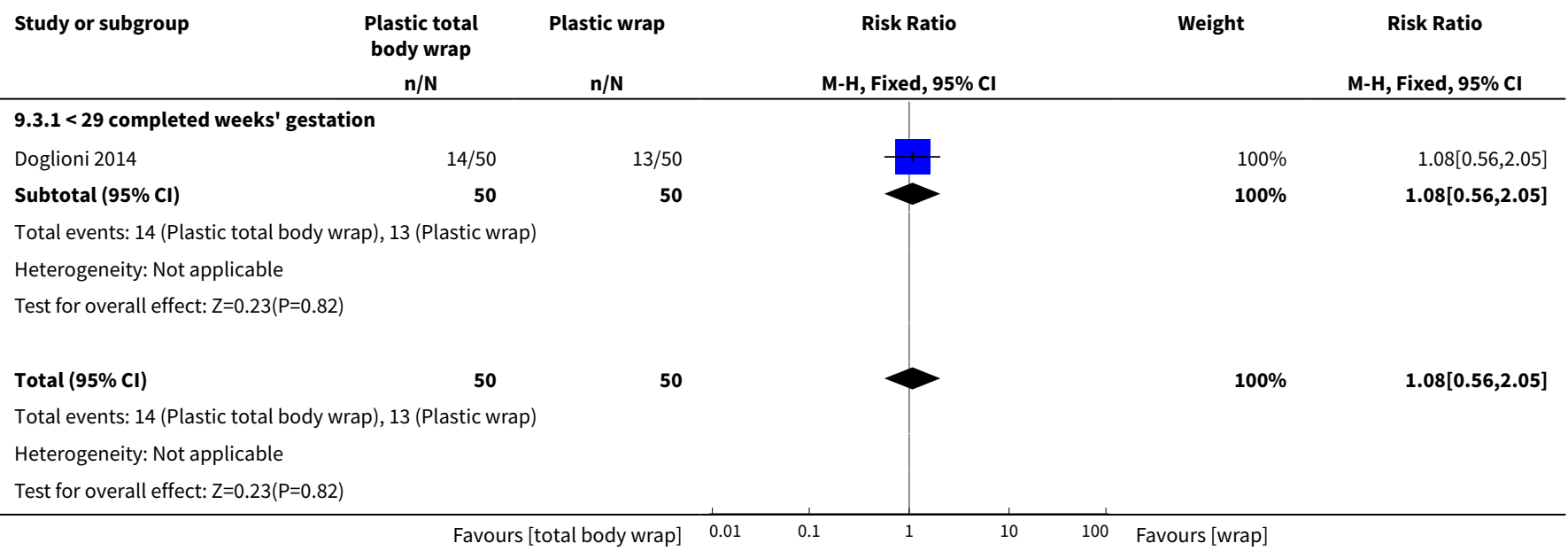

Analysis 9.4. Comparison 9 Plastic total body wrap (body + head) versus plastic body wrap (head uncovered), Outcome 4 Moderate hypothermia on admission to NICU: core body temperature $<32.0^{\circ} \mathrm{C}$ to $35.9^{\circ} \mathrm{C}$.

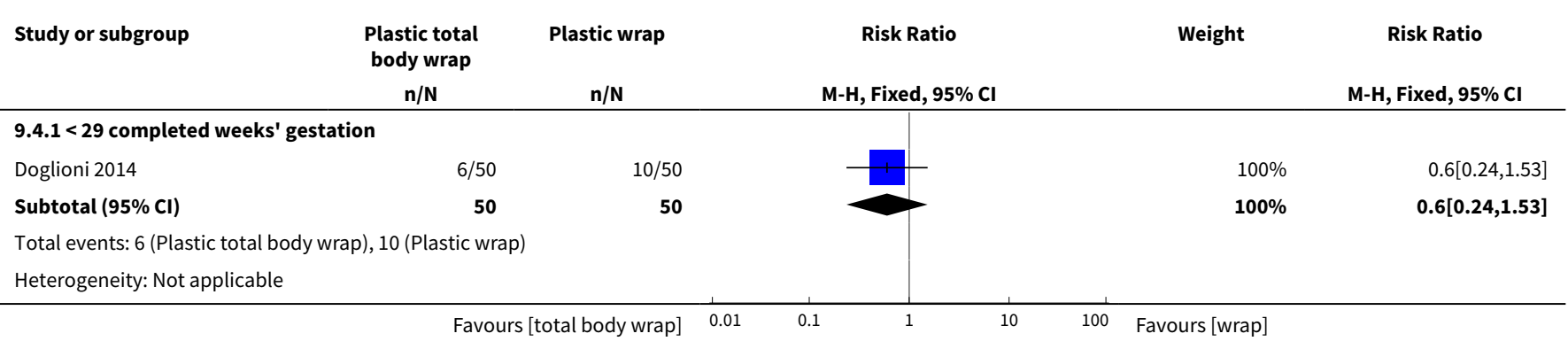




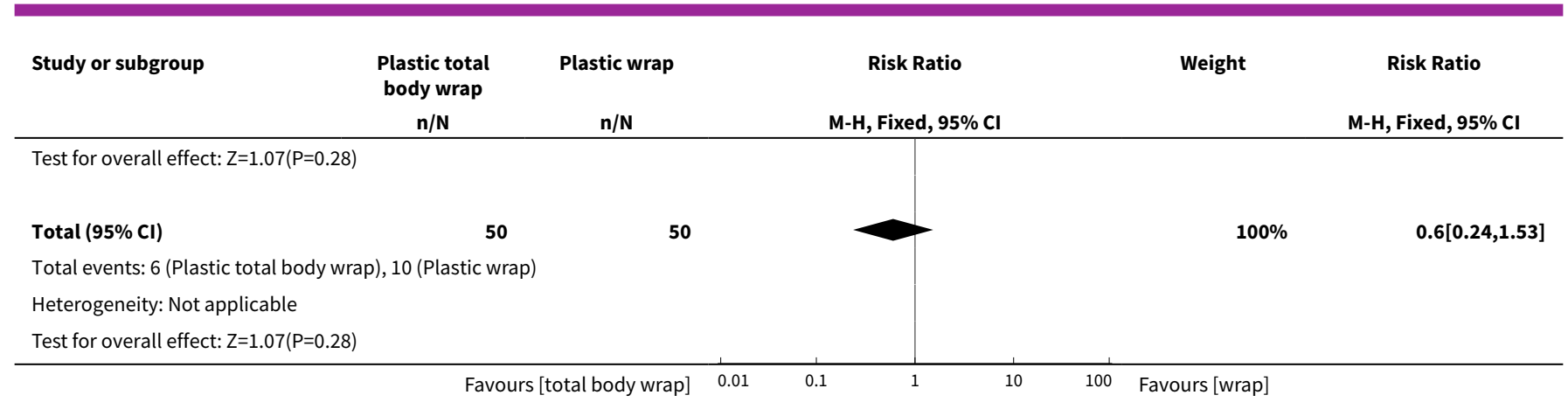

Analysis 9.5. Comparison 9 Plastic total body wrap (body + head) versus plastic body wrap (head uncovered), Outcome 5 Outside normothermic range on admission to NICU or within 2 hours after birth.

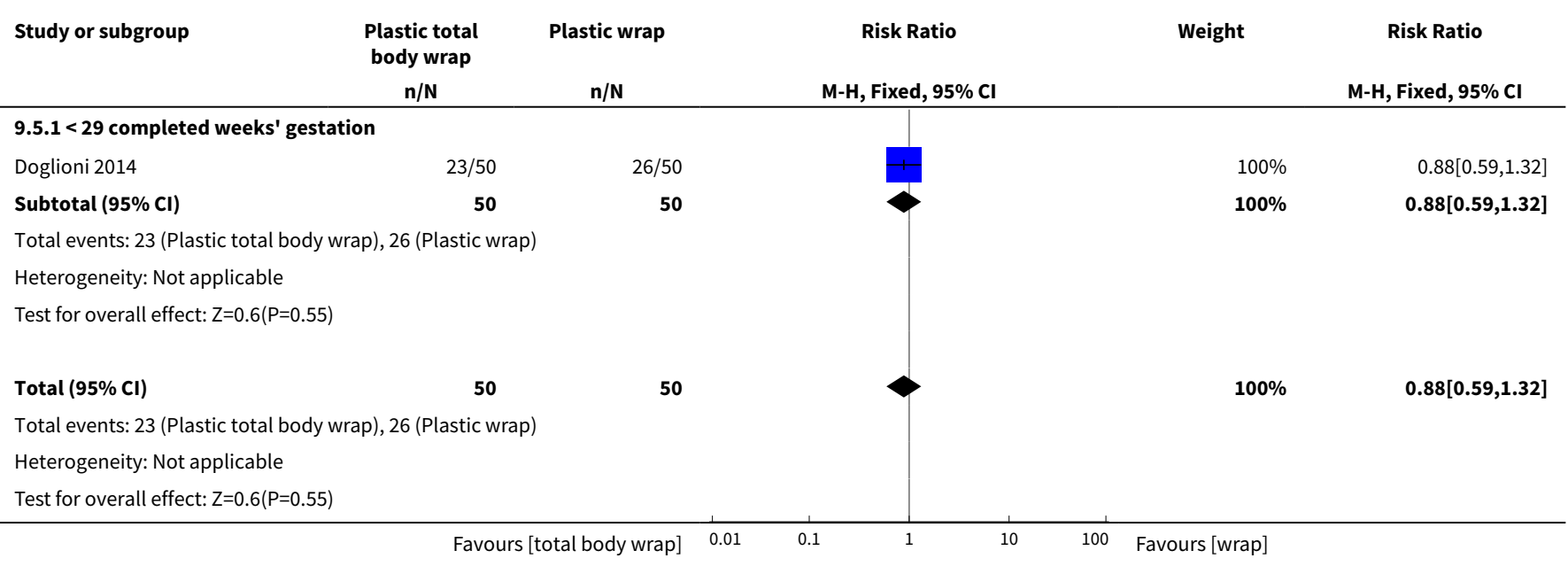

Analysis 9.6. Comparison 9 Plastic total body wrap (body + head) versus plastic body wrap (head uncovered), Outcome 6 Core body temperature $\left({ }^{\circ} \mathrm{C}\right) 1$ hour after admission to NICU.

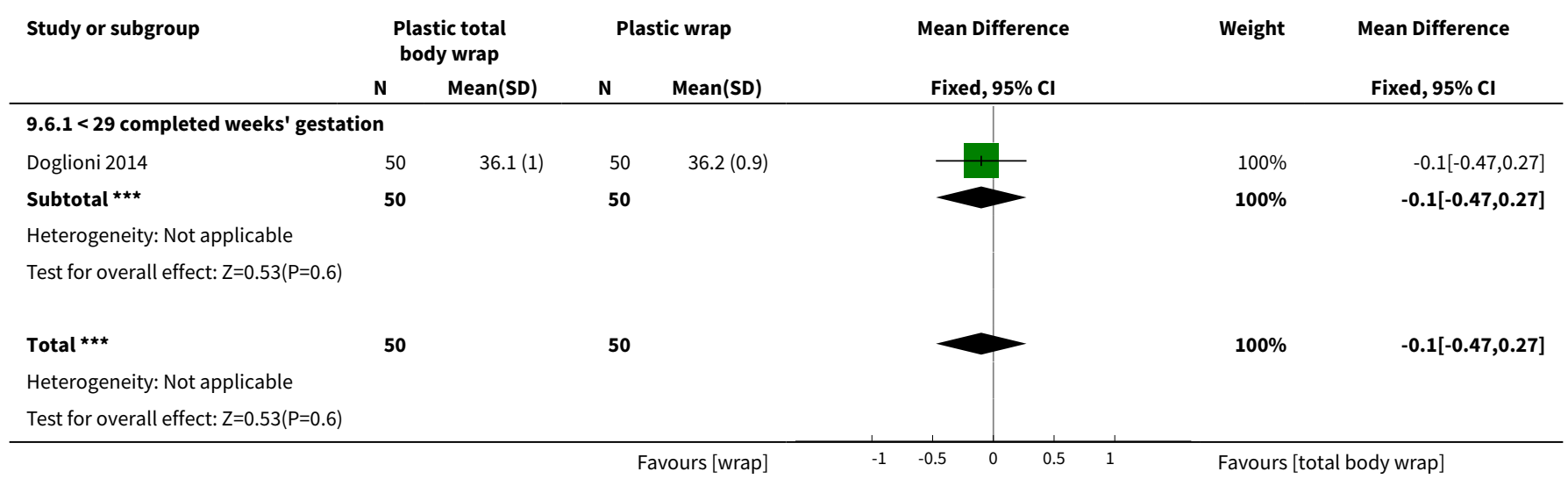


Analysis 9.7. Comparison 9 Plastic total body wrap (body + head) versus plastic body wrap (head uncovered), Outcome 7 Hyperthermia on admission to NICU: core body temperature $>37.5^{\circ} \mathrm{C}$.

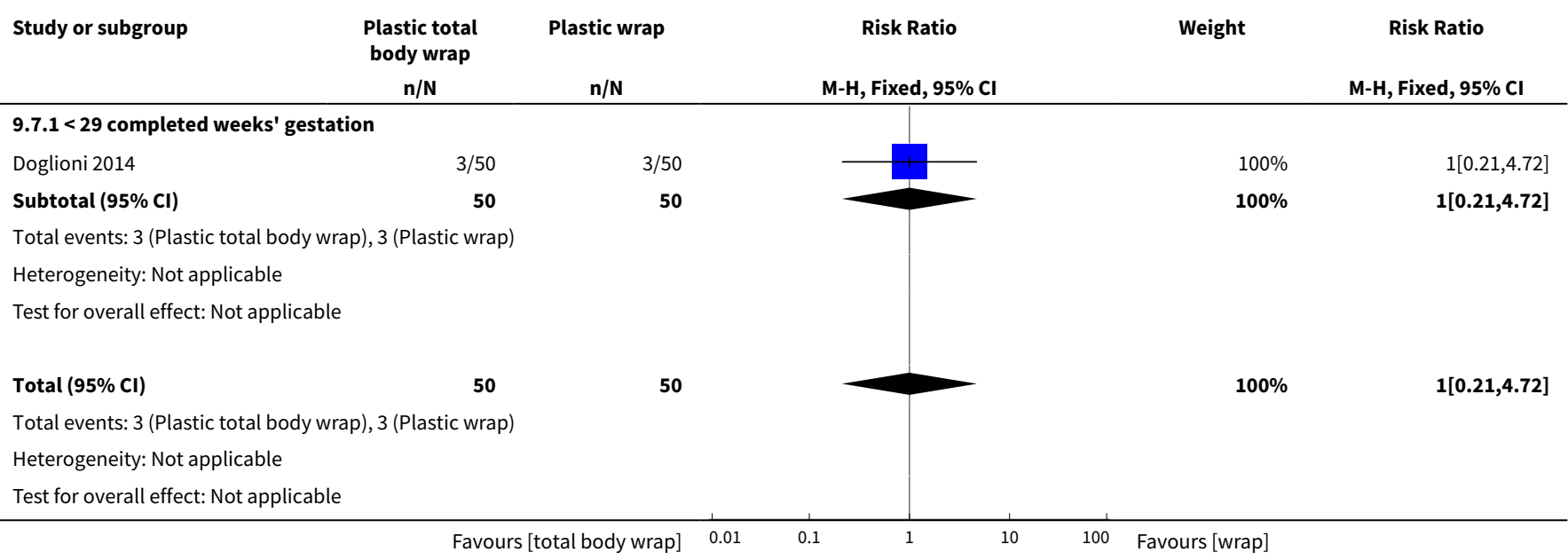

Analysis 9.8. Comparison 9 Plastic total body wrap (body + head) versus plastic body wrap (head uncovered), Outcome 8 Intraventricular haemorrhage.

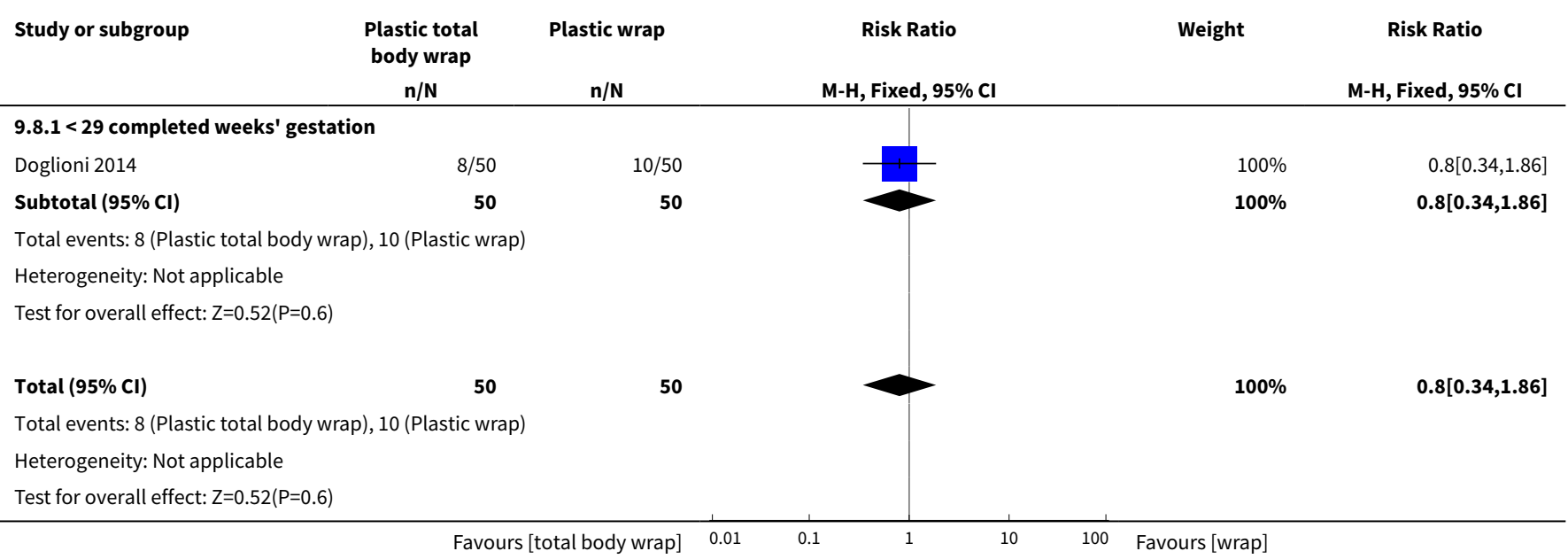

Analysis 9.9. Comparison 9 Plastic total body wrap (body + head) versus plastic body wrap (head uncovered), Outcome 9 Periventricular leukomalacia.

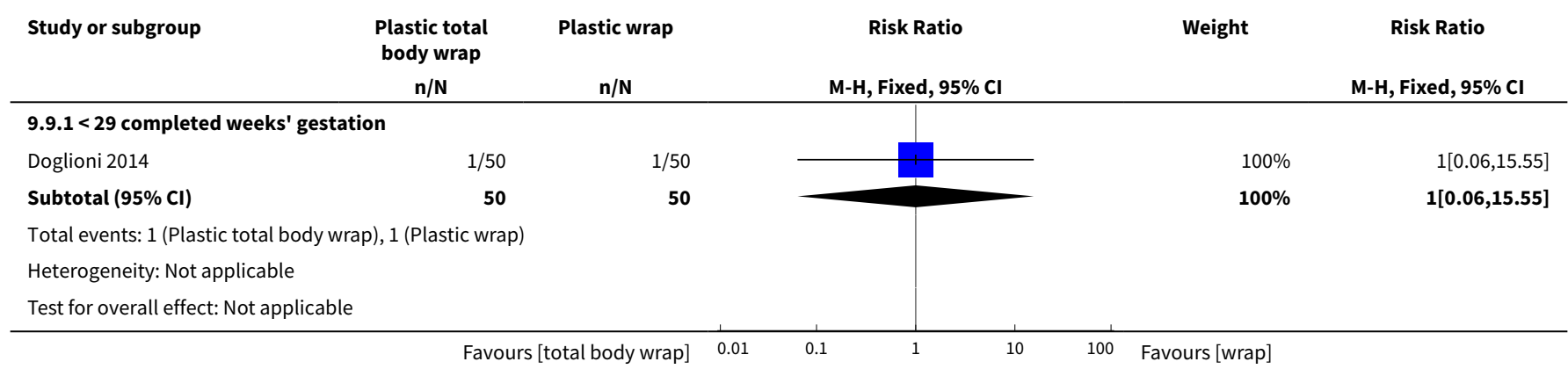




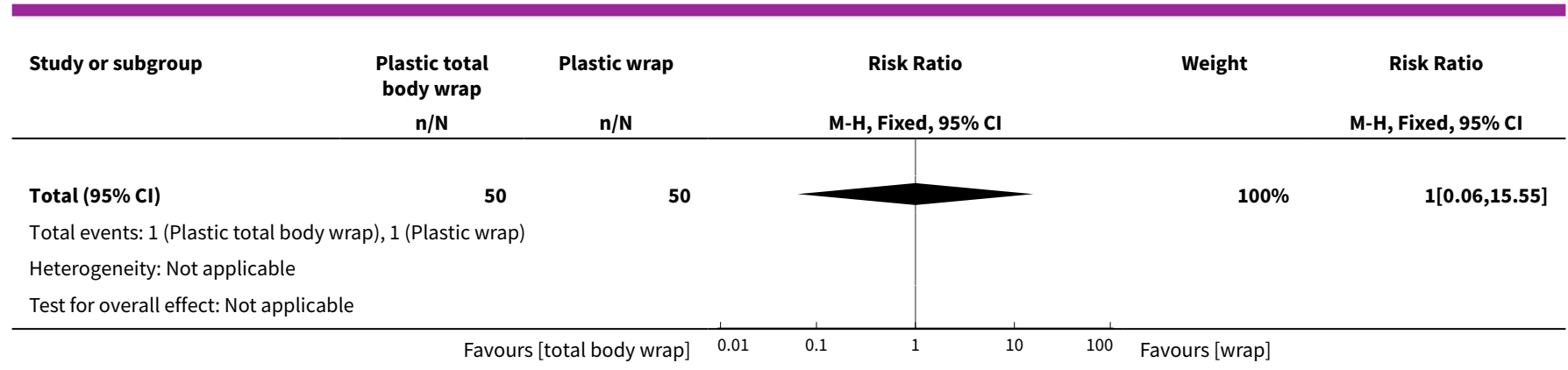

Analysis 9.10. Comparison 9 Plastic total body wrap (body + head) versus plastic body wrap (head uncovered), Outcome 10 Mortality.

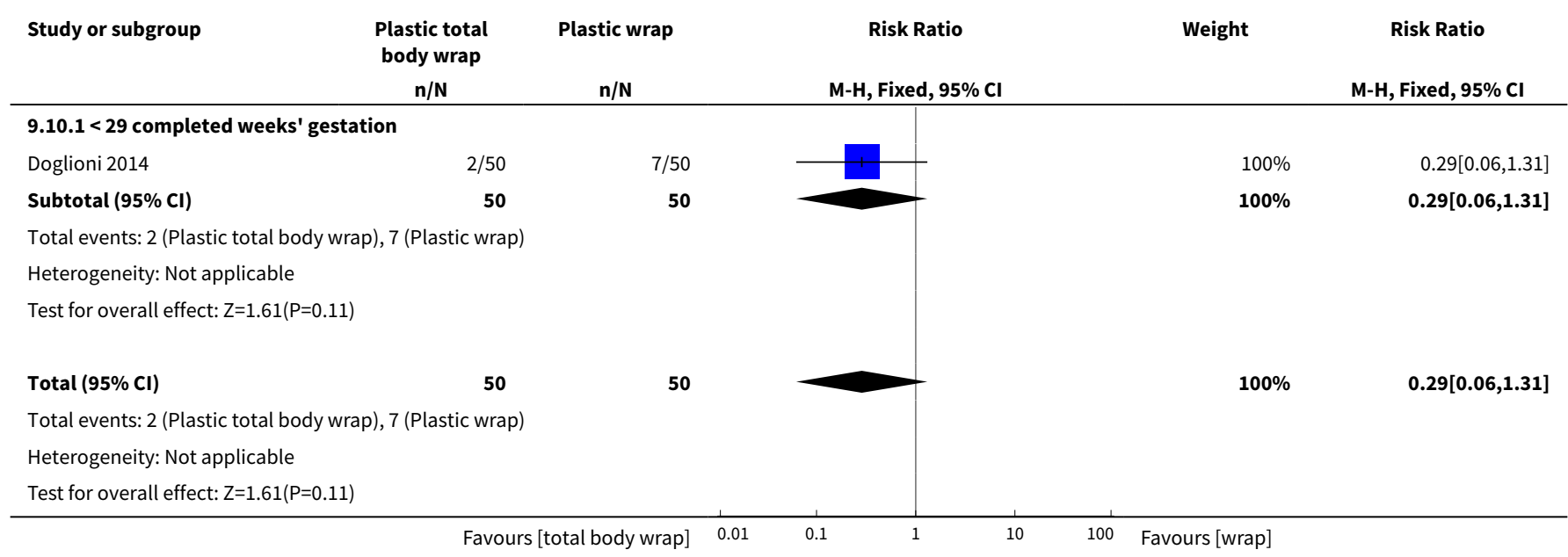

\section{Analysis 9.11. Comparison 9 Plastic total body wrap (body + head) versus plastic} body wrap (head uncovered), Outcome 11 Bronchopulmonary dysplasia (BPD).

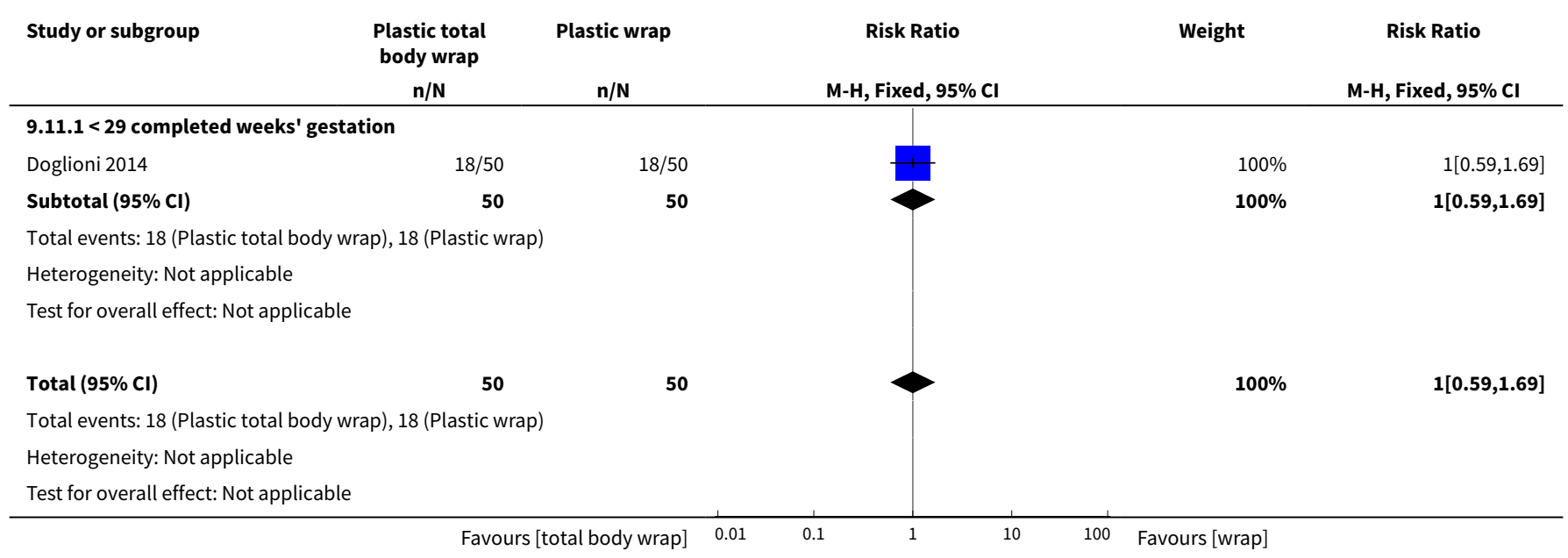


Analysis 9.12. Comparison 9 Plastic total body wrap (body + head) versus plastic body wrap (head uncovered), Outcome 12 Necrotising enterocolitis.

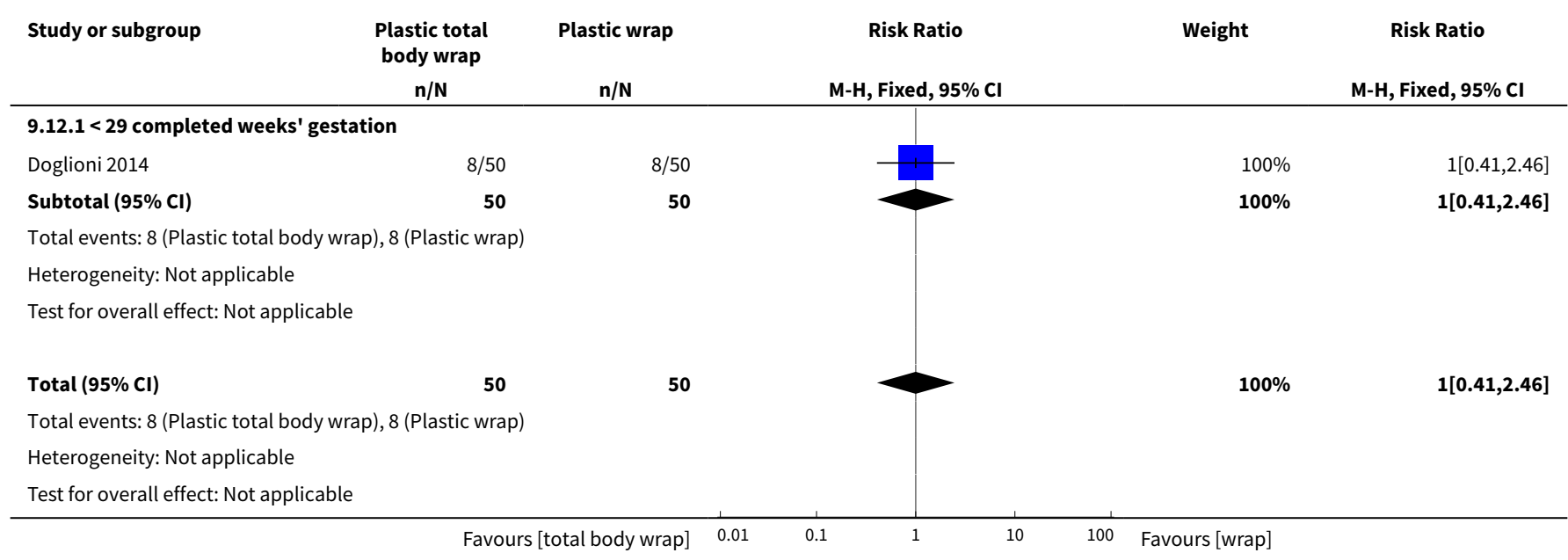

Comparison 10. Plastic bag and plastic hat versus plastic bag and cotton hat

\begin{tabular}{|c|c|c|c|c|}
\hline Outcome or subgroup title & No. of studies & $\begin{array}{l}\text { No. of partici- } \\
\text { pants }\end{array}$ & Statistical method & Effect size \\
\hline $\begin{array}{l}1 \text { Core body temperature }\left({ }^{\circ} \mathrm{C}\right) \text { on admis- } \\
\text { sion to NICU or up to } 2 \text { hours after birth }\end{array}$ & 1 & 64 & $\begin{array}{l}\text { Mean Difference (IV, Fixed, } \\
95 \% \mathrm{CI})\end{array}$ & $0.32[0.02,0.62]$ \\
\hline $\begin{array}{l}1.1 \geq 28 \text { and } \leq 32 \text { completed weeks' ges- } \\
\text { tation }\end{array}$ & 1 & 64 & $\begin{array}{l}\text { Mean Difference (IV, Fixed, } \\
95 \% \mathrm{CI} \text { ) }\end{array}$ & $0.32[0.02,0.62]$ \\
\hline $\begin{array}{l}2 \text { Core body temperature }\left({ }^{\circ} \mathrm{C}\right) 1 \text { hour af- } \\
\text { ter admission to NICU }\end{array}$ & 1 & 64 & $\begin{array}{l}\text { Mean Difference (IV, Fixed, } \\
95 \% \mathrm{CI})\end{array}$ & $0.37[0.03,0.71]$ \\
\hline $\begin{array}{l}2.1 \geq 28 \text { and } \leq 32 \text { completed weeks' ges- } \\
\text { tation }\end{array}$ & 1 & 64 & $\begin{array}{l}\text { Mean Difference (IV, Fixed, } \\
95 \% \mathrm{CI})\end{array}$ & $0.37[0.03,0.71]$ \\
\hline $\begin{array}{l}3 \text { Core body temperature }\left({ }^{\circ} \mathrm{C}\right) 2 \text { hours af- } \\
\text { ter admission to NICU }\end{array}$ & 1 & 64 & $\begin{array}{l}\text { Mean Difference (IV, Fixed, } \\
95 \% \mathrm{CI} \text { ) }\end{array}$ & $0.37[0.11,0.63]$ \\
\hline $\begin{array}{l}3.1 \geq 28 \text { and } \leq 32 \text { completed weeks' ges- } \\
\text { tation }\end{array}$ & 1 & 64 & $\begin{array}{l}\text { Mean Difference (IV, Fixed, } \\
95 \% \mathrm{CI} \text { ) }\end{array}$ & $0.37[0.11,0.63]$ \\
\hline
\end{tabular}

Analysis 10.1. Comparison 10 Plastic bag and plastic hat versus plastic bag and cotton hat, Outcome 1 Core body temperature $\left({ }^{\circ} \mathrm{C}\right)$ on admission to NICU or up to 2 hours after birth.

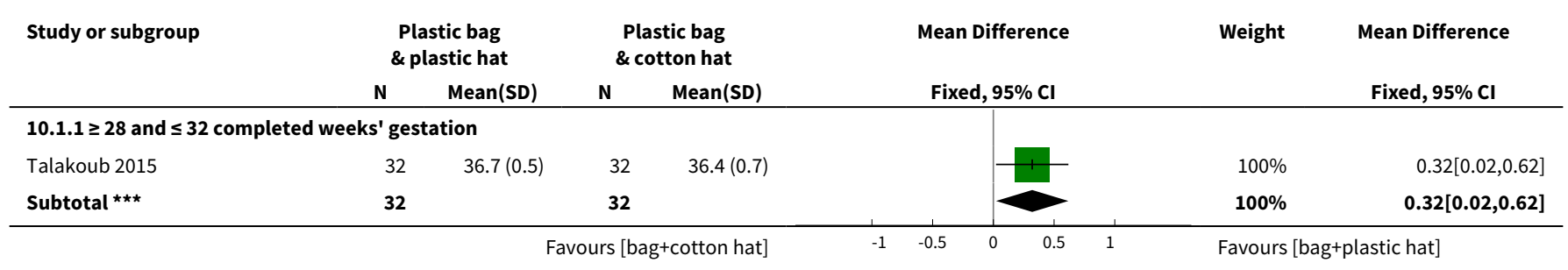




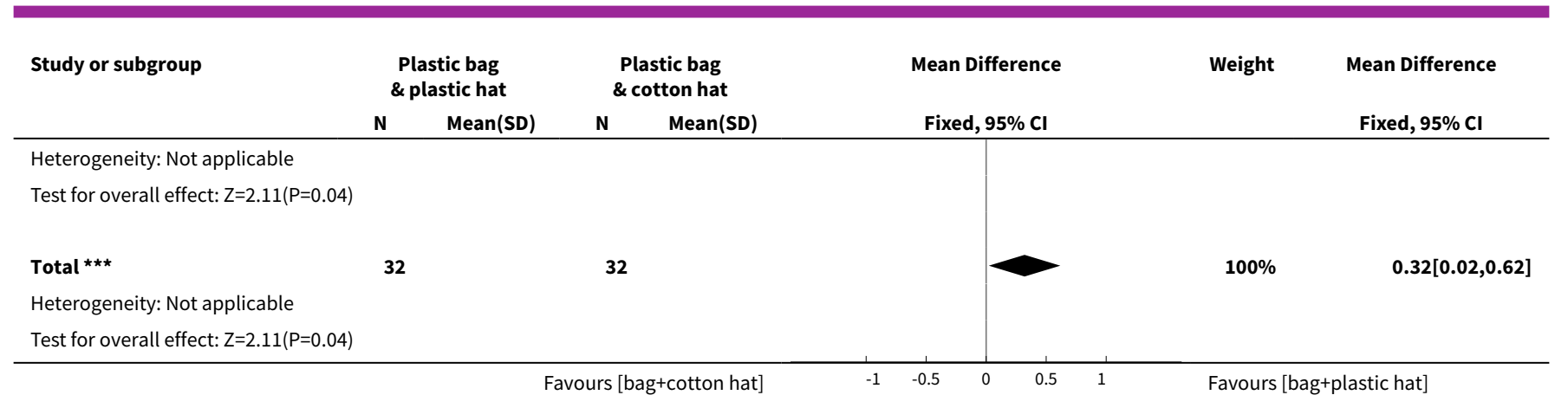

Analysis 10.2. Comparison 10 Plastic bag and plastic hat versus plastic bag and cotton hat, Outcome 2 Core body temperature $\left({ }^{\circ} \mathrm{C}\right) 1$ hour after admission to NICU.

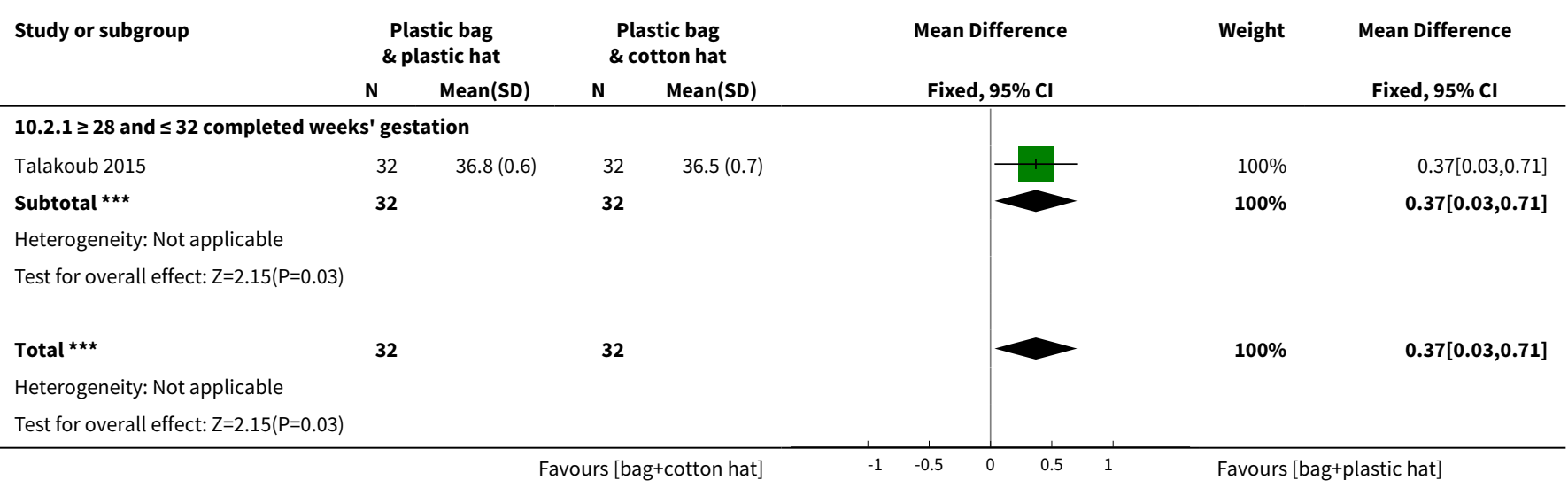

Analysis 10.3. Comparison 10 Plastic bag and plastic hat versus plastic bag and cotton hat, Outcome 3 Core body temperature $\left({ }^{\circ} \mathrm{C}\right) 2$ hours after admission to NICU.

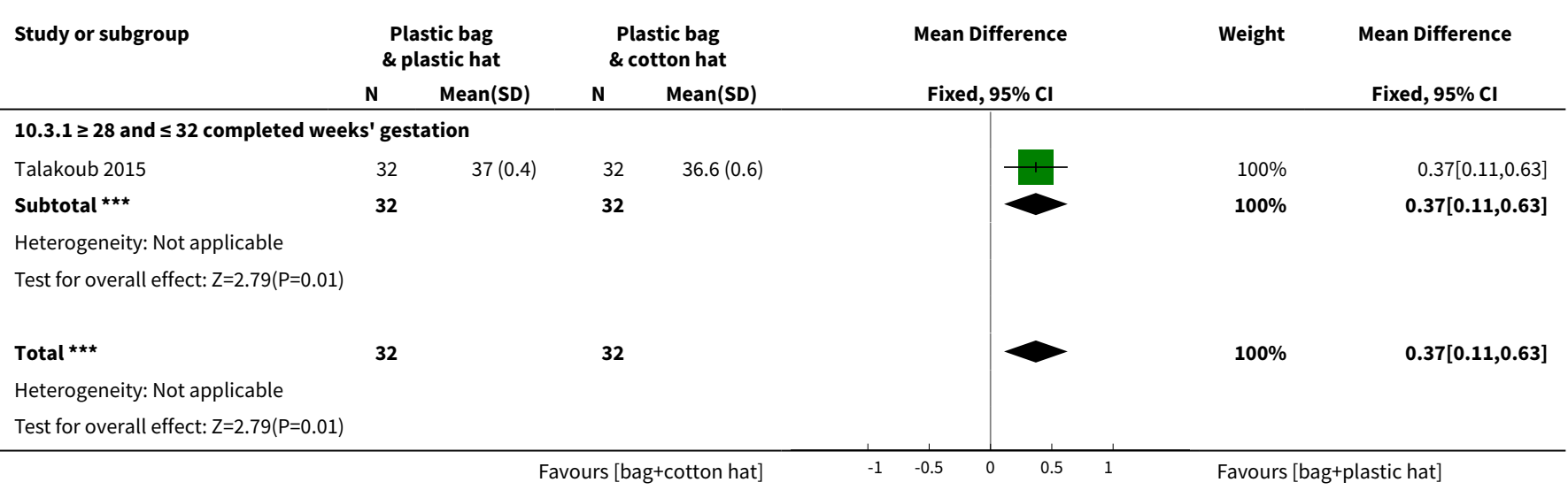


Comparison 11. Stockinette cap versus routine care (no cap)

\begin{tabular}{|c|c|c|c|c|}
\hline Outcome or subgroup title & No. of studies & $\begin{array}{l}\text { No. of partici- } \\
\text { pants }\end{array}$ & Statistical method & Effect size \\
\hline $\begin{array}{l}1 \text { Core body temperature }\left({ }^{\circ} \mathrm{C}\right) \text { on admis- } \\
\text { sion to NICU or up to } 2 \text { hours after birth }\end{array}$ & 1 & & $\begin{array}{l}\text { Mean Difference (IV, Fixed, } \\
95 \% \mathrm{CI})\end{array}$ & Subtotals only \\
\hline $\begin{array}{l}\text { 1.1 All infants ( } 32 \text { to } 36 \text { completed weeks' } \\
\text { gestation) }\end{array}$ & 1 & 40 & $\begin{array}{l}\text { Mean Difference (IV, Fixed, } \\
95 \% \mathrm{CI})\end{array}$ & $0.10[-0.21,0.41]$ \\
\hline $1.2<2000$ grams birth weight & 1 & 10 & $\begin{array}{l}\text { Mean Difference (IV, Fixed, } \\
95 \% \mathrm{CI} \text { ) }\end{array}$ & $0.70[-0.01,1.41]$ \\
\hline $1.3 \geq 2000$ grams birth weight & 1 & 30 & $\begin{array}{l}\text { Mean Difference (IV, Fixed, } \\
95 \% \mathrm{Cl} \text { ) }\end{array}$ & $0.0[-0.37,0.37]$ \\
\hline $\begin{array}{l}2 \text { Hypothermia on admission to NICU: } \\
\text { core body temperature }<36.5^{\circ} \mathrm{C} \text { or skin } \\
\text { temperature }<36^{\circ} \mathrm{C}\end{array}$ & 1 & 40 & $\begin{array}{l}\text { Risk Ratio (M-H, Fixed, } \\
95 \% \mathrm{Cl})\end{array}$ & $0.90[0.48,1.71]$ \\
\hline 2.132 to 36 completed weeks' gestation & 1 & 40 & $\begin{array}{l}\text { Risk Ratio (M-H, Fixed, } \\
95 \% \mathrm{Cl})\end{array}$ & $0.90[0.48,1.71]$ \\
\hline
\end{tabular}

\section{Analysis 11.1. Comparison 11 Stockinette cap versus routine care (no cap), Outcome 1 Core body temperature $\left({ }^{\circ} \mathrm{C}\right)$ on admission to NICU or up to 2 hours after birth.}

\begin{tabular}{|c|c|c|c|c|c|c|c|}
\hline \multirow[t]{2}{*}{ Study or subgroup } & \multicolumn{2}{|c|}{ Stockinet cap } & \multicolumn{2}{|c|}{ Routine care } & \multirow{2}{*}{$\begin{array}{c}\text { Mean Difference } \\
\text { Fixed, } 95 \% \mathrm{Cl}\end{array}$} & \multirow[t]{2}{*}{ Weight } & \multirow{2}{*}{$\begin{array}{c}\text { Mean Difference } \\
\text { Fixed, } 95 \% \mathrm{Cl}\end{array}$} \\
\hline & $\mathbf{N}$ & $\operatorname{Mean}(S D)$ & $\mathbf{N}$ & Mean(SD) & & & \\
\hline \multicolumn{8}{|c|}{ 11.1.1 All infants ( 32 to 36 completed weeks' gestation) } \\
\hline Roberts 1981 & 17 & $36(0.5)$ & 23 & $35.9(0.5)$ & & $100 \%$ & $0.1[-0.21,0.41]$ \\
\hline 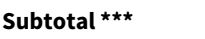 & 17 & & 23 & & & $100 \%$ & $0.1[-0.21,0.41]$ \\
\hline \multicolumn{8}{|c|}{ Heterogeneity: Not applicable } \\
\hline \multicolumn{8}{|c|}{ Test for overall effect: $Z=0.63(P=0.53)$} \\
\hline \multicolumn{8}{|c|}{ 11.1.2 $<2000$ grams birth weight } \\
\hline Roberts 1981 & 6 & $36.1(0.5)$ & 4 & $35.4(0.6)$ & & $100 \%$ & $0.7[-0.01,1.41]$ \\
\hline 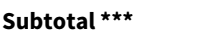 & 6 & & 4 & & & $100 \%$ & $0.7[-0.01,1.41]$ \\
\hline \multicolumn{8}{|c|}{ Heterogeneity: Not applicable } \\
\hline \multicolumn{8}{|c|}{ Test for overall effect: $Z=1.93(P=0.05)$} \\
\hline Roberts 1981 & 11 & $36(0.5)$ & 19 & $36(0.5)$ & - & $100 \%$ & $0[-0.37,0.37]$ \\
\hline 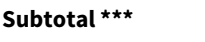 & 11 & & 19 & & & $100 \%$ & $0[-0.37,0.37]$ \\
\hline \multicolumn{8}{|c|}{ Heterogeneity: Not applicable } \\
\hline \multicolumn{8}{|c|}{ Test for overall effect: Not applicable } \\
\hline \multicolumn{8}{|c|}{ Test for subgroup differences: $\mathrm{Chi}^{2}=2.97, \mathrm{df}=1(\mathrm{P}=0.23), \mathrm{I}^{2}=32.68 \%$} \\
\hline
\end{tabular}


Analysis 11.2. Comparison 11 Stockinette cap versus routine care (no cap), Outcome 2 Hypothermia on admission to NICU: core body temperature $<36.5^{\circ} \mathrm{C}$ or skin temperature $<36^{\circ} \mathrm{C}$.

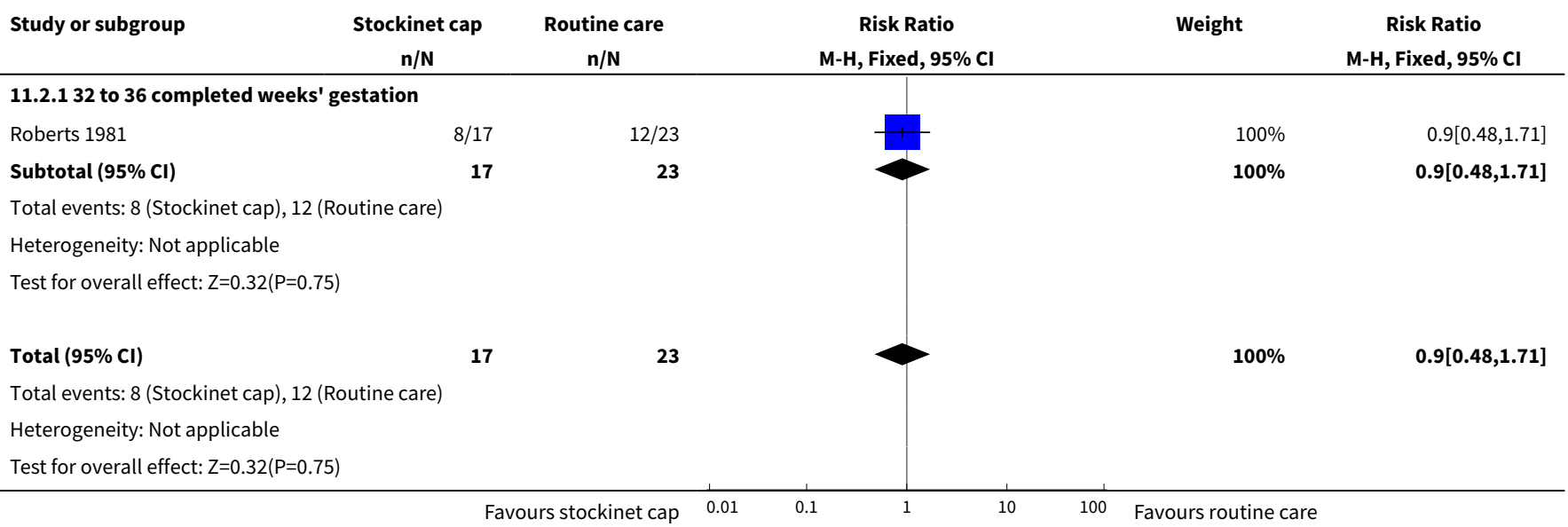

\section{Comparison 12. Skin-to-skin care versus routine care}

\begin{tabular}{|c|c|c|c|c|}
\hline Outcome or subgroup title & No. of studies & $\begin{array}{l}\text { No. of partici- } \\
\text { pants }\end{array}$ & Statistical method & Effect size \\
\hline $\begin{array}{l}1 \text { Hypothermia: skin temperature < } \\
35.5^{\circ} \mathrm{C} \text { for } 2 \text { consecutive recordings }\end{array}$ & 1 & 31 & $\begin{array}{l}\text { Risk Ratio (M-H, Fixed, 95\% } \\
\mathrm{Cl})\end{array}$ & $0.09[0.01,0.64]$ \\
\hline 1.11200 to 2199 grams birth weight & 1 & 31 & $\begin{array}{l}\text { Risk Ratio (M-H, Fixed, 95\% } \\
\mathrm{Cl} \text { ) }\end{array}$ & $0.09[0.01,0.64]$ \\
\hline $\begin{array}{l}2 \text { Hypoglycaemia: blood glucose level } \\
<2.6 \mathrm{mmol} / \mathrm{L}\end{array}$ & 1 & 31 & $\begin{array}{l}\text { Risk Ratio (M-H, Fixed, 95\% } \\
\mathrm{Cl})\end{array}$ & $0.24[0.03,2.06]$ \\
\hline 2.11200 to 2199 grams birth weight & 1 & 31 & $\begin{array}{l}\text { Risk Ratio (M-H, Fixed, 95\% } \\
\mathrm{Cl} \text { ) }\end{array}$ & $0.24[0.03,2.06]$ \\
\hline
\end{tabular}

Analysis 12.1. Comparison 12 Skin-to-skin care versus routine care, Outcome 1 Hypothermia: skin temperature $<35.5^{\circ} \mathrm{C}$ for 2 consecutive recordings.

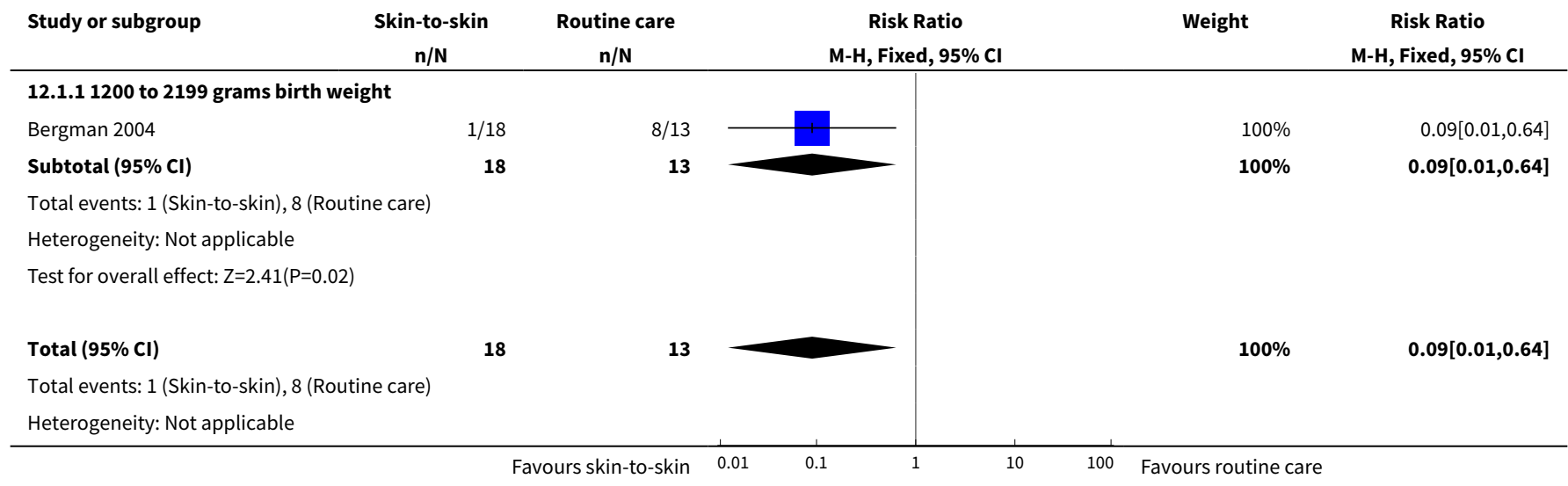




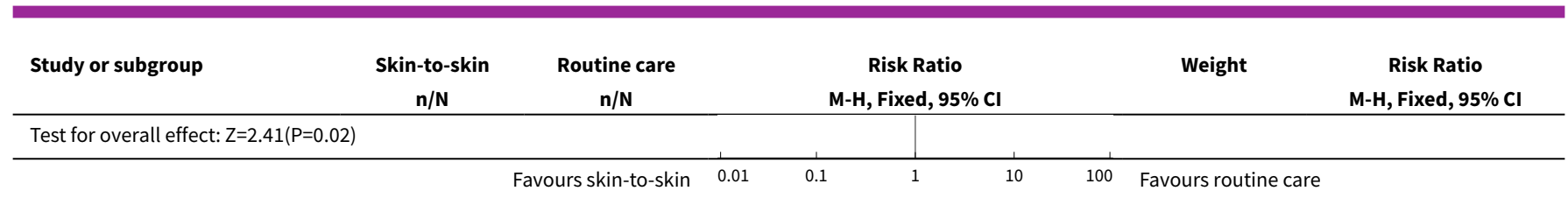

Analysis 12.2. Comparison 12 Skin-to-skin care versus routine care, Outcome 2 Hypoglycaemia: blood glucose level $<2.6 \mathrm{mmol} / \mathrm{L}$.

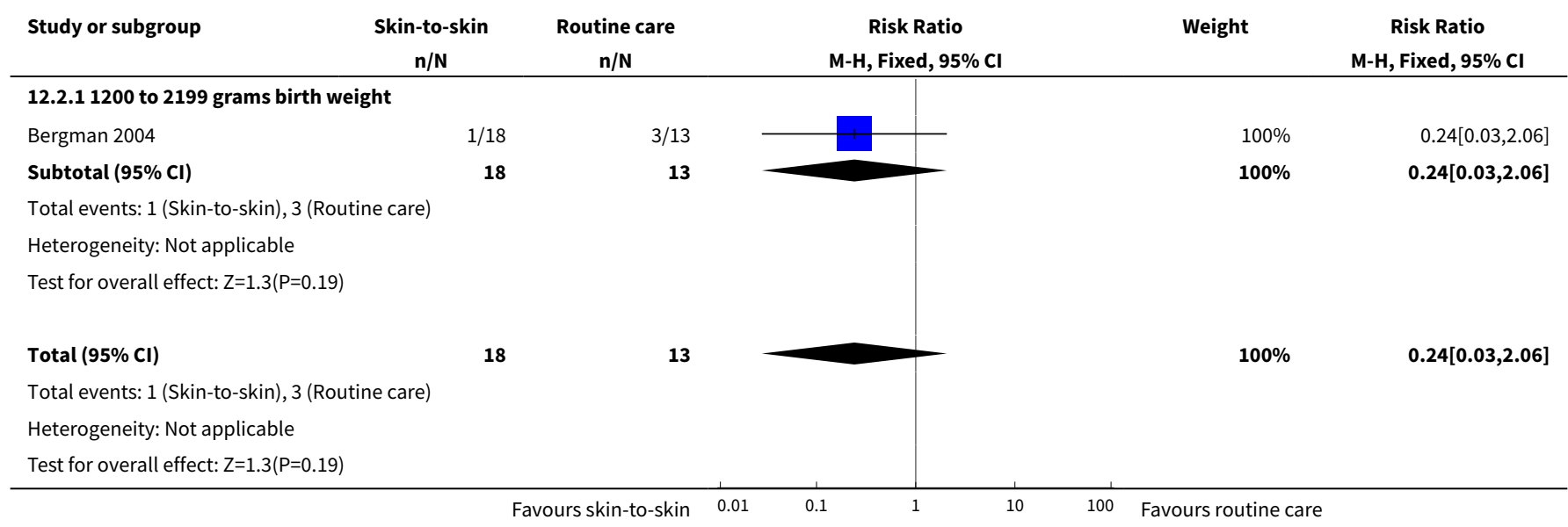

\section{Comparison 13. Thermal mattress versus routine care}

\begin{tabular}{|c|c|c|c|c|}
\hline Outcome or subgroup title & No. of studies & $\begin{array}{l}\text { No. of partici- } \\
\text { pants }\end{array}$ & Statistical method & Effect size \\
\hline $\begin{array}{l}1 \text { Core body temperature }\left({ }^{\circ} \mathrm{C}\right) \text { on } \\
\text { admission to NICU or up to } 2 \text { hours } \\
\text { after birth }\end{array}$ & 2 & 126 & $\begin{array}{l}\text { Mean Difference (IV, Fixed, 95\% } \\
\mathrm{CI})\end{array}$ & $0.65[0.36,0.94]$ \\
\hline $1.1 \leq 1500$ grams birth weight & 2 & 126 & $\begin{array}{l}\text { Mean Difference (IV, Fixed, 95\% } \\
\mathrm{CI} \text { ) }\end{array}$ & $0.65[0.36,0.94]$ \\
\hline $\begin{array}{l}2 \mathrm{Hypothermia} \text { on admission to } \\
\mathrm{NICU} \text { : core body temperature }< \\
36.5^{\circ} \mathrm{C} \text { or skin temperature }<36^{\circ} \mathrm{C}\end{array}$ & 1 & 24 & Risk Ratio (M-H, Fixed, 95\% Cl) & $0.3[0.11,0.83]$ \\
\hline $2.1 \leq 1500$ grams birth weight & 1 & 24 & Risk Ratio (M-H, Fixed, 95\% Cl) & $0.3[0.11,0.83]$ \\
\hline $\begin{array}{l}3 \text { Moderate hypothermia on ad- } \\
\text { mission to NICU: core body tem- } \\
\text { perature }<36^{\circ} \mathrm{C}\end{array}$ & 2 & 126 & Risk Ratio (M-H, Fixed, 95\% Cl) & $0.49[0.32,0.76]$ \\
\hline $3.1 \leq 1500$ grams birth weight & 2 & 126 & Risk Ratio (M-H, Fixed, 95\% Cl) & $0.49[0.32,0.76]$ \\
\hline $\begin{array}{l}4 \text { Hypothermia on admission to } \\
\mathrm{NICU} \text { : core body temperature }< \\
35^{\circ} \mathrm{C}\end{array}$ & 2 & 126 & Risk Ratio (M-H, Fixed, 95\% Cl) & $0.18[0.05,0.65]$ \\
\hline
\end{tabular}




\begin{tabular}{|c|c|c|c|c|}
\hline Outcome or subgroup title & No. of studies & $\begin{array}{l}\text { No. of partici- } \\
\text { pants }\end{array}$ & Statistical method & Effect size \\
\hline $4.1 \leq 1500$ grams birth weight & 2 & 126 & Risk Ratio (M-H, Fixed, 95\% Cl) & $0.18[0.05,0.65]$ \\
\hline $\begin{array}{l}5 \text { Outside normothermic range on } \\
\text { admission to NICU or up to } 2 \text { hours } \\
\text { after birth }\end{array}$ & 1 & 24 & Risk Ratio (M-H, Fixed, 95\% Cl) & $0.3[0.11,0.83]$ \\
\hline $5.1 \leq 1500$ grams birth weight & 1 & 24 & Risk Ratio (M-H, Fixed, 95\% Cl) & $0.3[0.11,0.83]$ \\
\hline $\begin{array}{l}6 \text { Hyperthermia on admission to } \\
\text { NICU: core body temperature }> \\
37.5^{\circ} \mathrm{C}\end{array}$ & 2 & 126 & Risk Ratio (M-H, Fixed, 95\% Cl) & $4.63[0.23,94.10]$ \\
\hline $\begin{array}{l}6.1<32 \text { completed weeks' gesta- } \\
\text { tion }\end{array}$ & 2 & 126 & Risk Ratio (M-H, Fixed, 95\% Cl) & $4.63[0.23,94.10]$ \\
\hline 7 Mortality & 1 & 102 & Risk Ratio (M-H, Fixed, 95\% Cl) & $0.31[0.01,7.40]$ \\
\hline $\begin{array}{l}7.1<32 \text { completed weeks' gesta- } \\
\text { tion }\end{array}$ & 1 & 102 & Risk Ratio (M-H, Fixed, 95\% Cl) & $0.31[0.01,7.40]$ \\
\hline $\begin{array}{l}8 \text { Major brain injury - IVH (grade } \mathbb{I} \\
\text { or } \mathbb{N} \text { ) }\end{array}$ & 1 & 102 & Risk Ratio (M-H, Fixed, 95\% Cl) & $4.62[0.56,38.19]$ \\
\hline $\begin{array}{l}8.1<32 \text { completed weeks' gesta- } \\
\text { tion }\end{array}$ & 1 & 102 & Risk Ratio (M-H, Fixed, 95\% Cl) & $4.62[0.56,38.19]$ \\
\hline $\begin{array}{l}9 \text { Major brain injury - cystic } \\
\text { periventricular leukomalacia }\end{array}$ & 1 & 102 & Risk Ratio (M-H, Fixed, 95\% Cl) & $1.39[0.24,7.95]$ \\
\hline $\begin{array}{l}9.1<32 \text { completed weeks' gesta- } \\
\text { tion }\end{array}$ & 1 & 102 & Risk Ratio (M-H, Fixed, 95\% Cl) & $1.39[0.24,7.95]$ \\
\hline $\begin{array}{l}10 \text { Bronchopulmonary dysplasia } \\
\text { (BPD) }\end{array}$ & 1 & 102 & Risk Ratio (M-H, Fixed, 95\% Cl) & $1.34[0.69,2.61]$ \\
\hline $\begin{array}{l}10.1<32 \text { completed weeks' gesta- } \\
\text { tion }\end{array}$ & 1 & 102 & Risk Ratio (M-H, Fixed, 95\% Cl) & $1.34[0.69,2.61]$ \\
\hline 11 Duration of hospital stay (days) & 1 & 102 & $\begin{array}{l}\text { Mean Difference (IV, Fixed, 95\% } \\
\mathrm{CI})\end{array}$ & $-5.0[-17.27,7.27]$ \\
\hline $\begin{array}{l}11.1<32 \text { completed weeks' gesta- } \\
\text { tion }\end{array}$ & 1 & 102 & $\begin{array}{l}\text { Mean Difference (IV, Fixed, 95\% } \\
\mathrm{CI} \text { ) }\end{array}$ & $-5.0[-17.27,7.27]$ \\
\hline 12 Duration of ventilation (days) & 1 & 102 & $\begin{array}{l}\text { Mean Difference (IV, Fixed, 95\% } \\
\mathrm{CI} \text { ) }\end{array}$ & $-4.0[-12.67,4.67]$ \\
\hline $\begin{array}{l}12.1<32 \text { completed weeks' gesta- } \\
\text { tion }\end{array}$ & 1 & 102 & $\begin{array}{l}\text { Mean Difference (IV, Fixed, 95\% } \\
\mathrm{CI})\end{array}$ & $-4.0[-12.67,4.67]$ \\
\hline $\begin{array}{l}13 \text { Duration of oxygen require- } \\
\text { ments (days) }\end{array}$ & 1 & 102 & $\begin{array}{l}\text { Mean Difference (IV, Fixed, 95\% } \\
\mathrm{CI} \text { ) }\end{array}$ & $-7.0[-19.66,5.66]$ \\
\hline $\begin{array}{l}13.1<32 \text { completed weeks' gesta- } \\
\text { tion }\end{array}$ & 1 & 102 & $\begin{array}{l}\text { Mean Difference (IV, Fixed, 95\% } \\
\mathrm{CI} \text { ) }\end{array}$ & $-7.0[-19.66,5.66]$ \\
\hline
\end{tabular}




\begin{tabular}{|c|c|c|c|c|}
\hline Outcome or subgroup title & No. of studies & $\begin{array}{l}\text { No. of partici- } \\
\text { pants }\end{array}$ & Statistical method & Effect size \\
\hline 14 Hypoglycaemia & 1 & 102 & Risk Ratio (M-H, Fixed, 95\% Cl) & $1.02[0.47,2.18]$ \\
\hline $\begin{array}{l}14.1<32 \text { completed weeks' gesta- } \\
\text { tion }\end{array}$ & 1 & 102 & Risk Ratio (M-H, Fixed, 95\% Cl) & $1.02[0.47,2.18]$ \\
\hline 15 Metabolic acidosis & 1 & 102 & Risk Ratio (M-H, Fixed, 95\% Cl) & $0.31[0.03,2.86]$ \\
\hline $\begin{array}{l}15.1<32 \text { completed weeks' gesta- } \\
\text { tion }\end{array}$ & 1 & 102 & Risk Ratio (M-H, Fixed, 95\% Cl) & $0.31[0.03,2.86]$ \\
\hline 16 Necrotising enterocolitis (NEC) & 1 & 102 & Risk Ratio (M-H, Fixed, 95\% Cl) & $0.64[0.33,1.23]$ \\
\hline $\begin{array}{l}16.1<32 \text { completed weeks' gesta- } \\
\text { tion }\end{array}$ & 1 & 102 & Risk Ratio (M-H, Fixed, 95\% Cl) & $0.64[0.33,1.23]$ \\
\hline 17 Sepsis & 1 & 102 & Risk Ratio (M-H, Fixed, 95\% Cl) & $0.92[0.48,1.79]$ \\
\hline $\begin{array}{l}17.1<32 \text { completed weeks' gesta- } \\
\text { tion }\end{array}$ & 1 & 102 & Risk Ratio (M-H, Fixed, 95\% Cl) & $0.92[0.48,1.79]$ \\
\hline
\end{tabular}

Analysis 13.1. Comparison 13 Thermal mattress versus routine care, Outcome 1 Core body temperature $\left({ }^{\circ} \mathrm{C}\right)$ on admission to NICU or up to 2 hours after birth.

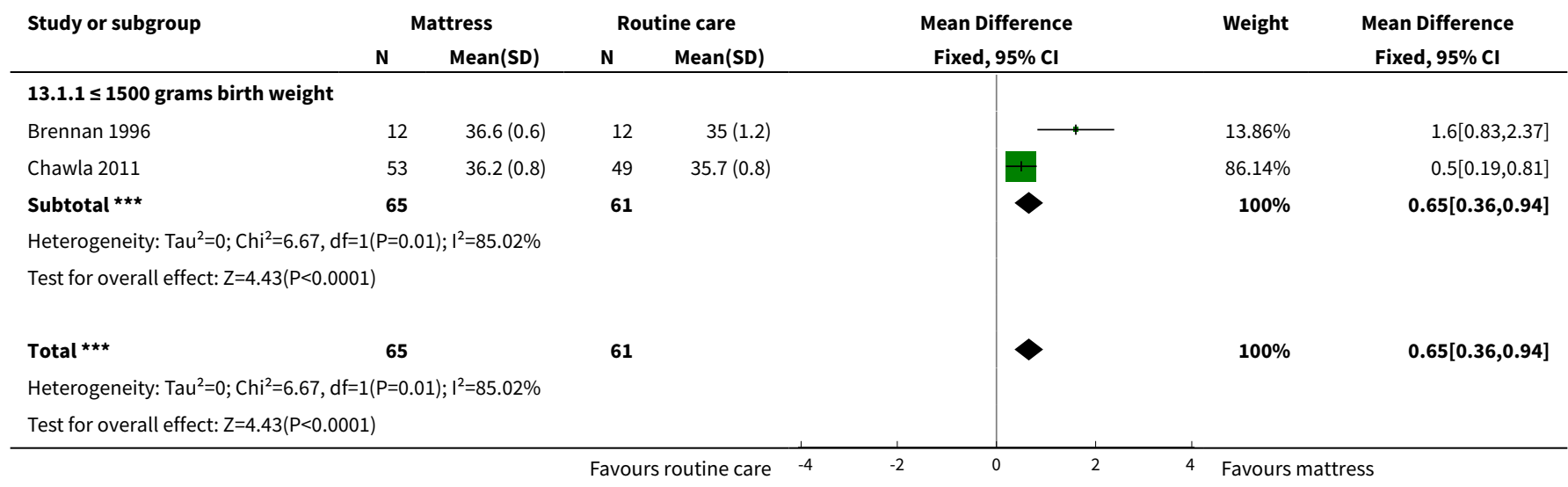

\section{Analysis 13.2. Comparison 13 Thermal mattress versus routine care, Outcome 2 Hypothermia} on admission to NICU: core body temperature $<36.5^{\circ} \mathrm{C}$ or skin temperature $<36^{\circ} \mathrm{C}$.

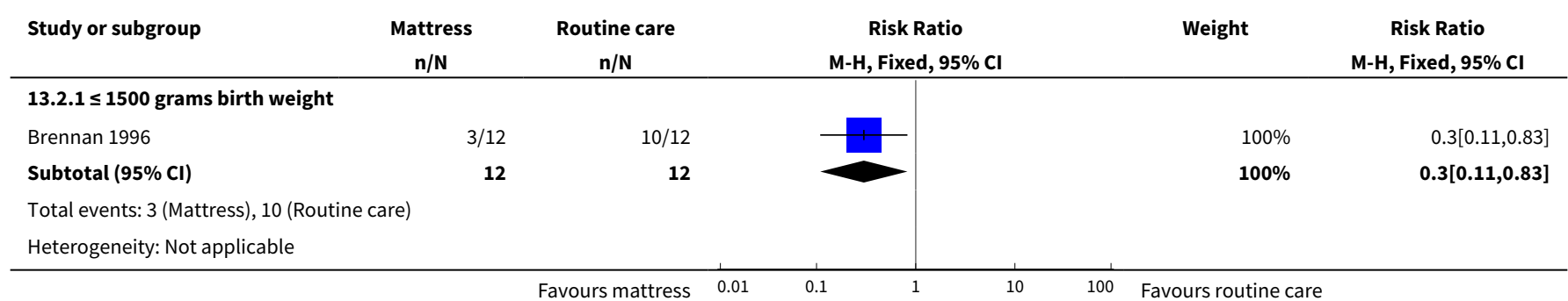




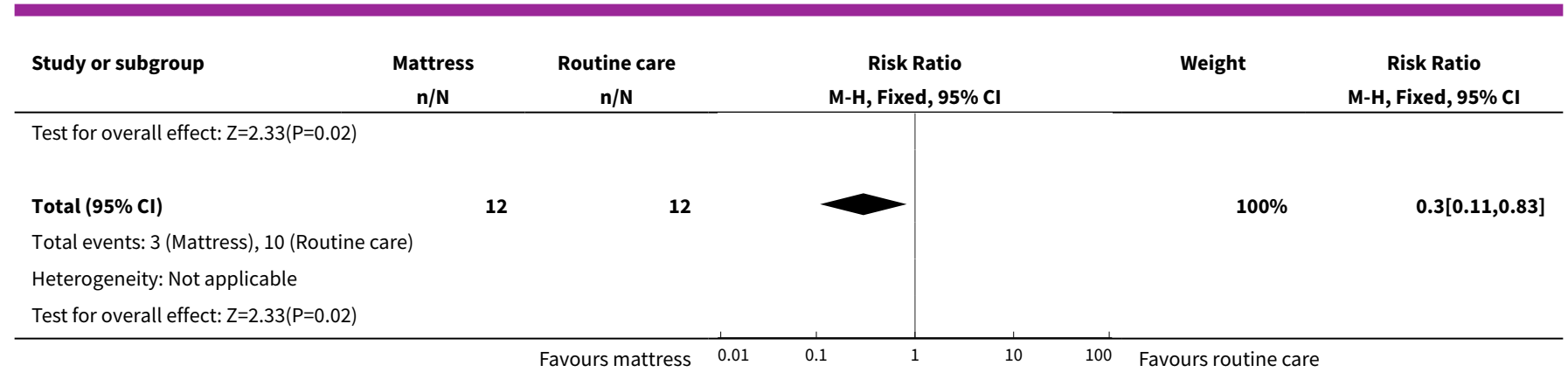

Analysis 13.3. Comparison 13 Thermal mattress versus routine care, Outcome 3 Moderate hypothermia on admission to NICU: core body temperature $<36^{\circ} \mathrm{C}$.

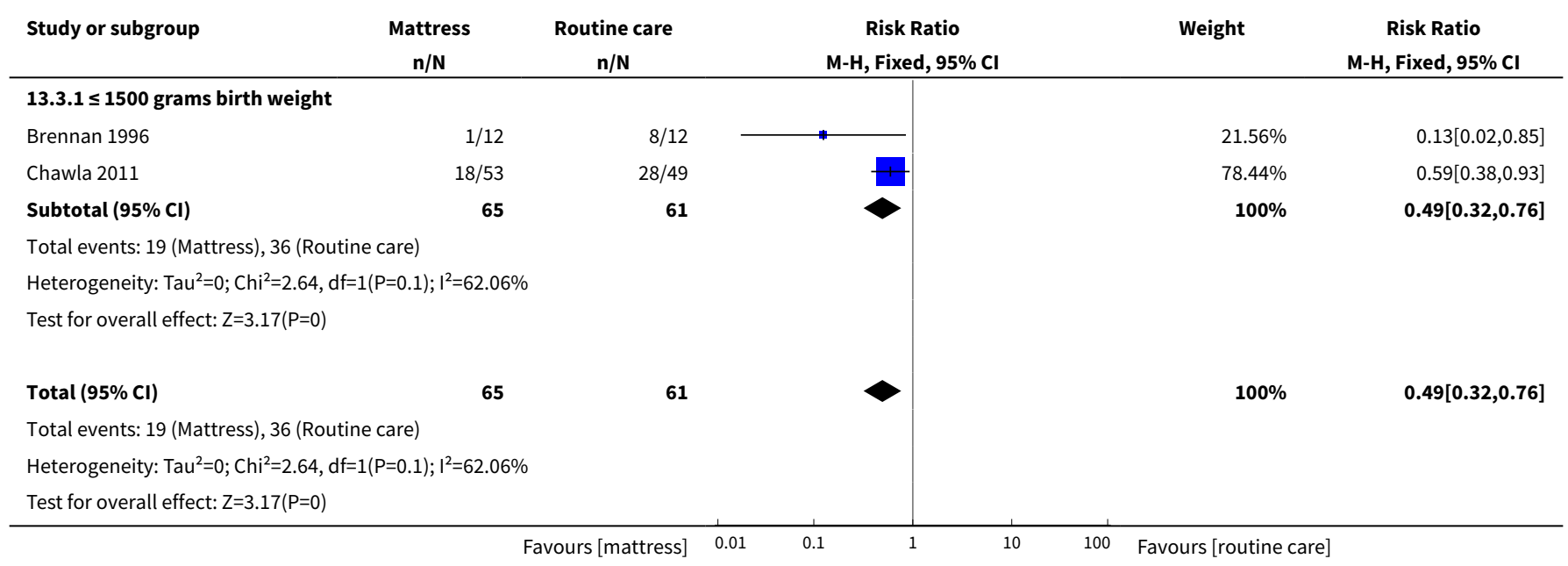

Analysis 13.4. Comparison 13 Thermal mattress versus routine care, Outcome 4 Hypothermia on admission to NICU: core body temperature $<35^{\circ} \mathrm{C}$.

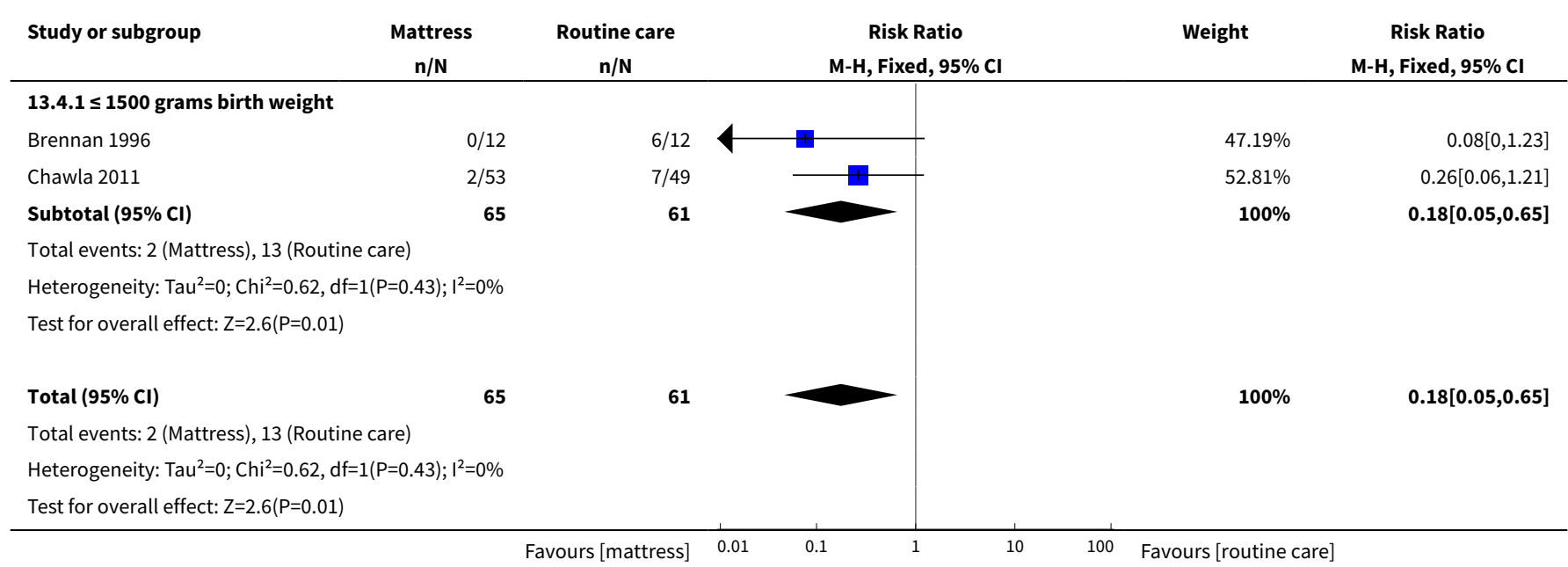


Analysis 13.5. Comparison 13 Thermal mattress versus routine care, Outcome 5 Outside normothermic range on admission to NICU or up to 2 hours after birth.

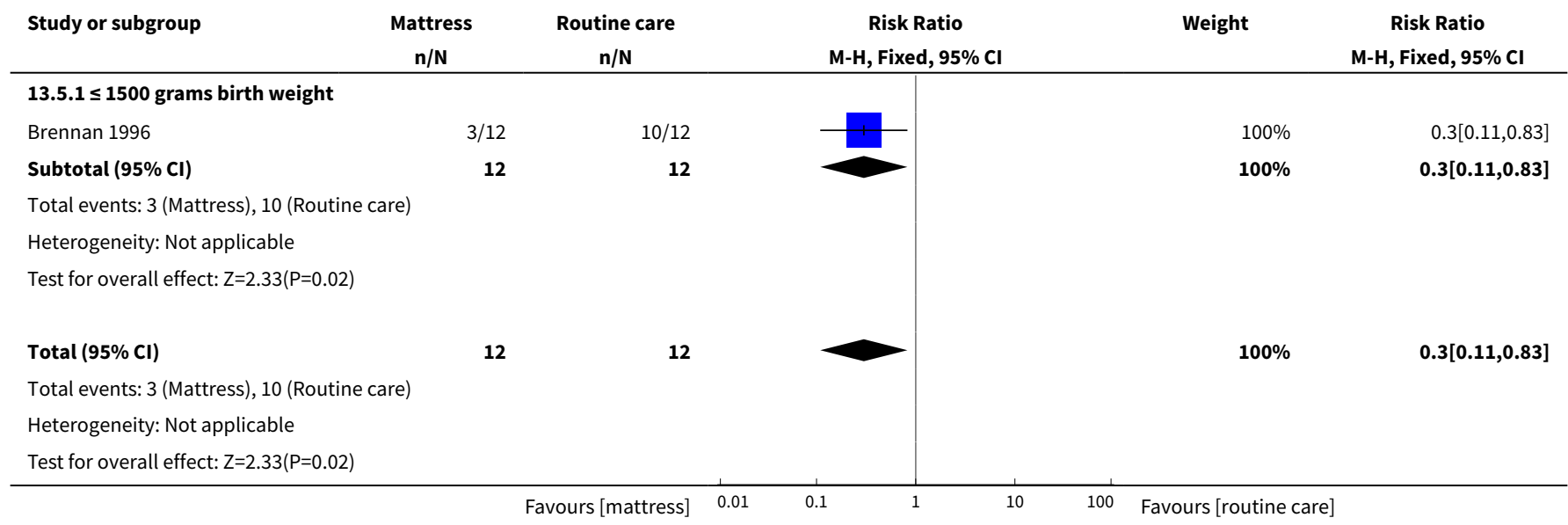

\section{Analysis 13.6. Comparison 13 Thermal mattress versus routine care, Outcome 6 Hyperthermia on admission to NICU: core body temperature $>37.5^{\circ} \mathrm{C}$.}

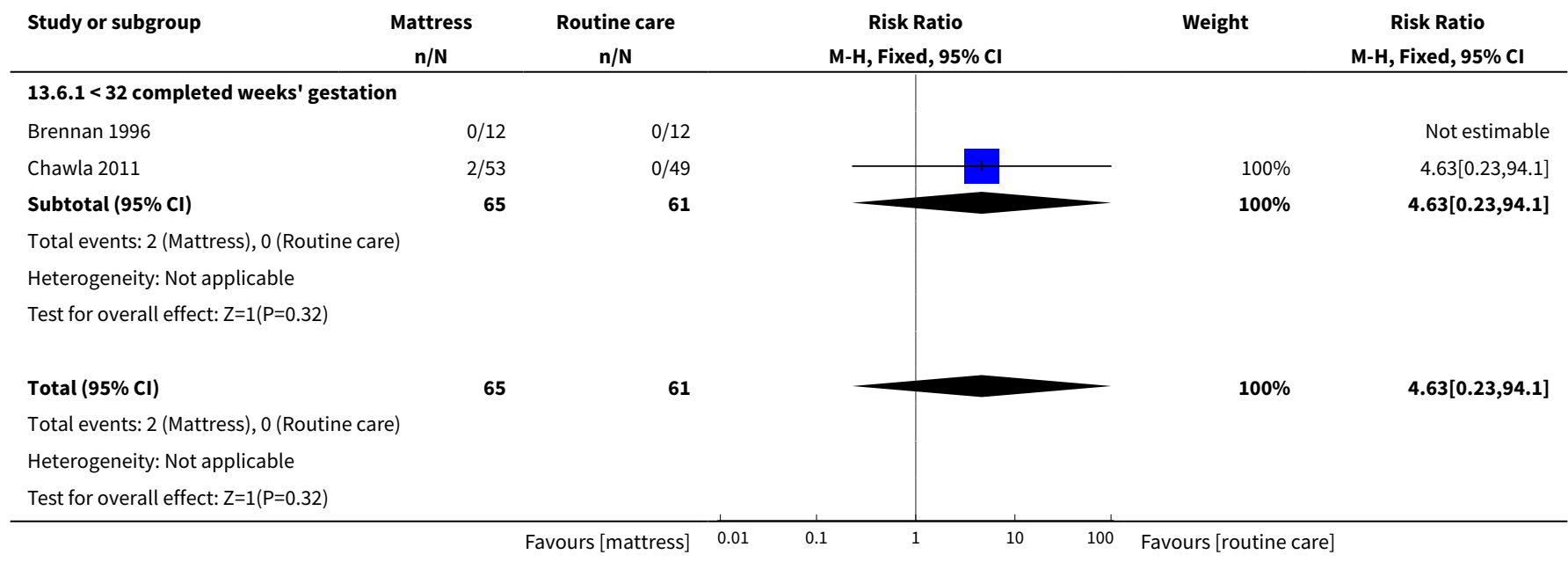

Analysis 13.7. Comparison 13 Thermal mattress versus routine care, Outcome 7 Mortality.

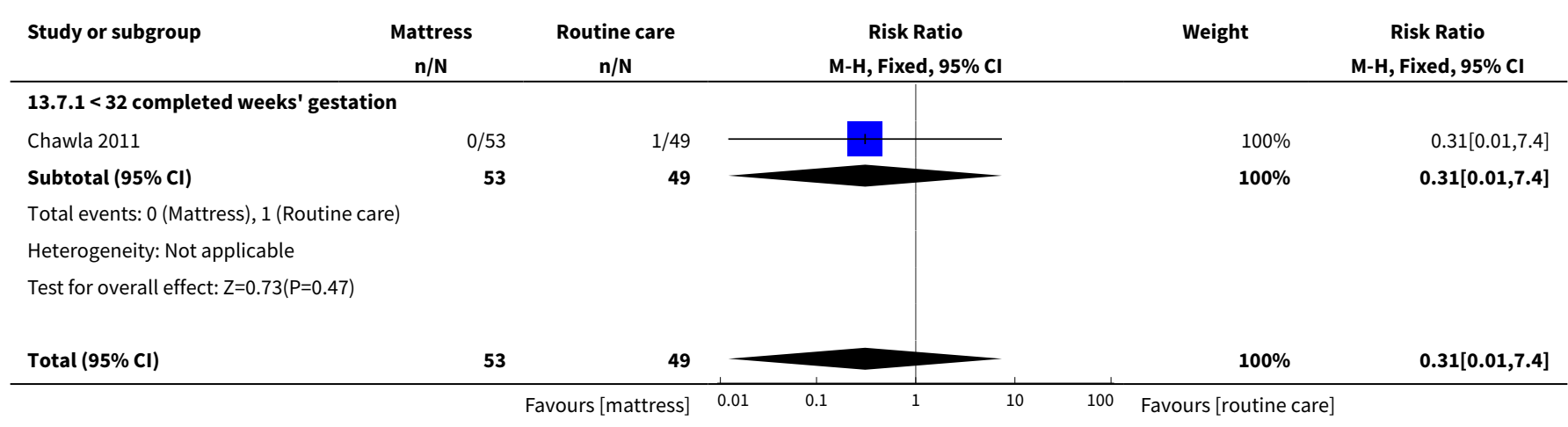




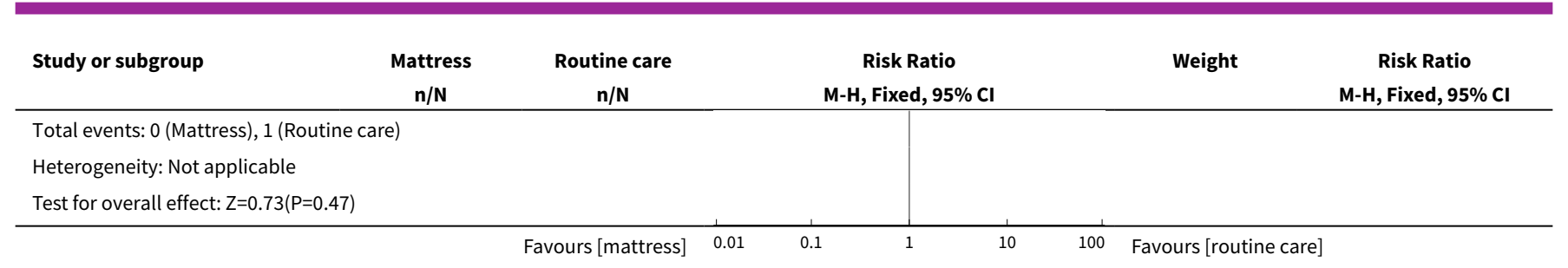

Analysis 13.8. Comparison 13 Thermal mattress versus routine care, Outcome 8 Major brain injury - IVH (grade III or IV).

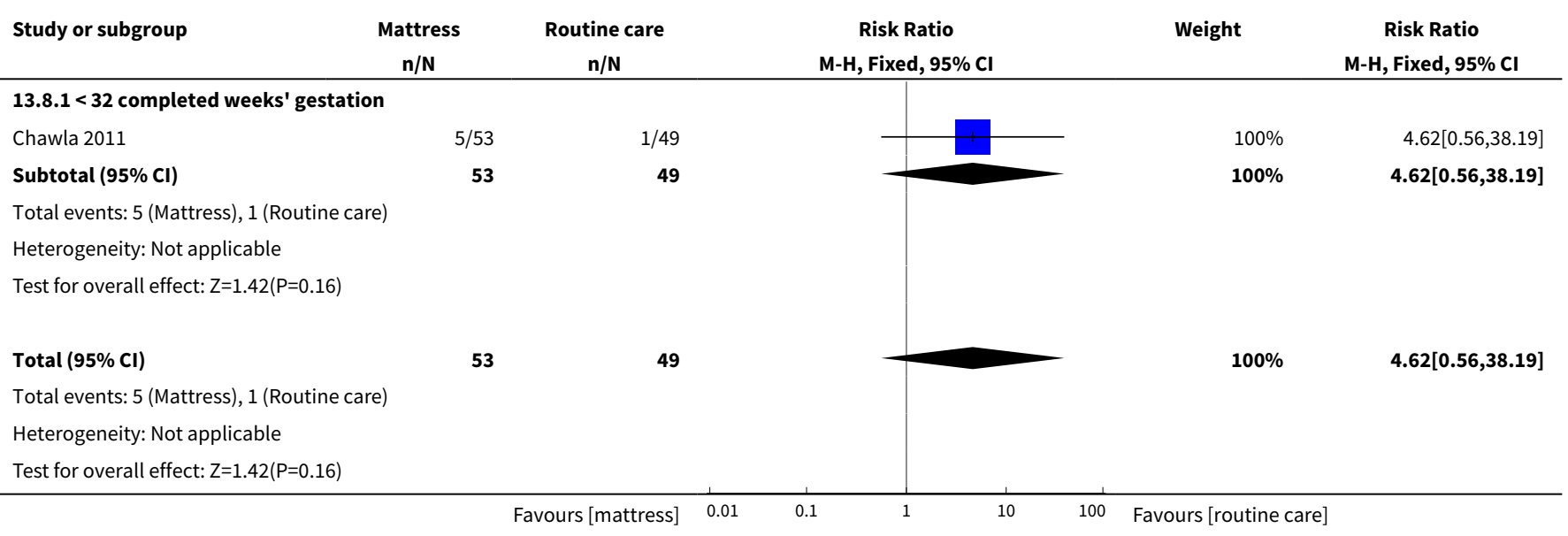

Analysis 13.9. Comparison 13 Thermal mattress versus routine care, Outcome 9 Major brain injury - cystic periventricular leukomalacia.

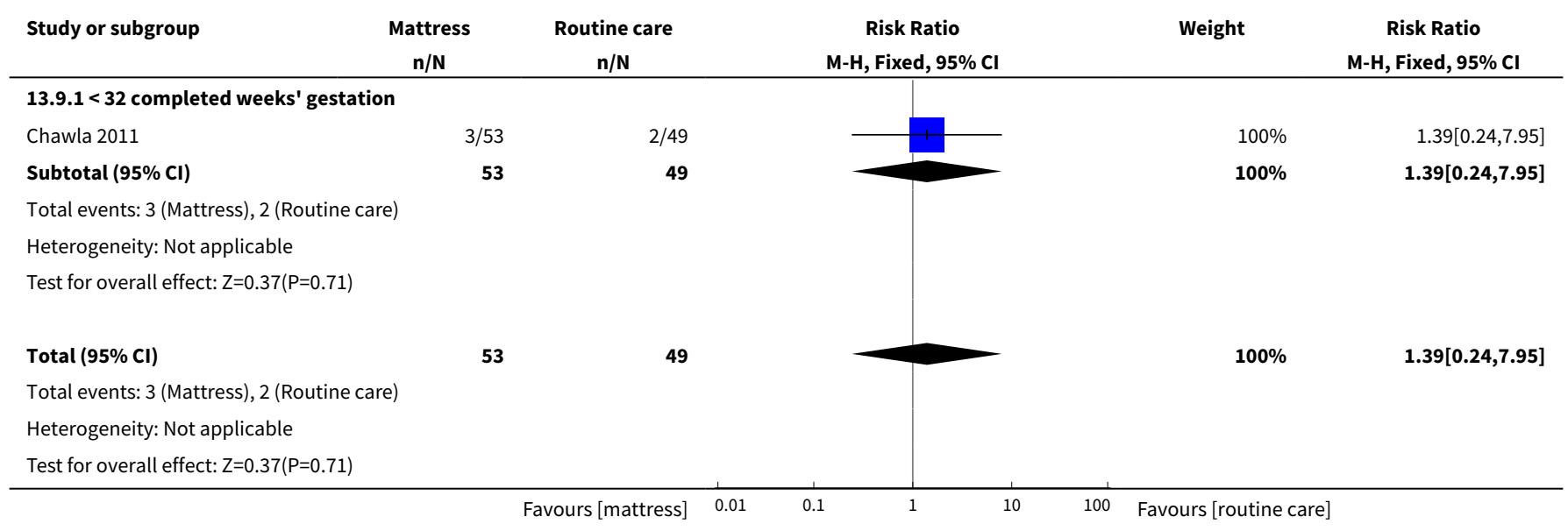


Analysis 13.10. Comparison 13 Thermal mattress versus routine care, Outcome 10 Bronchopulmonary dysplasia (BPD).

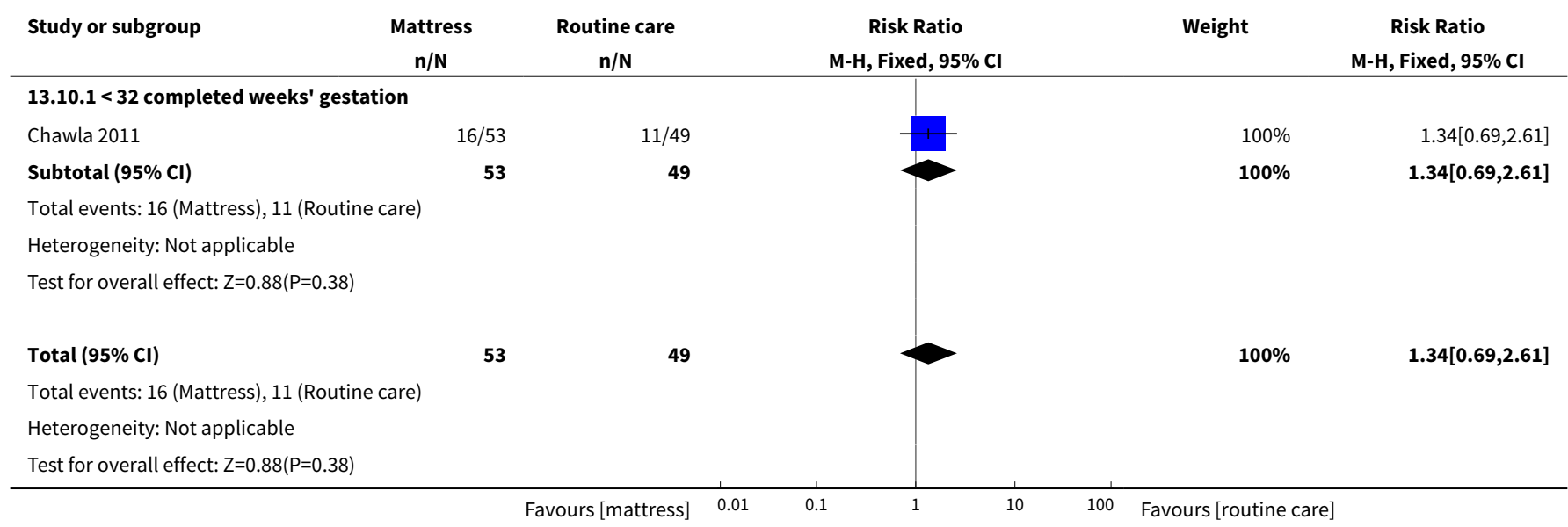

Analysis 13.11. Comparison 13 Thermal mattress versus routine care, Outcome 11 Duration of hospital stay (days).

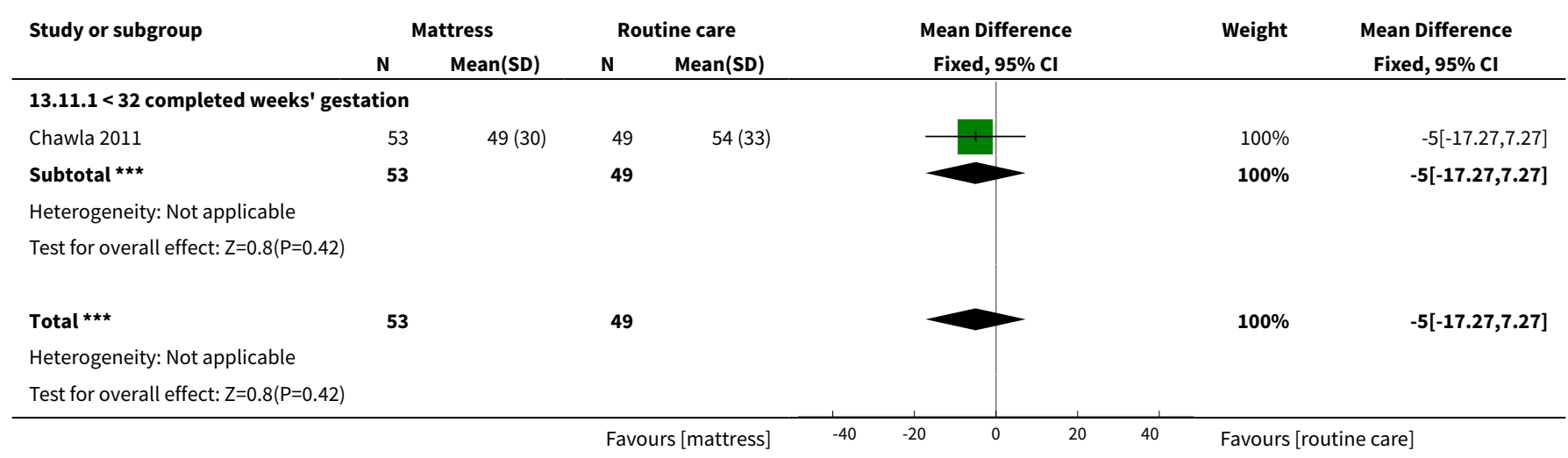

Analysis 13.12. Comparison 13 Thermal mattress versus routine care, Outcome 12 Duration of ventilation (days).

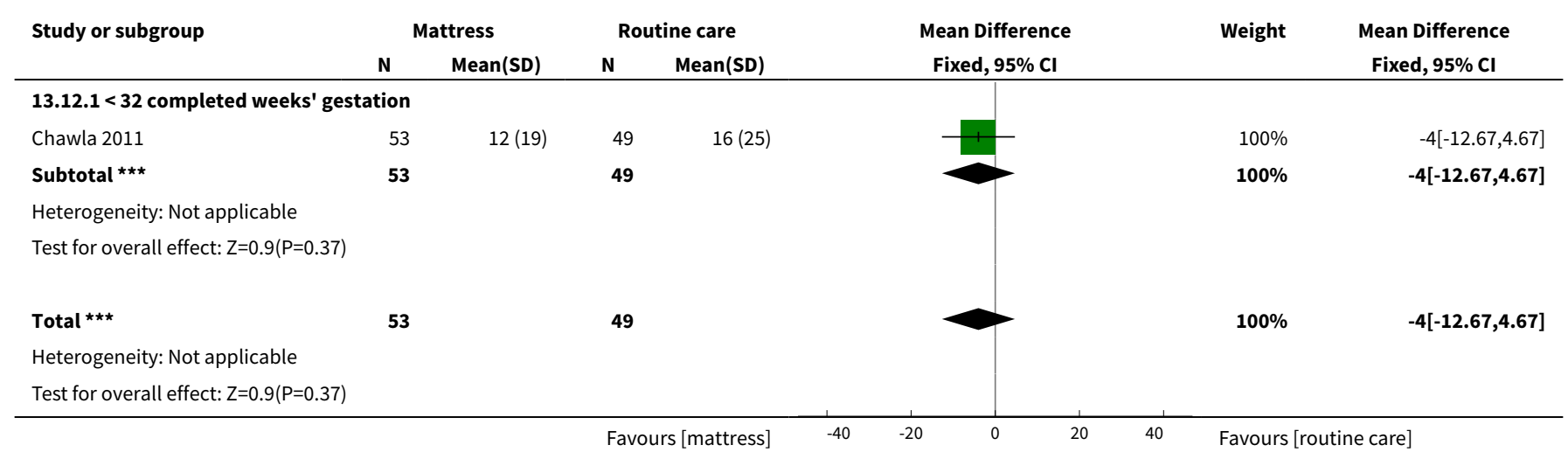




\section{Analysis 13.13. Comparison 13 Thermal mattress versus routine} care, Outcome 13 Duration of oxygen requirements (days).

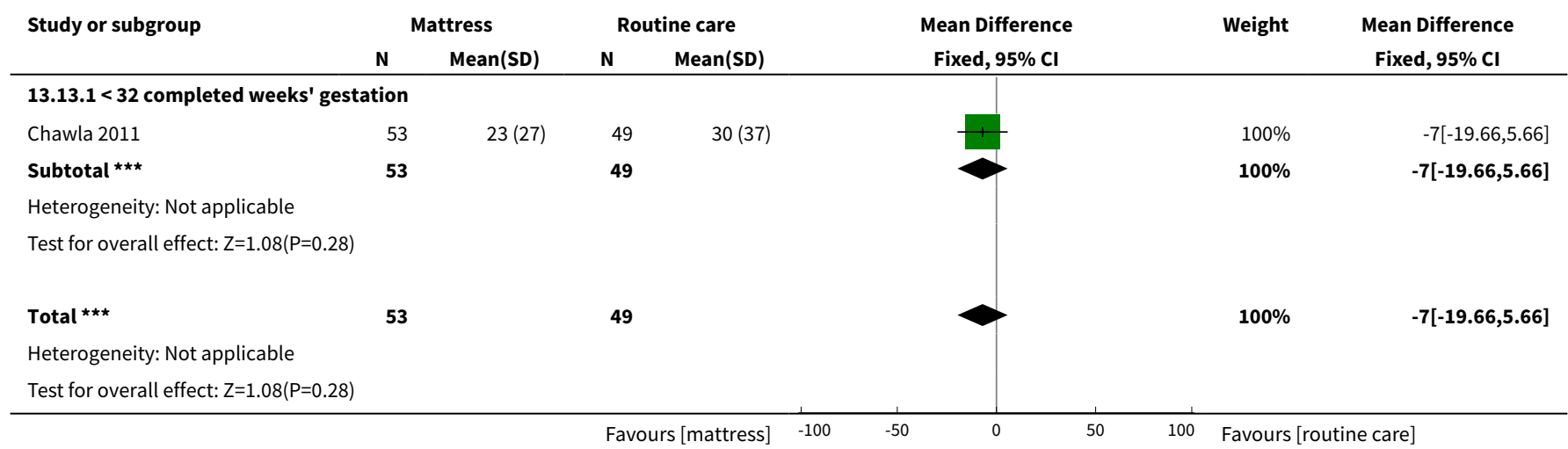

Analysis 13.14. Comparison 13 Thermal mattress versus routine care, Outcome 14 Hypoglycaemia.

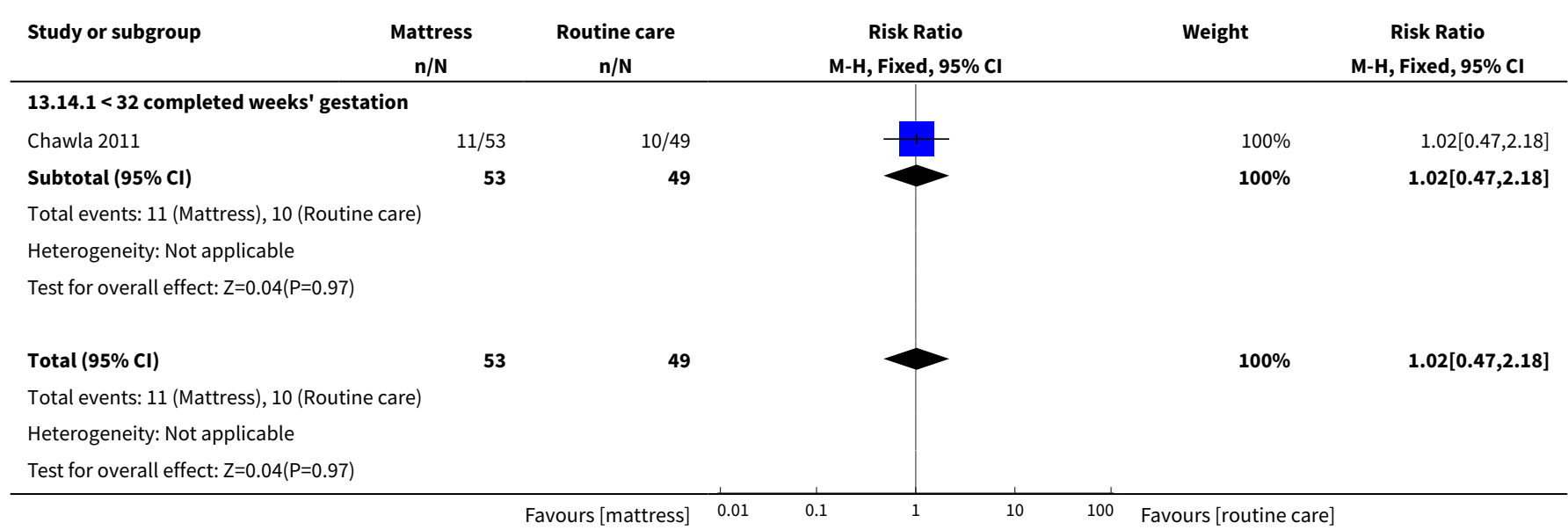

Analysis 13.15. Comparison 13 Thermal mattress versus routine care, Outcome 15 Metabolic acidosis.

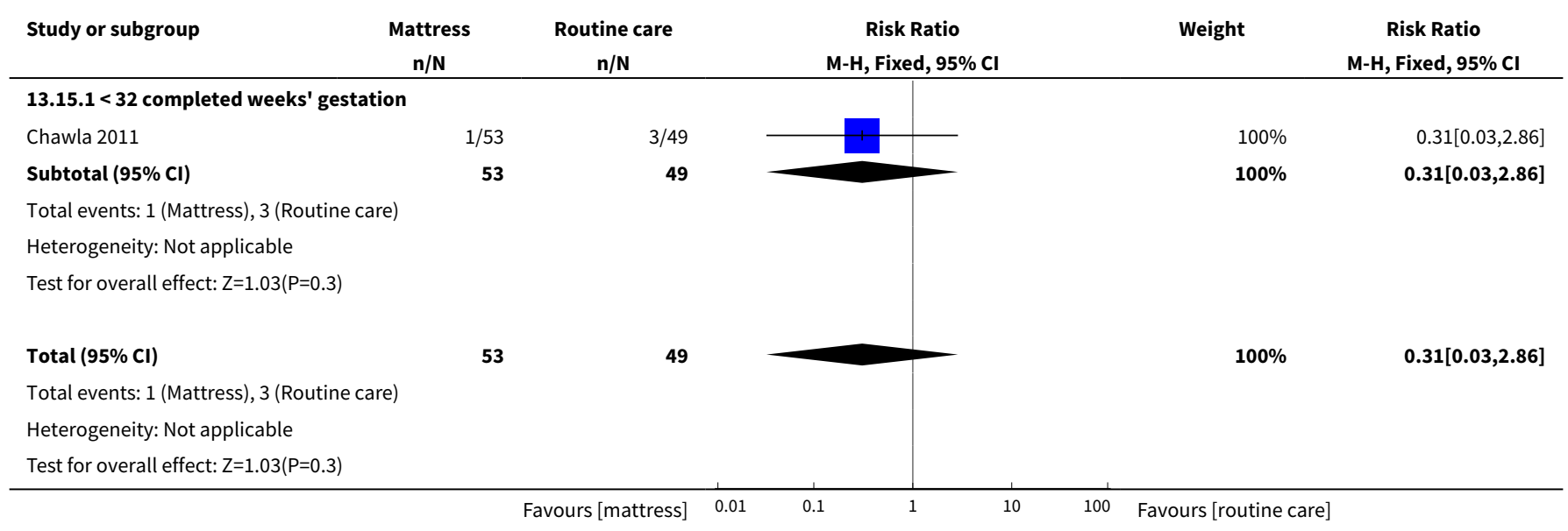


Analysis 13.16. Comparison 13 Thermal mattress versus routine care, Outcome 16 Necrotising enterocolitis (NEC).

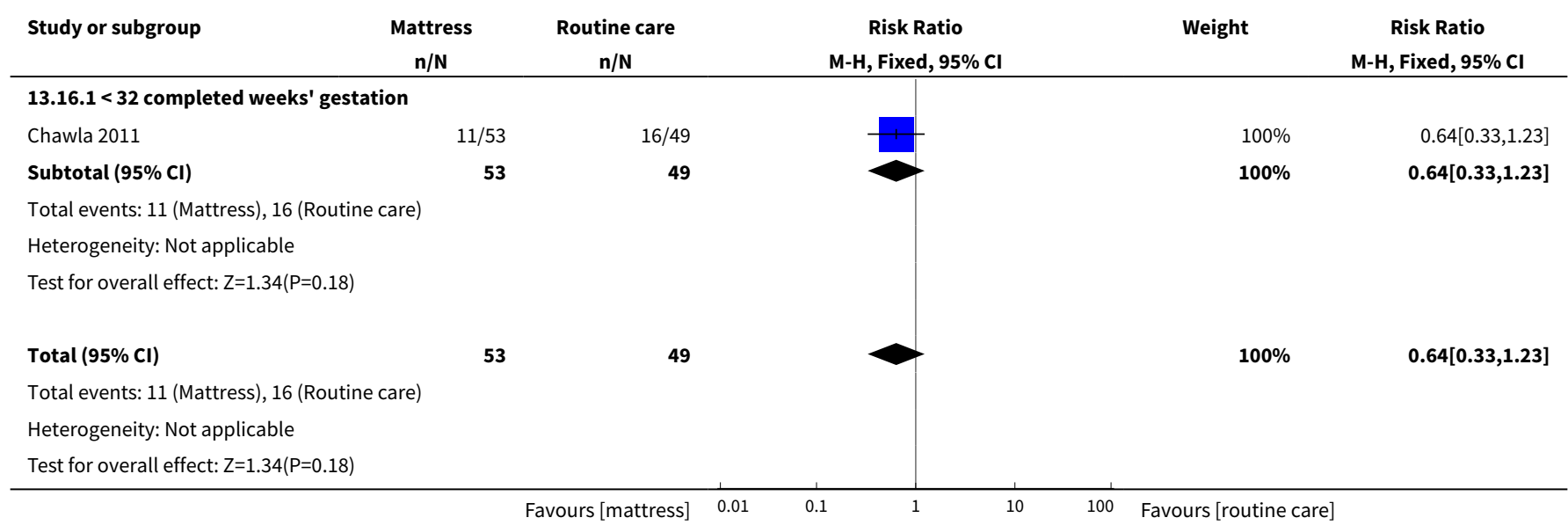

Analysis 13.17. Comparison 13 Thermal mattress versus routine care, Outcome 17 Sepsis.

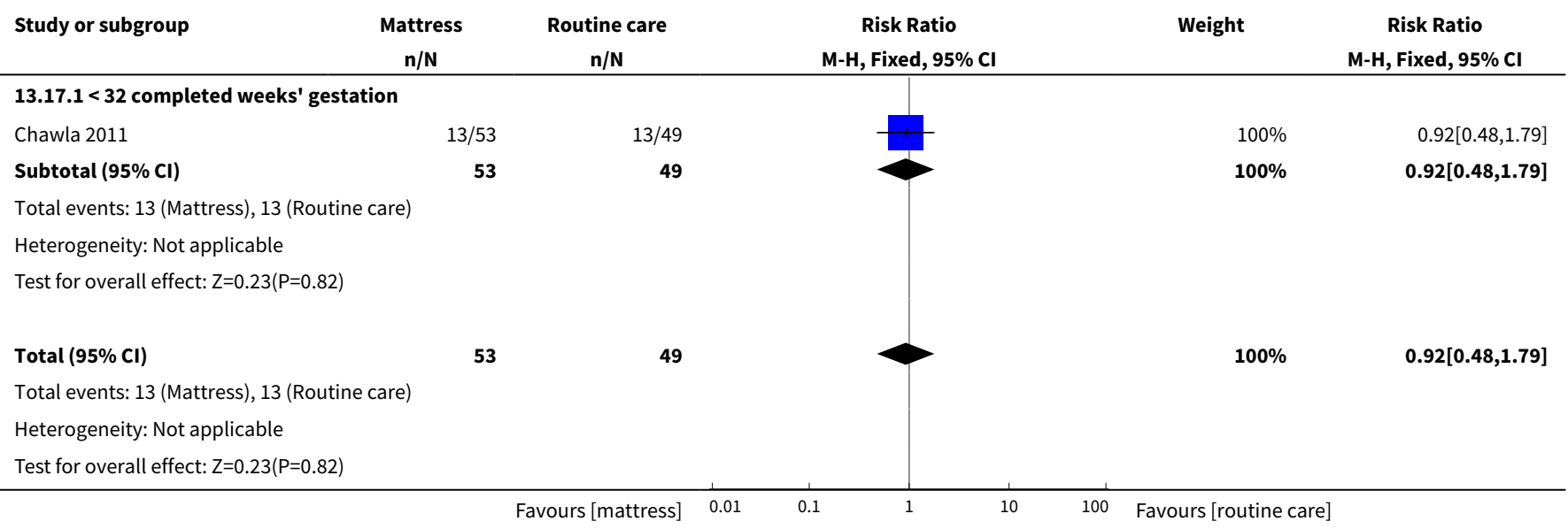

Comparison 14. Thermal mattress versus plastic wrap or bag

\begin{tabular}{lllll}
\hline Outcome or subgroup title & No. of studies & $\begin{array}{l}\text { No. of partici- } \\
\text { pants }\end{array}$ & Statistical method & Effect size \\
\hline $\begin{array}{l}1 \text { Core body temperature }\left({ }^{\circ} \mathrm{C}\right) \text { on admis- } \\
\text { sion to NICU or up to 2 hours after birth }\end{array}$ & 2 & 77 & $\begin{array}{l}\text { Mean Difference (IV, Fixed, } \\
95 \% \mathrm{Cl})\end{array}$ & $0.18[-0.18,0.54]$ \\
\hline $1.1 \leq 28$ completed weeks' gestation & 2 & 77 & $\begin{array}{l}\text { Mean Difference (IV, Fixed, } \\
95 \% \mathrm{Cl})\end{array}$ & $0.18[-0.18,0.54]$ \\
\hline $\begin{array}{l}2 \text { Hypothermia on admission to NICU } \\
\text { (core body temperature }<36.5^{\circ} \mathrm{C} \text { or skin } \\
\left.\text { temperature }<36^{\circ} \mathrm{C}\right)\end{array}$ & 1 & 36 & $\begin{array}{l}\text { Risk Ratio (M-H, Fixed, } \\
95 \% \mathrm{Cl})\end{array}$ & $0.60[0.32,1.15]$ \\
\hline
\end{tabular}




\begin{tabular}{|c|c|c|c|c|}
\hline Outcome or subgroup title & No. of studies & $\begin{array}{l}\text { No. of partici- } \\
\text { pants }\end{array}$ & Statistical method & Effect size \\
\hline $\begin{array}{l}2.1 \text { ( } \geq 24 \text { and } \leq 28 \text { completed weeks' ges- } \\
\text { tation and birth weight } \leq 1250 \text { grams) }\end{array}$ & 1 & 36 & $\begin{array}{l}\text { Risk Ratio (M-H, Fixed, } \\
95 \% \mathrm{Cl})\end{array}$ & $0.60[0.32,1.15]$ \\
\hline $\begin{array}{l}3 \text { Outside normothermic range on admis- } \\
\text { sion to NICU or up to } 2 \text { hours after birth }\end{array}$ & 1 & 36 & $\begin{array}{l}\text { Risk Ratio (M-H, Fixed, } \\
95 \% \mathrm{Cl})\end{array}$ & $0.69[0.38,1.24]$ \\
\hline $\begin{array}{l}3.1 \text { ( } \geq 24 \text { and } \leq 28 \text { completed weeks' ges- } \\
\text { tation and birth weight } \leq 1250 \text { grams) }\end{array}$ & 1 & 36 & $\begin{array}{l}\text { Risk Ratio (M-H, Fixed, } \\
95 \% \mathrm{Cl})\end{array}$ & $0.69[0.38,1.24]$ \\
\hline $\begin{array}{l}4 \text { Hyperthermia on admission to NICU: } \\
\text { core body temperature }>37.5^{\circ} \mathrm{C}\end{array}$ & 1 & 36 & $\begin{array}{l}\text { Risk Ratio (M-H, Fixed, } \\
95 \% \mathrm{Cl})\end{array}$ & $3.33[0.14,76.75]$ \\
\hline $\begin{array}{l}4.1 \text { ( } \geq 24 \text { and } \leq 28 \text { completed weeks' ges- } \\
\text { tation and birth weight } \leq 1250 \text { grams) }\end{array}$ & 1 & 36 & $\begin{array}{l}\text { Risk Ratio (M-H, Fixed, } \\
95 \% \mathrm{Cl})\end{array}$ & $3.33[0.14,76.75]$ \\
\hline 5 Brain injury (IVH grade I or $\mathbb{I}$ ) & 1 & 36 & $\begin{array}{l}\text { Risk Ratio (M-H, Fixed, } \\
95 \% \mathrm{Cl})\end{array}$ & $0.45[0.10,2.01]$ \\
\hline $\begin{array}{l}5.1 \text { ( } \geq 24 \text { and } \leq 28 \text { completed weeks' ges- } \\
\text { tation and birth weight } \leq 1250 \text { grams) }\end{array}$ & 1 & 36 & $\begin{array}{l}\text { Risk Ratio (M-H, Fixed, } \\
95 \% \mathrm{Cl})\end{array}$ & $0.45[0.10,2.01]$ \\
\hline 6 Major brain injury (IVH grade $\mathbb{I I}$ or $\mathbb{V}$ ) & 2 & 77 & $\begin{array}{l}\text { Risk Ratio (M-H, Fixed, } \\
95 \% \mathrm{Cl})\end{array}$ & $0.91[0.31,2.71]$ \\
\hline $6.1 \leq 28$ completed weeks' gestation & 2 & 77 & $\begin{array}{l}\text { Risk Ratio (M-H, Fixed, } \\
95 \% \mathrm{Cl})\end{array}$ & $0.91[0.31,2.71]$ \\
\hline 7 Mortality (death before discharge) & 2 & 77 & $\begin{array}{l}\text { Risk Ratio (M-H, Fixed, } \\
95 \% \mathrm{Cl})\end{array}$ & $1.10[0.39,3.17]$ \\
\hline $7.1 \leq 28$ completed weeks' gestation & 2 & 77 & $\begin{array}{l}\text { Risk Ratio (M-H, Fixed, } \\
95 \% \mathrm{Cl})\end{array}$ & $1.10[0.39,3.17]$ \\
\hline 8 Bronchopulmonary dysplasia (BPD) & 1 & 36 & $\begin{array}{l}\text { Risk Ratio (M-H, Fixed, } \\
95 \% \mathrm{Cl})\end{array}$ & $0.75[0.40,1.37]$ \\
\hline $\begin{array}{l}8.1 \text { ( } \geq 24 \text { and } \leq 28 \text { completed weeks' ges- } \\
\text { tation and birth weight } \leq 1250 \text { grams) }\end{array}$ & 1 & 36 & $\begin{array}{l}\text { Risk Ratio (M-H, Fixed, } \\
95 \% \mathrm{Cl})\end{array}$ & $0.75[0.40,1.37]$ \\
\hline 9 Hypotension during first 24 hours of life & 1 & 41 & $\begin{array}{l}\text { Risk Ratio (M-H, Fixed, } \\
95 \% \mathrm{Cl})\end{array}$ & $1.5[0.71,3.17]$ \\
\hline $9.1 \leq 28$ completed weeks' gestation & 1 & 41 & $\begin{array}{l}\text { Risk Ratio (M-H, Fixed, } \\
95 \% \mathrm{Cl})\end{array}$ & $1.5[0.71,3.17]$ \\
\hline 10 Necrotising enterocolitis (NEC) & 2 & 77 & $\begin{array}{l}\text { Risk Ratio (M-H, Fixed, } \\
95 \% \mathrm{Cl})\end{array}$ & $1.92[0.61,5.97]$ \\
\hline $10.1 \leq 28$ completed weeks' gestation & 2 & 77 & $\begin{array}{l}\text { Risk Ratio (M-H, Fixed, } \\
95 \% \mathrm{Cl})\end{array}$ & $1.92[0.61,5.97]$ \\
\hline 11 Patent ductus arteriosus (PDA) & 1 & 36 & $\begin{array}{l}\text { Risk Ratio (M-H, Fixed, } \\
95 \% \mathrm{Cl})\end{array}$ & $1.76[0.88,3.49]$ \\
\hline
\end{tabular}




\begin{tabular}{|c|c|c|c|c|}
\hline Outcome or subgroup title & No. of studies & $\begin{array}{l}\text { No. of partici- } \\
\text { pants }\end{array}$ & Statistical method & Effect size \\
\hline $\begin{array}{l}11.1 \text { ( } \geq 24 \text { and } \leq 28 \text { completed weeks' ges- } \\
\text { tation and birth weight } \leq 1250 \text { grams) }\end{array}$ & 1 & 36 & $\begin{array}{l}\text { Risk Ratio (M-H, Fixed, } \\
95 \% \mathrm{Cl})\end{array}$ & $1.76[0.88,3.49]$ \\
\hline 12 PDA - medication only & 1 & 36 & $\begin{array}{l}\text { Risk Ratio (M-H, Fixed, } \\
95 \% \mathrm{Cl})\end{array}$ & $1.44[0.69,3.01]$ \\
\hline $\begin{array}{l}12.1 \text { ( } \geq 24 \text { and } \leq 28 \text { completed weeks' ges- } \\
\text { tation and birth weight } \leq 1250 \text { grams) }\end{array}$ & 1 & 36 & $\begin{array}{l}\text { Risk Ratio (M-H, Fixed, } \\
95 \% \mathrm{CI})\end{array}$ & $1.44[0.69,3.01]$ \\
\hline 13 PDA - ligation & 1 & 36 & $\begin{array}{l}\text { Risk Ratio (M-H, Fixed, } \\
95 \% \mathrm{Cl})\end{array}$ & $\begin{array}{l}5.56[0.29 \\
108.16]\end{array}$ \\
\hline $\begin{array}{l}13.1 \text { ( } \geq 24 \text { and } \leq 28 \text { completed weeks' ges- } \\
\text { tation and birth weight } \leq 1250 \text { grams) }\end{array}$ & 1 & 36 & $\begin{array}{l}\text { Risk Ratio (M-H, Fixed, } \\
95 \% \mathrm{Cl})\end{array}$ & $\begin{array}{l}5.56[0.29 \\
108.16]\end{array}$ \\
\hline $\begin{array}{l}14 \text { Retinopathy of prematurity (ROP all } \\
\text { grades) }\end{array}$ & 1 & 36 & $\begin{array}{l}\text { Risk Ratio (M-H, Fixed, } \\
95 \% \mathrm{CI})\end{array}$ & $0.48[0.15,1.56]$ \\
\hline $\begin{array}{l}14.1 \text { ( } \geq 24 \text { and } \leq 28 \text { completed weeks' ges- } \\
\text { tation and birth weight } \leq 1250 \text { grams) }\end{array}$ & 1 & 36 & $\begin{array}{l}\text { Risk Ratio (M-H, Fixed, } \\
95 \% \mathrm{Cl})\end{array}$ & $0.48[0.15,1.56]$ \\
\hline $\begin{array}{l}15 \text { Retinopathy of prematurity (ROP laser } \\
\text { therapy) }\end{array}$ & 1 & 41 & $\begin{array}{l}\text { Risk Ratio }(\mathrm{M}-\mathrm{H}, \text { Fixed, } \\
95 \% \mathrm{Cl})\end{array}$ & $0.53[0.05,5.35]$ \\
\hline $15.1 \leq 28$ completed weeks' gestation & 1 & 41 & $\begin{array}{l}\text { Risk Ratio (M-H, Fixed, } \\
95 \% \mathrm{Cl})\end{array}$ & $0.53[0.05,5.35]$ \\
\hline $\begin{array}{l}16 \text { Spontaneous intestinal perforation } \\
\text { (SIP) }\end{array}$ & 1 & 41 & $\begin{array}{l}\text { Risk Ratio (M-H, Fixed, } \\
95 \% \mathrm{Cl})\end{array}$ & $0.53[0.11,2.56]$ \\
\hline $16.1 \leq 28$ completed weeks' gestation & 1 & 41 & $\begin{array}{l}\text { Risk Ratio (M-H, Fixed, } \\
95 \% \mathrm{Cl})\end{array}$ & $0.53[0.11,2.56]$ \\
\hline $\begin{array}{l}17 \text { Worst base deficit in first } 24 \text { hours of } \\
\text { life }\end{array}$ & 1 & 41 & $\begin{array}{l}\text { Mean Difference (IV, Fixed, } \\
95 \% \mathrm{CI})\end{array}$ & $-1.70[-3.99,0.59]$ \\
\hline $17.1 \leq 28$ completed weeks' gestation & 1 & 41 & $\begin{array}{l}\text { Mean Difference (IV, Fixed, } \\
95 \% \mathrm{CI} \text { ) }\end{array}$ & $-1.70[-3.99,0.59]$ \\
\hline 18 Worst $\mathrm{pH}$ in first 24 hours of life & 1 & 41 & $\begin{array}{l}\text { Mean Difference (IV, Fixed, } \\
95 \% \mathrm{CI} \text { ) }\end{array}$ & $\begin{array}{l}-0.09[-0.13 \\
-0.05]\end{array}$ \\
\hline $18.1 \leq 28$ completed weeks' gestation & 1 & 41 & $\begin{array}{l}\text { Mean Difference (IV, Fixed, } \\
95 \% \mathrm{CI})\end{array}$ & $\begin{array}{l}-0.09[-0.13 \\
-0.05]\end{array}$ \\
\hline
\end{tabular}

\section{Analysis 14.1. Comparison 14 Thermal mattress versus plastic wrap or bag, Outcome 1 Core body temperature $\left({ }^{\circ} \mathrm{C}\right)$ on admission to NICU or up to 2 hours after birth.}

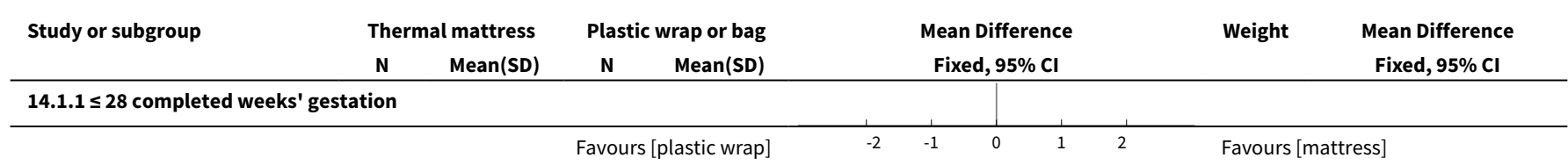




\begin{tabular}{|c|c|c|c|c|c|c|c|}
\hline \multirow[t]{2}{*}{ Study or subgroup } & \multicolumn{2}{|c|}{ Thermal mattress } & \multicolumn{2}{|c|}{ Plastic wrap or bag } & \multirow{2}{*}{$\begin{array}{c}\text { Mean Difference } \\
\text { Fixed, } 95 \% \mathrm{Cl}\end{array}$} & \multirow[t]{2}{*}{ Weight } & \multirow{2}{*}{$\begin{array}{c}\text { Mean Difference } \\
\text { Fixed, } 95 \% \mathrm{Cl}\end{array}$} \\
\hline & $\mathbf{N}$ & Mean(SD) & $\mathbf{N}$ & Mean(SD) & & & \\
\hline Mathew 2012 & 20 & $35.8(1.3)$ & 21 & $36.1(0.7)$ & 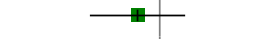 & $31.39 \%$ & $-0.3[-0.94,0.34]$ \\
\hline Simon 2011 & 17 & $36.5(0.7)$ & 19 & $36.1(0.7)$ & & $68.61 \%$ & $0.4[-0.04,0.84]$ \\
\hline Subtotal $\star \star \star$ & 37 & & 40 & & & $100 \%$ & $0.18[-0.18,0.54]$ \\
\hline \multicolumn{8}{|c|}{ Heterogeneity: $\mathrm{Tau}^{2}=0 ; \mathrm{Chi}^{2}=3.12, \mathrm{df}=1(\mathrm{P}=0.08) ; \mathrm{I}^{2}=67.93 \%$} \\
\hline \multicolumn{8}{|c|}{ Test for overall effect: $\mathrm{Z}=0.98(\mathrm{P}=0.33)$} \\
\hline Total $\star \star \star$ & 37 & & 40 & & & $100 \%$ & $0.18[-0.18,0.54]$ \\
\hline \multicolumn{8}{|c|}{ Heterogeneity: $\operatorname{Tau}^{2}=0 ; \mathrm{Chi}^{2}=3.12, \mathrm{df}=1(\mathrm{P}=0.08) ; \mathrm{I}^{2}=67.93 \%$} \\
\hline \multicolumn{8}{|c|}{ Test for overall effect: $Z=0.98(P=0.33)$} \\
\hline & & & Favours & astic wrap] & -2 & Favours & ess] \\
\hline
\end{tabular}

Analysis 14.2. Comparison 14 Thermal mattress versus plastic wrap or bag, Outcome 2 Hypothermia on admission to NICU (core body temperature $<36.5^{\circ} \mathrm{C}$ or skin temperature $<36^{\circ} \mathrm{C}$ ).

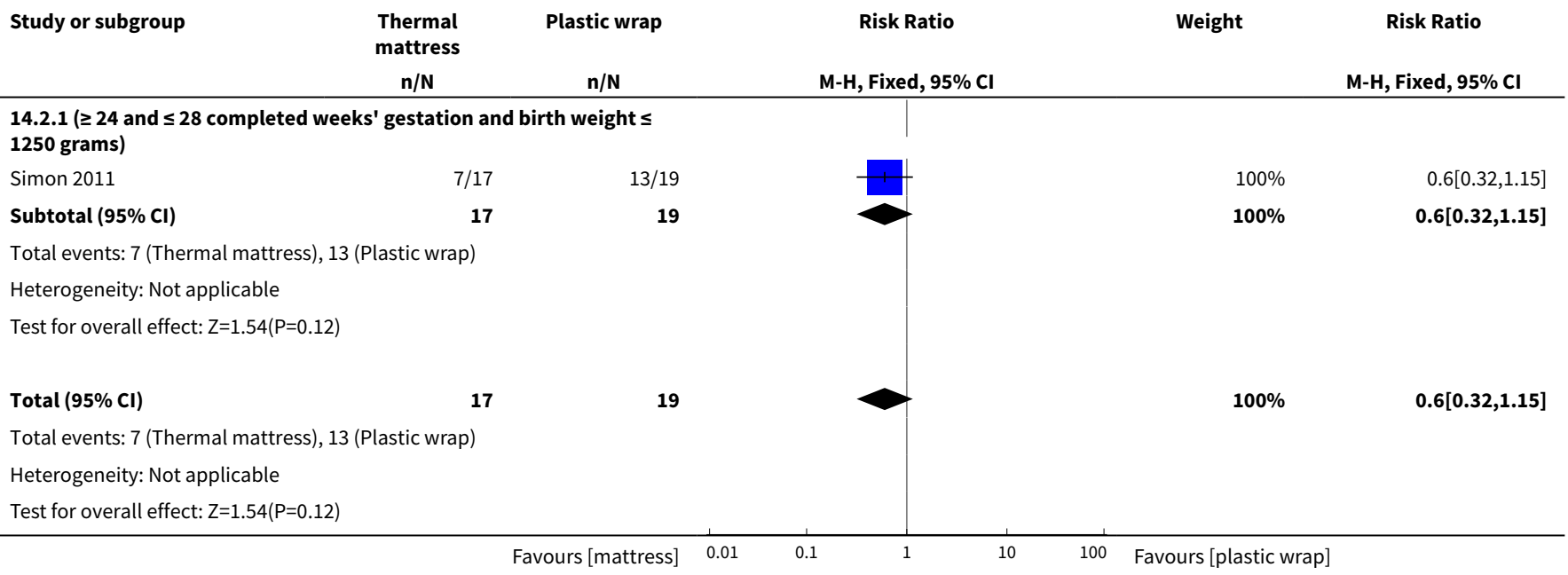

\section{Analysis 14.3. Comparison 14 Thermal mattress versus plastic wrap or bag, Outcome 3 Outside normothermic range on admission to NICU or up to 2 hours after birth.}

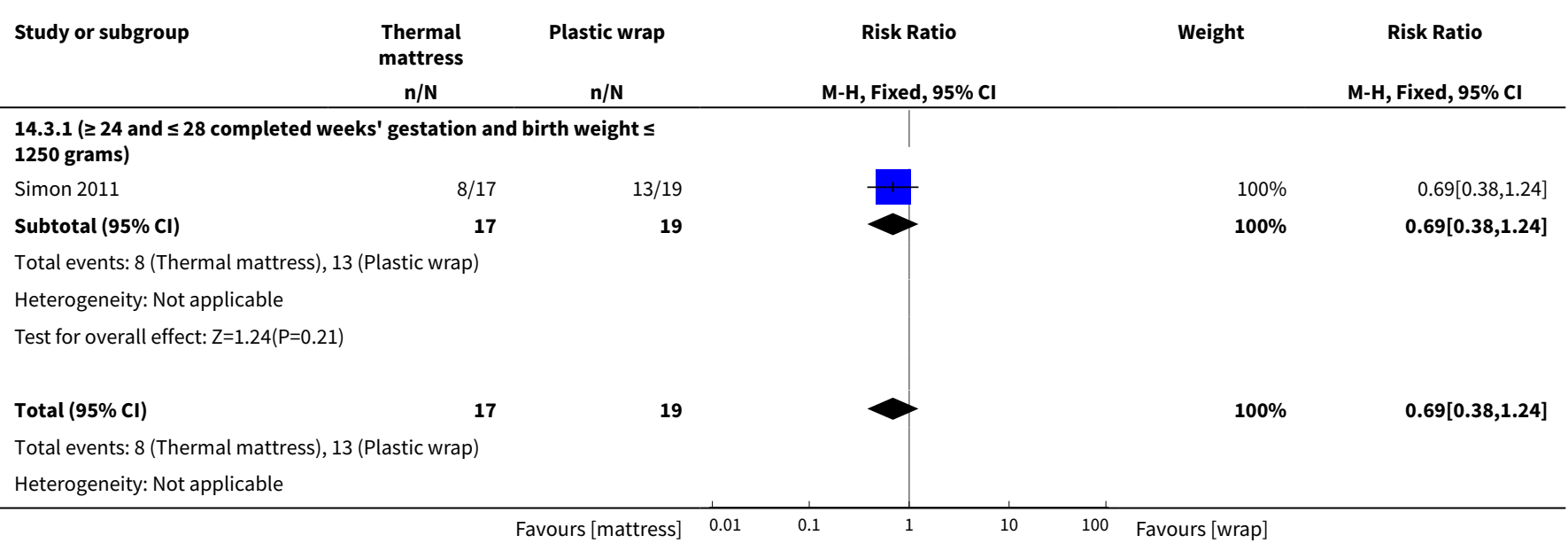




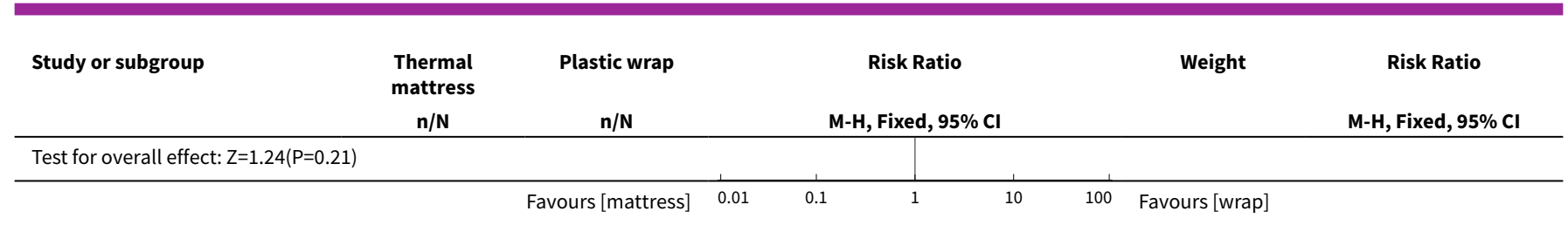

Analysis 14.4. Comparison 14 Thermal mattress versus plastic wrap or bag, Outcome 4 Hyperthermia on admission to NICU: core body temperature $>37.5^{\circ} \mathrm{C}$.

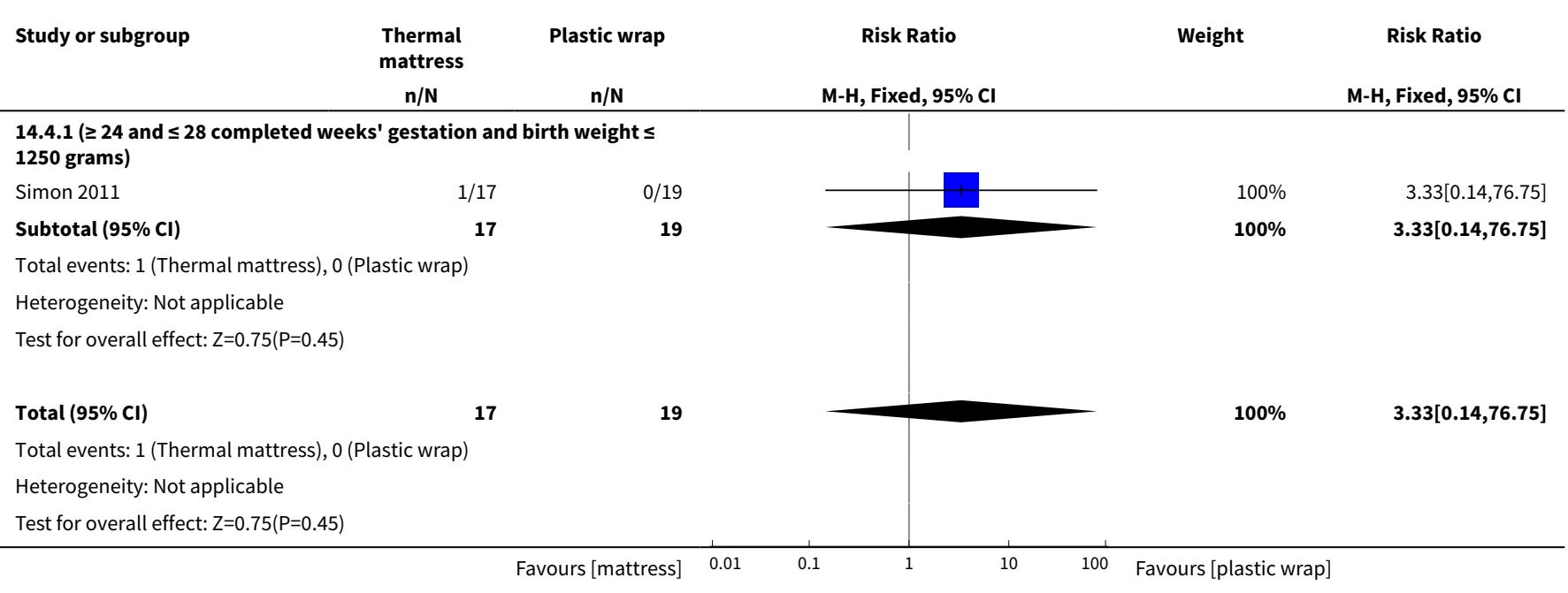

\section{Analysis 14.5. Comparison 14 Thermal mattress versus plastic wrap or bag, Outcome 5 Brain injury (IVH grade I or II ).}

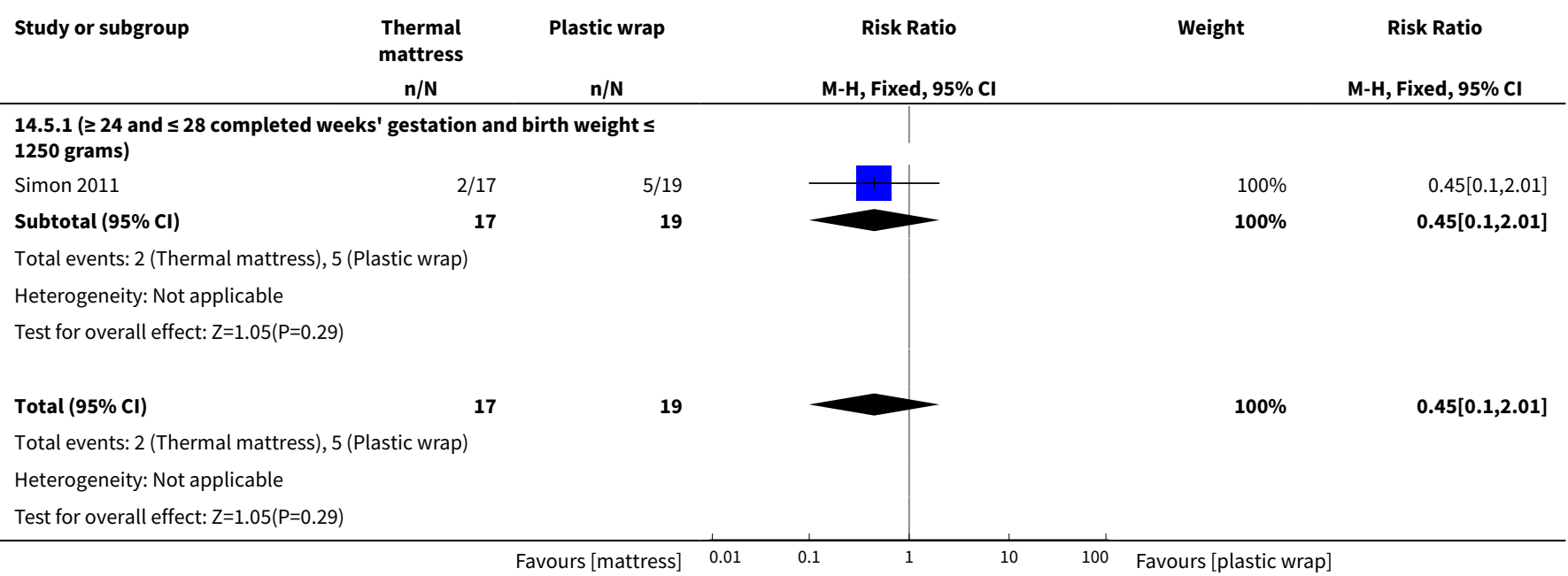


Analysis 14.6. Comparison 14 Thermal mattress versus plastic wrap or bag, Outcome 6 Major brain injury (IVH grade III or IV).

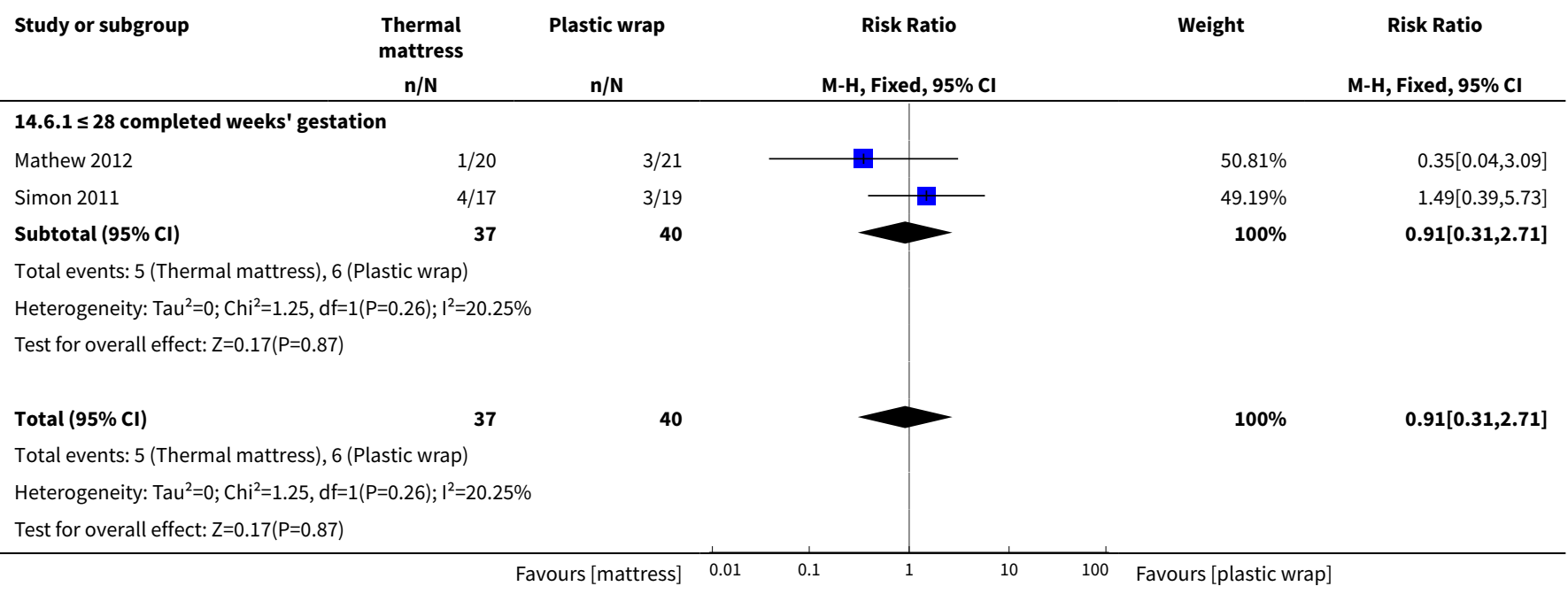

\section{Analysis 14.7. Comparison 14 Thermal mattress versus plastic wrap or bag, Outcome 7 Mortality (death before discharge).}

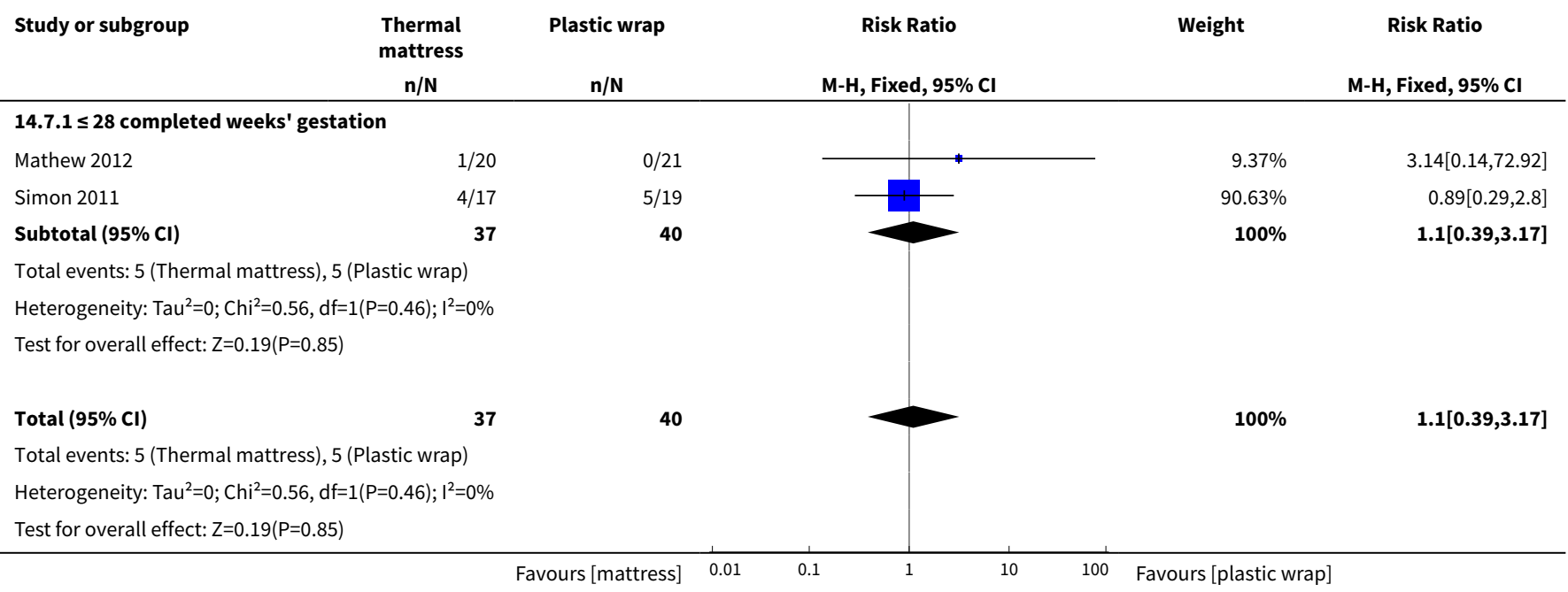

\section{Analysis 14.8. Comparison 14 Thermal mattress versus plastic wrap or bag, Outcome 8 Bronchopulmonary dysplasia (BPD).}

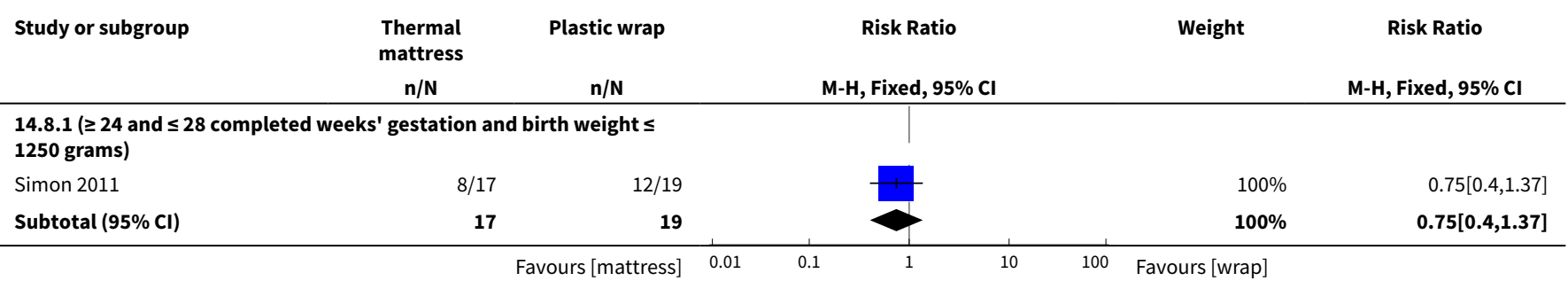




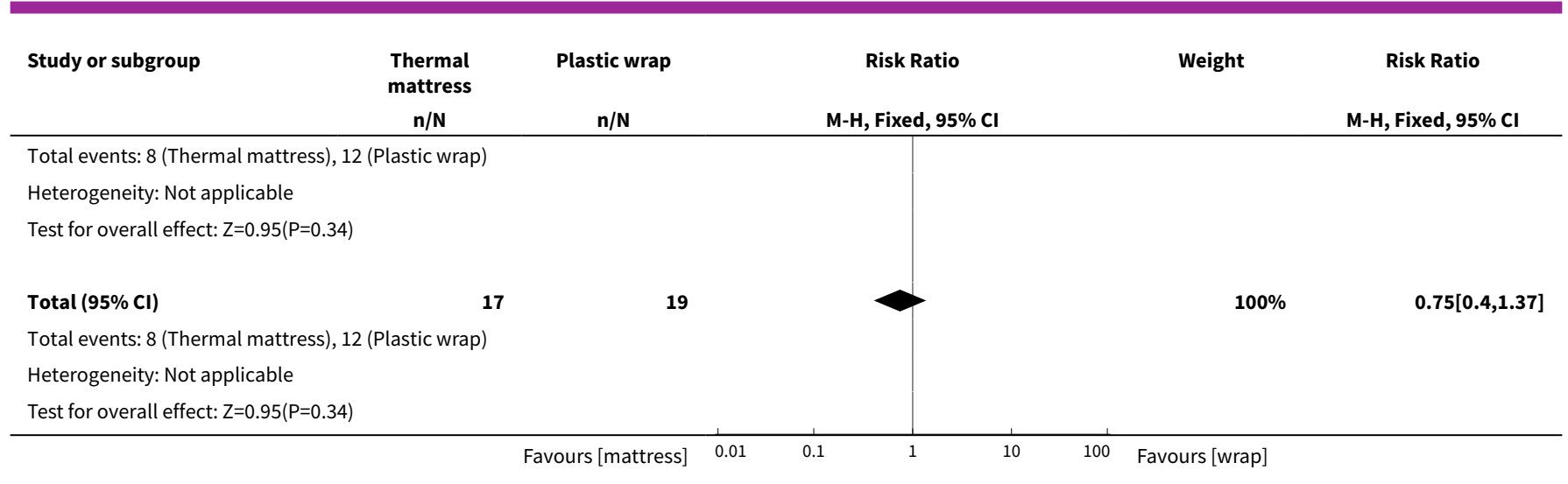

Analysis 14.9. Comparison 14 Thermal mattress versus plastic wrap or bag, Outcome 9 Hypotension during first 24 hours of life.

\begin{tabular}{|c|c|c|c|c|c|}
\hline Study or subgroup & $\begin{array}{c}\text { Thermal } \\
\text { mattress } \\
\mathrm{n} / \mathrm{N} \\
\end{array}$ & $\begin{array}{l}\text { Plastic wrap } \\
n / \mathbf{N} \\
\end{array}$ & $\begin{array}{c}\text { Risk Ratio } \\
\text { M-H, Fixed, 95\% Cl }\end{array}$ & Weight & $\begin{array}{c}\text { Risk Ratio } \\
\text { M-H, Fixed, } 95 \% \mathrm{Cl} \\
\end{array}$ \\
\hline \multicolumn{6}{|c|}{ 14.9.1 $\leq 28$ completed weeks' gestation } \\
\hline Mathew 2012 & $10 / 20$ & $7 / 21$ & & $100 \%$ & $1.5[0.71,3.17]$ \\
\hline Subtotal $(95 \% \mathrm{Cl})$ & 20 & 21 & & $100 \%$ & $1.5[0.71,3.17]$ \\
\hline \multicolumn{6}{|c|}{ Total events: 10 (Thermal mattress), 7 (Plastic wrap) } \\
\hline \multicolumn{6}{|c|}{ Test for overall effect: $Z=1.06(P=0.29)$} \\
\hline Total $(95 \% \mathrm{Cl})$ & 20 & 21 & & $100 \%$ & $1.5[0.71,3.17]$ \\
\hline \multicolumn{6}{|c|}{ Total events: 10 (Thermal mattress), 7 (Plastic wrap) } \\
\hline \multicolumn{6}{|c|}{ Heterogeneity: Not applicable } \\
\hline Test for overall effect & & & & & \\
\hline
\end{tabular}

Analysis 14.10. Comparison 14 Thermal mattress versus plastic wrap or bag, Outcome 10 Necrotising enterocolitis (NEC).

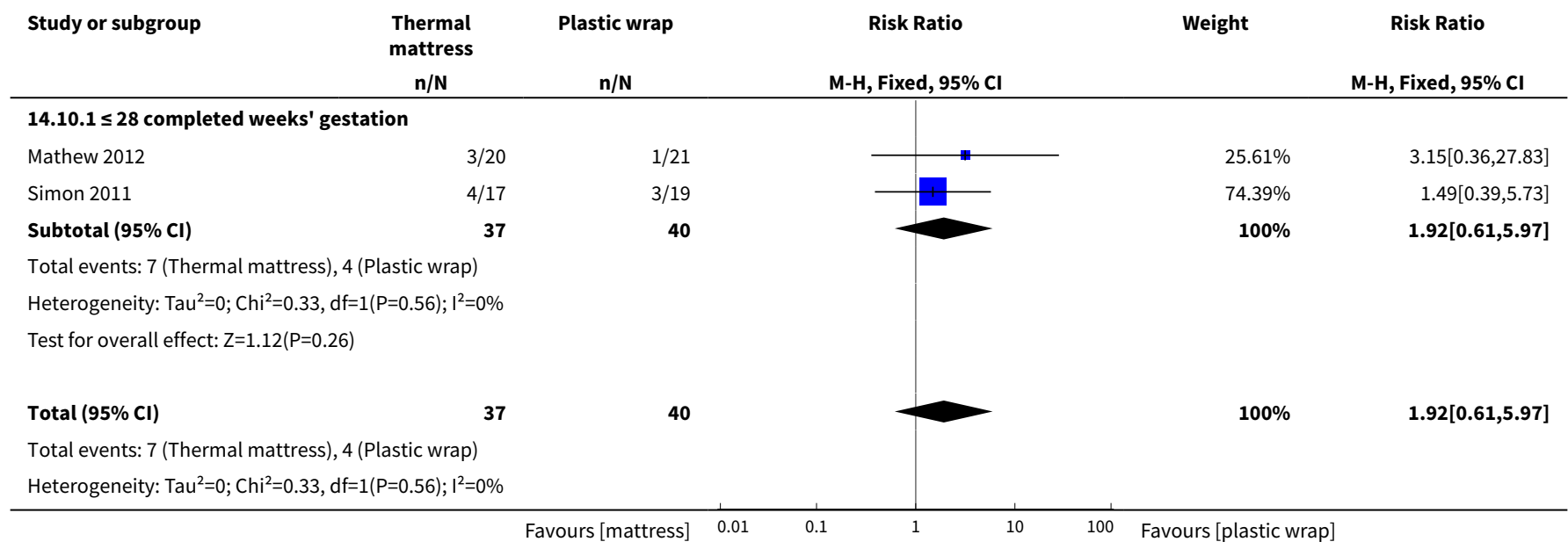




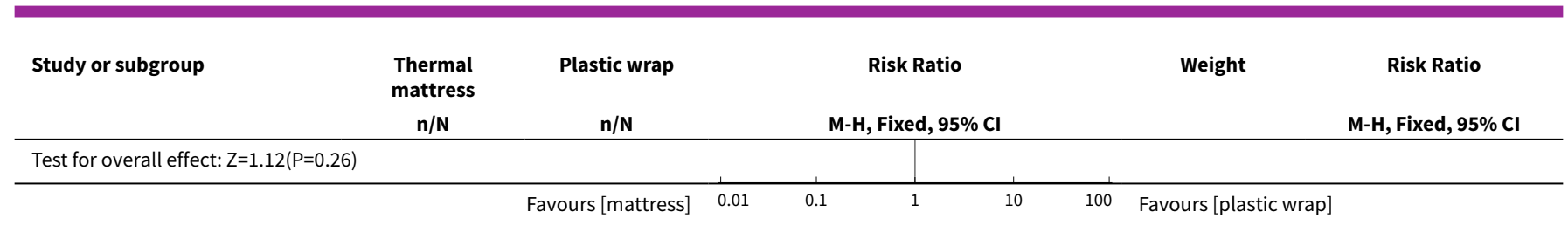

\section{Analysis 14.11. Comparison 14 Thermal mattress versus plastic wrap or bag, Outcome 11 Patent ductus arteriosus (PDA).}

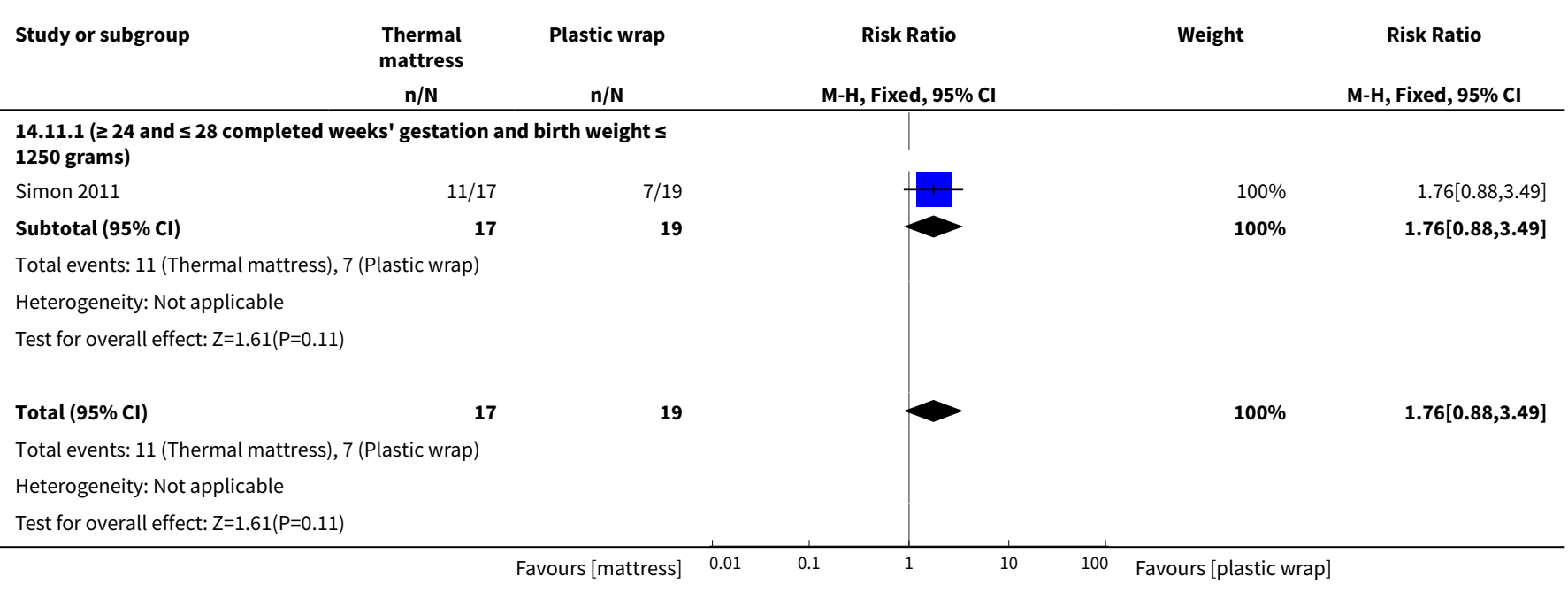

Analysis 14.12. Comparison 14 Thermal mattress versus plastic wrap or bag, Outcome 12 PDA - medication only.

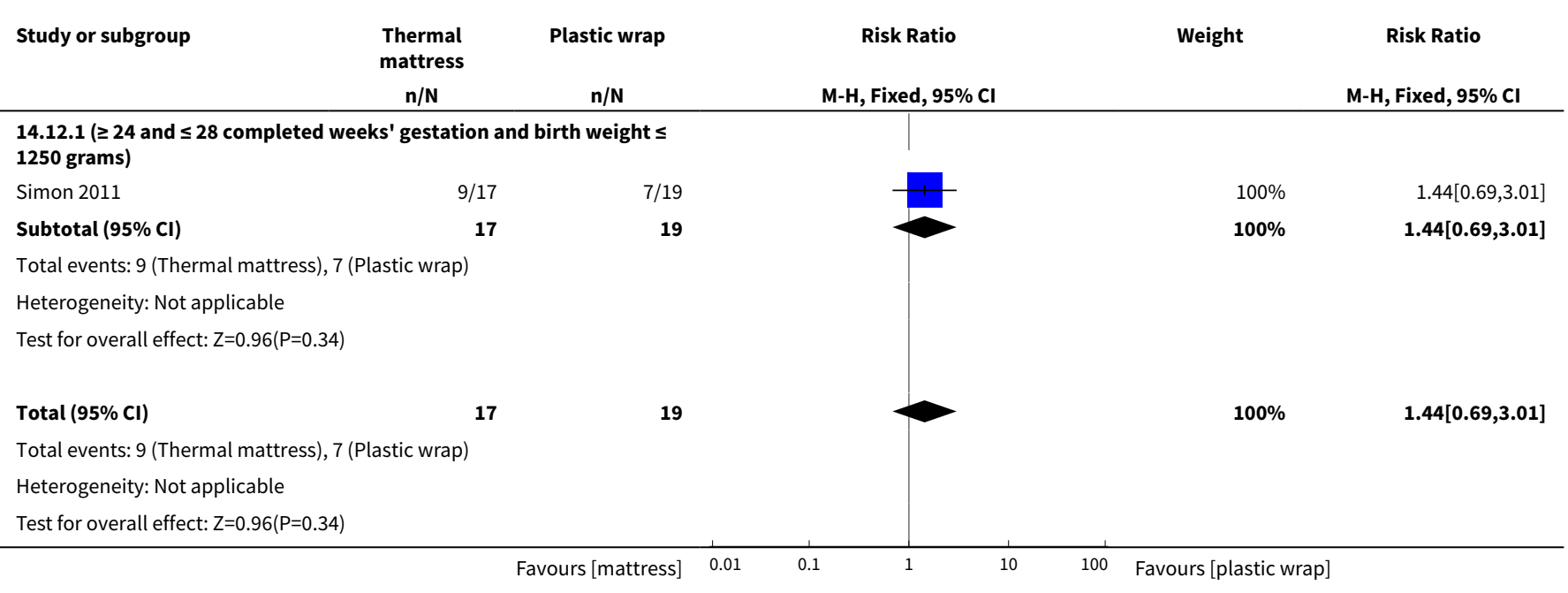


Analysis 14.13. Comparison 14 Thermal mattress versus plastic wrap or bag, Outcome 13 PDA - ligation.

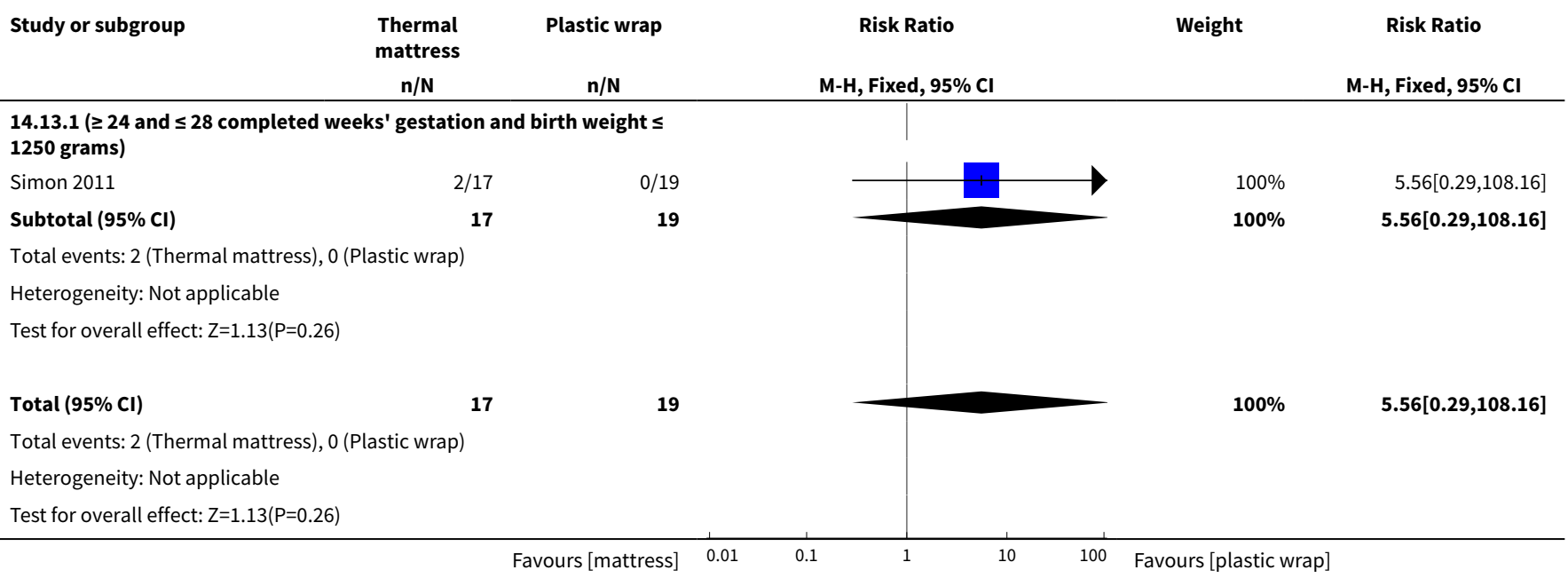

Analysis 14.14. Comparison 14 Thermal mattress versus plastic wrap or bag, Outcome 14 Retinopathy of prematurity (ROP all grades).

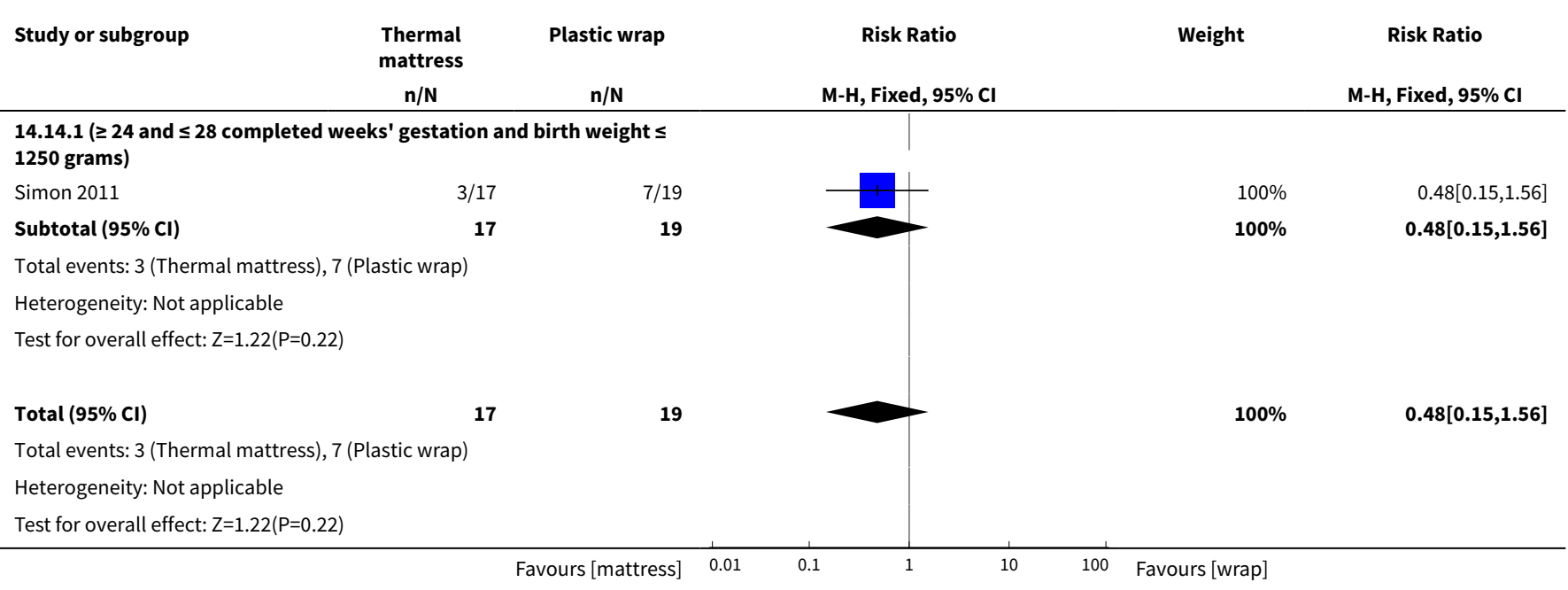

Analysis 14.15. Comparison 14 Thermal mattress versus plastic wrap or bag, Outcome 15 Retinopathy of prematurity (ROP laser therapy).

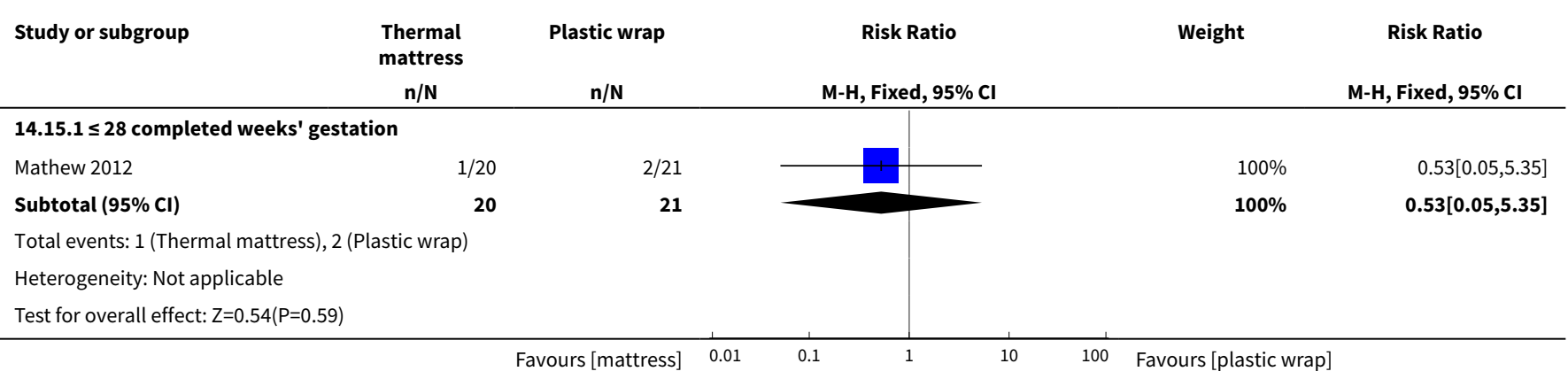




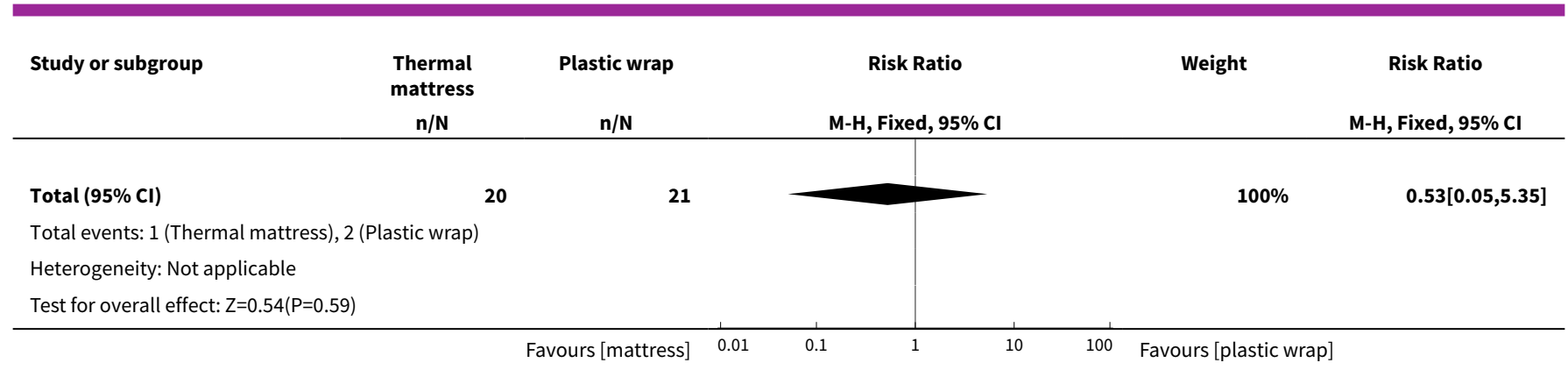

Analysis 14.16. Comparison 14 Thermal mattress versus plastic wrap or bag, Outcome 16 Spontaneous intestinal perforation (SIP).

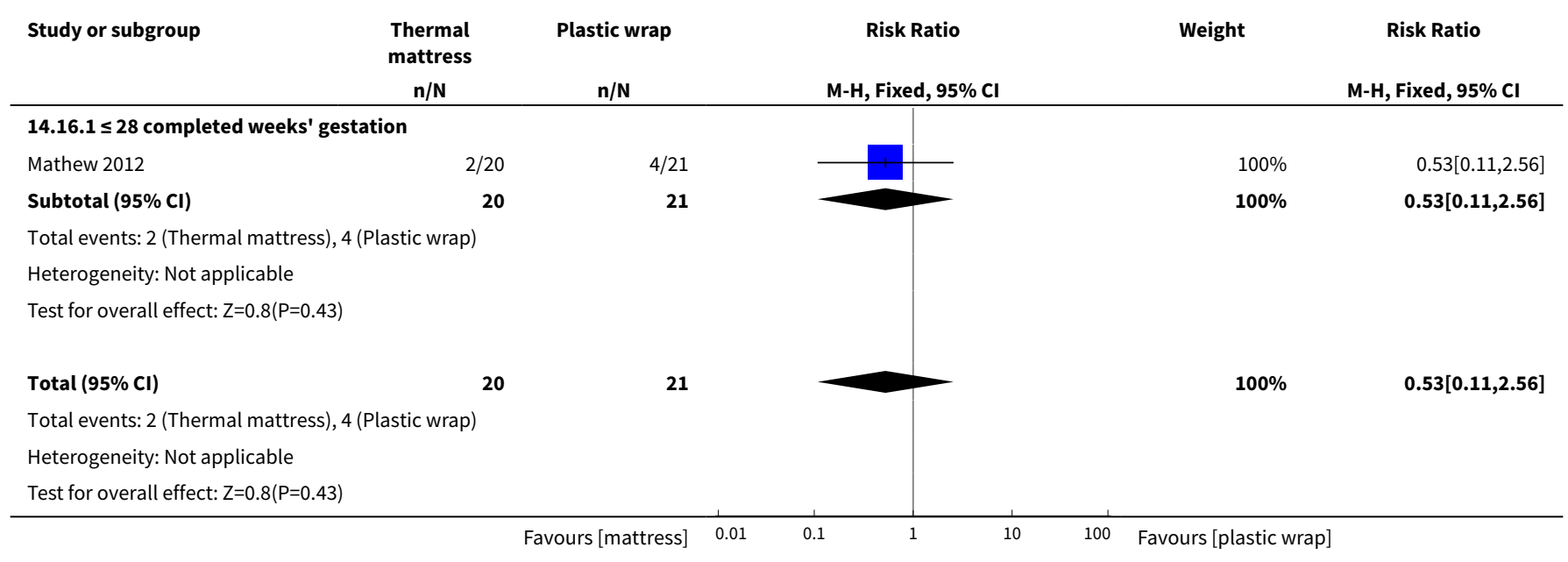

Analysis 14.17. Comparison 14 Thermal mattress versus plastic wrap or bag, Outcome 17 Worst base deficit in first 24 hours of life.

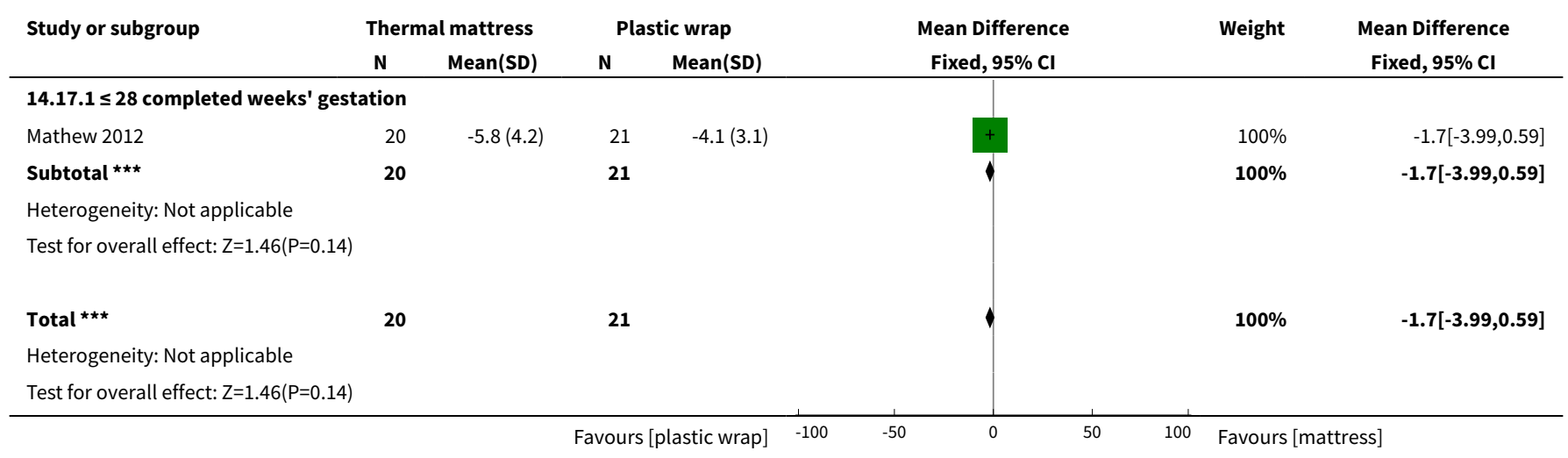




\section{Analysis 14.18. Comparison 14 Thermal mattress versus plastic} wrap or bag, Outcome 18 Worst pH in first 24 hours of life.

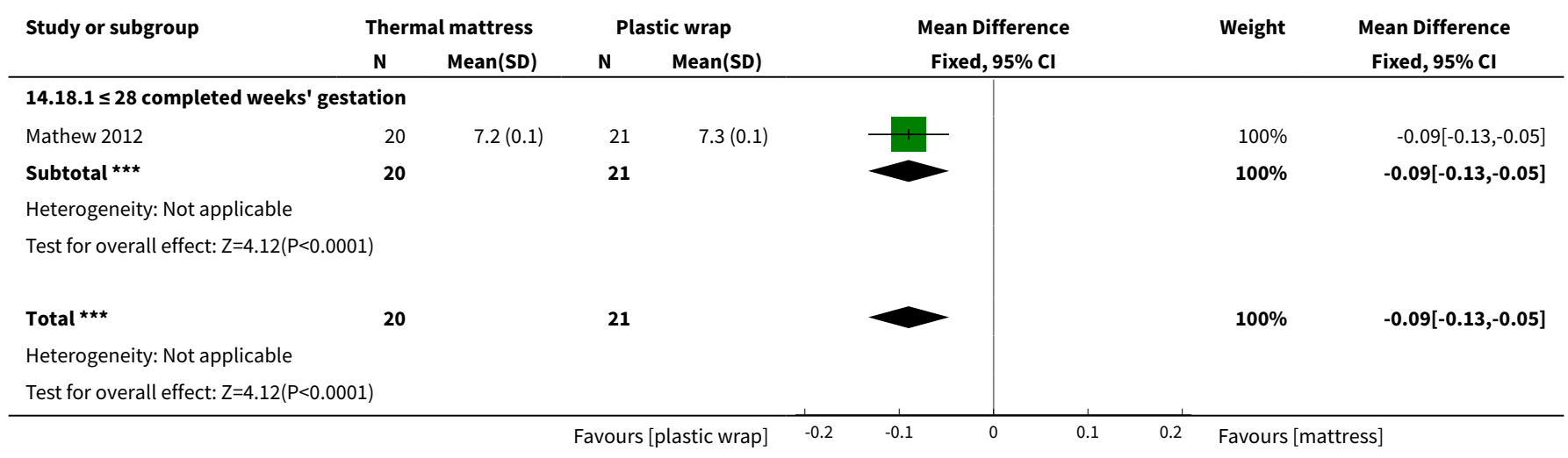

\section{Comparison 15. Plastic bag and thermal mattress versus plastic bag only}

\begin{tabular}{|c|c|c|c|c|}
\hline Outcome or subgroup title & No. of studies & $\begin{array}{l}\text { No. of partici- } \\
\text { pants }\end{array}$ & Statistical method & Effect size \\
\hline $\begin{array}{l}1 \text { Core body temperature }\left({ }^{\circ} \mathrm{C}\right) \text { on ad- } \\
\text { mission to NICU or up to } 2 \text { hours of af- } \\
\text { ter birth }\end{array}$ & 2 & & $\begin{array}{l}\text { Mean Difference (IV, Fixed, } \\
95 \% \mathrm{Cl} \text { ) }\end{array}$ & Subtotals only \\
\hline $\begin{array}{l}1.1 \text { All infants (<31 completed weeks' } \\
\text { gestation) }\end{array}$ & 2 & 119 & $\begin{array}{l}\text { Mean Difference (IV, Fixed, } \\
95 \% \mathrm{CI})\end{array}$ & $0.37[0.09,0.66]$ \\
\hline $1.2<28$ completed weeks' gestation & 2 & 76 & $\begin{array}{l}\text { Mean Difference (IV, Fixed, } \\
95 \% \mathrm{Cl})\end{array}$ & $0.57[0.20,0.94]$ \\
\hline $\begin{array}{l}1.328 \text { to } 30 \text { completed weeks' gesta- } \\
\text { tion }\end{array}$ & 1 & 43 & $\begin{array}{l}\text { Mean Difference (IV, Fixed, } \\
95 \% \mathrm{Cl} \text { ) }\end{array}$ & $0.10[-0.36,0.56]$ \\
\hline $\begin{array}{l}2 \text { Hypothermia: core body tempera- } \\
\text { ture }<36.5^{\circ} \mathrm{C} \text { on admission to NICU }\end{array}$ & 2 & & $\begin{array}{l}\text { Risk Ratio (M-H, Fixed, 95\% } \\
\mathrm{Cl})\end{array}$ & Subtotals only \\
\hline $\begin{array}{l}2.1 \text { All infants (<31 completed weeks' } \\
\text { gestation) }\end{array}$ & 2 & 119 & $\begin{array}{l}\text { Risk Ratio (M-H, Fixed, 95\% } \\
\mathrm{Cl})\end{array}$ & $0.93[0.45,1.90]$ \\
\hline $2.2<28$ completed weeks' gestation & 2 & 76 & $\begin{array}{l}\text { Risk Ratio (M-H, Fixed, 95\% } \\
\mathrm{Cl})\end{array}$ & $0.66[0.29,1.46]$ \\
\hline $\begin{array}{l}2.328 \text { to } 30 \text { completed weeks' gesta- } \\
\text { tion }\end{array}$ & 1 & 43 & $\begin{array}{l}\text { Risk Ratio (M-H, Fixed, 95\% } \\
\mathrm{Cl})\end{array}$ & $3.82[0.46,31.43]$ \\
\hline $\begin{array}{l}3 \text { Outside normothermic range on ad- } \\
\text { mission to NICU or up to } 2 \text { hours after } \\
\text { birth }\end{array}$ & 2 & & $\begin{array}{l}\text { Risk Ratio (M-H, Fixed, 95\% } \\
\mathrm{Cl})\end{array}$ & Subtotals only \\
\hline $\begin{array}{l}\text { 3.1 All infants (< } 31 \text { completed weeks' } \\
\text { gestation) }\end{array}$ & 2 & 119 & $\begin{array}{l}\text { Risk Ratio (M-H, Fixed, 95\% } \\
\mathrm{Cl})\end{array}$ & $1.46[0.94,2.27]$ \\
\hline $3.2<28$ completed weeks' gestation & 2 & 76 & $\begin{array}{l}\text { Risk Ratio (M-H, Fixed, 95\% } \\
\mathrm{Cl})\end{array}$ & $1.23[0.69,2.18]$ \\
\hline
\end{tabular}




\begin{tabular}{|c|c|c|c|c|}
\hline Outcome or subgroup title & No. of studies & $\begin{array}{l}\text { No. of partici- } \\
\text { pants }\end{array}$ & Statistical method & Effect size \\
\hline 3.328 to 30 weeks' gestation & 1 & 43 & $\begin{array}{l}\text { Risk Ratio (M-H, Fixed, 95\% } \\
\mathrm{Cl} \text { ) }\end{array}$ & $1.91[0.96,3.78]$ \\
\hline $\begin{array}{l}4 \text { Hyperthermia on admission to } \\
\text { NICU: core body temperature }>37.5^{\circ} \mathrm{C}\end{array}$ & 2 & & $\begin{array}{l}\text { Risk Ratio (M-H, Fixed, 95\% } \\
\mathrm{Cl})\end{array}$ & Subtotals only \\
\hline $\begin{array}{l}4.1 \text { All infants (<31 completed weeks' } \\
\text { gestation) }\end{array}$ & 2 & 119 & $\begin{array}{l}\text { Risk Ratio (M-H, Fixed, 95\% } \\
\mathrm{Cl})\end{array}$ & $2.15[1.07,4.32]$ \\
\hline $4.2<28$ completed weeks' gestation & 2 & 76 & $\begin{array}{l}\text { Risk Ratio (M-H, Fixed, 95\% } \\
\mathrm{Cl})\end{array}$ & $2.99[0.93,9.65]$ \\
\hline $\begin{array}{l}4.328 \text { to } 30 \text { completed weeks' gesta- } \\
\text { tion }\end{array}$ & 1 & 43 & $\begin{array}{l}\text { Risk Ratio (M-H, Fixed, 95\% } \\
\text { Cl) }\end{array}$ & $1.59[0.70,3.60]$ \\
\hline 5 Major brain injury & 1 & 72 & $\begin{array}{l}\text { Risk Ratio (M-H, Fixed, 95\% } \\
\mathrm{Cl})\end{array}$ & $0.32[0.03,2.89]$ \\
\hline $5.1<31$ completed weeks' gestation & 1 & 72 & $\begin{array}{l}\text { Risk Ratio (M-H, Fixed, 95\% } \\
\mathrm{Cl})\end{array}$ & $0.32[0.03,2.89]$ \\
\hline $\begin{array}{l}6 \text { Mortality (death before hospital dis- } \\
\text { charge) }\end{array}$ & 2 & 119 & $\begin{array}{l}\text { Risk Ratio (M-H, Fixed, 95\% } \\
\mathrm{Cl})\end{array}$ & $0.73[0.32,1.66]$ \\
\hline $6.1<31$ completed weeks' gestation & 2 & 119 & $\begin{array}{l}\text { Risk Ratio (M-H, Fixed, 95\% } \\
\mathrm{Cl})\end{array}$ & $0.73[0.32,1.66]$ \\
\hline 7 Chronic lung disease & 1 & 72 & $\begin{array}{l}\text { Risk Ratio (M-H, Fixed, 95\% } \\
\mathrm{Cl})\end{array}$ & $1.73[0.72,4.18]$ \\
\hline $7.1<31$ completed weeks' gestation & 1 & 72 & $\begin{array}{l}\text { Risk Ratio (M-H, Fixed, 95\% } \\
\text { Cl) }\end{array}$ & $1.73[0.72,4.18]$ \\
\hline 8 Coagulation support & 1 & 47 & $\begin{array}{l}\text { Risk Ratio (M-H, Fixed, 95\% } \\
\text { Cl) }\end{array}$ & $1.04[0.30,3.69]$ \\
\hline $8.1<29$ completed weeks' gestation & 1 & 47 & $\begin{array}{l}\text { Risk Ratio (M-H, Fixed, 95\% } \\
\mathrm{Cl})\end{array}$ & $1.04[0.30,3.69]$ \\
\hline 9 Inotrope use & 2 & 119 & $\begin{array}{l}\text { Risk Ratio (M-H, Fixed, 95\% } \\
\mathrm{Cl})\end{array}$ & $1.14[0.61,2.11]$ \\
\hline $9.1<31$ completed weeks' gestation & 2 & 119 & $\begin{array}{l}\text { Risk Ratio (M-H, Fixed, 95\% } \\
\mathrm{Cl})\end{array}$ & $1.14[0.61,2.11]$ \\
\hline 10 Intubated during admission & 1 & 72 & $\begin{array}{l}\text { Risk Ratio (M-H, Fixed, 95\% } \\
\mathrm{Cl} \text { ) }\end{array}$ & $0.98[0.73,1.32]$ \\
\hline $10.1<31$ completed weeks' gestation & 1 & 72 & $\begin{array}{l}\text { Risk Ratio (M-H, Fixed, 95\% } \\
\text { Cl) }\end{array}$ & $0.98[0.73,1.32]$ \\
\hline $\begin{array}{l}11 \geq 1 \text { dose surfactant during admis- } \\
\text { sion }\end{array}$ & 1 & 72 & $\begin{array}{l}\text { Risk Ratio (M-H, Fixed, 95\% } \\
\text { Cl) }\end{array}$ & $0.99[0.70,1.38]$ \\
\hline
\end{tabular}




\begin{tabular}{lllll}
\hline Outcome or subgroup title & No. of studies & $\begin{array}{l}\text { No. of partici- } \\
\text { pants }\end{array}$ & Statistical method & Effect size \\
\hline $11.1<31$ completed weeks' gestation & 1 & 72 & $\begin{array}{l}\text { Risk Ratio (M-H, Fixed, 95\% } \\
\text { Cl) }\end{array}$ & $0.99[0.70,1.38]$ \\
\hline
\end{tabular}

Analysis 15.1. Comparison 15 Plastic bag and thermal mattress versus plastic bag only, Outcome 1 Core body temperature $\left({ }^{\circ} \mathrm{C}\right)$ on admission to NICU or up to 2 hours of after birth.

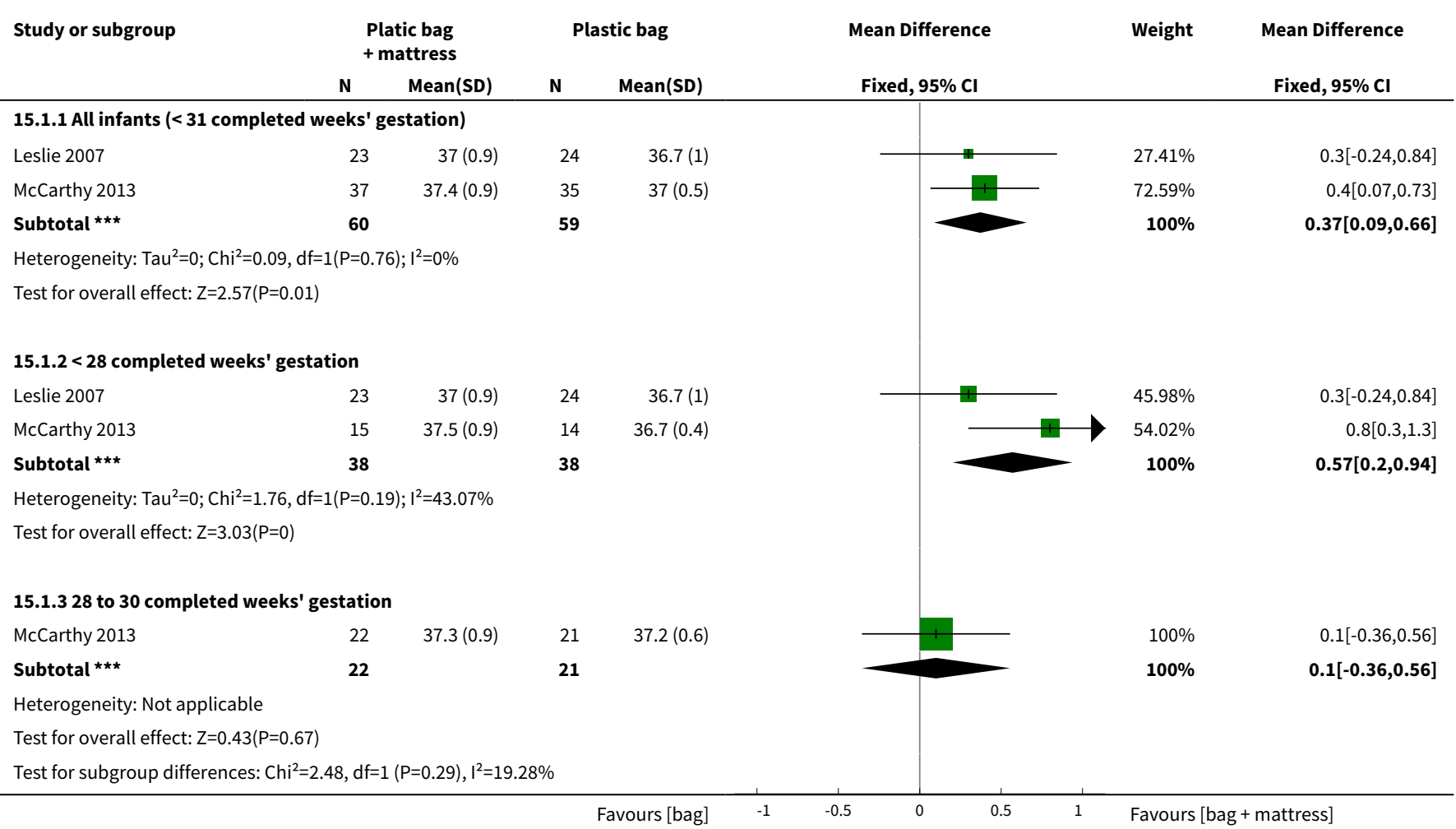

Analysis 15.2. Comparison 15 Plastic bag and thermal mattress versus plastic bag only, Outcome 2 Hypothermia: core body temperature $<36.5^{\circ} \mathrm{C}$ on admission to NICU.

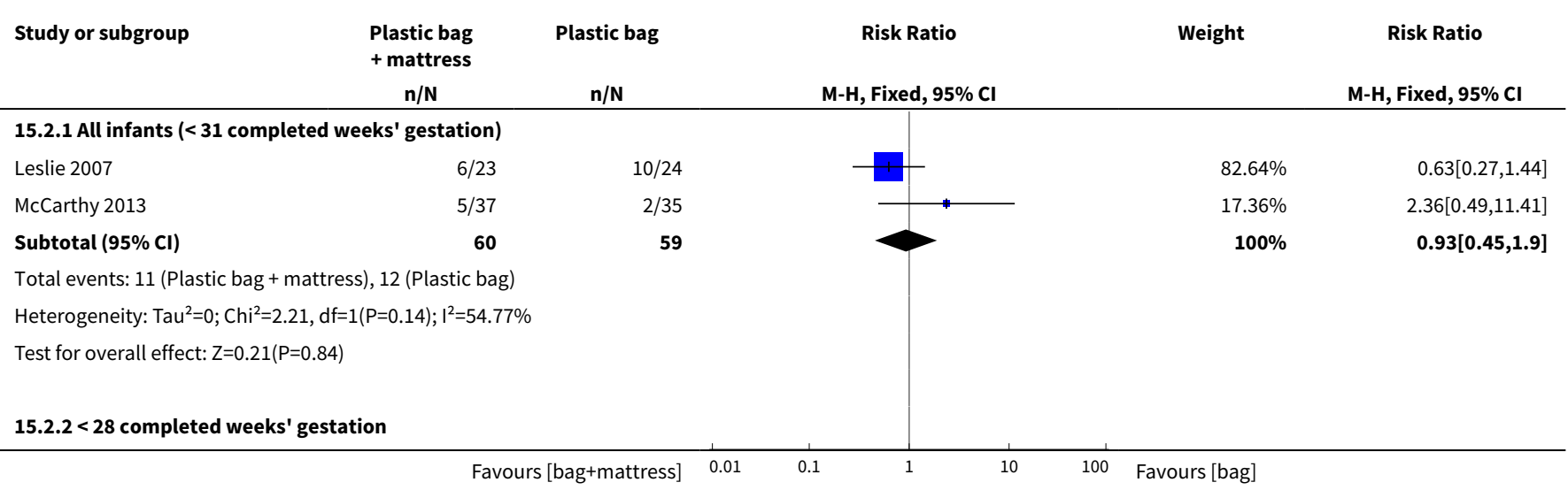




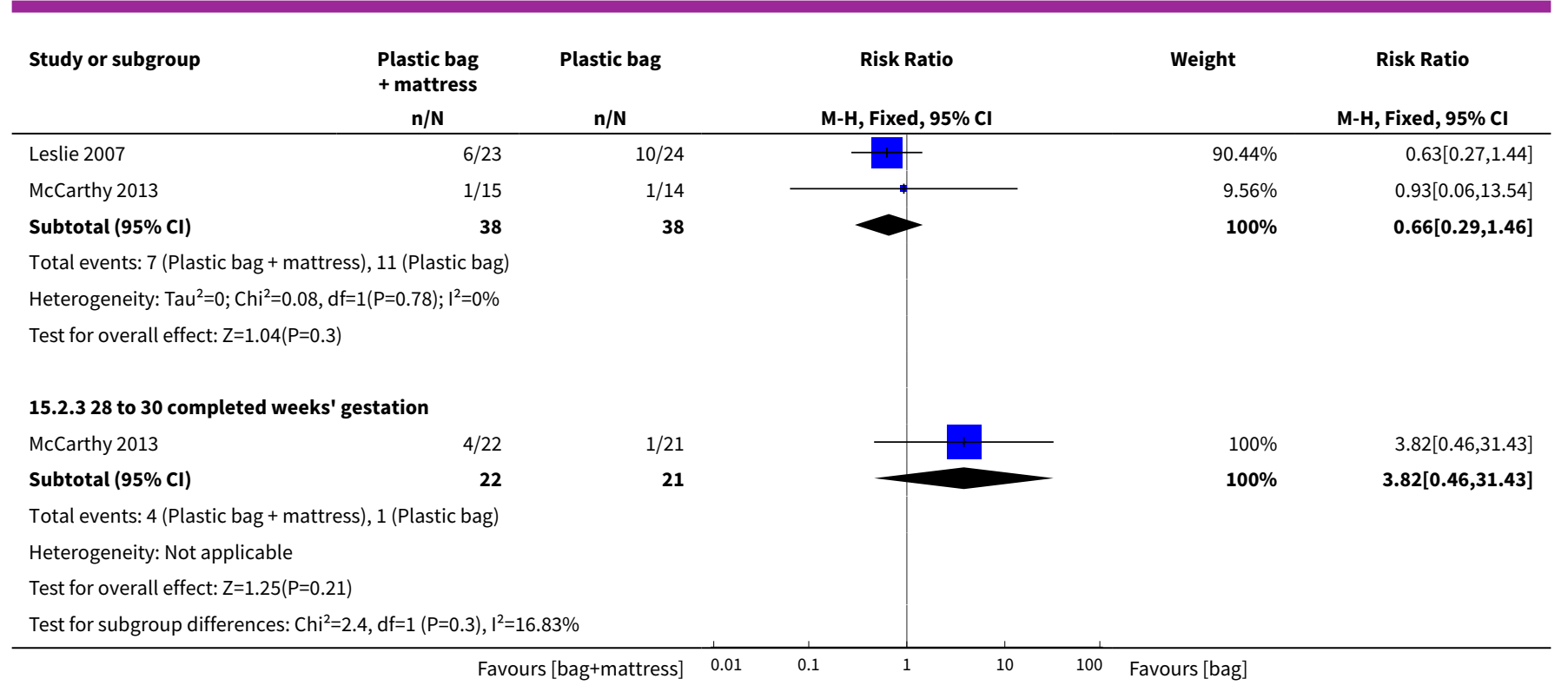

Analysis 15.3. Comparison 15 Plastic bag and thermal mattress versus plastic bag only, Outcome 3 Outside normothermic range on admission to NICU or up to 2 hours after birth.

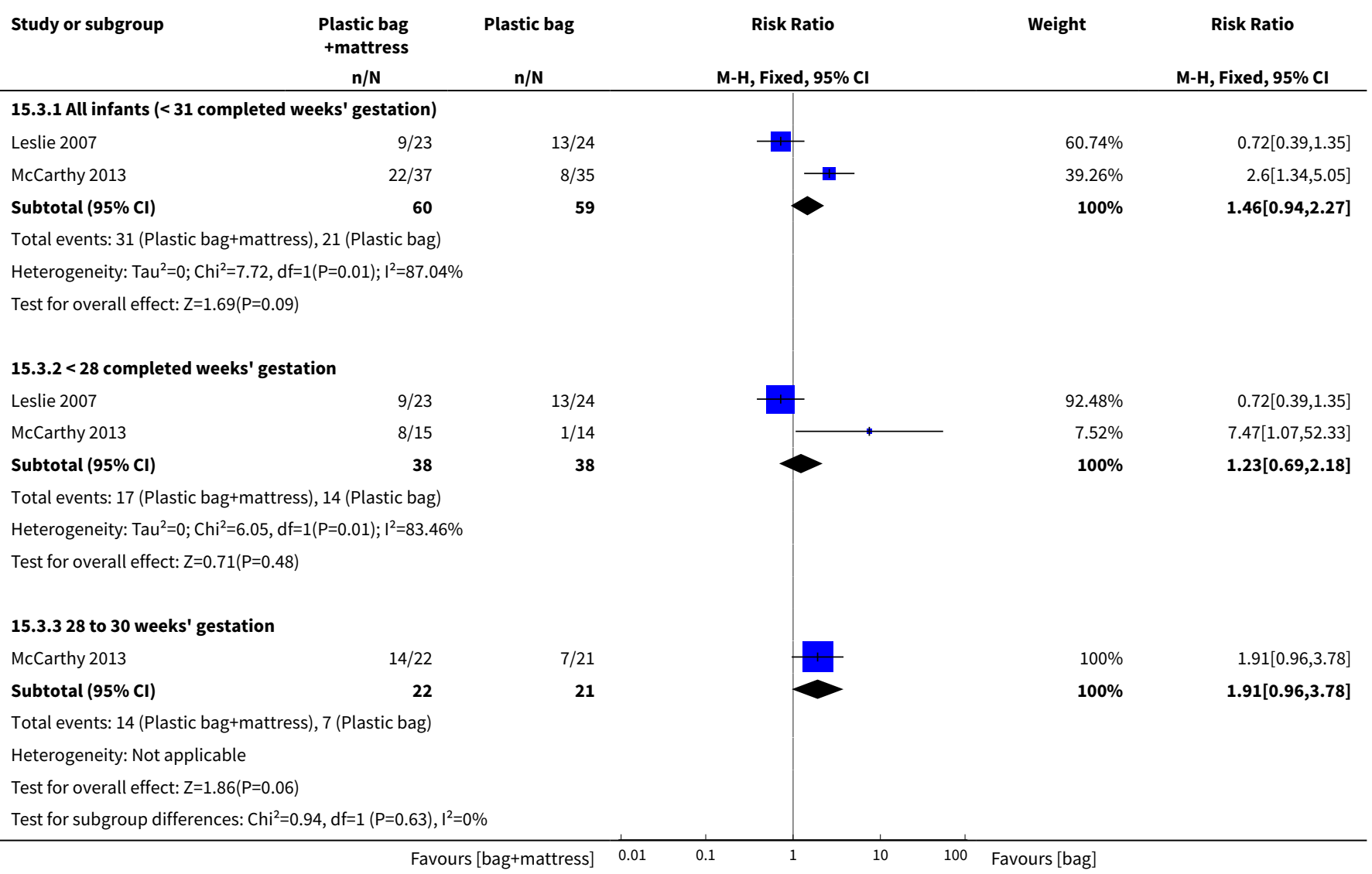


Analysis 15.4. Comparison 15 Plastic bag and thermal mattress versus plastic bag only, Outcome 4 Hyperthermia on admission to NICU: core body temperature $>37.5^{\circ} \mathrm{C}$.

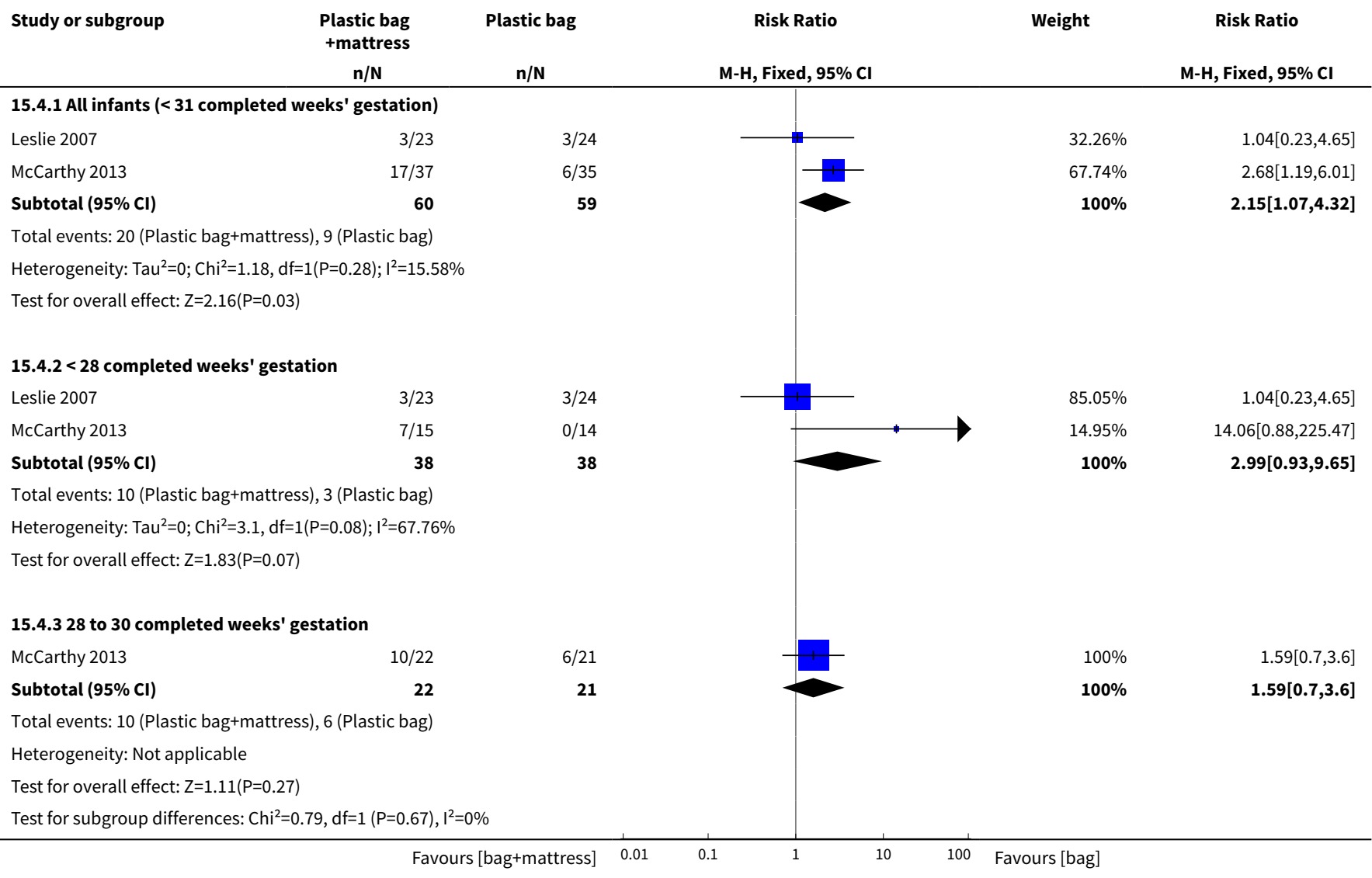

Analysis 15.5. Comparison 15 Plastic bag and thermal mattress versus plastic bag only, Outcome 5 Major brain injury.

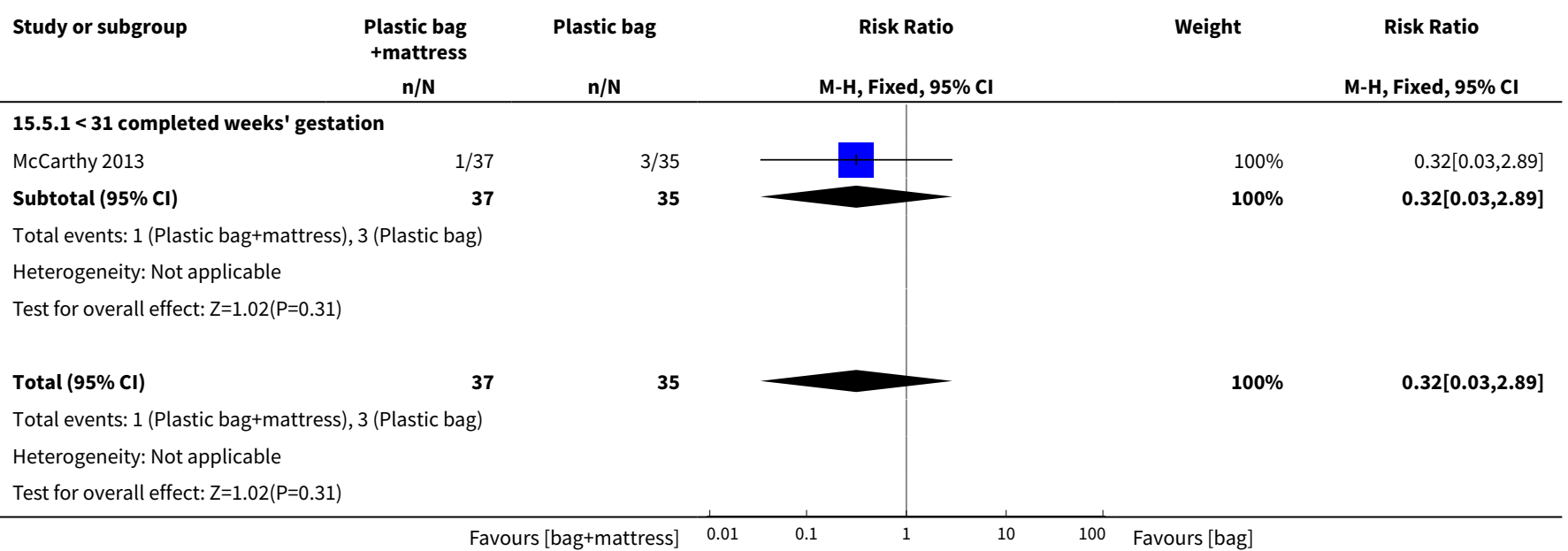


Analysis 15.6. Comparison 15 Plastic bag and thermal mattress versus plastic bag only, Outcome 6 Mortality (death before hospital discharge).

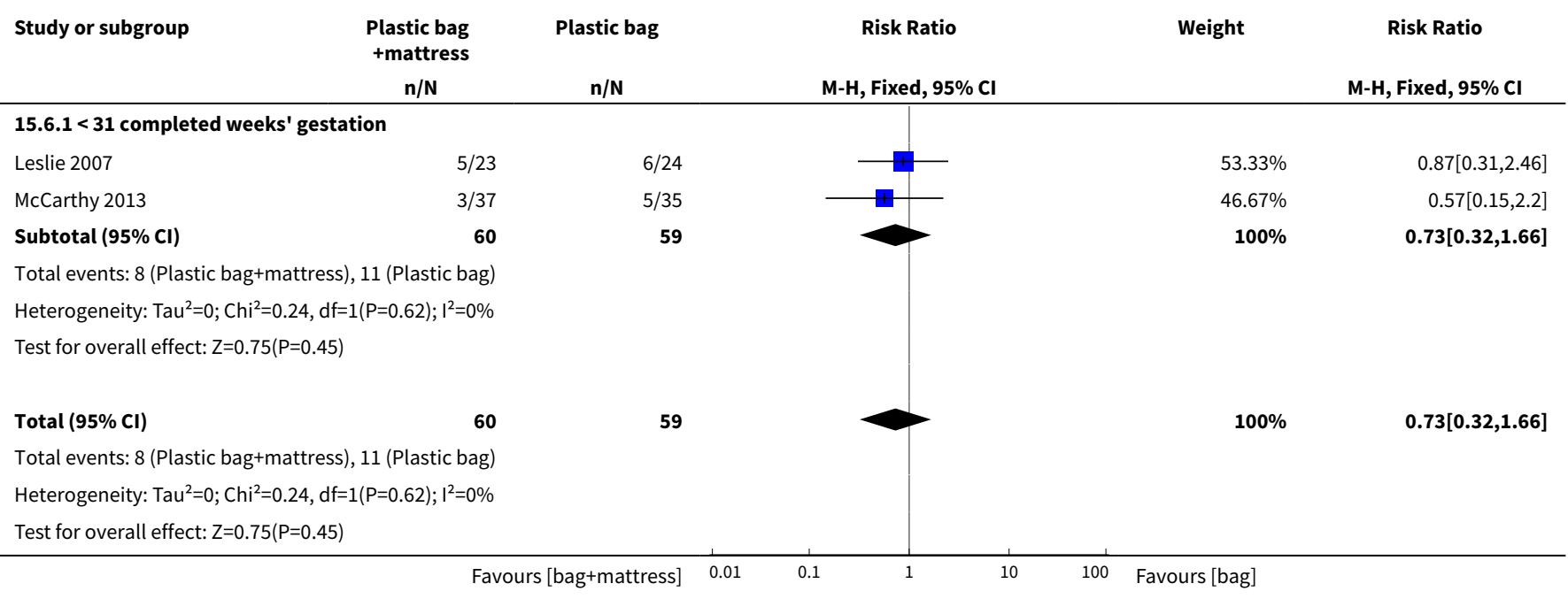

Analysis 15.7. Comparison 15 Plastic bag and thermal mattress versus plastic bag only, Outcome 7 Chronic lung disease.

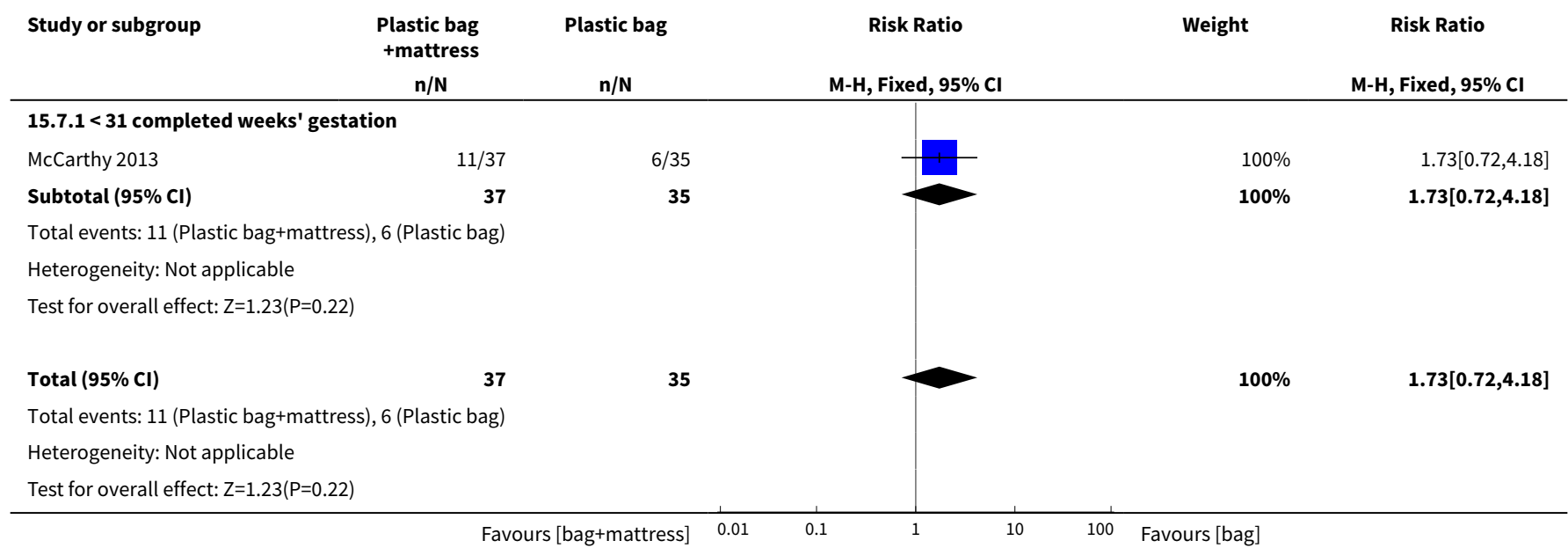

Analysis 15.8. Comparison 15 Plastic bag and thermal mattress versus plastic bag only, Outcome 8 Coagulation support.

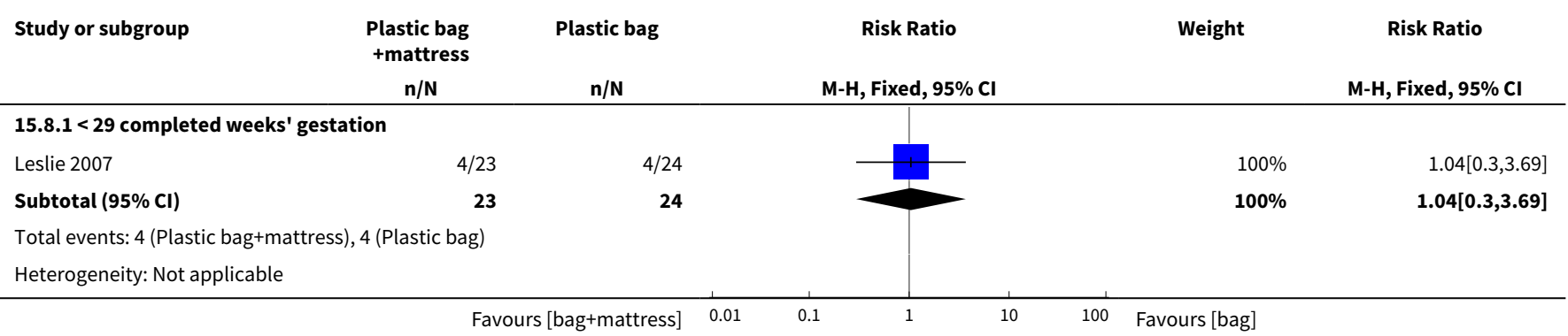




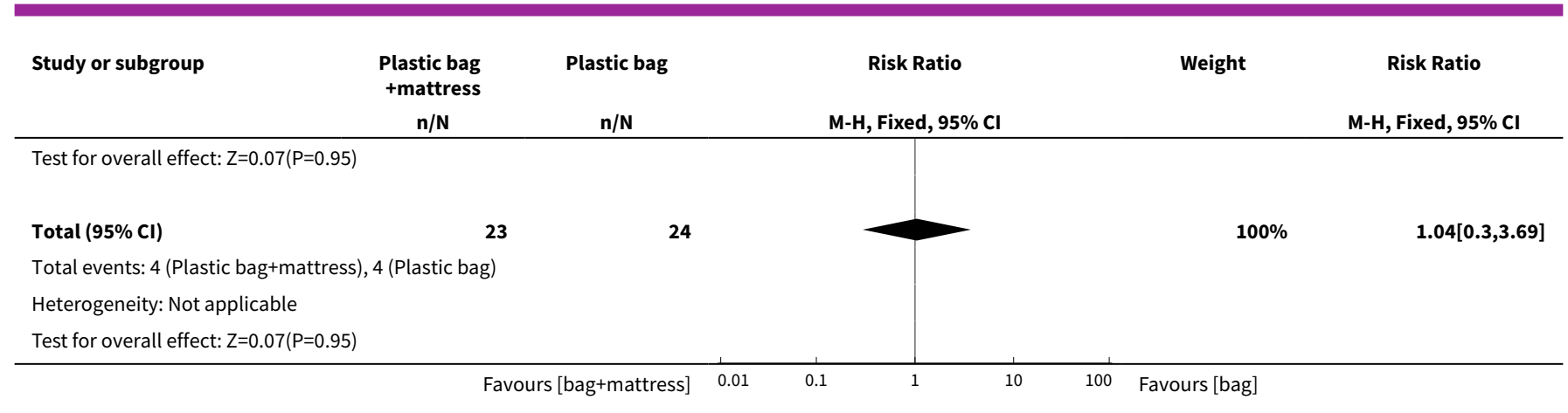

Analysis 15.9. Comparison 15 Plastic bag and thermal mattress versus plastic bag only, Outcome 9 Inotrope use.

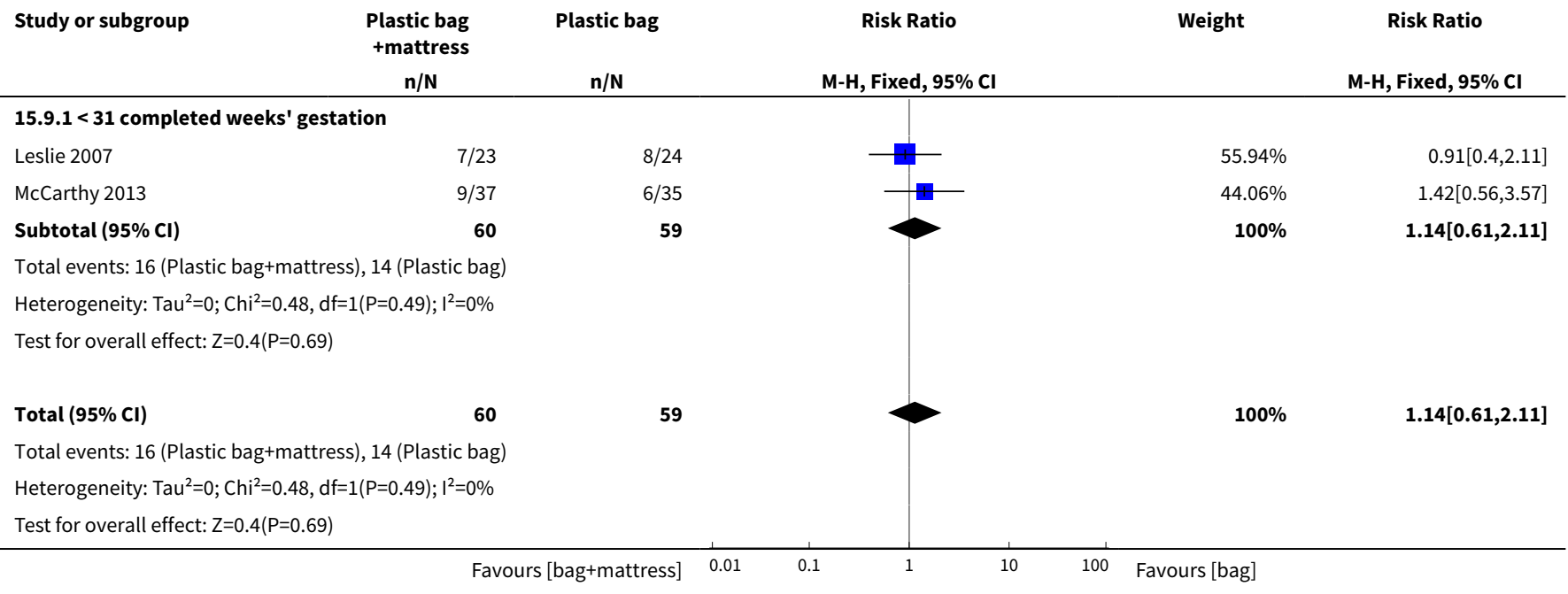

Analysis 15.10. Comparison 15 Plastic bag and thermal mattress versus plastic bag only, Outcome 10 Intubated during admission.

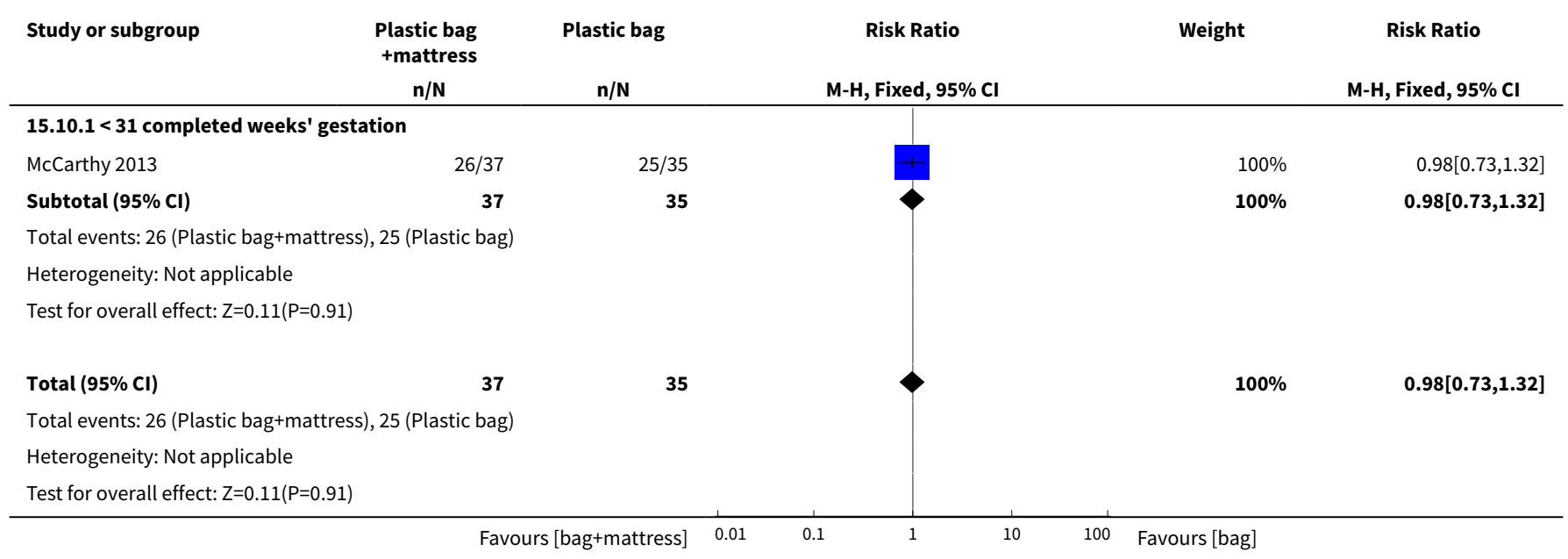




\section{Analysis 15.11. Comparison 15 Plastic bag and thermal mattress versus} plastic bag only, Outcome $11 \geq 1$ dose surfactant during admission.

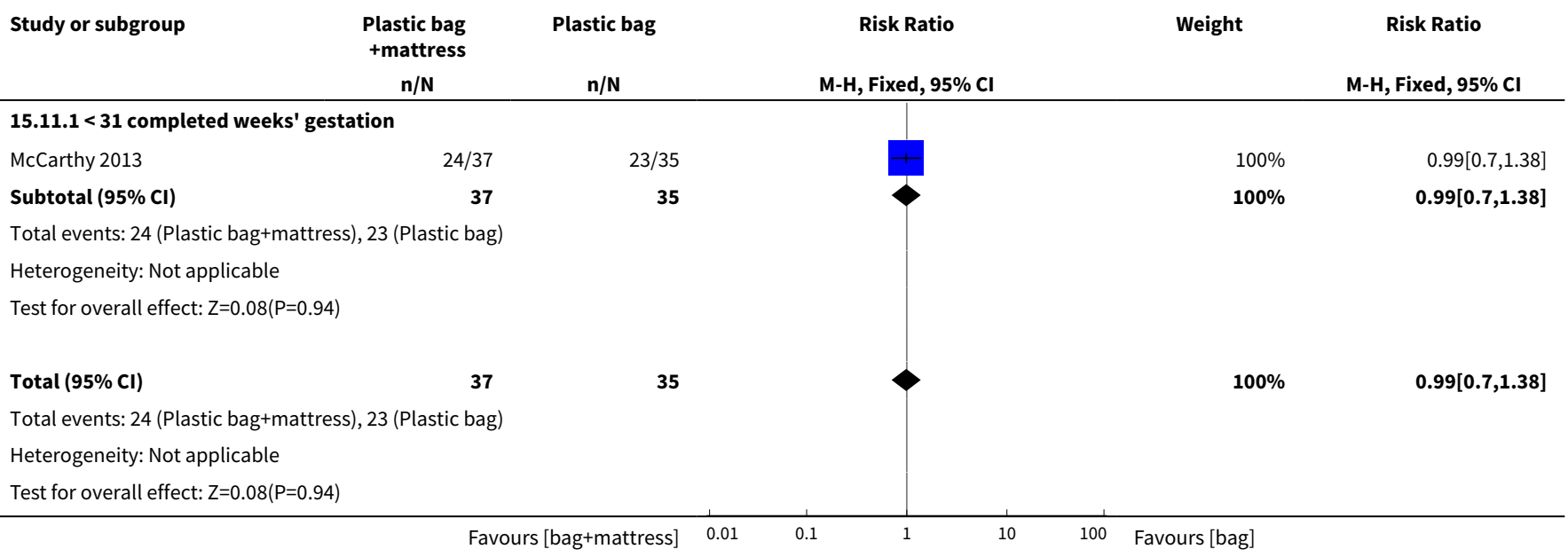

\section{APPENDICES}

\section{Appendix 1. MEDLINE search strategy}

1. plastic barrier*.ab,ti.

2. polyethylene ${ }^{\star}$.ab,ti.

3. (bubble wrap* or bubble-wrap* or bubblewrap*).ab,ti.

4. (clingfilm* or cling film* or cling-film*).ab,ti.

5. (plasticwrap* or plastic-wrap* or plastic wrap $\left.{ }^{\star}\right) \cdot a b, t i$.

6. exp Polyethylenes/

7. exp Polyvinyls/

8. (polyvinyl ${ }^{\star}$ or poly-vinyl $\left.{ }^{\star}\right) \cdot$ ab,ti.

9. (low density polyethylene* or low-density polyethylene*).ab,ti.

10. (gladwrap* or glad wrap* or glad-wrap*).ab,ti.

11. (polybag* or poly bag* or poly-bag*).ab,ti.

12. (saranwrap* or saran-wrap* or saran wrap*).ab,ti.

13. transparent baby bag*.ab,ti.

14. baby bag*.ab,ti.

15. (headwrap* or polyester headwrap*).ti,ab.

16. silver swaddling ${ }^{\star} . t i, a b$.

17. (silver and swaddling ${ }^{\star}$ ).ti,ab.

18. swaddling*.ti,ab.

19. exp Incubators, Infant/

20. radiant warmer*.ti,ab.

21. exp Membranes, Artificial/

22. (semi-permeable membrane ${ }^{\star}$ or semipermeable membrane*).ti,ab.

23. exp POLYURETHANES/

24. polyurethane ${ }^{\star} . t i, a b$.

25. kangaroo care $. t i, a b$.

26. skin to skin contact ${ }^{\star} . t i, a b$.

27. heat ${ }^{\star}$ mattress ${ }^{\star} . t i, a b$.

28. chemical gel mattress ${ }^{\star}$.ti,ab.

29. gel mattress ${ }^{\star} . t i, a b$.

30. chemical mattress ${ }^{\star} . t i, a b$.

31. head hood*.ti,ab.

32. head insulation*.ti,ab.

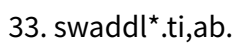

34. plastic bag*.ti,ab. 
35. early suck*.ti,ab.

36. Breast Feeding/

37. (breast feed ${ }^{\star}$ or breastfeed $\left.{ }^{\star}\right)$.ti,ab.

38. skin-to-skin.ti,ab.

39. suckl ${ }^{\star} . t i, a b$.

40. bath*.ti,ab.

41. radiant heater ${ }^{\star} . t i, a b$.

42. radiant heat lamp*.ti,ab.

43. (mother ${ }^{\star} \operatorname{adj} 5$ (bab* or infant $\left.^{\star}\right)$ adj5 (contact ${ }^{\star}$ or hold $\left.\left.{ }^{\star}\right)\right)$.ti,ab.

44. heated bed*.ti,ab.

45. resuscitat ${ }^{\star} . t i, a b$.

46. $\exp$ RESUSCITATION/

47. (intervention* or procedure* or method ${ }^{\star}$ ).ti,ab.

48. exp Infant, Low Birth Weight/

49. ((birth* or bab* or infant) and (premature or pre-mature or preterm or pre-term or low weight or low birth weight or very low birth weight or VLBW or LBW)).ab,ti.

50. Infant, Newborn/

51. Body Temperature Regulation/ or Body Temperature/

52. HYPOTHERMIA/

53. (hypothermia or cold stress or heat loss or temperature regulation or body temperature).ab,ti.

54. temperature drop ${ }^{\star} . \mathrm{ti}, \mathrm{ab}$.

55. heat loss.ti,ab.

56. or $/ 1-47$

57. or $/ 48-50$

58. or/51-55

59. 56 and 57 and 58

\section{Appendix 2. Standard search methods}

PubMed: ((infant, newborn[MeSH] OR newborn OR neonate OR neonatal OR premature OR low birth weight OR VLBW OR LBW or infan* or neonat ${ }^{\star}$ ) AND (randomized controlled trial [pt] OR controlled clinical trial [pt] OR Clinical Trial[ptyp] OR randomized [tiab] OR placebo [tiab] OR clinical trials as topic [mesh: noexp] OR randomly [tiab] OR trial [ti]) NOT (animals [mh] NOT humans [mh]))

Embase: (infant, newborn or newborn or neonate or neonatal or premature or very low birth weight or low birth weight or VLBW or LBW or Newborn or infan* or neonat*) AND (human not animal) AND (randomized controlled trial or controlled clinical trial or randomized or placebo or clinical trials as topic or randomly or trial or clinical trial)

CINAHL: (infant, newborn OR newborn OR neonate OR neonatal OR premature OR low birth weight OR VLBW OR LBW or Newborn or infan* or neonat ${ }^{\star}$ ) AND (randomized controlled trial OR controlled clinical trial OR randomized OR placebo OR clinical trials as topic OR randomly OR trial OR PT clinical trial)

Cochrane Library: (infant or newborn or neonate or neonatal or premature or very low birth weight or low birth weight or VLBW or LBW)

\section{WHAT'S NEW}

\begin{tabular}{lll}
\hline Date & Event & Description \\
\hline 16 January 2017 & New search has been performed & $\begin{array}{l}\text { We added } 18 \text { new studies and } 10 \text { new comparison groups to the } \\
\text { quantitative syntheses. }\end{array}$ \\
\hline 16 January 2017 & $\begin{array}{l}\text { New citation required and conclusions } \\
\text { have changed }\end{array}$ & $\begin{array}{l}\text { This updates the review "Interventions to prevent hypothermia } \\
\text { at birth in preterm and/or low birth weight infants", published in } \\
\text { the Cochrane Database of Systematic Reviews, Issue 3, 2010 (Mc- } \\
\text { Call 2010). }\end{array}$ \\
& $\begin{array}{l}\text { For this review update, we expanded our inclusion criteria to re- } \\
\text { flect changes in clinical practice. We remain focused on inter- } \\
\text { ventions to prevent hypothermia applied immediately at birth } \\
\text { apart from 'routine' care (as defined in our original protocol), } \\
\text { but we have also included randomised and quasi-randomised } \\
\text { controlled trials comparing any other single/combination of in- }\end{array}$ \\
& & \\
& &
\end{tabular}




$\begin{array}{lll}\text { Date Event Description } & \text { Den }\end{array}$

tervention(s) also designed for prevention of hypothermia in preterm and/or low birth weight infants, and applied within 10 minutes after birth in the delivery room.

We added the methods and plans for 'Summary of findings' tables and GRADE recommendations, which we did not include in the original protocol nor in the original review.

\section{H I S T O R Y}

Protocol first published: Issue 2, 2003

Review first published: Issue 1, 2005

\begin{tabular}{lll}
\hline Date & Event & Description \\
\hline 10 February 2010 & $\begin{array}{l}\text { New citation required but conclusions } \\
\text { have not changed }\end{array}$ & $\begin{array}{l}\text { We included a new citation and did not change the Review Con- } \\
\text { clusions. }\end{array}$ \\
\hline
\end{tabular}

10 February $2010 \quad$ New search has been performed

This updates the review "Interventions to prevent hypothermia at birth in preterm and/or low birthweight infants", published in the Cochrane Database of Systematic Reviews, Issue 1, 2008 (McCall 2008).

We included 1 additional eligible study.

We included data for the plastic wrap vs routine care comparison group and added a new comparison group - 'Plastic cap vs routine care'.

\begin{tabular}{lll}
\hline 3 July 2008 & Amended & We converted the review to new review format. \\
\hline 13 September 2007 & $\begin{array}{l}\text { New citation required but conclusions } \\
\text { have not changed }\end{array}$ & We made substantive amendments to the review. \\
\hline 13 September 2007 & New search has been performed & $\begin{array}{l}\text { This review updates the existing review "Interventions to prevent } \\
\text { hypothermia at birth in preterm and/or low birthweight babies", } \\
\text { published in the Cochrane Library, Issue 1, 2005 (McCall 2005). }\end{array}$ \\
& $\begin{array}{l}\text { We updated literature searches to July week 4, 2007, and found } \\
\text { no additional studies that fulfilled the criteria for inclusion in } \\
\text { this review. We updated secondary outcome measures for Vohra } \\
2004 \text { as a result of publication of the full manuscript. }\end{array}$ \\
& $\begin{array}{l}\text { We made no changes to the Review Conclusions nor to implica- } \\
\text { tions for practice or research. }\end{array}$ \\
\hline
\end{tabular}

\section{CONTRIBUTIONS OF AUTHORS}

This review was conceived by the Northern Ireland Neonatal Intensive Care Outcomes Research and Evaluation Group (NICORE).

The contact review author (EC) co-ordinated the review and prepared the manuscript in RevMan 5.3. EC and the QUB Medical Faculty Librarians designed the search strategy. EC drafted the data extraction proforma, which was edited by FA, HH, LJ, and SV. FA, HH, LJ, SV, and EC (this update, EC and FA) screened abstracts and papers against the inclusion criteria, appraised their quality, and extracted trial data. EC entered the data into RevMan 5.3. HH, LJ, and SV provided clinical input at all stages of the review process. FA and HH provided methodological input. All review authors were involved equally in development of content of the final manuscript. 


\section{DECLARATIONS OF INTEREST}

SV is principal investigator on three studies that met the review eligibility criteria and was excluded from the study appraisal process for these studies.

\section{SOURCES OF SUPPORT}

\section{Internal sources}

- Northern Ireland Neonatal Intensive Care Outcomes Research and Evaluation Group (NICORE), UK.

- Eunice Kennedy Shriver National Institute of Child Health and Human Development National Institutes of Health, Department of Health and Human Services, USA.

Editorial support of the Cochrane Neonatal Review Group has been funded with Federal funds from the Eunice Kennedy Shriver National Institute of Child Health and Human Development National Institutes of Health, Department of Health and Human Services, USA, under Contract No. HHSN275201600005C

\section{External sources}

- Research \& Development Office - Northern Ireland, UK.

\section{DIFFERENCES BETWEEN PROTOCOL AND REVIEW}

For this review update, we expanded our inclusion criteria to reflect changes in clinical practice. We remain focused on interventions to prevent hypothermia applied immediately at birth apart from 'routine' care (as defined in our original protocol) but have also included randomised and quasi-randomised controlled trials comparing any other single/combination of intervention(s) also designed for prevention of hypothermia in preterm and/or low birth weight infants, and applied within 10 minutes after birth in the delivery room.

We added the methods and the plan for 'Summary of findings' tables and GRADE recommendations, which were not included in the original protocol nor in the original review.

\section{INDEX TERMS}

\section{Medical Subject Headings (MeSH)}

*Infant, Low Birth Weight; Hypothermia [ ${ }^{*}$ prevention \& control]; Infant, Premature; Infant, Premature, Diseases [ ${ }^{\star}$ prevention \& control]; Perinatal Care [methods]; Randomized Controlled Trials as Topic

\section{MeSH check words}

Humans; Infant, Newborn 